\title{
Water-Quality Data for the Arkansas River Basin, Southeastern Colorado, 1990-93
}

by Russell G. Dash and Roderick F. Ortiz

\author{
U.S. GEOLOGICAL SURVEY
}

Open-File Report 95-464

Prepared in cooperation with the

COLORADO SPRINGS UTILITIES, WATER RESOURCES DEPARTMENT;

PUEBLO BOARD OF WATER WORKS;

SOUTHEASTERN COLORADO WATER CONSERVANCY DISTRICT;

PUEBLO COUNTY, DEPARTMENT OF PLANNING AND DEVELOPMENT;

CITY OF AURORA, DEPARTMENT OF UTILITIES;

ST. CHARLES MESA WATER DISTRICT;

UPPER ARKANSAS AREA COUNCIL OF GOVERNMENTS;

UPPER ARKANSAS WATER CONSERVANCY DISTRICT;

CITY OF PUEBLO, DEPARTMENT OF UTILITIES;

PUEBLO WEST METROPOLITAN DISTRICT;

FREMONT SANITATION DISTRICT;

CITY OF ROCKY FORD; CITY OF LAS ANIMAS; CITY OF LAMAR;

and the BUREAU OF RECLAMATION

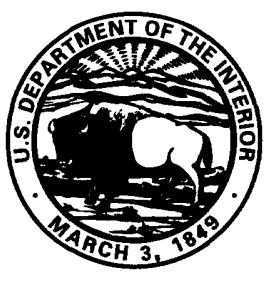




\section{U.S. DEPARTMENT OF THE INTERIOR \\ BRUCE BABBITT, Secretary}

U.S. GEOLOGICAL SURVEY

Gordon P. Eaton, Director

The use of trade, product, industry, or firm names is for descriptive purposes only and does not imply endorsement by the U.S. Government.

For additional information write to:

Copies of this report can be purchased from:

District Chief

U.S. Geological Survey

Box 25046, MS 415

Denver Federal Center

Denver, CO 80225
U.S. Geological Survey

Earth Science Information Center

Open-File Reports Section

Box 25286, MS 517

Denver Federal Center

Denver, CO 80225 


\section{CONTENTS}

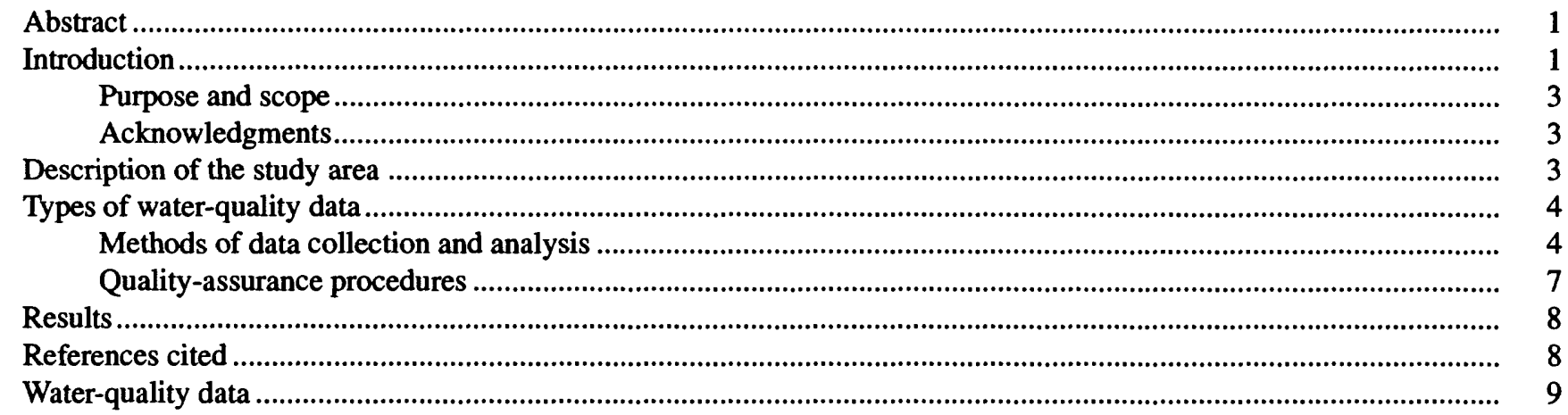

\section{PLATE}

[In pocket]

1. Map showing location of surface-water stations in the Arkansas River Basin of Colorado where water-quality data were collected between 1990 and 1993

\section{FIGURE}

1. Map showing location of study area.

\section{TABLES}

1. Selected surface-water stations in the Arkansas River Basin and water-quality constituents measured and analyzed between 1990 and 1993

2-7. Onsite measurements and selected inorganic data for:

2. Station 09061500, Columbine Ditch near Fremont Pass

3. Station 09062000, Ewing Ditch at Tennessee Pass

4. Station 09062500, Wurtz Ditch near Tennessee Pass ............................................................................. 13

5. Station 392005106130501, East Fork Arkansas River below French Gulch, near Climax .......................... 14

6. Station 391937106200301, Tennessee Creek at Highway 24, near Leadville .......................................... 15

7. Station 07079200, Leadville Mine Drainage Tunnel at Leadville......................................................... 16

8. Onsite measurements and bacteriological and selected inorganic data for station 07081200 ,

Arkansas River near Leadville

9-15. Onsite measurements and selected inorganic data for:

9. Station 07081800 , California Gulch at Malta..

10. Station 07082000, Lake Fork above Sugar Loaf Reservoir .................................................................... 23

11. Station 09063700, Homestake Tunnel near Leadville .......................................................................... 24

12. Station 09077160, Charles H. Bousted Tunnel near Leadville................................................................ 25

13. Station 09077500, Busk-Ivanhoe Tunnel at East Portal, near Malta .............................................................. 26

14. Station 07082500, Lake Fork below Sugar Loaf Reservoir ................................................................. 27

15. Station 391226106213201, Lake Fork above Halfmoon Creek, near Malta .............................................. 28

16. Onsite measurements and bacteriological and selected inorganic data for station 07083000 ,

Halfmoon Creek near Malta. 
17-18. Onsite measurements and selected inorganic data for:

17. Station 391120106194901, Iowa Gulch at Highway 24, near Malta......................................................... 31

18. Station 391013106190201, Empire Gulch near Malta ........................................................................ 32

19. Onsite measurements and bacteriological and selected inorganic data for station 07083710 ,

Arkansas River below Empire Gulch, near Malta

20-26. Onsite measurements and selected inorganic data for:

20. Station 09073000, Twin Lakes Tunnel at East Portal, near Twin Lakes .................................................... 36

21. Station 07084500, Lake Creek above Twin Lakes Reservoir........................................................................... 37

22. Station 390444106174900, Lake Creek at State Highway 82, below Twin Lakes Reservoir....................... 38

23. Station 07086000, Arkansas River at Granite ......................................................................................... 41

24. Station 07086500, Clear Creek above Clear Creek Reservoir .................................................................. 44

25. Station 07087000, Clear Creek below Clear Creek Reservoir .................................................................. 45

26. Station 390009106135001, Pine Creek at mouth, at Highway 24............................................................... 46

27. Onsite measurements and bacteriological and selected inorganic data for station 07087200 ,

Arkansas River at Buena Vista

28-29. Onsite measurements and selected inorganic data for:

28. Station 07089520, Cottonwood Creek at Buena Vista

29. Station 384427106040101 , Chalk Creek at mouth, at Nathrop.

30-31. Onsite measurements and bacteriological and selected inorganic data for:

30. Station 07091200, Arkansas River near Nathrop

31. Station 07091500, Arkansas River at Salida

32. Onsite measurements and selected inorganic data for station 07093500, South Arkansas River near Salida.

33. Onsite measurements and bacteriological and selected inorganic data for station 07093700 ,

Arkansas River near Wellsville.

34-36. Onsite measurements and selected inorganic data for:

34. Station 07093775, Badger Creek, Lower Station, near Howard

35. Station 07094000, Texas Creek at Texas Creek

36. Station 382917105225200 , Tallahassee Creek near Parkdale

37. Onsite measurements and bacteriological and selected inorganic data for station 07094500 ,

Arkansas River at Parkdale.

38-41. Onsite measurements and selected inorganic data for:

38. Station 383113105160401, Grape Creek at mouth, at Canon City …..................................................... 70

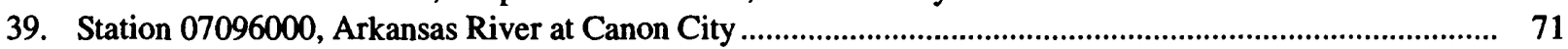

40. Station 07096500, Fourmile Creek near Canon City ..................................................................... 72

41. Station 382337105014600, Hardscrabble Creek at Highway 120, at Portland ......................................... 73

42. Onsite measurements and bacteriological and selected inorganic data for station 07097000 ,

Arkansas River at Portland

43. Onsite measurements and selected inorganic data for station 07099100, Beaver Creek near

Portland

44-48. Onsite measurements and bacteriological and selected inorganic data for:

44. Station 07099400, Arkansas River above Pueblo.

45. Station 07099970, Arkansas River at Moffat Street, at Pueblo ................................................................. 83

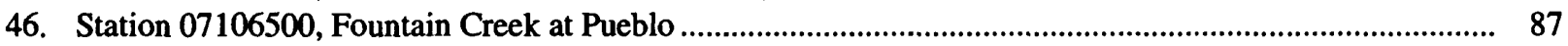

47. Station 381510104350601, Arkansas River below Highway 227, at Pueblo............................................ 90

48. Station 07109500, Arkansas River near Avondale ......................................................................... 93

49-51. Onsite measurements and selected inorganic data for:

49. Station 07116500, Huerfano River near Boone.

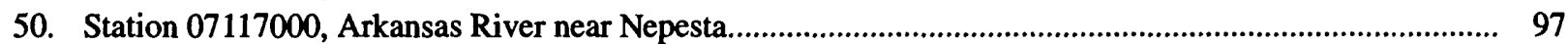

51. Station 380715103564701, Apishapa River at Highway 50, near Fowler ............................................... 98

52. Onsite measurements and bacteriological and selected inorganic data for station 07119700 ,

Arkansas River at Catlin Dam, near Fowler. 
53-56. Onsite measurements and selected inorganic data for:

53. Station 380111103382101, Timpas Creek at Highway 50, at Swink ..................................................... 103

54. Station 07122500, Crooked Arroyo near La Junta ............................................................................ 105

55. Station 07123000, Arkansas River at La Junta.................................................................................. 106

56. Station 380421103193101, Horse Creek at mouth, near Las Animas ....................................................... 107

57. Onsite measurements and bacteriological and selected inorganic data for station 07124000 ,

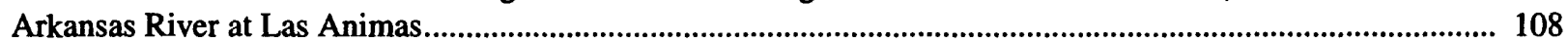

58. Onsite measurements and selected inorganic data for station 07128500 , Purgatoire River

near Las Animas

59. Onsite measurements and bacteriological and selected inorganic data for station 07130500 ,

Arkansas River below John Martin Reservoir

60. Onsite measurements and selected inorganic data for station 07134100, Big Sandy Creek

near Lamar.

61. Pesticide data for selected surface-water stations on the Arkansas River, 1990-92 _......................................... 117

62. Radiochemical data for selected surface-water stations on the Arkansas River, 1990-92................................. 123

63. Suspended-sediment data for selected surface-water stations on or near the Arkansas River, 1990-93.

64-65. Quality-assurance data for:

64. Source-solution blanks of deionized water for the Arkansas River Basin water-quality study, 1990-93...

65. Field-equipment blanks for the Arkansas River Basin water-quality study, 1990-93 136

\section{CONVERSION FACTORS, ABBREVIATIONS, AND VERTICAL DATUM}

\begin{tabular}{|c|c|c|}
\hline Multiply & By & To obtaln \\
\hline $\begin{array}{r}\text { acre } \\
\text { centimeter }\end{array}$ & $\begin{array}{l}0.4047 \\
0.3937\end{array}$ & $\begin{array}{l}\text { hectare } \\
\text { inch }\end{array}$ \\
\hline cubic foot per second $\left(\mathrm{ft}^{3} / \mathrm{s}\right)$ & $\begin{array}{l}0.02832 \\
1.9835\end{array}$ & $\begin{array}{l}\text { cubic meter per second } \\
\text { acre-foot per day }\end{array}$ \\
\hline foot (ft) & 0.3048 & meter \\
\hline inch (in.) & 2.54 & centimeter \\
\hline liter & 0.2642 & gallon (US) \\
\hline mile (mi) & 1.609 & kilometer \\
\hline million gallons per day (Mgal/d) & 0.043 & cubic meter per second \\
\hline milliliter & 0.3381 & fluid ounce \\
\hline millimeter $(\mathrm{mm})$ & 0.03937 & inches \\
\hline $\begin{array}{r}\text { square mile }\left(\mathrm{mi}^{2}\right) \\
\text { ton per day }(\mathrm{t} / \mathrm{day})\end{array}$ & $\begin{array}{l}2.59 \\
0.9072\end{array}$ & $\begin{array}{l}\text { square kilometer } \\
\text { megagram per day }\end{array}$ \\
\hline
\end{tabular}

Degree Celsius $\left({ }^{\circ} \mathrm{C}\right)$ may be converted to degree Fahrenheit $\left({ }^{\circ} \mathrm{F}\right)$ by using the following equation:

$$
{ }^{\circ} \mathrm{F}=9 / 5\left({ }^{\circ} \mathrm{C}\right)+32 \text {. }
$$

Degree Fahrenheit $\left({ }^{\circ} \mathrm{F}\right)$ may be converted to degree Celsius $\left({ }^{\circ} \mathrm{C}\right)$ by using the following equation:

$$
{ }^{\circ} \mathrm{C}=5 / 9\left({ }^{\circ} \mathrm{F}-32\right) \text {. }
$$

Sea level: In this report "sea level" refers to the National Geodetic Vertical Datum of 1929 (NGVD of 1929)-a geodetic datum derived from a general adjustment of the first-order level nets of both the United States and Canada, formerly called Sea Level Datum of 1929. 


\title{
Water-Quality Data for the Arkansas River Basin, Southeastern Colorado, 1990-93
}

\author{
By Russell G. Dash and Roderick F. Ortiz
}

\section{Abstract}

Water-quality data were collected and compiled for 59 surface-water stations in the Arkansas River Basin of Colorado. The purpose of the data collection was to describe selected water-quality characteristics of the Arkansas River from the headwaters downstream to the Colorado-Kansas State line. Data are presented for 19 Arkansas River stations, 31 tributary stations, 2 minedrainage stations, and 7 transmountain diversion stations. Water-quality data presented in this report include instantaneous discharge; onsite measurements of specific conductance, $\mathrm{pH}$, water temperature, and dissolved oxygen; analytical concentrations of bacteria, dissolved solids, major nutrients, trace elements, pesticides, radiochemicals, and suspended sediment; and qualityassurance data for selected water-quality constituents. Sampling began in April 1990 and continued through March 1993 at the 59 surface-water stations. The basinwide water-quality study was initiated in 1988 by the U.S. Geological Survey in cooperation with 14 local agencies and the Bureau of Reclamation.

\section{INTRODUCTION}

Water resources in the Arkansas River Basin of Colorado are critically important to southeastern Colorado. The Arkansas River originates in the Rocky Mountains near Leadville (fig. 1) and drains an area of about $25,400 \mathrm{mi}^{2}$. As the river flows southward and then eastward toward the State of Kansas, the flow is affected by diversions for agriculture, power development, and municipal, industrial, and recreational usage. These water uses can affect water quality in the drainage basin.

In recent years, operation of the existing watersupply system and proposed changes in water-use administration have focused attention on water quality in the Arkansas River Basin. The concern that water quality could limit some water uses resulted in the initiation of a U.S. Geological Survey study in 1988, in cooperation with the Colorado Springs Utilities, Water Resources Department; Pueblo Board of Water Works; Southeastern Colorado Water Conservancy District; Pueblo County, Department of Planning and Development; City of Aurora, Department of Utilities; St. Charles Mesa Water District; Upper Arkansas Area Council of Governments; Upper Arkansas Water Conservancy District; City of Pueblo, Department of Utilities; Pueblo West Metropolitan District; Fremont Sanitation District; Cities of Rocky Ford, Las Animas, and Lamar; and the Bureau of Reclamation.

In the early phases of the study, 13 water-quality issues in the Arkansas River Basin were identified by the cooperating agencies and the U.S. Geological Survey. The issues of highest priority were the determination of existing water-quality characteristics in the basin and the establishment of a consistent basinwide network to identify and monitor water-quality changes. Subsequently, a basinwide surface-water monitoring network was designed to provide reliable water-quality information that could be used to (1) evaluate downstream and seasonal variations in water quality;

(2) assess variations in water quality that occur during different flow periods; and (3) assess regional effects on water quality that might occur as a result of water and land uses, tributary inflows, point and nonpoint source discharges, and natural, climatic, and geological conditions. 

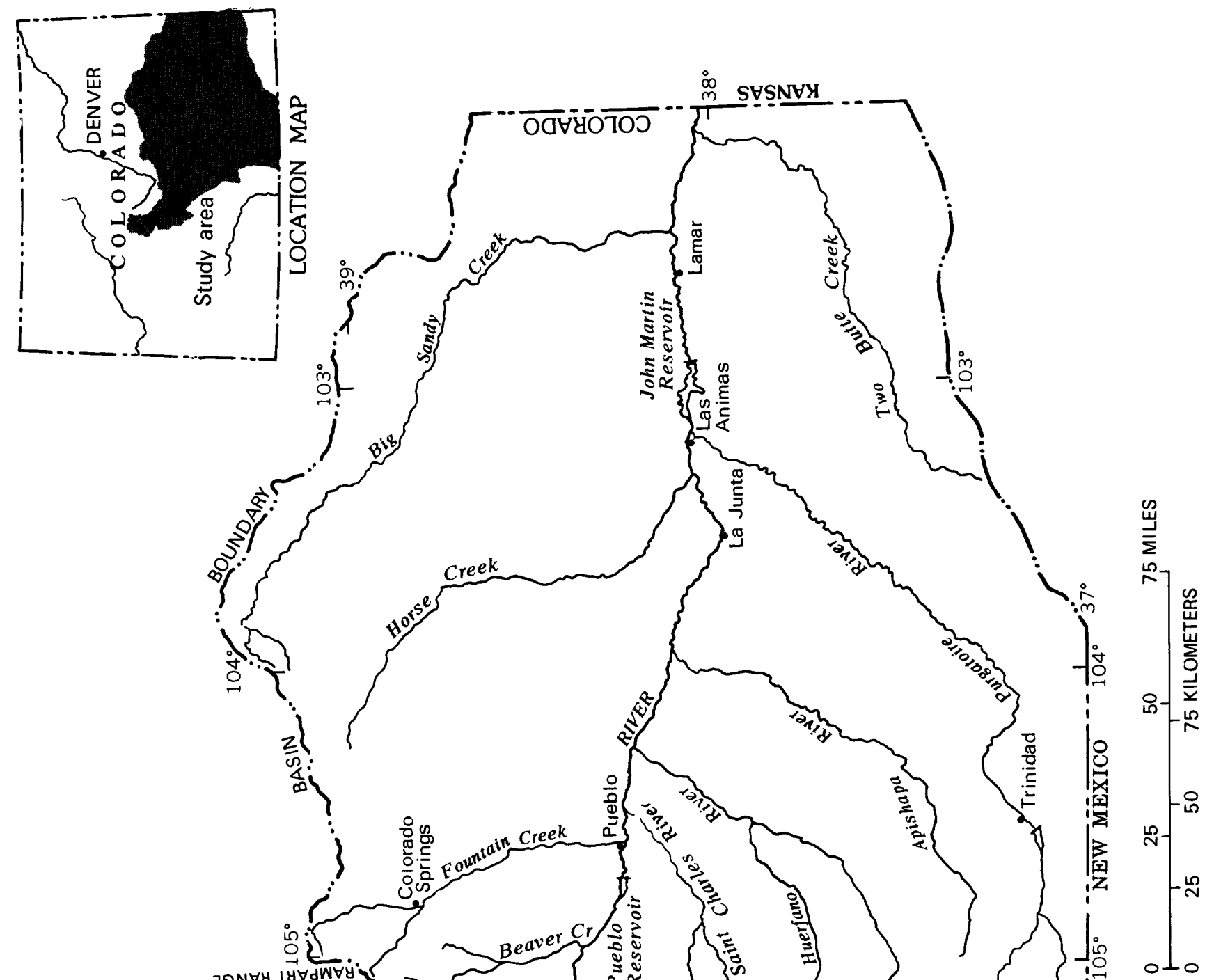

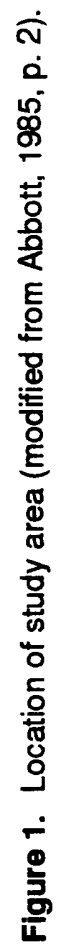




\section{Purpose and Scope}

This report lists the types of water-quality data collected, describes methods of data-collection, analysis, and quality-assurance procedures and presents a compilation of selected water-quality data. Data were collected from April 1990 through March 1993 at 59 surface-water stations in the Arkansas River Basin of Colorado (pl. 1) that included 19 Arkansas River stations, 31 tributary stations, 2 mine-drainage stations, and 7 transmountain diversion stations. Water-quality data presented in this report include onsite measurements of instantaneous discharge, specific conductance, $\mathrm{pH}$, water temperature, and dissolved oxygen; bacteriological analyses; chemical analyses of selected inorganic constituents, pesticides, and radiochemicals; analyses of suspended-sediment concentration; and analytical quality-assurance data for selected waterquality constituents.

\section{Acknowledgments}

The authors gratefully acknowledge the assistance of many government and local agencies that cooperated with the U.S. Geological Survey to develop a basinwide water-quality study. Appreciation is extended to the many landowners along the Arkansas River corridor who permitted access to their property to measure discharge and to collect water samples. The authors thank State hydrographers Frank Kipple and Tony Gutierrez, Colorado Department of Natural Resources, Division II of Water Resources, Office of the State Engineer, for their timely assistance with streamflow determination. Special thanks are extended to Jeffrey West, Charles (Chuck) Moore, and Matthew Kurchinski of the U.S. Geological Survey for the collection and compilation of water-quality data.

\section{DESCRIPTION OF THE STUDY AREA}

The study area includes the entire Arkansas River Basin of Colorado and consists of approximately the southeast one-quarter of the State (fig. 1). Elevations in the basin range from $3,350 \mathrm{ft}$ above sea level at the Colorado-Kansas State line to $14,433 \mathrm{ft}$ at the highest mountain peak in Colorado. Mean annual precipitation in the basin ranges from more than 40 in. in the mountains to less than $10 \mathrm{in}$. in areas of the eastern plains (Colorado Climate Center, 1984).
Streamflow in the Arkansas River primarily is from melting of snow that accumulates in the mountains from October through May each year. At lower elevations, runoff from summer thunderstorms can contribute substantial quantities of streamflow for short periods of time. Much of the streamflow that occurs east of La Junta (fig. 1) can be irrigation-return flow during parts of most years (Cain, 1985). Mean annual runoff decreases from more than $30 \mathrm{in}$. in the mountains to less than 0.1 in. downstream from Pueblo (Abbott, 1985). For the interested reader, a more comprehensive description of the historical water resources of the Arkansas River Basin of Colorado is reported in Crouch and others (1984), Abbott (1985), Burns (1985), Cain (1985), and Kuzmiak and Strickland (1994).

Land use in the study area predominantly is agricultural, consisting of rangeland and cropland areas throughout the drainage basin. National forests are located upstream from Canon City and cover about one-third of the upper Arkansas River Basin. The upper basin extends downstream to about Pueblo. Historically, substantial mining of precious metals occurred in the basin upstream from Canon City, but most of the mines are abandoned and ranching presently (1994) is a principal land use in the upper basin. Irrigation water use constitutes the largest withdrawals of surface water in the Arkansas River Basin. It was estimated that irrigation used about $1,730 \mathrm{Mgal} / \mathrm{d}$ of water during 1985 , with 88 percent of the total irrigation withdrawal from surfacewater sources (Litke and Appel, 1989). More than 411,000 acres of alluvial lands in the basin are irrigated, including about 56,000 irrigated acres located in the upper basin. Mainly alfalfa, hay, and pasture grass are irrigated in the upper basin, although fruits and some grain crops are grown on irrigated land in the foothills located around Canon City. Truck-crops, alfalfa, and grain-crops are grown on irrigated land in the lower basin downstream from Pueblo. Most of the nonirrigated agricultural lands in the basin are used for rangeland or dryland wheat production.

Population in the study area was determined by the 1990 census (U.S. Bureau of the Census, 1991) to be 641,700 , about 19 percent of the total population of Colorado. Most of the population is located near the cities of Colorado Springs and Pueblo or concentrated in small towns and rural areas along the Arkansas River corridor. 


\section{TYPES OF WATER-QUALITY DATA}

The types of water-quality data measured and analyzed at each surface-water station are listed in table I. The surface-water stations are presented in downstream order. Special samples at some stations indicate the irregular collection and analysis of additional water-quality constituents that were appended to routine samples normally collected during station visits. Continuous water-quality and discharge data have been collected at some of the stations, but are not published in this report. However, these data are published in the annual Colorado Water-Data Report series and are available upon request. The types of waterquality data presented in this report are:

1. Onsite measurements, including instantaneous discharge and field determination of specific conductance, $\mathrm{pH}$, water temperature, and dissolved-oxygen concentration.

2. Bacteriological field analyses, including total coliform, fecal coliform, and fecal streptococci.

3. Dissolved solids, including alkalinity, dissolved calcium, dissolved magnesium, dissolved sodium, dissolved sulfate, dissolved chloride, and dissolved-solids residue at $180^{\circ} \mathrm{C}$.

4. Major nutrients, including total nitrite plus nitrate as nitrogen and total ammonia as nitrogen for I990 through 1992; dissolved nitrite plus nitrate as nitrogen and dissolved ammonia as nitrogen for 1993; and total phosphorus for I990 through I 993.

5. Trace elements, including total and dissolved arsenic, cadmium, chromium, copper, iron, lead, manganese, mercury, nickel, selenium, silver, and zinc.

6. Pesticides, including organochlorine and organophosphorus insecticides and the chlorinated phenoxy-acid herbicides.

7. Radiochemical constituents, including total and dissolved gross alpha and gross beta constituents and dissolved natural uranium.

8. Suspended sediment, including suspendedsediment concentration and the percentage of the suspended sediment finer than sand (less than $0.062 \mathrm{~mm}$ ).

\section{Methods of Data Collection and Analysis}

Before each field trip, a standard cleaning and rinsing procedure was used to prepare the equipment for water-quality sampling. Sampling equipment was completely disassembled and washed thoroughly using a solution of nonphosphate laboratory detergent, followed by three thorough rinses using public-supplied tap water. Sampling equipment then was rinsed using a 1-percent hydrochloric acid solution and triple-rinsed using deionized water.

Upon arriving at each surface-water station, prior to a sample collection, the sampling equipment was rinsed once with deionized water, followed by a rinse with dilute hydrochloric acid, followed by a triple-rinse with deionized water. Finally, the sampling equipment was triple-rinsed thoroughly with native streamwater before the collection of a water-quality sample. At all stations sampled downstream from the Arkansas River near Avondale, station 07 109500 (table 1), east of Pueblo to the Colorado-Kansas State line (pl. 1), the dilute hydrochloric acid rinse was omitted because trace-level contamination of samples was not a concern in sampling the lower basin.

Onsite measurements were made during each site visit at surface-water stations using standardized procedures and techniques (U.S. Geological Survey, 1977). Stream discharge continuously was monitored at some stations or a discharge measurement was made at the time of sampling at other stations. Field waterquality analyses were made for specific conductance, $\mathrm{pH}$, water temperature, and dissolved oxygen (Fishman and Friedman, 1989). Water samples for bacteriological analyses were collected onsite at 17 surfacewater stations (table I) with clean sterilized glass bottles using a multivertical sampling procedure (Patrick Edelmann, U.S. Geological Survey, oral commun., 1990). Bacteriological samples were filtered and analyzed in the field using methods described in Britton and Greeson (1987).

Water samples for chemical analyses were collected using standardized U.S. Geological Survey guidelines (Sylvester and others, 1990). Except for extreme flow situations, surface-water samples from the stream were depth-integrated using the equalwidth-increment method (Sylvester and others, 1990) and were transferred into a churn splitter for a composite sample. The churn splitter allowed different subsample volumes to be obtained from the sample while still maintaining the basic chemical and physical properties of the original sample. Numerous aliquots were taken from the churn splitter, processed, and preserved onsite using methods described in Ward and Harr (1990). Water-quality samples were shipped on a regular basis to the laboratory for analysis of the chemical constituents. 
Table 1. Selected surface-water stations in the Arkansas River Basin and water-quality constituents measured and analyzed between 1990 and 1993

[X. routine sampling; S, special sampling; --, not collected or analyzed]

\begin{tabular}{|c|c|c|c|c|c|c|c|c|c|}
\hline $\begin{array}{l}\text { U.S. Geological } \\
\text { Survey station } \\
\text { number } \\
\text { (see plate 1) }\end{array}$ & $\begin{array}{l}\text { Station } \\
\text { name }\end{array}$ & $\begin{array}{c}\text { Onsite } \\
\text { measure- } \\
\text { ments }\end{array}$ & $\begin{array}{l}\text { Bac- } \\
\text { teria }\end{array}$ & $\begin{array}{l}\text { Dis- } \\
\text { solved } \\
\text { solids }\end{array}$ & $\begin{array}{l}\text { Major } \\
\text { nutri- } \\
\text { ents }\end{array}$ & $\begin{array}{l}\text { Trace } \\
\text { ele- } \\
\text { ments }\end{array}$ & $\begin{array}{l}\text { Pesti- } \\
\text { cides }\end{array}$ & $\begin{array}{l}\text { Radio- } \\
\text { chemi- } \\
\text { cals }\end{array}$ & $\begin{array}{l}\text { Sus- } \\
\text { pended } \\
\text { sedi- } \\
\text { ment }\end{array}$ \\
\hline 09061500 & Columbine Ditch near Fremont Pass & $\mathrm{X}$ & -- & $\mathrm{X}$ & $\mathrm{X}$ & $\mathrm{S}$ & -- & -- & -- \\
\hline 09062000 & Ewing Ditch at Tennessee Pass & $\mathrm{X}$ & -- & $\mathrm{X}$ & $\mathrm{X}$ & $\mathrm{s}$ & -- & -- & -- \\
\hline 09062500 & Wurtz Ditch near Tennessee Pass & $\mathrm{x}$ & -- & $\mathrm{x}$ & $\mathrm{x}$ & $\mathrm{X}$ & -- & -- & -- \\
\hline 392005106130501 & $\begin{array}{l}\text { East Fork Arkansas River below } \\
\text { French Gulch, near Climax }\end{array}$ & $\mathrm{x}$ & -- & $\mathrm{X}$ & $\mathrm{X}$ & $\mathrm{X}$ & -- & -- & -- \\
\hline 391937106200301 & $\begin{array}{l}\text { Tennessee Creek at Highway } 24 \text {, } \\
\text { near Leadville }\end{array}$ & $\mathrm{X}$ & -- & $\mathrm{X}$ & $\mathrm{X}$ & $\mathrm{x}$ & -- & -- & -- \\
\hline 07079200 & $\begin{array}{l}\text { Leadville Mine Drainage Tunnel } \\
\text { at Leadville }\end{array}$ & $\mathrm{X}$ & -- & $\mathrm{X}$ & $\mathrm{X}$ & $\mathrm{x}$ & -- & -- & -- \\
\hline 07081200 & Arkansas River near Leadville & $\mathrm{X}$ & $\mathrm{X}$ & $\mathrm{X}$ & $\mathrm{X}$ & $\mathrm{x}$ & -- & -- & $\mathrm{x}$ \\
\hline 07081800 & California Gulch at Malta & $\mathrm{X}$ & -- & $\mathrm{X}$ & $\mathrm{x}$ & $\mathrm{x}$ & -- & -- & -- \\
\hline 07082000 & $\begin{array}{l}\text { Lake Fork above Sugar Loaf } \\
\text { Reservoir }\end{array}$ & $\mathrm{X}$ & -- & $\mathrm{X}$ & $\mathrm{X}$ & $\mathrm{X}$ & -- & -- & - \\
\hline 09063700 & Homestake Tunnel near Leadville & $\mathrm{x}$ & -- & $\mathrm{x}$ & $\mathrm{X}$ & $\mathrm{X}$ & -- & -- & -- \\
\hline 09077160 & $\begin{array}{l}\text { Charles H. Bousted Tunnel near } \\
\text { Leadville }\end{array}$ & $\mathrm{X}$ & -- & $\mathrm{x}$ & $\mathrm{X}$ & $\mathrm{x}$ & -- & -- & -- \\
\hline 09077500 & $\begin{array}{l}\text { Busk-Ivanhoe Tunnel at East Portal, } \\
\text { near Malta }\end{array}$ & $\mathrm{X}$ & - & $\mathrm{x}$ & $\mathrm{X}$ & $\mathrm{x}$ & -- & -- & -- \\
\hline 07082500 & $\begin{array}{l}\text { Lake Fork below Sugar Loaf } \\
\text { Reservoir }\end{array}$ & $\mathrm{x}$ & -- & $\mathrm{x}$ & $\mathrm{X}$ & $\mathrm{X}$ & -- & -- & -- \\
\hline 391226106213201 & $\begin{array}{l}\text { Lake Fork above Halfmoon Creek, } \\
\text { near Malta }\end{array}$ & $\mathrm{x}$ & & $\mathrm{x}$ & $\mathrm{X}$ & $\mathrm{x}$ & -- & -- & -- \\
\hline 07083000 & Halfmoon Creek near Malta & $\mathrm{x}$ & $\mathrm{X}$ & $\mathrm{x}$ & $\mathrm{x}$ & $\mathrm{x}$ & -- & -- & -- \\
\hline 391120106194901 & $\begin{array}{l}\text { Iowa Gulch at Highway 24, } \\
\text { near Malta }\end{array}$ & $\mathrm{x}$ & -- & $\mathrm{x}$ & $\mathrm{x}$ & $\mathrm{x}$ & -- & -- & -- \\
\hline 391013106190201 & Empire Gulch near Malta & $\mathrm{x}$ & -- & $\mathrm{x}$ & $\mathrm{x}$ & $\mathrm{x}$ & -- & -- & -- \\
\hline 07083710 & $\begin{array}{l}\text { Arkansas River below Empire } \\
\text { Gulch, near Malta }\end{array}$ & $\mathrm{X}$ & $\mathrm{X}$ & $\mathrm{X}$ & $\mathrm{X}$ & $\mathrm{x}$ & -- & -- & $\mathrm{X}$ \\
\hline 09073000 & $\begin{array}{l}\text { Twin Lakes Tunnel at East Portal, } \\
\text { near Twin Lakes }\end{array}$ & $\mathrm{X}$ & -- & $\mathrm{X}$ & $\mathrm{X}$ & $\mathrm{x}$ & -- & -- & -- \\
\hline 07084500 & $\begin{array}{l}\text { Lake Creek above Twin Lakes } \\
\text { Reservoir }\end{array}$ & $\mathrm{x}$ & -- & $\mathrm{x}$ & $\mathrm{x}$ & $\mathrm{x}$ & -- & -- & -- \\
\hline 390444106174900 & $\begin{array}{l}\text { Lake Creek at State Highway } 82 \text {, } \\
\text { below Twin Lakes Reservoir }\end{array}$ & $\mathrm{x}$ & -- & $\mathrm{x}$ & $\mathrm{x}$ & $\mathrm{x}$ & -- & -- & -- \\
\hline 07086000 & Arkansas River at Granite & $\mathrm{x}$ & -- & $\mathrm{x}$ & $\mathrm{X}$ & $\mathrm{x}$ & -- & -- & $S$ \\
\hline 07086500 & $\begin{array}{l}\text { Clear Creek above Clear Creek } \\
\text { Reservoir }\end{array}$ & $\mathrm{x}$ & -- & $\mathrm{x}$ & $\mathrm{x}$ & $\mathrm{X}$ & -- & -- & -- \\
\hline 07087000 & $\begin{array}{l}\text { Clear Creek below Clear Creek } \\
\text { Reservoir }\end{array}$ & $\mathrm{x}$ & -- & $\mathrm{x}$ & $\mathrm{x}$ & $\mathrm{x}$ & -- & -- & -- \\
\hline 390009106135001 & Pine Creek at mouth, at Highway 24 & $\mathrm{x}$ & -- & $\mathrm{X}$ & $\mathrm{x}$ & $\mathrm{X}$ & -- & -- & -- \\
\hline 07087200 & Arkansas River at Buena Vista & $\mathrm{x}$ & $\mathrm{X}$ & $\mathrm{X}$ & $\mathrm{X}$ & $\mathrm{X}$ & -- & $\mathrm{X}$ & $\mathrm{X}$ \\
\hline 07089520 & Cottonwood Creek at Buena Vista & $\mathrm{x}$ & -- & $\mathrm{x}$ & $\mathrm{X}$ & $\mathrm{X}$ & -- & -- & -- \\
\hline 384427106040101 & Chalk Creek at mouth, at Nathrop & $\mathrm{x}$ & -- & $\mathrm{X}$ & $\mathrm{X}$ & $\mathrm{X}$ & -- & -- & -- \\
\hline 07091200 & Arkansas River near Nathrop & $\mathrm{x}$ & $\mathrm{X}$ & $\mathrm{x}$ & $\mathrm{X}$ & $\mathrm{X}$ &.- & -- & -- \\
\hline 07091500 & Arkansas River at Salida & $\mathrm{X}$ & $\mathrm{X}$ & $\mathrm{X}$ & $\mathrm{X}$ & - & -- & -- & -- \\
\hline
\end{tabular}


Table 1. Selected surface-water stations in the Arkansas River Basin and water-quality constituents measured and analyzed between 1990 and 1993--Continued

\begin{tabular}{|c|c|c|c|c|c|c|c|c|c|}
\hline $\begin{array}{l}\text { U.S. Geological } \\
\text { Survey station } \\
\text { number } \\
\text { (see plate 1) }\end{array}$ & $\begin{array}{l}\text { Station } \\
\text { name }\end{array}$ & $\begin{array}{c}\text { Onsite } \\
\text { measure- } \\
\text { ments }\end{array}$ & $\begin{array}{l}\text { Bac- } \\
\text { teria }\end{array}$ & $\begin{array}{l}\text { Dis- } \\
\text { solved } \\
\text { solids }\end{array}$ & $\begin{array}{l}\text { Major } \\
\text { nutri- } \\
\text { ents }\end{array}$ & $\begin{array}{l}\text { Trace } \\
\text { ele- } \\
\text { ments }\end{array}$ & $\begin{array}{l}\text { Pesti- } \\
\text { cides }\end{array}$ & $\begin{array}{l}\text { Radio- } \\
\text { chemi- } \\
\text { cals }\end{array}$ & $\begin{array}{l}\text { Sus- } \\
\text { pended } \\
\text { sedi- } \\
\text { ment }\end{array}$ \\
\hline 07093500 & South Arkansas River near Salida & $\mathrm{X}$ & -- & $\mathrm{X}$ & $\bar{X}$ & $\mathrm{X}$ & -- & -- & -- \\
\hline 07093700 & Arkansas River near Wellsville & $\mathrm{X}$ & $\mathrm{x}$ & $\mathrm{x}$ & $\mathrm{x}$ & $\mathrm{x}$ & -- & $\mathrm{X}$ & $\mathrm{X}$ \\
\hline 07093775 & $\begin{array}{l}\text { Badger Creek, Lower Station, near } \\
\text { Howard }\end{array}$ & $\mathrm{X}$ & -- & $\mathrm{X}$ & $\mathrm{X}$ & $\mathrm{X}$ & -- & -- & -- \\
\hline 07094000 & Texas Creek at Texas Creek & $\mathrm{X}$ & -- & $\mathrm{X}$ & $\mathrm{X}$ & -- & -- & -- & -- \\
\hline 382917105225200 & Tallahassee Creek near Parkdale & $\mathrm{x}$ & -- & $\mathrm{X}$ & $\mathrm{x}$ & $\mathrm{X}$ & -- & -- & -- \\
\hline 07094500 & Arkansas River at Parkdale & $\mathrm{X}$ & $\mathrm{X}$ & $\mathrm{X}$ & $\mathrm{X}$ & $\mathrm{X}$ & .. & $\mathrm{X}$ & $\mathrm{X}$ \\
\hline 383113105160401 & $\begin{array}{l}\text { Grape Creek at mouth, at } \\
\text { Canon City }\end{array}$ & $X$ & -. & $\mathrm{X}$ & $\mathrm{x}$ & $\mathrm{S}$ & $\cdots$ & -- & -- \\
\hline 07096000 & Arkansas River at Canon City & $\mathrm{X}$ & -. & $\mathrm{X}$ & $\mathrm{X}$ & -- & -- & -. & -- \\
\hline 07096500 & Fourmile Creek near Canon City & $\mathrm{x}$ & -- & $\mathrm{x}$ & $\mathrm{x}$ & $\mathrm{X}$ & -- & -- & -- \\
\hline 382337105014600 & $\begin{array}{l}\text { Hardscrabble Creek at Highway } 120 \text {, } \\
\text { at Portland }\end{array}$ & $\mathrm{x}$ & -- & $\mathrm{X}$ & $\mathrm{x}$ & -- & -- & -- & -- \\
\hline 07097000 & Arkansas River at Portland & $\mathrm{X}$ & $\mathrm{X}$ & $\mathrm{x}$ & $\mathrm{X}$ & $\mathrm{X}$ & $\mathrm{x}$ & $\mathrm{X}$ & $\mathrm{X}$ \\
\hline 07099100 & Beaver Creek near Portland & $\mathrm{x}$ & -- & $\mathrm{X}$ & $\mathrm{x}$ & $S$ & -- & -- & -- \\
\hline 07099400 & Arkansas River above Pueblo & $\mathrm{x}$ & $\mathrm{X}$ & $\mathrm{X}$ & $\mathrm{X}$ & $\mathrm{X}$ & -- & -- & $\mathrm{X}$ \\
\hline 07099970 & $\begin{array}{l}\text { Arkansas River at Moffat Street, } \\
\text { at Pueblo }\end{array}$ & $\mathrm{X}$ & $\mathrm{X}$ & $\mathrm{X}$ & $\mathrm{X}$ & $\mathrm{X}$ & -- & -- & $\mathrm{X}$ \\
\hline 07106500 & Fountain Creek at Pueblo & $\mathrm{x}$ & $\mathrm{x}$ & $\mathrm{x}$ & $\mathrm{x}$ & $\mathrm{x}$ & -- & -- & $\mathrm{x}$ \\
\hline 381510104350601 & $\begin{array}{l}\text { Arkansas River below Highway } 227 \text {, } \\
\text { at Pueblo }\end{array}$ & $\mathrm{x}$ & $\mathrm{X}$ & $\mathrm{x}$ & $\mathrm{X}$ & $\mathrm{X}$ & -- & -- & -- \\
\hline 07109500 & Arkansas River near Avondale & $\mathrm{X}$ & $\mathrm{X}$ & $\mathrm{X}$ & $\mathrm{X}$ & $\mathrm{X}$ & $\mathrm{X}$ & $\mathrm{X}$ & $\mathrm{X}$ \\
\hline 07116500 & Huerfano River near Boone & $\mathrm{x}$ & -- & $\mathrm{X}$ & $\mathrm{x}$ & -- & -- & -- & -- \\
\hline 07117000 & Arkansas River near Nepesta & $\mathrm{x}$ & -- & $\mathrm{x}$ & $\mathrm{X}$ & -- & -- & -- & -- \\
\hline 380715103564701 & $\begin{array}{l}\text { Apishapa River at Highway } 50 \text {, } \\
\text { near Fowler }\end{array}$ & $\mathrm{X}$ & -- & $\mathrm{X}$ & $\mathrm{X}$ & $s$ & -- & -- & $\mathbf{S}$ \\
\hline 07119700 & $\begin{array}{l}\text { Arkansas River at Catlin Dam, } \\
\text { near Fowler }\end{array}$ & $\mathrm{X}$ & $\mathrm{X}$ & $\mathrm{X}$ & $\mathrm{x}$ & $\mathrm{X}$ & $\mathrm{x}$ & $\mathrm{X}$ & $\mathrm{X}$ \\
\hline 380111103382101 & $\begin{array}{l}\text { Timpas Creek at Highway } 50 \text {, } \\
\text { at Swink }\end{array}$ & $\mathrm{X}$ & -- & $\mathrm{X}$ & $\mathrm{x}$ & $\mathrm{s}$ & -- & -- & -- \\
\hline 07122500 & Crooked Arroyo near La Junta & $\mathrm{X}$ & -- & $\mathrm{X}$ & $\mathrm{X}$ & -- & -. & -- & -- \\
\hline 07123000 & Arkansas River at La Junta & $\mathrm{x}$ & -- & $\mathrm{X}$ & $\mathrm{X}$ & -- & -- & -- & -- \\
\hline 380421103193101 & $\begin{array}{l}\text { Horse Creek at mouth, near } \\
\text { Las Animas }\end{array}$ & $\mathrm{x}$ & -- & $\mathrm{X}$ & $\mathrm{X}$ & -- & -- & -- & -- \\
\hline 07124000 & Arkansas River at Las Animas & $\mathrm{x}$ & $\mathrm{X}$ & $\mathrm{X}$ & $\mathrm{X}$ & $\mathrm{X}$ & $\mathrm{X}$ & $\mathrm{X}$ & $\mathrm{X}$ \\
\hline 07128500 & Purgatoire River near Las Animas & $\mathrm{x}$ & -- & $\mathrm{X}$ & $\mathrm{x}$ & $S$ & -- & -- & -- \\
\hline 07130500 & $\begin{array}{l}\text { Arkansas River below John Martin } \\
\text { Reservoir }\end{array}$ & $\mathrm{X}$ & $\mathrm{X}$ & $\mathrm{X}$ & $\mathrm{X}$ & $X$ & $\mathrm{X}$ & $\mathrm{X}$ & $\mathrm{X}$ \\
\hline 07134100 & Big Sandy Creek near Lamar & $\mathrm{X}$ & -- & $\mathrm{X}$ & $\mathrm{X}$ & -- & -- & -- & -- \\
\hline
\end{tabular}


The chemical constituents presented in this report were analyzed at the U.S. Geological Survey National Water Quality Laboratory (NWQL) in Arvada, Colo., using methods listed in Fishman and others (1994). The inorganic constituents, including dissolved solids, major nutrients, and trace elements, were analyzed using methods described by Fishman and Friedman (1989) and Fishman (1993). Pesticides were periodically collected at five surface-water stations (table 1) and were analyzed using methods described by Wershaw and others (1987) and Fishman (1993). Radiochemical constituents were collected at eight surface-water stations (table 1) and were analyzed using methods developed by Thatcher and others (1977).

The nitrogen data presented in the "WaterQuality Data" section at the back of this report indicate a change in the reporting of total nitrite plus nitrate as nitrogen and total ammonia as nitrogen after January 1, 1993. Following a thorough laboratory evaluation of the analytical method used to generate data for the above constituents on unfiltered and filtered samples, the NWQL concluded that "no valid basis exists for distinguishing between unfiltered and filtered determinations of nutrient species" using the current (1992) method, and "concentrations for the identified nutrient species are statistically indistinguishable" (U.S. Geological Survey, written commun., 1992). As a result, nutrient samples collected for the analysis of total nitrite plus nitrate as nitrogen and total ammonia as nitrogen were replaced by the analyses of the dissolved fraction in 1993. The analysis of total phosphorus was not affected by this change.

Suspended-sediment samples were periodically collected at 15 surface-water stations (table 1) using methods described by Guy and Norman (1970). The samples were collected using a DH-48 (hand-held sampler) or a D-74 (cable-and-reel sampler) depthintegrating sampler and the equal-width-increment method described by Sylvester and others (1990). Suspended-sediment samples were analyzed at the U.S. Geological Survey Sediment Laboratory in Cheyenne, Wyo., using methods described by Guy (1969). Samples containing sufficient suspendedsediment concentration were analyzed specifically for the percentage of suspended sediment finer than sand size (less than $0.062 \mathrm{~mm}$ ).

\section{Quality-Assurance Procedures}

Standard U.S. Geological Survey techniques were used during field collection and preservation of all water-quality samples to ensure that representative environmental samples were obtained for analysis. Standard field procedures included checking equipment operation and instrument calibrations before collecting onsite measurements. In addition to standard field procedures, specific quality-control procedures for the collection, treatment, and analysis of water samples were followed (Sylvester and others, 1990). The quality-assurance data presented in this report include laboratory analyses of source-solution blanks and field-equipment blanks. Source-solution blanks are samples obtained from the deionized water supply that is considered free of analytes of interest and that was used for rinsing and to develop fieldequipment blanks. Field-equipment blanks consisted of deionized water that was passed over all the surfaces of decontaminated sampling and processing equipment to evaluate the adequacy of field cleaning and rinsing procedures. Laboratory quality assurance included procedures described by Friedman and Erdmann (1982). The data generated from quality-assurance samples were reviewed by appropriate U.S. Geological Survey personnel associated with the investigation to identify potential sample contamination problems. Deficiencies in the quality-assurance data were documented and corrective actions were taken when required.

The cleaning and rinsing procedures described in the preceding "Methods of Data Collection and Analysis" section were designed to decrease the likelihood of equipment-caused contamination between surface-water sites. In an effort to evaluate fieldequipment cleaning procedures, a field-equipment blank was collected for laboratory analysis during each sampling trip. During the first year of sampling (1990), measured concentrations of some analytes in the fieldequipment blanks were higher than expected. These results prompted a reexamination of the collection process for the field-equipment blanks. It was determined that deionized water that passed over some metal equipment surfaces, which were not in contact with the environmental sample, could have biased the results of the field-equipment blanks. As a result, collection procedures for field-equipment blanks were modified in 1991 to better simulate the contact that occurs between native river water and samplingequipment surfaces during collection of the environmental sample. Subsequent concentrations of analytes determined in the quality-assurance samples were within acceptable levels throughout the remainder of the study. 


\section{RESULTS}

The results of the data collected and compiled during this study are presented in the "Water-Quality Data" section at the back of this report. Onsite measurements, bacteriological data at selected stations, and selected inorganic data for all stations sampled during the study are listed in tables 2-60. Selected surfacewater stations with pesticide data are listed in table 61; selected stations with radiochemical data are listed in table 62; and selected stations with suspendedsediment data are listed in table 63. Results of fieldcollected quality-assurance data for the purpose of assessing potential sample contamination are listed in tables 64 and 65.

\section{REFERENCES CITED}

Abbott, P.O., 1985, Description of water-systems operations in the Arkansas River Basin, Colorado: U.S. Geological Survey Water-Resources Investigations Report 85-4092, 67 p.

Britton, L.J., and Greeson, P.E., eds.,1987, Methods for collection and analysis of aquatic biological and microbiological samples: U.S. Geological Survey Techniques of Water-Resources Investigations, book 5, chap. A4, $363 \mathrm{p}$.

Burns, A.W., 1985, Selected hydrographs and statistical analyses characterizing the water resources of the Arkansas River Basin, Colorado: U.S. Geological Survey Water-Resources Investigations Report 85-4264, 199 p.

Cain, D.L., 1985, Quality of the Arkansas River and irrigation-return flows in the lower Arkansas River Valley, Colorado: U.S. Geological Survey WaterResources Investigations Report 84-4273, 85 p.

Colorado Climate Center, 1984, Colorado average annual precipitation map 1951-1980: scale 1:50,000.

Crouch, T.M., Cain, D.L., Abbott, P.O., Penley, R.D., and Hurr, R.T., 1984, Water-resources appraisal of the upper Arkansas River Basin from Leadville to Pueblo, Colorado: U.S. Geological Survey Water-Resources Investigations Report 82-4114, $123 \mathrm{p}$.

Fishman, M.J., 1993, Methods of analysis by the U.S. Geological Survey National Water Quality LaboratoryDetermination of inorganic and organic constituents in water and fluvial sediments: U.S. Geological Survey Open-File Report 93-125, 217 p.

Fishman, M.J., and Friedman, L.C., eds., 1989, Methods for determination of inorganic substances in water and fluvial sediments ( $3 \mathrm{~d}$ ed.): U.S. Geological Survey Techniques of Water-Resources Investigations, book 5 , chap. A1, $545 \mathrm{p}$.
Fishman, M.J., Raese, J.W., Gerlitz, C.N., and Husband, R.A., 1994, U.S. Geological Survey approved inorganic and organic methods for the analysis of water and fluvial sediment, 1954-94: U.S. Geological Survey OpenFile Report 94-351, $55 \mathrm{p}$.

Friedman, L.C., and Erdmann, D.E., 1982, Qualityassurance practices for the chemical and biological analyses of water and fluvial sediments: U.S. Geological Survey Techniques of Water-Resources Investigations, book 5, chap. A6, $181 \mathrm{p}$.

Guy, H.P., 1969, Laboratory theory and methods for sediment analysis: U.S. Geological Survey Techniques of Water-Resources Investigations, book 3, chap. C1, $58 \mathrm{p}$.

Guy, H.P., and Norman, V.W., 1970, Field methods for measurement of fluvial sediment: U.S. Geological Survey Techniques of Water-Resources Investigations, book 3, chap. C2, $59 \mathrm{p}$.

Kuzmiak, J.M., and Strickland, H.H., 1994, Bibliography of selected water-resources information for the Arkansas River Basin in Colorado through 1985: U.S. Geological Survey Open-File Report 94-331, 266 p.

Litke, D.W., and Appel, C.L., 1989, Estimated use of water in Colorado, 1985: U.S. Geological Survey WaterResources Investigations Report 88-4101, $157 \mathrm{p}$.

Sylvester, M.A., Kister, L.R., and Garrett, W.B., eds., 1990, Guidelines for the collection, treatment, and analysis of water samples-U.S. Geological Survey Western Region field manual: Unpublished report on file in the Pueblo, Colo., Water Resources Division Office of the U.S. Geological Survey, 144 p.

Thatcher, L.L., Janzer, V.J., and Edwards, K.W., 1977, Methods for determination of radioactive substances in water and fluvial sediments: U.S. Geological Survey Techniques of Water-Resources Investigations, book 5, chap. A5, $95 \mathrm{p}$.

U.S. Bureau of the Census, 1991, Census of population and housing, 1990-Public law (P.L.) 94-171 Data, Colorado: Washington, D.C., technical documentation and machine-readable data files.

U.S. Geological Survey, 1977, National handbook of recommended methods for water-data acquisition: various paging.

Ward, J.R., and Harr, C.A., eds., 1990, Methods for collection and processing of surface-water and bed-material samples for physical and chemical analyses: U.S. Geological Survey Open-File Report 90-140, 71 p.

Wershaw, R.L., Fishman, M.J., Grabbe, R.R., and Lowe, L.E., eds., 1987, Methods for determination of organic substances in water and fluvial sediments: U.S. Geological Survey Techniques of WaterResources Investigations, book 5, chap. A3, $80 \mathrm{p}$. 


\section{WATER-QUALITY DATA}


Time is in 24-hour units in tables 2-65.

The following abbreviations are used in tables $2-60$ :

$$
\begin{aligned}
\text { INST. } & =\text { instantaneous } \\
\mathrm{US} / \mathrm{CM} & =\text { microsiemens per centimeter at } 25 \text { degrees CeIsius } \\
\mathrm{PH} & =\mathrm{pH} \\
\mathrm{DEG} \mathrm{C} & =\text { degrees Celsius } \\
\mathrm{MG} / \mathrm{L} & =\text { milligrams per liter } \\
\mathrm{NO} 2+\mathrm{NO} 3 & =\text { nitrite plus nitrate } \\
\mathrm{UG} / \mathrm{L} & =\text { micrograms per liter } \\
-- & =\text { no data } \\
< & =\text { less than } \\
> & =\text { greater than } \\
\mathrm{IMMED} . & =\text { immediate } \\
\mathrm{UM}-\mathrm{MF} & =\text { microns per membrane filter } \\
\text { COLS. PER } 100 \mathrm{ML} & =\text { colonies per } 100 \text { milliliters } \\
\mathrm{K} \text {. } / 100 \mathrm{ML} & =\text { colonies per } 100 \text { milliliters } \\
\mathrm{E} & =\text { nonideal count }
\end{aligned}
$$

In addition to the above abbreviations, the following are used in table 61 :

$$
\begin{aligned}
\text { TOTAL RECOVER } & =\text { total recoverable } \\
\text { TOT. } \text { REC } & =\text { total recoverable } \\
\text { UNFLTRD REC } & =\text { unfiltered recoverable } \\
\text { WHLREC } & =\text { whole recoverable } \\
\text { RECOV. } & =\text { recoverable } \\
\text { REC } & =\text { recoverable } \\
\text { RECOVER } & =\text { recoverable }
\end{aligned}
$$

In addition to the above abbreviations, the following area used in table 62 :

$$
\begin{aligned}
\text { U-NAT } & =\text { uranium natural } \\
\text { SUSP. } & =\text { suspended } \\
\mathrm{PCI} / \mathrm{L} & =\text { picocuries per liter } \\
\mathrm{CS}-137 & =\text { cesium } 137 \\
\text { SR/YT-90 } & =\text { strontium/yttrium } 90
\end{aligned}
$$

In addition to the above abbreviations, the following are used in tables $63-65$ :

$$
\begin{aligned}
\text { T/DAY } & =\text { ton per day } \\
\text { SED. } & =\text { sediment } \\
\text { DIAM. } & =\text { diameter } \\
\% & =\text { percent } \\
\text { MM } & =\text { millimeter }
\end{aligned}
$$


Table 2. Onsite measurements and selected inorganic data for station 09061500, Columbine Ditch near Fremont Pass

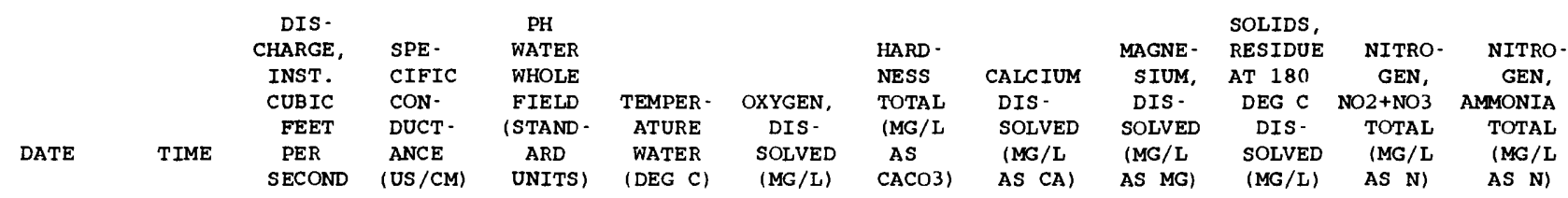

MAY 1990

\begin{tabular}{|c|c|c|c|c|c|c|c|c|c|c|c|c|}
\hline$\underset{\text { JUN }}{21 \ldots}$ & 1600 & $\cdots$ & 64 & 7.5 & $-\cdot$ & $\cdots$ & $\cdots$ & $\cdots$ & $\cdots$ & 38 & 0.100 & 0.050 \\
\hline $\begin{array}{c}04 \ldots \\
\pi N N \\
1991\end{array}$ & 1730 & 47 & 27 & 7.0 & 0.5 & 8.7 & $\cdots$ & $\cdots$ & $\cdots$ & 18 & 0.024 & 0.050 \\
\hline${ }_{\text {AUG }}^{17 \ldots}$ & 1845 & 38 & 34 & 7.2 & 5.0 & 8.3 & - & $\cdots$ & $\cdots$ & 20 & 0.019 & 0.026 \\
\hline $\begin{array}{c}12 \ldots \\
\text { JUN } 1992\end{array}$ & 1320 & 0.82 & 93 & 8.3 & 13.0 & $\cdots$ & $\cdots$ & -- & $\cdots$ & 61 & 0.010 & $<0.002$ \\
\hline$\underset{A U G}{24 \ldots}$ & 0945 & 12 & 56 & 7.8 & 7.0 & 8.0 & 28 & 5.2 & 3.7 & 36 & 0.025 & 0.016 \\
\hline $10 \ldots$ & 1500 & 0.82 & 107 & 8.4 & 13.0 & 6.3 & -- & $\cdots$ & $\cdots$ & 52 & 0.005 & 0.006 \\
\hline
\end{tabular}

\begin{tabular}{|c|c|c|c|c|c|c|c|c|c|c|c|c|}
\hline$T F$ & $\begin{array}{l}\text { CADMIUM } \\
\text { TOTAL } \\
\text { RECOV- } \\
\text { ERABLE } \\
\text { (UG/L }\end{array}$ & $\begin{array}{c}\text { CADMIUM } \\
\text { DIS - } \\
\text { SOLVED } \\
\text { (UG/L }\end{array}$ & $\begin{array}{c}\text { COPPER, } \\
\text { TOTAL } \\
\text { RECOV - } \\
\text { ERABLE } \\
\text { (UG/L }\end{array}$ & $\begin{array}{l}\text { COPPER, } \\
\text { DIS - } \\
\text { SOLVED } \\
\text { (UG / L }\end{array}$ & $\begin{array}{l}\text { IRON, } \\
\text { TOTAL } \\
\text { RECOV - } \\
\text { ERABLE } \\
\text { IUG/L }\end{array}$ & $\begin{array}{l}\text { IRON, } \\
\text { DIS- } \\
\text { SOLVED } \\
\text { /UG / L }\end{array}$ & $\begin{array}{l}\text { LEAD, } \\
\text { TOTAL } \\
\text { RECOV - } \\
\text { ERABLE } \\
\text { (UG / L }\end{array}$ & $\begin{array}{l}\text { LEAD, } \\
\text { DIS- } \\
\text { SOLVED } \\
\text { (UG / L }\end{array}$ & $\begin{array}{l}\text { MANGA - } \\
\text { NESE, } \\
\text { TOTAL } \\
\text { RECOV- } \\
\text { ERABLE } \\
\text { (UG/L }\end{array}$ & $\begin{array}{l}\text { MANGA - } \\
\text { NESE, } \\
\text { DIS - } \\
\text { SOLVED } \\
\text { (UG/L }\end{array}$ & $\begin{array}{l}\text { ZINC, } \\
\text { TOTAL } \\
\text { RECOV - } \\
\text { ERABLE } \\
\text { IUG/L }\end{array}$ & $\begin{array}{l}\text { ZINC, } \\
\text { DIS- } \\
\text { SOLVED } \\
\text { /UG /L }\end{array}$ \\
\hline & AS CD) & AS CD) & AS CU) & AS CU) & AS FE) & AS FE) & AS PB) & AS PB) & AS $\mathrm{MN}$ ) & AS $\mathbf{M N}$ ) & AS $\quad \mathrm{ZN}$ ) & AS $\mathrm{ZN}$ ) \\
\hline
\end{tabular}

MAY 1990

JUN 21.

$04 \ldots$

$17 \ldots$

AUG

$12 \ldots$

JUN 1992

24 ...

AUG

10 ... 
Table 3. Onsite measurements and selected inorganic data for station 09062000, Ewing Ditch at Tennessee Pass

\begin{tabular}{|c|c|c|c|c|c|c|c|c|c|c|c|c|}
\hline DATE & TIME & $\begin{array}{c}\text { DIS- } \\
\text { CHARGE, } \\
\text { INST. } \\
\text { CUBIC } \\
\text { FEET } \\
\text { PER } \\
\text { SECOND }\end{array}$ & $\begin{array}{l}\text { SPE- } \\
\text { CIFIC } \\
\text { CON- } \\
\text { DUCT - } \\
\text { ANCE } \\
\text { (US/CM) }\end{array}$ & $\begin{array}{c}\text { PH } \\
\text { WATER } \\
\text { WHOLE } \\
\text { FIELD } \\
\text { (STAND. } \\
\text { ARD } \\
\text { UNITS) }\end{array}$ & $\begin{array}{c}\text { TEMPER - } \\
\text { ATURE } \\
\text { WATER } \\
\text { (DEG C) }\end{array}$ & $\begin{array}{c}\text { OXYGEN, } \\
\text { DIS - } \\
\text { SOLVED } \\
\text { (MG/L) }\end{array}$ & $\begin{array}{l}\text { HARD - } \\
\text { NESS } \\
\text { TOTAL } \\
\text { (MG/L } \\
\text { AS } \\
\text { CACO3) }\end{array}$ & $\begin{array}{l}\text { CALCIUM } \\
\text { DIS - } \\
\text { SOLVED } \\
\text { (MG /L } \\
\text { AS CA) }\end{array}$ & $\begin{array}{l}\text { MAGNE- } \\
\text { SIUM, } \\
\text { DIS - } \\
\text { SOLVED } \\
\text { (MG/L } \\
\text { AS MG) }\end{array}$ & $\begin{array}{l}\text { SOLIDS, } \\
\text { RESIDUE } \\
\text { AT } 180 \\
\text { DEG C } \\
\text { DIS. } \\
\text { SOLVED } \\
\text { (MG/L) }\end{array}$ & $\begin{array}{c}\text { NITRO- } \\
\text { GEN, } \\
\text { NO2+NO3 } \\
\text { TOTAL } \\
\text { (MG/L } \\
\text { AS N) }\end{array}$ & $\begin{array}{l}\text { NITRO- } \\
\text { GEN, } \\
\text { AMMONIA } \\
\text { TOTAL } \\
\text { (MG /L } \\
\text { AS N) }\end{array}$ \\
\hline MAY 1990 & & & & & & & & & & & & \\
\hline$\underset{\pi U N}{21 \ldots}$ & 1700 & 5.4 & 60 & 8.1 & 5.5 & 8.8 & $\cdots$ & $\cdots$ & $\cdots$ & 42 & 0.123 & 0.040 \\
\hline $\begin{array}{c}04 \ldots \\
\pi N N 1991\end{array}$ & 2000 & 12 & 40 & 7.6 & 4.5 & 8.7 & $-\cdot$ & -- & $\cdots$ & 21 & $<0.010$ & 0.030 \\
\hline${ }_{A \cup G}^{17} \cdots$ & 1630 & 7.7 & 74 & 8.0 & 10.5 & 7.6 & -. & $\cdots$ & . & 41 & 0.023 & 0.017 \\
\hline $\begin{array}{c}12 \ldots \\
\text { JUN } 1992\end{array}$ & 1440 & 1.1 & 98 & 8.3 & 11.0 & $\cdots$ & - & $\cdots$ & $\cdots$ & 62 & 0.025 & $<0.002$ \\
\hline$\underset{A U G}{23} \ldots$ & 1645 & 4.7 & 81 & 8.0 & 9.0 & 7.6 & 34 & 9.3 & 2.5 & 52 & 0.047 & 0.009 \\
\hline $10 \ldots$ & 1310 & 1.3 & . & 8.2 & 11.0 & 7.4 & $\cdots$ & - & $\cdots$ & 58 & 0.069 & 0.008 \\
\hline DATE & $\begin{array}{l}\text { CADMIUM } \\
\text { TOTAL } \\
\text { RECOV- } \\
\text { ERABLE } \\
\text { (UG/L } \\
\text { AS CD) }\end{array}$ & $\begin{array}{l}\text { CADMIUM } \\
\text { DIS - } \\
\text { SOLVED } \\
\text { (UG / L } \\
\text { AS CD) }\end{array}$ & $\begin{array}{l}\text { COPPER, } \\
\text { TOTAL } \\
\text { RECOV- } \\
\text { ERABLE } \\
\text { (UG/L } \\
\text { AS CU) }\end{array}$ & $\begin{array}{l}\text { COPPER, } \\
\text { DIS - } \\
\text { SOLVED } \\
\text { (UG /L } \\
\text { AS CU) }\end{array}$ & $\begin{array}{l}\text { IRON, } \\
\text { TOTAL } \\
\text { RECOV- } \\
\text { ERABLE } \\
\text { (UG/L } \\
\text { AS FE) }\end{array}$ & $\begin{array}{l}\text { IRON, } \\
\text { DIS- } \\
\text { SOLVED } \\
\text { (UG/L } \\
\text { AS FE) }\end{array}$ & $\begin{array}{l}\text { LEAD, } \\
\text { TOTAL } \\
\text { RECOV- } \\
\text { ERABLE } \\
\text { (UG/L } \\
\text { AS PB) }\end{array}$ & $\begin{array}{l}\text { LEAD, } \\
\text { DIS- } \\
\text { SOLVED } \\
\text { (UG/L } \\
\text { AS PB) }\end{array}$ & $\begin{array}{l}\text { MANGA- } \\
\text { NESE, } \\
\text { TOTAL } \\
\text { RECOV- } \\
\text { ERABLE } \\
\text { (UG/L } \\
\text { AS MN) }\end{array}$ & $\begin{array}{l}\text { MANGA - } \\
\text { NESE, } \\
\text { DIS- } \\
\text { SOLVED } \\
\text { (UG /L } \\
\text { AS MN) }\end{array}$ & $\begin{array}{l}\text { ZINC, } \\
\text { TOTAL } \\
\text { RECOV- } \\
\text { ERABLE } \\
\text { (UG/L } \\
\text { AS ZN) }\end{array}$ & $\begin{array}{l}\text { ZINC, } \\
\text { DIS: } \\
\text { SOLVED } \\
\text { (UG/L } \\
\text { AS ZN) }\end{array}$ \\
\hline
\end{tabular}

$\begin{array}{lll}\text { MAY } 1990 & & \\ 21 \ldots & \ldots & - \\ \text { JUN } & & \\ 04 \ldots & <1 & <0.1 \\ \text { JUN } 1991 & & \\ 17 \ldots & \ldots & - \\ \text { AUG } & & \\ 12 \ldots & -. & - \\ \text { JUN } 1992 & & \\ 23 \ldots & \ldots & -. \\ \text { AUG } & & \\ 10 \ldots & \ldots & -.\end{array}$


Table 4. Onsite measurements and selected inorganic data for station 09062500, Wurtz Ditch near Tennessee Pass

\begin{tabular}{|c|c|c|c|c|c|c|c|c|c|c|c|c|}
\hline DATE & TIME & $\begin{array}{c}\text { DIS- } \\
\text { CHARGE, } \\
\text { INST. } \\
\text { CUBIC } \\
\text { FEET } \\
\text { PER } \\
\text { SECOND }\end{array}$ & $\begin{array}{l}\text { SPE- } \\
\text { CIFIC } \\
\text { CON- } \\
\text { DUCT - } \\
\text { ANCE } \\
\text { (US/CM) }\end{array}$ & $\begin{array}{c}\text { PH } \\
\text { WATER } \\
\text { WHOLE } \\
\text { FIELD } \\
\text { (STAND- } \\
\text { ARD } \\
\text { UNITS) }\end{array}$ & $\begin{array}{c}\text { TEMPER - } \\
\text { ATURE } \\
\text { WATER } \\
\text { (DEG C) }\end{array}$ & $\begin{array}{c}\text { OXYGEN, } \\
\text { DIS - } \\
\text { SOLVED } \\
\text { (MG /L) }\end{array}$ & $\begin{array}{l}\text { HARD - } \\
\text { NESS } \\
\text { TOTAL } \\
\text { (MG/L } \\
\text { AS } \\
\text { CACO3) }\end{array}$ & $\begin{array}{l}\text { CALCIUM } \\
\text { DIS - } \\
\text { SOLVED } \\
\text { (MG/L } \\
\text { AS CA) }\end{array}$ & $\begin{array}{l}\text { MAGNE- } \\
\text { SIUM, } \\
\text { DIS - } \\
\text { SOLVED } \\
\text { (MG/L } \\
\text { AS MG) }\end{array}$ & $\begin{array}{c}\text { SOLIDS, } \\
\text { RESIDUE } \\
\text { AT } 180 \\
\text { DEG C } \\
\text { DIS. } \\
\text { SOLVED } \\
\text { (MG } / L)\end{array}$ & $\begin{array}{c}\text { NITRO- } \\
\text { GEN, } \\
\text { NO2+NO3 } \\
\text { TOTAL } \\
\text { (MG / L } \\
\text { AS N) }\end{array}$ & $\begin{array}{l}\text { NITRO- } \\
\text { GEN, } \\
\text { AMMONIA } \\
\text { TOTAL } \\
\text { (MG / L } \\
\text { AS N) }\end{array}$ \\
\hline MAY 1990 & & & & & & & & & & & & \\
\hline$\underset{J U N}{21 \ldots}$ & 1740 & 5.6 & -- & 7.5 & 9.5 & 7.4 & $\cdots$ & $\cdots$ & $\cdots$ & 25 & 0.016 & 0.030 \\
\hline $\begin{array}{c}04 \ldots \\
\text { JUN } 1991\end{array}$ & 1910 & 37 & 25 & 7.1 & 8.0 & 7.8 & $\cdots$ & $\cdots$ & $-\cdot$ & 16 & 0.038 & 0.030 \\
\hline$\underset{A U G}{17} \cdots$ & 1715 & 18 & 26 & 8.0 & 14.5 & 6.7 & $\cdots$ & $\cdots$ & $\cdots$ & 19 & $<0.005$ & 0.025 \\
\hline $\begin{array}{l}12 \ldots \\
\text { JUN } 1992\end{array}$ & 1510 & 0.82 & 37 & 7.8 & 16.0 & $\cdots$ & $\cdots$ & $\cdots$ & - & 27 & 0.006 & $<0.002$ \\
\hline$\underset{\text { AUG }}{23} \ldots$ & 1600 & 6.3 & 28 & 7.8 & 9.0 & 7.7 & 12 & 3.2 & 0.90 & 26 & $<0.005$ & 0.029 \\
\hline $10 \ldots$ & 1350 & 0.0 & 44 & 7.8 & 14.5 & 6.0 & $\cdots$ & $\cdots$ & $\cdots$ & 28 & $<0.005$ & 0.037 \\
\hline
\end{tabular}

\begin{tabular}{|c|c|c|c|c|c|c|c|c|c|c|c|c|}
\hline & $\begin{array}{l}\text { CADMIUM } \\
\text { TOTAL } \\
\text { RECOV- } \\
\text { ERABLE } \\
\text { (UG/L }\end{array}$ & $\begin{array}{c}\text { CADMIUM } \\
\text { DIS - } \\
\text { SOLVED } \\
\text { (UG / L }\end{array}$ & $\begin{array}{l}\text { COPPER, } \\
\text { TOTAL } \\
\text { RECOV- } \\
\text { ERABLE } \\
\text { (UG/L }\end{array}$ & $\begin{array}{l}\text { COPPER, } \\
\text { DIS - } \\
\text { SOLVED } \\
\text { (UG / L }\end{array}$ & $\begin{array}{l}\text { IRON, } \\
\text { TOTAL } \\
\text { RECOV- } \\
\text { ERABLE } \\
\text { (UG/L }\end{array}$ & $\begin{array}{l}\text { IRON, } \\
\text { DIS- } \\
\text { SOLVED } \\
\text { IUG /L }\end{array}$ & $\begin{array}{l}\text { LEAD, } \\
\text { TOTAL } \\
\text { RECOV- } \\
\text { ERABLE } \\
\text { (UG/L }\end{array}$ & $\begin{array}{l}\text { LEAD, } \\
\text { DIS - } \\
\text { SOLVED } \\
\text { (UG/L }\end{array}$ & $\begin{array}{l}\text { MANGA- } \\
\text { NESE, } \\
\text { TOTAL } \\
\text { RECOV- } \\
\text { ERABLE } \\
\text { (UG/L }\end{array}$ & $\begin{array}{l}\text { MANGA - } \\
\text { NESE, } \\
\text { DIS- } \\
\text { SOLVED } \\
\text { (UG / L }\end{array}$ & $\begin{array}{l}\text { ZINC, } \\
\text { TOTAL } \\
\text { RECOV- } \\
\text { ERABLE } \\
\text { (UG/L }\end{array}$ & $\begin{array}{l}\text { ZINC, } \\
\text { DIS- } \\
\text { SOLVED } \\
\text { (UG / L }\end{array}$ \\
\hline & AS $(D)$ & AS CD & AS CU) & AS CU) & AS FE) & AS FE) & $A S \quad P B)$ & AS PB) & AS MN) & AS MN) & AS ZN & AS $\mathrm{ZN}$ ) \\
\hline
\end{tabular}

MAY 1990

$21 \ldots$

$04 \ldots$

JUN 1991

17 ...

AUG

JUN 1992

$23 \ldots$

AUG

10 ... 
Table 5. Onsite measurements and selected inorganic data for station 392005106130501 , East Fork Arkansas River below French Gulch, near Climax

\begin{tabular}{|c|c|c|c|c|c|c|c|c|c|c|c|}
\hline DATE & TIME & $\begin{array}{l}\text { DIS- } \\
\text { CHARGE, } \\
\text { INST. } \\
\text { CUBIC } \\
\text { FEET } \\
\text { PER } \\
\text { SECOND }\end{array}$ & $\begin{array}{l}\text { SPE- } \\
\text { CIFIC } \\
\text { CON- } \\
\text { DUCT- } \\
\text { ANCE } \\
\text { (US/CM) }\end{array}$ & $\begin{array}{c}\text { PH } \\
\text { WATER } \\
\text { WHOLE } \\
\text { FIELD } \\
\text { (STAND- } \\
\text { ARD } \\
\text { UNITS) }\end{array}$ & $\begin{array}{l}\text { TEMPER - } \\
\text { ATURE } \\
\text { WATER } \\
\text { (DEG C) }\end{array}$ & $\begin{array}{c}\text { OXYGEN, } \\
\text { DIS - } \\
\text { SOLVED } \\
\text { (MG/L) }\end{array}$ & $\begin{array}{l}\text { SOLIDS, } \\
\text { RESIDUE } \\
\text { AT } 180 \\
\text { DEG C } \\
\text { DIS - } \\
\text { SOLVED } \\
\text { (MG } / \mathrm{L} \text { ) }\end{array}$ & $\begin{array}{c}\text { NITRO- } \\
\text { GEN, } \\
\text { NO2+NO3 } \\
\text { TOTAL } \\
\text { (MG / L } \\
\text { AS N) }\end{array}$ & $\begin{array}{l}\text { NITRO- } \\
\text { GEN, } \\
\text { AMMONIA } \\
\text { TOTAL } \\
\text { (MG / L } \\
\text { AS N) }\end{array}$ & $\begin{array}{l}\text { CADMIUM } \\
\text { TOTAL } \\
\text { RECOV- } \\
\text { ERABLE } \\
\text { (UG/L } \\
\text { AS CD) }\end{array}$ & $\begin{array}{l}\text { CADMIUM } \\
\text { DIS - } \\
\text { SOLVED } \\
\text { (UG } / L \\
\text { AS CD) }\end{array}$ \\
\hline APR 1990 & & & & & & & & & & & \\
\hline${ }_{\text {JUN }} 17 \ldots$ & 0850 & 2.7 & 176 & $\cdots$ & 1.0 & $\cdots$ & 107 & 0.107 & $<0.010$ & $<1$ & 0.1 \\
\hline${ }_{\text {JUL }}^{05 \ldots}$ & 0815 & 129 & -. & 7.2 & 1.0 & 8.9 & 42 & 0.069 & 0.090 & 1 & 0.3 \\
\hline${ }_{A U G}^{16 \ldots}$ & 1515 & 19 & 103 & 7.8 & 11.5 & 6.5 & 56 & 0.033 & $<0.010$ & $<1$ & 0.2 \\
\hline$\underset{\mathrm{OCT}}{28 \ldots}$ & 0830 & 8.3 & 136 & 7.4 & 7.0 & 7.4 & 74 & 0.130 & $<0.010$ & $<1$ & $<0.1$ \\
\hline $\begin{array}{r}29 \ldots \\
A P R \\
1991\end{array}$ & 1215 & 4.8 & 167 & 7.8 & 5.0 & 7.5 & 93 & 0.123 & 0.020 & $<1$ & 0.5 \\
\hline$\underset{\text { JUN }}{22 \ldots}$ & 1545 & 3.4 & 217 & 7.7 & 5.0 & 8.4 & 95 & 0.106 & 0.018 & $<1$ & 0.4 \\
\hline JUL $18 .$. & 0630 & 97 & 71 & 7.2 & 4.0 & $\cdots$ & 29 & 0.090 & 0.017 & $<1$ & 0.1 \\
\hline${ }_{\text {OCT }}^{15 \ldots}$ & 1610 & 23 & 88 & 7.8 & 13.5 & 6.6 & 44 & 0.040 & 0.006 & $<1$ & 0.1 \\
\hline $\begin{array}{l}21 \ldots \\
A P R \\
1992\end{array}$ & 1530 & 6.0 & 156 & 7.8 & 7.0 & 7.5 & 90 & 0.097 & 0.022 & $<1$ & 0.1 \\
\hline$\underset{J U N}{20}$ & 1620 & 8.7 & 209 & 7.8 & 2.5 & 8.8 & 118 & 0.150 & 0.006 & $<1$ & 0.2 \\
\hline$\underset{A U G}{24} \ldots$ & 0830 & 89 & 67 & 7.7 & 6.0 & 8.0 & 36 & 0.081 & 0.011 & $<1$ & $<0.1$ \\
\hline$\underset{\mathrm{OCT}}{11 \ldots}$ & 0725 & 15 & 113 & 7.8 & 8.0 & 7.0 & 62 & 0.078 & 0.003 & $<1$ & $<0.1$ \\
\hline $27, \ldots$ & 0930 & 4.5 & 169 & 8.1 & 1.0 & 9.4 & 112 & 0.084 & 0.004 & $<1$ & 0.2 \\
\hline
\end{tabular}

\begin{tabular}{|c|c|c|c|c|c|c|c|c|c|c|}
\hline$T E$ & $\begin{array}{l}\text { COPPER, } \\
\text { TOTAL } \\
\text { RECOV- } \\
\text { ERABLE } \\
\text { (UG /L }\end{array}$ & $\begin{array}{l}\text { COPPER, } \\
\text { DIS - } \\
\text { SOLVED } \\
\text { IUG/L }\end{array}$ & $\begin{array}{l}\text { IRON, } \\
\text { TOTAL } \\
\text { RECOV- } \\
\text { ERABLE } \\
\text { (UG /L }\end{array}$ & $\begin{array}{l}\text { IRON, } \\
\text { DIS- } \\
\text { SOLVED } \\
\text { (UG/L }\end{array}$ & $\begin{array}{l}\text { LEAD, } \\
\text { TOTAL } \\
\text { RECOV- } \\
\text { ERABLE } \\
\text { (UG /L }\end{array}$ & $\begin{array}{l}\text { LEAD, } \\
\text { DIS- } \\
\text { SOLVED } \\
\text { (UG/L }\end{array}$ & $\begin{array}{l}\text { MANGA- } \\
\text { NESE, } \\
\text { TOTAL } \\
\text { RECOV- } \\
\text { ERABLE } \\
\text { (UG/L }\end{array}$ & $\begin{array}{l}\text { MANGA - } \\
\text { NESE, } \\
\text { DIS - } \\
\text { SOLVED } \\
\text { (UG/L }\end{array}$ & $\begin{array}{l}\text { ZINC, } \\
\text { TOTAL } \\
\text { RECOV- } \\
\text { ERABLE } \\
\text { /UG /L }\end{array}$ & $\begin{array}{l}\text { ZINC, } \\
\text { DIS- } \\
\text { SOLVED } \\
\text { (UG / L }\end{array}$ \\
\hline & AS CU) & AS CU) & AS FE) & AS FE) & AS PB) & AS PB) & AS MN) & AS MN) & AS $\mathrm{ZN}$ ) & AS $\mathrm{ZN}$ ) \\
\hline
\end{tabular}

\begin{tabular}{|c|c|c|c|c|c|c|c|c|c|c|}
\hline APR 1990 & & & & & & & & & & \\
\hline $17 \ldots$ & 2 & 1 & 170 & 83 & $<1$ & $<0.5$ & 30 & 27 & 90 & 78 \\
\hline JUN & & & & & & & & & & \\
\hline $05 \ldots$ & 9 & 5 & 2600 & 72 & 23 & 3 & 200 & 28 & 100 & 41 \\
\hline JUL & & & & & & & & & & \\
\hline $16 \ldots$ & 4 & 2 & 160 & 70 & 3 & 0.8 & 40 & 28 & 30 & 22 \\
\hline AUG & & & & & & & & & & \\
\hline $28 \ldots$ & 2 & 1 & 140 & 78 & 3 & 0.7 & 60 & 36 & 30 & 18 \\
\hline OCT & & & & & & & & & & \\
\hline $29 \ldots$ & 7 & 1 & 170 & 76 & $<1$ & 0.6 & 60 & 52 & 20 & 20 \\
\hline APR 1991 & & & & & & & & & & \\
\hline $22 \ldots$ & 3 & 1 & 290 & 87 & 3 & $<0.5$ & 50 & 34 & 40 & 15 \\
\hline JUN & & & & & & & & & & \\
\hline $18 \ldots$ & 4 & 3 & 190 & 55 & 5 & $<0.5$ & 20 & 14 & 20 & 26 \\
\hline JUL & & & & & & & & & & \\
\hline $15 \ldots$ & 3 & 1 & 80 & 57 & 5 & $<0.5$ & 20 & 20 & 10 & 15 \\
\hline OCT & & & & & & & & & & \\
\hline $21 \ldots$ & 5 & $<1$ & 150 & 100 & 4 & 0.6 & 40 & 35 & $<10$ & 10 \\
\hline APR 1992 & & & & & & & & & & \\
\hline $20 \ldots$ & 2 & 1 & 260 & 44 & 1 & $<0.5$ & 70 & 45 & 40 & 27 \\
\hline JUN & & & & & & & & & & \\
\hline $24 \ldots$ & 1 & 1 & 130 & 33 & 2 & 0.6 & 20 & 7 & 20 & 12 \\
\hline AUG & & & & & & & & & & \\
\hline $11 \ldots$ & 2 & $<1$ & 140 & 63 & 3 & 0.8 & 31 & 24 & 70 & 19 \\
\hline OCT & & & & & & & & & & \\
\hline 27 & 1 & $<1$ & 40 & 64 & 1 & 0.7 & $<10$ & 24 & $<10$ & 10 \\
\hline
\end{tabular}


Table 6. Onsite measurements and selected inorganic data for station 391937106200301 , Tennessee Creek at Highway 24, near Leadville

\begin{tabular}{|c|c|c|c|c|c|c|c|c|c|c|c|}
\hline DATE & TIME & $\begin{array}{c}\text { DIS - } \\
\text { CHARGE, } \\
\text { INST. } \\
\text { CUBIC } \\
\text { FEET } \\
\text { PER } \\
\text { SECOND }\end{array}$ & $\begin{array}{l}\text { SPE- } \\
\text { CIFIC } \\
\text { CON- } \\
\text { DUCT - } \\
\text { ANCE } \\
\text { (US/CM) }\end{array}$ & $\begin{array}{c}\text { PH } \\
\text { WATER } \\
\text { WHOLE } \\
\text { FIELD } \\
\text { (STAND- } \\
\text { ARD } \\
\text { UNITS) }\end{array}$ & $\begin{array}{c}\text { TEMPER- } \\
\text { ATURE } \\
\text { WATER } \\
\text { (DEG C) }\end{array}$ & $\begin{array}{c}\text { OXYGEN, } \\
\text { DIS - } \\
\text { SOLVED } \\
\text { (MG/L) }\end{array}$ & $\begin{array}{l}\text { SOLIDS, } \\
\text { RESIDUE } \\
\text { AT } 180 \\
\text { DEG C } \\
\text { DIS- } \\
\text { SOLVED } \\
\text { (MG/L) }\end{array}$ & $\begin{array}{c}\text { NITRO- } \\
\text { GEN, } \\
\text { NO2+NO3 } \\
\text { TOTAL } \\
\text { (MG/L } \\
\text { AS N) }\end{array}$ & $\begin{array}{c}\text { NITRO- } \\
\text { GEN, } \\
\text { AMMONIA } \\
\text { TOTAL } \\
\text { (MG / L } \\
\text { AS N) }\end{array}$ & $\begin{array}{l}\text { CADMIUM } \\
\text { TOTAL } \\
\text { RECOV - } \\
\text { ERABLE } \\
\text { (UG/L } \\
\text { AS CD) }\end{array}$ & $\begin{array}{l}\text { CADMIUM } \\
\text { DIS- } \\
\text { SOLVED } \\
\text { (UG/L } \\
\text { AS CD) }\end{array}$ \\
\hline 1990 & & & & & & & & & & & \\
\hline $\begin{array}{l}17 \ldots \\
\mathrm{N}\end{array}$ & 1030 & 4.1 & 68 & 8.0 & 0.0 & 8.5 & 60 & 0.119 & 0.020 & $<1$ & $<0.1$ \\
\hline$\frac{05 \ldots}{4}$ & 0820 & 128 & $\cdots$ & 7.1 & 2.0 & 9.7 & 23 & 0.037 & 0.020 & $<1$ & $<0.1$ \\
\hline $\begin{array}{l}17 \ldots \\
G\end{array}$ & 0830 & 12 & 42 & 7.4 & 7.5 & 8.3 & 21 & $<0.010$ & $<0.010$ & $<1$ & $<0.1$ \\
\hline $\begin{array}{l}28 \ldots \\
T\end{array}$ & 0815 & 1.3 & 80 & 7.9 & 7.5 & 7.6 & 48 & 0.021 & $<0.010$ & 2 & $<0.1$ \\
\hline $\begin{array}{l}29 \ldots \\
R 1991\end{array}$ & 1335 & 2.3 & 49 & 7.7 & 4.0 & 8.5 & 35 & $<0.010$ & 0.030 & $<1$ & $<0.1$ \\
\hline $\begin{array}{l}22 \ldots \\
\text { N }\end{array}$ & 1425 & 1.2 & 99 & 7.4 & 2.0 & 8.5 & 48 & 0.079 & 0.027 & $<1$ & $<0.1$ \\
\hline $10 \ldots$ & 0520 & $\cdots$ & $\cdots$ & $\cdots$ & $\cdots$ & $\cdots$ & 16 & $\cdots$ & - & $<1$ & 0.9 \\
\hline $\begin{array}{l}18 \ldots \\
L\end{array}$ & 0705 & 97 & 32 & 6.8 & 4.0 & 8.6 & 16 & 0.014 & 0.020 & $<1$ & $<0.1$ \\
\hline $\begin{array}{l}15 \ldots \\
T\end{array}$ & 1815 & 15 & 52 & 6.9 & 15.0 & 6.4 & 15 & $<0.005$ & 0.009 & $<1$ & 0.4 \\
\hline $\begin{array}{l}21 \ldots \\
R\end{array}$ & 1350 & 0.75 & 64 & 7.4 & 5.0 & 8.5 & 42 & 0.006 & 0.014 & $<1$ & 0.1 \\
\hline $\begin{array}{l}20 \ldots \\
N\end{array}$ & 1620 & 4.2 & 66 & 7.6 & 2.5 & $\cdots$ & 50 & 0.030 & 0.025 & $<1$ & 1.3 \\
\hline $\begin{array}{l}24 \cdots \\
G\end{array}$ & 1030 & 50 & 33 & 7.7 & 9.5 & 7.5 & 18 & $<0.005$ & 0.024 & $<1$ & $<0.1$ \\
\hline${ }_{T}^{11} \ldots$ & 0825 & 6.8 & 54 & 7.6 & 9.5 & 7.2 & 34 & $<0.005$ & 0.010 & $<1$ & $<0.1$ \\
\hline $27 \ldots$ & 0855 & 2.8 & 54 & 7.9 & 1.0 & 9.3 & 53 & 0.007 & 0.005 & $<1$ & 1.0 \\
\hline
\end{tabular}

\begin{tabular}{|c|c|c|c|c|c|c|c|c|c|}
\hline $\begin{array}{l}\text { COPPER, } \\
\text { TOTAL } \\
\text { RECOV- } \\
\text { ERABLE } \\
\text { (UG/L }\end{array}$ & $\begin{array}{l}\text { COPPER, } \\
\text { DIS - } \\
\text { SOLVED } \\
\text { (UG/L }\end{array}$ & $\begin{array}{l}\text { IRON, } \\
\text { TOTAL } \\
\text { RECOV- } \\
\text { ERABLE } \\
\text { (UG/L }\end{array}$ & $\begin{array}{l}\text { IRON, } \\
\text { DIS- } \\
\text { SOLVED } \\
\text { (UG /L }\end{array}$ & $\begin{array}{l}\text { LEAD, } \\
\text { TOTAL } \\
\text { RECOV- } \\
\text { ERABLE } \\
\text { (UG/L }\end{array}$ & $\begin{array}{l}\text { LEAD, } \\
\text { DIS - } \\
\text { SOLVED } \\
\text { /UG/L }\end{array}$ & $\begin{array}{l}\text { MANGA - } \\
\text { NESE, } \\
\text { TOTAL } \\
\text { RECOV - } \\
\text { ERABLE } \\
\text { (UG /L }\end{array}$ & $\begin{array}{l}\text { MANGA- } \\
\text { NESE, } \\
\text { DIS- } \\
\text { SOLVED } \\
\text { (UG/L }\end{array}$ & $\begin{array}{l}\text { ZINC, } \\
\text { TOTAL } \\
\text { RECOV. } \\
\text { ERABLE } \\
\text { (UG / L }\end{array}$ & $\begin{array}{c}\text { ZINC, } \\
\text { DIS- } \\
\text { SOLVED } \\
\text { (UG/L }\end{array}$ \\
\hline AS CU) & AS CU) & AS FE) & AS FE) & AS PB) & AS PB) & AS MN) & AS MN) & AS $\mathrm{ZN}$ ) & AS $\mathrm{ZN})$ \\
\hline
\end{tabular}

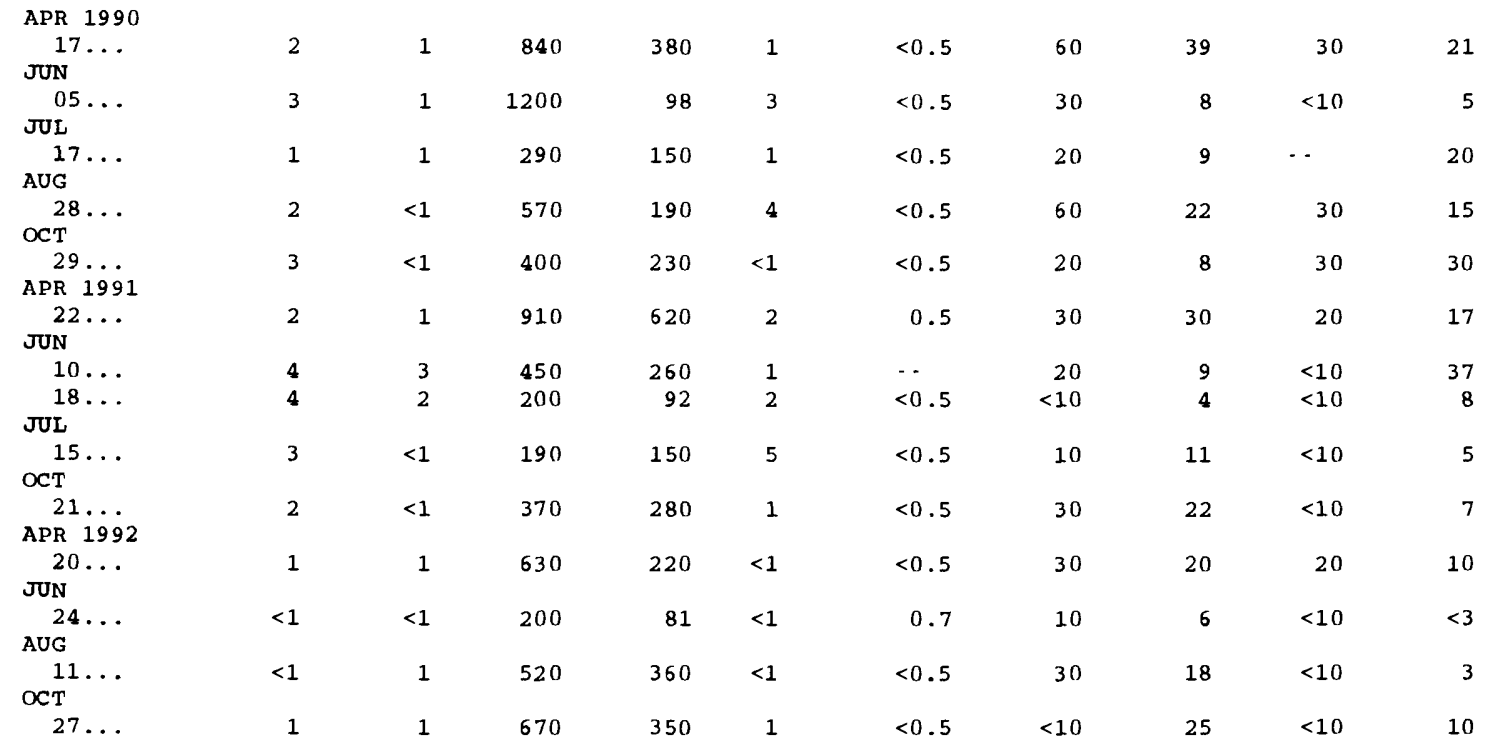


Table 7. Onsite measurements and selected inorganic data for station 07079200, Leadville Mine Drainage Tunnel at Leadville

\begin{tabular}{|c|c|c|c|c|c|c|c|c|c|c|}
\hline DATE & TIME & $\begin{array}{c}\text { DIS - } \\
\text { CHARGE, } \\
\text { INST. } \\
\text { CUBIC } \\
\text { FEET } \\
\text { PER } \\
\text { SECOND }\end{array}$ & $\begin{array}{l}\text { SPE - } \\
\text { CIFIC } \\
\text { CON- } \\
\text { DUCT- } \\
\text { ANCE } \\
\text { (US/CM) }\end{array}$ & $\begin{array}{c}\text { PH } \\
\text { WATER } \\
\text { WHOLE } \\
\text { FIELD } \\
\text { (STAND- } \\
\text { ARD } \\
\text { UNITS) }\end{array}$ & $\begin{array}{l}\text { TEMPER - } \\
\text { ATURE } \\
\text { WATER } \\
\text { (DEG C) }\end{array}$ & $\begin{array}{c}\text { OXYGEN, } \\
\text { DIS - } \\
\text { SOLVED } \\
\text { (MG/L) }\end{array}$ & $\begin{array}{l}\text { HARD - } \\
\text { NESS } \\
\text { TOTAL } \\
\text { (MG/L } \\
\text { AS } \\
\text { CACO3) }\end{array}$ & $\begin{array}{l}\text { CALCIUM } \\
\text { DIS - } \\
\text { SOLVED } \\
\text { (MG/L } \\
\text { AS CA) }\end{array}$ & $\begin{array}{l}\text { MAGNE - } \\
\text { SIUM, } \\
\text { DIS - } \\
\text { SOLVED } \\
\text { (MG/L } \\
\text { AS MG) }\end{array}$ & $\begin{array}{l}\text { SODIUM, } \\
\text { DIS - } \\
\text { SOLVED } \\
\text { (MG/L } \\
\text { AS NA) }\end{array}$ \\
\hline $\begin{array}{c}\text { APR } 1991 \\
22 \ldots \\
\text { MAY }\end{array}$ & 1640 & 2.5 & 1010 & $\cdots$ & 7.5 & 7.1 & 470 & 160 & 18 & 4.0 \\
\hline $\mathrm{JUN}^{13 \ldots}$ & 1310 & 1.4 & 1010 & 7.1 & 7.5 & 6.9 & 540 & 130 & 52 & 3.8 \\
\hline $\int_{\pi L}^{18} \cdots$ & 0725 & 2.7 & 859 & 7.0 & 8.0 & 7.1 & 450 & 110 & 43 & 3.3 \\
\hline${ }_{A U G}^{16} \ldots$ & 0840 & 2.8 & 732 & 7.3 & 7.5 & 7.8 & 380 & 94 & 36 & 2.7 \\
\hline$\underset{\mathrm{OCT}}{12} \cdots$ & 1600 & 1.5 & 661 & 7.5 & 8.0 & $\cdots$ & 350 & 85 & 34 & 3.2 \\
\hline DEC $21 \ldots$ & 1700 & 1.8 & 847 & 7.4 & 7.0 & 7.3 & 440 & 110 & 41 & 3.4 \\
\hline $\begin{array}{c}16 \\
A P R\end{array} 1992$ & 1300 & 2.3 & 847 & 7.4 & 7.0 & 7.3 & $\cdots$ & $\cdots$ & $\cdots$ & $\cdots$ \\
\hline$\underset{\operatorname{MAY}}{20 \ldots}$ & 1730 & 2.6 & 930 & 7.0 & 7.0 & 8.2 & $\cdots$ & $\cdots$ & $\cdots$ & $\cdots$ \\
\hline$\underset{J U N}{20} \cdots$ & 1230 & 1.9 & 1010 & 7.6 & 7.5 & 8.2 & $\cdots$ & $\cdots$ & $\cdots$ & $\cdots$ \\
\hline$\pi_{\text {JL }}^{24 \ldots}$ & 1100 & 2.0 & 805 & 7.4 & 7.5 & 8.6 & $\cdots$ & $\cdots$ & $\cdots$ & $\cdots$ \\
\hline${ }_{A U G}^{13} \ldots$ & 1030 & 2.3 & 712 & 7.5 & 7.5 & 8.3 & -- & $\cdots$ & $\cdots$ & - \\
\hline $\mathrm{OCT}^{11 \ldots}$ & 0845 & 2.5 & 657 & 7.4 & 7.5 & 8.4 & $\cdots$ & $\cdots$ & $\cdots$ & $\cdots$ \\
\hline 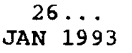 & 1210 & 2.5 & 764 & 7.4 & 7.0 & 8.7 & $\cdots$ & $\cdots$ & $\cdots$ & $\cdots$ \\
\hline $\operatorname{MAR}_{\operatorname{MA}}^{11}$ & 1040 & 2.3 & 835 & 7.6 & 7.0 & 8.5 & $\cdots$ & $\cdots$ & $\cdots$ & $\cdots$ \\
\hline $22 \ldots$ & 0900 & 2.4 & 890 & 7.6 & 7.0 & 8.2 & -- & $\cdots$ & $\cdots$ & $\cdots$ \\
\hline
\end{tabular}

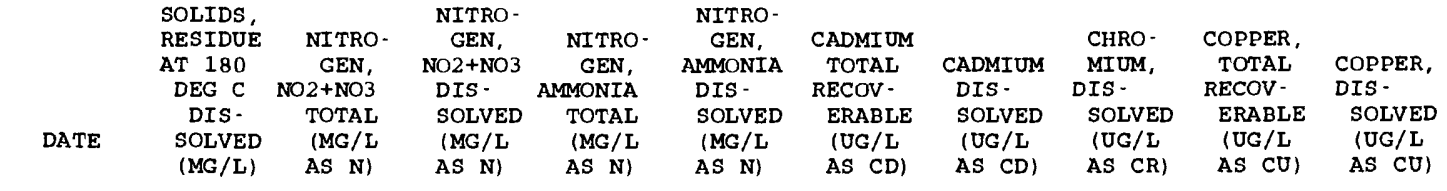

\begin{tabular}{|c|c|c|c|c|c|c|c|c|c|c|}
\hline $\begin{array}{c}\text { APR } 1991 \\
22 \ldots \\
\text { MAY }\end{array}$ & 600 & 0.400 & $\cdots$ & 0.020 & $\cdots$ & $\cdots$ & $<1$ & 11 & $<10$ & 2 \\
\hline${ }_{\text {JUN }}^{13} \cdots$ & 742 & 0.490 & $\cdots$ & 0.021 & $\cdots$ & 40 & 38 & $<5$ & 40 & $<10$ \\
\hline${ }_{\pi}^{18} \ldots$ & 601 & 0.420 & $\cdots$ & 0.030 & $\cdots$ & 36 & 31 & $<5$ & 20 & $<10$ \\
\hline${ }_{A U G}^{16} \cdots$ & 507 & 0.340 & $\cdots$ & $<0.010$ & $\cdots$ & 23 & 18 & $<5$ & 10 & $<10$ \\
\hline${ }_{\mathrm{OCT}}^{12 \ldots}$ & 468 & 0.410 & $\cdots$ & 0.030 & -- & 13 & 12 & $<5$ & 20 & $<10$ \\
\hline DEC $21 \cdots$ & 557 & 0.390 & $\cdots$ & 0.020 & $\cdots$ & 14 & 11 & $<5$ & $<10$ & $<10$ \\
\hline $\begin{array}{c}16 \ldots \\
\text { APR } 1992\end{array}$ & 643 & 0.381 & $\cdots$ & 0.024 & $\cdots$ & 10 & 10 & $\cdots$ & $\cdots$ & $<1$ \\
\hline $\operatorname{MAY}^{20} \cdots$ & 666 & 0.374 & $\cdots$ & 0.024 & $\cdots$ & $<1$ & $<0.1$ & $\cdots$ & $<1$ & $<1$ \\
\hline $\mathrm{JUN}^{20} \cdots$ & 760 & 0.419 & $-\cdot$ & 0.015 & -- & $<1$ & 0.4 & $\cdots$ & $<1$ & $<1$ \\
\hline $\mathrm{JUL}^{24 \ldots}$ & 624 & 0.333 & $\cdots$ & 0.010 & $\cdots$ & $<1$ & 0.2 & $\cdots$ & $<1$ & $<1$ \\
\hline${ }_{A U G}^{13} \cdots$ & 518 & 0.342 & $\cdots$ & 0.008 & $\cdots$ & $<1$ & 0.4 & $\cdots$ & $<1$ & $<1$ \\
\hline$\underset{\mathrm{OCT}}{11 \ldots}$ & 460 & 0.385 & $\cdots$ & 0.016 & $\cdots$ & 1 & 0.1 & $-\cdot$ & 1 & $<1$ \\
\hline $\begin{array}{l}26 \ldots \\
\text { JAN } 1993\end{array}$ & 572 & 0.410 & $\cdots$ & 0.014 & $\cdots$ & $<1$ & 0.3 & $\cdots$ & $<1$ & $<1$ \\
\hline${ }_{\operatorname{MAR}}^{11 \ldots}$ & 607 & $\cdots$ & 0.398 & $\cdots$ & 0.016 & $<1$ & 0.2 & $\cdots$ & 1 & $<1$ \\
\hline $22 \ldots$ & 648 & $\cdots$ & 0.389 & $\cdots$ & 0.015 & $<1$ & 0.3 & $\cdots$ & $<1$ & $<1$ \\
\hline
\end{tabular}


Table 7. Onsite measurements and selected inorganic data for station 07079200, Leadville Mine Drainage Tunnel at Leadville--Continued

\begin{tabular}{|c|c|c|c|c|c|c|c|c|c|c|}
\hline DATE & $\begin{array}{l}\text { IRON, } \\
\text { TOTAL } \\
\text { RECOV- } \\
\text { ERABLE } \\
\text { (UG/L } \\
\text { AS FE) }\end{array}$ & $\begin{array}{l}\text { IRON, } \\
\text { DIS- } \\
\text { SOLVED } \\
\text { (UG/L } \\
\text { AS FE) }\end{array}$ & $\begin{array}{l}\text { LEAD, } \\
\text { TOTAL } \\
\text { RECOV - } \\
\text { ERABLE } \\
\text { (UG/L } \\
\text { AS PB) }\end{array}$ & $\begin{array}{l}\text { LEAD, } \\
\text { DIS - } \\
\text { SOLVED } \\
\text { (UG/L }\end{array}$ & $\begin{array}{l}\text { MANGA- } \\
\text { NESE, } \\
\text { TOTAL } \\
\text { RECOV- } \\
\text { ERABLE } \\
\text { (UG / L }\end{array}$ & $\begin{array}{l}\text { MANGA- } \\
\text { NESE, } \\
\text { DIS. } \\
\text { SOLVED } \\
\text { UUG/L } \\
\text { AS MN }\end{array}$ & $\begin{array}{l}\text { NICKEL, } \\
\text { DIS - } \\
\text { SOLVED } \\
\text { (UG/L }\end{array}$ & $\begin{array}{l}\text { SILVER, } \\
\text { DIS - } \\
\text { SOLVED } \\
\text { (UG/L } \\
\text { AS AG) }\end{array}$ & $\begin{array}{l}\text { ZINC, } \\
\text { TOTAL } \\
\text { RECOV - } \\
\text { ERABLE } \\
\text { (UG/L } \\
\text { AS ZN) }\end{array}$ & $\begin{array}{l}\text { ZINC, } \\
\text { DIS: } \\
\text { SOLVED } \\
\text { (UG/L }\end{array}$ \\
\hline & AS FE) & AS FE) & AS PBI & AS PBI & AS MN) & AS MN) & AS NI) & AS AG) & AS ZN) & AS $\mathrm{ZN}$ ) \\
\hline PR 1991 & & & & & & & & & & \\
\hline$\underset{\operatorname{MAY}}{22 \cdots}$ & 42000 & $<10$ & 7 & $<1$ & 3800 & $<10$ & $<1$ & $<1$ & 4700 & $<10$ \\
\hline${ }_{\pi N}^{13} \cdots$ & 2300 & 870 & 7 & 10 & 3400 & 3400 & $<10$ & 2 & 7800 & 7400 \\
\hline${ }_{\pi U L}^{18 \ldots}$ & 1300 & 55 & 5 & $<10$ & 2600 & 2700 & $<10$ & $<1$ & 6100 & 6000 \\
\hline${ }_{\text {AUG }} 16 \ldots$ & 5500 & 7 & 20 & $<10$ & 1600 & 1400 & $<10$ & $<1$ & 3400 & 3600 \\
\hline$\underset{\text { OCT }}{12 \ldots}$ & 880 & 10 & 8 & $<10$ & 840 & 910 & $<10$ & $<1$ & 2300 & 2300 \\
\hline${ }_{D E C} 21 \cdots$ & 2100 & 56 & 9 & $<10$ & 1500 & 1500 & $<10$ & $<1$ & 3400 & 3500 \\
\hline $\begin{array}{c}16 \ldots \\
\text { APR } 1992\end{array}$ & 1500 & 400 & 4 & $<0.5$ & 1600 & 1800 & $\cdots$ & - & 3600 & 3800 \\
\hline$\underset{\operatorname{MAY}}{20 \ldots}$ & 30 & $<3$ & $<1$ & $<0.5$ & 20 & 13 & $\cdots$ & $\cdots$ & 20 & 8 \\
\hline${ }_{J U N}^{20} \cdots$ & 20 & 4 & $<1$ & $<0.5$ & 380 & 430 & $\cdots$ & . & 30 & 22 \\
\hline${ }_{\pi U L}^{24} \ldots$ & 20 & $<3$ & $<1$ & $<0.5$ & 180 & 180 & $\cdots$ & $\cdots$ & 20 & 10 \\
\hline${ }_{\text {AUG }}^{13 \ldots}$ & 40 & 3 & $<1$ & $<0.5$ & 150 & 130 & $\cdots$ & $\cdots$ & 20 & 14 \\
\hline${ }_{\mathrm{CT}}^{11 \ldots}$ & 2600 & 7 & 2 & $<0.5$ & 120 & 110 & $\cdots$ & .. & 170 & 15 \\
\hline $\begin{array}{c}26 \ldots \\
\text { JAN } 1993\end{array}$ & 10 & 4 & $<1$ & $<0.5$ & 190 & 230 & $\cdots$ & $\cdots$ & $<10$ & 13 \\
\hline${ }_{\operatorname{MAR}}^{11} \ldots$ & 10 & $<3$ & $<1$ & $<0.5$ & 730 & 830 & $\cdots$ & $\cdots$ & 60 & 43 \\
\hline $22 \ldots$ & 10 & 9 & $<1$ & $<0.5$ & 300 & 330 & $\cdots$ & - & $<10$ & 15 \\
\hline
\end{tabular}


Table 8. Onsite measurements and bacteriological and selected inorganic data for station 07081200 , Arkansas River near Leadville

\begin{tabular}{|c|c|c|c|c|c|c|c|c|c|c|}
\hline TIME & $\begin{array}{c}\text { DIS - } \\
\text { CHARGE, } \\
\text { INST. } \\
\text { CUBIC } \\
\text { FEET } \\
\text { PER }\end{array}$ & $\begin{array}{l}\text { SPE- } \\
\text { CIFIC } \\
\text { CON- } \\
\text { DUCT- } \\
\text { ANCE }\end{array}$ & $\begin{array}{c}\text { PH } \\
\text { WATER } \\
\text { WHOLE } \\
\text { FIELD } \\
\text { (STAND - } \\
\text { ARD }\end{array}$ & $\begin{array}{c}\text { TEMPER- } \\
\text { ATURE } \\
\text { WATER }\end{array}$ & $\begin{array}{c}\text { OXYGEN, } \\
\text { DIS - } \\
\text { SOLVED }\end{array}$ & $\begin{array}{c}\text { COLI - } \\
\text { FORM, } \\
\text { TOTAL, } \\
\text { IMMED. } \\
\text { /COLS. } \\
\text { PER }\end{array}$ & $\begin{array}{l}\text { COLI- } \\
\text { FORM, } \\
\text { FECAL, } \\
0.7 \\
\text { UM-MF } \\
\text { (COLS.) }\end{array}$ & $\begin{array}{c}\text { STREP- } \\
\text { TOCOCCI } \\
\text { FECAL, } \\
\text { KF AGAR } \\
\text { ICOLS. } \\
\text { PER }\end{array}$ & $\begin{array}{l}\text { HARD - } \\
\text { NESS } \\
\text { TOTAL } \\
\text { (MG /L } \\
\text { AS }\end{array}$ & $\begin{array}{l}\text { CALCIUM } \\
\text { DIS- } \\
\text { SOLVED } \\
\text { (MG/L }\end{array}$ \\
\hline & SECOND & (US/CM) & UNITS) & (DEG C) & (MG/L) & $100 \mathrm{ML})$ & $100 \mathrm{ML})$ & $100 \mathrm{ML})$ & СACO3) & AS $(\mathrm{A})$ \\
\hline
\end{tabular}

\begin{tabular}{|c|c|c|c|c|c|c|c|c|c|c|c|}
\hline \multicolumn{12}{|l|}{ APR 1990} \\
\hline $17 \ldots$ & 1315 & $\cdots$ & 170 & 8.2 & 5.0 & 8.2 & $\cdots$ & $<1$ & 56 & 82 & 20 \\
\hline \multicolumn{12}{|l|}{ MAY } \\
\hline $22 \ldots$ & 1230 & 128 & 121 & 7.7 & 9.0 & 8.0 & - & $<1$ & 44 & 53 & 13 \\
\hline \multicolumn{12}{|l|}{ JUN } \\
\hline $05 \ldots$ & 1135 & 493 & 76 & 7.7 & 7.5 & 8.0 & 880 & K10 & K50 & 33 & 9.4 \\
\hline $19 \ldots$ & 1200 & 271 & 101 & 7.9 & 9.5 & 8.0 & K120 & $<2$ & K10 & 46 & 11 \\
\hline \multicolumn{12}{|l|}{ JUL } \\
\hline $17 \ldots$ & 1100 & 70 & 153 & 8.2 & 11.5 & 7.8 & K57 & K1 & 20 & 65 & 14 \\
\hline \multicolumn{12}{|l|}{ AUG } \\
\hline $28 \ldots$ & 1030 & 32 & 223 & 8.5 & 10.0 & 7.8 & $<2$ & E1 & E3 & 120 & 28 \\
\hline \multicolumn{12}{|l|}{ OCT } \\
\hline $29 \ldots$ & 1445 & 27 & 219 & 8.4 & 7.0 & 8.1 & E45 & $<1$ & 86 & 110 & 26 \\
\hline \multicolumn{12}{|l|}{ DEC } \\
\hline $19 \ldots$ & 1210 & 18 & 273 & 7.7 & 0.5 & 9.5 & E33 & $<2$ & E8 & 140 & 33 \\
\hline \multicolumn{12}{|l|}{ MAR 1991} \\
\hline $25 \ldots$ & 1350 & 17 & 299 & 8.5 & 1.5 & 9.4 & $\cdots$ & $<1$ & $\cdots$ & 140 & 34 \\
\hline \multicolumn{12}{|l|}{ APR } \\
\hline $23 \ldots$ & 0755 & 27 & 221 & 7.9 & 1.5 & 9.1 & E32 & E1 & E7 & 100 & 25 \\
\hline \multicolumn{12}{|l|}{ MAY } \\
\hline $14 \ldots$ & 0820 & 94 & 121 & 8.2 & 1.0 & 9.4 & 250 & $<1$ & E11 & 52 & 13 \\
\hline $29 \ldots$ & 0930 & 295 & 79 & $\cdots$ & - & $\cdots$ & $\cdots$ & -- & $\cdots$ & $\cdots$ & - \\
\hline \multicolumn{12}{|l|}{ JUN } \\
\hline $10 \ldots$ & 0610 & 385 & - - & $\cdots$ & - & $\cdots$ & $\cdots$ & - - & $-\cdot$ & 35 & 8.9 \\
\hline $18 \ldots$ & 0945 & 325 & 87 & 7.7 & 7.0 & 8.4 & 280 & E2 & E4 & 41 & 9.7 \\
\hline \multicolumn{12}{|l|}{ JUL } \\
\hline $16 \ldots$ & 0945 & 89 & 133 & 8.1 & 10.5 & 7.7 & E31 & E14 & E8 & 66 & 16 \\
\hline \multicolumn{12}{|l|}{ AUG } \\
\hline $13 \ldots$ & 0615 & 73 & 167 & 7.9 & 9.0 & - & 56 & 28 & 51 & 78 & 19 \\
\hline \multicolumn{12}{|l|}{ OCT } \\
\hline $22 \ldots$ & 0850 & 20 & 249 & 8.1 & 1.5 & 9.1 & E3 & - & E3 & 120 & 29 \\
\hline \multicolumn{12}{|l|}{ DEC } \\
\hline $16 \ldots$ & 1445 & 16 & 265 & 7.8 & 1.0 & 9.4 & 60 & $<1$ & $-\cdot$ & 120 & 30 \\
\hline \multicolumn{12}{|l|}{ MAR 1992} \\
\hline $23 \ldots$ & 1340 & 32 & 289 & 8.1 & 1.5 & 9.4 & - & $\cdots$ & $\cdots$ & 130 & 31 \\
\hline \multicolumn{12}{|l|}{ APR } \\
\hline $20 \ldots$ & 1850 & 39 & 191 & 8.0 & 7.0 & 7.8 & 35 & $<1$ & E2 & 80 & 19 \\
\hline MAY & & & & & & & & & & & \\
\hline $20 \ldots$ & 1100 & 249 & 85 & 7.7 & 7.0 & 8.2 & E6 & E11 & E15 & 37 & 9.3 \\
\hline JUN & & & & & & & & & & & \\
\hline $24 \ldots$ & 1330 & 229 & 101 & 8.0 & 10.5 & 8.1 & E8 & E9 & E9 & 48 & 12 \\
\hline JUL & & & & & & & & & & & \\
\hline $13 \ldots$ & 1200 & 124 & 131 & 8.2 & 9.5 & 7.4 & E13 & E9 & E6 & 61 & 15 \\
\hline AUG & & & & & & & & & & & \\
\hline $11 \ldots$ & 1000 & 58 & 177 & 8.2 & 10.0 & 7.7 & 74 & E66 & 81 & 86 & 21 \\
\hline $26 \ldots$ & 0750 & 67 & 156 & 7.8 & 5.5 & 8.6 & 60 & 34 & 59 & 75 & 18 \\
\hline OCT & & & & & & & & & & & \\
\hline $26 \ldots$ & 1310 & 31 & 223 & 8.3 & 6.0 & 8.6 & 30 & $<1$ & E8 & 97 & 23 \\
\hline JAN 1993 & & & & & & & & & & & \\
\hline $11 \ldots$ & 1200 & E26 & 289 & 8.0 & 0.5 & 9.7 & E32 & $<1$ & $<1$ & 120 & 28 \\
\hline MAR & & & & & & & & & & & \\
\hline $22 \ldots$ & 1000 & E26 & 260 & 8.1 & 1.0 & 9.7 & $<2$ & $<1$ & $\cdots$ & 110 & 26 \\
\hline
\end{tabular}


Table 8. Onsite measurements and bacteriological and selected inorganic data for station 07081200 , Arkansas River near Leadville--Continued

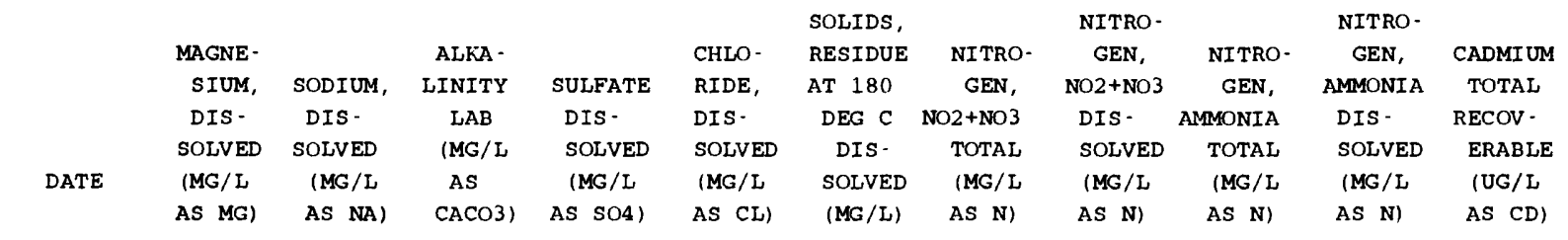

\begin{tabular}{|c|c|c|c|c|c|c|c|c|c|c|c|}
\hline APR 1990 & & & & & & & & & & & \\
\hline $17 \ldots$ & 7.9 & 2.4 & 50 & 42 & 0.90 & 121 & 0.040 & $\cdots$ & 0.010 & $\cdots$ & 2 \\
\hline MAY & & & & & & & & & & & \\
\hline $22 \ldots$ & 5.0 & 1.7 & 36 & 22 & 1.1 & 81 & 0.037 & $\cdots$ & $<0.010$ & $\cdots$ & 2 \\
\hline JUN & & & & & & & & & & & \\
\hline $05 \ldots$ & 2.4 & 1.4 & 23 & 14 & 0.30 & 48 & 0.026 & $\cdots$ & 0.020 & $\cdots$ & 2 \\
\hline $19 \ldots$ & 4.5 & 1.0 & 37 & 9.8 & 0.60 & 57 & 0.050 & $\cdots$ & $<0.010$ & $\cdots$ & 1 \\
\hline JUL & & & & & & & & & & & \\
\hline $17 \ldots$ & 7.3 & 0.50 & 60 & 20 & 0.20 & 94 & 0.057 & $\cdots$ & $<0.010$ & $-\cdot$ & $<1$ \\
\hline AUG & & & & & & & & & & & \\
\hline $28 \ldots$ & 11 & 1.9 & 80 & 33 & 1.7 & 131 & 0.105 & $-\cdot$ & $<0.010$ & $\cdots$ & $<1$ \\
\hline OCT & & & & & & & & & & & \\
\hline $29 \ldots$ & 10 & 2.3 & 75 & 36 & 2.1 & 131 & 0.078 & $\cdots$ & 0.020 & $\cdots$ & $<1$ \\
\hline DEC & & & & & & & & & & & \\
\hline $19 \ldots$ & 13 & 2.4 & 85 & 53 & 4.5 & 163 & 0.134 & $\cdots$ & 0.002 & $\cdots$ & 1 \\
\hline MAR 1991 & & & & & & & & & & & \\
\hline $25 \ldots$ & 13 & 2.5 & 83 & 61 & 5.1 & 156 & 0.126 & $\cdots$ & 0.030 & $\cdots$ & $<1$ \\
\hline APR & & & & & & & & & & & \\
\hline $23 \ldots$ & 9.9 & 2.2 & 67 & 43 & 1.8 & 120 & 0.072 & $\cdots$ & 0.018 & $\cdots$ & $<1$ \\
\hline MAY & & & & & & & & & & & \\
\hline $14 \ldots$ & 4.7 & 1.5 & 36 & 18 & 1.0 & 83 & 0.056 & $\cdots$ & 0.015 & $\cdots$ & 2 \\
\hline $29 \ldots$ & $\cdots$ & $\cdots$ & $\cdots$ & $\cdots$ & -. & $\cdots$ & $\cdots$ & $\cdots$ & $\cdots$ & $\cdots$ & $-\cdot$ \\
\hline JUN & & & & & & & & & & & \\
\hline $10 \ldots$ & 3.2 & 1.2 & 30 & 6.6 & $\cdots$ & 28 & $\cdots$ & $-\cdot$ & $\cdots$ & $\cdots$ & 1 \\
\hline $18 \ldots$ & 4.0 & 0.90 & 32 & 7.7 & 0.40 & 46 & 0.049 & $\cdots$ & 0.022 & $\cdots$ & 1 \\
\hline JUL & & & & & & & & & & & \\
\hline $16 \ldots$ & 6.4 & 1.3 & 52 & 16 & 1.5 & 92 & 0.058 & $-\cdot$ & 0.005 & $\cdots$ & $<1$ \\
\hline AUG & & & & & & & & & & & \\
\hline $13 \ldots$ & 7.3 & 1.6 & 65 & 22 & 0.20 & 100 & 0.080 & $\cdots$ & 0.003 & $\cdots$ & $<1$ \\
\hline ОСт & & & & & & & & & & & \\
\hline $22 \ldots$ & 11 & 2.1 & 89 & 40 & 1.2 & 139 & 0.086 & $\cdots$ & 0.021 & $\cdots$ & $<1$ \\
\hline DEC & & & & & & & & & & & \\
\hline $16 \ldots$ & 12 & 2.6 & 87 & 51 & $<0.10$ & 166 & 0.093 & $\cdots$ & 0.007 & $\cdots$ & $<1$ \\
\hline MAR 1992 & & & & & & & & & & & \\
\hline $23 \ldots$ & 13 & 5.9 & 66 & 82 & 3.6 & 174 & 0.370 & -- & 0.034 & $\cdots$ & $<1$ \\
\hline APR & & & & & & & & & & & \\
\hline $20 \ldots$ & 7.8 & 4.2 & 48 & 42 & 0.70 & 116 & 0.045 & $\cdots$ & 0.016 & $\cdots$ & $<1$ \\
\hline MAY & & & & & & & & & & & \\
\hline $20 \ldots$ & 3.4 & 1.3 & 27 & 12 & 0.50 & 78 & 0.054 & $\cdots$ & 0.011 & $\cdots$ & $<1$ \\
\hline JUN & & & & & & & & & & & \\
\hline $24 \ldots$ & 4.4 & 1.2 & 40 & 18 & 0.50 & 78 & 0.053 & $\cdots$ & 0.002 & $\cdots$ & 1 \\
\hline JUL & & & & & & & & & & & \\
\hline $13 \ldots$ & 5.8 & 1.5 & 49 & 16 & 0.40 & 108 & 0.049 & $\cdots$ & 0.005 & $\cdots$ & $<1$ \\
\hline AUG & & & & & & & & & & & \\
\hline $11 \ldots$ & 8.1 & 2.4 & 63 & 27 & 0.80 & 118 & 0.161 & $\cdots$ & 0.010 & $\cdots$ & $<1$ \\
\hline $26 \ldots$ & 7.3 & 2.3 & 54 & 19 & 1.2 & 84 & 0.057 & $\cdots$ & 0.008 & $\cdots$ & $<1$ \\
\hline OCT & & & & & & & & & & & \\
\hline $26 \ldots$ & 9.6 & 3.4 & 66 & 44 & 0.90 & 136 & 0.069 & $\cdots$ & 0.003 & $\cdots$ & $<1$ \\
\hline JAN 1993 & & & & & & & & & & & \\
\hline $11 \ldots$ & 12 & 5.1 & 71 & 66 & 0.90 & 172 & $\cdots$ & 0.164 & $\cdots$ & 0.020 & $<1$ \\
\hline MAR & & & & & & & & & & & \\
\hline $22 \ldots$ & 11 & 6.0 & 66 & 60 & 0.80 & 150 & $\cdots$ & 0.128 & $\cdots$ & 0.006 & $<1$ \\
\hline
\end{tabular}


Table 8. Onsite measurements and bacteriological and selected inorganic data for station 07081200 , Arkansas River near Leadville--Continued

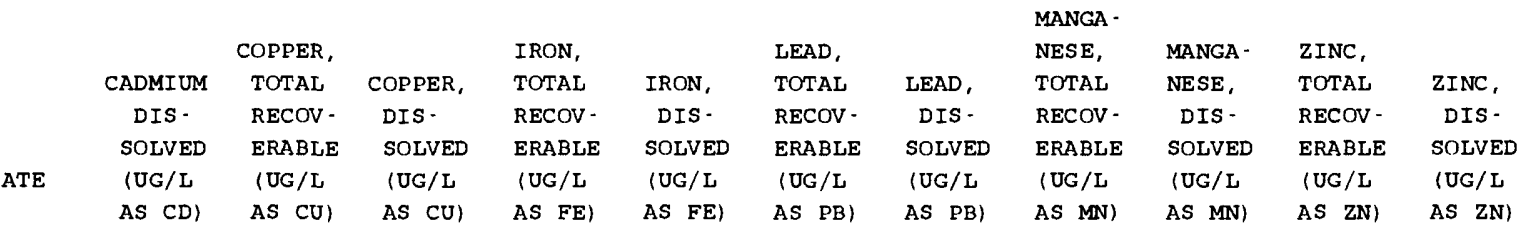

\begin{tabular}{|c|c|c|c|c|c|c|c|c|c|c|c|}
\hline \multicolumn{12}{|l|}{ APR 1990} \\
\hline $17 \ldots$ & 1.4 & 3 & 3 & 1800 & 710 & 3 & 0.8 & 290 & 230 & 420 & 370 \\
\hline \multicolumn{12}{|l|}{ MAY } \\
\hline $22 \ldots$ & 1.6 & 4 & 2 & 740 & 120 & 4 & $<0.5$ & 250 & 180 & 440 & 350 \\
\hline \multicolumn{12}{|l|}{ JUN } \\
\hline $05 \ldots$ & 0.3 & 15 & 3 & 2300 & 25 & 110 & 4.8 & 300 & 29 & 360 & 85 \\
\hline $19 \ldots$ & 0.4 & 6 & 2 & 580 & 68 & 4 & $<0.5$ & 80 & 47 & 120 & 87 \\
\hline \multicolumn{12}{|l|}{ JUL } \\
\hline $17 \ldots$ & 0.5 & 1 & 1 & 230 & 100 & 1 & 0.5 & 70 & 63 & 160 & 130 \\
\hline \multicolumn{12}{|l|}{ AUG } \\
\hline $28 \ldots$ & 0.3 & 2 & $<1$ & 130 & 52 & 1 & 0.9 & 90 & 76 & 210 & 180 \\
\hline \multicolumn{12}{|l|}{ OCT } \\
\hline $29 \ldots$ & 0.5 & 1 & $<1$ & 140 & 120 & 1 & $<0.5$ & 80 & 71 & 180 & 170 \\
\hline \multicolumn{12}{|l|}{ DEC } \\
\hline $19 \ldots$ & 0.6 & 4 & 1 & 130 & 70 & 2 & $<0.5$ & 130 & 130 & 330 & 320 \\
\hline \multicolumn{12}{|l|}{ MAR 1991} \\
\hline $25 \ldots$ & 0.7 & 2 & 1 & 210 & 65 & $<1$ & $<0.5$ & 150 & 140 & 240 & 310 \\
\hline \multicolumn{12}{|l|}{ APR } \\
\hline $23 \ldots$ & 0.4 & 2 & 1 & 410 & 230 & 3 & $<0.5$ & 130 & 130 & 220 & 210 \\
\hline \multicolumn{12}{|l|}{ MAY } \\
\hline $14 \ldots$ & 0.8 & 5 & 2 & 660 & 170 & 4 & 0.9 & 140 & 100 & 220 & 160 \\
\hline $29 \ldots$ & $\cdots$ & - & $\cdots$ & 1700 & 90 & - & $\cdots$ & $\cdots$ & $\cdots$ & 90 & 30 \\
\hline \multicolumn{12}{|l|}{ JUN } \\
\hline $10 \ldots$ & 1.8 & 6 & 3 & 670 & 500 & 5 & 6.7 & 60 & 63 & 120 & 140 \\
\hline $18 \ldots$ & 0.7 & 18 & 5 & 350 & 87 & 10 & $<0.5$ & 60 & 35 & 90 & 85 \\
\hline \multicolumn{12}{|l|}{ JUL } \\
\hline $16 \ldots$ & 0.3 & 3 & 1 & 210 & 88 & 16 & $<0.5$ & 50 & 52 & 90 & 87 \\
\hline \multicolumn{12}{|l|}{ AUG } \\
\hline $13 \ldots$ & 0.3 & 2 & $<1$ & 330 & 96 & 5 & $<0.5$ & 70 & 51 & 120 & 99 \\
\hline \multicolumn{12}{|l|}{ OCT } \\
\hline $22 \ldots$ & 0.5 & 2 & $<1$ & 120 & 68 & 1 & $<0.5$ & 100 & 94 & 210 & 200 \\
\hline \multicolumn{12}{|l|}{ DEC } \\
\hline $16 \ldots$ & 1.3 & - & $<1$ & 170 & 75 & $<1$ & $<0.5$ & 120 & 110 & 300 & 270 \\
\hline \multicolumn{12}{|l|}{ MAR 1992} \\
\hline $23 \ldots$ & 0.3 & $<1$ & $<1$ & 140 & 68 & $<1$ & $<0.5$ & 30 & 27 & 60 & 45 \\
\hline \multicolumn{12}{|l|}{ APR } \\
\hline $20 \ldots$ & 0.4 & 2 & 1 & 520 & 330 & 1 & $<0.5$ & 50 & 36 & 60 & 47 \\
\hline MAY & & & & & & & & & & & \\
\hline $20 \ldots$ & 0.4 & 4 & 2 & 740 & 82 & 5 & 0.8 & 210 & 34 & 120 & 69 \\
\hline JUN & & & & & & & & & & & \\
\hline $24 \ldots$ & 1.0 & 3 & 2 & 260 & 55 & 3 & $<0.5$ & 30 & 14 & 40 & 24 \\
\hline JUL & & & & & & & & & & & \\
\hline $13 \ldots$ & 0.1 & 1 & $<1$ & 210 & 80 & 1 & $<0.5$ & 30 & 18 & 20 & 16 \\
\hline AUG & & & & & & & & & & & \\
\hline $11 \ldots$ & 0.1 & 3 & $<1$ & 240 & 140 & 3 & 0.6 & 40 & 28 & 30 & 17 \\
\hline $26 \ldots$ & 0.1 & 9 & 1 & 330 & 180 & 3 & 0.5 & 50 & 28 & 70 & 55 \\
\hline OCT & & & & & & & & & & & \\
\hline $26 \ldots$ & 0.1 & $<1$ & 2 & 140 & 92 & $<1$ & $<0.5$ & $<10$ & 27 & 30 & 23 \\
\hline JAN 1993 & & & & & & & & & & & \\
\hline $11 \ldots$ & 0.1 & $<1$ & $<1$ & 160 & 88 & 1 & $<0.5$ & 60 & 38 & 50 & 35 \\
\hline MAR & & & & & & & & & & & \\
\hline $22 \ldots$ & 0.1 & $<1$ & $<1$ & 190 & 92 & $<1$ & $<0.5$ & 30 & 18 & 20 & 21 \\
\hline
\end{tabular}


Table 9. Onsite measurements and selected inorganic data for station 07081800 , California Gulch at Malta

\begin{tabular}{|c|c|c|c|c|c|c|c|c|c|c|}
\hline & & $\begin{array}{c}\text { DIS - } \\
\text { CHARGE, }\end{array}$ & SPE & $\begin{array}{c}\text { PH } \\
\text { WATER }\end{array}$ & & & HARD - & & AGNE - & \\
\hline DATE & TIME & $\begin{array}{l}\text { INST } \\
\text { CUBIC } \\
\text { FEET } \\
\text { PER }\end{array}$ & $\begin{array}{l}\text { CIFIC } \\
\text { CON- } \\
\text { DUCT - } \\
\text { ANCE }\end{array}$ & $\begin{array}{l}\text { WHOLE } \\
\text { FIELD } \\
\text { (STAND- } \\
\text { ARD }\end{array}$ & $\begin{array}{l}\text { TEMPER - } \\
\text { ATURE } \\
\text { WATER }\end{array}$ & $\begin{array}{c}\text { OXYGEN, } \\
\text { DIS - } \\
\text { SOLVED }\end{array}$ & $\begin{array}{l}\text { NESS } \\
\text { TOTAL } \\
\text { (MG/L } \\
\text { AS }\end{array}$ & $\begin{array}{l}\text { CALCIUM } \\
\text { DIS - } \\
\text { SOLVED } \\
\text { (MG /L }\end{array}$ & $\begin{array}{l}\text { SIUM, } \\
\text { DIS- } \\
\text { SOLVED } \\
\text { (MG/L }\end{array}$ & $\begin{array}{l}\text { SODIUM, } \\
\text { DIS- } \\
\text { SOLVED } \\
\text { /MG /L }\end{array}$ \\
\hline & & SECOND & (US/CM) & UNITS) & (DEG C) & $(\mathrm{MG} / \mathrm{L})$ & $(\mathrm{CACO} 3)$ & AS CA) & AS MG) & AS NA) \\
\hline
\end{tabular}

\begin{tabular}{|c|c|c|c|c|c|c|c|c|c|c|}
\hline $\begin{array}{c}\text { APR } 1991 \\
23 \ldots\end{array}$ & 0910 & $\cdots$ & 741 & 7.2 & 3.5 & 8.7 & 300 & 61 & 36 & 14 \\
\hline $\begin{array}{l}\text { MAY } \\
14 \ldots \\
\pi U N\end{array}$ & 1015 & 1.9 & 990 & 6.5 & 7.5 & 7.7 & 440 & 89 & 53 & 8.3 \\
\hline $\begin{array}{l}18 \ldots \\
\pi U L\end{array}$ & 1140 & 1.8 & 957 & 6.7 & 16.0 & 6.8 & 410 & 89 & 46 & 14 \\
\hline${ }_{A \cup G}^{16} \cdots$ & 1125 & 1.9 & 971 & 7.6 & 19.5 & 6.2 & 420 & 110 & 36 & 17 \\
\hline$\underset{\mathrm{OCT}}{13} \ldots$ & 0815 & 2.2 & 935 & 6.9 & 11.5 & $\cdots$ & 410 & 100 & 39 & 15 \\
\hline$\underset{\mathrm{DEC}}{22} \cdots$ & 1005 & 0.59 & 686 & 7.8 & 4.5 & 8.7 & 230 & 52 & 24 & 31 \\
\hline${ }_{A P R}^{16} 1992$ & 1610 & 1.3 & 716 & 7.3 & 1.0 & 9.2 & $\cdots$ & $\cdots$ & $\cdots$ & $\cdots$ \\
\hline$\underset{\operatorname{MAY}}{20} \cdots$ & 1750 & 1.8 & 971 & 6.9 & 8.5 & $\cdots$ & $\cdots$ & $\cdots$ & $\cdots$ & $\cdots$ \\
\hline$\underset{\pi N}{20} \cdots$ & 1700 & 3.3 & 518 & 8.1 & 16.0 & $\cdots$ & $\cdots$ & $\cdots$ & $\cdots$ & $\cdots$ \\
\hline$\underset{\pi L}{24} \ldots$ & 1500 & 2.0 & 628 & 8.0 & 17.5 & 6.8 & $\cdots$ & $\cdots$ & $\cdots$ & $\cdots$ \\
\hline$\underset{A U G}{13} \cdots$ & 1300 & 1.8 & 689 & 8.0 & 15.5 & 6.9 & $\cdots$ & $\cdots$ & $-\cdot$ & $\cdots$ \\
\hline $\operatorname{ocT}^{11} \cdots$ & 1045 & 2.5 & 792 & 7.5 & 13.0 & 7.0 & $-\cdot$ & $\cdots$ & $\cdots$ & $\cdots$ \\
\hline JAN 1993 & 1420 & 1.2 & 670 & 7.5 & 7.5 & 7.9 & $\cdots$ & $\cdots$ & $\cdots$ & $\cdots$ \\
\hline MAR & 1310 & 0.59 & 492 & 7.9 & 1.0 & 9.6 & $\cdots$ & $\cdots$ & $\cdots$ & $\cdots$ \\
\hline $22 \ldots$ & 1120 & 1.0 & 619 & 7.9 & 2.5 & 9.1 & $\cdots$ & $\cdots$ & $-\cdot$ & $-\cdot$ \\
\hline
\end{tabular}

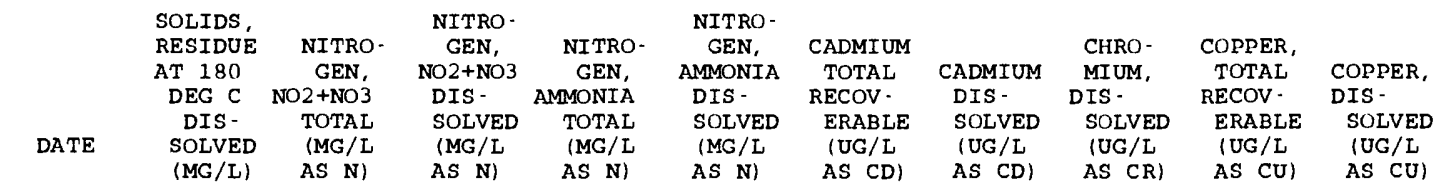

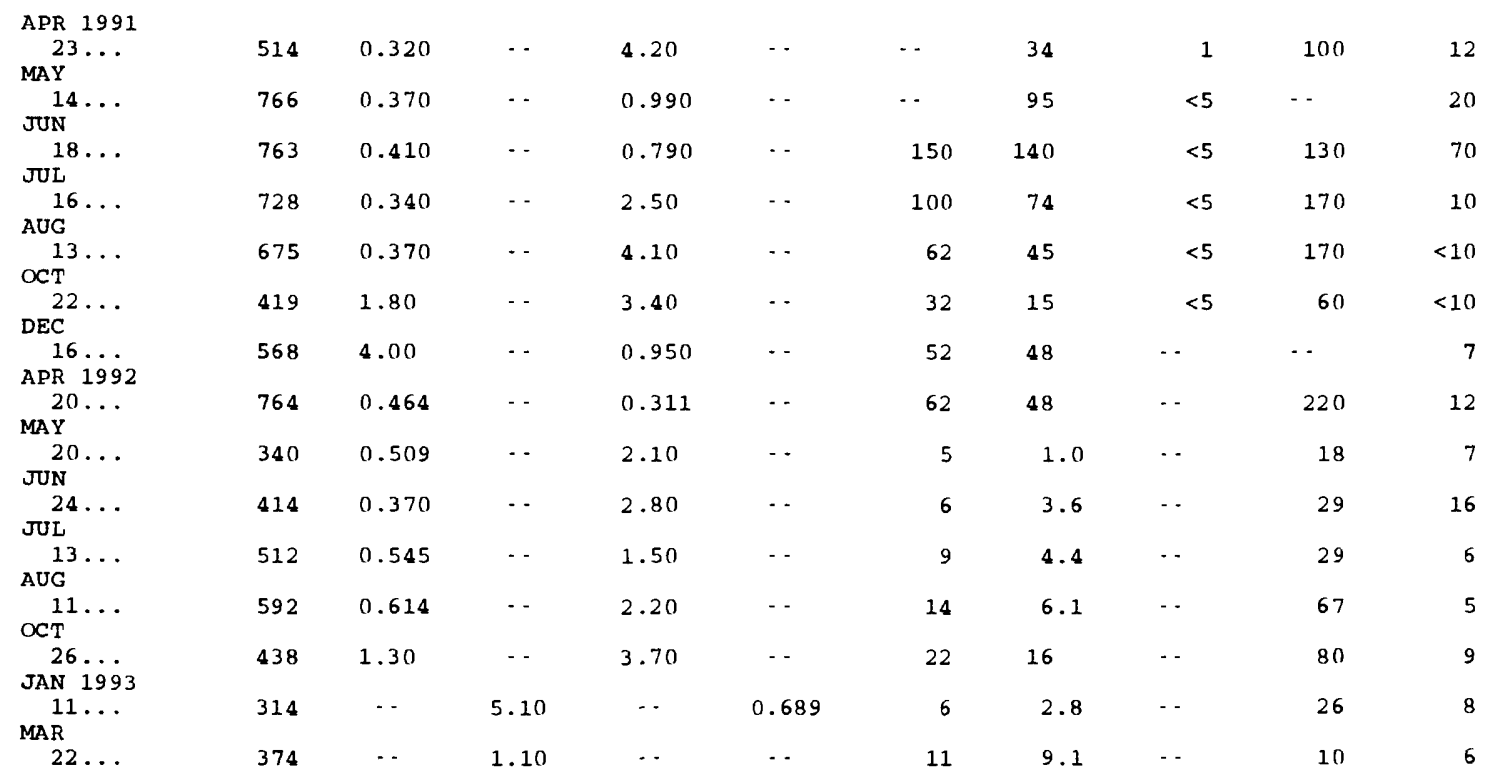


Table 9. Onsite measurements and selected inorganic data for station 07081800 , California Gulch at Malta--Continued

\begin{tabular}{|c|c|c|c|c|c|c|c|c|c|c|}
\hline DATE & $\begin{array}{l}\text { IRON, } \\
\text { TOTAL } \\
\text { RECOV - } \\
\text { ERABLE } \\
\text { (UG/L } \\
\text { AS FE) }\end{array}$ & $\begin{array}{l}\text { IRON, } \\
\text { DIS- } \\
\text { SOLVED } \\
\text { (UG/L } \\
\text { AS FE) }\end{array}$ & $\begin{array}{l}\text { LEAD, } \\
\text { TOTAL } \\
\text { RECOV - } \\
\text { ERABLE } \\
\text { (UG /L } \\
\text { AS PB) }\end{array}$ & $\begin{array}{l}\text { LEAD, } \\
\text { DIS - } \\
\text { SOLVED } \\
\text { (UG/L } \\
\text { AS PB) }\end{array}$ & $\begin{array}{l}\text { MANGA- } \\
\text { NESE, } \\
\text { TOTAL } \\
\text { RECOV- } \\
\text { ERABLE } \\
\text { (UG/L } \\
\text { AS MN) }\end{array}$ & $\begin{array}{l}\text { MANGA - } \\
\text { NESE, } \\
\text { DIS - } \\
\text { SOLVED } \\
\text { (UG/L } \\
\text { AS MN) }\end{array}$ & $\begin{array}{l}\text { NICKEL, } \\
\text { DIS - } \\
\text { SOLVED } \\
\text { (UG / L } \\
\text { AS NI) }\end{array}$ & $\begin{array}{l}\text { S I LVER, } \\
\text { DIS - } \\
\text { SOLVED } \\
\text { (UG / L } \\
\text { AS AG) }\end{array}$ & $\begin{array}{l}\text { ZINC, } \\
\text { TOTAL } \\
\text { RECOV - } \\
\text { ERABLE } \\
\text { \{UG/L } \\
\text { AS ZN }\end{array}$ & $\begin{array}{l}\text { ZINC, } \\
\text { DIS - } \\
\text { SOLVED } \\
\text { (UG/L } \\
\text { AS ZN) }\end{array}$ \\
\hline & & & & & & & & & & \\
\hline APR 1991 & & & & & & & & & & \\
\hline $23 \ldots$ & 5900 & 100 & 240 & 1 & 11000 & 9700 & $<1$ & $<1.0$ & 4300 & 1900 \\
\hline MAY & & & & & & & & & & \\
\hline $\operatorname{JUN}_{N}^{14} \cdots$ & $\cdots$ & 7100 & $\cdots$ & $<10$ & $\cdots$ & 19000 & 20 & 5.0 & $\cdots$ & 26000 \\
\hline$J_{J L}^{18 \ldots}$ & 4600 & 230 & 130 & 30 & 12000 & 14000 & 20 & 2.0 & 31000 & 32000 \\
\hline $16 \ldots$ & 3000 & 6 & 77 & $<10$ & 10000 & 11000 & 20 & 1.0 & 19000 & 15000 \\
\hline$\underset{\text { OCT }}{13} \ldots$ & 12000 & 69 & 1200 & $<10$ & 9100 & 10000 & 10 & 2.0 & 13000 & 10000 \\
\hline$\underset{\mathrm{DEC}}{22} \cdots$ & 1500 & 7 & 51 & $<10$ & 4700 & 5100 & $<10$ & 2.0 & 9200 & 4400 \\
\hline $\begin{array}{c}16 \ldots \\
\text { APR } 1992\end{array}$ & 2100 & 42 & 65 & $<0.5$ & 7500 & 8500 & - & - & 15000 & 14000 \\
\hline$\underset{\operatorname{MAY}}{20 \ldots}$ & 26000 & 5500 & 290 & $<0.5$ & 22000 & 24000 & $\cdots$ & $\cdots$ & 22000 & 22000 \\
\hline$\underset{J U N}{20 \ldots}$ & 1600 & 14 & 38 & $<0.5$ & 1800 & 1700 & $\cdots$ & $\cdots$ & 1800 & 320 \\
\hline $\mathrm{JUL}^{24 \ldots}$ & 2000 & 60 & 52 & $<0.5$ & 2700 & 2800 & $\cdots$ & $\cdots$ & 2000 & 1100 \\
\hline$\underset{\text { AUG }}{13} \ldots$ & 4300 & 43 & 120 & 0.6 & 3600 & 3800 & $\cdots$ & $\cdots$ & 3000 & 1500 \\
\hline $\mathrm{OCT}^{11 \ldots}$ & 8100 & 22 & 850 & $<0.5$ & 3700 & 3600 & $\cdots$ & $\cdots$ & 3600 & 2600 \\
\hline $\begin{array}{c}26 \ldots \\
\text { JAN } 1993\end{array}$ & 14000 & 1400 & 250 & 0.8 & 6000 & 5700 & $\cdots$ & $\cdots$ & 11000 & 7900 \\
\hline $11 \ldots$ & 2600 & 23 & 120 & 0.6 & 3100 & 3000 & $\cdots$ & $\cdots$ & 3500 & 2600 \\
\hline $22 \ldots$ & 3600 & 37 & 150 & $<0.5$ & 3800 & 3800 & $\cdots$ & $\cdots$ & 5200 & 4000 \\
\hline
\end{tabular}


Table 10. Onsite measurements and selected inorganic data for station 07082000 , Lake Fork above Sugar Loaf Reservoir

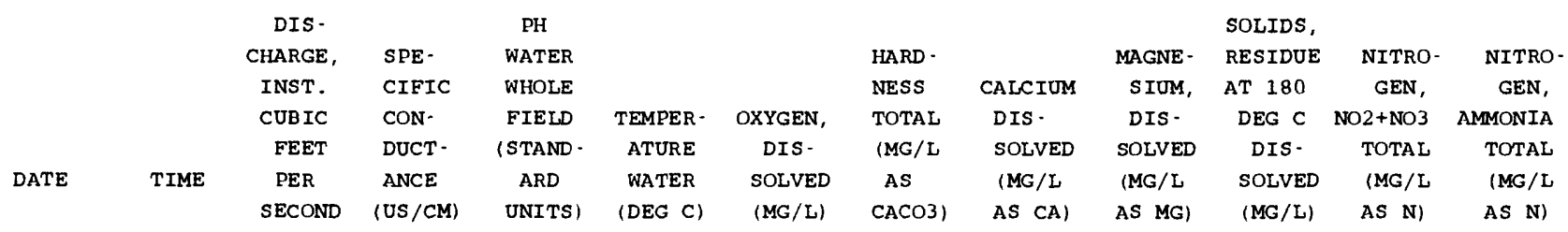

\begin{tabular}{|c|c|c|c|c|c|c|c|c|c|c|c|c|}
\hline \multicolumn{13}{|l|}{ APR 1990} \\
\hline $16 \ldots$ & 1745 & $\cdots$ & 27 & 7.9 & 0.0 & $\cdots$ & $\cdots$ & $\cdots$ & -- & 17 & 0.086 & $<0.010$ \\
\hline \multicolumn{13}{|l|}{ JUN } \\
\hline $04 \ldots$ & 1420 & 65 & & 6.8 & 6.0 & 8.3 & - - & $\cdots$ & $\cdots$ & 27 & 0.031 & 0.100 \\
\hline \multicolumn{13}{|l|}{ AUG } \\
\hline $27 \ldots$ & 1415 & 2.4 & 31 & 7.8 & 12.5 & 7.1 & $\cdots$ & - & $\cdots$ & 21 & 0.032 & $<0.010$ \\
\hline \multicolumn{13}{|l|}{ JUN 1991} \\
\hline $17 \ldots$ & 1805 & 95 & 16 & 6.7 & 7.5 & 8.2 & - & . & -. & 12 & 0.015 & 0.017 \\
\hline \multicolumn{13}{|l|}{ JUL } \\
\hline $15 \ldots$ & 1500 & 10 & 19 & 7.0 & 11.0 & 7.3 & $\cdots$ & $\cdots$ & $\cdots$ & 11 & 0.006 & 0.014 \\
\hline \multicolumn{13}{|l|}{ OCT } \\
\hline $22 \ldots$ & 0955 & $\cdots$ & 32 & 7.4 & 2.0 & 8.5 & $\cdots$ & $-\cdot$ & $\cdots$ & 21 & 0.062 & 0.019 \\
\hline \multicolumn{13}{|l|}{ MAY 1992} \\
\hline $20 \ldots$ & 1250 & 62 & 17 & 7.1 & 5.0 & 8.4 & 7 & 2.1 & 0.47 & 26 & 0.032 & 0.012 \\
\hline \multicolumn{13}{|l|}{ JUN } \\
\hline $23 \ldots$ & 1515 & 50 & 18 & 7.3 & 10.0 & 7.2 & $\cdots$ & $\cdots$ & $\cdots$ & 12 & 0.008 & 0.002 \\
\hline \multicolumn{13}{|l|}{ AUG } \\
\hline $10 \ldots$ & 1415 & 9.5 & 25 & 7.3 & 10.5 & - & - & $\cdots$ & $\cdots$ & 22 & 0.015 & 0.010 \\
\hline \multicolumn{13}{|l|}{ ХT } \\
\hline $27 \ldots$ & 1050 & 2.2 & 32 & 7.4 & 2.5 & 9.1 & $\cdots$ & $\cdots$ & $\cdots$ & 36 & 0.052 & 0.003 \\
\hline
\end{tabular}

\begin{tabular}{|c|c|c|c|c|c|c|c|c|c|c|c|c|}
\hline & \multirow[b]{2}{*}{$\begin{array}{l}\text { CADMIUM } \\
\text { TOTAL } \\
\text { RECOV - } \\
\text { ERABLE } \\
\text { (UG/L }\end{array}$} & \multirow[b]{2}{*}{$\begin{array}{c}\text { CADMIUM } \\
\text { DIS - } \\
\text { SOLVED } \\
\text { (UG } / L\end{array}$} & \multirow[b]{2}{*}{$\begin{array}{c}\text { COPPER, } \\
\text { TOTAL } \\
\text { RECOV- } \\
\text { ERABLE } \\
\text { (UG/L }\end{array}$} & \multirow[b]{2}{*}{$\begin{array}{l}\text { COPPER, } \\
\text { DIS - } \\
\text { SOLVED } \\
\text { /UG/L }\end{array}$} & \multirow[b]{2}{*}{$\begin{array}{l}\text { IRON, } \\
\text { TOTAL } \\
\text { RECOV - } \\
\text { ERABLE } \\
\text { (UG/L }\end{array}$} & \multirow[b]{2}{*}{$\begin{array}{l}\text { IRON, } \\
\text { DIS- } \\
\text { SOLVED } \\
\text { (UG/L }\end{array}$} & \multirow[b]{2}{*}{$\begin{array}{l}\text { LEAD, } \\
\text { TOTAL } \\
\text { RECOV- } \\
\text { ERABLE } \\
\text { (UG/L }\end{array}$} & \multirow[b]{2}{*}{$\begin{array}{l}\text { LEAD, } \\
\text { DIS - } \\
\text { SOLVED } \\
\text { (UG/L }\end{array}$} & \multirow[b]{2}{*}{$\begin{array}{l}\text { MANGA - } \\
\text { NESE, } \\
\text { TOTAL } \\
\text { RECOV- } \\
\text { ERABLE } \\
\text { (UG /L }\end{array}$} & \multirow[b]{2}{*}{$\begin{array}{l}\text { MANGA - } \\
\text { NESE, } \\
\text { DIS- } \\
\text { SOLVED } \\
\text { IUG /L }\end{array}$} & \multirow[b]{2}{*}{$\begin{array}{l}\text { ZINC. } \\
\text { TOTAL } \\
\text { RECOV- } \\
\text { ERABLE } \\
\text { (UG/L }\end{array}$} & \multirow{3}{*}{$\begin{array}{l}\text { ZINC, } \\
\text { DIS- } \\
\text { SOLVED } \\
\text { IUG /L }\end{array}$} \\
\hline & & & & & & & & & & & & \\
\hline & AS CD) & AS $(D)$ & AS CU) & AS CU) & AS FE) & AS FE) & AS $\quad$ PB) & AS $\mathrm{PB})$ & AS $M \mathbb{N}$ ) & AS MN) & AS $\mathrm{ZN})$ & \\
\hline
\end{tabular}

\begin{tabular}{|c|c|c|c|c|c|c|c|c|c|c|c|c|}
\hline APR 1990 & 1 & 50, & 3 & 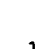 & 1000 & & 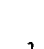 & 50 & 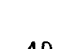 & 36 & 20 & 6 \\
\hline \multicolumn{13}{|l|}{ JUN } \\
\hline $04 \ldots$ & $<1$ & 0.3 & 9 & 5 & 140 & 93 & 3 & 1.2 & 10 & 8 & $\cdots$ & 23 \\
\hline \multicolumn{13}{|l|}{ AUG } \\
\hline $27 \ldots$ & $<1$ & 0.1 & 1 & 1 & 300 & 93 & 2 & 0.6 & 40 & 13 & 10 & 6 \\
\hline \multicolumn{13}{|l|}{ JUN 1991} \\
\hline $17 \ldots$ & $<1$ & $<0.1$ & 2 & $<1$ & 120 & 52 & 2 & $<0.5$ & 10 & 1 & $<10$ & 5 \\
\hline \multicolumn{13}{|l|}{ JUL } \\
\hline $15 \ldots$ & $<1$ & $<0.1$ & 3 & $<1$ & 60 & 52 & 5 & $<0.5$ & 10 & 5 & $<10$ & 4 \\
\hline OCT & & & & & & & & & & & & \\
\hline $22 \ldots$ & $<1$ & $<0.1$ & 3 & $<1$ & 80 & 79 & 2 & $<0.5$ & 10 & 12 & $<10$ & 5 \\
\hline \multicolumn{13}{|l|}{ MAY 1992} \\
\hline $20 \ldots$ & $<1$ & 0.1 & $<1$ & $<1$ & 120 & 50 & $<1$ & 0.5 & $<10$ & 2 & $<10$ & $<3$ \\
\hline \multicolumn{13}{|l|}{ JUN } \\
\hline $23 \ldots$ & $<1$ & $<0.1$ & $<1$ & $<1$ & 80 & 37 & $<1$ & $<0.5$ & 10 & 3 & $<10$ & $<3$ \\
\hline \multicolumn{13}{|l|}{ AUG } \\
\hline $10 \ldots$ & $<1$ & 0.1 & 17 & $<1$ & 130 & 81 & 20 & $<0.5$ & 20 & 14 & 1900 & $<3$ \\
\hline \multicolumn{13}{|l|}{ OCT } \\
\hline $27 \ldots$ & $<1$ & 0.2 & $<1$ & $<1$ & 110 & 98 & $<1$ & $<0.5$ & $<10$ & 17 & $<10$ & 5 \\
\hline
\end{tabular}


Table 11. Onsite measurements and selected inorganic data for station 09063700 , Homestake Tunnel near Leadville

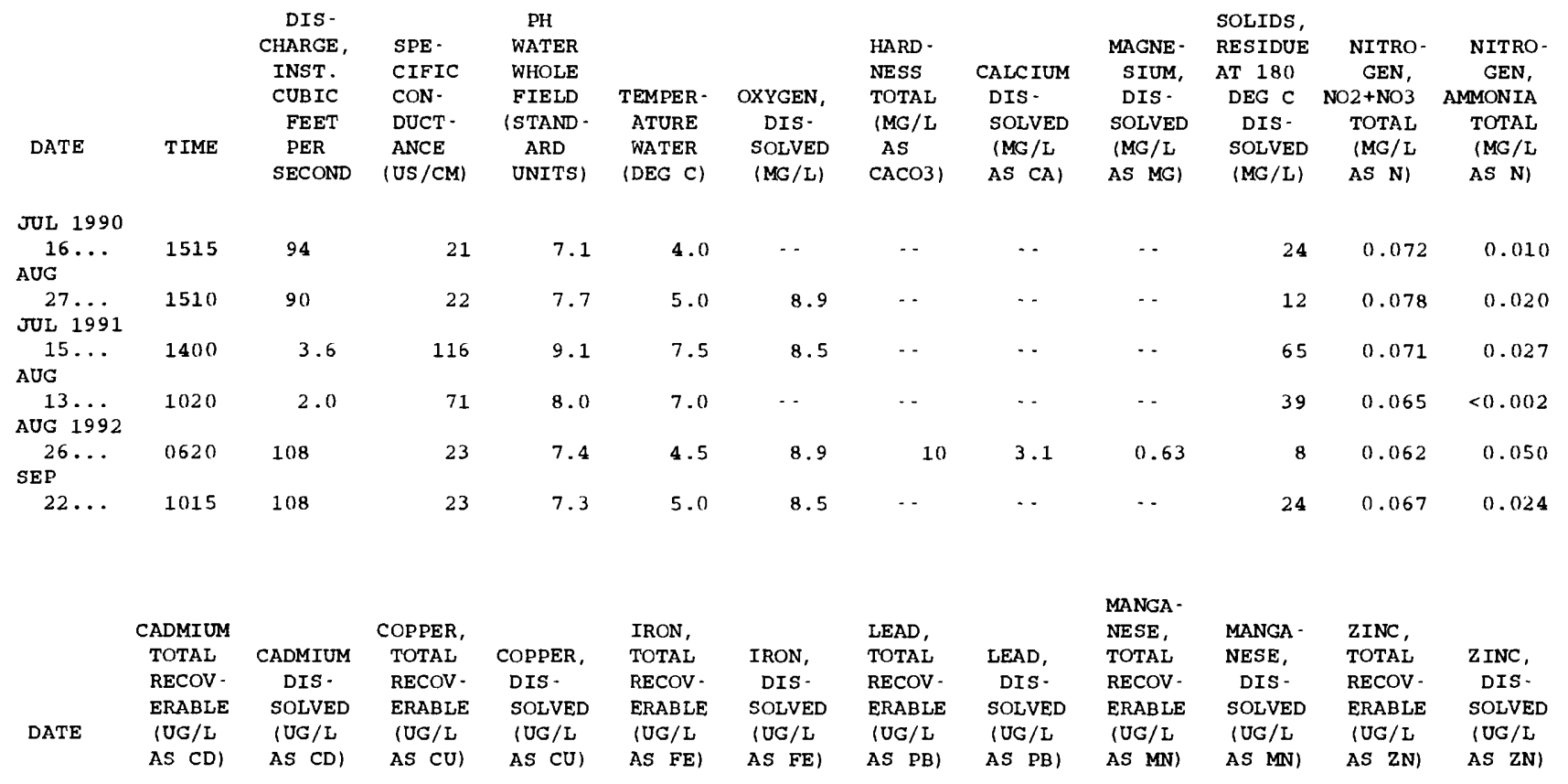

JUL 1990

\begin{tabular}{|c|c|c|c|c|c|c|c|c|c|c|c|c|}
\hline${ }_{A \cup G}^{16} \ldots$ & $<1$ & 0.1 & 2 & 2 & 80 & 38 & 1 & $<0.5$ & 20 & 4 & $<10$ & 4 \\
\hline $\begin{array}{l}27 \ldots \\
\text { JUL } 1991\end{array}$ & $<1$ & $<0.1$ & 2 & 1 & 80 & 41 & $<1$ & $<0.5$ & 20 & 2 & $<10$ & $<3$ \\
\hline${ }_{\text {AUG }}^{15} \cdots$ & $<1$ & $<0.1$ & 3 & $<1$ & 150 & 60 & 9 & $<0.5$ & 30 & 11 & $<10$ & $<3$ \\
\hline $\begin{array}{c}13 \ldots \\
\text { AUG } 1992\end{array}$ & $<1$ & $<0.1$ & 2 & $<1$ & 200 & 63 & 2 & $<0.5$ & 10 & 7 & $<10$ & $<3$ \\
\hline$\underset{\operatorname{seP}}{26 \ldots}$ & $<1$ & $<0.1$ & 2 & $<1$ & 30 & 46 & $<1$ & $\cdots$ & $<10$ & 4 & $<10$ & $<3$ \\
\hline $22 \ldots$ & $<1$ & $<0.1$ & 1 & $I$ & 70 & 39 & $<1$ & $<0.5$ & 10 & 3 & $<10$ & 4 \\
\hline
\end{tabular}


Table 12. Onsite measurements and selected inorganic data for station 09077160 , Charles $\mathrm{H}$. Bousted Tunnel near Leadville

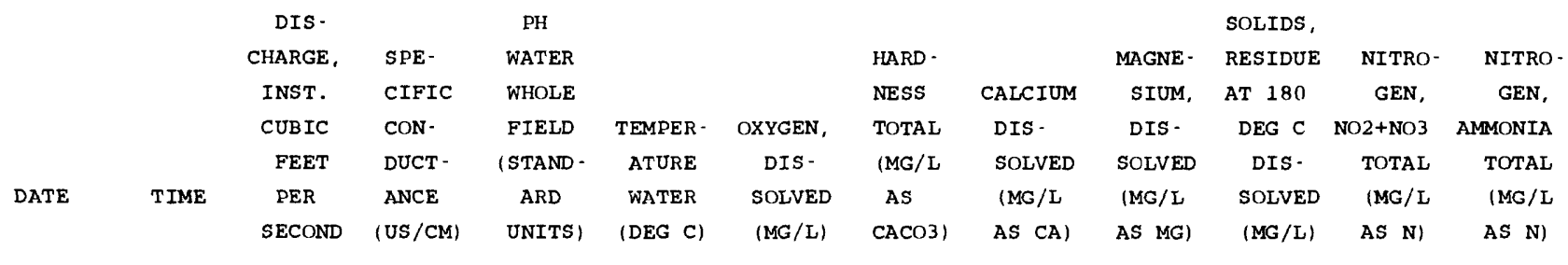

MAY 1990

\begin{tabular}{|c|c|c|c|c|c|c|c|c|c|c|c|c|}
\hline $22 \ldots$ & 0920 & 125 & 38 & 7.5 & 2.0 & 9.5 & $\cdots$ & $\cdots$ & $\cdots$ & 17 & 0.061 & $<0.010$ \\
\hline \multicolumn{13}{|l|}{ JUN } \\
\hline $04 \ldots$ & 1550 & 500 & $\cdots$ & 6.6 & 4.0 & 10.0 & $\cdots$ & $\cdots$ & $\cdots$ & 21 & 0.045 & 0.020 \\
\hline \multicolumn{13}{|l|}{ JUN 1991} \\
\hline $17 \ldots$ & 1930 & 908 & 22 & 6.6 & 5.5 & 9.0 & $\cdots$ & $\cdots$ & $\cdots$ & 17 & 0.035 & 0.016 \\
\hline \multicolumn{13}{|l|}{ JUL } \\
\hline $15 \ldots$ & 1615 & 75 & 39 & 7.1 & 9.0 & 7.8 & $\cdots$ & $\cdots$ & $\cdots$ & 12 & 0.033 & 0.012 \\
\hline \multicolumn{13}{|l|}{ AUG } \\
\hline $13 \ldots$ & 1050 & 10 & 77 & 7.9 & 8.5 & $\cdots$ & $\cdots$ & $\cdots$ & $\cdots$ & 57 & 0.087 & $<0.002$ \\
\hline \multicolumn{13}{|c|}{ MAY 1992} \\
\hline $20 \ldots$ & 1425 & 516 & 22 & 7.3 & 3.5 & 8.9 & 10 & 3.1 & 0.49 & 20 & 0.065 & 0.017 \\
\hline \multicolumn{13}{|l|}{ JUN } \\
\hline $23 \ldots$ & 1645 & 370 & 23 & 7.4 & 7.5 & 7.9 & $\cdots$ & $\cdots$ & $\cdots$ & 22 & 0.027 & 0.007 \\
\hline \multicolumn{13}{|l|}{ AUG } \\
\hline $10 \ldots$ & 1525 & 10 & 73 & 8.1 & 9.5 & $\cdots$ & $\cdots$ & $\cdots$ & $\cdots$ & 34 & 0.059 & 0.003 \\
\hline
\end{tabular}

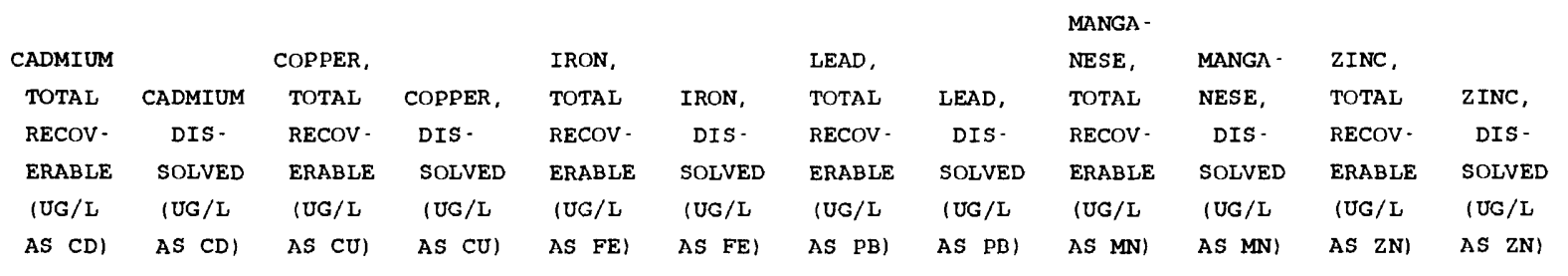

MAY 1990

\begin{tabular}{|c|c|c|c|c|c|c|c|c|c|c|c|c|}
\hline $22 \ldots$ & $<1$ & $<0.1$ & 1 & 1 & 280 & 92 & $<1$ & $<0.5$ & 30 & 4 & 10 & 3 \\
\hline \multicolumn{13}{|l|}{ JUN } \\
\hline $04 \ldots$ & $<1$ & $<0.1$ & 5 & 3 & 140 & 65 & 1 & $<0.5$ & $<10$ & 3 & $<10$ & 6 \\
\hline \multicolumn{13}{|l|}{ JUN 1991} \\
\hline $17 \ldots$ & $<1$ & 0.7 & 5 & 1 & 140 & 46 & 3 & $<0.5$ & $<10$ & $<1$ & $<10$ & 8 \\
\hline \multicolumn{13}{|l|}{ JUL } \\
\hline $15 \ldots$ & $<1$ & 0.1 & 3 & $<1$ & 20 & 25 & 5 & $<0.5$ & $<10$ & $<1$ & $<10$ & 4 \\
\hline \multicolumn{13}{|l|}{ AUG } \\
\hline $13 \ldots$ & $<1$ & $<0.1$ & 1 & $<1$ & 100 & 40 & 4 & $<0.5$ & $<10$ & $<1$ & $<10$ & $<3$ \\
\hline \multicolumn{13}{|l|}{ MAY 1992} \\
\hline $20 \ldots$ & $<1$ & $<0.1$ & $<1$ & $<1$ & 190 & 61 & 1 & $<0.5$ & 10 & 2 & $<10$ & $<3$ \\
\hline \multicolumn{13}{|l|}{ JUN } \\
\hline $23 \ldots$ & $<1$ & $<0.1$ & 1 & $<1$ & 100 & 46 & $<1$ & $<0.5$ & $<10$ & 1 & $<10$ & 16 \\
\hline \multicolumn{13}{|l|}{ AUG } \\
\hline $10 \ldots$ & $<1$ & $<0.1$ & $<1$ & $<1$ & 100 & 46 & $<1$ & $<0.5$ & 10 & 2 & 20 & 4 \\
\hline
\end{tabular}


Table 13. Onsite measurements and selected inorganic data for station 09077500, Busk-Ivanhoe Tunnel at East Portal, near Malta

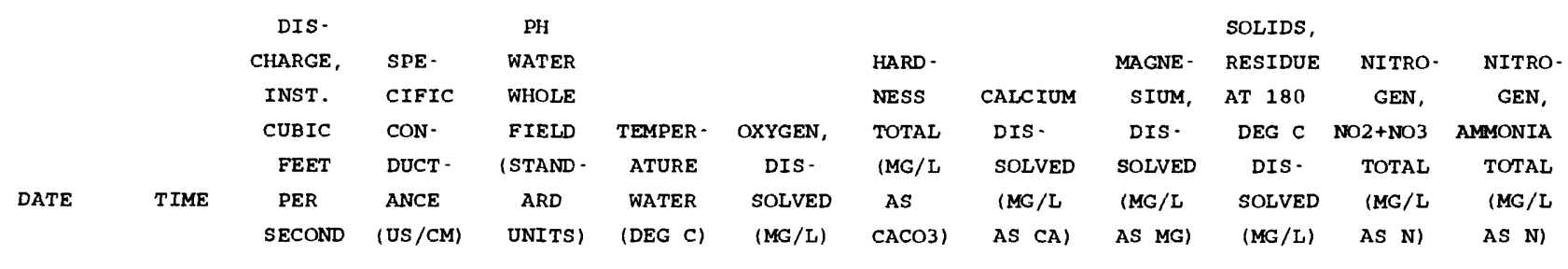

MAY 1990
$22 \ldots$
JUN
$04 \ldots$
JUN 1991
$17 \ldots$
JUL
$15 \ldots$
AUG
$13 \ldots$
MAY 1992
$20 \ldots$
JUN
$23 \ldots$
AUG
$11 \ldots$

\begin{tabular}{|c|c|c|c|c|c|c|c|c|c|c|c|c|}
\hline $22 \ldots$ & 1040 & 6.8 & 32 & 6.6 & 2.0 & 8.6 & $\cdots$ & $\cdots$ & $\cdots$ & 27 & 0.107 & 0.070 \\
\hline \multicolumn{13}{|l|}{ JUN } \\
\hline $\begin{array}{c}04 \ldots \\
\text { JUN } 1991\end{array}$ & 1745 & 48 & - & 6.6 & 1.0 & 8.6 & $\cdots$ & $\cdots$ & $\cdots$ & 14 & 0.074 & 0.090 \\
\hline $17 \ldots$ & 1640 & 94 & 14 & 6.6 & 7.0 & 7.6 & $\cdots$ & $\cdots$ & $\cdots$ & 1 & 0.018 & 0.035 \\
\hline \multicolumn{13}{|l|}{ JUL } \\
\hline $15 \ldots$ & 1245 & 7.1 & 19 & 6.8 & 13.0 & 6.7 & $\cdots$ & $\cdots$ & $-\cdot$ & 9 & 0.009 & 0.006 \\
\hline \multicolumn{13}{|l|}{ AUG } \\
\hline $13 \ldots$ & 0915 & 3.9 & 25 & 6.8 & 10.5 & $\cdots$ & $\cdots$ & $\cdots$ & $\cdots$ & 16 & 0.016 & $<0.002$ \\
\hline \multicolumn{13}{|l|}{ MAY 1992} \\
\hline $20 \ldots$ & 1120 & 47 & 15 & 6.8 & 2.5 & 8.6 & 6 & 1.7 & 0.32 & 20 & 0.074 & 0.012 \\
\hline \multicolumn{13}{|l|}{ JUN } \\
\hline $23 \ldots$ & 1340 & 7.3 & 20 & 7.0 & 9.5 & 6.8 & $\cdots$ & $\cdots$ & $\cdots$ & 11 & 0.014 & 0.009 \\
\hline \multicolumn{13}{|l|}{ AUG } \\
\hline $11 \ldots$ & 1635 & 6.5 & 22 & 6.9 & 14.0 & $\cdots$ & $-\cdot$ & $\cdots$ & $\cdots$ & 10 & 0.021 & 0.017 \\
\hline
\end{tabular}

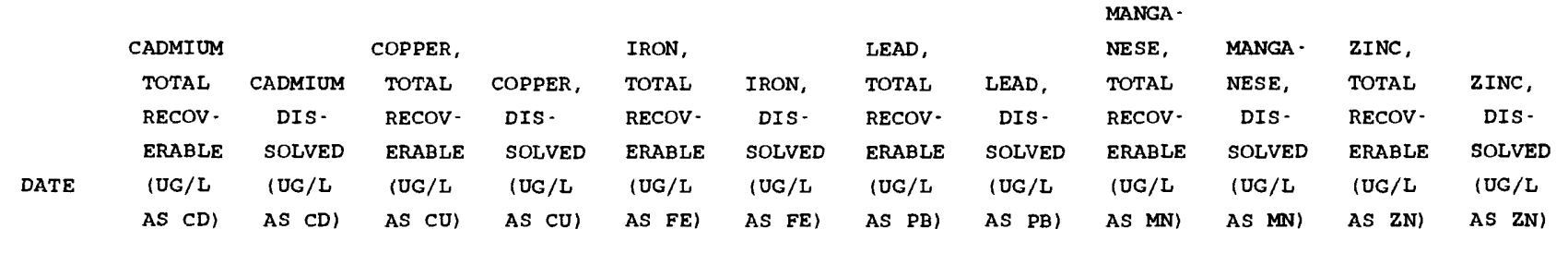

MAY 1990

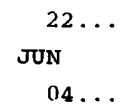

JUN 1991

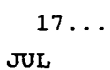

$$
<1
$$

(2.

$15 \ldots$

$13 \ldots$

MAY 1992

JUN

AUG

$11 \ldots$

$<1<0.1$

$<1 \quad 0.2$

$<1 \quad 0.1$

1

$<1$

220

$110 \quad 3$

$$
<0
$$$$
<0.5
$$

20

18

$<10$

8

4 
Table 14. Onsite measurements and selected inorganic data for station 07082500 , Lake Fork below Sugar Loaf Reservoir

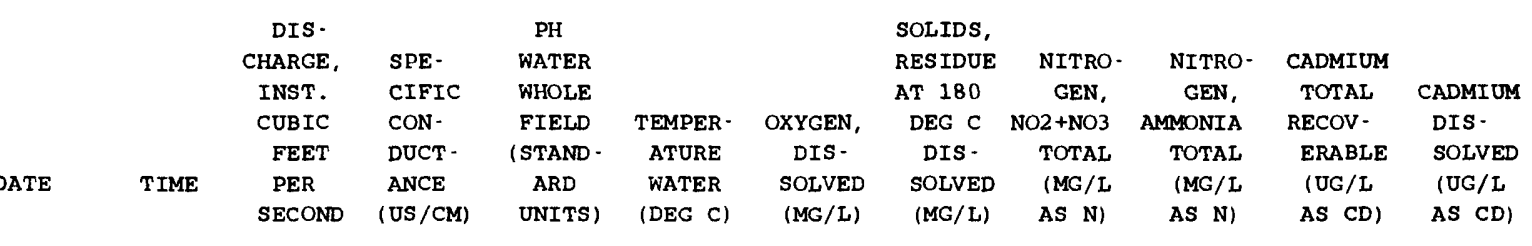

\begin{tabular}{|c|c|c|c|c|c|c|c|c|c|c|c|}
\hline \multicolumn{12}{|l|}{ JUN 1990} \\
\hline $04 \ldots$ & 1935 & 14 & $\cdots$ & 7.7 & 6.0 & 8.5 & 16 & $<0.010$ & 0.020 & $<1$ & 0.2 \\
\hline \multicolumn{12}{|l|}{ JUL } \\
\hline $16 \ldots$ & 1620 & 17 & 26 & 7.3 & 6.5 & 9.3 & 8 & 0.031 & 0.030 & $<1$ & $<0.1$ \\
\hline \multicolumn{12}{|l|}{ AUG } \\
\hline $27 \ldots$ & 1620 & 7.0 & 26 & 7.2 & 8.5 & 8.6 & 10 & 0.053 & 0.020 & 2 & 0.1 \\
\hline \multicolumn{12}{|l|}{ OCT } \\
\hline $29 \ldots$ & 1540 & 3.1 & 29 & 7.4 & 7.0 & 8.1 & 19 & 0.044 & 0.020 & $<1$ & $<0.1$ \\
\hline \multicolumn{12}{|l|}{ JUN 1991} \\
\hline $18 \ldots$ & 1110 & 15 & 27 & 7.1 & 7.0 & 9.3 & 18 & 0.037 & 0.036 & $<1$ & 1.3 \\
\hline \multicolumn{12}{|l|}{ JUL } \\
\hline $16 \ldots$ & 1045 & 17 & 25 & 6.8 & 7.0 & 8.5 & 13 & 0.044 & 0.012 & $<1$ & $<0.1$ \\
\hline \multicolumn{12}{|l|}{ OCT } \\
\hline $22 \ldots$ & 1115 & 3.1 & 26 & 7.0 & 7.0 & 8.2 & 22 & 0.068 & 0.033 & $<1$ & 0.1 \\
\hline \multicolumn{12}{|l|}{ MAY 1992} \\
\hline $20 \ldots$ & 1600 & 17 & 28 & 7.3 & 6.0 & 9.0 & 30 & 0.038 & 0.010 & $<1$ & $<0.1$ \\
\hline \multicolumn{12}{|l|}{ JUN } \\
\hline $23 \ldots$ & 1815 & 17 & 25 & 7.3 & 8.0 & 8.7 & 18 & 0.017 & 0.003 & $<1$ & $<0.1$ \\
\hline \multicolumn{12}{|l|}{ AUG } \\
\hline $10 \ldots$ & 1750 & 3.4 & 26 & 7.0 & 8.0 & $\cdots$ & 12 & 0.051 & 0.013 & $<1$ & $<0.1$ \\
\hline \multicolumn{12}{|l|}{ OCT } \\
\hline $27 \ldots$ & 1025 & 3.7 & 27 & 7.3 & 8.0 & 8.3 & 25 & 0.031 & 0.006 & $<1$ & $<0.1$ \\
\hline
\end{tabular}

\begin{tabular}{|c|c|c|c|c|c|c|c|c|c|c|}
\hline DATE & $\begin{array}{l}\text { COPPER, } \\
\text { TOTAL } \\
\text { RECOV- } \\
\text { ERABLE } \\
\text { (UG/L }\end{array}$ & $\begin{array}{l}\text { COPPER, } \\
\text { DIS - } \\
\text { SOLVED } \\
\text { (UG/L }\end{array}$ & $\begin{array}{l}\text { IRON, } \\
\text { TOTAL } \\
\text { RECOV- } \\
\text { ERABLE } \\
\text { (UG/L }\end{array}$ & $\begin{array}{l}\text { IRON, } \\
\text { DIS - } \\
\text { SOLVED } \\
\text { (UG/L }\end{array}$ & $\begin{array}{l}\text { LEAD, } \\
\text { TOTAL } \\
\text { RECOV- } \\
\text { ERABLE } \\
\text { (UG/L }\end{array}$ & $\begin{array}{l}\text { LEAD, } \\
\text { DIS - } \\
\text { SOLVED } \\
\text { (UG/L }\end{array}$ & $\begin{array}{l}\text { MANGA- } \\
\text { NESE, } \\
\text { TOTAL } \\
\text { RECOV- } \\
\text { ERABLE } \\
\text { (UG / L }\end{array}$ & $\begin{array}{l}\text { MANGA - } \\
\text { NESE, } \\
\text { DIS- } \\
\text { SOLVED } \\
\text { (UG/L }\end{array}$ & $\begin{array}{l}\text { ZINC, } \\
\text { TOTAL } \\
\text { RECOV- } \\
\text { ERABLE } \\
\text { (UG/L }\end{array}$ & $\begin{array}{l}\text { ZINC, } \\
\text { DIS- } \\
\text { SOLVED } \\
\text { (UG/L }\end{array}$ \\
\hline & AS $\mathrm{CU}$ ) & AS $(U)$ & AS FE) & AS FE) & AS PB) & AS PB) & AS MN) & AS MN) & AS $\mathrm{ZN}$ ) & AS $\mathrm{ZN}$ ) \\
\hline
\end{tabular}

\begin{tabular}{|c|c|c|c|c|c|c|c|c|c|c|}
\hline JUN 1990 & & & & & & & & & & \\
\hline $04 \ldots$ & 2 & 2 & 300 & 69 & 2 & $<0.5$ & 50 & 32 & $\cdots$ & 110 \\
\hline JUL & & & & & & & & & & \\
\hline $16 \ldots$ & 2 & 1 & 160 & 79 & $<1$ & $<0.5$ & 20 & 3 & $<10$ & 8 \\
\hline AUG & & & & & & & & & & \\
\hline $27 \ldots$ & 3 & 1 & 230 & 130 & 1 & $<0.5$ & 30 & 5 & 10 & 6 \\
\hline OCT & & & & & & & & & & \\
\hline $29 \ldots$ & 3 & 1 & 120 & 79 & $<1$ & $<0.5$ & 20 & 2 & 10 & 10 \\
\hline JUN 1991 & & & & & & & & & & \\
\hline $18 \ldots$ & 4 & 3 & 120 & 78 & 4 & $<0.5$ & 20 & 4 & $<10$ & 13 \\
\hline$\pi$ & & & & & & & & & & \\
\hline $16 \ldots$ & 3 & 1 & 110 & 96 & 7 & $<0.5$ & 20 & 2 & $<10$ & 7 \\
\hline OCT & & & & & & & & & & \\
\hline $22 \ldots$ & 4 & 1 & 210 & 130 & 5 & $<0.5$ & 20 & 4 & $<10$ & 9 \\
\hline MAY 1992 & & & & & & & & & & \\
\hline $20 \ldots$ & $<1$ & $<1$ & 220 & 70 & $<1$ & $<0.5$ & 50 & 29 & $<10$ & $<3$ \\
\hline$\pi U N$ & & & & & & & & & & \\
\hline $23 \ldots$ & 1 & 1 & 130 & 68 & $<1$ & $<0.5$ & $<10$ & 2 & 10 & $<3$ \\
\hline AUG & & & & & & & & & & \\
\hline $10 \ldots$ & 30 & $<1$ & 160 & 93 & 41 & $<0.5$ & 10 & 3 & 3300 & 7 \\
\hline OCT & & & & & & & & & & \\
\hline $27 \ldots$ & $<1$ & $<1$ & 70 & 41 & $<1$ & $<0.5$ & $<10$ & 2 & $<10$ & $<3$ \\
\hline
\end{tabular}


Table 15. Onsite measurements and selected inorganic data for station 391226106213201 , Lake Fork above Halfmoon Creek, near Malta

\begin{tabular}{|c|c|c|c|c|c|c|c|c|c|c|c|}
\hline DATE & TIME & $\begin{array}{c}\text { DIS- } \\
\text { CHARGE, } \\
\text { INST. } \\
\text { CUBIC } \\
\text { FEET } \\
\text { PER } \\
\text { SECOND }\end{array}$ & $\begin{array}{l}\text { SPE- } \\
\text { CIFIC } \\
\text { CON- } \\
\text { DUCT - } \\
\text { ANCE } \\
\text { (US/CM) }\end{array}$ & $\begin{array}{c}\text { PH } \\
\text { WATER } \\
\text { WHOLE } \\
\text { FIELD } \\
\text { (STAND- } \\
\text { ARD } \\
\text { UNITS) }\end{array}$ & $\begin{array}{c}\text { TEMPER- } \\
\text { ATURE } \\
\text { WATER } \\
\text { (DEG C) }\end{array}$ & $\begin{array}{c}\text { OXYGEN, } \\
\text { DIS - } \\
\text { SOLVED } \\
\text { (MG/L) }\end{array}$ & $\begin{array}{l}\text { SOLIDS, } \\
\text { RES IDUE } \\
\text { AT } 180 \\
\text { DEG C } \\
\text { DIS - } \\
\text { SOLVED } \\
\text { (MG/L) }\end{array}$ & $\begin{array}{c}\text { NITRO- } \\
\text { GEN, } \\
\text { NO2+NO3 } \\
\text { TOTAL } \\
\text { (MG / L } \\
\text { AS N) }\end{array}$ & $\begin{array}{c}\text { NITRO- } \\
\text { GEN, } \\
\text { AMMONIA } \\
\text { TOTAL } \\
\text { (MG / L } \\
\text { AS N) }\end{array}$ & $\begin{array}{l}\text { CADMIUM } \\
\text { TOTAL } \\
\text { RECOV- } \\
\text { ERABLE } \\
\text { (UG/L } \\
\text { AS CD) }\end{array}$ & $\begin{array}{l}\text { CADMIUM } \\
\text { DIS- } \\
\text { SOLVED } \\
\text { (UG/L } \\
\text { AS CD) }\end{array}$ \\
\hline APR 1990 & & & & & & & & & & & \\
\hline $\begin{array}{l}17 \ldots \\
\text { JUN }\end{array}$ & 1430 & 32 & 94 & 7.9 & 7.5 & 9.1 & 60 & 0.048 & $<0.010$ & $<1$ & 0.1 \\
\hline $\begin{array}{l}05 \ldots \\
\text { JUL }\end{array}$ & 1500 & 75 & .. & $\cdots$ & 14.5 & 6.5 & 39 & 0.022 & 0.030 & $<1$ & 0.3 \\
\hline$\underset{A \cup G}{17} \ldots$ & 1145 & 56 & 70 & $\cdots$ & 13.0 & 9.3 & 44 & 0.016 & $<0.010$ & $<1$ & $<0.1$ \\
\hline$\underset{O C T}{28 \cdots}$ & 1200 & 35 & 117 & 7.5 & 14.0 & 9.4 & 69 & 0.021 & $<0.010$ & $<1$ & 0.1 \\
\hline $\begin{array}{c}30 \ldots \\
\text { APR } 1991\end{array}$ & 0800 & 28 & 126 & 7.9 & 1.5 & 12.5 & 67 & 0.011 & 0.020 & $<1$ & $<0.1$ \\
\hline $\begin{array}{l}23 . \cdots \\
\text { JUN }\end{array}$ & 1115 & 29 & 100 & 7.7 & 5.5 & 9.5 & 38 & 0.044 & 0.025 & $<1$ & $<0.1$ \\
\hline $\begin{array}{l}18 \ldots \\
\text { JUL }\end{array}$ & 1145 & 82 & 67 & 7.4 & 11.0 & 7.8 & 32 & 0.030 & 0.026 & $<1$ & 0.2 \\
\hline${ }_{\text {OCT }}^{16 \ldots}$ & 1300 & 54 & 69 & 7.6 & 16.5 & 7.5 & 27 & 0.031 & 0.013 & $<1$ & 0.1 \\
\hline $\begin{array}{r}22 \ldots \\
\text { APR } 1992\end{array}$ & 1410 & 27 & 97 & 8.5 & 8.5 & 10.0 & 61 & 0.029 & 0.038 & $<1$ & 0.2 \\
\hline $\begin{array}{c}21 \ldots \\
\text { JUN }\end{array}$ & 0930 & 37 & 82 & 7.6 & 3.0 & 8.8 & 69 & 0.053 & 0.031 & $<1$ & 0.8 \\
\hline$\underset{A U G}{24 \ldots}$ & 1520 & 79 & 57 & 7.9 & 15.0 & 7.5 & 46 & 0.012 & 0.008 & $<1$ & $<0.1$ \\
\hline${ }_{\mathrm{OCT}}^{11 \ldots}$ & 1125 & 43 & 79 & 8.5 & 13.0 & 8.8 & 46 & 0.015 & 0.007 & $<1$ & $<0.1$ \\
\hline $27 \ldots$ & 1250 & 30 & 94 & 7.2 & 7.5 & 10.4 & 74 & 0.025 & 0.011 & $<1$ & $<0.1$ \\
\hline
\end{tabular}

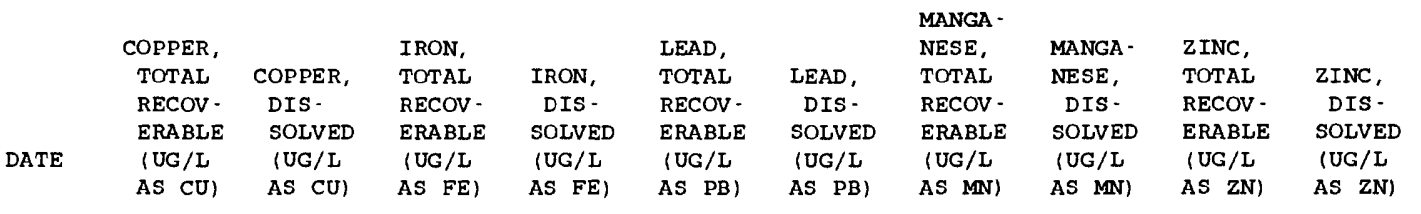

\begin{tabular}{|c|c|c|c|c|c|c|c|c|c|c|}
\hline \multicolumn{11}{|l|}{ APR 1990} \\
\hline $17 \ldots$ & 3 & 1 & 630 & 260 & 2 & $<0.5$ & 100 & 71 & 30 & 25 \\
\hline \multicolumn{11}{|l|}{ JUN } \\
\hline $05 \ldots$ & 7 & 4 & 650 & 150 & 2 & 0.7 & 100 & 69 & $\cdots$ & 37 \\
\hline \multicolumn{11}{|l|}{ JUL } \\
\hline $17 \ldots$ & 3 & 2 & 720 & 260 & 1 & $<0.5$ & 80 & 54 & 20 & 12 \\
\hline \multicolumn{11}{|l|}{$A D G$} \\
\hline $28 \ldots$ & 2 & 1 & 960 & 450 & 2 & $<0.5$ & 140 & 100 & 80 & 27 \\
\hline \multicolumn{11}{|l|}{ ост } \\
\hline $30 \ldots$ & 3 & 2 & $370^{\circ}$ & 170 & $<1$ & $<0.5$ & 60 & 44 & 20 & 20 \\
\hline \multicolumn{11}{|l|}{ APR 1991} \\
\hline $23 \ldots$ & 2 & 1 & 730 & 270 & 3 & $<0.5$ & 150 & 150 & 60 & 49 \\
\hline \multicolumn{11}{|l|}{ JUN } \\
\hline $18 \ldots$ & 8 & 3 & 1200 & 120 & 4 & $<0.5$ & 180 & 100 & 40 & 21 \\
\hline \multicolumn{11}{|l|}{ JUL } \\
\hline $16 \ldots$ & 3 & $<1$ & 510 & 220 & 6 & $<0.5$ & 80 & 50 & $<10$ & 11 \\
\hline \multicolumn{11}{|l|}{ OCT } \\
\hline $22 \ldots$ & 2 & $<1$ & 320 & 180 & 3 & $<0.5$ & 20 & 11 & $<10$ & 11 \\
\hline \multicolumn{11}{|l|}{ APR 1992} \\
\hline $21 \ldots$ & 2 & 2 & 670 & 210 & $<1$ & $<0.5$ & 160 & 120 & 70 & 62 \\
\hline \multicolumn{11}{|l|}{ JUN } \\
\hline $24 \ldots$ & 2 & 1 & 450 & 170 & $<1$ & $<0.5$ & 50 & 26 & 10 & $<3$ \\
\hline \multicolumn{11}{|l|}{ AUG } \\
\hline $11 \ldots$ & 4 & $<1$ & 490 & 250 & 1 & 0.7 & 40 & 28 & 20 & 5 \\
\hline \multicolumn{11}{|l|}{ OCT } \\
\hline $27 \ldots$ & 1 & $<1$ & 360 & 170 & $<1$ & $<0.5$ & 10 & 14 & 10 & 6 \\
\hline
\end{tabular}


Table 16. Onsite measurements and bacteriological and selected inorganic data for station 07083000 , Halfmoon Creek near Malta

\begin{tabular}{|c|c|c|c|c|c|c|c|c|c|c|}
\hline DATE & TIME & $\begin{array}{c}\text { DIS - } \\
\text { CHARGE, } \\
\text { INST. } \\
\text { CUBIC } \\
\text { FEET } \\
\text { PER } \\
\text { SECOND }\end{array}$ & $\begin{array}{l}\text { SPE- } \\
\text { CIFIC } \\
\text { CON- } \\
\text { DUCT- } \\
\text { ANCE } \\
\text { (US/CM) }\end{array}$ & $\begin{array}{c}\text { PH } \\
\text { WATER } \\
\text { WHOLE } \\
\text { FIELD } \\
\text { (STAND- } \\
\text { ARD } \\
\text { UNITS) }\end{array}$ & $\begin{array}{c}\text { TEMPER - } \\
\text { ATURE } \\
\text { WATER } \\
\text { (DEG C) }\end{array}$ & $\begin{array}{c}\text { OXYGEN, } \\
\text { DIS- } \\
\text { SOLVED } \\
\text { (MG/L) }\end{array}$ & $\begin{array}{l}\text { COLI- } \\
\text { FORM, } \\
\text { FECAL, } \\
0.7 \\
\text { UM-MF } \\
\text { (COLS./ } \\
100 \mathrm{ML} \text { ) }\end{array}$ & $\begin{array}{c}\text { STREP - } \\
\text { TOCOCCI } \\
\text { FECAL, } \\
\text { KF AGAR } \\
\text { (COLS. } \\
\text { PER } \\
100 \mathrm{ML} \text { ) }\end{array}$ & $\begin{array}{l}\text { HARD- } \\
\text { NESS } \\
\text { TOTAL } \\
\text { (MG/L } \\
\text { AS } \\
\text { CACO3) }\end{array}$ & $\begin{array}{l}\text { CALCIUM } \\
\text { DIS- } \\
\text { SOLVED } \\
\text { (MG/L } \\
\text { AS CA) }\end{array}$ \\
\hline $\mathrm{RR} 1990$ & 1530 & & & & & & & & & \\
\hline JN & 1530 & $\cdots$ & $\cdots$ & $\cdots$ & $\cdots$ & $\cdots$ & - & $\cdots$ & $\cdots$ & $\cdots$ \\
\hline${ }_{\mathrm{JL}}^{05 \ldots}$ & 1740 & 153 & 39 & 7.4 & 9.5 & 7.8 & $\cdots$ & $\cdots$ & $\cdots$ & - \\
\hline $17 \ldots$ & 1410 & 42 & 65 & 8.0 & 13.0 & 7.0 & $\cdots$ & $\cdots$ & $\cdots$ & $\cdots$ \\
\hline $28 \ldots$ & 1315 & 15 & 92 & 7.4 & 14.5 & - - & $\cdots$ & $\cdots$ & $\cdots$ & $\cdots$ \\
\hline${ }_{\pi N}^{30} 1991$ & 0940 & 9.1 & 85 & 8.1 & 0.5 & 9.2 & K2 & 21 & 40 & 10 \\
\hline $25 \cdots$ & 1205 & 89 & 50 & 7.6 & 8.5 & 7.8 & $<1$ & $<1$ & 24 & 6.3 \\
\hline $16 \ldots$ & 1200 & 43 & 63 & 7.8 & 12.0 & 6.9 & $\cdots$ & $\cdots$ & $\cdots$ & $\cdots$ \\
\hline $\mathrm{xT}^{13} \cdots$ & 1530 & 25 & 80 & 7.7 & 14.5 & $\cdots$ & $<1$ & K5 & 37 & 9.4 \\
\hline JuN 21992 & 1645 & 6.4 & 84 & 7.8 & 7.0 & 8.1 & $\cdots$ & $<1$ & 44 & 11 \\
\hline$\underset{A U G}{23} \cdots$ & 1425 & 94 & 58 & 7.9 & 10.0 & 7.8 & $\cdots$ & $\cdots$ & $\cdots$ & $\cdots$ \\
\hline$C_{C T}^{10} \cdots$ & 1700 & 34 & 76 & 8.0 & 10.0 & 7.4 & - & - & $\cdots$ & $\cdots$ \\
\hline $27 \ldots$ & 1210 & 7.9 & 90 & 8.1 & 4.0 & 8.7 & - - & -. & $\cdots$ & $\cdots$ \\
\hline
\end{tabular}

\begin{tabular}{cccc} 
MAGNE - & \multicolumn{3}{c}{ ALKA- } \\
SIUM, & SODIUM, & LINITY & SULFATE \\
DIS- & DIS- & LAB & DIS - \\
SOLVED & SOLVED & (MG/L & SOLVED \\
(MG/L & (MG/L & AS & (MG/L \\
AS MG) & AS NA) & CACO3) & AS SO4)
\end{tabular}

$\begin{array}{lccccc} & \text { SOLIDS, } & & \text { NITRO- } & & \text { NITRO- } \\ \text { CHLO- } & \text { RESIDUE } & \text { NITRO- } & \text { GEN, } & \text { NITRO- } & \text { GEN, } \\ \text { RIDE, } & \text { AT } 180 & \text { GEN, } & \text { NO2+NO3 } & \text { GEN, } & \text { AMMONIA } \\ \text { DIS- } & \text { DEG C } & \text { NO2+NO3 } & \text { DIS- } & \text { AMMONIA } & \text { DIS- } \\ \text { SOLVED } & \text { DIS - } & \text { TOTAL } & \text { SOLVED } & \text { TOTAL } & \text { SOLVED } \\ \text { (MG/L } & \text { SOLVED } & \text { (MG /L } & \text { (MG/L } & \text { (MG /L } & \text { (MG/L } \\ \text { AS CL) } & \text { (MG/L) } & \text { AS N) } & \text { AS N) } & \text { AS N) } & \text { AS N) }\end{array}$

APR 1990

JUN 17.

$05 \ldots$.

JUL

AUG

28 ...

OCT

$30 \ldots$

$25 \ldots$

JUL

16 ...

AUG

${ }_{\text {OCT }}^{13}$...

JN 1992

$23 \ldots$

AUG

$10 \ldots$

OCT

$27 \ldots$

\begin{tabular}{|c|c|c|c|c|c|c|c|c|c|c|}
\hline & $\begin{array}{r}\text { PHOS - } \\
\text { PHORUS } \\
\text { TOTAL } \\
\text { (MG/L }\end{array}$ & $\begin{array}{c}\text { ARSENIC } \\
\text { DIS - } \\
\text { SOLVED } \\
\text { (UG/L }\end{array}$ & $\begin{array}{l}\text { CADMIUM } \\
\text { TOTAL } \\
\text { RECOV - } \\
\text { ERABLE } \\
\text { /UG/L }\end{array}$ & $\begin{array}{c}\text { CADMIUM } \\
\text { DIS - } \\
\text { SOLVED } \\
\text { (UG/L }\end{array}$ & $\begin{array}{l}\text { CHRO- } \\
\text { MIUM, } \\
\text { DIS - } \\
\text { SOLVED } \\
\text { (UG / L }\end{array}$ & $\begin{array}{l}\text { COPPER, } \\
\text { TOTAL } \\
\text { RECOV- } \\
\text { ERABLE } \\
\text { (UG/L }\end{array}$ & $\begin{array}{l}\text { COPPER, } \\
\text { DIS - } \\
\text { SOLVED } \\
\text { (UG /L }\end{array}$ & $\begin{array}{l}\text { IRON, } \\
\text { TOTAL } \\
\text { RECOV- } \\
\text { ERABLE } \\
\text { (UG/L }\end{array}$ & $\begin{array}{l}\text { IRON, } \\
\text { DIS - } \\
\text { SOLVED } \\
\text { (UG/L }\end{array}$ & $\begin{array}{l}\text { LEAD, } \\
\text { TOTAL } \\
\text { RECOV - } \\
\text { ERABLE } \\
\text { (UG/L }\end{array}$ \\
\hline
\end{tabular}

\footnotetext{
APR 1990

JUN

05 ..

JUL $17 \ldots$

AUG

28 ...
}

$\begin{array}{llr}3.7 & 1.4 & 39 \\ 2.0 & 0.80 & 24 \\ \ldots & -. & - \\ 3.2 & 1.2 & 36 \\ 4.1 & 1.6 & 43 \\ \ldots & \ldots & \ldots \\ \ldots & \ldots & \ldots \\ \ldots & \ldots & \ldots\end{array}$

5.7
3.8

$<0.10$

48

0.100

0.100

0.020

0.010

0.30

250.089

0.089

0.010

0.010

$4.0 \quad 0.30$

46

0.098

0.098

$<0.010$

$<0.010$

$5.2 \quad 0.60$

510.078

0.078

$<0.010<0.010$

- -

- -

-

-

.

-

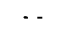


Table 16. Onsite measurements and bacteriological and selected inorganic data for station 07083000 , Halfmoon Creek near Malta--Continued

\begin{tabular}{|c|c|c|c|c|c|c|c|c|c|c|}
\hline \multirow[t]{2}{*}{ DATE } & $\begin{array}{l}\text { PHOS - } \\
\text { PHORUS } \\
\text { TOTAL } \\
\text { (MG /L }\end{array}$ & $\begin{array}{c}\text { ARSENIC } \\
\text { DIS - } \\
\text { SOLVED } \\
\text { (UG/L }\end{array}$ & $\begin{array}{l}\text { CADMIUM } \\
\text { TOTAL } \\
\text { RECOV - } \\
\text { ERABLE } \\
\text { (UG/L }\end{array}$ & $\begin{array}{l}\text { CADMIUM } \\
\text { DIS - } \\
\text { SOLVED } \\
\text { (UG/L }\end{array}$ & $\begin{array}{l}\text { CHRO- } \\
\text { MIUM, } \\
\text { DIS - } \\
\text { SOLVED } \\
\text { (UG /L }\end{array}$ & $\begin{array}{l}\text { COPPER, } \\
\text { TOTAL } \\
\text { RECOV- } \\
\text { ERABLE } \\
\text { (UG/L }\end{array}$ & $\begin{array}{l}\text { COPPER, } \\
\text { DIS - } \\
\text { SOLVED } \\
\text { (UG /L }\end{array}$ & $\begin{array}{l}\text { IRON, } \\
\text { TOTAL } \\
\text { RECOV - } \\
\text { ERABLE } \\
\text { /UG/L }\end{array}$ & $\begin{array}{l}\text { IRON, } \\
\text { DIS - } \\
\text { SOLVED } \\
\text { (UG/L }\end{array}$ & $\begin{array}{l}\text { LEAD, } \\
\text { TOTAL } \\
\text { RECOV- } \\
\text { ERABLE } \\
\text { (UG/L }\end{array}$ \\
\hline & AS P) & AS AS) & AS CDI & AS CDI & AS CRI & AS $\mathrm{CU}$ & AS (U) & AS FE) & AS FE) & AS $\mathrm{PB}$ ) \\
\hline \multicolumn{11}{|l|}{ OCT 1990} \\
\hline $30 \ldots$ & $<0.010$ & $<1$ & $<1$ & $<1.0$ & $<1$ & 1 & $<1$ & 100 & 45 & 1 \\
\hline \multicolumn{11}{|l|}{ JUN 1991} \\
\hline $25 \ldots$ & 0.010 & $<1$ & $\cdots$ & 2.0 & $<1$ & $\cdots$ & $<1$ & - & 32 & $\cdots$ \\
\hline \multicolumn{11}{|l|}{ JUL } \\
\hline $16 \ldots$ & $\cdots$ & $\cdots$ & $<1$ & $\cdots$ & $\cdots$ & 5 & $\cdots$ & $<10$ & 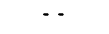 & 7 \\
\hline \multicolumn{11}{|l|}{ AUG } \\
\hline $13 \ldots$ & $<0.010$ & $<1$ & $<1$ & $<1.0$ & $<1$ & 1 & $<1$ & 140 & 76 & 3 \\
\hline \multicolumn{11}{|l|}{$\mathrm{OCT}$} \\
\hline $21 \ldots$ & $<0.010$ & $\cdots$ & $<1$ & $<1.0$ & $\cdots$ & 5 & $<1$ & $\cdots$ & 69 & 2 \\
\hline \multicolumn{11}{|l|}{ JUN 1992} \\
\hline $23 \ldots$ & $\cdots$ & -- & $<1$ & $<1.0$ & $-\cdots$ & 1 & $<1$ & 170 & 30 & 1 \\
\hline \multicolumn{11}{|l|}{ AUG } \\
\hline $10 \ldots$ & $\cdots$ & $\cdots$ & $<1$ & $<1.0$ & $\cdots$ & $<1$ & 1 & 100 & 90 & 1 \\
\hline \multicolumn{11}{|l|}{ OCT } \\
\hline $27 \ldots$ & $\cdots$ & -. & $<1$ & $<1.0$ & $\cdots$ & $<1$ & $<1$ & 110 & 70 & $<1$ \\
\hline
\end{tabular}

\begin{tabular}{|c|c|c|c|c|c|c|c|c|c|}
\hline \multirow{3}{*}{ DATE } & & AANGA - & & & & & & & \\
\hline & $\begin{array}{l}\text { LEAD, } \\
\text { DIS - } \\
\text { SOLVED } \\
\text { (UG/L }\end{array}$ & $\begin{array}{l}\text { NESE, } \\
\text { TOTAL } \\
\text { RECOV- } \\
\text { ERABLE } \\
\text { /UG/L }\end{array}$ & $\begin{array}{l}\text { MANGA - } \\
\text { NESE, } \\
\text { DIS - } \\
\text { SOLVED } \\
\text { /UG/L }\end{array}$ & $\begin{array}{c}\text { MERCURY } \\
\text { DIS - } \\
\text { SOLVED } \\
\text { (UG / L }\end{array}$ & $\begin{array}{l}\text { NICKEL, } \\
\text { DIS - } \\
\text { SOLVED } \\
\text { (UG /L }\end{array}$ & $\begin{array}{l}\text { SELE - } \\
\text { NIUM, } \\
\text { DIS - } \\
\text { SOLVED } \\
\text { (UG /L }\end{array}$ & $\begin{array}{l}\text { SILVER, } \\
\text { DIS - } \\
\text { SOLVED } \\
\text { /UG /L }\end{array}$ & $\begin{array}{l}\text { ZINC, } \\
\text { TOTAL } \\
\text { RECOV - } \\
\text { ERABLE } \\
\text { IUG/L }\end{array}$ & $\begin{array}{l}\text { ZINC, } \\
\text { DIS- } \\
\text { SOLVED } \\
\text { (UG /L }\end{array}$ \\
\hline & AS $\quad$ PB) & AS MN) & AS MN) & AS HG) & AS NI) & AS SE) & AS AG) & AS $\mathrm{ZN}$ ) & AS $\mathrm{ZN}$ ) \\
\hline
\end{tabular}

\begin{tabular}{|c|c|c|c|c|c|c|c|c|c|}
\hline \multicolumn{10}{|c|}{ APR 1990} \\
\hline $17 \ldots$ & $\cdots$ & $<10$ & $\cdots$ & $\cdots$ & $\cdots$ & - & $\cdots$ & $<10$ & $\cdots$ \\
\hline \multicolumn{10}{|l|}{ JUN } \\
\hline $05 \ldots$ & $\cdots$ & 40 & $\cdots$ & $\cdots$ & $\cdots$ & - & $\cdots$ & $<10$ & $\cdots$ \\
\hline \multicolumn{10}{|l|}{ JUL } \\
\hline $17 \ldots$ & $\cdots$ & $<10$ & $\cdots$ & -- & $\cdots$ & - & $\cdots$ & $<10$ & $\cdots$ \\
\hline \multicolumn{10}{|l|}{ AUG } \\
\hline $28 \ldots$ & $\cdots$ & 20 & $\cdots$ & $\cdots$ & $\cdots$ & $\cdots$ & $\cdots$ & $<10$ & $\cdots$ \\
\hline \multicolumn{10}{|l|}{ OCT } \\
\hline $30 \ldots$ & $<1$ & $<10$ & 5 & $<0.1$ & $<1$ & $<1$ & $<1.0$ & $<10$ & $<3$ \\
\hline \multicolumn{10}{|c|}{ JUN 1991} \\
\hline $25 \ldots$ & 1 & -. & 3 & $<0.1$ & 2 & $<1$ & $<1.0$ & $\cdots$ & 5 \\
\hline \multicolumn{10}{|l|}{ JUL } \\
\hline $16 \ldots$ & $\cdots$ & 10 & $\cdots$ & $\cdots$ & $\cdots$ & $\cdots$ & $\cdots$ & $<10$ & $\cdots$ \\
\hline \multicolumn{10}{|l|}{ AUG } \\
\hline $13 \ldots$ & $<1$ & $<10$ & 4 & $<0.1$ & $<1$ & $<1$ & $<1.0$ & $<10$ & $<3$ \\
\hline \multicolumn{10}{|l|}{ OCT } \\
\hline $21 \ldots$ & $<1$ & $\cdots$ & 5 & $\cdots$ & 1 & $<1$ & $<1.0$ & 40 & 40 \\
\hline \multicolumn{10}{|l|}{ JUN 1992} \\
\hline $23 \ldots$ & $<1$ & $<10$ & $<10$ & $\cdots$ & $\cdots$ & -. & $\cdots$ & $<10$ & $<10$ \\
\hline \multicolumn{10}{|l|}{ AUG } \\
\hline $10 \ldots$ & $<1$ & 20 & $<10$ & - & $-\cdot$ & $\cdots$ & $-\cdot$ & $<10$ & $<10$ \\
\hline \multicolumn{10}{|l|}{ OCT } \\
\hline $27 \ldots$ & $<1$ & $<10$ & $<10$ & $\cdots$ & $\cdots$ & $\cdots$ & $\cdots$ & $<10$ & $<10$ \\
\hline
\end{tabular}


Table 17. Onsite measurements and selected inorganic data for station 391120106194901, lowa Gulch at Highway 24, near Malta

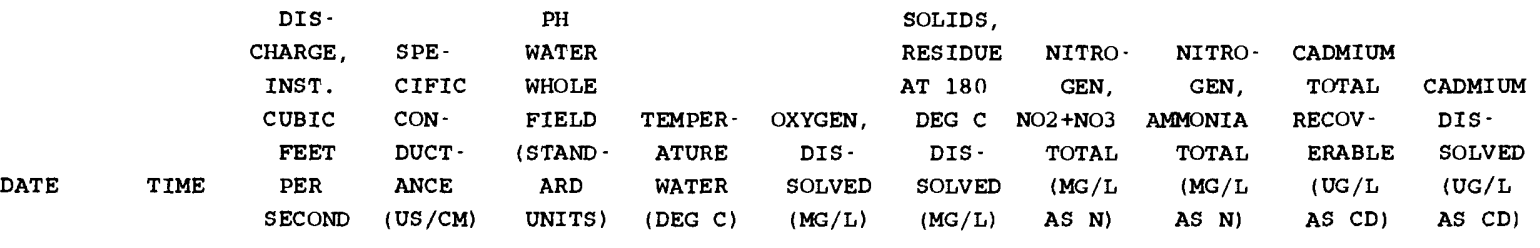

\begin{tabular}{|c|c|c|c|c|c|c|c|c|c|c|c|}
\hline \multicolumn{12}{|l|}{ APR 1990} \\
\hline $18 \ldots$ & 0800 & 1.2 & 1010 & 8.4 & 2.0 & $-\cdot$ & 826 & 0.189 & $<0.010$ & $<1$ & 0.2 \\
\hline \multicolumn{12}{|l|}{ JUN } \\
\hline $05 \ldots$ & 1340 & 21 & 418 & 8.2 & 13.5 & 6.9 & 289 & 0.737 & 0.030 & 1 & 0.3 \\
\hline \multicolumn{12}{|l|}{ JUL } \\
\hline $17 \ldots$ & 1515 & 1.1 & 685 & 8.4 & 19.5 & 6.3 & 480 & 0.397 & $<0.010$ & $<1$ & $<0.1$ \\
\hline \multicolumn{12}{|l|}{ JUN 1991} \\
\hline $18 \ldots$ & 1230 & 17 & 429 & 8.0 & 11.0 & 7.8 & 262 & 0.441 & 0.009 & $<1$ & 0.3 \\
\hline \multicolumn{12}{|l|}{ JUL } \\
\hline $16 \ldots$ & 1445 & 3.3 & 597 & 8.2 & 14.0 & 6.9 & 399 & 0.394 & 0.018 & $<1$ & 0.3 \\
\hline \multicolumn{12}{|l|}{ OCT } \\
\hline $22 \ldots$ & 1105 & 0.64 & 846 & 8.5 & 5.5 & 8.4 & 591 & 0.287 & 0.038 & $<1$ & 0.3 \\
\hline \multicolumn{12}{|l|}{ MAY 1992} \\
\hline $20 \ldots$ & 1400 & 8.4 & 702 & 8.3 & 13.5 & 7.2 & 507 & 0.890 & 0.015 & $<1$ & 0.2 \\
\hline \multicolumn{12}{|l|}{ JUN } \\
\hline $24 \ldots$ & 1600 & 17 & 384 & 8.2 & 12.0 & 7.3 & 262 & 0.361 & 0.045 & $<1$ & 0.2 \\
\hline \multicolumn{12}{|l|}{ AUG } \\
\hline $11 \ldots$ & 1140 & 7.8 & 604 & 8.4 & 13.0 & 7.3 & 414 & 0.311 & 0.025 & $<1$ & 0.2 \\
\hline \multicolumn{12}{|l|}{ OCT } \\
\hline $27 \ldots$ & 1350 & 1.0 & 814 & 8.4 & 8.5 & 7.9 & 625 & 0.272 & 0.022 & $<1$ & 0.2 \\
\hline
\end{tabular}

\begin{tabular}{|c|c|c|c|c|c|c|c|c|c|c|}
\hline & $\begin{array}{l}\text { COPPER, } \\
\text { TOTAL } \\
\text { RECOV - } \\
\text { ERABLE } \\
\text { (UG/L }\end{array}$ & $\begin{array}{l}\text { COPPER, } \\
\text { DIS - } \\
\text { SOLVED } \\
\text { (UG/L }\end{array}$ & $\begin{array}{l}\text { IRON, } \\
\text { TOTAL } \\
\text { RECOV - } \\
\text { ERABLE } \\
\text { (UG/L }\end{array}$ & $\begin{array}{l}\text { IRON, } \\
\text { DIS- } \\
\text { SOLVED } \\
\text { (UG/L }\end{array}$ & $\begin{array}{l}\text { LEAD, } \\
\text { TOTAL } \\
\text { RECOV- } \\
\text { ERABLE } \\
\text { IUG/L }\end{array}$ & $\begin{array}{l}\text { LEAD, } \\
\text { DIS - } \\
\text { SOLVED } \\
\text { /UG/L }\end{array}$ & $\begin{array}{l}\text { MANGA - } \\
\text { NESE, } \\
\text { TOTAL } \\
\text { RECOV - } \\
\text { ERABLE } \\
\text { (UG/L }\end{array}$ & $\begin{array}{l}\text { MANGA - } \\
\text { NESE, } \\
\text { DIS- } \\
\text { SOLVED } \\
\text { (UG/L }\end{array}$ & $\begin{array}{l}\text { ZINC, } \\
\text { TOTAL } \\
\text { RECOV- } \\
\text { ERABLE } \\
\text { IUG/L }\end{array}$ & $\begin{array}{l}\text { ZINC, } \\
\text { DIS - } \\
\text { SOLVED } \\
\text { UUG /L }\end{array}$ \\
\hline & AS $\mathrm{CU})$ & AS $\mathrm{CU}$ ) & AS FE) & AS FE) & AS PB) & AS PB) & AS MN) & AS MN) & AS $\mathrm{ZN}$ ) & AS $\mathrm{ZN}$ ) \\
\hline
\end{tabular}

\begin{tabular}{|c|c|c|c|c|c|c|c|c|c|c|}
\hline \multicolumn{11}{|l|}{ APR 1990} \\
\hline $18 \ldots$ & 2 & $<1$ & 100 & 5 & 3 & $<0.5$ & 40 & 14 & 130 & 100 \\
\hline \multicolumn{11}{|l|}{ JUN } \\
\hline $05 \ldots$ & 10 & 2 & 1200 & 11 & 51 & 0.7 & 270 & 17 & 230 & 93 \\
\hline \multicolumn{11}{|l|}{ JUL } \\
\hline $17 \ldots$ & 2 & 1 & 150 & 13 & 6 & $<0.5$ & 60 & 10 & 80 & 45 \\
\hline \multicolumn{11}{|l|}{ JUN 1991} \\
\hline $18 \ldots$ & 5 & 2 & 190 & 17 & 14 & $<0.5$ & 60 & 13 & 70 & 57 \\
\hline \multicolumn{11}{|l|}{ JUL } \\
\hline $16 \ldots$ & 4 & 2 & 100 & 20 & 11 & 0.8 & 50 & 20 & 50 & 47 \\
\hline \multicolumn{11}{|l|}{ OCT } \\
\hline $22 \ldots$ & 2 & $<1$ & 90 & 12 & 7 & 0.6 & 40 & 11 & 60 & 57 \\
\hline \multicolumn{11}{|l|}{ MAY 1992} \\
\hline $20 \ldots$ & 4 & $<1$ & 540 & 22 & 30 & 0.6 & 220 & 13 & 130 & 40 \\
\hline \multicolumn{11}{|l|}{ JUN } \\
\hline $24 \ldots$ & 3 & 1 & 280 & 16 & 16 & $<0.5$ & 80 & 11 & 90 & 41 \\
\hline \multicolumn{11}{|l|}{ AUG } \\
\hline $11 \ldots$ & 10 & $<1$ & 230 & 31 & 21 & 0.5 & 100 & 31 & 1100 & 63 \\
\hline \multicolumn{11}{|l|}{ OCT } \\
\hline $27 \ldots$ & $<1$ & $<1$ & 10 & 22 & 3 & $<0.5$ & 20 & 24 & 70 & 65 \\
\hline
\end{tabular}


Table 18. Onsite measurements and selected inorganic data for station 391013106190201 , Empire Gulch near Malta

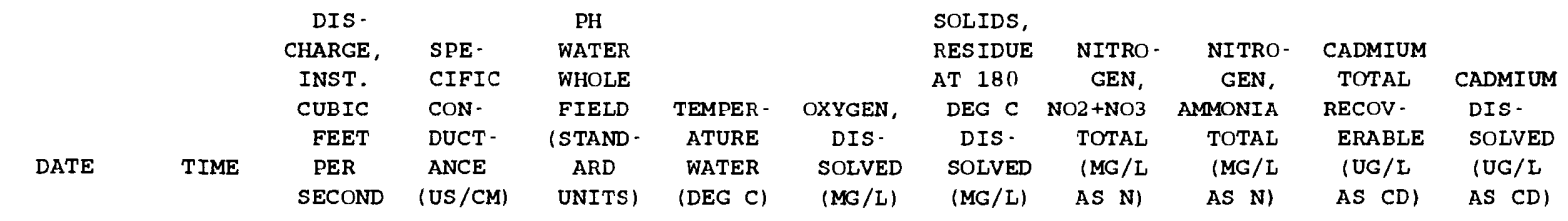

\begin{tabular}{|c|c|c|c|c|c|c|c|c|c|c|c|}
\hline APR 1990 & & & & & & & & & & & \\
\hline $\begin{array}{c}17 \ldots \\
\text { JUN }\end{array}$ & 1555 & 0.50 & $\cdots$ & 8.5 & 6.0 & $\cdots$ & 100 & 0.014 & $<0.010$ & $<1$ & $<0.1$ \\
\hline $05 \ldots$ & 1610 & 5.0 & 155 & 8.1 & 16.0 & 6.6 & 95 & 0.020 & 0.030 & $<1$ & $<0.1$ \\
\hline $17 \ldots$ & 1300 & 2.8 & 173 & 8.2 & 17.0 & 6.8 & 98 & 0.011 & 0.010 & $<1$ & $<0.1$ \\
\hline AUG & & & & & & & & & & & \\
\hline $\begin{array}{l}28 \ldots \\
\text { ост }\end{array}$ & 1400 & 0.28 & 202 & 8.0 & 18.0 & 6.5 & 106 & $<0.010$ & $<0.010$ & $<1$ & $<0.1$ \\
\hline $\begin{array}{c}30 \ldots \\
A P R \quad 1991\end{array}$ & 1140 & 1.1 & 203 & 8.4 & 5.0 & 8.6 & 115 & $<0.010$ & 0.020 & $<1$ & $<0.1$ \\
\hline JUN $23 \ldots$ & 1235 & $\cdots$ & 243 & 7.7 & 1.0 & 8.5 & 143 & 0.016 & 0.019 & 3 & 0.1 \\
\hline${ }_{\text {JUL }} 18 \ldots$ & 1315 & 6.5 & 171 & 7.9 & 13.5 & 7.2 & 90 & $<0.005$ & 0.013 & $<1$ & $<0.1$ \\
\hline $\begin{array}{l}16 \ldots \\
\text { ост }\end{array}$ & 1530 & 1.9 & $\cdots$ & $\cdots$ & 14.5 & 6.9 & 99 & 0.022 & 0.018 & $<1$ & 2.4 \\
\hline $\begin{array}{c}22 \ldots \\
\text { APR } 1992\end{array}$ & 1215 & 0.18 & 213 & 8.2 & 4.5 & 8.5 & 119 & $<0.005$ & 0.028 & $<1$ & $<0.1$ \\
\hline$\underset{\text { JUN }}{21 \ldots}$ & 0900 & 0.28 & 225 & 8.4 & 1.0 & 9.6 & 128 & 0.023 & 0.021 & $<1$ & $<0.1$ \\
\hline$\underset{A U G}{24} \ldots$ & 1640 & 6.9 & 166 & 8.4 & 14.0 & 6.6 & 98 & 0.006 & 0.017 & $<1$ & $<0.1$ \\
\hline $11 \ldots$ & 1245 & 1.3 & 230 & 8.3 & 13.0 & 6.9 & 142 & 0.054 & 0.013 & 3 & $<0.1$ \\
\hline
\end{tabular}

\begin{tabular}{|c|c|c|c|c|c|c|c|c|c|c|}
\hline DATE & $\begin{array}{l}\text { COPPER, } \\
\text { TOTAL } \\
\text { RECOV - } \\
\text { ERABLE } \\
\text { IUG /L }\end{array}$ & $\begin{array}{l}\text { COPPER, } \\
\text { DIS - } \\
\text { SOLVED } \\
\text { /UG/L }\end{array}$ & $\begin{array}{l}\text { IRON, } \\
\text { TOTAL } \\
\text { RECOV- } \\
\text { ERABLE } \\
\text { (UG/L }\end{array}$ & $\begin{array}{l}\text { IRON, } \\
\text { DIS- } \\
\text { SOLVED } \\
\text { (UG /L }\end{array}$ & $\begin{array}{l}\text { LEAD, } \\
\text { TOTAL } \\
\text { RECOV - } \\
\text { ERABLE } \\
\text { (UG / L }\end{array}$ & $\begin{array}{l}\text { LEAD, } \\
\text { DIS - } \\
\text { SOLVED } \\
\text { UUG /L }\end{array}$ & $\begin{array}{l}\text { MANGA - } \\
\text { NESE, } \\
\text { TOTAL } \\
\text { RECOV - } \\
\text { ERABLE } \\
\text { (UG /L }\end{array}$ & $\begin{array}{l}\text { MANGA- } \\
\text { NESE, } \\
\text { DIS- } \\
\text { SOLVED } \\
\text { (UG/L }\end{array}$ & $\begin{array}{l}\text { ZINC, } \\
\text { TOTAL } \\
\text { RECOV- } \\
\text { ERABLE } \\
\text { IUG/L }\end{array}$ & $\begin{array}{l}\text { ZINC, } \\
\text { DIS - } \\
\text { SOLVED } \\
\text { (UG /L }\end{array}$ \\
\hline & AS CU) & AS CU) & AS FE) & AS FE) & AS PB) & AS PB) & AS MN) & AS MN) & AS $\mathrm{ZN})$ & AS $\mathrm{ZN}$ ) \\
\hline
\end{tabular}

APR 1990
$17 \ldots$
JUN
$05 \ldots$
JUL $\ldots$
$17 \ldots$
AUG
$28 \ldots$
OCT $\ldots$
$30 \ldots$
APR 1991
$23 \ldots$
JUN
$18 \ldots$
JUL
$16 \ldots$
OCT
$22 \ldots$
APR 1992
$21 \ldots$
JUN
$24 \ldots$
AUG
$11 \ldots$

$\begin{array}{rrrrrrrrrr}2 & 1 & 510 & 26 & 5 & <0.5 & 50 & 7 & 30 & 25 \\ 5 & 1 & 1600 & 55 & 20 & 0.8 & 350 & 10 & 50 & 10 \\ 2 & 1 & 510 & 110 & 8 & <0.5 & 50 & 5 & 20 & 5 \\ 2 & <1 & 300 & 66 & 5 & <0.5 & 20 & 2 & 20 & 5 \\ 1 & <1 & 270 & 89 & <1 & <0.5 & 20 & 2 & <10 & <3 \\ 10 & 1 & 1800 & 49 & 180 & 3.2 & 180 & 47 & 420 & 14 \\ 2 & <1 & 630 & 87 & 5 & <0.5 & 80 & 4 & <10 & <3 \\ 5 & 1 & 1900 & 82 & 28 & <0.5 & 250 & 7 & 50 & <3 \\ 2 & <1 & 190 & 130 & 3 & <0.5 & 10 & <1 & <10 & 9 \\ <1 & <1 & 220 & 71 & <1 & <0.5 & 20 & 1 & <10 & <3 \\ 1 & <1 & 630 & 110 & 4 & 0.9 & 100 & 4 & 10 & <3 \\ 1 & <1 & 520 & 120 & 3 & <0.5 & 60 & 5 & <10 & <3\end{array}$


Table 19. Onsite measurements and bacteriological and selected inorganic data for station 07083710 , Arkansas River below Empire Gulch, near Malta

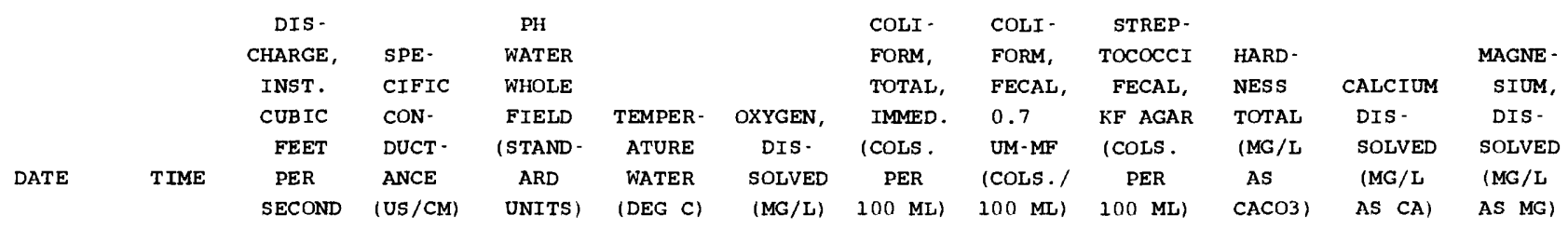

\begin{tabular}{|c|c|c|c|c|c|c|c|c|c|c|c|c|}
\hline \multicolumn{13}{|l|}{ APR 1990} \\
\hline $17 \ldots$ & 1720 & 91 & 191 & 8.2 & 8.5 & - & - & $<2$ & K400 & 88 & 21 & 8.6 \\
\hline \multicolumn{13}{|l|}{ MAY } \\
\hline $22 \ldots$ & 1615 & 140 & 154 & 7.9 & 13.0 & 7.7 & $\cdots$ & K6 & $\mathrm{k} 12$ & 65 & 16 & 6.0 \\
\hline \multicolumn{13}{|l|}{ JUN } \\
\hline $05 \ldots$ & 1430 & 512 & 115 & 7.7 & 12.0 & 7.8 & K380 & K16 & $\mathrm{K} 40$ & 51 & 13 & 4.5 \\
\hline $19 \ldots$ & 1510 & 360 & 136 & 7.9 & 14.5 & 7.5 & K150 & $<2$ & K10 & 65 & 16 & 6.1 \\
\hline \multicolumn{13}{|l|}{ JUL } \\
\hline $17 \ldots$ & 1420 & 139 & 179 & 8.3 & 17.5 & 7.4 & K16 & $<1$ & K9 & 78 & 18 & 8.1 \\
\hline \multicolumn{13}{|l|}{ AUG } \\
\hline $28 \ldots$ & 1245 & 72 & 210 & 8.4 & 15.0 & 8.1 & $\mathrm{~K} 2$ & $<1$ & $<1$ & 98 & 24 & 9.2 \\
\hline \multicolumn{13}{|l|}{ OCT } \\
\hline $30 \ldots$ & 1300 & 99 & 200 & $-\cdot$ & 7.5 & 9.2 & $\cdots$ & $\cdots$ & $\cdots$ & 92 & 23 & 8.3 \\
\hline \multicolumn{13}{|l|}{ DEC } \\
\hline $19 \ldots$ & 1415 & 48 & 210 & 7.7 & 0.5 & 10.3 & 2 & $<1$ & E100 & 94 & 23 & 8.9 \\
\hline \multicolumn{13}{|c|}{ MAR 1991} \\
\hline $25 \ldots$ & 1550 & 57 & 239 & 8.5 & 5.0 & 9.2 & $\cdots$ & $<1$ & $\cdots$ & 100 & 26 & 9.5 \\
\hline \multicolumn{13}{|l|}{ APR } \\
\hline $23 \ldots$ & 1325 & 79 & 206 & 7.8 & 10.0 & 8.0 & 230 & $<1$ & $<1$ & 87 & 21 & 8.3 \\
\hline \multicolumn{13}{|l|}{ MAY } \\
\hline $14 \ldots$ & 1400 & 156 & 150 & 7.6 & 11.0 & 7.6 & 160 & $<2$ & E1 & 62 & 15 & 5.9 \\
\hline \multicolumn{13}{|l|}{ JUN } \\
\hline $18 \ldots$ & 1345 & 427 & 129 & $\cdots$ & 12.0 & 7.7 & 290 & $<2$ & E8 & 56 & 14 & 5.1 \\
\hline \multicolumn{13}{|l|}{ JUL } \\
\hline $16 \ldots$ & 1600 & 137 & 174 & 8.0 & 15.5 & 6.5 & 140 & E27 & 54 & 80 & 20 & 7.4 \\
\hline \multicolumn{13}{|l|}{ AUG } \\
\hline $13 \ldots$ & 1315 & 132 & 194 & 8.1 & 16.0 & - & E17 & E3 & E17 & 90 & 23 & 8.0 \\
\hline \multicolumn{13}{|l|}{ OCT } \\
\hline $22 \ldots$ & 1430 & 71 & 212 & 8.3 & 9.0 & 8.4 & E50 & $\cdots$ & $<3$ & 92 & 23 & 8.3 \\
\hline \multicolumn{13}{|l|}{$\mathrm{DEC}$} \\
\hline $17 \ldots$ & 0900 & 77 & 250 & 7.6 & 0.5 & 9.4 & E2 & E2 & $<1$ & 110 & 27 & 9.7 \\
\hline \multicolumn{13}{|l|}{ MAR 1992} \\
\hline $23 \ldots$ & 1530 & 61 & 215 & 8.5 & 5.5 & 9.1 & - & - & - & 89 & 22 & 8.2 \\
\hline \multicolumn{13}{|l|}{ APR } \\
\hline $21 \ldots$ & 1030 & 95 & 179 & 7.9 & 3.0 & 9.8 & 390 & E1 & E3 & 78 & 19 & 7.3 \\
\hline \multicolumn{13}{|l|}{ MAY } \\
\hline $20 \ldots$ & 1520 & 297 & 124 & 8.0 & 12.5 & 7.7 & E14 & E6 & E14 & 54 & 14 & 4.7 \\
\hline$\pi U N$ & & & & & & & & & & & & \\
\hline $24 \ldots$ & 1710 & 350 & 138 & 8.0 & 12.5 & 7.6 & 23 & E17 & 57 & 63 & 16 & 5.5 \\
\hline JUL & & & & & & & & & & & & \\
\hline $13 \ldots$ & 1615 & 219 & 168 & 8.5 & 16.0 & 6.7 & E7 & E1 & E6 & 77 & 20 & 6.6 \\
\hline AUG & & & & & & & & & & & & \\
\hline $11 \ldots$ & 1330 & 134 & 215 & 8.4 & 14.0 & 8.2 & 54 & 32 & 51 & 100 & 27 & 8.3 \\
\hline $26 \ldots$ & 1000 & 180 & 205 & 8.2 & 7.5 & 8.6 & E34 & E13 & 40 & 95 & 25 & 8.0 \\
\hline OCT & & & & & & & & & & & & \\
\hline $26 \ldots$ & 1515 & 95 & 209 & 8.7 & 9.0 & 8.9 & E4 & E5 & E14 & 92 & 23 & 8.5 \\
\hline JAN 1993 & & & & & & & & & & & & \\
\hline $11 \ldots$ & 1440 & 45 & 211 & 8.0 & 0.0 & $\cdots$ & $\cdots$ & $<1$ & $<1$ & 86 & 21 & 8.1 \\
\hline MAR & & & & & & & & & & & & \\
\hline $22 \ldots$ & 1330 & 58 & 219 & 8.2 & 5.5 & 9.1 & E7 & $<1$ & -. & 89 & 22 & 8.2 \\
\hline
\end{tabular}


Table 19. Onsite measurements and bacteriological and selected inorganic data for station 07083710 , Arkansas River below Empire Gulch, near Malta--Continued

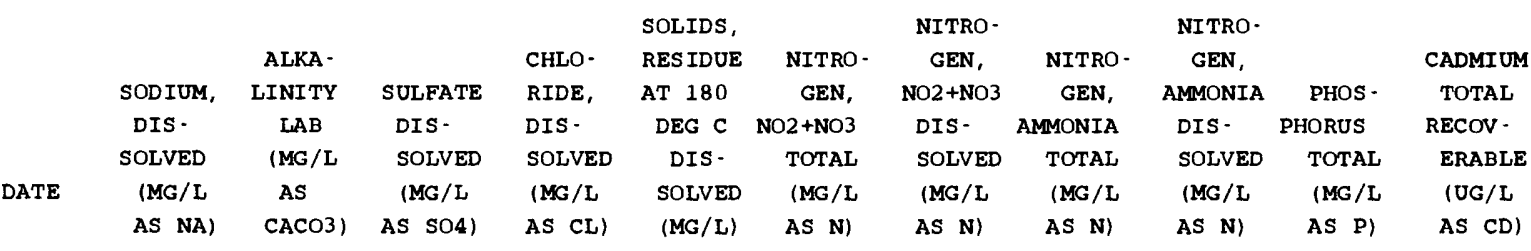

\begin{tabular}{|c|c|c|c|c|c|c|c|c|c|c|c|}
\hline \multicolumn{12}{|l|}{ APR 1990} \\
\hline $17 \ldots$ & 4.0 & 47 & 42 & 2.2 & 128 & 0.095 & $\cdots$ & $<0.010$ & $\cdots$ & 0.083 & 3 \\
\hline \multicolumn{12}{|l|}{ MAY } \\
\hline $22 \ldots$ & 3.3 & 39 & 30 & 2.7 & 95 & 0.037 & $\cdots$ & $<0.010$ & $\cdots$ & 0.057 & 3 \\
\hline \multicolumn{12}{|l|}{ JUN } \\
\hline $05 \ldots$ & 1.6 & 31 & 22 & 0.50 & 77 & 0.075 & $\cdots$ & 0.030 & $\cdots$ & 0.026 & 5 \\
\hline $19 \ldots$ & 1.9 & 42 & 24 & 1.9 & 77 & 0.060 & $\cdots$ & $<0.010$ & $\cdots$ & 0.024 & 2 \\
\hline \multicolumn{12}{|l|}{ JOL } \\
\hline $17 \ldots$ & 3.2 & 54 & 37 & 1.1 & 108 & $\cdots$ & $\cdots$ & $\cdots$ & $\cdots$ & $\cdots$ & 2 \\
\hline \multicolumn{12}{|l|}{ AUG } \\
\hline $28 \ldots$ & 4.2 & 64 & 37 & 2.9 & 122 & 0.026 & $\cdots$ & $<0.010$ & $\cdots$ & 0.021 & 2 \\
\hline \multicolumn{12}{|l|}{ OCT } \\
\hline $30 \ldots$ & 4.4 & 64 & 35 & 3.1 & 115 & 0.040 & $\cdots$ & 0.020 & $\cdots$ & 0.020 & $<1$ \\
\hline \multicolumn{12}{|l|}{ DEC } \\
\hline $19 \ldots$ & 4.8 & 63 & 41 & 4.4 & 125 & 0.206 & $\cdots$ & 0.026 & $\cdots$ & 0.053 & 1 \\
\hline \multicolumn{12}{|l|}{ MAR 1991} \\
\hline $25 \ldots$ & 5.7 & 64 & 48 & 3.6 & 129 & 0.180 & $\cdots$ & 0.053 & $\cdots$ & 0.034 & 1 \\
\hline \multicolumn{12}{|l|}{ APR } \\
\hline $23 \ldots$ & 4.4 & 56 & 41 & 2.9 & 109 & 0.151 & $\cdots$ & 0.051 & $\cdots$ & 0.028 & 2 \\
\hline \multicolumn{12}{|l|}{ MAY } \\
\hline $14 \ldots$ & 2.7 & 36 & 30 & 1.4 & 84 & 0.074 & $\cdots$ & 0.053 & $\cdots$ & 0.023 & 4 \\
\hline \multicolumn{12}{|l|}{ JUN } \\
\hline $18 \ldots$ & 1.8 & 37 & 17 & 0.30 & 66 & 0.058 & $\cdots$ & 0.010 & $\cdots$ & 0.028 & 2 \\
\hline \multicolumn{12}{|l|}{ JUL } \\
\hline $16 \ldots$ & 2.9 & 53 & 35 & 2.5 & 96 & 0.090 & $\cdots$ & 0.010 & $\cdots$ & 0.027 & 2 \\
\hline \multicolumn{12}{|l|}{$A \cup G$} \\
\hline $13 \ldots$ & 3.3 & 59 & 36 & 1.1 & 116 & 0.108 & $\cdots$ & 0.011 & $\cdots$ & 0.021 & 1 \\
\hline \multicolumn{12}{|l|}{ OCT } \\
\hline $22 \ldots$ & 4.5 & 67 & 30 & 3.6 & 124 & 0.096 & $\cdots$ & 0.028 & $\cdots$ & 0.025 & 1 \\
\hline \multicolumn{12}{|l|}{ DEC } \\
\hline $17 \ldots$ & 5.3 & 71 & 48 & 2.1 & 158 & 0.267 & $\cdots$ & 0.011 & $\cdots$ & 0.029 & 2 \\
\hline \multicolumn{12}{|l|}{ MAR 1992} \\
\hline $23 \ldots$ & 5.5 & 58 & 44 & 3.6 & 128 & 0.168 & $\cdots$ & 0.016 & $\cdots$ & 0.047 & $<1$ \\
\hline \multicolumn{12}{|l|}{ APR } \\
\hline $21 \ldots$ & 4.4 & 46 & 37 & 1.5 & 88 & 0.110 & $\cdots$ & 0.029 & $\cdots$ & 0.023 & 1 \\
\hline \multicolumn{12}{|l|}{ MAY } \\
\hline $20 \ldots$ & 2.4 & 33 & 24 & 0.70 & 90 & 0.077 & $\cdots$ & 0.015 & $\cdots$ & 0.020 & 2 \\
\hline JUN & & & & & & & & & & & \\
\hline $24 \ldots$ & 2.0 & 45 & 23 & 0.50 & 90 & 0.065 & $\cdots$ & 0.004 & $\cdots$ & 0.017 & $<1$ \\
\hline JUL & & & & & & & & & & & \\
\hline $13 \ldots$ & 2.5 & 52 & 28 & 1.2 & 92 & 0.043 & $\cdots$ & 0.003 & $\cdots$ & 0.015 & $<1$ \\
\hline AUG & & & & & & & & & & & \\
\hline $11 \ldots$ & 3.4 & 61 & 45 & 1.5 & 134 & 0.095 & $\cdots$ & 0.017 & $\cdots$ & 0.024 & $<1$ \\
\hline $26 \ldots$ & 3.6 & 59 & 40 & 2.0 & 120 & 0.101 & -. & 0.025 & $\cdots$ & 0.019 & 1 \\
\hline OCT & & & & & & & & & & & \\
\hline $26 \ldots$ & 4.5 & 61 & 38 & 2.8 & 133 & 0.066 & $-\cdot$ & 0.004 & $\cdots$ & 0.021 & $<1$ \\
\hline JAN 1993 & & & & & & & & & & & \\
\hline $11 \ldots$ & 5.1 & 60 & 40 & 5.0 & 108 & $\cdots$ & 0.265 & $\cdots$ & 0.018 & 0.030 & $<1$ \\
\hline MAR & & & & & & & & & & & \\
\hline $22 \ldots$ & 6.1 & 61 & 42 & 2.6 & 134 & $\cdots$ & 0.213 & $\cdots$ & 0.079 & 0.050 & $<1$ \\
\hline
\end{tabular}


Table 19. Onsite measurements and bacteriological and selected inorganic data for station 07083710 , Arkansas River below Empire Gulch, near Malta--Continued

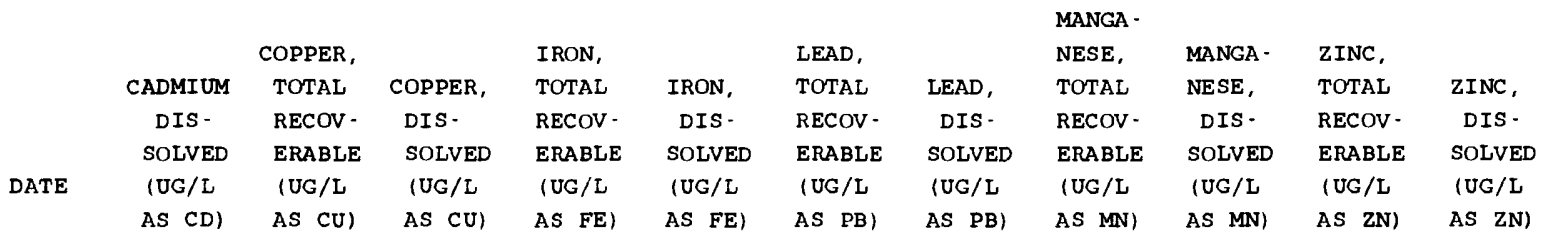

\begin{tabular}{|c|c|c|c|c|c|c|c|c|c|c|c|}
\hline \multicolumn{12}{|l|}{ APR 1990} \\
\hline $17 \ldots$ & 1.9 & 8 & 4 & 1900 & 440 & 22 & 1.7 & 570 & 480 & 730 & 470 \\
\hline \multicolumn{12}{|l|}{ MAY } \\
\hline $22 \ldots$ & 1.5 & 8 & 5 & 1200 & 110 & 22 & 1.1 & 390 & 220 & 640 & 330 \\
\hline \multicolumn{12}{|l|}{ JUN } \\
\hline $05 \ldots$ & 1.2 & 17 & 6 & 3200 & 87 & 80 & 1.4 & 580 & 100 & 890 & 310 \\
\hline $19 \ldots$ & 1.4 & 8 & 4 & 420 & 67 & 8 & $<0.5$ & 150 & 110 & 360 & 290 \\
\hline \multicolumn{12}{|l|}{ JUL } \\
\hline $17 \ldots$ & 1.4 & 12 & 9 & 360 & 150 & 6 & 1.1 & 180 & 170 & 290 & 230 \\
\hline \multicolumn{12}{|l|}{ AUG } \\
\hline $28 \ldots$ & 0.9 & 7 & 4 & 330 & 120 & 6 & $<0.5$ & 130 & 100 & 250 & 180 \\
\hline \multicolumn{12}{|l|}{ OCT } \\
\hline $30 \ldots$ & 0.5 & 4 & 1 & 270 & 77 & 4 & $<0.5$ & 90 & 58 & 170 & 110 \\
\hline \multicolumn{12}{|l|}{ DEC } \\
\hline $19 \ldots$ & 0.9 & 6 & 2 & 490 & 60 & 11 & $<0.5$ & 190 & 110 & 370 & 290 \\
\hline \multicolumn{12}{|l|}{ MAR 1991} \\
\hline $25 \ldots$ & 0.4 & 4 & 2 & 260 & 71 & 5 & 0.5 & 110 & 87 & 200 & 160 \\
\hline \multicolumn{12}{|l|}{ APR } \\
\hline $23 \ldots$ & 1.2 & 4 & 2 & 540 & 170 & 14 & 0.8 & 250 & 250 & 330 & 270 \\
\hline \multicolumn{12}{|l|}{ MAY } \\
\hline $14 \ldots$ & 1.8 & 10 & 3 & 940 & 240 & 29 & 1.7 & 360 & 310 & 570 & 290 \\
\hline \multicolumn{12}{|l|}{ JUN } \\
\hline $18 \ldots$ & 1.2 & 17 & 14 & 530 & 100 & 13 & $<0.5$ & 150 & 87 & 240 & 210 \\
\hline \multicolumn{12}{|l|}{ JUL } \\
\hline $16 \ldots$ & 1.2 & 5 & 2 & 360 & 140 & 13 & $<0.5$ & 140 & 130 & 240 & 230 \\
\hline \multicolumn{12}{|l|}{ AUG } \\
\hline $13 \ldots$ & 0.9 & 5 & 2 & 430 & 92 & 17 & 0.5 & 120 & 120 & 160 & 110 \\
\hline \multicolumn{12}{|l|}{ OCT } \\
\hline $22 \ldots$ & 0.6 & 3 & 1 & 200 & 78 & 4 & 0.7 & 50 & 41 & 130 & 100 \\
\hline \multicolumn{12}{|l|}{$\mathrm{DEC}$} \\
\hline $17 \ldots$ & 1.7 & $\cdots$ & 2 & 180 & 68 & $<1$ & 0.8 & 170 & 170 & 500 & 530 \\
\hline \multicolumn{12}{|l|}{ MAR 1992} \\
\hline $23 \ldots$ & 0.4 & 1 & 1 & 270 & 70 & 4 & $<0.5$ & 80 & 57 & 130 & 82 \\
\hline \multicolumn{12}{|l|}{$A P R$} \\
\hline $21 \ldots$ & 0.9 & 4 & 2 & 640 & 190 & 7 & $<0.5$ & 300 & 280 & 360 & 320 \\
\hline \multicolumn{12}{|l|}{ MAY } \\
\hline $20 \ldots$ & 0.5 & 7 & 3 & 1100 & 91 & 27 & 2 & 240 & 58 & 270 & 110 \\
\hline JUN & & & & & & & & & & & \\
\hline $24 \ldots$ & 0.5 & 3 & 2 & 330 & 88 & 6 & $<0.5$ & 70 & 36 & 120 & 90 \\
\hline JUL & & & & & & & & & & & \\
\hline $13 \ldots$ & 0.3 & 2 & 1 & 290 & 130 & 5 & 0.6 & 60 & 38 & 70 & 48 \\
\hline AUG & & & & & & & & & & & \\
\hline $11 \ldots$ & 0.9 & 3 & 1 & 380 & 110 & 10 & $<0.5$ & 90 & 67 & 150 & 95 \\
\hline $26 \ldots$ & 0.7 & 3 & 1 & 380 & 160 & 9 & 0.6 & 140 & 110 & 240 & 180 \\
\hline OCT & & & & & & & & & & & \\
\hline $26 \ldots$ & 0.1 & 2 & $<1$ & 460 & 89 & 8 & $<0.5$ & 80 & 24 & 100 & 45 \\
\hline JAN 1993 & & & & & & & & & & & \\
\hline $11 \ldots$ & 0.2 & 3 & $<1$ & 470 & 66 & 12 & 0.5 & 160 & 60 & 230 & 150 \\
\hline MAR & & & & & & & & & & & \\
\hline $22 \ldots$ & 0.5 & 1 & $<1$ & 440 & 70 & 8 & $<0.5$ & 150 & 80 & 180 & 120 \\
\hline
\end{tabular}


Table 20. Onsite measurements and selected inorganic data for station 09073000, Twin Lakes Tunnel at East Portal, near Twin Lakes

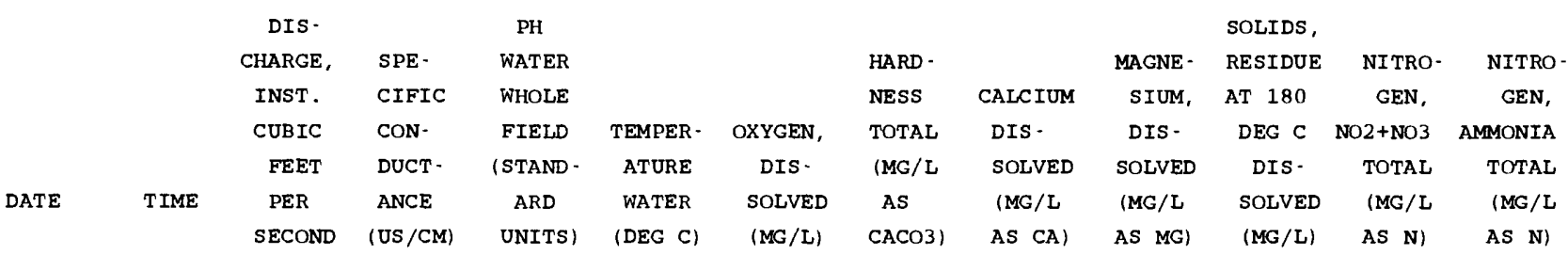

MAY 1990

\begin{tabular}{|c|c|c|c|c|c|c|c|c|c|c|c|c|}
\hline $22 \ldots$ & 1815 & 159 & 44 & $\cdots$ & 1.0 & 8.8 & $\cdots$ & $\cdots$ & $\cdots$ & 38 & 0.087 & $<0.010$ \\
\hline \multicolumn{13}{|l|}{ JUN } \\
\hline $04 \ldots$ & 1350 & 302 & 40 & 7.3 & 2.0 & 9.6 & $\cdots$ & $\cdots$ & $\cdots$ & 39 & 0.053 & 0.030 \\
\hline \multicolumn{13}{|l|}{ JUN 1991} \\
\hline $17 \ldots$ & 1500 & 461 & 37 & 7.1 & 5.0 & 8.6 & $\cdots$ & $\cdots$ & $\cdots$ & 21 & 0.054 & 0.027 \\
\hline \multicolumn{13}{|l|}{ JUL } \\
\hline $15 \ldots$ & 1420 & 83 & 47 & 7.8 & 9.0 & 7.6 & $\cdots$ & $\cdots$ & $\cdots$ & 23 & 0.024 & $<0.002$ \\
\hline \multicolumn{13}{|l|}{ AUG } \\
\hline $13 \ldots$ & 1805 & 1.8 & 86 & 7.3 & 9.5 & $\cdots$ & $\cdots$ & $\cdots$ & $\cdots$ & 58 & 0.058 & $<0.002$ \\
\hline \multicolumn{13}{|l|}{ JUN 1992} \\
\hline $23 \ldots$ & 1240 & 374 & 36 & 7.5 & 5.0 & 8.6 & 13 & 4.3 & 0.65 & 34 & 0.041 & $<0.002$ \\
\hline \multicolumn{13}{|l|}{ JUL } \\
\hline $13 \ldots$ & 1820 & 145 & 48 & 7.6 & 8.0 & 7.9 & $\cdots$ & $\cdots$ & $\cdots$ & 36 & 0.022 & 0.006 \\
\hline \multicolumn{13}{|l|}{ AUG } \\
\hline $11 \ldots$ & 1555 & 0.66 & 95 & 8.3 & 10.5 & 7.1 & $\cdots$ & $\cdots$ & $\cdots$ & 64 & 0.151 & 0.007 \\
\hline
\end{tabular}

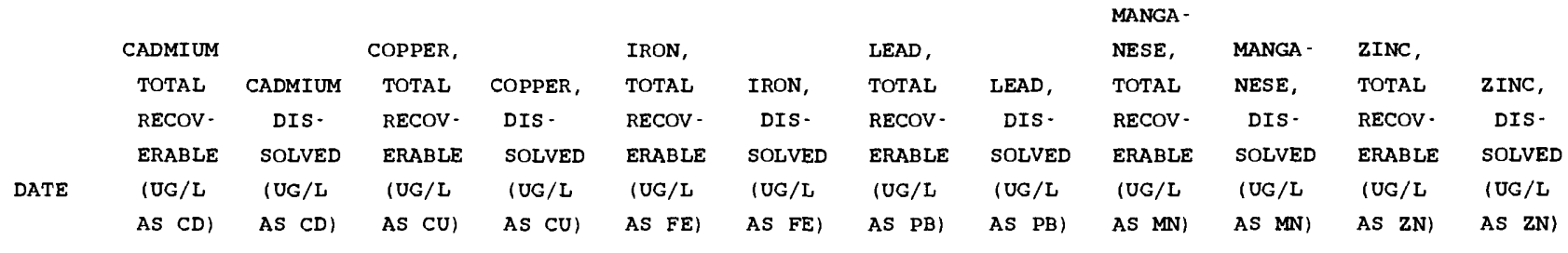

MAY 1990

$\begin{array}{llll}22 \ldots & \cdots & <0.1 & \cdots \\ \operatorname{JUN} & & & \\ 04 \ldots & <1 & <0.1 & \\ \operatorname{JUN} 1991 & & & \\ 17 \ldots & 1 & 0.3 & \\ \operatorname{JUL} & & & \\ 15 \ldots & <1 & 0.1 & \\ \text { AUG } & & & \\ 13 \ldots & <1 & <0.1 & \\ \operatorname{JUN} 1992 & & & \\ 23 \ldots & <1 & <0.1 \\ \operatorname{JUL} & & & \\ 13 \ldots & <1 & <0.1 & 12 \\ \text { AUG } & & <1 & <0.1\end{array}$


Table 21. Onsite measurements and selected inorganic data for station 07084500 , Lake Creek above Twin Lakes Reservoir

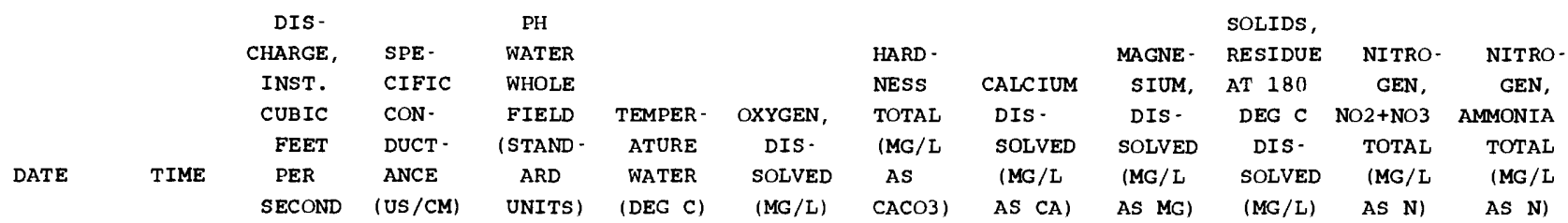

APR 1990

\begin{tabular}{|c|c|c|c|c|c|c|c|c|c|c|c|c|}
\hline$\underset{J U N}{16 \ldots}$ & 1545 & E23 & 131 & 7.8 & 6.5 & $\cdots$ & $\cdots$ & $\cdots$ & $\cdots$ & 71 & 0.164 & $<0.010$ \\
\hline $04 \ldots$ & 1525 & 706 & -. & 7.5 & 7.5 & 8.4 & -. & - & - & 37 & 0.079 & 0.030 \\
\hline \multicolumn{13}{|l|}{ AUG } \\
\hline $27 \ldots$ & 1800 & 42 & 127 & 7.9 & 14.5 & 7.1 & $\cdots$ & $\cdots$ & $\cdots$ & 64 & 0.117 & $<0.010$ \\
\hline \multicolumn{13}{|l|}{ JUN 1991} \\
\hline \multicolumn{13}{|l|}{ JUL } \\
\hline $15 \ldots$ & 1320 & 188 & 69 & 7.5 & 12.0 & 7.4 & $\cdots$ & $\cdots$ & $\cdots$ & 35 & 0.072 & 0.004 \\
\hline \multicolumn{13}{|l|}{ OCT } \\
\hline $21 \ldots$ & 1445 & 16 & 137 & 7.7 & 5.0 & 9.1 & $-\cdot$ & $\cdots$ & $\cdots$ & 86 & 0.131 & 0.017 \\
\hline \multicolumn{13}{|l|}{ JUN 1992} \\
\hline $23 \ldots$ & 1115 & 632 & 48 & 7.5 & 7.0 & 9.9 & 19 & 6.1 & 0.95 & 27 & 0.061 & $<0.002$ \\
\hline \multicolumn{13}{|l|}{ JUL } \\
\hline $13 \ldots$ & 1735 & 314 & 62 & 7.6 & 10.5 & 8.0 & $\cdots$ & $\cdots$ & $\cdots$ & 56 & 0.038 & $<0.002$ \\
\hline \multicolumn{13}{|l|}{ AUG } \\
\hline $11 \ldots$ & 1525 & 77 & 100 & 7.8 & 12.0 & 7.5 & $\cdots$ & $\cdots$ & $\cdots$ & 56 & 0.096 & 0.005 \\
\hline \multicolumn{13}{|l|}{ OCT } \\
\hline $27 \ldots$ & 1500 & 27 & 133 & 7.8 & 4.5 & 8.7 & -. & $\cdots$ & - & 76 & 0.142 & $<0.002$ \\
\hline
\end{tabular}

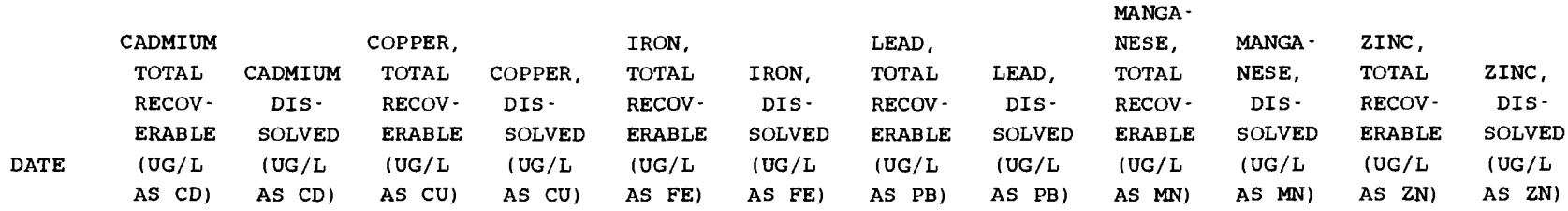

\begin{tabular}{|c|c|c|c|c|c|c|c|c|c|c|c|c|}
\hline \multicolumn{13}{|l|}{ APR 1990} \\
\hline $16 \ldots$ & $<1$ & 0.2 & 39 & 4 & 2200 & $<3$ & 1 & $<0.5$ & 40 & 25 & 40 & 6 \\
\hline \multicolumn{13}{|l|}{ JUN } \\
\hline $04 \ldots$ & $<1$ & 1.4 & 18 & 6 & 800 & 72 & 2 & $<0.5$ & 10 & 7 & $<10$ & 8 \\
\hline \multicolumn{13}{|l|}{ AUG } \\
\hline $27 \ldots$ & $<1$ & $<0.1$ & 33 & 4 & 400 & 11 & $<1$ & $<0.5$ & 30 & 19 & 20 & $<3$ \\
\hline \multicolumn{13}{|l|}{ JUN 1991} \\
\hline $17 \ldots$ & $<1$ & $<0.1$ & 42 & 11 & 640 & 34 & 9 & 0.6 & 20 & 4 & $<10$ & 14 \\
\hline \multicolumn{13}{|l|}{ JUL } \\
\hline $15 \ldots$ & $<1$ & $<0.1$ & 19 & 9 & 260 & 44 & 9 & $<0.5$ & 10 & 8 & $<10$ & 5 \\
\hline \multicolumn{13}{|l|}{ OCT } \\
\hline $21 \ldots$ & $<1$ & 0.2 & 30 & 5 & 340 & $<3$ & $<1$ & $<0.5$ & 30 & 22 & $<10$ & 10 \\
\hline \multicolumn{13}{|l|}{ JUN 1992} \\
\hline $23 \ldots$ & $<1$ & $<0.1$ & 49 & 9 & 410 & 30 & 2 & $<0.5$ & 20 & 5 & 10 & 6 \\
\hline \multicolumn{13}{|c|}{$\pi L$} \\
\hline $13 \ldots$ & $<1$ & $<0.1$ & 16 & 9 & 250 & 57 & $<1$ & $<0.5$ & $<10$ & 7 & 20 & 4 \\
\hline \multicolumn{13}{|l|}{ AUG } \\
\hline $11 \ldots$ & $<1$ & 0.1 & 30 & 6 & 450 & 12 & $<1$ & $<0.5$ & 10 & 14 & 20 & 5 \\
\hline \multicolumn{13}{|l|}{ O T } \\
\hline $27 \ldots$ & $<1$ & 0.1 & 32 & 5 & 360 & $<3$ & $<1$ & $<0.5$ & $<10$ & 20 & $<10$ & 7 \\
\hline
\end{tabular}


Table 22. Onsite measurements and selected inorganic data for station 390444106174900 , Lake Creek at State Highway 82, below Twin Lakes Reservoir

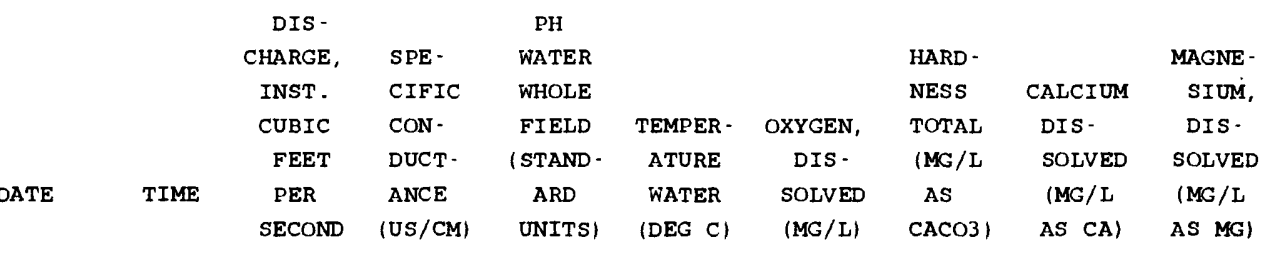

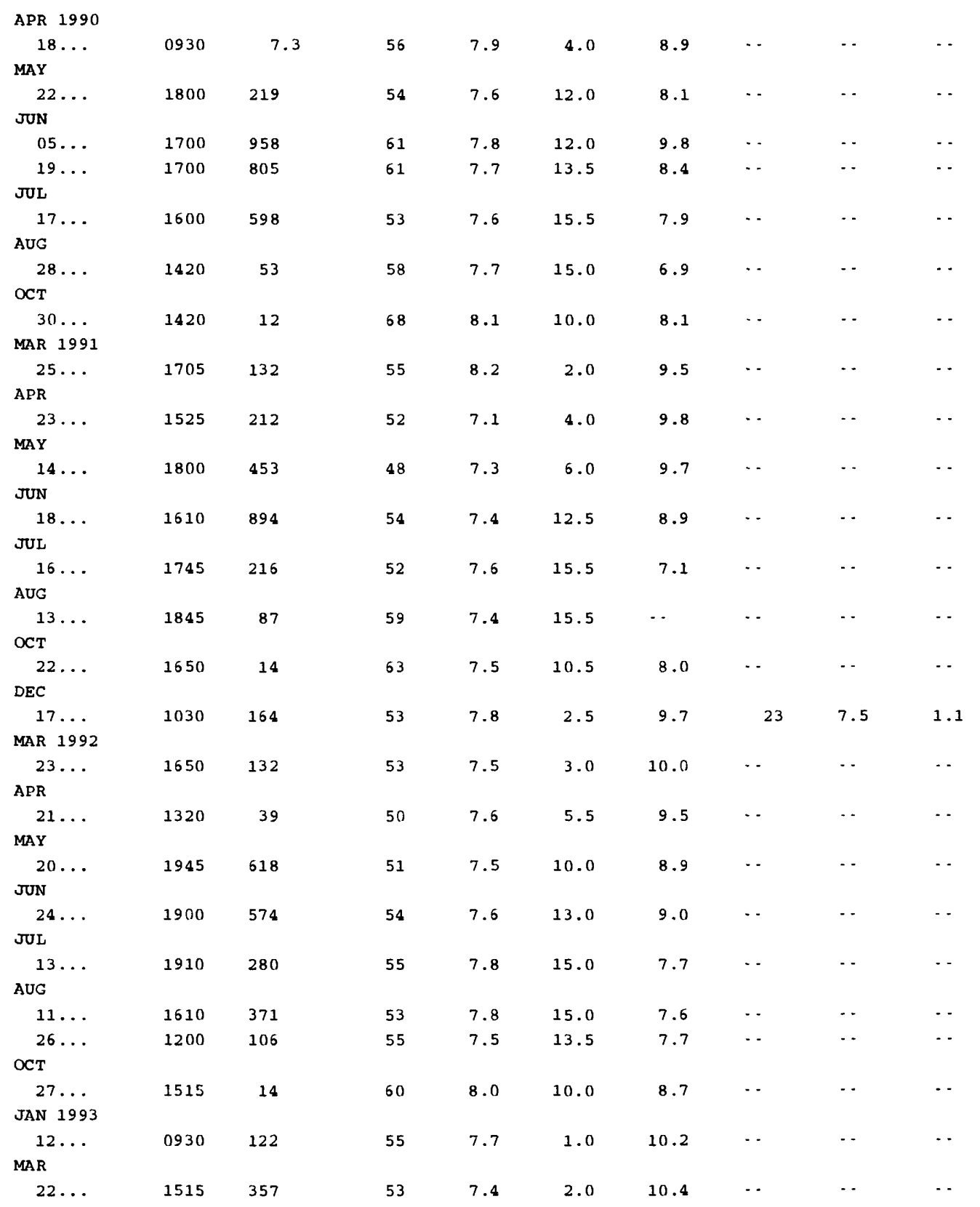


Table 22. Onsite measurements and selected inorganic data for station 390444106174900 , Lake Creek at State Highway 82, below Twin Lakes Reservoir--Continued

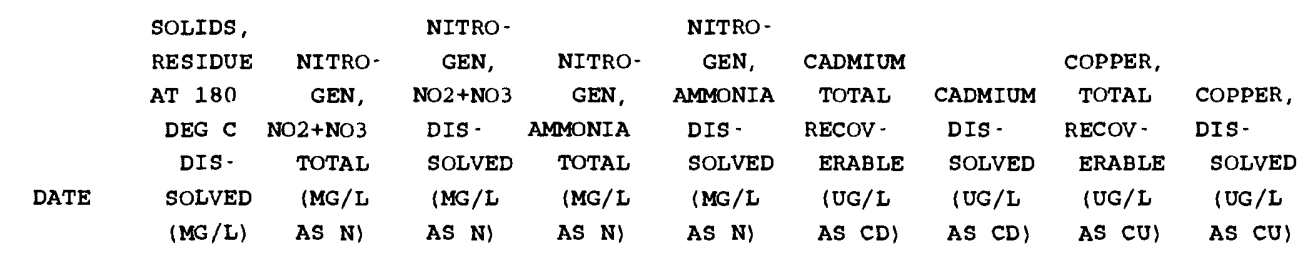

\begin{tabular}{|c|c|c|c|c|c|c|c|c|c|}
\hline APR 1990 & & & & & & & & & \\
\hline $18 \ldots$ & 26 & 0.034 & $\cdots$ & $<0.010$ & $\cdots$ & $<1$ & $<0.1$ & 2 & 1 \\
\hline MAY & & & & & & & & & \\
\hline $22 \ldots$ & 30 & $<0.010$ & $\cdots$ & $<0.010$ & $\cdots$ & $<1$ & $<0.1$ & 2 & 2 \\
\hline JUN & & & & & & & & & \\
\hline $05 \ldots$ & 24 & $<0.010$ & $\cdots$ & 0.020 & $\cdots$ & $<1$ & $<0.1$ & 5 & 2 \\
\hline $19 \ldots$ & 29 & 0.010 & $\cdots$ & $<0.010$ & .. & $<1$ & 0.4 & 5 & 2 \\
\hline JUL & & & & & & & & & \\
\hline $17 \ldots$ & 23 & 0.021 & $\cdots$ & $<0.010$ & $\cdots$ & $<1$ & $<0.1$ & 4 & 3 \\
\hline AUG & & & & & & & & & \\
\hline $28 \ldots$ & 28 & 0.023 & $-\cdot$ & 0.010 & - & $<1$ & $<0.1$ & 4 & 2 \\
\hline OCT & & & & & & & & & \\
\hline $30 \ldots$ & 41 & 0.019 & $\cdots$ & 0.020 & -. & $<1$ & $<0.1$ & 4 & 2 \\
\hline MAR 1991 & & & & & & & & & \\
\hline $25 \ldots$ & 42 & 0.025 & $\cdots$ & 0.025 & $\cdots$ & - - & $-\cdot$ & $\cdots$ & $\cdots$ \\
\hline APR & & & & & & & & & \\
\hline $23 \ldots$ & 23 & 0.030 & $\cdots$ & 0.022 & $\cdots$ & $<1$ & $<0.1$ & 3 & 2 \\
\hline MAY & & & & & & & & & \\
\hline $14 \ldots$ & 27 & 0.009 & $\cdots$ & 0.018 & $\cdots$ & $\cdots$ & $\cdots$ & $\cdots$ & $\cdots$ \\
\hline JUN & & & & & & & & & \\
\hline $18 \ldots$ & 29 & 0.014 & $\cdots$ & 0.015 & $\cdots$ & $<1$ & $<0.1$ & 6 & 3 \\
\hline$\pi U L$ & & & & & & & & & \\
\hline $16 \ldots$ & 16 & 0.024 & - & 0.004 & $\cdots$ & $<1$ & 0.1 & 6 & 3 \\
\hline AUG & & & & & & & & & \\
\hline $13 \ldots$ & 34 & 0.042 & - & 0.016 & $\cdots$ & $\cdots$ & $\cdots$ & $\cdots$ & $\cdots$ \\
\hline OCT & & & & & & & & & \\
\hline $22 \ldots$ & 48 & 0.020 & $\cdots$ & 0.029 & $\cdots$ & $<1$ & 0.2 & 4 & 2 \\
\hline $\mathrm{DEC}$ & & & & & & & & & \\
\hline $17 \ldots$ & 29 & 0.009 & $\cdots$ & 0.005 & $\cdots$ & $<1$ & 0.6 & $\cdots$ & 2 \\
\hline MAR 1992 & & & & & & & & & \\
\hline $23 \ldots$ & 31 & 0.032 & $\cdots$ & 0.024 & $\cdots$ & - & - & - & $\cdots$ \\
\hline $\mathrm{APR}$ & & & & & & & & & \\
\hline $21 \ldots$ & 22 & 0.016 & $\cdots$ & 0.009 & - & $<1$ & $<0.1$ & 2 & 2 \\
\hline MAY & & & & & & & & & \\
\hline $20 \ldots$ & 30 & 0.013 & - - & 0.010 & $\cdots$ & - & $\cdots$ & - - & - \\
\hline$\pi U N$ & & & & & & & & & \\
\hline $24 \ldots$ & 40 & 0.008 & $\cdots$ & 0.036 & $\cdots$ & $<1$ & $<0.1$ & 3 & 3 \\
\hline$\pi U L$ & & & & & & & & & \\
\hline $13 \ldots$ & 34 & 0.007 & - & 0.009 & -. & -. & $\cdots$ & $\cdots$ & $\cdots$ \\
\hline AUG & & & & & & & & & \\
\hline $11 \ldots$ & 30 & 0.018 & - & 0.018 & $\cdots$ & $<1$ & $<0.1$ & 4 & 3 \\
\hline $26 \ldots$ & 22 & 0.010 & $\cdots$ & 0.016 & $\cdots$ & - & $\cdots$ & - & $\cdots$ \\
\hline OCT & & & & & & & & & \\
\hline $27 \ldots$ & 42 & 0.013 & - & 0.006 & $\cdots$ & $<1$ & 0.1 & 2 & 2 \\
\hline JAN 1993 & & & & & & & & & \\
\hline $12 \ldots$ & 37 & - - & 0.013 & - - & 0.020 & $\cdots$ & -. & - & $\cdots$ \\
\hline MAR & & & & & & & & & \\
\hline $22 \ldots$ & 14 & $\cdots$ & 0.013 & -. & 0.007 & $<1$ & $<0.1$ & 2 & 2 \\
\hline
\end{tabular}


Table 22. Onsite measurements and selected inorganic data for station 390444106174900 , Lake Creek at State Highway 82, below Twin Lakes Reservoir--Continued

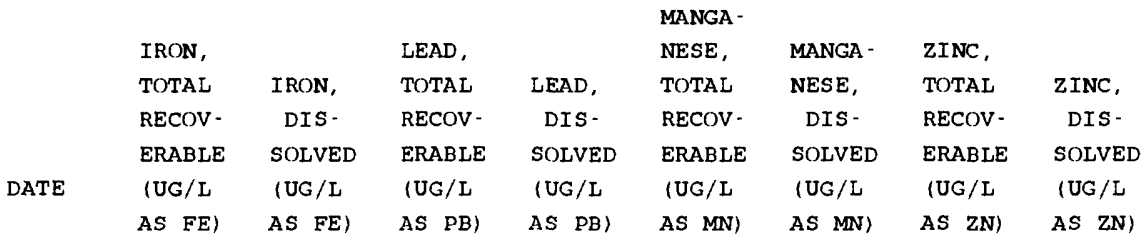

\begin{tabular}{|c|c|c|c|c|c|c|c|c|}
\hline \multicolumn{9}{|l|}{ APR 1990} \\
\hline $18 \ldots$ & 140 & 6 & $<1$ & $<0.5$ & 20 & 7 & $<10$ & $<3$ \\
\hline \multicolumn{9}{|l|}{ MAY } \\
\hline $22 \ldots$ & 170 & 8 & 1 & $<0.5$ & 30 & 6 & 10 & $<3$ \\
\hline \multicolumn{9}{|l|}{ JUN } \\
\hline $05 \ldots$ & 360 & 13 & 4 & $<0.5$ & 20 & 4 & $<10$ & 7 \\
\hline $19 \ldots$ & 400 & 9 & 1 & $<0.5$ & 20 & 4 & $<10$ & $<3$ \\
\hline \multicolumn{9}{|l|}{ JUL } \\
\hline $17 \ldots$ & 140 & 16 & 1 & $<0.5$ & 20 & 4 & $<10$ & 6 \\
\hline \multicolumn{9}{|l|}{ AUG } \\
\hline $28 \ldots$ & 100 & 19 & 3 & $<0.5$ & 20 & 2 & 20 & 8 \\
\hline \multicolumn{9}{|l|}{ ОСт } \\
\hline $30 \ldots$ & 70 & 7 & $<1$ & $<0.5$ & 20 & 9 & $<10$ & $<3$ \\
\hline \multicolumn{9}{|l|}{ MAR 1991} \\
\hline $25 \ldots$ & $\cdots$ & $\cdots$ & - & $\cdots$ & $\cdots$ & $\cdots$ & $-\cdot$ & $\cdots$ \\
\hline \multicolumn{9}{|l|}{$\mathrm{APR}$} \\
\hline $23 \ldots$ & 80 & 15 & 2 & $<0.5$ & $<10$ & 3 & $<10$ & 5 \\
\hline \multicolumn{9}{|l|}{ MAY } \\
\hline $14 \ldots$ & -. & $\cdots$ & -. & $\cdots$ & $-\cdot$ & $\cdots$ & $\cdots$ & $\cdots$ \\
\hline \multicolumn{9}{|l|}{ JUN } \\
\hline $18 \ldots$ & 80 & 16 & 24 & 0.6 & 20 & $<1$ & $<10$ & $<3$ \\
\hline \multicolumn{9}{|l|}{ JUL } \\
\hline $16 \ldots$ & 60 & 21 & 7 & $<0.5$ & 10 & 1 & $<10$ & $<3$ \\
\hline \multicolumn{9}{|l|}{ AUG } \\
\hline $13 \ldots$ & - & $\cdots$ & - & - & $-\cdot$ & $\cdots$ & $\cdots$ & -. \\
\hline \multicolumn{9}{|l|}{ OCT } \\
\hline $22 \ldots$ & 40 & 10 & 2 & 1.1 & 30 & 10 & $<10$ & 6 \\
\hline \multicolumn{9}{|l|}{ DEC } \\
\hline $17 \ldots$ & 60 & 9 & $<1$ & $<0.5$ & 10 & 2 & 30 & $<3$ \\
\hline \multicolumn{9}{|l|}{ MAR 1992} \\
\hline $23 \ldots$ & - - & $\cdots$ & $\cdots$ & $-\cdot$ & - - & $\cdots$ & -- & - \\
\hline \multicolumn{9}{|l|}{$\mathrm{APR}$} \\
\hline $21 \ldots$ & 200 & 15 & $<1$ & $<0.5$ & 20 & 8 & $<10$ & $<3$ \\
\hline \multicolumn{9}{|l|}{ MAY } \\
\hline $20 \ldots$ & - & $\cdots$ & - & - - & $-\cdot$ & $-\cdot$ & -- & - \\
\hline \multicolumn{9}{|l|}{ JUN } \\
\hline $24 \ldots$ & 90 & 14 & $<1$ & 0.5 & 10 & 3 & $<10$ & $<3$ \\
\hline \multicolumn{9}{|l|}{ JUL } \\
\hline $13 \ldots$ & $\cdots$ & $\cdots$ & $\cdots$ & $\cdots$ & $\cdots$ & -- & $\cdots$ & - \\
\hline \multicolumn{9}{|l|}{ AUG } \\
\hline $11 \ldots$ & 140 & 22 & 1 & $<0.5$ & 10 & 3 & 40 & 3 \\
\hline $26 \ldots$ & $\cdots$ & $\cdots$ & - & $\cdots$ & -. & - & $-\cdot$ & $\cdots$ \\
\hline \multicolumn{9}{|l|}{ OCT } \\
\hline $27 \ldots$ & 40 & 11 & $<1$ & $<0.5$ & $<10$ & 3 & $<10$ & $<3$ \\
\hline JAN 1993 & & & & & & & & \\
\hline $12 \ldots$ & . & $\cdots$ & $\cdots$ & $\cdots$ & -. & $\cdots$ & $-\cdot$ & 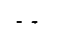 \\
\hline MAR & & & & & & & & \\
\hline $22 \ldots$ & 50 & 15 & $<1$ & $<0.5$ & $<10$ & 2 & $<10$ & 5 \\
\hline
\end{tabular}


Table 23. Onsite measurements and selected inorganic data for station 07086000 , Arkansas River at Granite

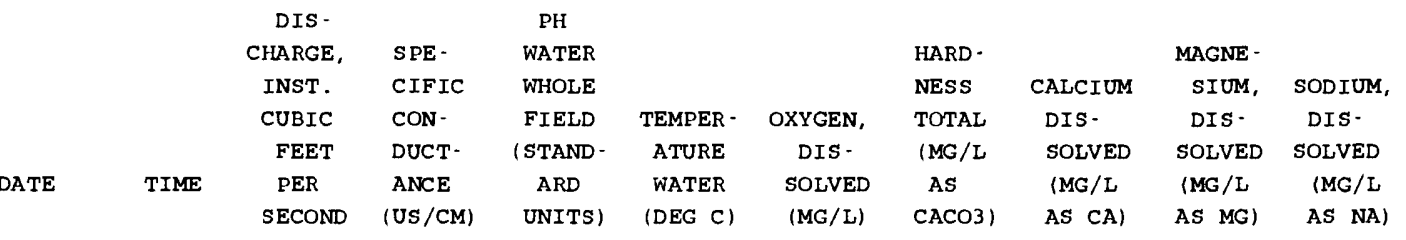

\begin{tabular}{|c|c|c|c|c|c|c|c|c|c|c|}
\hline \multicolumn{11}{|l|}{ APR 1990} \\
\hline $18 \ldots$ & 1030 & 118 & 178 & 8.4 & 5.0 & 9.2 & 82 & 20 & 7.9 & 3.9 \\
\hline \multicolumn{11}{|l|}{ MAY } \\
\hline $22 \ldots$ & 1920 & 408 & 100 & 7.9 & 12.5 & 7.4 & 41 & 11 & 3.3 & 2.2 \\
\hline \multicolumn{11}{|l|}{ JUN } \\
\hline $05 \ldots$ & 1810 & 1720 & 81 & 7.6 & 12.5 & 7.4 & 33 & 8.0 & 3.1 & 0.90 \\
\hline $19 \ldots$ & 1920 & 1220 & 88 & 7.9 & 14.0 & 7.1 & 39 & 11 & 2.8 & 1.5 \\
\hline \multicolumn{11}{|l|}{ JUL } \\
\hline $17 \ldots$ & 1700 & 867 & 81 & 8.0 & 16.5 & 6.8 & 36 & 10 & 2.6 & 1.6 \\
\hline \multicolumn{11}{|l|}{ AUG } \\
\hline $28 \ldots$ & 1545 & 150 & 162 & 8.2 & 16.5 & 6.8 & 70 & 18 & 6.1 & 3.4 \\
\hline \multicolumn{11}{|l|}{ OCT } \\
\hline $30 \ldots$ & 1525 & 118 & 185 & 8.6 & 8.0 & 8.5 & 87 & 22 & 7.7 & 4.5 \\
\hline \multicolumn{11}{|l|}{ DEC } \\
\hline $19 \ldots$ & 1605 & 227 & 155 & 7.7 & 1.0 & 10.0 & 66 & 17 & 5.6 & 3.5 \\
\hline \multicolumn{11}{|l|}{ MAR 1991} \\
\hline $25 \ldots$ & 1800 & 223 & 112 & 8.3 & 3.5 & 9.5 & 47 & 13 & 3.5 & 2.5 \\
\hline \multicolumn{11}{|l|}{$\mathrm{APR}$} \\
\hline $23 \ldots$ & 1640 & 313 & 105 & 7.7 & 6.5 & 8.9 & 42 & 12 & 2.9 & 2.2 \\
\hline \multicolumn{11}{|l|}{ MAY } \\
\hline $14 \ldots$ & 1930 & 748 & 88 & 7.6 & 7.5 & 8.0 & 34 & 9.6 & 2.5 & 1.7 \\
\hline \multicolumn{11}{|l|}{ JUN } \\
\hline $10 \ldots$ & 1520 & 1500 & $\cdots$ & - & $\cdots$ & - & 33 & 9.4 & 2.3 & 1.4 \\
\hline $18 \ldots$ & 1730 & 1450 & 82 & 7.7 & 13.5 & 7.5 & 36 & 10 & 2.6 & 1.4 \\
\hline \multicolumn{11}{|l|}{ JUL } \\
\hline $16 \ldots$ & 1930 & 410 & 109 & 8.0 & 15.5 & 7.1 & 48 & 13 & 3.8 & 2.1 \\
\hline \multicolumn{11}{|l|}{ AUG } \\
\hline $13 \ldots$ & 2000 & 241 & 148 & 7.9 & $\cdots$ & - & 65 & 17 & 5.4 & 2.8 \\
\hline \multicolumn{11}{|l|}{ OCT } \\
\hline $22 \ldots$ & 1710 & 94 & 183 & 8.5 & 9.0 & 8.2 & 82 & 21 & 7.1 & 4.4 \\
\hline \multicolumn{11}{|l|}{ DEC } \\
\hline $17 \ldots$ & 1155 & 229 & 99 & 8.2 & 2.5 & 9.8 & 42 & 12 & 2.9 & 2.1 \\
\hline \multicolumn{11}{|l|}{ MAR 1992} \\
\hline $23 \ldots$ & 1750 & 207 & 106 & 8.4 & 4.0 & 9.5 & 44 & 12 & 3.3 & 2.7 \\
\hline \multicolumn{11}{|l|}{ APR } \\
\hline $21 \ldots$ & 1245 & 147 & 147 & 8.3 & 6.0 & 8.7 & 59 & 15 & 5.3 & 3.8 \\
\hline \multicolumn{11}{|l|}{ MAY } \\
\hline $20 \ldots$ & 1820 & 990 & 80 & 8.0 & 10.5 & 7.8 & 34 & 9.5 & 2.4 & 1.7 \\
\hline JUN & & & & & & & & & & \\
\hline $24 \ldots$ & 2010 & 951 & 102 & 8.0 & 14.0 & 7.3 & 39 & 11 & 2.8 & 1.6 \\
\hline JUL & & & & & & & & & & \\
\hline $13 \ldots$ & 2015 & 536 & 117 & 8.3 & 15.0 & 6.8 & 48 & 13 & 3.7 & 1.9 \\
\hline AUG & & & & & & & & & & \\
\hline $11 \ldots$ & 1730 & 567 & 100 & 8.1 & 15.0 & 6.8 & 43 & 12 & 3.2 & 2.0 \\
\hline $26 \ldots$ & 1315 & 313 & 156 & 8.3 & 12.5 & 7.4 & 71 & 19 & 5.8 & 3.1 \\
\hline OCT & & & & & & & & & & \\
\hline $27 \ldots$ & 1630 & 117 & 191 & 8.6 & 8.0 & 8.2 & 83 & 21 & 7.3 & 4.1 \\
\hline JAN 1993 & & & & & & & & & & \\
\hline $12 \ldots$ & 1030 & 227 & 116 & 8.0 & 1.0 & 10.5 & 48 & 13 & 3.7 & 2.7 \\
\hline MAR & & & & & & & & & & \\
\hline $22 \ldots$ & 1630 & 470 & 84 & 8.5 & 3.5 & 10.1 & 34 & 9.6 & 2.4 & 2.1 \\
\hline
\end{tabular}


Table 23. Onsite measurements and selected inorganic data for station 07086000 , Arkansas River at Granite--Continued

\begin{tabular}{|c|c|c|c|c|c|c|c|c|c|c|}
\hline & $\begin{array}{l}\text { ALKA- } \\
\text { LINITY } \\
\text { LAB } \\
\text { IMG / L } \\
\text { AS }\end{array}$ & $\begin{array}{l}\text { SULFATE } \\
\text { DIS - } \\
\text { SOLVED } \\
\text { (MG / L }\end{array}$ & $\begin{array}{l}\text { CHLO- } \\
\text { RIDE, } \\
\text { DIS - } \\
\text { SOLVED } \\
\text { (MG / L }\end{array}$ & $\begin{array}{c}\text { SOLIDS, } \\
\text { RESIDUE } \\
\text { AT } 180 \\
\text { DEG C } \\
\text { DIS - } \\
\text { SOLVED }\end{array}$ & $\begin{array}{l}\text { NITRO- } \\
\text { GEN, } \\
\text { NO2+NO3 } \\
\text { TOTAL } \\
\text { (MG /L }\end{array}$ & $\begin{array}{l}\text { NITRO- } \\
\text { GEN, } \\
\text { NO2+NO3 } \\
\text { DIS- } \\
\text { SOLVED } \\
\text { (MG/L }\end{array}$ & $\begin{array}{l}\text { NITRO- } \\
\text { GEN, } \\
\text { AMMONIA } \\
\text { TOTAL } \\
\text { (MG/L }\end{array}$ & $\begin{array}{l}\text { NITRO- } \\
\text { GEN, } \\
\text { AMMONIA } \\
\text { DIS - } \\
\text { SOLVED } \\
\text { (MG/L }\end{array}$ & $\begin{array}{l}\text { CADMIUM } \\
\text { TOTAL } \\
\text { RECOV - } \\
\text { ERABLE } \\
\text { (UG / L }\end{array}$ & $\begin{array}{l}\text { CADMIUM } \\
\text { DIS - } \\
\text { SOLVED } \\
\text { (UG / L }\end{array}$ \\
\hline DATE & (АAC3) & AS SO4) & AS CL) & $(M G / L)$ & AS N) & AS N) & AS N) & AS N) & & AS $C D)$ \\
\hline \multicolumn{11}{|l|}{ APR 1990} \\
\hline $18 \ldots$ & 45 & 34 & 1.5 & 117 & 0.055 & $\cdots$ & $<0.010$ & $\cdots$ & 4 & 2.0 \\
\hline \multicolumn{11}{|l|}{ MAY } \\
\hline $22 \ldots$ & 29 & 19 & 1.3 & 60 & 0.018 & $\cdots$ & $<0.010$ & $\cdots$ & 2 & 0.5 \\
\hline \multicolumn{11}{|l|}{ JUN } \\
\hline $05 \ldots$ & 24 & 9.2 & 0.90 & 48 & 0.046 & $\cdots$ & 0.020 & $\cdots$ & 2 & 0.5 \\
\hline $19 \ldots$ & 26 & 16 & $<0.10$ & 63 & 0.014 & $\cdots$ & 0.010 & $\cdots$ & 1 & 0.4 \\
\hline \multicolumn{11}{|l|}{ JUL } \\
\hline $17 \ldots$ & 26 & 14 & 0.20 & 50 & 0.013 & - & 0.010 & $\cdots$ & $<1$ & 0.2 \\
\hline \multicolumn{11}{|l|}{ AUG } \\
\hline $28 \ldots$ & 50 & 26 & 2.2 & 86 & 0.010 & $\cdots$ & 0.020 & $\cdots$ & 2 & 0.5 \\
\hline \multicolumn{11}{|l|}{ OCT } \\
\hline $30 \ldots$ & 64 & 29 & 3.3 & 115 & $<0.010$ & . & 0.020 & - & $<1$ & 0.6 \\
\hline \multicolumn{11}{|l|}{$\mathrm{DEC}$} \\
\hline $19 \ldots$ & 47 & 27 & 2.8 & 91 & 0.100 & -. & 0.070 & $\cdots$ & $<1$ & 0.5 \\
\hline \multicolumn{11}{|l|}{ MAR 1991} \\
\hline $25 \ldots$ & 34 & 17 & 1.7 & 72 & 0.014 & -. & 0.025 & -. & $<1$ & 0.4 \\
\hline \multicolumn{11}{|l|}{ APR } \\
\hline $23 \ldots$ & 30 & 17 & 1.4 & 55 & 0.033 & - & 0.021 & -. & $<1$ & $<0.1$ \\
\hline \multicolumn{11}{|l|}{ MAY } \\
\hline $14 \ldots$ & 24 & 15 & 1.0 & 35 & 0.017 & - & 0.024 & - - & 1 & 0.6 \\
\hline \multicolumn{11}{|l|}{ JUN } \\
\hline $10 \ldots$ & 23 & 12 & -. & - - & - & - - & - & $\cdots$ & 1 & 0.3 \\
\hline $18 \ldots$ & 25 & 11 & $<0.10$ & 33 & 0.020 & - & 0.018 & - & $<1$ & 0.3 \\
\hline \multicolumn{11}{|l|}{ JUL } \\
\hline $16 \ldots$ & 34 & 19 & 1.5 & 61 & 0.018 & $\cdots$ & 0.011 & $-\cdot$ & $<1$ & 0.6 \\
\hline \multicolumn{11}{|l|}{ AUG } \\
\hline $13 \ldots$ & 48 & 25 & 0.70 & 89 & 0.023 & $\cdots$ & 0.005 & -. & $<1$ & 0.4 \\
\hline \multicolumn{11}{|l|}{ OCT } \\
\hline $22 \ldots$ & 63 & 22 & 2.5 & 113 & 0.008 & $\cdots$ & 0.019 & $\cdots$ & $<1$ & 0.2 \\
\hline \multicolumn{11}{|l|}{ DEC } \\
\hline $17 \ldots$ & 32 & 16 & $<0.10$ & 62 & 0.038 & $\cdots$ & 0.011 & $\cdots$ & $<1$ & 0.7 \\
\hline \multicolumn{11}{|l|}{ MAR 1992} \\
\hline $23 \ldots$ & 32 & 18 & 1.7 & 49 & 0.025 & $\cdots$ & 0.009 & $\cdots$ & $<1$ & 0.2 \\
\hline APR & & & & & & & & & & \\
\hline $21 \ldots$ & 42 & 30 & 1.4 & 104 & 0.021 & - & 0.013 & $\cdots$ & $<1$ & 0.6 \\
\hline MAY & & & & & & & & & & \\
\hline $20 \ldots$ & 23 & 13 & 0.50 & 46 & 0.024 & - - & 0.009 & $\cdots$ & 2 & 0.3 \\
\hline JUN & & & & & & & & & & \\
\hline $24 \ldots$ & 29 & 15 & 0.50 & 54 & 0.013 & $-\cdot$ & 0.002 & $\cdots$ & $<1$ & 0.2 \\
\hline JUL & & & & & & & & & & \\
\hline $13 \ldots$ & 35 & 16 & 0.70 & 64 & 0.005 & $\cdots$ & $<0.002$ & -. & $<1$ & 0.1 \\
\hline AUG & & & & & & & & & & \\
\hline $11 \ldots$ & 31 & 16 & 0.60 & 56 & 0.018 & $-\cdot$ & 0.013 & $-\cdot$ & $<1$ & $<0.1$ \\
\hline $26 \ldots$ & 48 & 32 & 1.6 & 104 & 0.044 & - & 0.030 & $-\cdot$ & $<1$ & 0.5 \\
\hline OCT & & & & & & & & & & \\
\hline $27 \ldots$ & 59 & 31 & 1.8 & 122 & 0.015 & $\cdots$ & 0.004 & -. & $<1$ & 0.2 \\
\hline JAN 1993 & & & & & & & & & & \\
\hline $12 \ldots$ & 35 & 19 & 1.0 & 72 & $\cdots$ & 0.075 & $-\cdot$ & 0.009 & $<1$ & 0.1 \\
\hline MAR & & & & & & & & & & \\
\hline $22 \ldots$ & 27 & 13 & 0.70 & 36 & $\cdots$ & 0.033 & $\cdots$ & $<0.002$ & $<1$ & 0.2 \\
\hline
\end{tabular}


Table 23. Onsite measurements and selected inorganic data for station 07086000 , Arkansas River at Granite--Continued

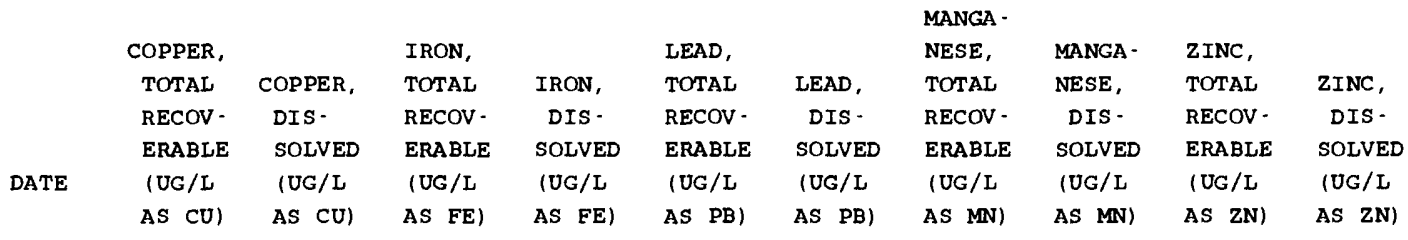

\begin{tabular}{|c|c|c|c|c|c|c|c|c|c|c|}
\hline \multicolumn{11}{|l|}{ APR 1990} \\
\hline $18 \ldots$ & 9 & 5 & 2200 & 570 & 38 & 6 & 640 & 500 & 940 & 620 \\
\hline \multicolumn{11}{|l|}{ MAY } \\
\hline $22 \ldots$ & 6 & 4 & 1400 & 48 & 37 & 0.7 & 280 & 82 & 380 & 96 \\
\hline \multicolumn{11}{|l|}{ JUN } \\
\hline $05 \ldots$ & 17 & 5 & 3300 & 110 & 72 & 14 & 390 & 60 & 370 & 130 \\
\hline $19 \ldots$ & 8 & 3 & 390 & 25 & 8 & 0.9 & 40 & 22 & 80 & 55 \\
\hline \multicolumn{11}{|l|}{ JUL } \\
\hline $17 \ldots$ & 5 & 4 & 190 & 31 & 2 & $<0.5$ & 30 & 20 & 40 & 31 \\
\hline \multicolumn{11}{|l|}{ AUG } \\
\hline $28 \ldots$ & 4 & 2 & 180 & 57 & 4 & $<0.5$ & 60 & 41 & 150 & 96 \\
\hline \multicolumn{11}{|l|}{ OCT } \\
\hline $30 \ldots$ & 5 & 1 & 210 & 74 & 5 & $<0.5$ & 80 & 47 & 170 & 120 \\
\hline \multicolumn{11}{|l|}{ DEC } \\
\hline $19 \ldots$ & 4 & 2 & 280 & 57 & 4 & $<0.5$ & 90 & 70 & 200 & 180 \\
\hline \multicolumn{11}{|l|}{ MAR 1991} \\
\hline $25 \ldots$ & 3 & 2 & 150 & 35 & 3 & $<0.5$ & 50 & 22 & 110 & 85 \\
\hline \multicolumn{11}{|l|}{$\mathrm{APR}$} \\
\hline $23 \ldots$ & 5 & 2 & 250 & 69 & 13 & $<0.5$ & 70 & 43 & 100 & 52 \\
\hline \multicolumn{11}{|l|}{ MAY } \\
\hline $14 \ldots$ & 17 & 4 & 490 & 77 & 18 & 2.4 & 110 & 63 & 160 & 110 \\
\hline \multicolumn{11}{|l|}{ JUN } \\
\hline $10 \ldots$ & 8 & 5 & 1000 & 330 & 19 & $<0.5$ & 90 & 80 & 150 & 140 \\
\hline $18 \ldots$ & 7 & 4 & 330 & 46 & 11 & $<0.5$ & 60 & 20 & 80 & 58 \\
\hline \multicolumn{11}{|l|}{ JUL } \\
\hline $16 \ldots$ & 8 & 2 & 160 & 59 & 8 & $<0.5$ & 50 & 33 & 90 & 73 \\
\hline \multicolumn{11}{|l|}{ AUG } \\
\hline $13 \ldots$ & 3 & 2 & 220 & 50 & 7 & 0.6 & 50 & 35 & 80 & 48 \\
\hline \multicolumn{11}{|l|}{ OCT } \\
\hline $22 \ldots$ & 4 & $<1$ & 110 & 39 & 5 & 1.5 & 40 & 23 & 80 & 60 \\
\hline \multicolumn{11}{|l|}{ DEC } \\
\hline $17 \ldots$ & $\cdots$ & 2 & 120 & 21 & 7 & 0.9 & 30 & 20 & 130 & 73 \\
\hline \multicolumn{11}{|l|}{ MAR 1992} \\
\hline $23 \ldots$ & $<1$ & 2 & 160 & 56 & $<1$ & $<0.5$ & 40 & 17 & 50 & 36 \\
\hline \multicolumn{11}{|l|}{ APR } \\
\hline $21 \ldots$ & 2 & 2 & 490 & 150 & 6 & 0.6 & 160 & 120 & 220 & 140 \\
\hline \multicolumn{11}{|l|}{ MAY } \\
\hline $20 \ldots$ & 9 & 3 & 790 & 47 & 20 & $<0.5$ & 170 & 23 & 200 & 43 \\
\hline$\pi U N$ & & & & & & & & & & \\
\hline $24 \ldots$ & 75 & 7 & 220 & 45 & 8 & $<0.5$ & 40 & 15 & 60 & 41 \\
\hline JUL & & & & & & & & & & \\
\hline $13 \ldots$ & 3 & 2 & 120 & 52 & 3 & 0.6 & 30 & 14 & 40 & 24 \\
\hline AUG & & & & & & & & & & \\
\hline $11 \ldots$ & 12 & 2 & 440 & 37 & 15 & $<0.5$ & 40 & 12 & 1000 & 24 \\
\hline $26 \ldots$ & 4 & 2 & 270 & 100 & 7 & 0.9 & 80 & 48 & 130 & 96 \\
\hline OCT & & & & & & & & & & \\
\hline $27 \ldots$ & 2 & 1 & 260 & 61 & 4 & 0.6 & 40 & 24 & 90 & 55 \\
\hline JAN 1993 & & & & & & & & & & \\
\hline $12 \ldots$ & 3 & 2 & 190 & 35 & 6 & $<0.5$ & 60 & 19 & 100 & 61 \\
\hline MAR & & & & & & & & & & \\
\hline $22 \ldots$ & 2 & 2 & 370 & 26 & 10 & $<0.5$ & 90 & 16 & 80 & 26 \\
\hline
\end{tabular}


Table 24. Onsite measurements and selected inorganic data for station 07086500 , Clear Creek above Clear Creek Reservoir

\begin{tabular}{|c|c|c|c|c|c|c|c|c|c|c|c|c|}
\hline DATE & TIME & $\begin{array}{c}\text { DIS - } \\
\text { CHARGE, } \\
\text { INST. } \\
\text { CUBIC } \\
\text { FEET } \\
\text { PER } \\
\text { SECOND }\end{array}$ & $\begin{array}{l}\text { SPE- } \\
\text { CIFIC } \\
\text { CON- } \\
\text { DUCT - } \\
\text { ANCE } \\
\text { (US/CM) }\end{array}$ & $\begin{array}{c}\text { PH } \\
\text { WATER } \\
\text { WHOLE } \\
\text { FIELD } \\
\text { (STAND - } \\
\text { ARD } \\
\text { UNITS) }\end{array}$ & $\begin{array}{l}\text { TEMPER- } \\
\text { ATURE } \\
\text { WATER } \\
\text { (DEG C) }\end{array}$ & $\begin{array}{c}\text { OXYGEN, } \\
\text { DIS - } \\
\text { SOLVED } \\
\text { (MG/L) }\end{array}$ & $\begin{array}{l}\text { HARD- } \\
\text { NESS } \\
\text { TOTAL } \\
\text { (MG/L } \\
\text { AS } \\
\text { CACO3) }\end{array}$ & $\begin{array}{l}\text { CALC IUM } \\
\text { DIS - } \\
\text { SOLVED } \\
\text { (MG /L } \\
\text { AS CA) }\end{array}$ & $\begin{array}{l}\text { MAGNE- } \\
\text { SIUM, } \\
\text { DIS - } \\
\text { SOLVED } \\
\text { (MG/L } \\
\text { AS MG) }\end{array}$ & $\begin{array}{c}\text { SOLIDS, } \\
\text { RESIDUE } \\
\text { AT } 180 \\
\text { DEG C } \\
\text { DIS- } \\
\text { SOLVED } \\
\text { (MG/L) }\end{array}$ & $\begin{array}{c}\text { NITRO- } \\
\text { GEN, } \\
\text { NO2+NO3 } \\
\text { TOTAL } \\
\text { (MG/L } \\
\text { AS N) }\end{array}$ & $\begin{array}{r}\text { NITRO- } \\
\text { GEN, } \\
\text { AMMONIA } \\
\text { TOTAL } \\
\text { (MG/L } \\
\text { AS N) }\end{array}$ \\
\hline APR 1990 & & & & & & & & & & & & \\
\hline $\begin{array}{l}16 \ldots \\
\text { JUN }\end{array}$ & 1420 & 17 & 144 & 8.0 & 9.5 & 7.4 & $\cdots$ & - & - & 91 & 0.117 & $<0.010$ \\
\hline$\underset{A U G}{04} \ldots$ & 1200 & 199 & 80 & 7.7 & 8.0 & 8.5 & $\cdots$ & $\cdots$ & $\cdots$ & 51 & 0.103 & 0.010 \\
\hline $\begin{array}{c}27 \ldots \\
\text { APR } 1991\end{array}$ & 1615 & 41 & 133 & 7.4 & 16.5 & 7.2 & $\cdots$ & $\cdots$ & $\cdots$ & 69 & 0.093 & $<0.010$ \\
\hline$\pi_{\pi N}^{22} \cdots$ & 1215 & 11 & 155 & 7.7 & 6.5 & 9.3 & $\cdots$ & $\cdots$ & $\cdots$ & 90 & 0.136 & 0.013 \\
\hline$\underset{\text { JUL }}{17 \ldots}$ & 1410 & 240 & 76 & 7.4 & 10.5 & 7.8 & $\cdots$ & $\cdots$ & $\cdots$ & 37 & 0.105 & 0.024 \\
\hline$\underset{\mathrm{OCT}}{15 \ldots}$ & 1205 & 91 & 100 & 7.9 & 14.0 & 7.3 & $\cdots$ & $\cdots$ & $\cdots$ & 68 & 0.086 & 0.008 \\
\hline $\begin{array}{c}21 \ldots \\
\text { APR } 1992\end{array}$ & 1305 & 15 & 142 & 8.0 & 7.5 & 8.2 & $\cdots$ & $\cdots$ & $\cdots$ & 86 & 0.120 & 0.015 \\
\hline$\underset{\text { JUN }}{20 \ldots}$ & 1315 & 16 & 149 & 8.0 & 7.5 & 8.2 & $\cdots$ & $\cdots$ & $\cdots$ & 84 & 0.113 & 0.007 \\
\hline$\underset{\text { AUG }}{23 \ldots}$ & 1115 & 162 & 81 & 7.8 & 10.0 & 8.0 & 37 & 13 & 1.0 & 60 & 0.087 & $<0.002$ \\
\hline$\underset{\text { OCT }}{10 \ldots}$ & 1200 & 46 & 121 & 8.0 & 12.0 & - & - & $\cdots$ & $\cdots$ & 56 & 0.081 & 0.005 \\
\hline $28 \ldots$ & 0715 & 19 & 143 & 7.9 & 4.0 & 9.0 & $\cdots$ & $\cdots$ & $\cdots$ & 89 & 0.140 & 0.007 \\
\hline
\end{tabular}

\begin{tabular}{|c|c|c|c|c|c|c|c|c|c|c|c|c|}
\hline ITE & $\begin{array}{l}\text { CADMIUM } \\
\text { TOTAL } \\
\text { RECOV- } \\
\text { ERABLE } \\
\text { /UG/L }\end{array}$ & $\begin{array}{c}\text { CADMIUM } \\
\text { DIS - } \\
\text { SOLVED } \\
\text { /UG/L }\end{array}$ & $\begin{array}{c}\text { COPPER, } \\
\text { TOTAL } \\
\text { RECOV- } \\
\text { ERABLE } \\
\text { /UG/L }\end{array}$ & $\begin{array}{l}\text { COPPER, } \\
\text { DIS - } \\
\text { SOLVED } \\
\text { (UG } / L\end{array}$ & $\begin{array}{l}\text { IRON, } \\
\text { TOTAL } \\
\text { RECOV - } \\
\text { ERABLE } \\
\text { (UG/L }\end{array}$ & $\begin{array}{l}\text { IRON, } \\
\text { DIS - } \\
\text { SOLVED } \\
\text { (UG/L }\end{array}$ & $\begin{array}{l}\text { LEAD, } \\
\text { TOTAL } \\
\text { RECOV- } \\
\text { ERABLE } \\
\text { (UG/L }\end{array}$ & $\begin{array}{l}\text { LEAD, } \\
\text { DIS- } \\
\text { SOLVED } \\
\text { (UG/L }\end{array}$ & $\begin{array}{l}\text { MANGA- } \\
\text { NESE, } \\
\text { TOTAL } \\
\text { RECOV- } \\
\text { ERABLE } \\
\text { (UG/L }\end{array}$ & $\begin{array}{l}\text { MANGA- } \\
\text { NESE, } \\
\text { DIS- } \\
\text { SOLVED } \\
\text { (UG/L }\end{array}$ & $\begin{array}{l}\text { ZINC, } \\
\text { TOTAL } \\
\text { RECOV- } \\
\text { ERABLE } \\
\text { (UG/L }\end{array}$ & $\begin{array}{l}\text { ZINC, } \\
\text { DIS - } \\
\text { SOLVED } \\
\text { (UG } / L\end{array}$ \\
\hline & AS $C D$ ) & AS $C D$ ) & AS CU) & AS CU) & AS FE) & AS FE) & AS PB) & AS PB) & AS $\mathrm{MN}$ ) & AS MN) & AS $\mathrm{zN}$ ) & AS $\mathrm{zN}$ ) \\
\hline
\end{tabular}

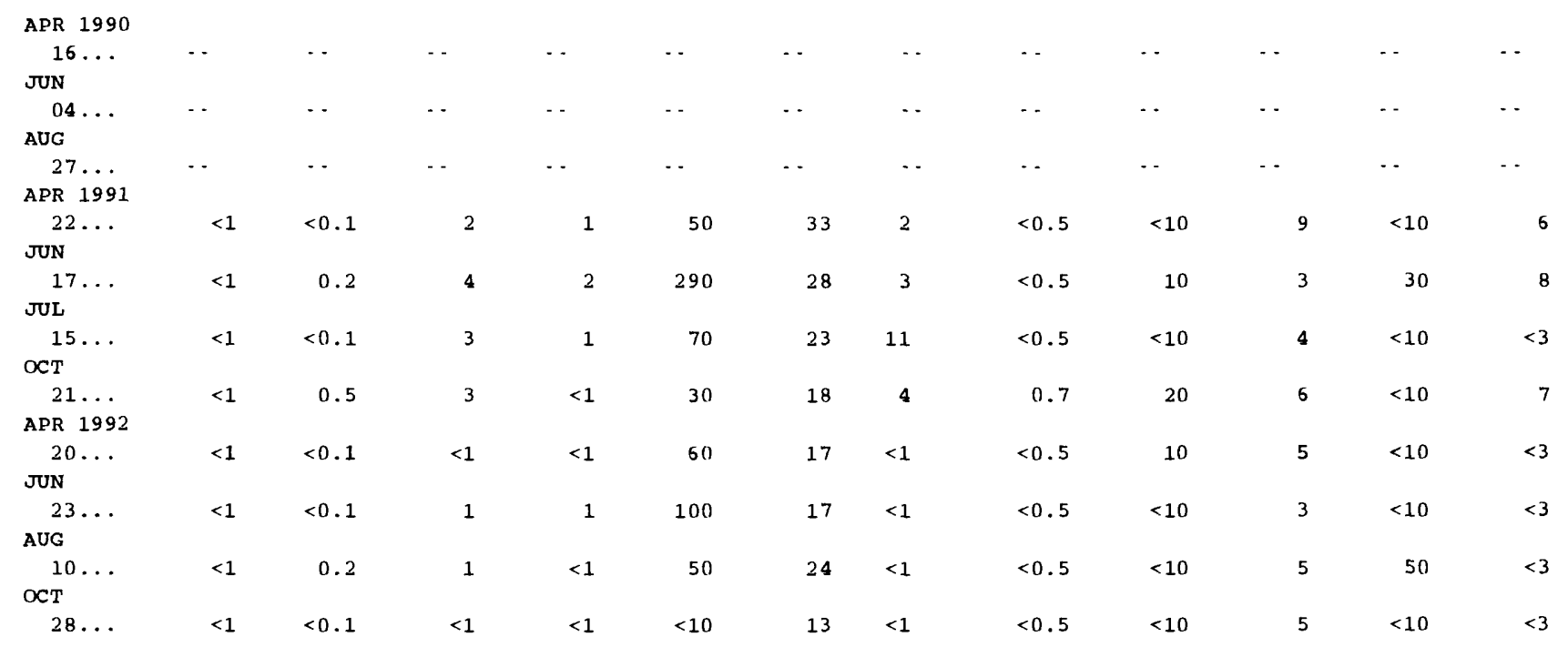


Table 25. Onsite measurements and selected inorganic data for station 07087000 , Clear Creek below Clear Creek Reservoir

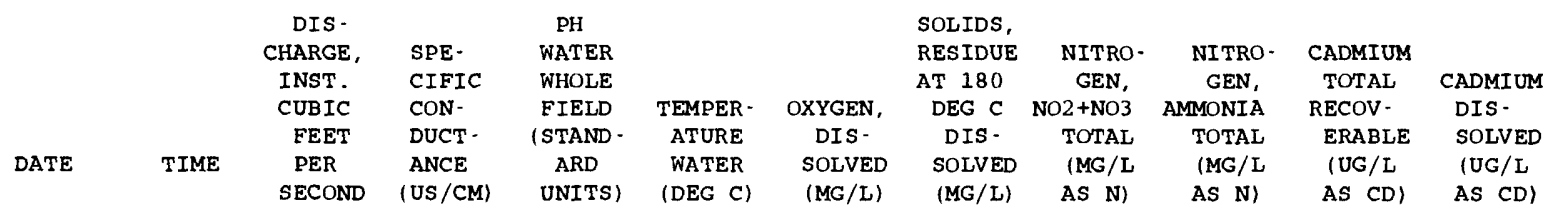

\begin{tabular}{|c|c|c|c|c|c|c|c|c|c|c|c|}
\hline APR 1990 & & & & & & & & & & & \\
\hline${ }_{J U N}^{18 \ldots}$ & 1150 & 15 & 134 & 8.5 & 5.0 & 9.0 & 72 & $<0.010$ & $<0.010$ & $<1$ & $<0.1$ \\
\hline${ }_{\text {JUL }}^{05 \ldots}$ & 1940 & 304 & 111 & 8.0 & 9.5 & 8.6 & 65 & $<0.010$ & 0.020 & $<1$ & $<0.1$ \\
\hline$\underset{A \cup G}{17} \cdots$ & 1800 & 92 & 83 & 7.7 & 12.5 & 7.5 & 56 & 0.014 & $<0.010$ & $<1$ & $<0.1$ \\
\hline$\underset{\mathrm{OCT}}{28 \ldots}$ & 1530 & 41 & 112 & 8.1 & 14.5 & 7.5 & 54 & 0.011 & $<0.010$ & $<1$ & $<0.1$ \\
\hline $\begin{array}{c}30 \ldots \\
\text { APR } 1991\end{array}$ & 1620 & 28 & 119 & 8.3 & 7.5 & 8.5 & 67 & $<0.010$ & 0.020 & $<1$ & $<0.1$ \\
\hline$\underset{J U N}{24 \ldots}$ & 0755 & 18 & 144 & 7.8 & 5.0 & 9.1 & 94 & 0.052 & 0.016 & $<1$ & $<0.1$ \\
\hline $\int_{\pi L}^{18 \ldots}$ & 1820 & 218 & 78 & 7.3 & 9.0 & 8.4 & 41 & 0.114 & 0.016 & $<1$ & 0.2 \\
\hline${ }_{\text {OCT }}^{16 \ldots}$ & 1920 & 171 & 87 & 7.3 & 15.5 & 7.2 & 44 & 0.010 & 0.007 & $<1$ & $<0: 1$ \\
\hline $\begin{array}{rl}22 \ldots \\
\mathrm{APR} & 1992\end{array}$ & 1815 & 1.7 & 145 & 7.8 & 9.0 & 7.5 & 83 & 0.030 & 0.021 & $<1$ & 0.3 \\
\hline$\underset{\text { JUN }}{21 \ldots}$ & 1500 & 25 & 137 & 8.0 & 4.5 & 9.1 & 82 & $<0.005$ & 0.014 & $<1$ & $<0.1$ \\
\hline$\underset{A U G}{24 \ldots}$ & 2005 & 124 & 97 & 8.1 & 11.5 & 7.7 & 70 & 0.013 & 0.009 & $<1$ & $<0.1$ \\
\hline $\mathrm{OCT}^{11 \cdots}$ & 1845 & 40 & 108 & 8.0 & 14.5 & 7.2 & 58 & 0.005 & 0.007 & $<1$ & $<0.1$ \\
\hline $28 \ldots$ & 0825 & 19 & 124 & 8.2 & 7.5 & 8.6 & 82 & 0.005 & 0.008 & $<1$ & $<0.1$ \\
\hline
\end{tabular}

\begin{tabular}{|c|c|c|c|c|c|c|c|c|c|}
\hline $\begin{array}{l}\text { COPPER, } \\
\text { TOTAL } \\
\text { RECOV- } \\
\text { ERABLE } \\
\text { (UG/L }\end{array}$ & $\begin{array}{l}\text { COPPER, } \\
\text { DIS - } \\
\text { SOLVED } \\
\text { (UG/L }\end{array}$ & $\begin{array}{l}\text { IRON, } \\
\text { TOTAL } \\
\text { RECOV- } \\
\text { ERABLE } \\
\text { (UG/L }\end{array}$ & $\begin{array}{l}\text { IRON, } \\
\text { DIS - } \\
\text { SOLVED } \\
\text { (UG/L }\end{array}$ & $\begin{array}{l}\text { LEAD, } \\
\text { TOTAL } \\
\text { RECOV- } \\
\text { ERABLE } \\
\text { (UG/L }\end{array}$ & $\begin{array}{l}\text { LEAD, } \\
\text { DIS - } \\
\text { SOLVED } \\
\text { (UG/L }\end{array}$ & $\begin{array}{l}\text { MANGA - } \\
\text { NESE, } \\
\text { TOTAL } \\
\text { RECOV - } \\
\text { ERABLE } \\
\text { (UG / L }\end{array}$ & $\begin{array}{l}\text { MANGA - } \\
\text { NESE, } \\
\text { DIS- } \\
\text { SOLVED } \\
\text { (UG/L }\end{array}$ & $\begin{array}{l}\text { ZINC, } \\
\text { TOTAL } \\
\text { RECOV. } \\
\text { ERABLE } \\
\text { l UG /L }\end{array}$ & $\begin{array}{l}\text { ZINC, } \\
\text { DIS - } \\
\text { SOLVED } \\
\text { (UG/L }\end{array}$ \\
\hline AS CUl & AS CU) & AS FE) & AS FE) & AS PB) & AS PB) & AS MN) & AS MN) & AS $\mathrm{ZN}$ ) & AS ZN) \\
\hline
\end{tabular}

APR 1990
$18 \ldots$
JUN
$05 \ldots$
JUL
$17 \ldots$
AUG
$28 \ldots$
OCT $\ldots$
$30 \ldots$
APR 1991
$24 \ldots$
JUN
$18 \ldots$
JUL
$16 \ldots$
OCT
$22 \ldots$
APR 1992
$21 \ldots$
JUN
$24 \ldots$
AUG
$11 \ldots$
OCT
$28 \ldots$

$\begin{array}{rrrrrrrrrr}2 & <1 & 60 & <3 & 1 & <0.5 & 10 & 5 & 10 & <3 \\ 5 & 1 & 130 & 19 & 2 & <0.5 & 10 & <1 & <10 & 8 \\ 1 & 1 & 170 & 55 & <1 & <0.5 & 20 & 7 & <10 & 5 \\ 3 & <1 & 170 & 79 & 4 & <0.5 & 60 & 15 & <10 & <3 \\ 2 & <1 & 40 & 10 & <1 & <0.5 & <10 & 4 & <10 & 5 \\ 3 & 1 & 70 & 8 & 5 & <0.5 & 20 & 7 & 10 & <3 \\ 6 & 4 & 220 & 33 & 4 & <0.5 & <10 & 1 & <10 & 9 \\ 3 & <1 & 100 & 67 & 5 & <0.5 & 20 & <1 & <10 & <3 \\ 3 & <1 & 240 & 34 & 6 & 1.4 & 80 & 76 & 70 & 62 \\ <1 & <1 & 120 & 13 & <1 & <0.5 & 40 & 10 & <10 & <3 \\ <1 & <1 & 70 & 13 & <1 & <0.5 & 10 & <1 & <10 & <3 \\ 16 & <1 & 90 & 33 & 18 & <0.5 & 20 & 6 & 1700 & <3 \\ <1 & <1 & 60 & 17 & <1 & <0.5 & <10 & 4 & <10 & <3\end{array}$


Table 26. Onsite measurements and selected inorganic data for station 390009106135001 , Pine Creek at mouth, at Highway 24

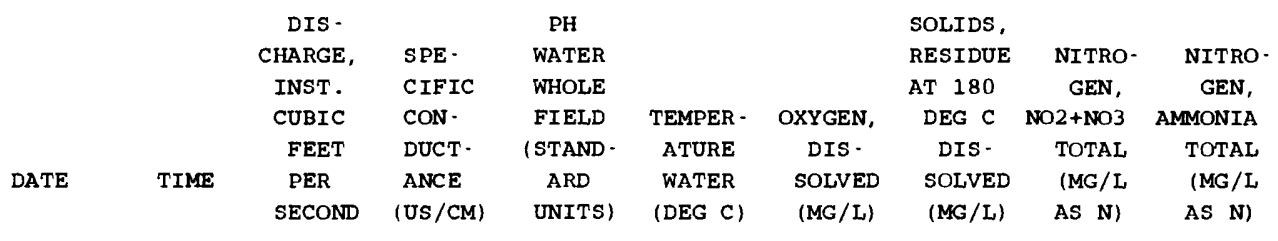

\begin{tabular}{|c|c|c|c|c|c|c|c|c|c|}
\hline \multicolumn{10}{|l|}{ APR 1990} \\
\hline $18 \ldots$ & 1245 & - - & 113 & 7.9 & 5.5 & 9.8 & 64 & $\cdots$ & $\cdots$ \\
\hline \multicolumn{10}{|l|}{ JUN } \\
\hline $06 \ldots$ & 1405 & 84 & $\cdots$ & 7.6 & 8.5 & 8.3 & 24 & $\cdots$ & -- \\
\hline \multicolumn{10}{|l|}{ JUL } \\
\hline $17 \ldots$ & 1840 & 27 & 73 & 7.8 & 12.5 & 7.3 & 43 & 0.078 & 0.020 \\
\hline \multicolumn{10}{|l|}{ AUG } \\
\hline $28 \ldots$ & 1620 & 13 & 99 & 7.6 & 13.5 & 7.4 & 57 & 0.105 & 0.020 \\
\hline \multicolumn{10}{|l|}{ OCT } \\
\hline $31 \ldots$ & 0800 & 6.6 & 96 & $\cdots$ & 1.5 & 9.8 & 63 & 0.131 & 0.020 \\
\hline \multicolumn{10}{|l|}{ APR 1991} \\
\hline $24 \ldots$ & 0835 & 3.1 & 117 & 7.5 & 1.5 & 9.8 & 63 & 0.130 & 0.022 \\
\hline \multicolumn{10}{|l|}{ JUN } \\
\hline $18 \ldots$ & 1830 & 56 & 59 & 7.3 & 9.5 & 8.0 & 22 & 0.091 & 0.023 \\
\hline \multicolumn{10}{|l|}{ JUL } \\
\hline $17 \ldots$ & 1010 & 19 & 77 & 7.9 & 10.0 & 7.9 & 34 & 0.051 & 0.014 \\
\hline \multicolumn{10}{|l|}{ OCT } \\
\hline $22 \ldots$ & 1845 & 3.5 & 109 & 8.0 & 5.5 & 9.0 & 66 & 0.069 & 0.047 \\
\hline \multicolumn{10}{|l|}{ APR 1992} \\
\hline $21 \ldots$ & 1500 & 2.0 & 113 & 8.1 & 8.5 & 7.8 & 60 & 0.086 & 0.012 \\
\hline \multicolumn{10}{|l|}{ JUN } \\
\hline $25 \ldots$ & 1030 & 32 & 66 & 8.0 & 9.5 & 8.0 & 32 & 0.053 & 0.010 \\
\hline \multicolumn{10}{|l|}{ AUG } \\
\hline $11 \ldots$ & 1915 & 15 & 88 & 8.0 & 11.5 & 7.6 & 70 & 0.050 & 0.010 \\
\hline \multicolumn{10}{|l|}{ OCT } \\
\hline $28 \ldots$ & 0815 & 5.9 & 107 & 8.1 & 3.0 & 9.7 & 69 & 0.084 & 0.009 \\
\hline
\end{tabular}


Table 27. Onsite measurements and bacteriological and selected inorganic data for station 07087200 , Arkansas River at Buena Vista

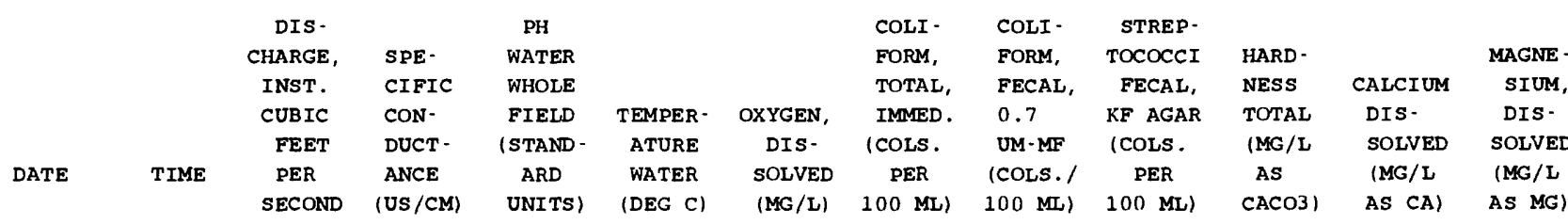

\begin{tabular}{|c|c|c|c|c|c|c|c|c|c|c|c|c|}
\hline \multicolumn{13}{|l|}{ APR 1990} \\
\hline $18 \ldots$ & 1530 & 171 & 176 & 8.3 & 9.0 & 8.8 & $\cdots$ & $<2$ & 350 & 80 & 21 & 6.8 \\
\hline \multicolumn{13}{|l|}{ MAY } \\
\hline $23 \ldots$ & 0900 & 519 & 108 & 7.9 & 9.5 & 8.5 & $\cdots$ & K7 & K37 & 45 & 13 & 3.1 \\
\hline \multicolumn{13}{|l|}{ JUN } \\
\hline $06 \ldots$ & 1100 & 2240 & 89 & 7.6 & 11.0 & 8.4 & 1300 & K20 & 130 & 37 & 11 & 2.3 \\
\hline $20 \ldots$ & 1240 & 1690 & 88 & 7.8 & 12.5 & 8.7 & 410 & $<2$ & K6 & 38 & 11 & 2.5 \\
\hline \multicolumn{13}{|l|}{ JUL } \\
\hline $18 \ldots$ & 1110 & 970 & 90 & 7.8 & 15.0 & 7.6 & 170 & К2 & 56 & 37 & 11 & 2.4 \\
\hline \multicolumn{13}{|l|}{ AUG } \\
\hline $29 \ldots$ & 0820 & 227 & 143 & 7.8 & 12.5 & 7.8 & $\mathrm{k} 3$ & $<1$ & 22 & 55 & 15 & 4.3 \\
\hline \multicolumn{13}{|l|}{ OCT } \\
\hline $31 \ldots$ & 1000 & 167 & 168 & 8.3 & 5.0 & 9.9 & $\cdots$ & $\cdots$ & $\cdots$ & 80 & 22 & 6.1 \\
\hline \multicolumn{13}{|l|}{ DEC } \\
\hline $20 \ldots$ & 0900 & 219 & 159 & 7.7 & 0.0 & 10.0 & 220 & $<1$ & 23 & 71 & 19 & 5.7 \\
\hline \multicolumn{13}{|l|}{ JAN 1991} \\
\hline $16 \ldots$ & 1310 & 517 & 100 & 8.3 & 0.0 & $\cdots$ & $<2$ & $<1$ & E4 & 45 & 13 & 3.1 \\
\hline \multicolumn{13}{|l|}{ MAR } \\
\hline $26 \ldots$ & 0830 & 252 & 133 & 7.9 & 2.5 & 10.0 & $\cdots$ & $<1$ & E9 & 49 & 14 & 3.3 \\
\hline \multicolumn{13}{|l|}{ APR } \\
\hline $24 \ldots$ & 0945 & E378 & 110 & 7.5 & 4.5 & 9.7 & 230 & $<1$ & E2 & 46 & 13 & 3.2 \\
\hline \multicolumn{13}{|l|}{ MAY } \\
\hline $15 \ldots$ & 1130 & 868 & 99 & 7.2 & 7.5 & 8.7 & 420 & $<2$ & E9 & 38 & 11 & 2.5 \\
\hline $30 \ldots$ & 0810 & 1420 & 85 & $\cdots$ & $-\cdot$ & $\cdots$ & $\cdots$ & $\cdots$ & $\cdots$ & $\cdots$ & $\cdots$ & $\cdots$ \\
\hline \multicolumn{13}{|l|}{ JUN } \\
\hline $19 \ldots$ & 0830 & 1780 & 86 & 7.6 & 11.5 & 8.3 & 510 & E4 & E11 & 38 & 11 & 2.5 \\
\hline \multicolumn{13}{|l|}{$\pi U$} \\
\hline $17 \ldots$ & 0730 & 648 & 105 & 7.9 & 13.0 & 7.7 & E19 & E6 & E15 & 48 & 14 & 3.2 \\
\hline \multicolumn{13}{|l|}{ AUG } \\
\hline $14 \ldots$ & 0800 & 423 & 133 & 7.7 & 13.0 & $\cdots$ & E22 & E3 & E22 & 59 & 17 & 4.0 \\
\hline \multicolumn{13}{|l|}{ SEP } \\
\hline $03 \ldots$ & 1045 & 213 & 146 & 8.1 & 14.0 & 8.0 & $\cdots$ & $\cdots$ & $\cdots$ & $\cdots$ & $\cdots$ & $\cdots$ \\
\hline OCT & & & & & & & & & & & & \\
\hline $23 \ldots$ & 0825 & 114 & 172 & 8.2 & 5.0 & 9.4 & $<5$ & $\cdots$ & E1 & 78 & 21 & 6.1 \\
\hline DEC & & & & & & & & & & & & \\
\hline $17 \ldots$ & 1320 & 254 & 105 & 7.7 & 0.5 & 10.5 & 55 & E13 & $<1$ & 45 & 13 & 3.0 \\
\hline MAR 1992 & & & & & & & & & & & & \\
\hline $24 \ldots$ & 0830 & 219 & 113 & 8.0 & 2.0 & 10.5 & $\cdots$ & $\cdots$ & $\cdots$ & 46 & 13 & 3.3 \\
\hline APR & & & & & & & & & & & & \\
\hline $22 \ldots$ & 0815 & 192 & 146 & 8.0 & 6.0 & 8.8 & 220 & E1 & $<1$ & 64 & 18 & 4.7 \\
\hline MAY & & & & & & & & & & & & \\
\hline $21 \ldots$ & 0840 & 1210 & 83 & 7.8 & 10.0 & 7.7 & E2 & E7 & 24 & 37 & 11 & 2.4 \\
\hline JUN & & & & & & & & & & & & \\
\hline $25 \ldots$ & 1020 & 1250 & 97 & 7.9 & 12.5 & 8.2 & E8 & E11 & 30 & 42 & 12 & 2.8 \\
\hline JUL & & & & & & & & & & & & \\
\hline $14 \ldots$ & 0715 & 634 & 111 & 7.9 & 12.0 & 7.9 & E5 & E6 & E14 & 46 & 13 & 3.2 \\
\hline AUG & & & & & & & & & & & & \\
\hline $12 \ldots$ & 0830 & 595 & 105 & 8.0 & 13.0 & 8.0 & E11 & E3 & E16 & 45 & 13 & 3.1 \\
\hline $25 \ldots$ & 1745 & 462 & 128 & 8.0 & 13.0 & 7.8 & E19 & 26 & 38 & 59 & 17 & 4.0 \\
\hline OCT & & & & & & & & & & & & \\
\hline $28 \ldots$ & 0950 & 154 & 173 & 8.3 & 7.0 & 9.2 & E8 & E3 & E9 & 76 & 21 & 5.7 \\
\hline JAN 1993 & & & & & & & & & & & & \\
\hline $12 \ldots$ & 1200 & 229 & 120 & 8.0 & 0.0 & 11.4 & 41 & $<1$ & 72 & 47 & 13 & 3.5 \\
\hline MAR & & & & & & & & & & & & \\
\hline $23 \ldots$ & 0730 & 450 & 90 & 7.8 & 0.5 & 10.9 & 130 & E2 & $\cdots$ & 35 & 10 & 2.4 \\
\hline
\end{tabular}


Table 27. Onsite measurements and bacteriological and selected inorganic data for station 07087200 , Arkansas River at Buena Vista--Continued

\begin{tabular}{|c|c|c|c|c|c|c|c|c|c|c|}
\hline & ALKA- & & CHLO- & $\begin{array}{l}\text { SOLIDS, } \\
\text { RES IDUE }\end{array}$ & NITRO- & $\begin{array}{l}\text { NITRO- } \\
\text { GEN, }\end{array}$ & NITRO- & $\begin{array}{l}\text { NITRO- } \\
\text { GEN, }\end{array}$ & & \\
\hline SOD IUM, & LINITY & SULFATE & RIDE, & AT 180 & GEN, & $\mathrm{NO} 2+\mathrm{NO} 3$ & GEN, & AMMONIA & & ARS ENIC \\
\hline DIS- & LAB & DIS - & DIS - & DEG C & $\mathrm{NO} 2+\mathrm{NO} 3$ & DIS - & AMMONIA & DIS - & ARSENIC & DIS - \\
\hline $\begin{array}{r}\text { SOLVED } \\
\text { (MG/L }\end{array}$ & $\begin{array}{c}\text { (MG / L } \\
\text { AS }\end{array}$ & $\begin{array}{l}\text { SOLVED } \\
\text { (MG/L }\end{array}$ & $\begin{array}{l}\text { SOLVED } \\
\text { (MG/L }\end{array}$ & $\begin{array}{l}\text { DIS- } \\
\text { SOLVED }\end{array}$ & $\begin{array}{l}\text { TOTAL } \\
\text { (MG/L }\end{array}$ & $\begin{array}{l}\text { SOLVED } \\
\text { (MG/L }\end{array}$ & $\begin{array}{l}\text { TOTAL } \\
\text { (MG/L }\end{array}$ & $\begin{array}{l}\text { SOLVED } \\
\text { (MG/L }\end{array}$ & $\begin{array}{l}\text { TOTAL } \\
\text { (UG/L }\end{array}$ & $\begin{array}{l}\text { SOLVED } \\
\text { (UG/L }\end{array}$ \\
\hline AS NA) & (ACO3) & AS SO4) & AS CL) & (MG/I) & AS N) & AS N) & AS N) & AS N) & AS AS) & AS AS) \\
\hline
\end{tabular}

\begin{tabular}{|c|c|c|c|c|c|c|c|c|c|c|c|}
\hline APR 1990 & & & & & & & & & & & \\
\hline $18 \ldots$ & 4.1 & 51 & 31 & 1.3 & 111 & 0.051 & $\cdots$ & $<0.010$ & $\cdots$ & $-\cdot$ & $\cdots$ \\
\hline MAY & & & & & & & & & & & \\
\hline $23 \ldots$ & 2.5 & 32 & 18 & 1.3 & 65 & 0.018 & $\cdots$ & $<0.010$ & $\cdots$ & $\cdots$ & $-\cdot$ \\
\hline JUN & & & & & & & & & & & \\
\hline $06 \ldots$ & 1.5 & 25 & 15 & 0.30 & 41 & 0.035 & $\cdots$ & 0.030 & $\cdots$ & $\cdots$ & $\cdots$ \\
\hline $20 \ldots$ & 1.5 & 27 & 17 & 1.6 & 43 & 0.032 & $-\cdot$ & $<0.010$ & - - & $\cdots$ & . \\
\hline JUL & & & & & & & & & & & \\
\hline $18 \ldots$ & 1.7 & 29 & 13 & 0.40 & 54 & 0.020 & $\cdots$ & 0.010 & $\cdots$ & $\cdots$ & $\cdots$ \\
\hline AUG & & & & & & & & & & & \\
\hline $29 \ldots$ & 3.2 & 47 & 22 & 2.2 & 87 & 0.064 & $\cdots$ & $<0.010$ & $\cdots$ & $<1$ & $<1$ \\
\hline OCT & & & & & & & & & & & \\
\hline $31 \ldots$ & 4.2 & 59 & 23 & 2.3 & 102 & 0.033 & $\cdots$ & 0.020 & $\cdots$ & $\cdots$ & $\cdots$ \\
\hline DEC & & & & & & & & & & & \\
\hline $20 \ldots$ & 3.8 & 43 & 28 & 8.2 & 96 & 0.146 & $\cdots$ & 0.016 & $\cdots$ & $\cdots$ & $\cdots$ \\
\hline JAN 1991 & & & & & & & & & & & \\
\hline $16 \ldots$ & 2.3 & 33 & 15 & 3.7 & 59 & 0.058 & $\cdots$ & 0.013 & $\cdots$ & $\cdots$ & $\cdots$ \\
\hline MAR & & & & & & & & & & & \\
\hline $26 \ldots$ & 2.6 & 38 & 17 & 4.7 & 71 & 0.015 & $\cdots$ & 0.022 & $\cdots$ & $\cdots$ & $\cdots$ \\
\hline APR & & & & & & & & & & & \\
\hline $24 \ldots$ & 2.4 & 34 & 18 & 1.5 & 65 & 0.035 & $\cdots$ & 0.018 & $\cdots$ & $\cdots$ & $\cdots$ \\
\hline MAY & & & & & & & & & & & \\
\hline $15 \ldots$ & 1.9 & 27 & 14 & 0.60 & 57 & 0.026 & $\cdots$ & 0.012 & $\cdots$ & $\cdots$ & $\cdots$ \\
\hline $30 \ldots$ & $\cdots$ & - & -. & $\cdots$ & $\cdots$ & $-\cdot$ & $\cdots$ & - - & $\cdots$ & $\cdots$ & $\cdots$ \\
\hline JUN & & & & & & & & & & & \\
\hline $19 \ldots$ & 1.4 & 26 & 11 & $<0.10$ & 44 & 0.036 & $\cdots$ & 0.013 & $\cdots$ & $<1$ & $<1$ \\
\hline JUL & & & & & & & & & & & \\
\hline $17 \ldots$ & 2.0 & 35 & 17 & 1.4 & 68 & 0.040 & $\cdots$ & 0.005 & $\cdots$ & $\cdots$ & $\cdots$ \\
\hline AUG & & & & & & & & & & & \\
\hline $14 \ldots$ & 2.6 & 45 & 22 & 0.50 & 90 & 0.050 & $\cdots$ & $<0.002$ & $\cdots$ & $\cdots$ & $\cdots$ \\
\hline SEP & & & & & & & & & & & \\
\hline $03 \ldots$ & $\cdots$ & .. & -. & - - & . & $\cdots$ & - - & $\cdots$ & $\cdots$ & $<1$ & $<1$ \\
\hline OCT & & & & & & & & & & & \\
\hline $23 \ldots$ & 4.2 & 63 & 26 & 2.1 & 102 & 0.044 & - - & 0.020 & $\cdots$ & $\cdots$ & $\cdots$ \\
\hline DEC & & & & & & & & & & & \\
\hline $17 \ldots$ & 2.5 & 36 & 16 & 1.1 & 85 & 0.055 & - & 0.006 & $\cdots$ & $\cdots$ & $\cdots$ \\
\hline MAR 1992 & & & & & & & & & & & \\
\hline $24 \ldots$ & 2.9 & 36 & 18 & 1.3 & 62 & 0.020 & $\cdots$ & 0.010 & $\cdots$ & $\cdots$ & $\cdots$ \\
\hline APR & & & & & & & & & & & \\
\hline $22 \ldots$ & 3.6 & 44 & 27 & 1.2 & 68 & 0.021 & $-\cdot$ & 0.010 & $\cdots$ & $\cdots$ & $\cdots$ \\
\hline MAY & & & & & & & & & & & \\
\hline $21 \ldots$ & 1.7 & 26 & 14 & 0.40 & 50 & 0.036 & $\cdots$ & 0.006 & $-\cdot$ & $\cdots$ & $\cdots$ \\
\hline JUN & & & & & & & & & & & \\
\hline $25 \ldots$ & 1.7 & 31 & 20 & 0.60 & 56 & 0.040 & $\cdots$ & 0.002 & $\cdots$ & $<1$ & $<1$ \\
\hline JUL & & & & & & & & & & & \\
\hline $14 \ldots$ & 2.0 & 36 & 16 & 0.90 & 58 & 0.020 & $\cdots$ & $<0.002$ & $\cdots$ & $\cdots$ & $\cdots$ \\
\hline AUG & & & & & & & & & & & \\
\hline $12 \ldots$ & 2.2 & 35 & 15 & 1.3 & 66 & 0.026 & $\cdots$ & 0.003 & $\cdots$ & $<1$ & $<1$ \\
\hline $25 \ldots$ & 2.7 & 41 & 20 & 1.4 & 64 & 0.042 & $\cdots$ & 0.034 & $\cdots$ & $\cdots$ & $\cdots$ \\
\hline OCT & & & & & & & & & & & \\
\hline $28 \ldots$ & 4.2 & 57 & 27 & 1.6 & 98 & 0.045 & - & 0.012 & $\cdots$ & $\cdots$ & $\cdots$ \\
\hline JAN 1993 & & & & & & & & & & & \\
\hline $12 \ldots$ & 2.8 & 38 & 18 & 0.90 & 73 & $\cdots$ & 0.094 & $\cdots$ & 0.011 & $\cdots$ & $-\cdot$ \\
\hline MAR & & & & & & & & & & & \\
\hline $23 \ldots$ & 2.2 & 28 & 13 & 0.70 & 34 & $\cdots$ & 0.040 & $\cdots$ & 0.002 & $\cdots$ & $\cdots$ \\
\hline
\end{tabular}


Table 27. Onsite measurements and bacteriological and selected inorganic data for station 07087200 , Arkansas River at Buena Vista--Continued

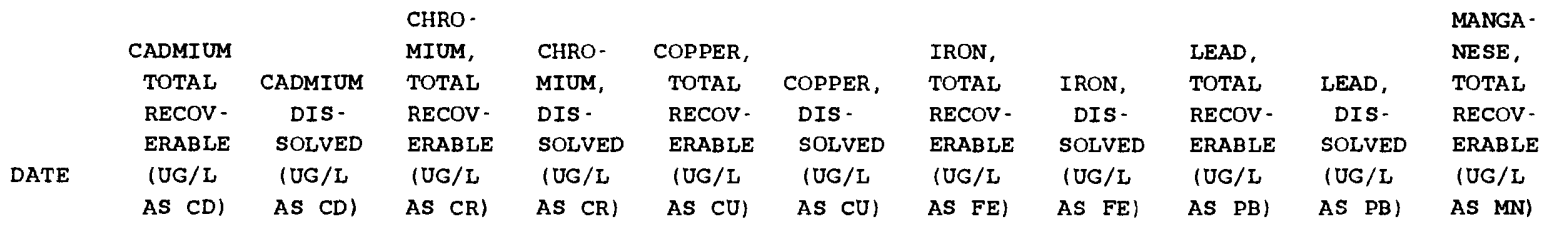

\begin{tabular}{|c|c|c|c|c|c|c|c|c|c|c|c|}
\hline \multicolumn{12}{|l|}{ APR 1990} \\
\hline $18 \ldots$ & 2 & 0.8 & $\cdots$ & $\cdots$ & 6 & 3 & 730 & 180 & 13 & 1.7 & 200 \\
\hline \multicolumn{12}{|l|}{ MAY } \\
\hline $23 \ldots$ & 3 & 0.4 & $\cdots$ & $\cdots$ & 8 & 4 & 2000 & 28 & 42 & $<0.5$ & 410 \\
\hline \multicolumn{12}{|l|}{ JUN } \\
\hline $06 \ldots$ & 5 & 0.3 & $\cdots$ & $\cdots$ & 31 & 3 & 6900 & 46 & 110 & 0.9 & 750 \\
\hline $20 \ldots$ & $<1$ & 0.4 & $\cdots$ & $\cdots$ & 14 & 4 & 440 & 20 & 23 & 6.8 & 50 \\
\hline \multicolumn{12}{|l|}{ JUL } \\
\hline $18 \ldots$ & $<1$ & 0.3 & $\cdots$ & $\cdots$ & 4 & 3 & 190 & 34 & 2 & $<0.5$ & 40 \\
\hline \multicolumn{12}{|l|}{ AUG } \\
\hline $29 \ldots$ & $<1$ & 0.3 & $<1$ & $<1$ & 4 & 3 & 110 & 31 & 1 & $<0.5$ & 30 \\
\hline \multicolumn{12}{|l|}{ Ост } \\
\hline $31 \ldots$ & $<1$ & 0.5 & $\cdots$ & $\cdots$ & 3 & 1 & 80 & 39 & 1 & $<0.5$ & 20 \\
\hline \multicolumn{12}{|l|}{ DEC } \\
\hline $20 \ldots$ & $<1$ & 0.5 & $\cdots$ & $\cdots$ & 4 & 2 & 70 & 36 & 2 & $<0.5$ & 30 \\
\hline \multicolumn{12}{|l|}{ JAN 1991} \\
\hline $16 \ldots$ & $<1$ & 0.2 & $\cdots$ & $\cdots$ & 7 & 1 & 590 & 18 & 9 & $<0.5$ & 130 \\
\hline \multicolumn{12}{|l|}{ MAR } \\
\hline $26 \ldots$ & $<1$ & 0.4 & $\cdots$ & $\cdots$ & 4 & 3 & 70 & 24 & 1 & $<0.5$ & 20 \\
\hline \multicolumn{12}{|l|}{ APR } \\
\hline $24 \ldots$ & $<1$ & 0.7 & $\cdots$ & $\cdots$ & 3 & 2 & 190 & 56 & 5 & $<0.5$ & 40 \\
\hline \multicolumn{12}{|l|}{ MAY } \\
\hline $15 \ldots$ & 2 & 0.7 & $\cdots$ & $\cdots$ & 21 & 13 & 870 & 88 & 43 & 2 & 160 \\
\hline $30 \ldots$ & . & $\cdots$ & $\cdots$ & $\cdots$ & . & $\cdots$ & 2300 & 50 & - & $\cdots$ & $-\cdot$ \\
\hline \multicolumn{12}{|l|}{ JUN } \\
\hline $19 \ldots$ & $<1$ & 0.3 & $<1$ & $<1$ & 64 & 32 & 350 & 40 & 17 & 0.6 & 60 \\
\hline \multicolumn{12}{|l|}{ JUL } \\
\hline $17 \ldots$ & $<1$ & 0.3 & $\cdots$ & $\cdots$ & 4 & 2 & 160 & 50 & 24 & $<0.5$ & 30 \\
\hline \multicolumn{12}{|l|}{ AUG } \\
\hline $14 \ldots$ & $<1$ & 0.3 & $\cdots$ & $\cdots$ & 3 & 1 & 200 & 41 & 5 & $<0.5$ & 30 \\
\hline \multicolumn{12}{|l|}{ SEP } \\
\hline $03 \ldots$ & & $\cdots$ & $<1$ & $<1$ & $\cdots$ & $\cdots$ & $\cdots$ & - & $\cdots$ & $\cdots$ & $\cdots$ \\
\hline \multicolumn{12}{|l|}{$\mathrm{OCT}$} \\
\hline $23 \ldots$ & $<1$ & 0.4 & $\cdots$ & $\cdots$ & 6 & $<1$ & 60 & 26 & 29 & $<0.5$ & 20 \\
\hline DEC & & & & & & & & & & & \\
\hline $17 \ldots$ & $<1$ & 1.2 & $\cdots$ & - & $\cdots$ & 2 & 150 & 21 & 2 & 0.7 & 40 \\
\hline MAR 1992 & & & & & & & & & & & \\
\hline $24 \ldots$ & $<1$ & 0.3 & $\cdots$ & $\cdots$ & $<1$ & 2 & 80 & 23 & $<1$ & $<0.5$ & 50 \\
\hline APR & & & & & & & & & & & \\
\hline $22 \ldots$ & $<1$ & 0.5 & -- & $\cdots$ & $<1$ & 2 & 210 & 90 & 2 & 0.8 & 70 \\
\hline MAY & & & & & & & & & & & \\
\hline $21 \ldots$ & 1 & 0.3 & $\cdots$ & -- & 9 & 4 & 1300 & 41 & 28 & $<0.5$ & 300 \\
\hline JUN & & & & & & & & & & & \\
\hline $25 \ldots$ & $<1$ & 0.3 & $<1$ & $<1$ & 31 & 17 & 300 & 55 & 7 & 0.6 & 50 \\
\hline JUL & & & & & & & & & & & \\
\hline $14 \ldots$ & $<1$ & 0.1 & $\cdots$ & $\cdots$ & 2 & 2 & 120 & 41 & 2 & 0.6 & 20 \\
\hline AUG & & & & & & & & & & & \\
\hline $12 \ldots$ & $<1$ & 0.2 & $<1$ & $<1$ & 3 & 2 & 160 & 32 & 2 & 0.7 & 30 \\
\hline $25 \ldots$ & $<1$ & 0.3 & $\cdots$ & $\cdot-$ & 3 & 3 & 440 & 98 & 13 & 1.3 & 80 \\
\hline Ост & & & & & & & & & & & \\
\hline $28 \ldots$ & $<1$ & 0.2 & $\cdots$ & $\cdots$ & $<1$ & $<1$ & 120 & 38 & $<1$ & $<0.5$ & $<10$ \\
\hline JAN 1993 & & & & & & & & & & & \\
\hline $12 \ldots$ & $<1$ & 0.1 & $\cdots$ & $\cdots$ & 2 & 2 & 120 & 23 & 2 & $<0.5$ & 40 \\
\hline MAR & & & & & & & & & & & \\
\hline $23 \ldots$ & $<1$ & 0.3 & $\cdots$ & $-\cdot$ & 1 & 2 & 170 & 26 & 4 & $<0.5$ & 50 \\
\hline
\end{tabular}


Table 27. Onsite measurements and bacteriological and selected inorganic data for station 07087200 , Arkansas River at Buena Vista--Continued

\begin{tabular}{|c|c|c|c|c|c|c|c|c|c|c|}
\hline $\begin{array}{l}\text { MANGA - } \\
\text { NESE, } \\
\text { DIS - } \\
\text { SOLVED } \\
\text { (UG/L }\end{array}$ & $\begin{array}{c}\text { MERCURY } \\
\text { TOTAL } \\
\text { RECOV - } \\
\text { ERABLE } \\
\text { (UG/L }\end{array}$ & $\begin{array}{c}\text { MERCURY } \\
\text { DIS - } \\
\text { SOLVED } \\
\text { (UG/L }\end{array}$ & $\begin{array}{c}\text { NICKEL, } \\
\text { TOTAL } \\
\text { RECOV - } \\
\text { ERABLE } \\
\text { (UG /L }\end{array}$ & $\begin{array}{l}\text { NICKEL, } \\
\text { DIS - } \\
\text { SOLVED } \\
\text { (UG /L }\end{array}$ & $\begin{array}{l}\text { SELE- } \\
\text { NIUM, } \\
\text { TOTAL } \\
\text { (UG /L }\end{array}$ & $\begin{array}{l}\text { SELE- } \\
\text { NIUM， } \\
\text { DIS - } \\
\text { SOLVED } \\
\text { (UG /L }\end{array}$ & $\begin{array}{c}\text { SILVER, } \\
\text { TOTAL } \\
\text { RECOV - } \\
\text { ERABLE } \\
\text { (UG/L }\end{array}$ & $\begin{array}{c}\text { SILVER, } \\
\text { DIS - } \\
\text { SOLVED } \\
\text { (UG/L }\end{array}$ & $\begin{array}{l}\text { ZINC, } \\
\text { TOTAL } \\
\text { RECOV - } \\
\text { ERABLE } \\
\text { /UG /L }\end{array}$ & $\begin{array}{l}\text { ZINC, } \\
\text { DIS - } \\
\text { SOLVED } \\
\text { (UG/L }\end{array}$ \\
\hline AS MN) & AS HG) & AS HG) & AS NI) & AS NI) & AS SE) & AS SE) & AS AG) & AS $\mathrm{AG})$ & AS ZN) & AS $\mathrm{ZN}$ ) \\
\hline
\end{tabular}

\begin{tabular}{|c|c|c|c|c|c|c|c|c|c|c|c|}
\hline \multicolumn{12}{|l|}{ APR 1990} \\
\hline $18 \ldots$ & 130 & $\cdots$ & $\cdots$ & $\cdots$ & - & $\cdots$ & - & - & - & 350 & 200 \\
\hline \multicolumn{12}{|l|}{ MAY } \\
\hline $23 \ldots$ & 70 & $\cdots$ & .. & $\cdots$ & .. & $\cdots$ & .. & $\cdots$ & $\cdots$ & 580 & 130 \\
\hline \multicolumn{12}{|l|}{ JUN } \\
\hline $06 \ldots$ & 28 & $\cdots$ & -. & $\cdots$ & - & $\cdots$ & $\cdots$ & $\cdots$ & $\cdots$ & 840 & 86 \\
\hline $20 \ldots$ & 14 & $\cdots$ & -- & $\cdots$ & - & - & - & - & -. & 110 & 66 \\
\hline \multicolumn{12}{|l|}{ JUL } \\
\hline $18 \ldots$ & 15 & $\cdots$ & $\cdots$ & - - & $\cdots$ & $\cdots$ & $\cdots$ & - & - & 80 & 53 \\
\hline \multicolumn{12}{|l|}{ AUG } \\
\hline $29 \ldots$ & 11 & $<0.10$ & $<0.1$ & 2 & $<1$ & $<1$ & $<1$ & $<1$ & $<1.0$ & 100 & 83 \\
\hline \multicolumn{12}{|l|}{ OCT } \\
\hline $31 \ldots$ & 16 & $\cdots$ & $\cdots$ & - & - & $\cdots$ & $\cdots$ & $\cdots$ & -- & 130 & 120 \\
\hline \multicolumn{12}{|l|}{ DEC } \\
\hline $20 \ldots$ & 25 & $\cdots$ & $\cdots$ & $\cdots$ & -. & $\cdots$ & $\cdots$ & $\cdots$ & $\cdots$ & 190 & 190 \\
\hline \multicolumn{12}{|l|}{ JAN 1991} \\
\hline $16 \ldots$ & 7 & $\cdots$ & $\cdots$ & $\cdots$ & $\cdots$ & $\cdots$ & $\cdots$ & $\cdots$ & $\cdots$ & 170 & 72 \\
\hline \multicolumn{12}{|l|}{ MAR } \\
\hline $26 \ldots$ & 18 & $\cdots$ & $\cdots$ & $\cdots$ & - & $\cdots$ & -. & $\cdots$ & $\cdots$ & 120 & 110 \\
\hline \multicolumn{12}{|l|}{$\mathrm{APR}$} \\
\hline $24 \ldots$ & 32 & $\cdots$ & $\cdots$ & $\cdots$ & $\cdots$ & $\cdots$ & $\cdots$ & $\cdots$ & $\cdots$ & 130 & 110 \\
\hline \multicolumn{12}{|l|}{ MAY } \\
\hline $15 \ldots$ & 67 & - & $\cdots$ & $\cdots$ & -. & $\cdots$ & $\cdots$ & $\cdots$ & $\cdots$ & 250 & 150 \\
\hline $30 \ldots$ & - & $\cdots$ & - & - & .. & - & $\cdots$ & $\cdots$ & - & 140 & 60 \\
\hline \multicolumn{12}{|l|}{ JUN } \\
\hline $19 \ldots$ & 11 & $<0.10$ & $<0.1$ & 5 & 3 & $<1$ & $<1$ & $<1$ & $<1.0$ & 90 & 64 \\
\hline \multicolumn{12}{|l|}{ JUL } \\
\hline $17 \ldots$ & 11 & $\cdots$ & $-\cdot$ & $\cdots$ & $\cdots$ & $\cdots$ & $\cdots$ & $\cdots$ & $\cdots$ & 60 & 62 \\
\hline \multicolumn{12}{|l|}{ AUG } \\
\hline $14 \ldots$ & 12 & - - & - - & $\cdots$ & $\cdots$ & - & $\cdots$ & $\cdots$ & $\cdots$ & 60 & 51 \\
\hline \multicolumn{12}{|l|}{ SEP } \\
\hline \multicolumn{12}{|l|}{ OCT } \\
\hline $23 \ldots$ & 10 & $\cdots$ & $\cdots$ & $\cdots$ & $\cdots$ & $\cdots$ & $\cdots$ & $\cdots$ & $\cdots$ & 130 & 120 \\
\hline DEC & & & & & & & & & & & \\
\hline $17 \ldots$ & 14 & $\cdots$ & $\cdots$ & - - & - & $\cdots$ & $\cdots$ & $\cdots$ & $\cdots$ & 130 & 85 \\
\hline MAR 1992 & & & & & & & & & & & \\
\hline $24 \ldots$ & 13 & $\cdots$ & - & $\cdots$ & $\cdots$ & $\cdots$ & $\cdots$ & $\cdots$ & $\cdots$ & 90 & 59 \\
\hline APR & & & & & & & & & & & \\
\hline $22 \ldots$ & 63 & $\cdots$ & $\cdots$ & $\cdots$ & $\cdots$ & $\cdots$ & $\cdots$ & $\cdots$ & $\cdots$ & 160 & 130 \\
\hline MAY & & & & & & & & & & & \\
\hline $21 \ldots$ & 23 & - & -. & $\cdots$ & $\cdots$ & - & - & $\cdots$ & $\cdots$ & 240 & 58 \\
\hline JUN & & & & & & & & & & & \\
\hline $25 \ldots$ & 13 & $<0.10$ & $<0.1$ & $<1$ & $<1$ & $<1$ & $<1$ & $<1$ & $<1.0$ & 80 & 50 \\
\hline JUL & & & & & & & & & & & \\
\hline $14 \ldots$ & 7 & $\cdots$ & $\cdots$ & $\cdots$ & $-\cdot$ & $\cdots$ & $\cdots$ & $\cdots$ & $\cdots$ & 40 & 30 \\
\hline AUG & & & & & & & & & & & \\
\hline $12 \ldots$ & 7 & $<0.10$ & $<0.1$ & $<1$ & $<1$ & $<1$ & $<1$ & $<1$ & $<1.0$ & 60 & 38 \\
\hline $25 \ldots$ & 27 & - & $\cdots$ & $\cdots$ & -. & $\cdots$ & .. & $\cdots$ & $\cdots$ & 140 & 85 \\
\hline OCT & & & & & & & & & & & \\
\hline $28 \ldots$ & 8 & $\cdots$ & $\cdots$ & $\cdots$ & $\cdots$ & $\cdots$ & $\cdots$ & $\cdots$ & $\cdots$ & 70 & 69 \\
\hline JAN 1993 & & & & & & & & & & & \\
\hline $12 \ldots$ & 9 & $\cdots$ & $\cdots$ & $\cdots$ & - & $\cdots$ & $\cdot \cdot$ & $\cdots$ & $\cdots$ & 90 & 64 \\
\hline MAR & & & & & & & & & & & \\
\hline $23 \ldots$ & 12 & $\cdots$ & $\cdots$ & $\cdots$ & $\cdots$ & $\cdots$ & $\cdots$ & $\cdots$ & $\cdots$ & 70 & 51 \\
\hline
\end{tabular}


Table 28. Onsite measurements and selected inorganic data for station 07089520 , Cottonwood Creek at Buena Vista

\begin{tabular}{|c|c|c|c|c|c|c|c|c|c|c|c|}
\hline DATE & TIME & $\begin{array}{l}\text { DIS - } \\
\text { CHARGE, } \\
\text { INST. } \\
\text { CUBIC } \\
\text { FEET } \\
\text { PER } \\
\text { SECOND }\end{array}$ & $\begin{array}{l}\text { SPE- } \\
\text { CIFIC } \\
\text { CON- } \\
\text { DUCT- } \\
\text { ANCE } \\
\text { (US/CM) }\end{array}$ & $\begin{array}{c}\text { PH } \\
\text { WATER } \\
\text { WHOLE } \\
\text { FIELD } \\
\text { (STAND- } \\
\text { ARD } \\
\text { UNITS) }\end{array}$ & $\begin{array}{l}\text { TEMPER- } \\
\text { ATURE } \\
\text { WATER } \\
\text { (DEG C) }\end{array}$ & $\begin{array}{c}\text { OXYGEN, } \\
\text { DIS - } \\
\text { SOLVED } \\
\text { (MG/L) }\end{array}$ & $\begin{array}{c}\text { SOLIDS, } \\
\text { RESIDUE } \\
\text { AT } 180 \\
\text { DEG C } \\
\text { DIS - } \\
\text { SOLVED } \\
\text { (MG/L) }\end{array}$ & $\begin{array}{c}\text { NITRO- } \\
\text { GEN, } \\
\text { NO2+NO3 } \\
\text { TOTAL } \\
\text { (MG /L } \\
\text { AS N) }\end{array}$ & $\begin{array}{c}\text { NITRO- } \\
\text { GEN, } \\
\text { AMMONIA } \\
\text { TOTAL } \\
\text { (MG/L } \\
\text { AS N) }\end{array}$ & $\begin{array}{l}\text { CADMIUM } \\
\text { TOTAL } \\
\text { RECOV- } \\
\text { ERABLE } \\
\text { (UG/L } \\
\text { AS CD) }\end{array}$ & $\begin{array}{l}\text { CADMIUM } \\
\text { DIS - } \\
\text { SOLVED } \\
\text { (UG / L } \\
\text { AS CD) }\end{array}$ \\
\hline $\begin{array}{l}R \quad 1990 \\
18 \ldots \\
\text { N }\end{array}$ & 1710 & 2.0 & 140 & 7.6 & 10.0 & 8.9 & 81 & 0.073 & $<0.010$ & $<1$ & $<0.1$ \\
\hline $06 \ldots$ & 1040 & $\cdots$ & 64 & 7.6 & 7.0 & 9.1 & 48 & 0.063 & 0.020 & $<1$ & 0.2 \\
\hline $18 \ldots$ & 1100 & 17 & $\cdots$ & 8.1 & 13.0 & 8.2 & 65 & 0.061 & $<0.010$ & $<1$ & $<0.1$ \\
\hline $29 \ldots$ & 0815 & 6.0 & 140 & 7.5 & 11.5 & 7.8 & 77 & 0.135 & $<0.010$ & $<1$ & $<0.1$ \\
\hline $\begin{array}{l}31 \ldots \\
P_{R} \quad 1991\end{array}$ & 1100 & 25 & 117 & 8.2 & 5.0 & 9.5 & 84 & 0.109 & 0.020 & $<1$ & $<0.1$ \\
\hline $24 \cdots$ & 1100 & 0.95 & 140 & 7.3 & 9.5 & 9.3 & 90 & 0.141 & 0.018 & $<1$ & $<0.1$ \\
\hline $\begin{array}{l}11 \ldots \\
19 \ldots\end{array}$ & $\begin{array}{l}0830 \\
0830\end{array}$ & $41^{\cdots}$ & ${ }_{81}$ & $\begin{array}{l}- \\
7.7\end{array}$ & 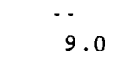 & $\begin{array}{l}\cdots \\
8.6\end{array}$ & $\begin{array}{l}68 \\
27\end{array}$ & $\begin{array}{c}\cdots \\
0.062\end{array}$ & $\begin{array}{c}\cdots \\
0.022\end{array}$ & $\begin{array}{l}<1 \\
<1\end{array}$ & $\begin{array}{r}0.4 \\
<0.1\end{array}$ \\
\hline $17 \ldots$ & 0800 & 1.1 & 147 & 7.4 & 10.5 & 6.9 & 79 & 0.321 & 0.009 & $<1$ & $<0.1$ \\
\hline $\begin{array}{l}23 \ldots \\
\text { PR } 1992\end{array}$ & 1000 & 5.6 & 136 & 7.9 & 7.0 & 9.2 & 85 & 0.103 & 0.017 & $<1$ & $<0.1$ \\
\hline $22 \ldots$ & 1025 & 13 & 139 & 8.1 & 7.0 & 8.7 & 78 & 0.090 & 0.016 & $<1$ & $<0.1$ \\
\hline $\begin{array}{l}25 \ldots \\
{ }_{G}\end{array}$ & 1145 & 18 & 100 & 8.1 & 12.5 & 7.7 & 70 & 0.056 & 0.010 & $<1$ & $<0.1$ \\
\hline $12 \ldots$ & 0910 & 4.4 & 132 & 7.8 & 11.5 & 8.1 & 86 & 0.113 & 0.010 & $<1$ & $<0.1$ \\
\hline $28 \ldots$ & 1120 & 25 & 139 & 8.1 & 7.5 & 9.1 & 88 & 0.074 & 0.013 & $<1$ & $<0.1$ \\
\hline
\end{tabular}

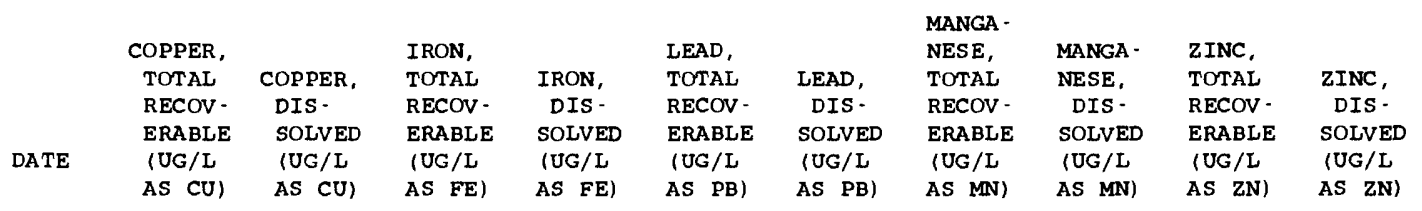

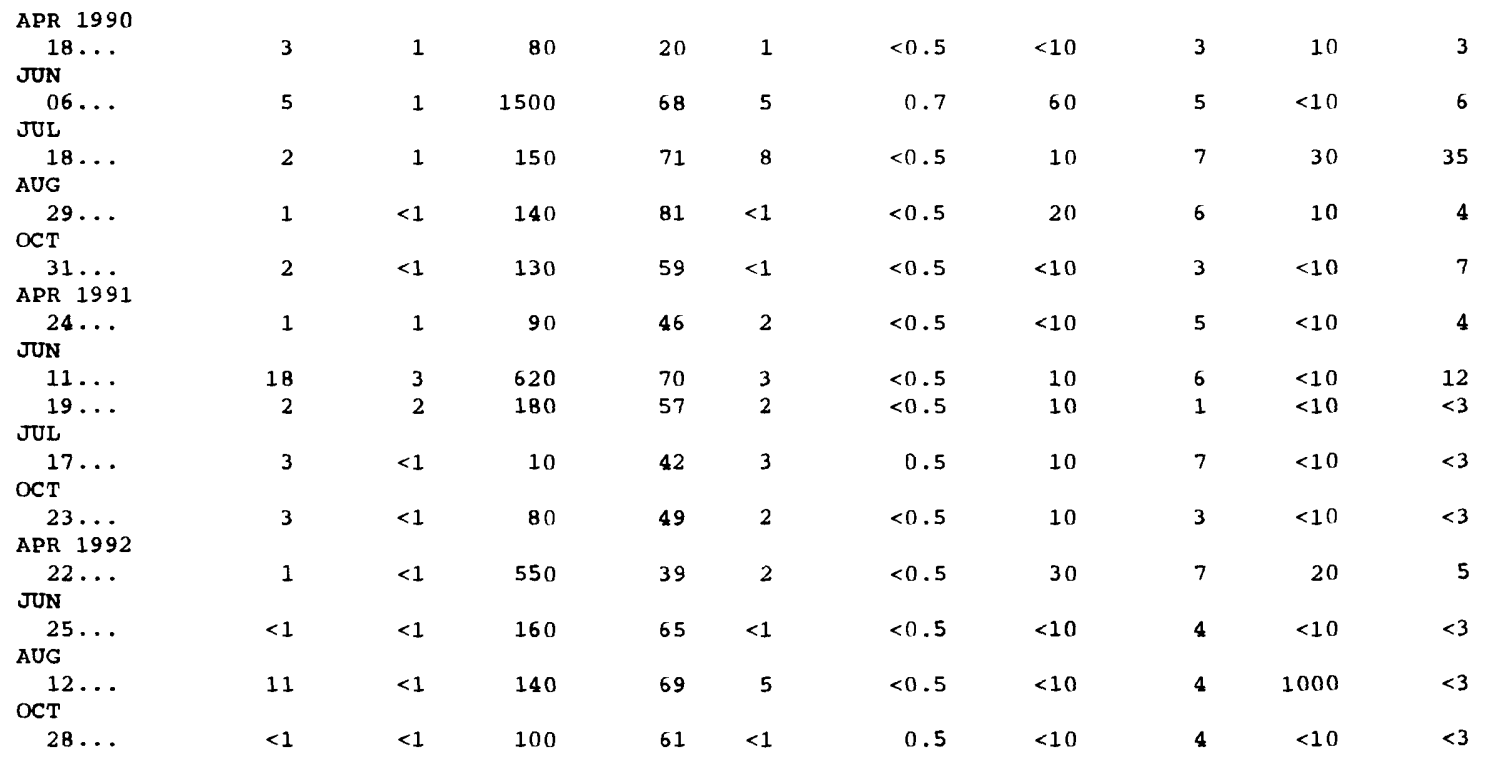


Table 29. Onsite measurements and selected inorganic data for station 384427106040101 , Chalk Creok at mouth, at Nathrop

\begin{tabular}{|c|c|c|c|c|c|c|c|c|c|c|c|}
\hline DATE & T IME & $\begin{array}{c}\text { DIS- } \\
\text { CHARGE, } \\
\text { INST. } \\
\text { CUBIC } \\
\text { FEET } \\
\text { PER } \\
\text { SECOND }\end{array}$ & $\begin{array}{l}\text { SPE- } \\
\text { CIFIC } \\
\text { CON- } \\
\text { DUCT- } \\
\text { ANCE } \\
\text { (US/CM) }\end{array}$ & $\begin{array}{c}\text { PH } \\
\text { WATER } \\
\text { WHOLE } \\
\text { FIELD } \\
\text { (STAND- } \\
\text { ARD } \\
\text { UNITS) }\end{array}$ & $\begin{array}{l}\text { TEMPER- } \\
\text { ATURE } \\
\text { WATER } \\
\text { (DEG C) }\end{array}$ & $\begin{array}{c}\text { OXYGEN, } \\
\text { DIS - } \\
\text { SOLVED } \\
\text { (MG/L) }\end{array}$ & $\begin{array}{l}\text { SOLIDS, } \\
\text { RESIDUE } \\
\text { AT } 180 \\
\text { DEG C } \\
\text { DIS- } \\
\text { SOLVED } \\
(\mathrm{MG} / \mathrm{L})\end{array}$ & $\begin{array}{l}\text { NITRO- } \\
\text { GEN, } \\
\text { NO2+NO3 } \\
\text { TOTAL } \\
\text { (MC / L } \\
\text { AS N) }\end{array}$ & $\begin{array}{l}\text { NITRO- } \\
\text { GEN, } \\
\text { AMMONIA } \\
\text { TOTAL } \\
\text { (MG/L } \\
\text { AS N) }\end{array}$ & $\begin{array}{l}\text { CADMIUM } \\
\text { TOTAL } \\
\text { RECOV- } \\
\text { ERABLE } \\
\text { (UG/L } \\
\text { AS CD) }\end{array}$ & $\begin{array}{l}\text { CADMIUM } \\
\text { DIS- } \\
\text { SOLVED } \\
\text { (UG/L } \\
\text { AS CD) }\end{array}$ \\
\hline $\begin{array}{c}\text { APR } 1990 \\
19 \ldots \\
\text { JUN }\end{array}$ & 1015 & 2.9 & 237 & 8.4 & 7.5 & 9.3 & 139 & 0.055 & $<0.010$ & $<1$ & $<0.1$ \\
\hline $\begin{array}{l}06 \ldots \\
\text { JUL }\end{array}$ & 0800 & 150 & $\cdots$ & 7.5 & 6.0 & 9.1 & 42 & 0.066 & 0.120 & 2 & 0.3 \\
\hline$\underset{A U G}{18}$ & 0810 & 69 & 123 & 7.7 & 12.5 & 8.6 & 82 & 0.089 & 0.010 & $<1$ & $<0.1$ \\
\hline$\underset{\text { Ост }}{29 \ldots}$ & 0930 & 34 & 169 & 7.9 & 13.0 & 7.9 & 87 & 0.129 & $<0.010$ & $<1$ & $<0.1$ \\
\hline $\begin{array}{c}31 \ldots \\
A P R \\
1991\end{array}$ & 1225 & 30 & 156 & 8.1 & 10.5 & 8.4 & 93 & 0.097 & 0.030 & $<1$ & 0.1 \\
\hline$\underset{\text { JUN }}{24 \ldots}$ & 1245 & 3.9 & 235 & 8.5 & 14.0 & 8.2 & 135 & 0.024 & 0.012 & $<1$ & $<0.1$ \\
\hline $\begin{array}{l}11 \ldots \\
19 \ldots\end{array}$ & $\begin{array}{l}1200 \\
1045\end{array}$ & $173^{\cdots}$ & 81 & $\begin{array}{l}-1 \\
7.5\end{array}$ & $\overline{10} .0$ & $\begin{array}{c}- \\
8.4\end{array}$ & $\begin{array}{l}52 \\
45\end{array}$ & $\begin{array}{c}\cdots \\
0.059\end{array}$ & $\begin{array}{l}\cdots \\
0.029\end{array}$ & $\begin{array}{l}<1 \\
<1\end{array}$ & $\begin{array}{l}0.7 \\
0.5\end{array}$ \\
\hline JUL & & & & & & & & & & & \\
\hline${ }_{\mathrm{CT}}^{17 \ldots}$ & 1300 & 59 & 125 & 8.1 & 17.0 & 7.1 & 70 & 0.062 & 0.006 & $<1$ & 0.2 \\
\hline $\begin{array}{l}23 \ldots \\
A P R \quad 1992\end{array}$ & 1240 & 13 & 182 & 8.3 & 10.0 & 8.4 & 115 & 0.096 & 0.018 & $<1$ & $<0.1$ \\
\hline JUN & 1255 & 2.7 & 224 & 8.6 & 11.5 & 8.2 & 148 & 0.047 & 0.019 & $<1$ & $<0.1$ \\
\hline$\underset{\text { AUG }}{25 \ldots}$ & 0805 & 133 & 99 & 7.8 & 10.5 & 8.1 & 62 & 0.079 & 0.046 & $<1$ & 0.1 \\
\hline$\underset{\mathrm{CT}}{12}$ & 1000 & 46 & 145 & 8.1 & 12.5 & 8.1 & 78 & 0.085 & 0.009 & $<1$ & 0.1 \\
\hline $28 \ldots$ & 1220 & 23 & 177 & 8.4 & 11.0 & 8.4 & 113 & 0.081 & 0.010 & $<1$ & 0.1 \\
\hline
\end{tabular}

DATE

\begin{tabular}{|c|c|c|c|c|c|}
\hline $\begin{array}{c}\text { COPPER, } \\
\text { TOTAL } \\
\text { RECOY - }\end{array}$ & $\begin{array}{c}\text { COPPER, } \\
\text { DIS - }\end{array}$ & $\begin{array}{l}\text { IRON, } \\
\text { TOTAL }\end{array}$ & $\begin{array}{l}\text { IRON, } \\
\text { DIS - }\end{array}$ & $\begin{array}{l}\text { LEAD, } \\
\text { TOTAL } \\
\text { RECOY }\end{array}$ & $\begin{array}{r}\text { LEAD, } \\
\text { DIS - }\end{array}$ \\
\hline $\begin{array}{l}\text { ERABLE } \\
\text { /UG /L }\end{array}$ & $\begin{array}{l}\text { SOLVED } \\
\text { \{UG/L }\end{array}$ & $\begin{array}{l}\text { ERABLE } \\
\text { (UG /L }\end{array}$ & $\begin{array}{l}\text { SOLVED } \\
\text { (UG/L }\end{array}$ & $\begin{array}{l}\text { ERABLE } \\
\text { (UG /L }\end{array}$ & $\begin{array}{l}\text { SOLVED } \\
\text { /UG / L }\end{array}$ \\
\hline AS CU) & AS CU) & AS FE) & AS FE) & AS PB) & AS PBI \\
\hline
\end{tabular}

MANGA -

NESE, MANGA- ZINC,

TOTAL NESE, TOTAL ZINC,

RECOV - DIS - RECOV - DIS -

ERABLE SOLVED ERABLE SOLVED

(UG/L IUG/L IUG/L (UG/L

AS CU) AS CU) AS FE) AS FE) AS PB) AS PBI AS MN) AS MN) AS ZN) AS ZN)

APR 1990
$19 \ldots$
JUN
$06 \ldots$
JUL
$18 \ldots$
AUG
$29 \ldots$
OCT
$31 \ldots$
APR 1991
$24 \ldots$
JUN
$11 \ldots$
$19 \ldots$
JUL
$17 \ldots$
OCT
$23 \ldots$
APR 1992
$22 \ldots$
JUN
$25 \ldots$
AUG
$12 \ldots$
OCT
$28 \ldots$

$\begin{array}{rrrrr}3 & 1 & 320 & 71 & 2 \\ 35 & 5 & 12000 & 85 & <1 \\ 3 & 2 & 200 & 58 & 3 \\ 2 & <1 & 220 & 78 & 3 \\ 3 & 1 & 260 & 97 & 3 \\ 2 & 2 & 170 & 79 & 3 \\ 6 & 4 & 1600 & 82 & 19 \\ 6 & 4 & 690 & 60 & 14 \\ 3 & 1 & 120 & 65 & 7 \\ 2 & <1 & 150 & 110 & 1 \\ 1 & <1 & 210 & 100 & 1 \\ 2 & 2 & 270 & 44 & 4 \\ 2 & <1 & 380 & 83 & 5 \\ <1 & <1 & 160 & 97 & <1\end{array}$

$\begin{array}{rrrrr}<0.5 & 60 & 27 & 20 & 7 \\ 3.1 & 620 & 21 & 360 & 45 \\ 0.8 & 30 & 20 & 60 & 50 \\ 1.0 & 40 & 27 & 40 & 24 \\ 1.4 & 40 & 23 & 40 & 33 \\ <0.5 & 20 & 12 & 20 & 7 \\ 1.7 & 80 & 16 & 130 & 74 \\ 1.2 & 60 & 13 & 90 & 76 \\ 0.8 & 20 & 12 & 30 & 44 \\ 0.5 & 20 & 18 & <10 & 14 \\ <0.5 & 40 & 24 & 20 & 6 \\ <0.5 & 30 & 12 & 70 & 63 \\ 0.6 & 50 & 22 & 80 & 30 \\ 0.7 & <10 & 11 & <10 & 7\end{array}$


Table 30. Onsite measurements and bacteriological and selected inorganic data for station 07091200 , Arkansas River near Nathrop

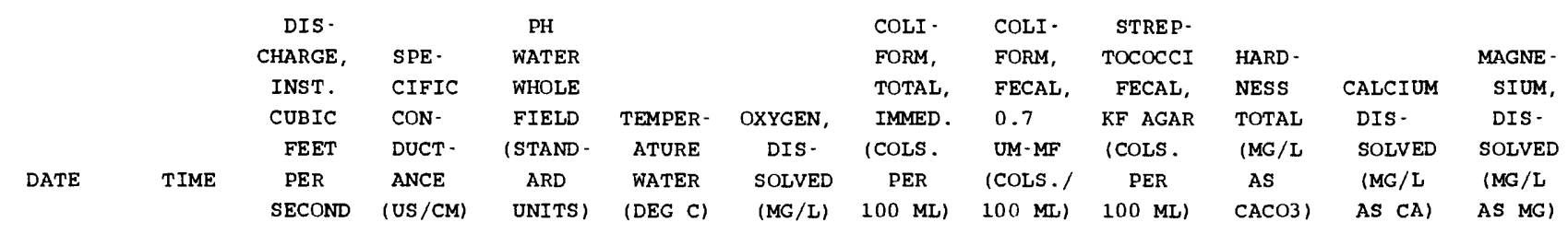

\begin{tabular}{|c|c|c|c|c|c|c|c|c|c|c|c|c|}
\hline \multicolumn{13}{|l|}{ APR 1990} \\
\hline $19 \ldots$ & 0800 & 247 & 191 & 8.3 & 6.0 & 9.4 & $\cdots$ & 48 & 110 & 85 & 23 & 6.7 \\
\hline \multicolumn{13}{|l|}{ MAY } \\
\hline $23 \ldots$ & 1210 & 615 & 125 & 8.0 & 12.5 & 8.2 & $\cdots$ & K40 & 130 & 51 & 15 & 3.4 \\
\hline \multicolumn{13}{|l|}{ JUN } \\
\hline $06 \ldots$ & 0830 & 2600 & 91 & 7.7 & 10.0 & 8.9 & K1900 & K48 & 260 & 37 & 11 & 2.2 \\
\hline $20 \ldots$ & 0925 & 1820 & 92 & 7.8 & 11.5 & 8.4 & 450 & $\mathrm{k} 12$ & 82 & 40 & 12 & 2.5 \\
\hline \multicolumn{13}{|l|}{ JUL } \\
\hline $18 \ldots$ & 0830 & 1060 & 108 & 7.8 & 14.0 & 7.8 & $>270$ & K14 & K150 & 44 & 13 & 2.9 \\
\hline \multicolumn{13}{|l|}{ AUG } \\
\hline $29 \ldots$ & 1040 & 373 & 184 & 8.1 & 13.0 & 7.9 & K6 & K10 & 30 & 82 & 24 & 5.3 \\
\hline \multicolumn{13}{|l|}{ OCT } \\
\hline $31 \ldots$ & 1355 & 328 & 189 & $\cdots$ & 7.0 & 9.4 & $\cdots$ & $\cdot$ & $\cdots$ & 88 & 26 & 5.7 \\
\hline \multicolumn{13}{|l|}{ JAN 1991} \\
\hline $16 \ldots$ & 1530 & 433 & 126 & 8.0 & 1.0 & 10.6 & $<3$ & $<1$ & 2 & 57 & 17 & 3.5 \\
\hline \multicolumn{13}{|l|}{ MAR } \\
\hline $26 \ldots$ & 1030 & 343 & 157 & 8.6 & 4.5 & 9.7 & -- & $<2$ & E8 & 61 & 18 & 3.9 \\
\hline \multicolumn{13}{|l|}{$\mathrm{APR}$} \\
\hline $24 \ldots$ & 1420 & 441 & 130 & 7.7 & 9.0 & 8.7 & E16 & $<1$ & E4 & 54 & 16 & 3.5 \\
\hline \multicolumn{13}{|l|}{ MAY } \\
\hline $15 \ldots$ & 0830 & 988 & 113 & 7.7 & 7.5 & 8.8 & 400 & E10 & 40 & 44 & 13 & 2.8 \\
\hline \multicolumn{13}{|l|}{ JUN } \\
\hline $11 \ldots$ & 1300 & 2200 & -- & - & $\cdots$ & -- & -- & -- & $-\cdot$ & -- & $\cdots$ & $\cdots$ \\
\hline $11 \ldots$ & 1540 & 2180 & $\cdots$ & - - & -- & - & - & $\cdots$ & $-\cdot$ & $\cdots$ & $-\cdot$ & $\cdots$ \\
\hline $19 \ldots$ & 1315 & 2020 & 93 & 7.8 & 12.5 & 8.3 & 600 & E19 & E29 & 40 & 12 & 2.4 \\
\hline \multicolumn{13}{|l|}{ JUL } \\
\hline $17 \ldots$ & 1530 & 771 & 126 & 8.3 & 18.0 & 7.0 & 96 & E3 & E16 & 57 & 17 & 3.5 \\
\hline \multicolumn{13}{|l|}{ AUG } \\
\hline $14 \ldots$ & 1515 & 511 & 162 & 8.4 & 18.0 & - & E4 & E2 & 28 & 70 & 21 & 4.2 \\
\hline \multicolumn{13}{|l|}{ OCT } \\
\hline $23 \ldots$ & 0730 & 208 & 218 & 8.3 & 7.0 & 9.1 & E11 & -- & E11 & 97 & 28 & 6.5 \\
\hline \multicolumn{13}{|c|}{ MAR 1992} \\
\hline $24 \ldots$ & 1030 & 311 & 136 & 8.1 & 3.5 & 11.0 & $-\cdot$ & - & $-\cdot$ & 57 & 17 & 3.5 \\
\hline \multicolumn{13}{|l|}{ APR } \\
\hline $22 \ldots$ & 0815 & 244 & 171 & 8.1 & 10.0 & 9.2 & E6 & E3 & E9 & 76 & 22 & 5.2 \\
\hline \multicolumn{13}{|l|}{ MAY } \\
\hline $21 \ldots$ & 0800 & 1290 & 92 & 7.9 & 10.5 & 8.5 & E43 & E37 & 95 & 39 & 12 & 2.3 \\
\hline \multicolumn{13}{|l|}{ JUN } \\
\hline $25 \ldots$ & 0800 & 1240 & 104 & 7.9 & 12.5 & 8.3 & E41 & E17 & 61 & 46 & 14 & 2.7 \\
\hline JUL & & & & & & & & & & & & \\
\hline $14 \ldots$ & 1120 & 733 & 130 & 8.2 & 13.5 & 8.2 & E18 & E4 & 31 & 57 & 17 & 3.6 \\
\hline AUG & & & & & & & & & & & & \\
\hline $12 \ldots$ & 1110 & 701 & 131 & 8.1 & 14.0 & 8.0 & E18 & E16 & 45 & 57 & 17 & 3.5 \\
\hline $25 \ldots$ & 1450 & 844 & 155 & 8.1 & 13.0 & 8.1 & 100 & 89 & 230 & 68 & 20 & 4.5 \\
\hline OCT & & & & & & & & & & & & \\
\hline $28 \ldots$ & 1330 & 283 & 202 & 8.6 & 8.5 & 9.4 & E7 & E3 & E7 & 88 & 25 & 6.1 \\
\hline JAN 1993 & & & & & & & & & & & & \\
\hline $12 \ldots$ & 1400 & $\cdots$ & 157 & 8.2 & 0.0 & 11.1 & E4 & $<1$ & $<1$ & 62 & 18 & 4.1 \\
\hline MAR & & & & & & & & & & & & \\
\hline $23 \ldots$ & 0910 & 562 & 112 & 8.1 & 3.5 & 10.1 & 56 & E2 & - & 44 & 13 & 2.8 \\
\hline
\end{tabular}


Table 30. Onsite measurements and bacteriological and selected inorganic data for station 07091200 , Arkansas River near Nathrop--Continued

\begin{tabular}{|c|c|c|c|c|c|c|c|c|c|c|c|}
\hline DATE & $\begin{array}{l}\text { SODIUM, } \\
\text { DIS - } \\
\text { SOLVED } \\
\text { (MG/L } \\
\text { AS NA) }\end{array}$ & $\begin{array}{c}\text { ALKA- } \\
\text { LINITY } \\
\text { LAB } \\
\text { (MG / L } \\
\text { AS } \\
\text { CACO3) }\end{array}$ & $\begin{array}{l}\text { SULFATE } \\
\text { DIS - } \\
\text { SOLVED } \\
\text { (MG/L } \\
\text { AS SO4) }\end{array}$ & $\begin{array}{l}\text { CHLO- } \\
\text { RIDE, } \\
\text { DIS - } \\
\text { SOLVED } \\
\text { (MG/L } \\
\text { AS CL) }\end{array}$ & $\begin{array}{l}\text { SOLIDS, } \\
\text { RESIDUE } \\
\text { AT } 180 \\
\text { DEG C } \\
\text { DIS - } \\
\text { SOLVED } \\
\text { (MG/L) }\end{array}$ & $\begin{array}{c}\text { NITRO- } \\
\text { GEN, } \\
\text { NO2+NO3 } \\
\text { TOTAL } \\
\text { (MG / L } \\
\text { AS N) }\end{array}$ & $\begin{array}{l}\text { NITRO- } \\
\text { GEN, } \\
\text { NO2+NO3 } \\
\text { DIS- } \\
\text { SOLVED } \\
\text { (MG/L } \\
\text { AS N) }\end{array}$ & $\begin{array}{l}\text { NITRO- } \\
\text { GEN, } \\
\text { AMMONIA } \\
\text { TOTAL } \\
\text { (MG /L } \\
\text { AS N) }\end{array}$ & $\begin{array}{l}\text { NITRO- } \\
\text { GEN, } \\
\text { AMMONIA } \\
\text { DIS- } \\
\text { SOLVED } \\
\text { (MG/L } \\
\text { AS N) }\end{array}$ & $\begin{array}{l}\text { PHOS - } \\
\text { PHORUS } \\
\text { TOTAL } \\
\text { (MG } / L \\
\text { AS P) }\end{array}$ & $\begin{array}{l}\text { CADMIUM } \\
\text { TOTAL } \\
\text { RECOV- } \\
\text { ERABLE } \\
\text { (UG/L } \\
\text { AS CD) }\end{array}$ \\
\hline \multicolumn{12}{|l|}{ APR 1990} \\
\hline \multicolumn{12}{|l|}{ MAY } \\
\hline \multicolumn{12}{|l|}{ JUN } \\
\hline $\begin{array}{l}06 \ldots \\
20 \ldots\end{array}$ & $\begin{array}{l}1.8 \\
2.0\end{array}$ & $\begin{array}{l}28 \\
31\end{array}$ & $\begin{array}{l}13 \\
9.6\end{array}$ & $\begin{array}{l}0.30 \\
0.30\end{array}$ & $\begin{array}{l}55 \\
52\end{array}$ & $\begin{array}{l}0.051 \\
0.047\end{array}$ & $\cdots$ & $\begin{array}{r}0.020 \\
<0.010\end{array}$ & $\begin{array}{l}\cdots \\
\cdots\end{array}$ & $\begin{array}{l}0.016 \\
0.016\end{array}$ & $\begin{array}{l}3 \\
1\end{array}$ \\
\hline \multicolumn{12}{|l|}{ JUL } \\
\hline \multicolumn{11}{|l|}{ AUG } & $<1$ \\
\hline $\begin{array}{l}29 \ldots \\
\text { OCT }\end{array}$ & 5.5 & 70 & 19 & 2.1 & 105 & 0.146 & $\cdots$ & 0.010 & $\cdots$ & 0.007 & 1 \\
\hline \multicolumn{11}{|l|}{ JAN 1991} & $<1$ \\
\hline $16 \ldots$ & 4.0 & 47 & 14 & 1.3 & 61 & 0.119 & $\cdots$ & 0.008 & $\cdots$ & 0.006 & $<1$ \\
\hline$\underset{A P R}{26 \ldots}$ & 4.1 & 52 & 18 & 4.7 & 86 & 0.073 & \multicolumn{3}{|c|}{ APR } & 0.003 & \\
\hline \multicolumn{11}{|l|}{ MAY } & $<1$ \\
\hline \multicolumn{12}{|l|}{ JUN } \\
\hline $11 \ldots$ & $\begin{array}{l}\cdots \\
\cdots\end{array}$ & - & $\cdots$ & $\because$ & $\cdots$ & $\cdots$ & $\begin{array}{l}\cdots \\
\cdots\end{array}$ & - & $\begin{array}{l}\cdots \\
\cdots\end{array}$ & $\cdots$ & 2 \\
\hline JuL $19 .$. & 1.9 & 30 & 9.4 & 0.20 & 49 & 0.045 & - & 0.017 & - & 0.019 & $<1$ \\
\hline $17 \ldots$ & 3.3 & 47 & 17 & 2.0 & 80 & 0.049 & -. & 0.014 & $\cdots$ & 0.016 & $<1$ \\
\hline $14 \ldots$ & 4.3 & 61 & 20 & 0.60 & 102 & 0.085 & $\cdots$ & 0.005 & $\cdots$ & 0.010 & $<1$ \\
\hline $\begin{array}{l}\text { OCT } \\
\quad 23 \ldots\end{array}$ & 7.2 & 89 & 22 & 2.6 & 131 & 0.163 & $\cdots$ & 0.027 & $\cdots$ & 0.008 & $<1$ \\
\hline \multicolumn{12}{|l|}{ MAR 1992} \\
\hline \multicolumn{12}{|l|}{$\mathrm{APR}$} \\
\hline \multicolumn{11}{|l|}{ MAY } & $<1$ \\
\hline \multicolumn{12}{|l|}{ JUN } \\
\hline \multicolumn{12}{|l|}{ JUL } \\
\hline $\begin{array}{l}14 \ldots \\
\text { AUG }\end{array}$ & 3.1 & 47 & 16 & 0.80 & 76 & 0.041 & $\cdots$ & $<0.002$ & $\cdots$ & 0.004 & $<1$ \\
\hline $12 \ldots$ & 3.9 & 47 & 16 & 1.3 & 88 & 0.076 & $\cdots$ & 0.017 & $\ldots$ & 0.013 & $<1$ \\
\hline $25 \ldots$ & 4.2 & 54 & 21 & 1.7 & 82 & 0.091 & $\cdots$ & 0.024 & $\cdots$ & 0.013 & 1 \\
\hline \multicolumn{12}{|l|}{ OCT } \\
\hline $\begin{array}{c}28 \ldots \\
\text { JAN } 1993\end{array}$ & 6.5 & 78 & 22 & 1.8 & 113 & 0.135 & $\cdots$ & 0.010 & $\cdots$ & 0.005 & $<1$ \\
\hline $\operatorname{MAR}_{\operatorname{MAR}}^{12 \ldots}$ & 5.0 & 57 & 18 & 1.2 & 91 & $\cdots$ & 0.161 & $\cdots$ & 0.016 & $\cdots$ & $<1$ \\
\hline $23 \ldots$ & 3.6 & 40 & 14 & 0.90 & 44 & $\cdots$ & 0.052 & $\cdots$ & $<0.002$ & 0.006 & $<1$ \\
\hline
\end{tabular}


Table 30. Onsite measurements and bacteriological and selected inorganic data for station 07091200 , Arkansas River near Nathrop--Continued

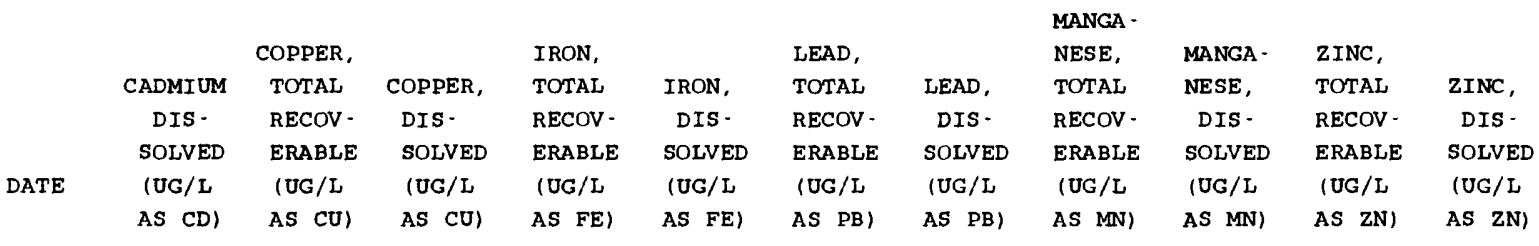

\begin{tabular}{|c|c|c|c|c|c|c|c|c|c|c|c|}
\hline \multicolumn{12}{|l|}{ APR 1990} \\
\hline $19 \ldots$ & 0.6 & 4 & 2 & 540 & 130 & 6 & 1.1 & 100 & 73 & 270 & 190 \\
\hline \multicolumn{12}{|l|}{ MAY } \\
\hline $23 \ldots$ & 0.1 & 8 & 2 & 3000 & 33 & 50 & 0.7 & 480 & 60 & 670 & 72 \\
\hline \multicolumn{12}{|l|}{ JUN } \\
\hline $06 \ldots$ & 0.2 & 25 & 4 & 7300 & 38 & 85 & 2.5 & 580 & 23 & 520 & 65 \\
\hline $20 \ldots$ & 0.5 & 23 & 19 & 820 & 20 & 54 & 26 & 60 & 11 & 120 & 62 \\
\hline \multicolumn{12}{|l|}{ JUL } \\
\hline $18 \ldots$ & 0.2 & 26 & 7 & 290 & 28 & 28 & 15 & 30 & 9 & 60 & 40 \\
\hline \multicolumn{12}{|l|}{ AUG } \\
\hline $29 \ldots$ & 0.3 & 3 & 2 & 130 & 34 & 2 & 0.9 & 30 & 15 & 70 & 51 \\
\hline \multicolumn{12}{|l|}{ OCT } \\
\hline $31 \ldots$ & 0.2 & 4 & 1 & 100 & 33 & 4 & $<0.5$ & 20 & 10 & 70 & 54 \\
\hline \multicolumn{12}{|l|}{ JAN 1991} \\
\hline $16 \ldots$ & 0.1 & 4 & 1 & 350 & 15 & 6 & $<0.5$ & 60 & 7 & 100 & 54 \\
\hline \multicolumn{12}{|l|}{ MAR } \\
\hline $26 \ldots$ & 0.2 & 3 & 2 & 90 & 27 & 2 & $<0.5$ & 20 & 12 & 60 & 59 \\
\hline \multicolumn{12}{|l|}{ APR } \\
\hline $24 \ldots$ & 0.3 & 3 & 2 & 170 & 48 & 4 & 0.5 & 40 & 23 & 90 & 74 \\
\hline \multicolumn{12}{|l|}{ MAY } \\
\hline $15 \ldots$ & 0.4 & 11 & 2 & 1900 & 67 & 80 & 2.8 & 200 & 36 & 280 & 91 \\
\hline \multicolumn{12}{|l|}{ JUN } \\
\hline $11 \ldots$ & 0.9 & 21 & 3 & 3000 & 30 & 44 & 1.2 & 240 & $<10$ & 320 & 40 \\
\hline $11 \ldots$ & 0.6 & 13 & 4 & 2000 & 50 & 52 & 1.2 & 220 & 20 & 210 & 60 \\
\hline $19 \ldots$ & 0.3 & 60 & 49 & 480 & 40 & 18 & $<0.5$ & 60 & 10 & 80 & 52 \\
\hline \multicolumn{12}{|l|}{ JUL } \\
\hline $17 \ldots$ & 0.2 & 4 & 2 & 160 & 41 & 8 & $<0.5$ & 20 & 8 & 40 & 28 \\
\hline \multicolumn{12}{|l|}{ AUG } \\
\hline $14 \ldots$ & 0.2 & 4 & 1 & 260 & 42 & 4 & 1.1 & 20 & 10 & 50 & 29 \\
\hline \multicolumn{12}{|l|}{ OCT } \\
\hline $23 \ldots$ & 0.1 & 11 & $<1$ & 60 & 23 & 10 & $<0.5$ & 20 & 8 & $7 n$ & 48 \\
\hline \multicolumn{12}{|l|}{ MAR 1992} \\
\hline $24 \ldots$ & 0.2 & $<1$ & 2 & 120 & 23 & $<1$ & $<0.5$ & 70 & 11 & 50 & 37 \\
\hline \multicolumn{12}{|l|}{ APR } \\
\hline $22 \ldots$ & 0.3 & 2 & 1 & 230 & 71 & 2 & $<0.5$ & 70 & 48 & 130 & 100 \\
\hline \multicolumn{12}{|l|}{ MAY } \\
\hline $21 \ldots$ & 0.2 & $<1$ & 2 & 1900 & 53 & 43 & 0.8 & 330 & 20 & 310 & 49 \\
\hline \multicolumn{12}{|l|}{ JUN } \\
\hline $25 \ldots$ & 0.2 & 50 & 41 & 460 & 32 & 8 & $<0.5$ & 50 & 7 & 80 & 35 \\
\hline JUL & & & & & & & & & & & \\
\hline $14 \ldots$ & 0.1 & 2 & 2 & 130 & 37 & 2 & $<0.5$ & 20 & 7 & 50 & 23 \\
\hline AUG & & & & & & & & & & & \\
\hline $12 \ldots$ & $<0.1$ & 8 & 2 & 370 & 32 & 5 & 0.6 & 30 & 6 & 240 & 26 \\
\hline $25 \ldots$ & 0.3 & 10 & 3 & 1000 & 120 & 25 & 2 & 120 & 18 & 170 & 29 \\
\hline OCT & & & & & & & & & & & \\
\hline $28 \ldots$ & $<0.1$ & $<1$ & $<1$ & 70 & 34 & $<1$ & 0.6 & $<10$ & 7 & 30 & 28 \\
\hline JAN 1993 & & & & & & & & & & & \\
\hline $12 \ldots$ & $<0.1$ & 2 & 1 & 120 & 21 & 2 & $<0.5$ & 30 & 7 & 70 & 43 \\
\hline MAR & & & & & & & & & & & \\
\hline $23 \ldots$ & 0.1 & 1 & $<1$ & 150 & 23 & 3 & $<0.5$ & 30 & 9 & 40 & 30 \\
\hline
\end{tabular}


Table 31. Onsite measurements and bacteriological and selected inorganic data for station 07091500, Arkansas River at Salida

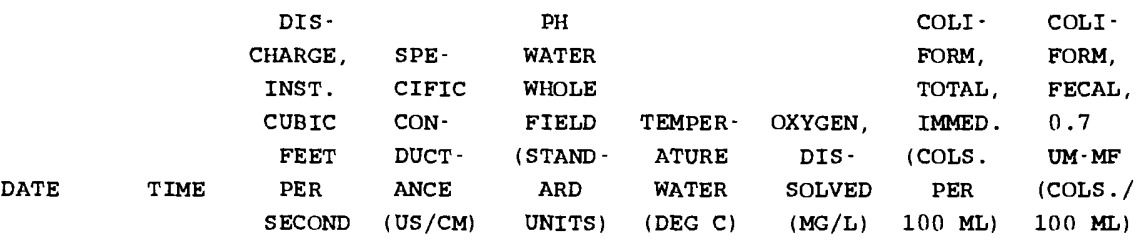

\begin{tabular}{|c|c|c|c|c|c|c|c|c|}
\hline \multicolumn{9}{|c|}{ APR 1990} \\
\hline $19 \ldots$ & 1200 & 205 & 203 & 8.3 & 10.0 & 9.1 & -. & E1 \\
\hline \multicolumn{9}{|l|}{ MAY } \\
\hline $23 \ldots$ & 1430 & 515 & 140 & 8.1 & 16.0 & 7.5 & - & $\mathrm{K} 20$ \\
\hline \multicolumn{9}{|l|}{ JUN } \\
\hline $06 \ldots$ & 1525 & 2410 & 93 & 7.7 & 13.5 & 8.2 & K60 & K13 \\
\hline $20 \ldots$ & 1530 & 1260 & 101 & 7.7 & 15.5 & 8.2 & $<10$ & $<2$ \\
\hline \multicolumn{9}{|l|}{ JUL } \\
\hline $18 \ldots$ & 1430 & 1040 & 118 & 8.0 & 18.0 & 7.5 & $>200$ & 22 \\
\hline \multicolumn{9}{|l|}{ AUG } \\
\hline $29 \ldots$ & 1300 & 323 & 211 & 8.0 & 18.0 & 7.4 & К2 & K7 \\
\hline \multicolumn{9}{|l|}{ OCT } \\
\hline $31 \ldots$ & 1505 & 359 & 212 & $\cdots$ & 9.0 & 9.1 & -- & $\cdots$ \\
\hline \multicolumn{9}{|l|}{ DEC } \\
\hline $20 \ldots$ & 1100 & 283 & 226 & $\cdot$ & 0.0 & 10.1 & E46 & E1 \\
\hline \multicolumn{9}{|c|}{ MAR 1991} \\
\hline $26 \ldots$ & 1245 & 317 & 162 & 8.2 & 8.0 & 9.0 & $\cdots$ & $<1$ \\
\hline \multicolumn{9}{|l|}{ APR } \\
\hline $24 \ldots$ & 1600 & 391 & 142 & 8.0 & 11.0 & 8.5 & E5 & E1 \\
\hline \multicolumn{9}{|l|}{ MAY } \\
\hline $15 \ldots$ & 1400 & 886 & 121 & 7.7 & 12.0 & 7.9 & 26 & E9 \\
\hline \multicolumn{9}{|l|}{ JUN } \\
\hline $19 \ldots$ & 1645 & 1810 & 98 & 7.9 & 14.0 & 8.0 & - & - \\
\hline \multicolumn{9}{|l|}{ JUL } \\
\hline $17 \ldots$ & 1925 & 655 & 139 & 8.3 & 19.0 & 7.1 & E18 & E12 \\
\hline \multicolumn{9}{|l|}{ AUG } \\
\hline $14 \ldots$ & 1945 & 493 & 186 & 8.1 & 17.0 & $\cdot$ & -. & $\cdots$ \\
\hline \multicolumn{9}{|l|}{ OCT } \\
\hline $23 \ldots$ & 1010 & 225 & 242 & 8.3 & 8.5 & 9.1 & E17 & - \\
\hline \multicolumn{9}{|l|}{ DEC } \\
\hline $17 \ldots$ & 1515 & 442 & 162 & 8.3 & 1.5 & 10.6 & E4 & E7 \\
\hline \multicolumn{9}{|l|}{ MAR 1992} \\
\hline $24 \ldots$ & 1200 & 351 & 156 & 8.4 & 7.0 & 9.6 & $\cdots$ & $\cdots$ \\
\hline \multicolumn{9}{|l|}{ APR } \\
\hline $22 \ldots$ & 1015 & 225 & 180 & 8.2 & 8.5 & 8.9 & E8 & E3 \\
\hline \multicolumn{9}{|l|}{ MAY } \\
\hline $21 \ldots$ & 1000 & 1160 & 97 & 7.8 & 12.0 & 8.3 & E30 & 83 \\
\hline \multicolumn{9}{|l|}{ JUN } \\
\hline $25 \ldots$ & 1215 & 1240 & 110 & 8.0 & 14.5 & 8.1 & $<2$ & E3 \\
\hline \multicolumn{9}{|l|}{ JUL } \\
\hline $14 \ldots$ & 1730 & 702 & 141 & 8.3 & 17.0 & 7.2 & -. & E2 1 \\
\hline AUG & & & & & & & & \\
\hline $12 \ldots$ & 1230 & 655 & 139 & 8.2 & 16.5 & 7.6 & E8 & E10 \\
\hline $25 \ldots$ & 1300 & 905 & 162 & 8.3 & 13.0 & 8.4 & & 160 \\
\hline OCT & & & & & & & & \\
\hline $28 \ldots$ & 1500 & 282 & 218 & 8.6 & 10.0 & 8.8 & E3 & E3 \\
\hline JAN 1993 & & & & & & & & \\
\hline $12 \ldots$ & 1530 & 322 & 167 & 8.3 & 0.0 & 11.3 & E6 & E2 \\
\hline MAR & & & & & & & & \\
\hline $23 \ldots$ & 1030 & 570 & 110 & 8.1 & 6.0 & 10.4 & $\cdots$ & E1 \\
\hline
\end{tabular}


Table 31. Onsite measurements and bacteriological and selected inorganic data for station 07091500, Arkansas River at Salida--Continued

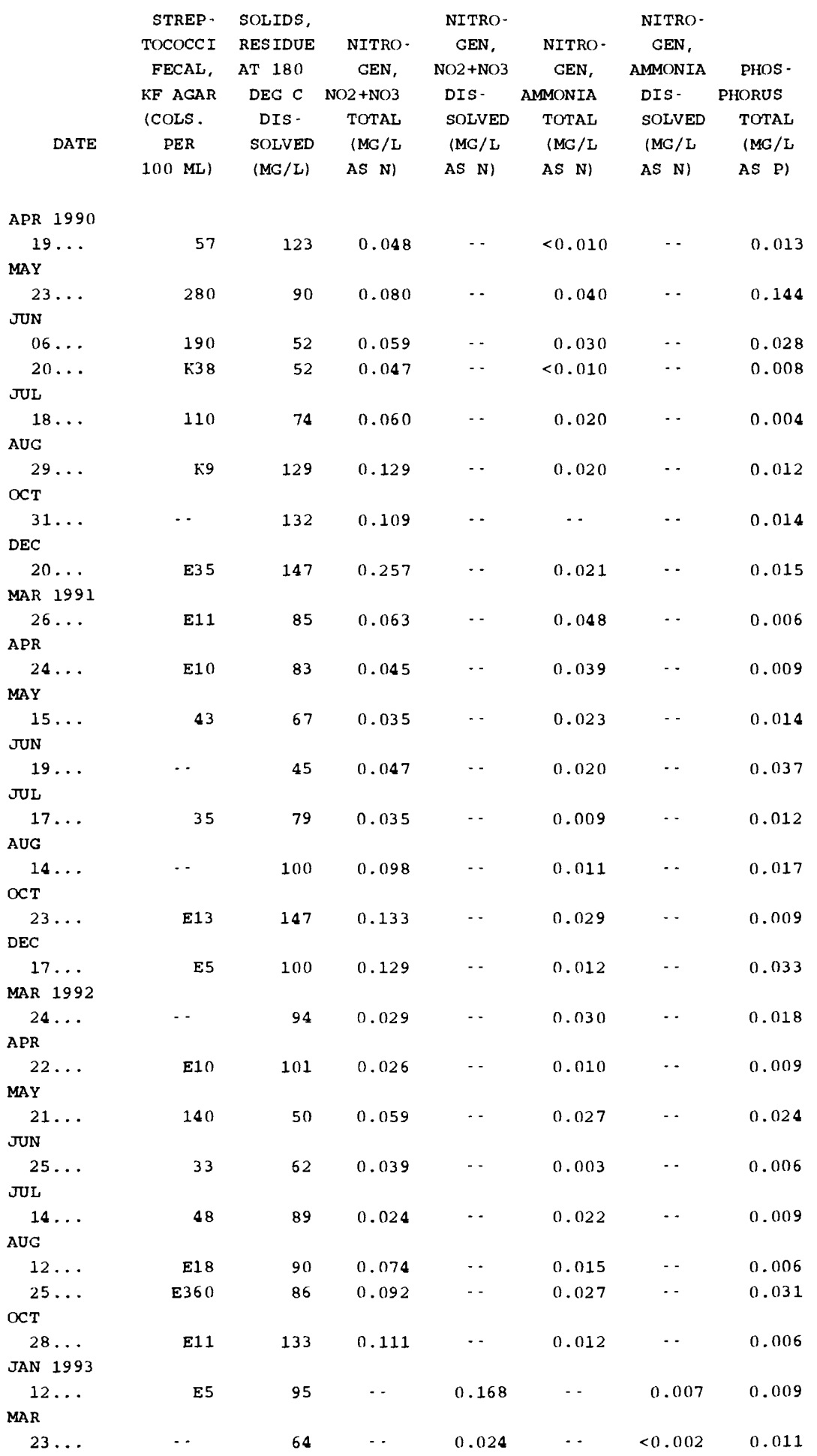


Table 32. Onsite measurements and selected inorganic data for station 07093500 , South Arkansas River near Salida

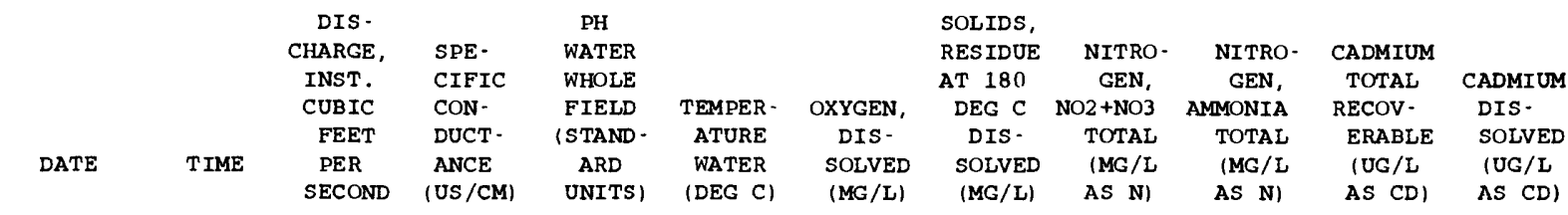

\begin{tabular}{|c|c|c|c|c|c|c|c|c|c|c|c|}
\hline $\begin{array}{c}\text { APR } 1990 \\
19 \ldots \\
\text { JUN }\end{array}$ & 1320 & 5.1 & 391 & 8.3 & 10.0 & 8.2 & 233 & 0.072 & $<0.010$ & $<1$ & $<0.1$ \\
\hline JUL & 1605 & 118 & $\cdots$ & - & 15.0 & 7.7 & 104 & 0.123 & 0.040 & $<1$ & $<0.1$ \\
\hline${ }_{A \cup G}^{18 \ldots}$ & 1250 & 5.2 & 408 & 8.7 & 20.5 & 6.5 & 220 & 0.033 & $<0.010$ & $<1$ & $<0.1$ \\
\hline$\underset{\text { OCT }}{29 . .}$ & 1115 & 5.8 & 433 & 8.0 & 16.5 & 10.4 & 271 & 0.184 & $<0.010$ & $<1$ & $<0.1$ \\
\hline $\begin{array}{c}31 \ldots \\
A P R \quad 1991\end{array}$ & 1600 & 29 & 331 & 8.6 & 11.0 & 8.3 & 198 & 0.019 & 0.020 & $<1$ & 0.1 \\
\hline JUN & 1730 & 2.9 & 449 & 8.2 & 14.0 & 9.2 & 260 & 0.461 & 0.036 & $<1$ & $<0.1$ \\
\hline JUL $^{19 . .}$ & 1700 & 33 & 267 & 7.9 & 15.0 & 8.0 & 147 & 0.062 & 0.017 & $<1$ & 0.1 \\
\hline${ }_{\mathrm{OCT}}^{17 \ldots}$ & 2025 & 5.1 & 445 & 7.7 & 19.0 & $\cdots$ & 241 & 0.282 & 0.014 & $<1$ & 0.2 \\
\hline $\begin{array}{c}23 \\
\mathrm{APR} \\
1992\end{array}$ & 1300 & 11 & 420 & 8.5 & 12.5 & 10.0 & 252 & 0.053 & 0.018 & $<1$ & $<0.1$ \\
\hline$\underset{\text { JUN }}{22 \ldots}$ & 1300 & 11 & 337 & 8.7 & 9.5 & 10.5 & 184 & 0.075 & 0.014 & $<1$ & $<0.1$ \\
\hline$\underset{A U G}{25 \ldots}$ & 1335 & 46 & 245 & 8.4 & 16.0 & 7.4 & 148 & 0.047 & 0.022 & $<1$ & $<0.1$ \\
\hline${ }_{\mathrm{OCT}} 12 \ldots$ & 1340 & 9.7 & 392 & 8.2 & 18.0 & 8.1 & 236 & 0.181 & 0.032 & $<1$ & 0.1 \\
\hline $28 \ldots$ & 1555 & 19 & 400 & 8.6 & 10.5 & 8.5 & 217 & 0.087 & 0.022 & $<1$ & $<0.1$ \\
\hline
\end{tabular}

\begin{tabular}{|c|c|c|c|c|c|c|c|c|c|c|}
\hline & $\begin{array}{l}\text { COPPER, } \\
\text { TOTAL } \\
\text { RECOV- } \\
\text { ERABLE } \\
\text { (UG/L }\end{array}$ & $\begin{array}{l}\text { COPPER, } \\
\text { DIS - } \\
\text { SOLVED } \\
\text { (UG/L }\end{array}$ & $\begin{array}{l}\text { IRON, } \\
\text { TOTAL } \\
\text { RECOV- } \\
\text { ERABLE } \\
\text { (UG/L }\end{array}$ & $\begin{array}{l}\text { IRON, } \\
\text { DIS- } \\
\text { SOLVED } \\
\text { (UG/L }\end{array}$ & $\begin{array}{l}\text { LEAD, } \\
\text { TOTAL } \\
\text { RECOV. } \\
\text { ERABLE } \\
\text { (UG/L }\end{array}$ & $\begin{array}{l}\text { LEAD, } \\
\text { DIS - } \\
\text { SOLVED } \\
\text { (UG/L }\end{array}$ & $\begin{array}{l}\text { MANGA- } \\
\text { NESE, } \\
\text { TOTAL } \\
\text { RECOV- } \\
\text { ERABLE } \\
\text { (UG/L }\end{array}$ & $\begin{array}{l}\text { MANGA- } \\
\text { NESE, } \\
\text { DIS - } \\
\text { SOLVED } \\
\text { IUG } / L\end{array}$ & $\begin{array}{l}\text { ZINC, } \\
\text { TOTAL } \\
\text { RECOV- } \\
\text { ERABLE } \\
\text { (UG / }\end{array}$ & $\begin{array}{l}\text { ZINC, } \\
\text { DIS - } \\
\text { SOLVED } \\
\text { (UG/L }\end{array}$ \\
\hline & AS CUI & AS CUl & AS FE) & AS FE) & AS PB) & AS PB) & AS MN) & AS MN) & AS ZN) & AS $\mathrm{ZN}$ ) \\
\hline
\end{tabular}

APR 1990
$19 \ldots$
JUN
$06 \ldots$
JUL $\ldots$
$18 \ldots$
AUG
$29 \ldots$
OCT
$31 \ldots$
APR 1991
$24 \ldots$
JUN
$19 \ldots$
JUL
$17 \ldots$
OCT
$23 \ldots$
APR 1992
$22 \ldots$
JUN $\ldots$
$25 \ldots$
AUG
$12 \ldots$
OCT

$\begin{array}{rrrrr}3 & 1 & 290 & 14 & 1 \\ 15 & 2 & 12000 & 58 & 12 \\ 2 & 2 & 110 & 36 & <1 \\ 2 & 1 & 120 & 33 & 2 \\ 1 & 1 & 220 & 59 & <1 \\ 3 & 2 & 130 & 49 & 2 \\ 6 & 2 & 480 & 68 & 3 \\ 3 & 1 & 140 & 61 & 7 \\ 3 & <1 & 120 & 36 & 2 \\ 1 & <1 & 190 & 34 & <1 \\ 2 & 1 & 570 & 62 & <1 \\ 2 & 1 & 320 & 44 & <1 \\ <1 & <1 & 100 & 44 & <1\end{array}$

$\begin{array}{rrrrr}<0.5 & 40 & 18 & 20 & 5 \\ 0.9 & 360 & 12 & 50 & 3 \\ <0.5 & 10 & 6 & <10 & 4 \\ <0.5 & 30 & 10 & <10 & 6 \\ <0.5 & 20 & 8 & <10 & 4 \\ <0.5 & 30 & 26 & <10 & 9 \\ <0.5 & 40 & 15 & 80 & <3 \\ 0.5 & 40 & 20 & <10 & 5 \\ <0.5 & 30 & 10 & <10 & 4 \\ <0.5 & 30 & 9 & <10 & <3 \\ <0.5 & 40 & 10 & <10 & <3 \\ <0.5 & 50 & 12 & <10 & <3 \\ <0.5 & <10 & 8 & <10 & <3\end{array}$


Table 33. Onsite measurements and bacteriological and selected inorganic data for station 07093700 , Arkansas River near Wellsville

\begin{tabular}{|c|c|c|c|c|c|c|c|c|c|c|c|c|}
\hline & TIME & $\begin{array}{c}\text { DIS - } \\
\text { CHARGE, } \\
\text { INST. } \\
\text { CUBIC } \\
\text { FEET } \\
\text { PER }\end{array}$ & $\begin{array}{l}\text { SPE- } \\
\text { CIFIC } \\
\text { CON- } \\
\text { DUCT- } \\
\text { ANCE }\end{array}$ & $\begin{array}{c}\text { PH } \\
\text { WATER } \\
\text { WHOLE } \\
\text { FIELD } \\
\text { (STAND- } \\
\text { ARD }\end{array}$ & $\begin{array}{l}\text { TEMPER - } \\
\text { ATURE } \\
\text { WATER }\end{array}$ & $\begin{array}{c}\text { OXYGEN, } \\
\text { DIS - } \\
\text { SOLVED }\end{array}$ & $\begin{array}{c}\text { COLI - } \\
\text { FORM, } \\
\text { TOTAL, } \\
\text { IMMED. } \\
\text { ICOLS . } \\
\text { PER }\end{array}$ & $\begin{array}{l}\text { COLI- } \\
\text { FORM, } \\
\text { FECAL, } \\
0.7 \\
\text { UM-MF } \\
\text { ICOLS./ }\end{array}$ & $\begin{array}{c}\text { STREP- } \\
\text { TOCOCCI } \\
\text { FECAL, } \\
\text { KF AGAR } \\
\text { (COLS. } \\
\text { PER }\end{array}$ & $\begin{array}{l}\text { HARD - } \\
\text { NESS } \\
\text { TOTAL } \\
\text { (MG /L } \\
\text { AS }\end{array}$ & $\begin{array}{l}\text { CALCIUM } \\
\text { DIS - } \\
\text { SOLVED } \\
\text { (MG/L }\end{array}$ & $\begin{array}{l}\text { MAGNE - } \\
\text { SIUM, } \\
\text { DIS - } \\
\text { SOLVED } \\
\text { (MG /L }\end{array}$ \\
\hline & & SECOND & (US/CM) & UNITS। & (DEG C) & (MG/L) & $100 \mathrm{ML})$ & $100 \mathrm{MLI}$ & $100 \mathrm{ML})$ & (ACO3) & AS CA) & AS MG) \\
\hline
\end{tabular}

\begin{tabular}{|c|c|c|c|c|c|c|c|c|c|c|c|c|}
\hline \multicolumn{13}{|l|}{ APR 1990} \\
\hline $19 \ldots$ & 1430 & 250 & 230 & 8.3 & 9.5 & 8.7 & $\cdots$ & K15 & 68 & 110 & 31 & 7.5 \\
\hline \multicolumn{13}{|l|}{ MAY } \\
\hline $23 \ldots$ & 1630 & 585 & 162 & 8.1 & 17.0 & 7.2 & $\cdots$ & 48 & 370 & 71 & 21 & 4.5 \\
\hline \multicolumn{13}{|l|}{ JUN } \\
\hline $06 \ldots$ & 1700 & 2620 & 103 & 7.9 & 14.0 & 8.2 & $\mathrm{~K} 180$ & K27 & 280 & 43 & 13 & 2.5 \\
\hline $20 \ldots$ & 1730 & 1690 & 105 & 7.9 & 16.5 & 8.0 & $<10$ & $\mathrm{~K} 4$ & 96 & 46 & 14 & 2.8 \\
\hline \multicolumn{13}{|l|}{ JOL } \\
\hline $18 \ldots$ & 1550 & 1160 & 131 & 8.1 & 18.0 & 7.2 & 72 & $\mathrm{~K} 2$ & 34 & 59 & 18 & 3.4 \\
\hline \multicolumn{13}{|l|}{ AUG } \\
\hline $29 \ldots$ & 1400 & 404 & 236 & 8.2 & 19.0 & 7.2 & $<3$ & $\mathrm{k} 3$ & 60 & 100 & 31 & 6.1 \\
\hline \multicolumn{13}{|l|}{ NOV } \\
\hline $01 \ldots$ & 0745 & 421 & 241 & 8.5 & 6.5 & 9.5 & $\cdots$ & $\cdots$ & $\cdots$ & 110 & 33 & 6.8 \\
\hline \multicolumn{13}{|l|}{ JAN 1991} \\
\hline $17 \ldots$ & 0900 & 498 & 172 & 8.0 & 0.0 & 11.0 & 51 & $<1$ & 10 & 76 & 23 & 4.6 \\
\hline \multicolumn{13}{|l|}{ MAR } \\
\hline $26 \ldots$ & 1405 & 372 & 196 & 8.5 & 8.5 & 8.7 & $\cdots$ & $<1$ & 21 & 77 & 23 & 4.7 \\
\hline \multicolumn{13}{|l|}{ APR } \\
\hline $25 \ldots$ & 0745 & 405 & 168 & 7.8 & 7.0 & 9.0 & 47 & 25 & 73 & 69 & 21 & 4.1 \\
\hline \multicolumn{13}{|l|}{ MAY } \\
\hline $15 \ldots$ & 1620 & 1030 & 132 & 7.7 & 13.5 & 8.0 & E110 & E6 & 80 & 53 & 16 & 3.1 \\
\hline \multicolumn{13}{|l|}{ JUN } \\
\hline $12 \ldots$ & 0545 & 2290 & $\cdots$ & $\cdots$ & $\cdots$ & $\cdots$ & $\cdots$ & $\cdots$ & $\cdots$ & $-\cdot$ & -- & $\cdots$ \\
\hline $19 \ldots$ & 1845 & 1930 & 108 & 8.0 & 14.0 & 7.9 & 190 & E4 & 78 & 46 & 14 & 2.6 \\
\hline \multicolumn{13}{|l|}{ JUL } \\
\hline $17 \ldots$ & 2015 & 728 & 154 & 8.4 & 19.5 & 6.8 & $>330$ & E20 & 45 & 71 & 21 & 4.4 \\
\hline \multicolumn{13}{|l|}{ AUG } \\
\hline $15 \ldots$ & 0615 & 547 & 206 & 7.9 & 15.0 & $\cdots$ & E35 & 21 & E120 & 91 & 28 & 5.0 \\
\hline \multicolumn{13}{|l|}{ SEP } \\
\hline $03 \ldots$ & 1340 & 347 & 239 & 8.6 & 18.5 & 8.3 & $\cdots$ & $\cdots$ & $\cdots$ & $\cdots$ & $\cdots$ & $\cdots$ \\
\hline \multicolumn{13}{|l|}{ OCT } \\
\hline $23 \ldots$ & 1420 & 270 & 266 & 8.8 & 10.5 & 9.5 & E8 & $\cdots$ & E5 & 130 & 38 & 7.5 \\
\hline \multicolumn{13}{|l|}{$\mathrm{DEC}$} \\
\hline $18 \ldots$ & 0900 & 500 & 194 & 8.1 & 0.0 & 11.2 & E33 & E6 & 46 & 87 & 26 & 5.3 \\
\hline MAR 1992 & & & & & & & & & & & & \\
\hline $24 \ldots$ & 1315 & 397 & 175 & 8.9 & 7.5 & 10.7 & $\cdots$ & $\cdots$ & $\cdots$ & 74 & 22 & 4.6 \\
\hline APR & & & & & & & & & & & & \\
\hline $22 \ldots$ & 1520 & 270 & 202 & 8.8 & 11.0 & 8.8 & E1 & E2 & E10 & 92 & 27 & 5.9 \\
\hline MAY & & & & & & & & & & & & \\
\hline $21 \ldots$ & 1200 & 1400 & 101 & 8.0 & 12.0 & 8.1 & 56 & 92 & 150 & 46 & 14 & 2.8 \\
\hline JUN & & & & & & & & & & & & \\
\hline $25 \ldots$ & 1430 & 1330 & 127 & 8.3 & 15.5 & 7.6 & E30 & E4 & 90 & 56 & 17 & 3.3 \\
\hline JUL & & & & & & & & & & & & \\
\hline $14 \ldots$ & 2000 & 784 & 160 & 8.3 & 17.5 & 7.8 & $\cdots$ & 32 & 85 & 73 & 22 & 4.3 \\
\hline AUG & & & & & & & & & & & & \\
\hline $12 \ldots$ & 1450 & 742 & 163 & 8.4 & 17.5 & 7.4 & E140 & 40 & 93 & 70 & 21 & 4.3 \\
\hline $25 \ldots$ & 1020 & 1180 & 178 & 8.2 & 11.0 & 8.6 & $\cdots$ & E470 & $>560$ & 81 & 24 & 5.1 \\
\hline OCT & & & & & & & & & & & & \\
\hline $28 \ldots$ & 1620 & 378 & 256 & 8.7 & 10.0 & 8.9 & $\cdots$ & E6 & 39 & 110 & 34 & 7.1 \\
\hline JAN 1993 & & & & & & & & & & & & \\
\hline $12 \ldots$ & 1640 & 408 & 195 & 8.3 & 0.0 & 11.0 & 150 & $<1$ & E4 & 81 & 24 & 5.0 \\
\hline MAR & & & & & & & & & & & & \\
\hline $23 \ldots$ & 1200 & 638 & 134 & 8.4 & 6.0 & 10.1 & $\cdots$ & E2 & $\cdots$ & 53 & 16 & 3.2 \\
\hline
\end{tabular}


Table 33. Onsite measurements and bacteriological and selected inorganic data for station 07093700 , Arkansas River near Wellsville--Continued

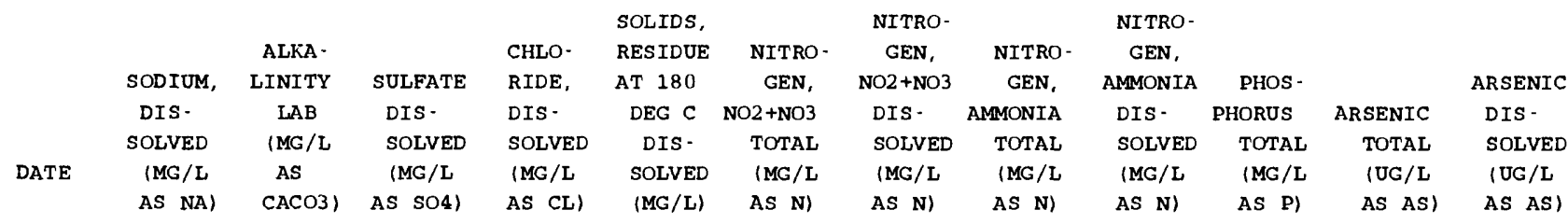

\begin{tabular}{|c|c|c|c|c|c|c|c|c|c|c|c|c|}
\hline APR 1990 & & & & & & & & & & & & \\
\hline $19 \ldots$ & 7.6 & 85 & 26 & 2.8 & 139 & 0.133 & $\cdots$ & 0.030 & $-\cdot$ & 0.011 & $\cdots$ & $\cdots$ \\
\hline MAY & & & & & & & & & & & & \\
\hline $23 \ldots$ & 5.1 & 59 & 21 & 2.2 & 99 & 0.129 & $\cdots$ & 0.010 & $\cdots$ & $\cdots$ & $\cdots$ & $\cdots$ \\
\hline JUN & & & & & & & & & & & & \\
\hline $06 \ldots$ & 2.3 & 33 & 13 & 0.40 & 64 & 0.069 & $\cdots$ & 0.030 & $\cdots$ & 0.023 & $\cdots$ & $\cdots$ \\
\hline $20 \ldots$ & 2.5 & 37 & 12 & 1.2 & - & 0.059 & $\cdots$ & $<0.010$ & $\cdots$ & 0.006 & $\cdots$ & $\cdots$ \\
\hline JUL & & & & & & & & & & & & \\
\hline $18 \ldots$ & 3.5 & 50 & 14 & 0.70 & 77 & 0.060 & $\cdots$ & 0.020 & $\cdots$ & 0.012 & $\cdots$ & $\cdots$ \\
\hline AUG & & & & & & & & & & & & \\
\hline $29 \ldots$ & 7.4 & 94 & 18 & 2.9 & 132 & 0.193 & $\cdots$ & 0.020 & $\cdots$ & 0.032 & $<1$ & $<1$ \\
\hline NOV & & & & & & & & & & & & \\
\hline $01 \ldots$ & 8.2 & 103 & 20 & 4.5 & 146 & 0.139 & $\cdots$ & 0.030 & $\cdots$ & 0.015 & $\cdots$ & $\cdots$ \\
\hline JAN 1991 & & & & & & & & & & & & \\
\hline $17 \ldots$ & 5.6 & 67 & 15 & 2.0 & 96 & 0.188 & $\cdots$ & 0.030 & $\cdots$ & 0.026 & $\cdots$ & $\cdots$ \\
\hline MAR & & & & & & & & & & & & \\
\hline $26 \ldots$ & 5.5 & 73 & 19 & 3.7 & 102 & 0.109 & $\cdots$ & 0.044 & $\cdots$ & 0.032 & $\cdots$ & $\cdots$ \\
\hline APR & & & & & & & & & & & & \\
\hline $25 \ldots$ & 4.7 & 61 & 17 & 2.1 & 93 & 0.075 & $\cdots$ & 0.021 & $\cdots$ & 0.026 & $\cdots$ & $\cdots$ \\
\hline MAY & & & & & & & & & & & & \\
\hline $15 \ldots$ & 3.5 & 44 & 18 & 1.4 & 68 & 0.052 & $\cdots$ & 0.027 & $\cdots$ & 0.020 & $\cdots$ & $\cdots$ \\
\hline JUN & & & & & & & & & & & & \\
\hline $12 \ldots$ & $\cdots$ & $\cdots$ & - & $\cdots$ & - & $\cdots$ & $\cdots$ & $\cdots$ & $\cdots$ & $\cdots$ & $\cdots$ & $\cdots$ \\
\hline $19 \ldots$ & 2.4 & 37 & 10 & 0.20 & 57 & 0.069 & $-\cdot$ & 0.027 & $-\cdot$ & 0.014 & $<1$ & $<1$ \\
\hline JUL & & & & & & & & & & & & \\
\hline $17 \ldots$ & 4.2 & 61 & 17 & 0.50 & 86 & 0.069 & $\cdots$ & 0.018 & -- & 0.022 & $\cdots$ & $\cdots$ \\
\hline AUG & & & & & & & & & & & & \\
\hline $15 \ldots$ & 6.0 & 85 & 20 & 2.2 & 125 & 0.155 & $\cdots$ & 0.016 & $\cdots$ & 0.018 & $\cdots$ & $\cdots$ \\
\hline SEP & & & & & & & & & & & & \\
\hline $03 \ldots$ & $\cdots$ & $\cdots$ & $\cdots$ & $\cdots$ & $\cdots$ & $\cdots$ & - & $\cdots$ & - & $\cdots$ & $<1$ & $<1$ \\
\hline OCT & & & & & & & & & & & & \\
\hline $23 \ldots$ & 9.1 & 121 & 22 & 2.9 & 163 & 0.186 & $\cdots$ & 0.035 & $-\cdot$ & 0.035 & $\cdots$ & $\cdots$ \\
\hline DEC & & & & & & & & & & & & \\
\hline $18 \ldots$ & 6.5 & 81 & 14 & 1.4 & 121 & 0.182 & $\cdots$ & 0.028 & $\cdots$ & 0.023 & $\cdots$ & $\cdots$ \\
\hline MAR 1992 & & & & & & & & & & & & \\
\hline $24 \ldots$ & 6.0 & 70 & 18 & 2.1 & 111 & 0.053 & $\cdots$ & 0.023 & $\cdots$ & 0.035 & $\cdots$ & $\cdots$ \\
\hline APR & & & & & & & & & & & & \\
\hline $22 \ldots$ & 6.5 & 80 & 23 & 1.3 & 126 & 0.076 & $-\cdot$ & 0.032 & $\cdots$ & 0.027 & $\cdots$ & $\cdots$ \\
\hline MAY & & & & & & & & & & & & \\
\hline $21 \ldots$ & 2.9 & 38 & 13 & 0.50 & 62 & 0.079 & $\cdots$ & 0.026 & $\cdots$ & 0.030 & $\cdots$ & $\cdots$ \\
\hline JUN & & & & & & & & & & & & \\
\hline $25 \ldots$ & 3.3 & 49 & 14 & 0.50 & 72 & 0.064 & -. & 0.017 & $\cdots$ & 0.013 & $<1$ & $<1$ \\
\hline JUL & & & & & & & & & & & & \\
\hline $14 \ldots$ & 4.0 & 62 & 16 & 1.2 & 90 & 0.050 & $\cdots$ & 0.006 & $\cdots$ & 0.013 & $\cdots$ & $\cdots$ \\
\hline AUG & & & & & & & & & & & & \\
\hline $12 \ldots$ & 4.8 & 65 & 16 & 1.1 & 98 & 0.190 & $\cdots$ & 0.023 & $\cdots$ & 0.021 & $<1$ & $<1$ \\
\hline $25 \ldots$ & 5.1 & 70 & 14 & 1.9 & 110 & 0.141 & $\cdots$ & 0.056 & $\cdots$ & 0.026 & $\cdots$ & - \\
\hline OCT & & & & & & & & & & & & \\
\hline $28 \ldots$ & 8.4 & 107 & 22 & 2.5 & 146 & 0.201 & $\cdots$ & 0.029 & $\cdots$ & 0.031 & $\cdots$ & $\cdots$ \\
\hline JAN 1993 & & & & & & & & & & & & \\
\hline $12 \ldots$ & 6.3 & 78 & 17 & 1.6 & 129 & $\cdots$ & 0.222 & $\cdots$ & 0.049 & 0.032 & $\cdots$ & $\cdots$ \\
\hline MAR & & & & & & & & & & & & \\
\hline $23 \ldots$ & 4.3 & 52 & 13 & 1.1 & 64 & $\cdots$ & 0.055 & $\cdots$ & 0.025 & 0.027 & $\cdots$ & $\cdots$ \\
\hline
\end{tabular}


Table 33. Onsite measurements and bacteriological and selected inorganic data for station 07093700 , Arkansas River near Wellsville--Continued

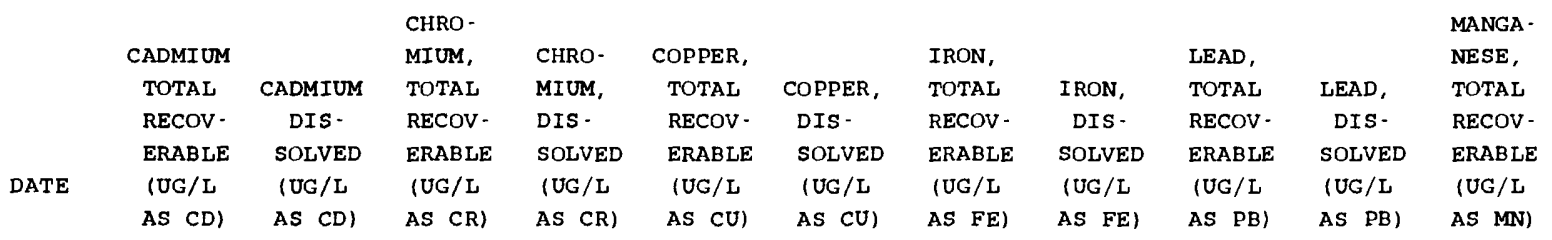

APR 1990

$19 . .$.

MAY

$23 .$.

JUN

$06 \ldots$

$20 \ldots$

JUL

$18 \ldots$

AUG

29 ..

NOV

$01 .$.

JAN 1991

$17 \ldots$

MAR

$26 \ldots$

APR

$25 \ldots$

MAY

$15 \ldots$

JUN

$12 \ldots$

$19 .$.

JUL

$17 .$.

AUG

$15 .$.

SEP

$03 .$.

OCT

$23 .$.

DEC

$18 \ldots$

MAR 1992

$24 \ldots$

APR

$22 \ldots$

MAY

$21 \ldots$

JUN

$25 \ldots$

JUL

$14 .$.

AUG

$12 \ldots$

$25 \ldots$

S CD) AS CD)

AS $(R)$

AS CR)

AS CU) AS FE)

AS FE)

AS PB)

AS PB)

MN)

\begin{tabular}{|c|c|c|c|c|c|c|c|c|c|c|}
\hline$<1$ & 0.1 & $\cdots$ & $\cdots$ & 4 & 2 & 350 & 54 & 4 & $<0.5$ & 60 \\
\hline 4 & $<0.1$ & $\cdots$ & $\cdots$ & 9 & 3 & 4200 & 22 & 63 & 0.6 & 560 \\
\hline 9 & 0.1 & $\cdots$ & $\cdots$ & 30 & 7 & 8000 & 64 & 92 & 1.2 & 610 \\
\hline 1 & 0.2 & $\cdots$ & $\cdots$ & 10 & 3 & 1200 & 26 & 11 & 0.8 & 70 \\
\hline$<1$ & 0.2 & $\cdots$ & $\cdots$ & 4 & 3 & 370 & 22 & 4 & $<0.5$ & 40 \\
\hline$<1$ & $<0.1$ & $<1$ & $<1$ & 3 & 2 & 150 & 25 & 1 & $<0.5$ & 40 \\
\hline$<1$ & 0.1 & $\cdots$ & $\cdots$ & 2 & 2 & 130 & 33 & $<1$ & 1.5 & 20 \\
\hline$<1$ & $<0.1$ & - & $\cdots$ & 4 & 2 & 210 & 18 & 2 & $<0.5$ & 10 \\
\hline$<1$ & 0.1 & $\cdots$ & $\cdots$ & 3 & 2 & 140 & 25 & 1 & $<0.5$ & 20 \\
\hline$<1$ & 0.2 & $\cdots$ & $\cdots$ & 3 & 2 & 180 & 45 & 3 & $<0.5$ & 40 \\
\hline 2 & 0.1 & $\cdots$ & $\cdots$ & 9 & 2 & 1200 & 54 & 22 & 1.0 & 150 \\
\hline 2 & 0.5 & $\cdots$ & $\cdots$ & 19 & 5 & 3100 & 70 & 90 & 2.5 & 270 \\
\hline$<1$ & 0.2 & $<1$ & $<1$ & 27 & 16 & 740 & 38 & 12 & $<0.5$ & 70 \\
\hline$<1$ & 0.1 & $\cdots$ & $\cdots$ & 7 & 1 & 250 & 34 & 7 & $<0.5$ & 30 \\
\hline$<1$ & 0.2 & $\cdots$ & $\cdots$ & 4 & 1 & 360 & 35 & 5 & $<0.5$ & 40 \\
\hline & $\cdots$ & $<1$ & $<1$ & $\cdots$ & $\cdots$ & $\cdots$ & $\cdots$ & $\cdots$ & $\cdots$ & - - \\
\hline$<1$ & 0.1 & $\cdots$ & $\cdots$ & 5 & $<1$ & 90 & 28 & 7 & $<0.5$ & 20 \\
\hline$<1$ & 0.6 & $\cdots$ & $\cdots$ & $\cdots$ & 1 & 120 & 23 & $<1$ & $<0.5$ & 20 \\
\hline$<1$ & $<0.1$ & $-\cdot$ & $\cdots$ & 2 & 1 & 120 & 23 & 3 & $<0.5$ & 30 \\
\hline$<1$ & 0.1 & $\cdots$ & $\cdots$ & 3 & 2 & 220 & 55 & 2 & 0.5 & 50 \\
\hline 2 & 0.2 & - & $\cdots$ & 10 & 2 & 2600 & 43 & 38 & $<0.5$ & 300 \\
\hline$<1$ & 0.2 & $<1$ & $<1$ & 61 & 39 & 350 & 38 & 6 & $<0.5$ & 50 \\
\hline$<1$ & $<0.1$ & $\cdots$ & $\cdots$ & 2 & 2 & 140 & 33 & 2 & $<0.5$ & 20 \\
\hline$<1$ & $<0.1$ & 1 & $<1$ & 2 & 2 & 170 & 45 & 2 & $<0.5$ & 30 \\
\hline$<1$ & $<0.1$ & $\cdots$ & - & 7 & 2 & 1500 & 70 & 13 & 0.9 & 130 \\
\hline$<1$ & $<0.1$ & - & $\cdots$ & 2 & 2 & 130 & 36 & 1 & 0.6 & $<10$ \\
\hline$<1$ & 0.6 & . & $\cdots$ & 2 & 1 & 180 & 24 & 2 & $<0.5$ & 40 \\
\hline$<1$ & 0.1 & $\cdots$ & $\cdots$ & 1 & 1 & 170 & 23 & 2 & $<0.5$ & 30 \\
\hline
\end{tabular}


Table 33. Onsite measurements and bacteriological and selected inorganic data for station 07093700 , Arkansas River near Wellsville--Continued

\begin{tabular}{|c|c|c|c|c|c|c|c|c|c|c|}
\hline $\begin{array}{l}\text { MANGA- } \\
\text { NESE, } \\
\text { DIS- } \\
\text { SOLVED } \\
\text { (UG / L }\end{array}$ & $\begin{array}{l}\text { MERCURY } \\
\text { TOTAL } \\
\text { RECOV- } \\
\text { ERABLE } \\
\text { (UG/L }\end{array}$ & $\begin{array}{c}\text { MERCURY } \\
\text { DIS - } \\
\text { SOLVED } \\
\text { (UG / L }\end{array}$ & $\begin{array}{l}\text { NICKEL, } \\
\text { TOTAL } \\
\text { RECOV - } \\
\text { ERABLE } \\
\text { (UG / L }\end{array}$ & $\begin{array}{l}\text { NICKEL, } \\
\text { DIS - } \\
\text { SOLVED } \\
\text { (UG / L }\end{array}$ & $\begin{array}{l}\text { SELE- } \\
\text { NIUM, } \\
\text { TOTAL } \\
\text { (UG/L }\end{array}$ & $\begin{array}{l}\text { SELE- } \\
\text { NIUM, } \\
\text { DIS - } \\
\text { SOLVED } \\
\text { UUG /L }\end{array}$ & $\begin{array}{l}\text { SILVER, } \\
\text { TOTAL } \\
\text { RECOV- } \\
\text { ERABLE } \\
\text { (UG /L }\end{array}$ & $\begin{array}{c}\text { SILVER, } \\
\text { DIS - } \\
\text { SOLVED } \\
\text { (UG / L }\end{array}$ & $\begin{array}{l}\text { ZINC, } \\
\text { TOTAL } \\
\text { RECOV - } \\
\text { ERABLE } \\
\text { (UG /L }\end{array}$ & $\begin{array}{l}\text { ZINC, } \\
\text { DIS - } \\
\text { SOLVED } \\
\text { IUG/L }\end{array}$ \\
\hline AS MN) & AS HG) & AS HG) & AS NI) & AS NI) & AS SE) & AS SE) & AS $A G)$ & AS AG) & AS $\mathbf{Z N}$ ) & AS $\mathrm{ZN}$ ) \\
\hline
\end{tabular}

\begin{tabular}{|c|c|c|c|c|c|c|c|c|c|c|c|}
\hline APR 1990 & & & & & & & & & & & \\
\hline $19 \ldots$ & 30 & $\cdots$ & $\cdots$ & $\cdots$ & $\cdots$ & $\cdots$ & $\cdots$ & $\cdots$ & . & 110 & 59 \\
\hline MAY & & & & & & & & & & & \\
\hline $23 \ldots$ & 39 & $\cdots$ & $\cdots$ & $\cdots$ & $\cdots$ & $\cdots$ & $\cdots$ & $\cdots$ & $\cdots$ & 800 & 35 \\
\hline JUN & & & & & & & & & & & \\
\hline $06 \ldots$ & 23 & $\cdots$ & $\cdots$ & $\cdots$ & $\cdots$ & $\cdots$ & $\cdots$ & $\cdots$ & $\cdots$ & 520 & 52 \\
\hline $20 \ldots$ & 12 & $\cdots$ & $\cdots$ & $\cdots$ & $\cdots$ & $\cdots$ & $\cdots$ & $\cdots$ & $\cdots$ & 100 & 35 \\
\hline JOL & & & & & & & & & & & \\
\hline $18 \ldots$ & 8 & $\cdots$ & $\cdots$ & $\cdots$ & $\cdots$ & $\cdots$ & $\cdots$ & $-\cdot$ & $\cdots$ & 60 & 24 \\
\hline$A \cup G$ & & & & & & & & & & & \\
\hline $29 \ldots$ & 16 & $<0.10$ & $<0.1$ & $<1$ & $<1$ & $<1$ & $<1$ & $<1$ & $<1.0$ & 50 & 26 \\
\hline NOV & & & & & & & & & & & \\
\hline $01 \ldots$ & 14 & $\cdots$ & $\cdots$ & $\cdots$ & $\cdots$ & $\cdots$ & $\cdots$ & $\cdots$ & $\cdots$ & 60 & 52 \\
\hline JAN 1991 & & & & & & & & & & & \\
\hline $17 \ldots$ & 11 & $\cdots$ & $\cdots$ & $\cdots$ & $\cdots$ & $\cdots$ & -- & $\cdots$ & $\cdots$ & 80 & 59 \\
\hline MAR & & & & & & & & & & & \\
\hline $26 \ldots$ & 12 & $\cdots$ & $\cdots$ & $\cdots$ & $\cdots$ & $\cdots$ & $\cdots$ & $\cdots$ & $\cdots$ & 40 & 31 \\
\hline APR & & & & & & & & & & & \\
\hline $25 \ldots$ & 26 & $\cdots$ & $\cdots$ & $\cdots$ & $\cdots$ & $\cdots$ & $\cdots$ & $\cdots$ & $\cdots$ & 80 & 73 \\
\hline MAY & & & & & & & & & & & \\
\hline $15 \ldots$ & 23 & $\cdots$ & $\cdots$ & $\cdots$ & $\cdots$ & $\cdots$ & $\cdots$ & $\cdots$ & $\cdots$ & 170 & 41 \\
\hline JUN & & & & & & & & & & & \\
\hline $12 \ldots$ & 10 & $\cdots$ & $\cdots$ & $\cdots$ & $\cdots$ & $-\cdot$ & $\cdots$ & $\cdots$ & $\cdots$ & 240 & 50 \\
\hline $19 \ldots$ & 10 & $<0.10$ & $<0.1$ & 2 & $<1$ & $<1$ & $<1$ & $<1$ & $<1.0$ & 90 & 42 \\
\hline JUL & & & & & & & & & & & \\
\hline $17 \ldots$ & 9 & $\cdots$ & $\cdots$ & $\cdots$ & $\cdots$ & $\cdots$ & $\cdots$ & $\cdots$ & $\cdots$ & 30 & 26 \\
\hline AUG & & & & & & & & & & & \\
\hline $15 \ldots$ & 15 & $\cdots$ & $\cdots$ & $\cdots$ & $\cdots$ & $\cdots$ & $\cdots$ & $\cdots$ & $-\cdot$ & 60 & 41 \\
\hline SEP & & & & & & & & & & & \\
\hline $03 \ldots$ & $\cdots$ & $<0.10$ & $<0.1$ & $<1$ & $<1$ & $<1$ & $<1$ & $<1$ & $<1.0$ & $\cdots$ & - \\
\hline OCT & & & & & & & & & & & \\
\hline $23 \ldots$ & 12 & $\cdots$ & -- & $\cdots$ & $\cdots$ & $\cdots$ & $\cdots$ & $-\cdot$ & $\cdots$ & 30 & 23 \\
\hline DEC & & & & & & & & & & & \\
\hline $18 \ldots$ & 13 & $\cdots$ & $\cdots$ & $\cdots$ & - & $\cdots$ & $\cdots$ & $\cdots$ & $\cdots$ & 80 & 44 \\
\hline MAR 1992 & & & & & & & & & & & \\
\hline $24 \ldots$ & 12 & $\cdots$ & $\cdots$ & $\cdots$ & $\cdots$ & $\cdots$ & $\cdots$ & $\cdots$ & $\cdots$ & 40 & 10 \\
\hline APR & & & & & & & & & & & \\
\hline $22 \ldots$ & 22 & $\cdots$ & - & - & -- & -- & $\cdots$ & $\cdots$ & $\cdots$ & 70 & 30 \\
\hline MAY & & & & & & & & & & & \\
\hline $21 \ldots$ & 17 & $\cdots$ & $\cdots$ & - & - & $\cdots$ & $-\cdot$ & $\cdots$ & - & 280 & 31 \\
\hline JUN & & & & & & & & & & & \\
\hline $25 \ldots$ & 7 & $<0.10$ & 0.3 & 1 & $<1$ & $<1$ & $<1$ & $<1$ & $<1.0$ & 60 & 23 \\
\hline JUL & & & & & & & & & & & \\
\hline $14 \ldots$ & 9 & $\cdots$ & $\cdots$ & $\cdots$ & $\cdots$ & -- & - & $\cdots$ & $\cdots$ & 30 & 16 \\
\hline AUG & & & & & & & & & & & \\
\hline $12 \ldots$ & 7 & $<0.10$ & $<0.1$ & $<1$ & $<1$ & $<1$ & $<1$ & $<1$ & $<1.0$ & 30 & 16 \\
\hline $25 \ldots$ & 9 & - & $\cdots$ & $\cdots$ & -. & $\cdots$ & -. & $\cdot$ & $\cdots$ & 100 & 4 \\
\hline OCT & & & & & & & & & & & \\
\hline $28 \ldots$ & 10 & $\cdots$ & $\cdots$ & $\cdots$ & $\cdots$ & $\cdots$ & $\cdots$ & $\cdots$ & $\cdots$ & 20 & 20 \\
\hline JAN 1993 & & & & & & & & & & & \\
\hline $12 \ldots$ & 10 & $\cdots$ & $\cdots$ & $\cdots$ & $\cdots$ & $\cdots$ & $\cdots$ & $\cdots$ & $\cdots$ & 60 & 33 \\
\hline MAR & & & & & & & & & & & \\
\hline $23 \ldots$ & 10 & $\cdots$ & $\cdots$ & $\cdots$ & $\cdots$ & $\cdots$ & -. & $\cdots$ & $\cdots$ & 30 & 18 \\
\hline
\end{tabular}


Table 34. Onsite measurements and selected inorganic data for station 07093775, Badger Creek, Lower Station, near Howard

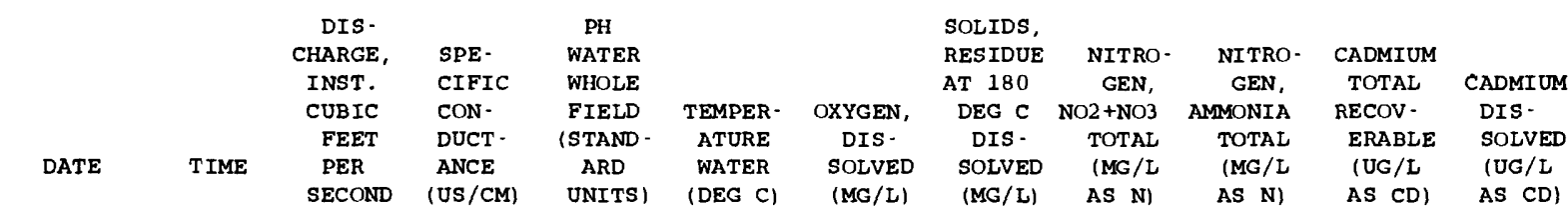

\begin{tabular}{|c|c|c|c|c|c|c|c|c|c|c|c|}
\hline APR 1990 & & & & & & & & & & & \\
\hline$\pi_{\pi N}^{19}$ & 1610 & 12 & 850 & 8.7 & 7.0 & 9.4 & 510 & 0.014 & $<0.010$ & 2 & $<0.1$ \\
\hline JUL $07 \ldots$ & 0730 & 8.6 & -. & 8.3 & 8.5 & 9.0 & 556 & $<0.010$ & 0.010 & $<1$ & $<0.1$ \\
\hline${ }_{A U G}^{18} \ldots$ & 1545 & 5.5 & 970 & 8.9 & 24.0 & 6.7 & 560 & 0.012 & $<0.010$ & $<1$ & $<0.1$ \\
\hline$\underset{\text { Nov }}{29}$ & 1400 & 7.4 & 980 & 8.3 & 26.0 & 6.0 & 606 & $<0.010$ & 0.010 & $<1$ & $<0.1$ \\
\hline $\begin{array}{c}01 \ldots \\
\text { APR } 1991\end{array}$ & 0910 & 7.8 & 1020 & 8.7 & 7.0 & 9.3 & 572 & $<0.010$ & 0.020 & $<1$ & $<0,1$ \\
\hline $\begin{array}{l}25 \ldots \\
\text { JoN }\end{array}$ & 0900 & 14 & 808 & 8.4 & 1.5 & 10.8 & 456 & 0.018 & 0.016 & $<1$ & 1.5 \\
\hline${ }_{\text {JUL }}^{19} \ldots$ & 1815 & 7.4 & 978 & 8.7 & 16.5 & 7.4 & 544 & 0.008 & 0.017 & $<1$ & 0.1 \\
\hline$\underset{\mathrm{OCT}}{18 \ldots}$ & 0810 & 4.6 & 1030 & 8.5 & 14.0 & 7.7 & 578 & 0.008 & 0.009 & $<1$ & $<0.1$ \\
\hline $\begin{array}{r}23 \ldots \\
\text { APR } 1992\end{array}$ & 1620 & 5.9 & 1050 & 8.3 & 12.5 & 8.0 & 608 & 0.008 & 0.019 & $<1$ & $<0.1$ \\
\hline$\underset{\text { JUN }}{22 \ldots}$ & 1710 & 12 & 920 & 8.7 & 13.0 & 8.0 & 540 & 0.015 & 0.011 & $<1$ & $<0.1$ \\
\hline${ }_{A U G}^{25} \ldots$ & 1640 & 7.4 & 953 & 8.8 & 19.5 & 7.4 & 538 & $<0.005$ & 0.021 & $<1$ & 0.2 \\
\hline$\underset{\text { OCT }}{13 \ldots}$ & 0730 & 8.2 & 971 & 8.5 & 12.0 & 8.5 & 564 & 0.008 & 0.026 & $<1$ & $<0.1$ \\
\hline $29 \ldots$ & 0730 & 7.4 & 1020 & 8.6 & 6.0 & 9.5 & 708 & $<0.005$ & 0.019 & $<1$ & $<0.1$ \\
\hline
\end{tabular}

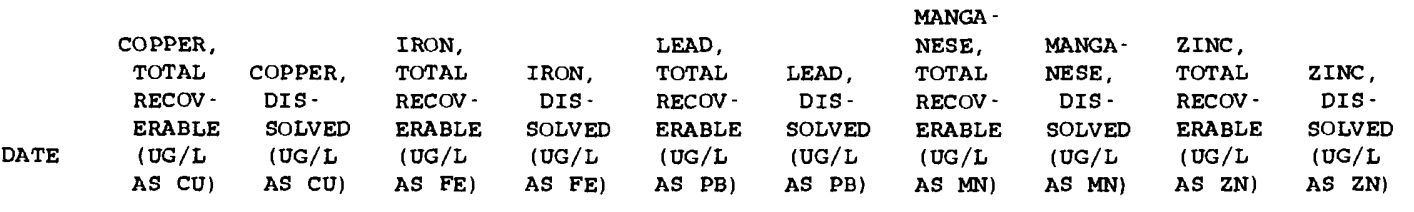

\begin{tabular}{|c|c|c|c|c|c|c|c|c|c|c|}
\hline \multicolumn{11}{|l|}{ APR 1990} \\
\hline $19 \ldots$ & 4 & 1 & 1400 & 9 & 3 & $<0.5$ & 60 & 5 & 30 & 12 \\
\hline JUN & & & & & & & & & & \\
\hline $07 \ldots$ & 4 & 1 & 280 & 7 & 1 & $<0.5$ & 10 & 4 & $<10$ & 4 \\
\hline $\begin{array}{l}\text { JUL } \\
18 \ldots\end{array}$ & & & & & & & & & & \\
\hline${ }_{\text {AUG }}^{18} \cdots$ & 3 & 2 & 1000 & 41 & 2 & $<0.5$ & 60 & 5 & $<10$ & $<3$ \\
\hline $\operatorname{Nov}^{29} \ldots$ & 4 & $<1$ & 1400 & 64 & 2 & $<0.5$ & 170 & 75 & 20 & $<3$ \\
\hline $\begin{array}{c}01 \ldots \\
A P R \\
A 991\end{array}$ & 4 & $<1$ & 200 & 12 & $<1$ & $<0.5$ & 30 & 14 & $<10$ & 7 \\
\hline$\underset{\pi N N}{25} \cdots$ & 3 & 1 & 1700 & 30 & 3 & $<0.5$ & 50 & 4 & 10 & 6 \\
\hline JUL $^{19} \cdots$ & 13 & 1 & 390 & 14 & 2 & $<0.5$ & 30 & 6 & $<10$ & $<3$ \\
\hline$\underset{O C T}{18 \cdots}$ & 4 & 1 & 590 & 10 & 5 & $<0.5$ & 160 & 140 & $<10$ & 6 \\
\hline $\begin{array}{c}23 \ldots \\
A P R \quad 1992\end{array}$ & 3 & $<1$ & 190 & 7 & 2 & 0.8 & 30 & 8 & $<10$ & 8 \\
\hline$J_{J N} 22 \cdots$ & $<1$ & $<1$ & 360 & 7 & $<1$ & $<0.5$ & 30 & 5 & 10 & $<3$ \\
\hline$\underset{A U G}{25 \ldots}$ & 3 & 2 & 200 & 10 & $<1$ & 0.7 & 20 & 5 & $<10$ & 4 \\
\hline $\mathrm{OCT}^{13}$ & 2 & $<1$ & 1000 & 10 & 1 & 0.6 & 40 & 6 & $<10$ & 4 \\
\hline $29 \ldots$ & $<1$ & $<1$ & 110 & 7 & $<1$ & $<0.5$ & $<10$ & 4 & $<10$ & $<3$ \\
\hline
\end{tabular}


Table 35. Onsite measurements and selected inorganic data for station 07094000, Texas Creek at Texas Creek

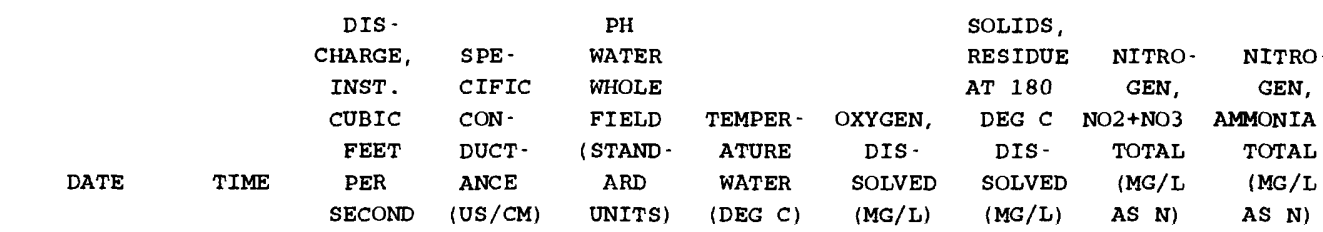

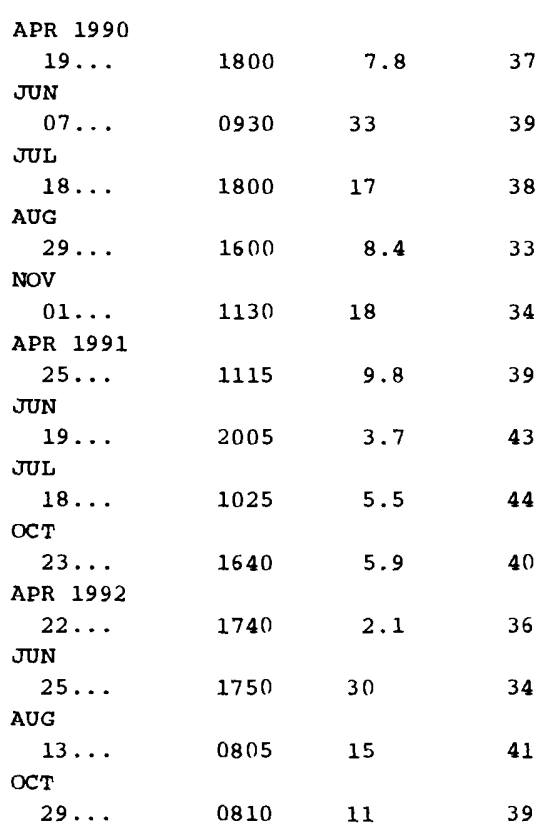

$\begin{array}{rrrrrrr}370 & 8.8 & 8.5 & 9.4 & 233 & 0.087 & <0.010 \\ 399 & 8.4 & 12.5 & 8.0 & 215 & 0.131 & 0.020 \\ 382 & 8.6 & 19.5 & 7.1 & 254 & 0.025 & <0.010 \\ 337 & 8.4 & 23.0 & 6.7 & 219 & <0.010 & <0.010 \\ 343 & 8.8 & 7.5 & 9.5 & 205 & <0.010 & 0.020 \\ 92 & 8.5 & 10.5 & 9.0 & 230 & 0.014 & 0.021 \\ 31 & 8.4 & 17.0 & 6.8 & 243 & 0.011 & 0.020 \\ 44 & 8.6 & 18.0 & 7.3 & 254 & 0.007 & 0.018 \\ 41 & 8.7 & 11.5 & 8.6 & 254 & 0.010 & 0.021 \\ 365 & 8.8 & 14.5 & 7.6 & 219 & 0.012 & 0.020 \\ 34 & 8.7 & 18.0 & 7.5 & 216 & 0.019 & 0.047 \\ 16 & 8.6 & 14.0 & 8.5 & 256 & 0.005 & 0.022 \\ 91 & 8.7 & 7.0 & 9.6 & 258 & <0.005 & 0.011\end{array}$


Table 36. Onsite measurements and selected inorganic data for station 382917105225200 , Tallahassee Creek near Parkdale

\begin{tabular}{|c|c|c|c|c|c|c|c|c|c|c|c|}
\hline DATE & TIME & $\begin{array}{c}\text { DIS- } \\
\text { CHARGE, } \\
\text { INST. } \\
\text { CUBIC } \\
\text { FEET } \\
\text { PER } \\
\text { SECOND }\end{array}$ & $\begin{array}{l}\text { SPE- } \\
\text { CIFIC } \\
\text { CON- } \\
\text { DUCT - } \\
\text { ANCE } \\
\text { (US /CM) }\end{array}$ & $\begin{array}{c}\text { PH } \\
\text { WATER } \\
\text { WHOLE } \\
\text { FIELD } \\
\text { (STAND- } \\
\text { ARD } \\
\text { UNITS) }\end{array}$ & $\begin{array}{c}\text { TEMPER - } \\
\text { ATURE } \\
\text { WATER } \\
\text { (DEG C) }\end{array}$ & $\begin{array}{c}\text { OXYGEN, } \\
\text { DIS- } \\
\text { SOLVED } \\
\text { (MG/L) }\end{array}$ & $\begin{array}{l}\text { SOLIDS, } \\
\text { RESIDUE } \\
\text { AT } 180 \\
\text { DEG C } \\
\text { DIS - } \\
\text { SOLVED } \\
\text { (MG/L) }\end{array}$ & $\begin{array}{l}\text { NITRO- } \\
\text { GEN, } \\
\text { NO2+NO3 } \\
\text { TOTAL } \\
\text { (MG/L } \\
\text { AS N) }\end{array}$ & $\begin{array}{l}\text { NITRO- } \\
\text { GEN, } \\
\text { AMMONIA } \\
\text { TOTAL } \\
\text { (MG/L } \\
\text { AS N) }\end{array}$ & $\begin{array}{l}\text { CADMIUM } \\
\text { TOTAL } \\
\text { RECOV - } \\
\text { ERABLE } \\
\text { (UG/L } \\
\text { AS CD) }\end{array}$ & $\begin{array}{l}\text { CADMIUM } \\
\text { DIS - } \\
\text { SOLVED } \\
\text { (UG/L } \\
\text { AS CD) }\end{array}$ \\
\hline PR 1990 & & & & & & & & & & & \\
\hline $20 \ldots$ & 0730 & 0.54 & 708 & 8.3 & 8.0 & 8.6 & 451 & 0.104 & $<0.010$ & $<1$ & $<0.1$ \\
\hline JUL & 1000 & 2.7 & 600 & 8.5 & 20.0 & 7.4 & 365 & 0.062 & 0.030 & $<1$ & $<0.1$ \\
\hline$\underset{A U G}{19} \cdots$ & 0755 & 4.7 & 691 & 8.5 & 15.0 & 7.8 & 428 & 0.203 & 0.030 & $<1$ & 0.1 \\
\hline $\begin{array}{l}30 \ldots \\
\text { Nov }\end{array}$ & 0840 & 2.8 & 698 & 8.4 & 15.0 & 7.9 & 431 & 0.190 & 0.020 & $<1$ & $<0.1$ \\
\hline $\begin{array}{c}01 \ldots \\
A P R\end{array}$ & 1300 & 3.6 & 689 & 8.7 & 11.5 & 8.6 & 416 & 0.020 & 0.020 & $<1$ & $<0.1$ \\
\hline$\underset{\text { JUN }}{25 \ldots}$ & 1315 & 0.14 & 744 & 8.1 & 13.0 & 8.3 & 456 & 0.103 & 0.019 & $<1$ & $<0.1$ \\
\hline${ }_{\text {JUL }}^{20 \ldots}$ & 0905 & 0.40 & 806 & 8.3 & 15.0 & 8.5 & 488 & 0.018 & 0.019 & $<1$ & $<0.1$ \\
\hline $\begin{array}{c}18 \ldots \\
\text { OCT }\end{array}$ & 0845 & 0.70 & 783 & 8.2 & 17.5 & 7.3 & 460 & 0.040 & 0.020 & $<1$ & $<0.1$ \\
\hline $\begin{array}{c}24 \ldots \\
\text { APR } 1992\end{array}$ & 0825 & 3.8 & 734 & 8.5 & 8.0 & 8.7 & 428 & 0.034 & 0.029 & $<1$ & $<0.1$ \\
\hline$\underset{\text { JUN }}{23 \ldots}$ & 0845 & 4.2 & 679 & 8.6 & 7.0 & 9.2 & 406 & 0.133 & 0.018 & $<1$ & 0.1 \\
\hline$\underset{A U G}{26 \ldots}$ & 0845 & 32 & 386 & 8.4 & 14.0 & 7.7 & 220 & 0.122 & 0.137 & $<1$ & $<0.1$ \\
\hline${ }_{\text {OCT }}^{13 \ldots}$ & 1010 & 0.68 & 682 & 8.4 & 17.0 & 7.8 & 402 & 0.055 & 0.017 & $<1$ & $<0.1$ \\
\hline $29 \ldots$ & 0950 & 0.20 & 706 & 8.4 & 10.5 & 8.6 & 411 & 0.039 & 0.024 & $<1$ & $<0.1$ \\
\hline
\end{tabular}

\begin{tabular}{|c|c|c|c|c|c|c|c|c|c|c|}
\hline ATE & $\begin{array}{l}\text { COPPER, } \\
\text { TOTAL } \\
\text { RECOV- } \\
\text { ERABLE } \\
\text { (UG/L }\end{array}$ & $\begin{array}{l}\text { COPPER, } \\
\text { DIS- } \\
\text { SOLVED } \\
\text { (UG/L }\end{array}$ & $\begin{array}{l}\text { IRON, } \\
\text { TOTAL } \\
\text { RECOV- } \\
\text { ERABLE } \\
\text { (UG/L }\end{array}$ & $\begin{array}{l}\text { IRON, } \\
\text { DIS - } \\
\text { SOLVED } \\
\text { (UG/L }\end{array}$ & $\begin{array}{l}\text { LEAD, } \\
\text { TOTAL } \\
\text { RECOV- } \\
\text { ERABLE } \\
\text { (UG/L }\end{array}$ & $\begin{array}{l}\text { LEAD, } \\
\text { DIS: } \\
\text { SOLVED } \\
\text { (UG/L }\end{array}$ & $\begin{array}{l}\text { MANGA- } \\
\text { NESE, } \\
\text { TOTAL } \\
\text { RECOV - } \\
\text { ERABLE } \\
\text { (UG /L }\end{array}$ & $\begin{array}{l}\text { MANGA- } \\
\text { NESE, } \\
\text { DIS - } \\
\text { SOLVED } \\
\text { /UG/L }\end{array}$ & $\begin{array}{l}\text { ZINC, } \\
\text { TOTAL } \\
\text { RECOV- } \\
\text { ERABLE } \\
\text { (UG/L }\end{array}$ & $\begin{array}{l}\text { ZINC, } \\
\text { DIS- } \\
\text { SOLVED } \\
\text { IUG /L }\end{array}$ \\
\hline & AS CU) & AS CU) & AS FE) & AS FE) & AS PB) & AS PB) & AS MN) & AS MN) & AS $\mathrm{ZN}$ ) & AS $\mathrm{ZN}$ ) \\
\hline
\end{tabular}

\begin{tabular}{|c|c|c|c|c|c|c|c|c|c|c|}
\hline $\begin{array}{c}\text { APR } 1990 \\
20 \ldots \\
\text { JUN }\end{array}$ & 3 & 2 & 370 & 11 & 1 & $<0.5$ & 190 & 130 & 20 & 5 \\
\hline $\begin{array}{l}07 \ldots \\
\text { JUL }\end{array}$ & 5 & 1 & 650 & 16 & 2 & $<0.5$ & 100 & 69 & $<10$ & 9 \\
\hline$\underset{A U G}{19} \cdots$ & 7 & 1 & 6300 & 10 & 5 & $<0.5$ & 360 & 120 & 30 & $<3$ \\
\hline${ }_{\text {Nov }}^{30 \ldots}$ & 2 & 1 & 510 & 15 & 1 & $<0.5$ & 140 & 100 & 10 & $<3$ \\
\hline $\begin{array}{c}01 \ldots \\
\text { APR } 1991\end{array}$ & 1 & 1 & 120 & 18 & $<1$ & $<0.5$ & 60 & 39 & $<10$ & 6 \\
\hline$\underset{\text { JUN }}{25 \ldots}$ & 5 & 1 & 220 & 16 & 1 & $<0.5$ & 50 & 38 & 10 & 8 \\
\hline${ }_{\text {JUL }}^{20 . . .}$ & 3 & 1 & 150 & 13 & 1 & $<0.5$ & 90 & 73 & $<10$ & $<3$ \\
\hline $\mathrm{OCT}^{18 \ldots}$ & 6 & $<1$ & 160 & 18 & 5 & $<0.5$ & 150 & 120 & $<10$ & $<3$ \\
\hline $\begin{array}{r}24 \ldots \\
\text { APR } 1992\end{array}$ & 2 & $<1$ & 170 & 31 & 1 & 0.6 & 80 & 62 & $<10$ & 3 \\
\hline $23 \ldots$ & 2 & $<1$ & 870 & 8 & $<1$ & $<0.5$ & 120 & 60 & 20 & 4 \\
\hline$\underset{A U G}{26 \ldots}$ & 20 & 2 & 7600 & 71 & 4 & $<0.5$ & 1800 & 16 & 150 & $<3$ \\
\hline$\underset{\text { OCT }}{13 \ldots}$ & 1 & $<1$ & 110 & 24 & $<1$ & $<0.5$ & 80 & 67 & $<10$ & $<3$ \\
\hline $29 \ldots$ & $<1$ & $<1$ & 30 & 17 & $<1$ & $<0.5$ & $<10$ & 29 & $<10$ & $<3$ \\
\hline
\end{tabular}


Table 37. Onsite measurements and bacteriological and selected inorganic data for station 07094500 , Arkansas River at Parkdale

\begin{tabular}{|c|c|c|c|c|c|c|c|c|c|c|c|c|}
\hline DATE & TIME & $\begin{array}{c}\text { DIS - } \\
\text { CHARGE, } \\
\text { INST. } \\
\text { CUBIC } \\
\text { FEET } \\
\text { PER } \\
\text { SECOND }\end{array}$ & $\begin{array}{l}\text { SPE- } \\
\text { CIFIC } \\
\text { CON- } \\
\text { DUCT - } \\
\text { ANCE } \\
\text { (US/CM) }\end{array}$ & $\begin{array}{c}\text { PH } \\
\text { WATER } \\
\text { WHOLE } \\
\text { FIELD } \\
\text { (STAND - } \\
\text { ARD } \\
\text { UNITS) }\end{array}$ & $\begin{array}{c}\text { TEMPER - } \\
\text { ATURE } \\
\text { WATER } \\
\text { (DEG C) }\end{array}$ & $\begin{array}{c}\text { OXYGEN, } \\
\text { DIS - } \\
\text { SOLVED } \\
\text { (MG /L) }\end{array}$ & $\begin{array}{l}\text { COLI - } \\
\text { FORM, } \\
\text { TOTAL, } \\
\text { IMMED. } \\
\text { (COLS. } \\
\text { PER } \\
100 \mathrm{ML} \text { ) }\end{array}$ & $\begin{array}{l}\text { COLI - } \\
\text { FORM, } \\
\text { FECAL, } \\
0.7 \\
\text { UM-MF } \\
\text { (COLS./ } \\
100 \mathrm{ML} \text { ) }\end{array}$ & $\begin{array}{c}\text { STREP- } \\
\text { TOCOCCI } \\
\text { FECAL, } \\
\text { KF AGAR } \\
\text { (COLS. } \\
\text { PER } \\
\text { (00 ML) }\end{array}$ & $\begin{array}{l}\text { HARD - } \\
\text { NESS } \\
\text { TOTAL } \\
\text { (MG /L } \\
\text { AS } \\
\text { CACO3) }\end{array}$ & $\begin{array}{l}\text { CALCIUM } \\
\text { DIS - } \\
\text { SOLVED } \\
\text { (MG /L } \\
\text { AS CA) }\end{array}$ & $\begin{array}{l}\text { MAGNE - } \\
\text { SIUM, } \\
\text { DIS- } \\
\text { SOLVED } \\
\text { (MG /L } \\
\text { AS MG) }\end{array}$ \\
\hline APR 1990 & & & & & & & & & & & & \\
\hline$\underset{M A Y}{20} \ldots$ & 0845 & 343 & 306 & 8.4 & 9.0 & 9.4 & -- & $\mathrm{K} 2$ & 41 & 140 & 39 & 10 \\
\hline${ }_{\text {JUN }}^{24 \ldots}$ & 0900 & 674 & 242 & 8.2 & 15.0 & 7.9 & $\cdots$ & 62 & $\mathrm{~K} 490$ & 98 & 28 & 6.8 \\
\hline $07 \ldots$ & 0815 & 3110 & 125 & 8.0 & 13.5 & 8.5 & K40 & K70 & 690 & 53 & 16 & 3.2 \\
\hline${ }_{\text {JUL }}^{21 \ldots}$ & 0840 & 1990 & 135 & 8.0 & 15.0 & 8.0 & $<10$ & K16 & 200 & 56 & 16 & 3.8 \\
\hline${ }_{\text {AUG }}^{19} \ldots$ & 0940 & 1110 & 181 & 8.2 & 17.5 & 7.7 & K36 & K23 & 62 & 75 & 22 & 4.8 \\
\hline${ }_{\mathrm{Nov}}^{30 \ldots}$ & 1110 & 395 & 296 & 8.3 & 20.0 & 7.5 & $<2$ & $\mathrm{~K} 1$ & K5 & 120 & 36 & 8.0 \\
\hline $\begin{array}{c}01 \ldots \\
\text { JAN } 1991\end{array}$ & 1400 & 476 & 310 & 8.6 & 9.0 & 9.9 & $\cdots$ & $\cdots$ & $\cdots$ & 130 & 38 & 9.1 \\
\hline${ }_{\operatorname{MAR}}^{17 \ldots}$ & 1200 & 515 & 214 & 8.1 & 0.0 & 12.1 & $<5$ & $<1$ & 8 & 96 & 28 & 6.4 \\
\hline $\begin{array}{l}26 \ldots \\
A P R\end{array}$ & 1610 & 410 & 259 & 8.8 & 7.5 & 9.4 & $\cdots$ & $<2$ & E6 & 100 & 30 & 6.8 \\
\hline${ }_{\operatorname{MAY}}^{25 \ldots}$ & 1400 & 443 & 229 & 8.1 & 11.5 & 9.0 & 120 & E1 & E8 & 90 & 26 & 6.0 \\
\hline $\begin{array}{c}16 \ldots \\
\text { JUN }\end{array}$ & 0830 & 956 & 161 & 8.0 & 12.0 & 8.6 & E17 & E4 & 60 & 64 & 19 & 3.9 \\
\hline $\begin{array}{l}12 \ldots \\
20 \ldots\end{array}$ & $\begin{array}{l}1700 \\
0910\end{array}$ & $\begin{array}{l}2620 \\
1970\end{array}$ & $\begin{array}{l}-\cdot \\
133\end{array}$ & $\begin{array}{l}-- \\
7.8\end{array}$ & 14.0 & $\cdots$ & ${ }^{-}$ & ${ }_{\text {E2 }}$ & $\cdots$ & ${ }^{\cdots}$ & $16^{-\cdot}$ & $\begin{array}{l}\cdots \\
3.7\end{array}$ \\
\hline JUL & & & & & & & & & & & & \\
\hline$\underbrace{18 \ldots}_{\text {AUG }}$ & 0945 & 848 & 199 & 8.4 & 19.5 & 7.5 & $<2$ & E6 & 36 & 91 & 27 & 5.8 \\
\hline $\operatorname{SEP}^{15 \ldots}$ & 1100 & 641 & 265 & 8.5 & 19.0 & $\cdots$ & $<3$ & E5 & 89 & 110 & 33 & 7.6 \\
\hline OCT & 1625 & 410 & 307 & 8.7 & 20.5 & 7.7 & $\cdots$ & $\cdots$ & $\cdots$ & $-\cdot$ & $\cdots$ & $\cdots$ \\
\hline${ }_{\mathrm{DEC}}^{24 \ldots}$ & 0820 & 308 & 344 & 8.5 & 9.0 & 8.9 & 11 & $\cdots$ & 8 & 150 & 43 & 10 \\
\hline $\begin{array}{c}18 \ldots \\
\text { MAR } 1992\end{array}$ & 1120 & 524 & 254 & 8.3 & 0.5 & 12.0 & $<1$ & E7 & E4 & 100 & 30 & 7.0 \\
\hline$\underset{A P R}{24 \ldots}$ & 1630 & 469 & 236 & 8.5 & 8.0 & 10.0 & - & $\cdots$ & $\cdots$ & 97 & 28 & 6.5 \\
\hline $\operatorname{MAY}^{23 \ldots}$ & 0820 & 321 & 302 & 8.4 & 9.5 & 9.3 & $<2$ & E1 & E9 & 110 & 32 & 8.2 \\
\hline$\underset{J U N}{22 \ldots}$ & 0730 & 1550 & 139 & 8.1 & 12.0 & 8.7 & E28 & 50 & 130 & 60 & 18 & 3.7 \\
\hline $\begin{array}{l}26 \ldots \\
\pi U L\end{array}$ & 0900 & 1610 & 173 & 8.2 & 15.0 & 8.0 & $\cdots$ & 930 & 2700 & 76 & 22 & 5.0 \\
\hline${ }_{A U G}^{15 \ldots}$ & 0915 & 880 & 210 & 8.3 & 17.0 & 8.0 & 72 & E7 & E13 & 92 & 27 & 6.0 \\
\hline $13 \ldots$ & 1000 & 843 & 213 & 8.4 & 17.0 & 7.9 & E24 & E15 & 33 & 92 & 27 & 6.0 \\
\hline$\underset{\text { OCT }}{25 \ldots}$ & 0700 & 1770 & 242 & 8.3 & 12.0 & 8.9 & $\cdots$ & E3500 & $>3300$ & 100 & 31 & 5.9 \\
\hline $\begin{array}{c}29 \ldots \\
\text { JAN } 1993\end{array}$ & 0920 & 438 & 312 & 8.6 & 9.5 & 9.7 & E5 & E4 & $\cdots$ & 130 & 38 & 9.1 \\
\hline $13 \ldots$ & 0815 & 459 & 255 & 8.2 & 0.0 & 11.3 & $<2$ & $<1$ & E3 & 100 & 30 & 7.0 \\
\hline $\begin{array}{l}\text { MAR } \\
23 \ldots\end{array}$ & 1410 & 732 & 179 & 8.5 & 10.0 & 9.3 & $\cdots$ & $<1$ & $\cdots$ & 72 & 21 & 4.8 \\
\hline
\end{tabular}


Table 37. Onsite measurements and bacteriological and selected inorganic data for station 07094500 , Arkansas River at Parkdale--Continued

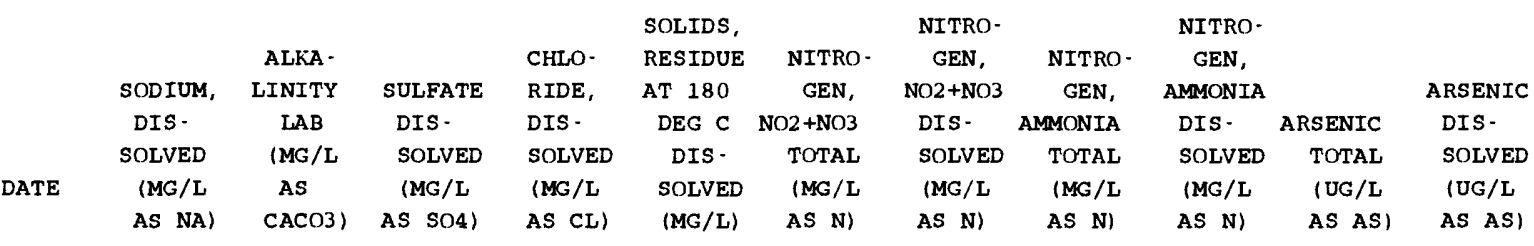

\begin{tabular}{|c|c|c|c|c|c|c|c|c|c|c|c|}
\hline \multicolumn{12}{|l|}{ APR 1990} \\
\hline $20 \ldots$ & 14 & 105 & 36 & 9.9 & 185 & 0.040 & $\cdots$ & $<0.010$ & $\cdots$ & - & $\cdots$ \\
\hline \multicolumn{12}{|l|}{ MAY } \\
\hline $24 \ldots$ & 9.2 & 81 & 26 & 7.0 & 140 & 0.166 & $\cdots$ & 0.020 & $\cdots$ & $\cdots$ & $\cdots$ \\
\hline \multicolumn{12}{|l|}{ JON } \\
\hline $07 \ldots$ & 3.2 & 41 & 16 & 1.2 & 73 & 0.088 & $\cdots$ & 0.060 & $\cdots$ & -- & $\cdots$ \\
\hline $21 \ldots$ & 3.7 & 45 & 1.7 & 2.5 & 72 & 0.067 & $\cdots$ & $<0.010$ & - & -. & $\cdots$ \\
\hline \multicolumn{12}{|l|}{ JUL } \\
\hline $19 \ldots$ & 5.8 & 63 & 20 & 4.0 & 102 & 0.013 & $\cdots$ & $<0.010$ & $\cdots$ & $\cdots$ & $\cdots$ \\
\hline \multicolumn{12}{|l|}{ AUG } \\
\hline $30 \ldots$ & 12 & 111 & 33 & 8.8 & 172 & 0.025 & $\cdots$ & $<0.010$ & $\cdots$ & $<1$ & $<1$ \\
\hline \multicolumn{12}{|l|}{ NOV } \\
\hline $01 \ldots$ & 13 & 118 & 28 & 9.9 & 179 & 0.030 & $\cdots$ & 0.020 & $\cdots$ & $\cdots$ & $\cdots$ \\
\hline \multicolumn{12}{|l|}{ JAN 1991} \\
\hline $17 \ldots$ & 8.9 & 81 & 25 & 6.0 & 120 & 0.170 & $\cdots$ & 0.024 & $-\cdot$ & $\cdots$ & $\cdots$ \\
\hline \multicolumn{12}{|l|}{ MAR } \\
\hline $26 \ldots$ & 9.7 & 90 & 30 & 8.1 & 149 & 0.008 & $\cdots$ & 0.026 & $\cdots$ & $\cdots$ & $\cdots$ \\
\hline \multicolumn{12}{|l|}{ APR } \\
\hline $25 \ldots$ & 9.1 & 78 & 27 & 8.7 & 127 & 0.006 & $\cdots$ & 0.013 & $\cdots$ & $-\cdot$ & $\cdots$ \\
\hline \multicolumn{12}{|l|}{ MAY } \\
\hline $16 \ldots$ & 5.5 & 54 & 21 & 3.7 & 93 & 0.052 & $\cdots$ & 0.021 & $\cdots$ & $\cdots$ & $\cdots$ \\
\hline \multicolumn{12}{|l|}{ JUN } \\
\hline $12 \ldots$ & -. & $\cdots$ & $\cdots$ & $\cdots$ & - & $\cdots$ & - & $\cdots$ & -- & $\cdots$ & $\cdots$ \\
\hline $20 \ldots$ & 3.6 & 45 & 12 & 2.1 & 84 & 0.070 & $\cdots$ & 0.019 & -. & $<1$ & $<1$ \\
\hline \multicolumn{12}{|l|}{ JUL } \\
\hline $18 \ldots$ & 6.7 & 75 & 21 & 4.9 & 112 & 0.006 & $\cdots$ & 0.016 & $\cdots$ & $\cdots$ & $\cdots$ \\
\hline \multicolumn{12}{|l|}{ AUG } \\
\hline $15 \ldots$ & 9.4 & 104 & 29 & 6.4 & 169 & 0.066 & $\cdots$ & $<0.002$ & $\cdots$ & $\cdots$ & $\cdots$ \\
\hline \multicolumn{12}{|l|}{ SEP } \\
\hline $03 \ldots$ & $\cdots$ & $\cdots$ & $\cdots$ & $\cdots$ & $\cdots$ & $\cdots$ & $\cdots$ & $\cdots$ & $\cdots$ & $<1$ & $<1$ \\
\hline \multicolumn{12}{|l|}{ OCT } \\
\hline $24 \ldots$ & 14 & 136 & 37 & 8.4 & 201 & 0.028 & $\cdots$ & 0.024 & $\cdots$ & $\cdots$ & $\cdots$ \\
\hline \multicolumn{12}{|l|}{ DEC } \\
\hline $18 \ldots$ & 9.9 & 97 & 26 & 6.6 & 149 & 0.141 & $\cdots$ & 0.007 & $\cdots$ & $\cdots$ & $\cdots$ \\
\hline \multicolumn{12}{|l|}{ MAR 1992} \\
\hline $24 \ldots$ & 9.4 & 86 & 29 & 6.7 & 141 & 0.017 & $\cdots$ & 0.013 & $\cdots$ & $\cdots$ & $\cdots$ \\
\hline APR & & & & & & & & & & & \\
\hline $23 \ldots$ & 12 & 101 & 36 & 12 & 168 & 0.010 & $\cdots$ & 0.011 & $\cdots$ & $\cdots$ & $-\cdot$ \\
\hline MAY & & & & & & & & & & & \\
\hline $22 \ldots$ & 4.3 & 50 & 17 & 2.6 & 88 & 0.104 & $\cdots$ & 0.031 & $\cdots$ & $\cdots$ & $\cdots$ \\
\hline JUN & & & & & & & & & & & \\
\hline $26 \ldots$ & 5.1 & 69 & 20 & 2.8 & 96 & 0.044 & $\cdots$ & 0.008 & $\cdots$ & $<1$ & $<1$ \\
\hline JUL & & & & & & & & & & & \\
\hline $15 \ldots$ & 6.5 & 77 & 23 & 4.0 & 118 & 0.024 & $\cdots$ & 0.018 & $\cdots$ & $\cdots$ & -- \\
\hline AUG & & & & & & & & & & & \\
\hline $13 \ldots$ & 7.7 & 80 & 22 & 4.3 & 118 & 0.196 & $\cdots$ & 0.013 & $-\cdot$ & 1 & $<1$ \\
\hline $25 \ldots$ & 10 & 105 & 26 & 5.2 & 148 & 0.190 & $\cdots$ & 0.075 & -. & $\cdots$ & $\cdots$ \\
\hline OCT & & & & & & & & & & & \\
\hline $29 \ldots$ & 12 & 120 & 32 & 7.7 & 192 & 0.057 & $\cdots$ & 0.015 & $\cdots$ & $\cdots$ & $\cdots$ \\
\hline JAN 1993 & & & & & & & & & & & \\
\hline $13 \ldots$ & 9.1 & 95 & 26 & 4.9 & 146 & $\cdots$ & 0.206 & $\cdots$ & 0.020 & $\cdots$ & $\cdots$ \\
\hline MAR & & & & & & & & & & & \\
\hline $23 \ldots$ & 7.2 & 67 & 19 & 0.80 & 116 & $\cdots$ & 0.018 & $\cdots$ & 0.008 & $\cdots$ & $\cdots$ \\
\hline
\end{tabular}


Table 37. Onsite measurements and bacteriological and selected inorganic data for station 07094500 , Arkansas River at Parkdale--Continued

\begin{tabular}{|c|c|c|c|c|c|c|c|c|c|c|}
\hline $\begin{array}{l}\text { CADMIUM } \\
\text { TOTAL } \\
\text { RECOV- } \\
\text { ERABLE } \\
\text { (UG/L }\end{array}$ & $\begin{array}{l}\text { CADMIUM } \\
\text { DIS- } \\
\text { SOLVED } \\
\text { (UG /L }\end{array}$ & $\begin{array}{l}\text { CHRO- } \\
\text { MIUM, } \\
\text { TOTAL } \\
\text { RECOV- } \\
\text { ERABLE } \\
\text { (UG/L }\end{array}$ & $\begin{array}{l}\text { CHRO- } \\
\text { MIUM, } \\
\text { DIS- } \\
\text { SOLVED } \\
\text { IUG /L }\end{array}$ & $\begin{array}{c}\text { COPPER, } \\
\text { TOTAL } \\
\text { RECOV- } \\
\text { ERABLE } \\
\text { (UG/L }\end{array}$ & $\begin{array}{l}\text { COPPER, } \\
\text { DIS - } \\
\text { SOLVED } \\
\text { (UG } / L\end{array}$ & $\begin{array}{l}\text { IRON, } \\
\text { TOTAL } \\
\text { RECOV- } \\
\text { ERABLE } \\
\text { lUG } / L\end{array}$ & $\begin{array}{l}\text { IRON, } \\
\text { DIS - } \\
\text { SOLVED } \\
\text { lUG /L }\end{array}$ & $\begin{array}{l}\text { LEAD, } \\
\text { TOTAL } \\
\text { RECOV - } \\
\text { ERABLE } \\
\text { (UG/L }\end{array}$ & $\begin{array}{l}\text { LEAD, } \\
\text { DIS- } \\
\text { SOLVED } \\
\text { (UG/L }\end{array}$ & $\begin{array}{l}\text { MANGA- } \\
\text { NESE, } \\
\text { TOTAL } \\
\text { RECOV- } \\
\text { ERABLE } \\
\text { (UG/L }\end{array}$ \\
\hline AS $(D)$ & AS $C D$ ) & AS (R) & AS CR) & AS $\mathrm{CU})$ & AS CU) & AS FE) & AS FE) & AS PB) & AS PB) & AS MN) \\
\hline
\end{tabular}

APR 1990

$20 \ldots$

MAY

24...

JUN

$07 .$.

$21 .$.

JUL

19 ...

AUG

30 ...

NOV

$01 .$.

JAN 1991

$17 \ldots$

MAR

26 ...

APR

25...

MAY

$16 \ldots$

JUN

$12 \ldots$

$20 \ldots$

JUL

$18 \ldots$

AUG

$15 \ldots$

SEP

$03 \ldots$

OCT

24 ...

DEC

18...

MAR 1992

24 ...

APR

$23 \ldots$

MAY

22 ...

JUN

26 ...

JUL

15 ...

AUG

$13 \ldots$

$25 \ldots$

OCT

$29 \ldots$

JAN 1993

$13 \ldots$

MAR

$23 .$.
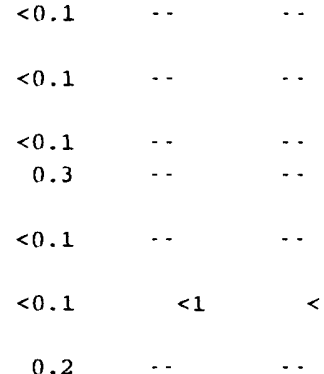

$<1 \quad 0.2$

$<1<0$.

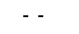

$\cdots$

-

$\begin{array}{ll}- & 19\end{array}$

-

$<1$

0.4

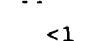

9
22

$$
<1
$$

$$
<1
$$$$
<0.1
$$$$
<0.1
$$

$$
<1
$$$$
<
$$$$
<0.1
$$$$
0.4
$$$$
<1<0
$$$$
<1
$$$$
5
$$$$
<1
$$$$
<0 \text {. }
$$$$
14
$$$$
<1
$$$$
<0 \text {. }
$$$$
<0.1
$$$$
<0.1
$$$$
<1
$$$$
<0.1
$$$$
<1<0.1
$$

$<1$

$<0.1$

\section{11
80
25}

8
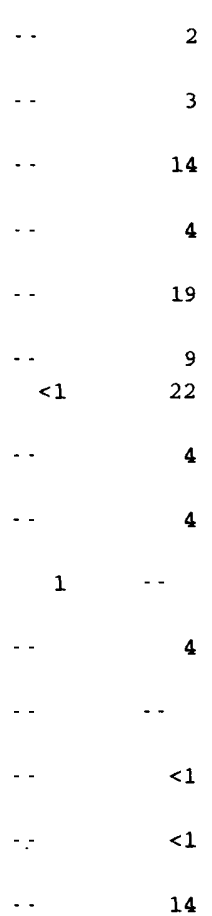

14

$<1 \quad 38$

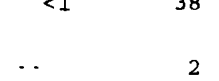

2

$\begin{array}{lr}<1 & 3 \\ \cdots & 45\end{array}$

1

1

$\begin{array}{rr}2 & 43 \\ 1 & 440 \\ 4 & 1500 \\ 3 & 270\end{array}$
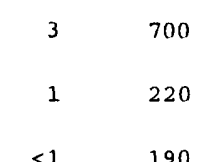

$\begin{array}{rr}17 & 3 \\ 17 & 32 \\ 51 & 120 \\ 16 & 22\end{array}$

$\begin{array}{rr}<0.5 & 60 \\ <0.5 & 490 \\ 1.6 & 890 \\ 1.2 & 140\end{array}$

$\begin{array}{ll}18 & 4 \\ 17 & 2\end{array}$

$\begin{array}{rr}0.6 & 40 \\ <0.5 & 30 \\ <0.5 & 20\end{array}$

2420

$18<1$

157

$$
<0.5 \quad 10
$$$$
<0.5 \quad 30
$$$$
<0.5 \quad 30
$$$$
1.1 \quad 180
$$$$
0.9260
$$$$
\begin{array}{rr}
0.9 & 260 \\
<0.5 & <10
\end{array}
$$$$
<0.5 \quad 40
$$$$
<0.5 \quad 50
$$$$
165
$$

$\begin{array}{ll}20 & 8 \\ 19 & 4\end{array}$
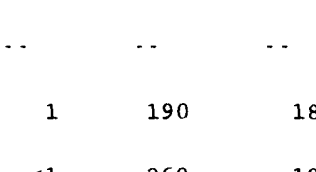

1

1

2320

$\begin{array}{rr}18 & 2 \\ 19 & <1 \\ 18 & <1 \\ 31 & 2 \\ 50 & 34 \\ 43 & 15 \\ 22 & 3 \\ 23 & 3 \\ 30 & 56 \\ 23 & <1 \\ 21 & 1 \\ 18 & 2\end{array}$

$\begin{array}{rr}<0.5 & 40 \\ 0.7 & 20 \\ <0.5 & 30 \\ <0.5 & 30 \\ 0.7 & 320 \\ <0.5 & 430 \\ 0.8 & 40 \\ <0.5 & 50 \\ <0.5 & 1300 \\ 0.6 & <10 \\ <0.5 & 30 \\ <0.5 & 40\end{array}$


Table 37. Onsite measurements and bacteriological and selected inorganic data for station 07094500 , Arkansas River at Parkdale--Continued

\begin{tabular}{|c|c|c|c|c|c|c|c|c|c|c|c|}
\hline DATE & $\begin{array}{l}\text { MANGA - } \\
\text { NESE, } \\
\text { DIS - } \\
\text { SOLVED } \\
\text { (UG/L }\end{array}$ & $\begin{array}{c}\text { MERCURY } \\
\text { TOTAL } \\
\text { RECOV - } \\
\text { ERABLE } \\
\text { IUG/L }\end{array}$ & $\begin{array}{c}\text { MERCURY } \\
\text { DIS - } \\
\text { SOLVED } \\
\text { (UG/L }\end{array}$ & $\begin{array}{l}\text { NICKEL, } \\
\text { TOTAL } \\
\text { RECOV - } \\
\text { ERABLE } \\
\text { (UG /L }\end{array}$ & $\begin{array}{l}\text { NICKEL, } \\
\text { DIS - } \\
\text { SOLVED } \\
\text { (UG/L }\end{array}$ & $\begin{array}{l}\text { SELE- } \\
\text { NIUM, } \\
\text { TOTAL } \\
\text { IUG/L }\end{array}$ & $\begin{array}{l}\text { SELE - } \\
\text { NIUM, } \\
\text { DIS - } \\
\text { SOLVED } \\
\text { (UG/L }\end{array}$ & $\begin{array}{l}\text { SILVER, } \\
\text { TOTAL } \\
\text { RECOV - } \\
\text { ERABLE } \\
\text { (UG/L }\end{array}$ & $\begin{array}{c}\text { SILVER, } \\
\text { DIS - } \\
\text { SOLVED } \\
\text { IUG/L }\end{array}$ & $\begin{array}{l}\text { ZINC, } \\
\text { TOTAL } \\
\text { RECOV - } \\
\text { ERABLE } \\
\text { (UG/L }\end{array}$ & $\begin{array}{l}\text { ZINC, } \\
\text { DIS - } \\
\text { SOLVED } \\
\text { (UG/L }\end{array}$ \\
\hline & AS MN) & AS HG) & AS HG) & AS NI) & AS NI) & AS SE) & AS SE) & AS AG) & AS AG) & AS $2 \mathrm{~N}$ ) & AS $2 \mathrm{~N}$ ) \\
\hline
\end{tabular}

\begin{tabular}{|c|c|c|c|c|c|c|c|c|c|c|c|}
\hline APR 1990 & & & & & & & & & & & \\
\hline $20 \ldots$ & 10 & $\cdots$ & $\cdots$ & $-\cdot$ & $\cdots$ & $\cdots$ & - & - & - & 60 & 12 \\
\hline MAY & & & & & & & & & & & \\
\hline $24 \ldots$ & 4 & $\cdots$ & $\cdots$ & $\cdots$ & $\cdots$ & $\cdots$ & -- & $\cdots$ & $\cdots$ & 580 & 6 \\
\hline JUN & & & & & & & & & & & \\
\hline $07 \ldots$ & 9 & $\cdots$ & $\cdots$ & $\cdots$ & $\cdots$ & $\cdots$ & $\cdots$ & $\cdots$ & $\cdots$ & 820 & 30 \\
\hline $21 \ldots$ & 3 & $\cdots$ & $\cdots$ & $\cdots$ & $\cdots$ & $\cdots$ & - & $\cdots$ & $\cdots$ & 150 & 25 \\
\hline JUL & & & & & & & & & & & \\
\hline $19 \ldots$ & 4 & $\cdots$ & $\cdots$ & $-\cdot$ & $\cdots$ & $\cdots$ & -. & $\cdots$ & - & 50 & 12 \\
\hline AUG & & & & & & & & & & & \\
\hline $30 \ldots$ & 7 & $<0.10$ & $<0.1$ & $<1$ & $<1$ & $<1$ & $<1$ & $<1$ & $<1.0$ & 30 & 7 \\
\hline NOV & & & & & & & & & & & \\
\hline $01 \ldots$ & 5 & - & $-\cdot$ & -. & - & $\cdots$ & -. & - - & $\cdots$ & 40 & 20 \\
\hline JAN 1991 & & & & & & & & & & & \\
\hline $17 \ldots$ & 4 & -- & -- & -- & - & . & $-\cdot$ & $\cdots$ & - - & 50 & 33 \\
\hline MAR & & & & & & & & & & & \\
\hline $26 \ldots$ & 11 & $\cdots$ & - & - & $\cdots$ & $\ldots$ & - & $-\cdot$ & - & 30 & 15 \\
\hline APR & & & & & & & & & & & \\
\hline $25 \ldots$ & 10 & - & - - & - & - & - & - . & - & - - & 50 & 23 \\
\hline MAY & & & & & & & & & & & \\
\hline $16 \ldots$ & 5 & $-\cdot$ & $\cdots$ & $\cdots$ & $\cdots$ & $\cdots$ & -. & - & -. & 200 & 30 \\
\hline JUN & & & & & & & & & & & \\
\hline $12 \ldots$ & $<10$ & - & $\cdots$ & -- & $\cdots$ & - & $\cdots$ & -- & - & 280 & 20 \\
\hline $20 \ldots$ & 6 & $<0.10$ & $<0.1$ & 3 & 2 & $<1$ & $<1$ & $<1$ & $<1.0$ & $<10$ & 8 \\
\hline JUL & & & & & & & & & & & \\
\hline $18 \ldots$ & 3 & $-\cdot$ & $-\cdot$ & - & -- & $\cdots$ & $\cdots$ & $\cdots$ & -- & 20 & 9 \\
\hline AUG & & & & & & & & & & & \\
\hline $15 \ldots$ & 5 & $\cdots$ & - - & - - & $\cdots$ & $\cdots$ & -- & $\cdots$ & -- & 30 & 7 \\
\hline SEP & & & & & & & & & & & \\
\hline $03 \ldots$ & - & $<0.10$ & $<0.1$ & 2 & $<1$ & 2 & 1 & $<1$ & $<1.0$ & -- & $\cdots$ \\
\hline OCT & & & & & & & & & & & \\
\hline $24 \ldots$ & 7 & $\cdots$ & - & $\cdots$ & - - & $\cdots$ & - & - & - - & 20 & 14 \\
\hline DEC & & & & & & & & & & & \\
\hline $18 \ldots$ & 6 & - & - & $\cdots$ & - & $\cdots$ & - - & . & - & 60 & 30 \\
\hline MAR 1992 & & & & & & & & & & & \\
\hline $24 \ldots$ & 9 & - & - & - - & - & - & -. & - & $\cdots$ & 40 & 9 \\
\hline APR & & & & & & & & & & & \\
\hline $23 \ldots$ & 12 & $\cdots$ & - & - & $\cdots$ & - & $\cdots$ & - & - - & 40 & 13 \\
\hline MAY & & & & & & & & & & & \\
\hline $22 \ldots$ & 5 & $\cdots$ & $\cdots$ & - & -. & $\cdots$ & $\cdots$ & - & - - & 280 & 16 \\
\hline JUN & & & & & & & & & & & \\
\hline $26 \ldots$ & 4 & $<0.10$ & $<0.1$ & $<1$ & $<1$ & $<1$ & $<1$ & $<1$ & $<1.0$ & 130 & 3 \\
\hline JUL & & & & & & & & & & & \\
\hline $15 \ldots$ & 4 & $\cdots$ & $\cdots$ & - & $\cdots$ & - & - & $\cdots$ & - & 40 & 9 \\
\hline AUG & & & & & & & & & & & \\
\hline $13 \ldots$ & 4 & $<0.10$ & $<0.1$ & $<1$ & $<1$ & $<1$ & $<1$ & $<1$ & $<1.0$ & 50 & 10 \\
\hline $25 \ldots$ & 2 & - & - & . & - & $\cdots$ & -. & - & $\cdots$ & 450 & 9 \\
\hline OCT & & & & & & & & & & & \\
\hline $29 \ldots$ & 5 & $\cdots$ & $\cdots$ & - & $\cdots$ & $\cdots$ & .. & - & - & 20 & 8 \\
\hline JAN 1993 & & & & & & & & & & & \\
\hline $13 \ldots$ & 9 & $\cdots$ & - & . & - & - & - & . & . & 50 & 27 \\
\hline MAR & & & & & & & & & & & \\
\hline $23 \ldots$ & 7 & $\cdots$ & - & $\cdots$ & - & $\cdots$ & . & - & $\cdots$ & 30 & 9 \\
\hline
\end{tabular}


Table 38. Onsite measurements and selected inorganic data for station 383113105160401, Grape Creek at mouth, at Canon City

\begin{tabular}{|c|c|c|c|c|c|c|c|c|c|c|c|}
\hline DATE & TIME & $\begin{array}{c}\text { DIS- } \\
\text { CHARGE, } \\
\text { INST. } \\
\text { CUBIC } \\
\text { FEET } \\
\text { PER } \\
\text { SECOND }\end{array}$ & $\begin{array}{l}\text { SPE- } \\
\text { CIFIC } \\
\text { CON- } \\
\text { DUCT - } \\
\text { ANCE } \\
\text { (US/CM) }\end{array}$ & $\begin{array}{c}\text { PH } \\
\text { WATER } \\
\text { WHOLE } \\
\text { FIELD } \\
\text { (STAND- } \\
\text { ARD } \\
\text { UNITS) }\end{array}$ & $\begin{array}{c}\text { TEMPER- } \\
\text { ATURE } \\
\text { WATER } \\
\text { (DEG C) }\end{array}$ & $\begin{array}{c}\text { OXYGEN, } \\
\text { DIS - } \\
\text { SOLVED } \\
\text { (MG/L) }\end{array}$ & $\begin{array}{l}\text { SOLIDS, } \\
\text { RESIDUE } \\
\text { AT } 180 \\
\text { DEG C } \\
\text { DIS - } \\
\text { SOLVED } \\
\text { (MG/L) }\end{array}$ & $\begin{array}{l}\text { NITRO- } \\
\text { GEN, } \\
\text { NO2 +NO3 } \\
\text { TOTAL } \\
\text { (MG/L } \\
\text { AS N) }\end{array}$ & $\begin{array}{c}\text { NITRO- } \\
\text { GEN, } \\
\text { AMMONIA } \\
\text { TOTAL } \\
\text { (MG/L } \\
\text { AS N) }\end{array}$ & $\begin{array}{l}\text { CADMIUM } \\
\text { TOTAL } \\
\text { RECOV- } \\
\text { ERABLE } \\
\text { (UG/L } \\
\text { AS CD) }\end{array}$ & $\begin{array}{l}\text { CADMIUM } \\
\text { DIS - } \\
\text { SOLVED } \\
\text { (UG/L } \\
\text { AS CD) }\end{array}$ \\
\hline $\begin{array}{c}\text { APR } 1990 \\
20 \ldots\end{array}$ & 1045 & 32 & 419 & 8.7 & 12.0 & 9.7 & 262 & 0.013 & $<0.010$ & $\cdots$ & $\cdots$ \\
\hline JUN & & & & & 2.0 & & & 0.00 & (6.0 & & \\
\hline JUL $07 .$. & 1320 & 7.1 & 444 & 8.4 & 25.5 & 6.5 & 280 & $<0.010$ & 0.020 & $\cdots$ & $\cdots$ \\
\hline${ }_{A O G}^{19} \cdots$ & 0800 & 25 & 432 & 8.5 & 16.5 & 7.8 & 244 & 0.044 & 0.020 & $\cdots$ & $\cdots$ \\
\hline$\underset{\text { NOV }}{30 \ldots}$ & 0940 & 16 & 351 & 8.0 & 16.5 & 8.6 & 207 & $\cdots$ & $<0.010$ & $\cdots$ & $\cdots$ \\
\hline $\begin{array}{l}01 \ldots \\
\text { APR } 1991\end{array}$ & 1525 & 44 & 350 & 8.4 & 10.0 & 8.8 & 197 & 0.012 & 0.030 & $\cdots$ & $\cdots$ \\
\hline $\mathrm{JUN}^{25 \ldots}$ & 1535 & 9.6 & 407 & 8.3 & 15.5 & 8.2 & 235 & 0.024 & 0.022 & $\cdots$ & -. \\
\hline JUL 20. & 1200 & 5.5 & 420 & 8.6 & 20.5 & 8.2 & 226 & $<0.005$ & 0.023 & $\cdots$ & $\cdots$ \\
\hline $\begin{array}{c}18 \ldots \\
\text { ост }\end{array}$ & 1125 & 24 & 382 & 8.6 & 22.5 & 6.9 & 232 & 0.009 & 0.018 & $\cdots$ & $\cdots$ \\
\hline $\begin{array}{r}24 \ldots \\
\text { MAR } 1992\end{array}$ & 1040 & 9.0 & 407 & 8.3 & 7.0 & 9.5 & 238 & 0.014 & 0.025 & $\cdots$ & $\cdots$ \\
\hline${ }_{\mathrm{APR}}^{31 \cdots}$ & 1020 & 227 & 441 & 8.6 & 4.5 & 10.5 & 273 & 0.093 & 0.021 & $<1$ & $<0.1$ \\
\hline$\underset{\pi N N}{23} \cdots$ & 1115 & 44 & 424 & 8.6 & 11.0 & 8.7 & 250 & 0.007 & 0.023 & $\cdots$ & $\cdots$ \\
\hline${ }_{A U G}^{26} \cdots$ & 1235 & 59 & 391 & 8.8 & 21.0 & 7.1 & 230 & $<0.005$ & 0.036 & $\cdots$ & $\cdots$ \\
\hline $13 \ldots$ & 1210 & 46 & 312 & 8.7 & 20.0 & 7.3 & 184 & 0.165 & 0.031 & $\cdots$ & $\cdots$ \\
\hline $29 \ldots$ & 1200 & 18 & 400 & 8.5 & 7.0 & 9.8 & 239 & 0.261 & 0.368 & $\cdots$ & $\cdots$ \\
\hline
\end{tabular}

DATE

$\begin{array}{cclc}\text { COPPER, } & & \text { IRON, } \\ \text { TOTAL } & \text { COPPER, } & \text { TOTAL } & \text { IRON, } \\ \text { RECOV- } & \text { DIS- } & \text { RECOV- } & \text { DIS- } \\ \text { ERABLE } & \text { SOLVED } & \text { ERABLE } & \text { SOLVED } \\ \text { (UG/L } & \text { (UG/L } & \text { (UG/L } & \text { (UG/L } \\ \text { AS CU) } & \text { AS CO) } & \text { AS FE) } & \text { AS FE) }\end{array}$

$\begin{array}{lcllll} & & \text { MANGA- } & & \\ \text { LEAD, } & & \text { NESE, } & \text { MANGA- } & \text { ZINC, } \\ \text { TOTAL } & \text { LEAD, } & \text { TOTAL } & \text { NESE, } & \text { TOTAL } & \text { ZINC, } \\ \text { RECOV- } & \text { DIS- } & \text { RECOV- } & \text { DIS- } & \text { RECOV- } & \text { DIS- } \\ \text { ERABLE } & \text { SOLVED } & \text { ERABLE } & \text { SOLVED } & \text { ERABLE } & \text { SOLVED } \\ \text { (UG/L } & \text { (UG/L } & \text { (UG/L } & \text { (UG/L } & \text { (UG/L } & \text { (UG/L } \\ \text { AS PB) } & \text { AS PB) } & \text { AS MN) } & \text { AS MN) } & \text { AS ZN) } & \text { AS ZN) }\end{array}$

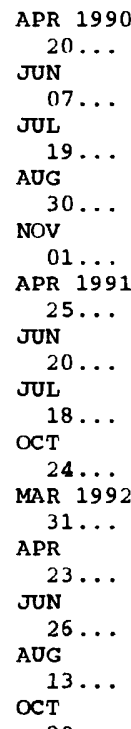
$29 \ldots$ 
Table 39. Onsite measurements and selected inorganic data for station 07096000, Arkansas River at Canon City

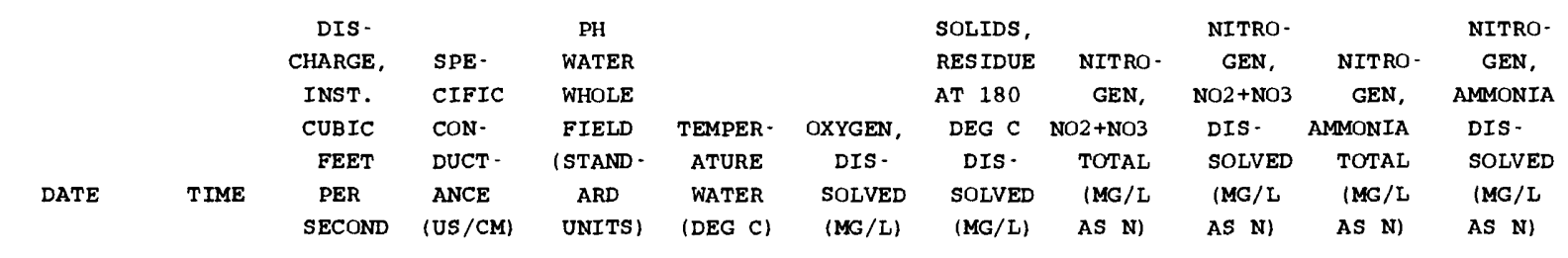

\begin{tabular}{|c|c|c|c|c|c|c|c|c|c|c|c|}
\hline \multicolumn{12}{|l|}{ APR 1990} \\
\hline $20 \ldots$ & 1215 & 240 & 333 & 8.5 & 11.5 & 9.7 & 195 & 0.020 & $\cdots$ & $<0.010$ & - \\
\hline \multicolumn{12}{|l|}{ MAY } \\
\hline $24 \ldots$ & 1145 & 533 & 249 & 8.2 & 17.0 & 7.8 & 148 & 0.165 & $\cdots$ & 0.070 & $\cdots$ \\
\hline \multicolumn{12}{|l|}{ JUN } \\
\hline $07 \ldots$ & 1200 & 2920 & 130 & 7.8 & 15.0 & 8.3 & 70 & 0.096 & $\cdots$ & 0.030 & $\cdots$ \\
\hline $21 \ldots$ & 1030 & 1650 & 142 & 7.9 & 16.0 & 8.5 & 75 & 0.064 & $\cdots$ & $<0.010$ & $\cdots$ \\
\hline \multicolumn{12}{|l|}{ JUL } \\
\hline $19 \ldots$ & 1215 & 843 & 185 & 8.2 & 19.5 & 7.8 & 103 & 0.035 & $\cdots$ & 0.020 & $\cdots$ \\
\hline \multicolumn{12}{|l|}{ AUG } \\
\hline $30 \ldots$ & 1250 & 280 & 294 & 8.3 & 21.5 & 7.4 & 170 & 0.014 & $\cdots$ & $<0.010$ & $\cdots$ \\
\hline \multicolumn{12}{|l|}{ Nov } \\
\hline $01 \ldots$ & 1645 & 405 & 315 & 8.8 & 9.0 & 9.0 & 177 & 0.024 & $\cdots$ & 0.020 & $\cdots$ \\
\hline \multicolumn{12}{|l|}{ JAN 1991} \\
\hline $17 \ldots$ & 1430 & 519 & 222 & 7.0 & 0.0 & 12.2 & 134 & 0.154 & $\cdots$ & 0.012 & $\cdots$ \\
\hline \multicolumn{12}{|l|}{ MAR } \\
\hline $27 \ldots$ & 0815 & 387 & 276 & 8.3 & 5.0 & 9.8 & 154 & 0.007 & $\cdots$ & 0.020 & $\cdots$ \\
\hline \multicolumn{12}{|l|}{ APR } \\
\hline $25 \ldots$ & 1655 & 296 & 238 & 8.3 & 13.0 & 8.7 & 126 & 0.007 & $\cdots$ & 0.014 & $\cdots$ \\
\hline \multicolumn{12}{|l|}{ MAY } \\
\hline $16 \ldots$ & 1115 & 800 & 179 & 7.9 & 13.0 & 8.5 & 91 & 0.062 & $\cdots$ & 0.040 & $\cdots$ \\
\hline \multicolumn{12}{|l|}{ JUN } \\
\hline $20 \ldots$ & 1130 & 1780 & 137 & 8.1 & 15.0 & 8.3 & 69 & 0.070 & $\cdots$ & 0.019 & $\cdots$ \\
\hline \multicolumn{12}{|l|}{ JUL } \\
\hline $18 \ldots$ & 1245 & 663 & 206 & 8.3 & 21.5 & 7.4 & 117 & 0.005 & $\cdots$ & $<0.002$ & $\cdots$ \\
\hline \multicolumn{12}{|l|}{ AUG } \\
\hline $15 \ldots$ & 1300 & 655 & 270 & 8.4 & 19.0 & $\cdots$ & 154 & 0.048 & $\cdots$ & 0.003 & $\cdots$ \\
\hline \multicolumn{12}{|l|}{ OCT } \\
\hline $24 \ldots$ & 1115 & 221 & 356 & 8.2 & 9.0 & 9.5 & 214 & 0.024 & $\cdots$ & 0.024 & $\cdots$ \\
\hline \multicolumn{12}{|l|}{ DEC } \\
\hline $18 \ldots$ & 1320 & 464 & 255 & 8.3 & 0.5 & 12.2 & 179 & 0.137 & $\cdots$ & 0.008 & $\cdots$ \\
\hline \multicolumn{12}{|l|}{ MAR 1992} \\
\hline $25 \ldots$ & 0820 & 519 & 297 & 8.5 & 5.5 & 10.8 & 177 & 0.043 & $\cdots$ & 0.012 & $\cdots$ \\
\hline \multicolumn{12}{|l|}{ APR } \\
\hline $23 \ldots$ & 1040 & 275 & 332 & 8.4 & 11.5 & 8.7 & 186 & 0.013 & $\cdots$ & 0.012 & - \\
\hline \multicolumn{12}{|l|}{ MAY } \\
\hline $22 \ldots$ & 1000 & 1380 & 144 & 8.1 & 13.5 & 8.6 & 84 & 0.108 & $\cdots$ & 0.024 & $\cdots$ \\
\hline JUN & & & & & & & & & & & \\
\hline $26 \ldots$ & 1105 & 1530 & 190 & 8.3 & 17.0 & 7.6 & 112 & 0.045 & $\cdots$ & 0.008 & $\cdots$ \\
\hline JUL & & & & & & & & & & & \\
\hline $15 \ldots$ & 1030 & 251 & 226 & 8.4 & 17.5 & 7.9 & 138 & 0.030 & -. & 0.004 & $\cdots$ \\
\hline AUG & & & & & & & & & & & \\
\hline $13 \ldots$ & 1115 & 743 & 221 & 8.4 & 18.5 & 7.8 & 182 & 0.126 & $\cdots$ & 0.024 & $\cdots$ \\
\hline $24 \ldots$ & 1810 & 1860 & 226 & 8.4 & 15.5 & 8.1 & 136 & 0.157 & - & 0.034 & $\cdots$ \\
\hline OCT & & & & & & & & & & & \\
\hline $29 \ldots$ & 1050 & 306 & 320 & 8.5 & 8.5 & 9.5 & 195 & 0.075 & $\cdots$ & 0.039 & $\cdots$ \\
\hline JAN 1993 & & & & & & & & & & & \\
\hline $13 \ldots$ & 0935 & 381 & 268 & 8.3 & 0.0 & 12.1 & 156 & $\cdots$ & 0.196 & $\cdots$ & 0.022 \\
\hline MAR & & & & & & & & & & & \\
\hline $23 \ldots$ & 1520 & 647 & 194 & 8.5 & 11.5 & 9.2 & 104 & $\cdots$ & 0.017 & $\cdots$ & 0.099 \\
\hline
\end{tabular}


Table 40. Onsite measurements and selected inorganic data for station 07096500 , Fourmile Creek near Canon City

\begin{tabular}{|c|c|c|c|c|c|c|c|c|c|c|c|}
\hline DATE & TIME & $\begin{array}{c}\text { DIS - } \\
\text { CHARGE, } \\
\text { INST. } \\
\text { CUBIC } \\
\text { FEET } \\
\text { PER } \\
\text { SECOND }\end{array}$ & $\begin{array}{l}\text { SPE- } \\
\text { CIFIC } \\
\text { CON- } \\
\text { DUCT- } \\
\text { ANCE } \\
\text { (US/CM) }\end{array}$ & $\begin{array}{c}\text { PH } \\
\text { WATER } \\
\text { WHOLE } \\
\text { FIELD } \\
\text { (STAND - } \\
\text { ARD } \\
\text { UNITS) }\end{array}$ & $\begin{array}{c}\text { TEMPER - } \\
\text { ATURE } \\
\text { WATER } \\
\text { (DEG C) }\end{array}$ & $\begin{array}{c}\text { OXYGEN, } \\
\text { DIS - } \\
\text { SOLVED } \\
\text { (MG/L) }\end{array}$ & $\begin{array}{c}\text { SOLIDS, } \\
\text { RESIDUE } \\
\text { AT } 180 \\
\text { DEG C } \\
\text { DIS- } \\
\text { SOLVED } \\
\text { (MG/L) }\end{array}$ & $\begin{array}{c}\text { NITRO- } \\
\text { GEN, } \\
\text { NO2+NO3 } \\
\text { TOTAL } \\
\text { (MG / L } \\
\text { AS N) }\end{array}$ & $\begin{array}{l}\text { NITRO- } \\
\text { GEN, } \\
\text { AMMONIA } \\
\text { TOTAL } \\
\text { (MG /L } \\
\text { AS N) }\end{array}$ & $\begin{array}{l}\text { CADMIUM } \\
\text { TOTAL } \\
\text { RECOV- } \\
\text { ERABLE } \\
\text { (UG/L } \\
\text { AS CD) }\end{array}$ & $\begin{array}{l}\text { CADMIUM } \\
\text { DIS- } \\
\text { SOLVED } \\
\text { (UG/L } \\
\text { AS CD) }\end{array}$ \\
\hline APR 1990 & & & & & & & & & & & \\
\hline$\underset{\pi N}{20} \ldots$ & 1320 & 14 & 1020 & 8.3 & 17.5 & 9.2 & 740 & 0.326 & 0.040 & $<1$ & $<0.1$ \\
\hline $\begin{array}{l}07 \ldots \\
\text { JUL }\end{array}$ & 1205 & 47 & 880 & 8.0 & 19.5 & 6.8 & 593 & 0.214 & 0.090 & 1 & 0.8 \\
\hline${ }_{A U G}^{19} \ldots$ & 1045 & 28 & 800 & 8.1 & 19.5 & 7.7 & 550 & 0.135 & $<0.010$ & $<1$ & $<0.1$ \\
\hline $\begin{array}{l}30 \ldots \\
\text { Nov }\end{array}$ & 1100 & 25 & 906 & 8.0 & 19.0 & 8.5 & 709 & 0.180 & 0.010 & $<1$ & $<0.1$ \\
\hline $\begin{array}{r}02 \ldots \\
A P R \quad 1991\end{array}$ & 0750 & 12 & 845 & 8.3 & 9.0 & 9.1 & 570 & 0.103 & 0.030 & $<1$ & 0.3 \\
\hline $\int_{\text {JN }}^{26 \ldots}$ & 0730 & 17 & 999 & 7.9 & 11.0 & 8.1 & 673 & 0.412 & 0.040 & $<1$ & $<0.1$ \\
\hline $\begin{array}{l}20 \ldots \\
\text { JUL }\end{array}$ & 1230 & 39 & 916 & 8.4 & 20.5 & 8.6 & 621 & 0.130 & 0.030 & $<1$ & 0.5 \\
\hline$\underset{\mathrm{OCT}}{18 \ldots}$ & 1205 & 22 & 1170 & 8.1 & 20.0 & 7.5 & 828 & 0.348 & 0.019 & $<1$ & $<0.1$ \\
\hline $\begin{array}{c}24 \ldots \\
\text { APR } 1992\end{array}$ & 1210 & 37 & 749 & 8.2 & 11.0 & 8.6 & 499 & 0.213 & 0.038 & $<1$ & 0.1 \\
\hline $\mathrm{JUN}^{23 \ldots}$ & 1215 & 70 & 557 & 8.2 & 12.0 & 8.9 & 376 & 0.227 & 0.036 & $<1$ & $<0.1$ \\
\hline${ }_{A U G}^{26 \ldots}$ & 1200 & 77 & 744 & 8.1 & 19.0 & 6.9 & 524 & 0.334 & 0.032 & $<1$ & 0.2 \\
\hline $\begin{array}{l}13 \ldots \\
\text { OCT }\end{array}$ & 1210 & 30 & 1090 & 8.1 & 20.5 & 7.4 & 816 & 0.537 & 0.023 & $<1$ & $<0.1$ \\
\hline $29 \ldots$ & 1240 & 19 & 1220 & 8.2 & 12.0 & 8.7 & 860 & 0.657 & 0.018 & $<1$ & $<0.1$ \\
\hline
\end{tabular}

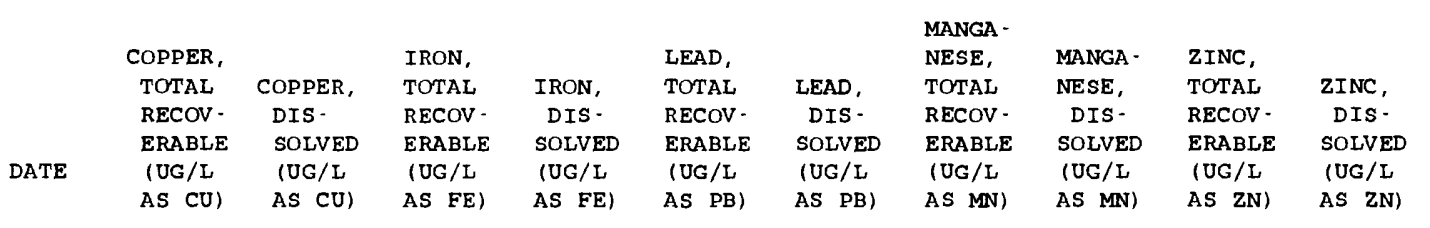

\begin{tabular}{|c|c|c|c|c|c|c|c|c|c|c|}
\hline $\begin{array}{c}20 \ldots \\
\pi U N\end{array}$ & 8 & 1 & 1900 & 7 & 5 & $<0.5$ & 110 & 31 & 50 & 5 \\
\hline $07 \ldots$ & 11 & 3 & 3500 & 14 & 10 & 0.6 & 220 & 35 & 50 & 9 \\
\hline JUL & & & & & & & & & & \\
\hline${ }_{A \cup G}^{19} \ldots$ & 5 & 2 & 750 & 13 & 5 & 0.9 & 80 & 24 & 40 & 7 \\
\hline $\begin{array}{l}30 \ldots \\
\text { NOV }\end{array}$ & 4 & 2 & 660 & 120 & 3 & $<0.5$ & 70 & 34 & 20 & 9 \\
\hline $\begin{array}{r}02 \ldots \\
\text { APR } 1991\end{array}$ & 4 & 1 & 420 & 41 & $<1$ & $<0.5$ & 50 & 27 & 20 & 17 \\
\hline${ }_{J U N}^{26} \ldots$ & 4 & $<1$ & 530 & 36 & 3 & $<0.5$ & 50 & 34 & 30 & 16 \\
\hline $\int_{\pi L}^{20 \ldots}$ & 8 & 4 & 540 & 53 & 6 & $<0.5$ & 60 & 30 & 10 & $<3$ \\
\hline$\underset{O C T}{18 \ldots}$ & 3 & $<1$ & 400 & 15 & 5 & $<0.5$ & 60 & 43 & $<10$ & 10 \\
\hline $\begin{array}{r}24 \ldots \\
\text { APR } 1992\end{array}$ & 9 & $<1$ & 1800 & 21 & 9 & 0.5 & 110 & 27 & 40 & 4 \\
\hline$\underset{\pi U N}{23} \ldots$ & 4 & 2 & 3500 & 30 & 5 & $<0.5$ & 310 & 31 & 40 & 4 \\
\hline$\underset{A U G}{26} \ldots$ & 12 & 2 & 7900 & 22 & 12 & $<0.5$ & 390 & 23 & 50 & $<3$ \\
\hline$\underset{\mathrm{OCT}}{13 \ldots}$ & 2 & 1 & 230 & 18 & 1 & $<0.5$ & 40 & 22 & 10 & 4 \\
\hline $29 \ldots$ & 1 & $<1$ & 200 & 25 & $<1$ & $<0.5$ & 20 & 25 & $<10$ & $<3$ \\
\hline
\end{tabular}


Table 41. Onsite measurements and selected inorganic data for station 382337105014600 , Hardscrabble Creek at Highway 120, at Portland

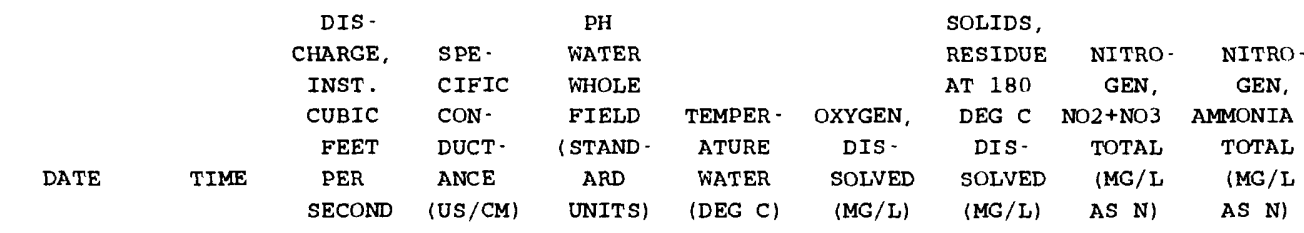

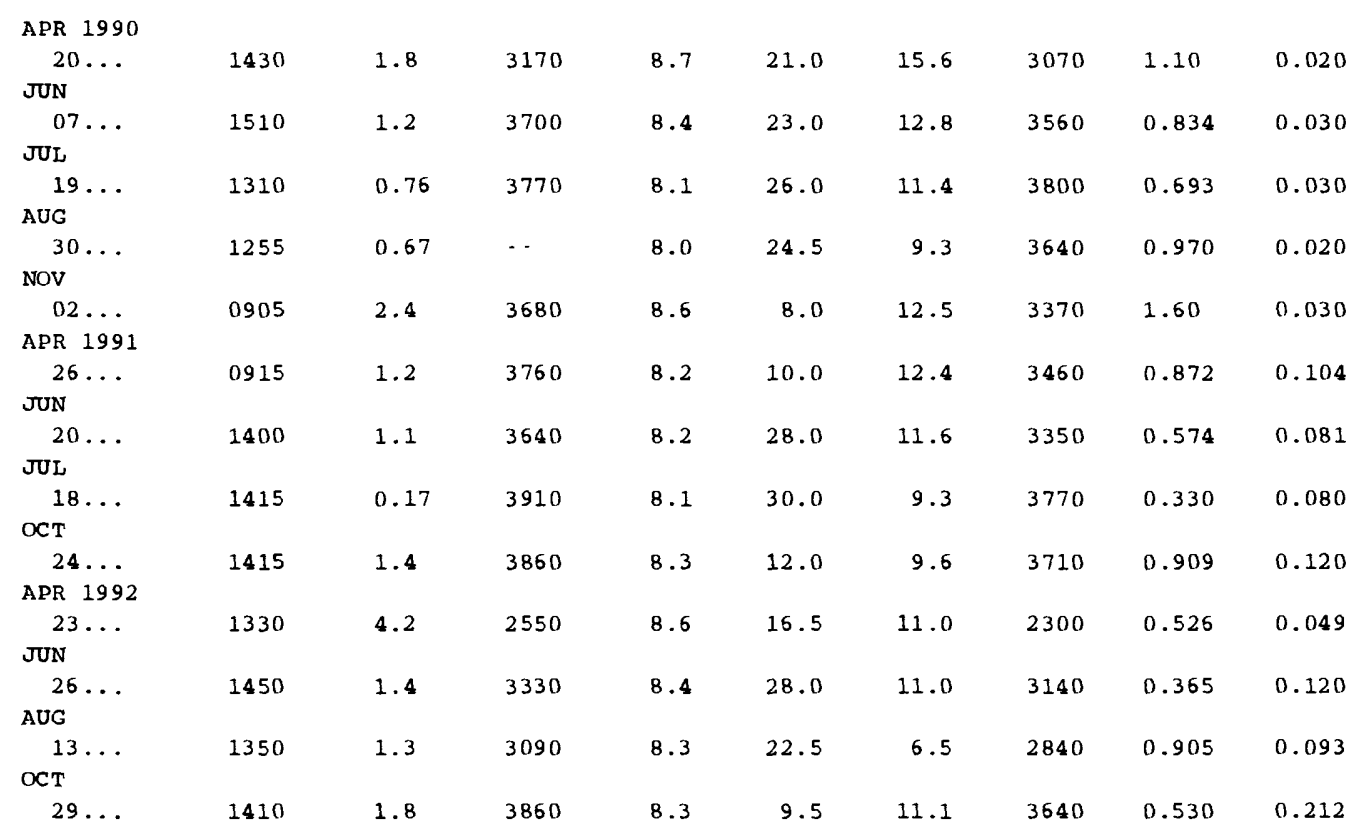


Table 42. Onsite measurements and bacteriological and selected inorganic data for station 07097000 , Arkansas River at Portland

\begin{tabular}{|c|c|c|c|c|c|c|c|c|c|c|c|c|}
\hline TE & TIME & $\begin{array}{c}\text { DIS - } \\
\text { CHARGE, } \\
\text { INST. } \\
\text { CUBIC } \\
\text { FEET } \\
\text { PER }\end{array}$ & $\begin{array}{l}\text { SPE- } \\
\text { CIFIC } \\
\text { CON- } \\
\text { DUCT - } \\
\text { ANCE }\end{array}$ & $\begin{array}{c}\text { PH } \\
\text { WATER } \\
\text { WHOLE } \\
\text { FIELD } \\
\text { (STAND - } \\
\text { ARD }\end{array}$ & $\begin{array}{l}\text { TEMPER- } \\
\text { ATURE } \\
\text { WATER }\end{array}$ & $\begin{array}{c}\text { OXYGEN, } \\
\text { DIS - } \\
\text { SOLVED }\end{array}$ & $\begin{array}{c}\text { COLI- } \\
\text { FORM, } \\
\text { TOTAL, } \\
\text { IMMED. } \\
\text { ICOLS. } \\
\text { PER }\end{array}$ & $\begin{array}{l}\text { COLI- } \\
\text { FORM, } \\
\text { FECAL, } \\
0.7 \\
\text { UM-MF } \\
\text { (COLS.) }\end{array}$ & $\begin{array}{c}\text { STREP- } \\
\text { TOCOCCI } \\
\text { FECAL, } \\
\text { KF AGAR } \\
\text { ICOLS. } \\
\text { PER }\end{array}$ & $\begin{array}{l}\text { HARD - } \\
\text { NESS } \\
\text { TOTAL } \\
\text { (MG / L } \\
\text { AS }\end{array}$ & $\begin{array}{l}\text { CALCIUM } \\
\text { DIS - } \\
\text { SOLVED } \\
\text { (MG/L }\end{array}$ & $\begin{array}{l}\text { MAGNE - } \\
\text { SIUM, } \\
\text { DIS - } \\
\text { SOLVED } \\
\text { (MG / L }\end{array}$ \\
\hline & & SECOND & (US /CM) & UNITS) & (DEG C) & (MG/L) & $100 \mathrm{ML})$ & $100 \mathrm{ML})$ & $100 \mathrm{MU})$ & (ACO3) & AS CA) & AS MG) \\
\hline
\end{tabular}

APR 1990

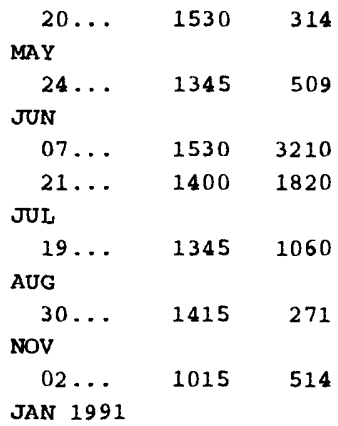

$17 \ldots \quad 1550 \quad 470$

MAR

$27 \ldots \quad 1010 \quad 400$

APR

$26 \ldots \quad 1015 \quad 291$

MAY

$16 \ldots \quad 1315 \quad 728$

JUN

$20 \ldots 1420 \quad 1920$

JUL

$18 \ldots \quad 1400 \quad 642$

$15 \ldots \quad 1500 \quad 858$

SEP

$03 \ldots \quad 1900 \quad 384$

OCT

24... $\quad 1315 \quad 339$

DEC

$18 \ldots \quad 1500 \quad 460$

MAR 1992

$\begin{array}{lll}25 \ldots & 1005 & 642\end{array}$

$23 \ldots \quad 1400 \quad 318$

MAY

$22 \ldots \quad 1230 \quad 1450$

JUN

$26 \ldots 1400 \quad 1730$

JUL

15... $1200 \quad 805$

AUG

$13 \ldots \quad 1330 \quad 800$

$24 \ldots \quad 1500 \quad 917$

OCT

$29 \ldots \quad 1400 \quad 343$

JAN 1993

$13 \ldots \quad 1050 \quad 539$

MAR

$24 \ldots \quad 0855 \quad 587$
555

471

173

199

274

504

482

352

394

490

320

197

331

418

495

581

413

379

540

230

269

327

351

435

521

401

278
$8.9 \quad 15.5$

8.2

19.5

7.9

18.0

$7.4 \quad K 160$

$\begin{array}{llll}8.6 & 23.0 & 8.6 & 130\end{array}$

$\begin{array}{llll}8.7 & 25.0 & 10.2 & \text { K2 }\end{array}$

$8.8 \quad 8.5$

8.53.

11.

12.0

$8.6 \quad 8.5$

-

$-\cdot$

$\begin{array}{llll}8.7 & 11.0 & 12.7 & 140\end{array}$

$\begin{array}{llll}8.8 & 14.0 & 8.2 & 110\end{array}$

$\begin{array}{llll}8.0 & 18.5 & 7.9 & 140\end{array}$

$9.1 \quad 24.0 \quad 10.2 \quad E 100$

8.3

23.0

$\cdots$

E3

$8.3 \quad 22.5$

8.5

11.0

10.5

$\begin{array}{llll}8.4 & 1.0 & 12.2 & 75\end{array}$

$\begin{array}{llll}8.5 & 7.5 & 10.9 & \ldots\end{array}$

$\begin{array}{lllll}8.6 & 15.0 & 10.0 & \text { E32 } & \text { E2 } 1\end{array}$

$\begin{array}{lllll}8.2 & 15.5 & 8.1 & \text { E56 } & 160\end{array}$

$8.3 \quad 20.0$

7.5

8.620 .0

$9.1 \quad 110$

8.621 .0

$7.6 \quad-$.

8.2

16.5

7.9

$>4000$

150

$8.4 \quad 10.5$

$9.7 \quad 280$

8.3

0.0

$12.2 \quad$ E26

8.2

8.0

9.9

K17

K2

$<2$

80

57

63
570

180

280

130

K12

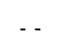

E3

45

290

380

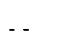

-

150

25

$170 \quad 46$

$160 \quad 44$

$220 \quad 60$

$94 \quad 27$

6.5

7.8

10

$140 \quad 40$

150

$150 \quad 41 \quad 11$

$220 \quad 60$

17

12

8.0 
Table 42. Onsite measurements and bacteriological and selected inorganic data for station 07097000 , Arkansas River at Portland--Continued

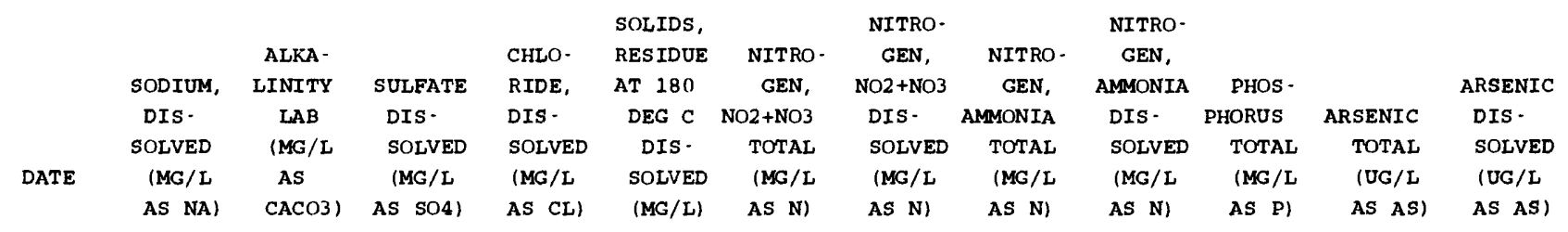

\begin{tabular}{|c|c|c|c|c|c|c|c|c|c|c|c|c|}
\hline \multicolumn{13}{|l|}{ APR 1990} \\
\hline $20 \ldots$ & 32 & 135 & 120 & 13 & 352 & 0.143 & $\cdots$ & $<0.010$ & - & 0.097 & $\cdots$ & - \\
\hline \multicolumn{13}{|l|}{ MAY } \\
\hline $24 \ldots$ & 24 & 114 & 120 & 12 & 300 & 0.266 & $\cdots$ & $<0.010$ & $\cdots$ & $\cdots$ & - & - \\
\hline \multicolumn{13}{|l|}{ JUN } \\
\hline $07 \ldots$ & 5.8 & 49 & 31 & 1.9 & 109 & 0.131 & -. & 0.080 & -. & $\cdots$ & $\cdots$ & $\cdots$ \\
\hline $21 \ldots$ & 7.2 & 56 & 37 & 3.9 & 113 & 0.099 & -. & $<0.010$ & -. & $\cdots$ & $-\cdot$ & . \\
\hline \multicolumn{13}{|l|}{ JUL } \\
\hline $19 \ldots$ & 12 & 81 & 45 & 5.8 & 164 & 0.033 & $\cdots$ & 0.030 & -. & 0.028 & $-\cdot$ & $\cdots$ \\
\hline \multicolumn{13}{|l|}{ AUG } \\
\hline $30 \ldots$ & 26 & 140 & 98 & 0.26 & 408 & 0.040 & $\cdots$ & $<0.010$ & -. & 0.064 & $<1$ & $<1$ \\
\hline \multicolumn{13}{|l|}{ NOV } \\
\hline $02 \ldots$ & 23 & 144 & 92 & 12 & 267 & 0.037 & $\cdots$ & 0.020 & - - & 0.034 & $-\cdot$ & $\cdots$ \\
\hline \multicolumn{13}{|l|}{ JAN 1991} \\
\hline $17 \ldots$ & 17 & 101 & 68 & 11 & 216 & 0.249 & -. & 0.009 & -. & 0.030 & $\cdots$ & $\cdots$ \\
\hline \multicolumn{13}{|l|}{ MAR } \\
\hline $27 \ldots$ & 18 & 111 & 70 & 9.0 & 231 & 0.032 & $\cdots$ & 0.041 & -. & 0.024 & - - & $\cdots$ \\
\hline \multicolumn{13}{|l|}{ APR } \\
\hline $26 \ldots$ & 25 & 113 & 130 & 13 & 297 & 0.013 & $\cdots$ & 0.018 & -. & 0.044 & -. & - \\
\hline \multicolumn{13}{|l|}{ MAY } \\
\hline $16 \ldots$ & 15 & 76 & 70 & 5.2 & 194 & 0.130 & $\cdots$ & 0.043 & - & 0.060 & $\cdots$ & $\cdots$ \\
\hline \multicolumn{13}{|l|}{ JUN } \\
\hline $20 \ldots$ & 6.9 & 56 & 29 & 3.2 & 103 & 0.096 & -- & 0.022 & $\cdots$ & 0.085 & $<1$ & $<1$ \\
\hline \multicolumn{13}{|l|}{ JUL } \\
\hline $18 \ldots$ & 15 & 100 & 74 & 7.3 & 185 & 0.007 & $\cdots$ & 0.011 & - & 0.052 & -. & $-\cdot$ \\
\hline \multicolumn{13}{|l|}{ AUG } \\
\hline $15 \ldots$ & 19 & 125 & 90 & 7.8 & 280 & 0.201 & $-\cdot$ & 0.005 & - & 0.065 & -. & $\cdots$ \\
\hline \multicolumn{13}{|l|}{ SEP } \\
\hline $03 \ldots$ & $\cdots$ & $\cdots$ & -. & - & - & $\cdots$ & $\cdots$ & - & - & - & $<1$ & $<1$ \\
\hline \multicolumn{13}{|l|}{ OCT } \\
\hline $24 \ldots$ & 30 & 170 & 150 & 11 & 371 & 0.132 & $\cdots$ & 0.036 & - & 0.088 & - & $\cdots$ \\
\hline \multicolumn{13}{|l|}{ DEC } \\
\hline $18 \ldots$ & 20 & 115 & 87 & 9.6 & 265 & 0.242 & $\cdots$ & 0.009 & -. & 0.061 & -. & $-\cdot$ \\
\hline \multicolumn{13}{|l|}{ MAR 1992} \\
\hline $25 \ldots$ & 17 & 122 & 70 & 11 & 230 & 0.090 & $\cdots$ & 0.020 & $\cdots$ & 0.032 & $\cdots$ & $\cdots$ \\
\hline APR & & & & & & & & & & & & \\
\hline $23 \ldots$ & 28 & 130 & 130 & 13 & 348 & 0.160 & $\cdots$ & 0.019 & - & 0.080 & $\cdots$ & $\cdots$ \\
\hline MAY & & & & & & & & & & & & \\
\hline $22 \ldots$ & 9.5 & 68 & 46 & 4.0 & 136 & 0.180 & . & 0.033 & $\cdots$ & 0.060 & $\cdots$ & $\cdots$ \\
\hline JUN & & & & & & & & & & & & \\
\hline $26 \ldots$ & 11 & 85 & 47 & 4.2 & 176 & 0.089 & $\cdots$ & 0.029 & $\cdots$ & 0.041 & $<1$ & $<1$ \\
\hline JUL & & & & & & & & & & & & \\
\hline $15 \ldots$ & 14 & 101 & 60 & 6.5 & 202 & 0.063 & $\cdots$ & 0.008 & -. & 0.033 & $\cdots$ & $\cdots$ \\
\hline$A \cup G$ & & & & & & & & & & & & \\
\hline $13 \ldots$ & 16 & 103 & 70 & 7.6 & 216 & 0.178 & $\cdots$ & 0.017 & - & 0.054 & 1 & $<1$ \\
\hline $24 \ldots$ & 25 & 107 & 110 & 8.0 & 270 & 0.282 & . & 0.056 & - & 0.059 & -. & . \\
\hline OCT & & & & & & & & & & & & \\
\hline $29 \ldots$ & 25 & 150 & 110 & 10 & 312 & 0.288 & $\cdots$ & 0.030 & $\cdots$ & 0.052 & $\cdots$ & $\cdots$ \\
\hline JAN 1993 & & & & & & & & & & & & \\
\hline $13 \ldots$ & 18 & 114 & 85 & 8.0 & 238 & - & 0.304 & $\cdots$ & 0.014 & 0.032 & $-\cdot$ & $\cdots$ \\
\hline MAR & & & & & & & & & & & & \\
\hline $24 \ldots$ & 12 & 80 & 53 & - & 148 & $\cdots$ & 0.049 & $\cdots$ & 0.019 & 0.054 & $-\cdot$ & $\cdots$ \\
\hline
\end{tabular}


Table 42. Onsite measurements and bacteriological and selected inorganic data for station 07097000 , Arkansas River at Portland--Continued

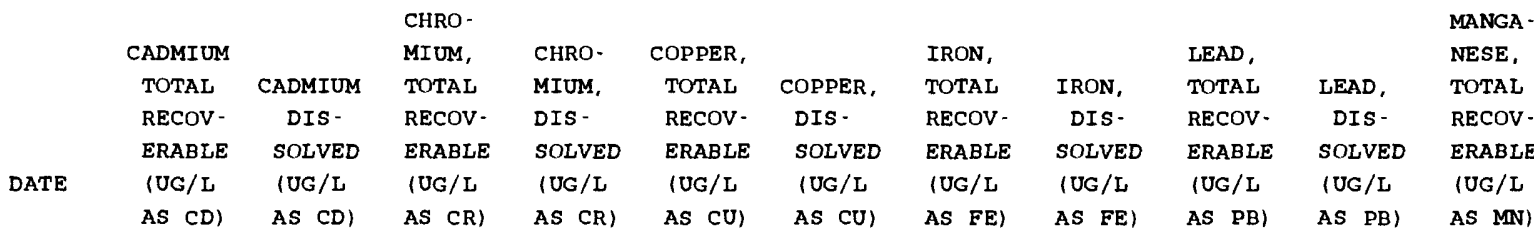

\begin{tabular}{|c|c|c|c|c|c|c|c|c|c|c|c|}
\hline \multicolumn{12}{|l|}{ APR 1990} \\
\hline $20 \ldots$ & $<1$ & $<0.1$ & $\cdots$ & $\cdots$ & 4 & 2 & 700 & 10 & 3 & $<0.5$ & 70 \\
\hline \multicolumn{12}{|l|}{ MAY } \\
\hline $24 \ldots$ & 2 & $<0.1$ & $-\cdot$ & $\cdots$ & 10 & 2 & 12000 & 21 & 15 & $<0.5$ & 510 \\
\hline \multicolumn{12}{|l|}{ JUN } \\
\hline $07 \ldots$ & 5 & 0.1 & $\cdots$ & $\cdots$ & 70 & 2 & 19000 & 44 & 110 & 1.3 & 990 \\
\hline $21 \ldots$ & $<1$ & 0.1 & $\cdots$ & - - & 68 & 25 & 3300 & 17 & 32 & 1.2 & 150 \\
\hline \multicolumn{12}{|l|}{ JUL } \\
\hline $19 \ldots$ & $<1$ & 0.2 & $\cdots$ & $\cdots$ & 5 & 3 & 1100 & 17 & 4 & $<0.5$ & 60 \\
\hline \multicolumn{12}{|l|}{ AUG } \\
\hline $30 \ldots$ & $<1$ & $<0.1$ & 1 & $<1$ & 3 & 1 & 180 & 17 & 1 & $<0.5$ & 40 \\
\hline \multicolumn{12}{|l|}{ NOV } \\
\hline $02 \ldots$ & $<1$ & $<0.1$ & $\cdots$ & $\cdots$ & 4 & 1 & 520 & 36 & 2 & 0.5 & 60 \\
\hline \multicolumn{12}{|l|}{ JAN 1991} \\
\hline $17 \ldots$ & $<1$ & $<0.1$ & $\cdots$ & $\cdots$ & 4 & 1 & 640 & 24 & 2 & $<0.5$ & 60 \\
\hline \multicolumn{12}{|l|}{ MAR } \\
\hline $27 \ldots$ & $<1$ & $<0.1$ & $\cdots$ & $\cdots$ & 4 & 2 & 360 & 17 & 1 & $<0.5$ & 40 \\
\hline \multicolumn{12}{|l|}{$\mathrm{APR}$} \\
\hline $26 \ldots$ & $<1$ & $<0.1$ & $\cdots$ & $-\cdot$ & 4 & 2 & 290 & 36 & 4 & $<0.5$ & 60 \\
\hline \multicolumn{12}{|l|}{ MAY } \\
\hline $16 \ldots$ & 2 & $<0.1$ & - & $\cdots$ & 18 & 2 & 3100 & 37 & 24 & 1.4 & 210 \\
\hline \multicolumn{12}{|l|}{ JUN } \\
\hline $20 \ldots$ & $<1$ & 0.1 & 1 & $<1$ & 22 & 11 & 1700 & 33 & 14 & $<0.5$ & 110 \\
\hline \multicolumn{12}{|l|}{ JUL } \\
\hline $18 \ldots$ & $<1$ & $<0.1$ & -- & $\cdots$ & 6 & $<1$ & 330 & 19 & 5 & 0.9 & 40 \\
\hline \multicolumn{12}{|l|}{ AUG } \\
\hline $15 \ldots$ & $<1$ & 0.4 & - - & -- & 6 & 1 & 3200 & 19 & 14 & $<0.5$ & 150 \\
\hline \multicolumn{12}{|l|}{ SEP } \\
\hline $03 \ldots$ & $\cdots$ & - & $<1$ & $<1$ & $\cdots$ & $\cdot$ & - & - & $\cdots$ & $\cdots$ & $\cdots$ \\
\hline \multicolumn{12}{|l|}{ OCT } \\
\hline $24 \ldots$ & $<1$ & $<0.1$ & $\cdots$ & $\cdots$ & 11 & $<1$ & 600 & 18 & 11 & 0.5 & 70 \\
\hline \multicolumn{12}{|l|}{ DEC } \\
\hline $18 \ldots$ & $<1$ & $<0.1$ & $\cdots$ & $\cdots$ & & $<1$ & 440 & 22 & $<1$ & $<0.5$ & 50 \\
\hline \multicolumn{12}{|l|}{ MAR 1992} \\
\hline $25 \ldots$ & $<1$ & 0.1 & - & - & 2 & 1 & 770 & 21 & 2 & $<0.5$ & 70 \\
\hline APR & & & & & & & & & & & \\
\hline $23 \ldots$ & $<1$ & $<0.1$ & $\cdots$ & $\cdots$ & 2 & 1 & 640 & 37 & 2 & 0.7 & 100 \\
\hline MAY & & & & & & & & & & & \\
\hline $22 \ldots$ & 2 & $<0.1$ & - & - - & 15 & 1 & 6800 & 54 & 40 & $<0.5$ & 570 \\
\hline JUN & & & & & & & & & & & \\
\hline $26 \ldots$ & $<1$ & $<0.1$ & $<1$ & $<1$ & 17 & 4 & 4600 & 25 & 13 & $<0.5$ & 220 \\
\hline JUL & & & & & & & & & & & \\
\hline $15 \ldots$ & $<1$ & $<0.1$ & - & $\cdots$ & 3 & 2 & 540 & 19 & 4 & 0.6 & 60 \\
\hline AUG & & & & & & & & & & & \\
\hline $13 \ldots$ & $<1$ & $<0.1$ & $<1$ & $<1$ & 5 & 1 & 1400 & 17 & 6 & 0.6 & 90 \\
\hline $24 \ldots$ & 2 & 0.2 & . & . & 34 & 2 & 25000 & 23 & 43 & 0.5 & 680 \\
\hline OCT & & & & & & & & & & & \\
\hline $29 \ldots$ & $<1$ & $<0.1$ & - & $\cdots$ & 4 & 1 & 2600 & 15 & 12 & $<0.5$ & 90 \\
\hline JAN 1993 & & & & & & & & & & & \\
\hline $13 \ldots$ & $<1$ & $<0.1$ & $\cdots$ & -. & 3 & 1 & 1200 & 16 & 4 & 1.0 & 80 \\
\hline MAR & & & & & & & & & & & \\
\hline $24 \ldots$ & $<1$ & $<0.1$ & $\cdots$ & - - & 2 & 1 & 650 & 22 & 3 & $<0.5$ & 60 \\
\hline
\end{tabular}


Table 42. Onsite measurements and bacteriological and selected inorganic data for station 07097000 , Arkansas River at Portland---Continued

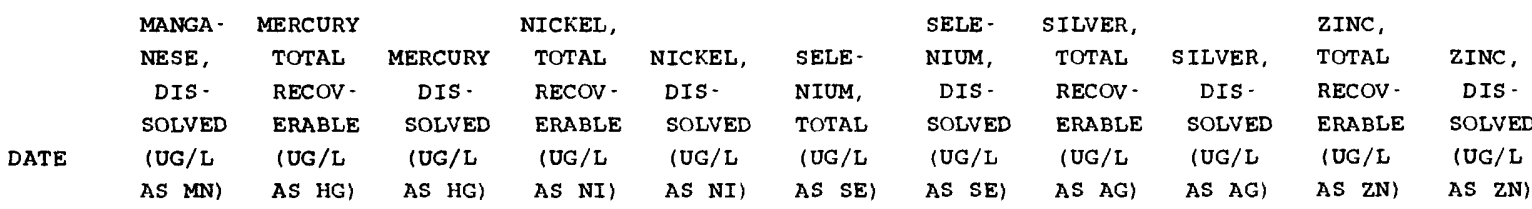

APR 1990

$20 .$.

MAY

24...

JUN

$07 .$.

$21 \ldots$

JUL

$19 .$.

AUG

$30 .$.

NOV

$02 \ldots$

JAN 1991

17 ...

MAR

27...

APR

$26 \ldots$

MAY

$16 \ldots$

JUN

$20 \ldots$

JUL

18 ...

AUG

$15 \ldots$

SEP

$03 \ldots$

OCT

$24 \ldots$

DEC

$18 \ldots$

MAR 1992

$25 \ldots$

APR

$23 \ldots$

MAY

$22 \ldots$

JUN

$26 \ldots$

JUL

$15 \ldots$

AUG

$13 . .$.

$24 \ldots$

OCT

$29 . .$.

JAN 1993

$13 .$.

MAR

$24 \ldots$

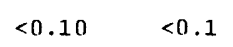

18

$<0$.

21

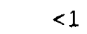

$<1.0$

10

16

$\cdots$

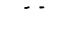

$$
\text { - } \quad \text { - }
$$$$
\cdots
$$

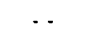

-

$$
\text { - }
$$
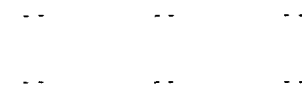

$<1.0$

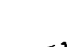

$$
<1
$$$$
\cdots
$$

$<1.0$ 
Table 43. Onsite measurements and selected inorganic data for station 07099100, Beaver Creek near Portland

\begin{tabular}{|c|c|c|c|c|c|c|c|c|c|c|c|}
\hline DATE & TIME & $\begin{array}{c}\text { DIS- } \\
\text { CHARGE, } \\
\text { INST. } \\
\text { CUBIC } \\
\text { FEET } \\
\text { PER } \\
\text { SECOND }\end{array}$ & $\begin{array}{l}\text { SPE- } \\
\text { CIFIC } \\
\text { CON- } \\
\text { DUCT- } \\
\text { ANCE } \\
\text { (US/CM) }\end{array}$ & $\begin{array}{c}\text { PH } \\
\text { WATER } \\
\text { WHOLE } \\
\text { FIELD } \\
\text { (STAND- } \\
\text { ARD } \\
\text { UNITS) }\end{array}$ & $\begin{array}{c}\text { TEMPER - } \\
\text { ATURE } \\
\text { WATER } \\
\text { (DEG C) }\end{array}$ & $\begin{array}{l}\text { OXYGEN, } \\
\text { DIS - } \\
\text { SOLVED } \\
\text { (MG /L) }\end{array}$ & $\begin{array}{c}\text { SOLIDS, } \\
\text { RESIDUE } \\
\text { AT } 180 \\
\text { DEG C } \\
\text { DIS. } \\
\text { SOLVED } \\
\text { (MG/L) }\end{array}$ & $\begin{array}{l}\text { NITRO- } \\
\text { GEN, } \\
\text { NO2+NO3 } \\
\text { TOTAL } \\
\text { (MG/L } \\
\text { AS N) }\end{array}$ & $\begin{array}{c}\text { NITRO- } \\
\text { GEN, } \\
\text { AMMONIA } \\
\text { TOTAL } \\
\text { (MG/L } \\
\text { AS N) }\end{array}$ & $\begin{array}{l}\text { CADMIUM } \\
\text { TOTAL } \\
\text { RECOV- } \\
\text { ERABLE } \\
\text { (UG/L } \\
\text { AS CD) }\end{array}$ & $\begin{array}{l}\text { CADMTUM } \\
\text { DIS - } \\
\text { SOLVED } \\
\text { (UG/L } \\
\text { AS CD) }\end{array}$ \\
\hline APR 1990 & & & & & & & & & & & \\
\hline$\underset{\text { JUN }}{20 \ldots}$ & 1700 & E0.22 & 2210 & 7.8 & 23.5 & 7.4 & 1990 & 0.014 & $<0.010$ & $\cdots$ & $\cdots$ \\
\hline JUL $07 .$. & 1625 & E12 & $\cdots$ & 8.3 & 28.5 & 6.3 & 588 & $\cdots$ & $\cdots$ & $\cdots$ & $\cdots$ \\
\hline$\underset{A U G}{19} \ldots$ & 1440 & $E 0.44$ & 1650 & 7.9 & 29.0 & 7.4 & 1400 & 0.010 & $<0.010$ & $\cdots$ & $\cdots$ \\
\hline $\begin{array}{l}30 \ldots \\
\text { Nov }\end{array}$ & 1350 & E3.9 & 1400 & 7.7 & 28.0 & 7.5 & 1250 & 0.045 & $<0.010$ & $\cdots$ & $\cdots$ \\
\hline $\begin{array}{r}02 \ldots \\
\text { APR } 1991\end{array}$ & 1130 & E1.2 & 1720 & 8.1 & 11.0 & 9.2 & 1380 & 0.077 & 0.030 & $\cdots$ & $\cdots$ \\
\hline $\begin{array}{l}26 \ldots \\
\text { JUN }\end{array}$ & 1130 & E0.90 & 1410 & 8.1 & 9.5 & 9.5 & 1070 & 0.016 & 0.052 & $\cdots$ & $\cdots$ \\
\hline$J_{J L}^{20 \ldots}$ & 1445 & E64 & 251 & 8.0 & 25.5 & 7.0 & 155 & 0.018 & 0.015 & $\cdots$ & $\cdots$ \\
\hline${ }_{\text {OCT }}^{18 \ldots}$ & 1525 & E1.2 & 1770 & 8.1 & 30.5 & 6.5 & 1470 & 0.010 & 0.036 & $\cdots$ & +- \\
\hline $\begin{array}{c}24 \ldots \\
\text { APR } 1992\end{array}$ & 1510 & E4. 4 & 1940 & 8.0 & 14.5 & 8.4 & 1680 & 3.40 & 0.064 & - - & - \\
\hline $\mathrm{JUN}^{23} \cdots$ & 1430 & E66 & 290 & 8.1 & 16.0 & 7.9 & 192 & 0.067 & 0.012 & $\cdots$ & $\cdots$ \\
\hline $\begin{array}{l}11 \ldots \\
26 . .\end{array}$ & $\begin{array}{l}0805 \\
1520\end{array}$ & $\begin{array}{l}\text { E112 } \\
\text { E130 }\end{array}$ & $\begin{array}{l}192 \\
175\end{array}$ & $\begin{array}{l}8.1 \\
8.2\end{array}$ & $\begin{array}{l}13.5 \\
23.0\end{array}$ & $\begin{array}{l}8.2 \\
7.3\end{array}$ & $\begin{array}{l}108 \\
110\end{array}$ & $\begin{array}{l}0.102 \\
0.055\end{array}$ & $\begin{array}{l}0.008 \\
0.007\end{array}$ & $\begin{array}{l}<1 \\
<1\end{array}$ & $\begin{array}{l}<0.1 \\
<0.1\end{array}$ \\
\hline$A \cup G$ & & & & & & & & & & & \\
\hline${ }_{\text {OCT }}^{13} \ldots$ & 1450 & E2.2 & 979 & 8.2 & 22.0 & 6.7 & 726 & 0.104 & 0.042 & $\cdots$ & $\cdots$ \\
\hline $29 \ldots$ & 1510 & E0.80 & 1810 & 8.0 & 11.0 & 9.7 & 1570 & 0.817 & 0.104 & $\cdots$ & $\cdots$ \\
\hline
\end{tabular}

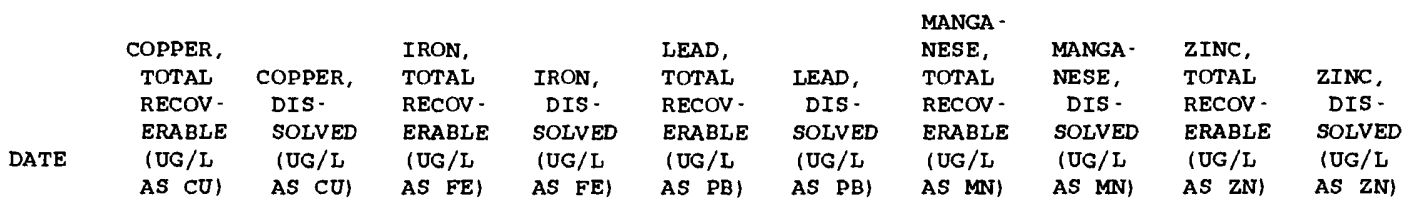

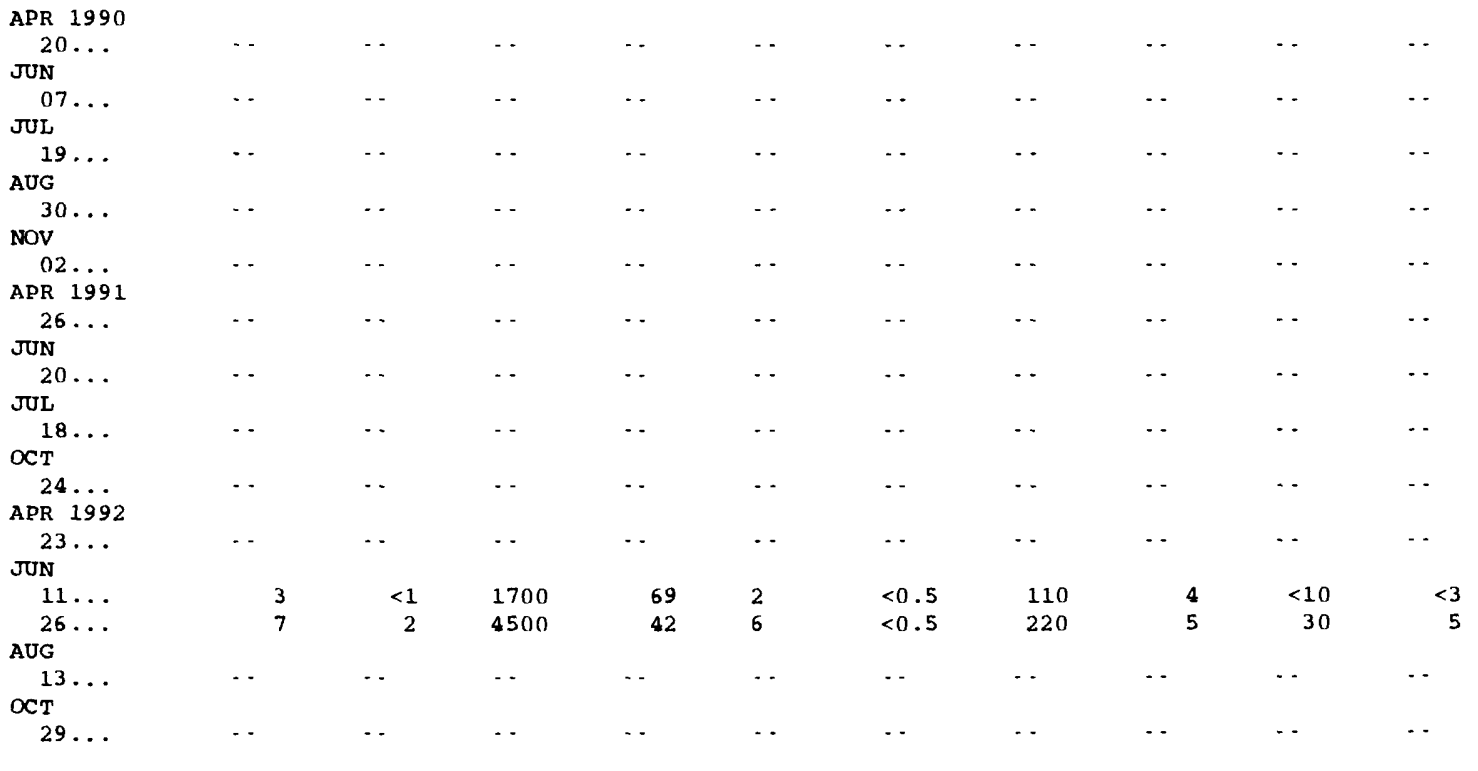


Table 44. Onsite measurements and bacteriological and selected inorganic data for station 07099400 ,

Arkansas River above Pueblo

\begin{tabular}{|c|c|c|c|c|c|c|c|c|c|c|}
\hline TIME & $\begin{array}{c}\text { DIS- } \\
\text { CHARGE, } \\
\text { INST. } \\
\text { CUBIC } \\
\text { FEET } \\
\text { PER }\end{array}$ & $\begin{array}{l}\text { SPE- } \\
\text { CIFIC } \\
\text { CON- } \\
\text { DUCT - } \\
\text { ANCE }\end{array}$ & $\begin{array}{c}\text { PH } \\
\text { WATER } \\
\text { WHOLE } \\
\text { FIELD } \\
\text { (STAND - } \\
\text { ARD }\end{array}$ & $\begin{array}{c}\text { TEMPER- } \\
\text { ATURE } \\
\text { WATER }\end{array}$ & $\begin{array}{c}\text { OXYGEN, } \\
\text { DIS- } \\
\text { SOLVED }\end{array}$ & $\begin{array}{l}\text { COLI- } \\
\text { FORM, } \\
\text { FECAL, } \\
0.7 \\
\text { UM.MF } \\
\text { (COLS.) }\end{array}$ & $\begin{array}{c}\text { STREP- } \\
\text { TOCOCCI } \\
\text { FECAL, } \\
\text { KF AGAR } \\
\text { (COLS. } \\
\text { PER }\end{array}$ & $\begin{array}{l}\text { HARD - } \\
\text { NESS } \\
\text { TOTAL } \\
\text { (MG / L } \\
\text { AS }\end{array}$ & $\begin{array}{l}\text { CALCIUM } \\
\text { DIS - } \\
\text { SOLVED } \\
\text { (MG/L }\end{array}$ & $\begin{array}{l}\text { MAGNE- } \\
\text { SIUM, } \\
\text { DIS - } \\
\text { SOLVED } \\
\text { (MG/L }\end{array}$ \\
\hline & SECOND & (US/CM) & UNITS) & (DEG C) & $(M G / L)$ & $100 \mathrm{ML})$ & $100 \mathrm{ML})$ & (АСО 3$)$ & AS CA) & AS MG) \\
\hline
\end{tabular}

\begin{tabular}{|c|c|c|c|c|c|c|c|c|c|c|c|}
\hline \multicolumn{12}{|l|}{ APR 1990} \\
\hline $09 \ldots$ & 1440 & 228 & 571 & 8.4 & 6.0 & 9.2 & -. & - & 250 & 66 & 20 \\
\hline \multicolumn{12}{|l|}{ MAY } \\
\hline $14 \ldots$ & 0825 & 360 & $\cdots$ & 8.3 & 10.0 & 11.6 & K2 & K12 & 270 & 70 & 22 \\
\hline \multicolumn{12}{|l|}{ JUN } \\
\hline $11 \ldots$ & 0915 & 3180 & 603 & 8.3 & 15.0 & 9.0 & K3 & K9 & 240 & 63 & 20 \\
\hline \multicolumn{12}{|l|}{ JUL } \\
\hline $20 \ldots$ & 1920 & 1260 & 355 & 8.0 & 20.0 & $\cdots$ & $\cdots$ & $\cdots$ & 150 & 42 & 11 \\
\hline $24 \ldots$ & 0950 & 695 & 377 & 8.2 & 20.5 & 7.9 & K9 & K35 & 160 & 45 & 11 \\
\hline \multicolumn{12}{|l|}{ AUG } \\
\hline $20 \ldots$ & 1035 & 968 & 426 & 7.9 & 21.0 & 8.0 & $<5$ & K12 & 170 & 49 & 12 \\
\hline \multicolumn{12}{|l|}{ SEP } \\
\hline $28 \ldots$ & 1510 & 328 & 478 & 8.1 & 19.5 & $\cdots$ & $<1$ & K6 & 200 & 54 & 15 \\
\hline \multicolumn{12}{|l|}{ OCT } \\
\hline $22 \ldots$ & 0915 & E555 & 553 & 8.5 & 14.0 & 9.2 & E15 & 29 & 220 & 61 & 17 \\
\hline \multicolumn{12}{|l|}{ DEC } \\
\hline $17 \ldots$ & 0910 & 112 & 569 & 8.5 & 4.5 & 10.9 & E1 & 92 & 250 & 68 & 19 \\
\hline \multicolumn{12}{|l|}{ MAR 1991} \\
\hline $27 \ldots$ & 1150 & 368 & 561 & 8.5 & 7.5 & 10.4 & $<1$ & 23 & 240 & 65 & 19 \\
\hline \multicolumn{12}{|l|}{ APR } \\
\hline $15 \ldots$ & 0820 & 292 & 574 & 7.7 & 8.5 & 10.2 & $<1$ & E1 & 230 & 63 & 18 \\
\hline \multicolumn{12}{|l|}{ MAY } \\
\hline $20 \ldots$ & 0900 & 560 & 575 & 8.2 & 11.0 & 9.8 & $<1$ & E2 & 240 & 66 & 19 \\
\hline \multicolumn{12}{|l|}{ JUN } \\
\hline $10 \ldots$ & 0930 & 1500 & 516 & 8.2 & 15.5 & 8.8 & $<1$ & E6 & 210 & 57 & 16 \\
\hline \multicolumn{12}{|l|}{ JUL } \\
\hline $22 \ldots$ & 0830 & 1520 & 379 & 7.8 & 21.0 & 8.2 & $<1$ & E18 & 180 & 48 & 14 \\
\hline \multicolumn{12}{|l|}{ AUG } \\
\hline $15 \ldots$ & 0740 & 1380 & 475 & 8.1 & 21.0 & $\cdots$ & 29 & 72 & 200 & 57 & 13 \\
\hline \multicolumn{12}{|l|}{ OCT } \\
\hline $28 \ldots$ & 0935 & 196 & 656 & 8.4 & 13.0 & 9.3 & E9 80 & 35 & 260 & 73 & 20 \\
\hline \multicolumn{12}{|l|}{ DEC } \\
\hline $16 \ldots$ & 0920 & 133 & 643 & 8.3 & 4.5 & 10.6 & E2 & 15 & 280 & 74 & 22 \\
\hline \multicolumn{12}{|l|}{ MAR 1992} \\
\hline $25 \ldots$ & 1145 & 407 & 549 & 8.3 & 6.0 & 10.6 & $\cdots$ & - & 230 & 63 & 18 \\
\hline \multicolumn{12}{|l|}{ APR } \\
\hline $28 \ldots$ & 0825 & 620 & 564 & 8.3 & 8.0 & 10.8 & $<1$ & $<1$ & 240 & 67 & 18 \\
\hline MAY & & & & & & & & & & & \\
\hline $18 \ldots$ & 0855 & 670 & 562 & 8.2 & 11.0 & 11.3 & $<1$ & $<1$ & 230 & 63 & 18 \\
\hline JUN & & & & & & & & & & & \\
\hline $16 \ldots$ & 0730 & 1660 & 472 & 8.2 & 16.0 & 8.8 & E4 & E3 & 190 & 53 & 15 \\
\hline JUL & & & & & & & & & & & \\
\hline $16 \ldots$ & 0800 & 1140 & 396 & 8.2 & 19.5 & 8.2 & E4 & E7 & 160 & 45 & 12 \\
\hline AUG & & & & & & & & & & & \\
\hline $17 \ldots$ & 0845 & 645 & 425 & 8.2 & 20.5 & 8.1 & E1 & E10 & 180 & 52 & 13 \\
\hline Ост & & & & & & & & & & & \\
\hline $20 \ldots$ & 0830 & 288 & 511 & 8.3 & 14.0 & 9.3 & E3 & 24 & 220 & 60 & 16 \\
\hline JAN 1993 & & & & & & & & & & & \\
\hline $13 \ldots$ & 1320 & 241 & 560 & 8.4 & 3.5 & 12.1 & $<1$ & E1 & 230 & 63 & 18 \\
\hline MAR & & & & & & & & & & & \\
\hline $24 \ldots$ & 1000 & 253 & 517 & 8.4 & 7.0 & 10.8 & $<1$ & $\cdots$ & 210 & 58 & 17 \\
\hline
\end{tabular}


Table 44. Onsite measurements and bacteriological and selected inorganic data for station 07099400 , Arkansas River above Pueblo--Continued

\begin{tabular}{|c|c|c|c|c|c|c|c|c|c|c|}
\hline DATE & $\begin{array}{l}\text { SODIUM, } \\
\text { DIS - } \\
\text { SOLVED } \\
\text { (MG/L } \\
\text { AS NA) }\end{array}$ & $\begin{array}{c}\text { ALKA- } \\
\text { LINITY } \\
\text { LAB } \\
\text { (MG/L } \\
\text { AS } \\
\text { CACO3) }\end{array}$ & $\begin{array}{l}\text { SULFATE } \\
\text { DIS - } \\
\text { SOLVED } \\
\text { (MG/L } \\
\text { AS SO4) }\end{array}$ & $\begin{array}{l}\text { CHLO- } \\
\text { RIDE, } \\
\text { DIS - } \\
\text { SOLVED } \\
\text { (MG/L } \\
\text { AS CL) }\end{array}$ & $\begin{array}{c}\text { SOLIDS, } \\
\text { RESIDUE } \\
\text { AT } 180 \\
\text { DEG C } \\
\text { DIS - } \\
\text { SOLVED } \\
\text { (MG } / L)\end{array}$ & $\begin{array}{l}\text { NITRO- } \\
\text { GEN, } \\
\text { NO2+NO3 } \\
\text { TOTAL } \\
\text { (MG/L } \\
\text { AS N) }\end{array}$ & $\begin{array}{l}\text { NITRO- } \\
\text { GEN, } \\
\text { NO2+NO3 } \\
\text { DIS - } \\
\text { SOLVED } \\
\text { (MG/L } \\
\text { AS N) }\end{array}$ & $\begin{array}{l}\text { NITRO- } \\
\text { GEN, } \\
\text { AMMONIA } \\
\text { TOTAL } \\
\text { (MG/L } \\
\text { AS N) }\end{array}$ & $\begin{array}{l}\text { NITRO- } \\
\text { GEN, } \\
\text { AMMONIA } \\
\text { DIS - } \\
\text { SOLVED } \\
\text { (MG/L } \\
\text { AS N) }\end{array}$ & $\begin{array}{l}\text { PHOS - } \\
\text { PHORUS } \\
\text { TOTAL } \\
\text { (MG/L } \\
\text { AS P) }\end{array}$ \\
\hline \multicolumn{11}{|l|}{ APR 1990} \\
\hline${ }_{\operatorname{MAY}}^{09} \ldots$ & \multicolumn{8}{|c|}{ MAY } & & 0.040 \\
\hline${ }_{\text {JUN }}^{14 \ldots}$ & \multicolumn{8}{|c|}{ JUN } & $\cdots$ & 0.040 \\
\hline${ }_{\text {JUL }} 11 \ldots$ & 29 & 126 & 170 & 11 & 369 & 0.200 & $\cdots$ & 0.080 & $\cdots$ & 0.020 \\
\hline $20 \ldots$ & 15 & 87 & 81 & 6.1 & 220 & 0.200 & $\cdots$ & 0.050 & $\cdots$ & 0.060 \\
\hline $24 \ldots$ & 16 & 91 & 89 & 6.1 & 243 & 0.200 & - & 0.040 & $\cdots$ & 0.040 \\
\hline \multicolumn{11}{|l|}{ AUG } \\
\hline$\underset{\operatorname{SEP}}{20 \ldots}$ & 18 & 94 & 100 & 9.5 & 265 & 0.100 & $\cdots$ & 0.110 & $\cdots$ & 0.030 \\
\hline $28 \ldots$ & 22 & 107 & 120 & 8.9 & 305 & 0.200 & - & 0.020 & - & 0.020 \\
\hline $\begin{array}{c}\text { OCT } \\
22 \ldots\end{array}$ & 25 & 120 & 150 & 8.7 & 355 & 0.330 & $\cdots$ & 0.030 & . & 0.030 \\
\hline $\begin{array}{l}\text { DEC } \\
17 \ldots\end{array}$ & 27 & 136 & 150 & 12 & 378 & 0.250 & $\cdots$ & 0.060 & - & 0.020 \\
\hline $\begin{array}{c}\text { MAR } 1991 \\
27 \ldots\end{array}$ & 26 & 128 & 150 & 9.0 & 360 & 0.210 & $\cdots$ & 0.030 & $\cdots$ & 0.020 \\
\hline \multicolumn{5}{|l|}{ APR } & 366 & 0.210 & $\cdots$ & 0.030 & $\cdots$ & 0.020 \\
\hline \multicolumn{11}{|l|}{ MAY } \\
\hline $\begin{array}{l}20 \ldots \\
\text { JuN }\end{array}$ & \multicolumn{7}{|c|}{ JUN } & & & 0.020 \\
\hline & 23 & 114 & 140 & 9.0 & 314 & 0.140 & \multicolumn{2}{|c|}{ JUL } & & 0.030 \\
\hline$\underset{A U G}{22 \ldots}$ & 23 & 94 & 130 & 9.5 & $\cdots$ & 0.470 & \multicolumn{2}{|c|}{ AUG } & & 0.310 \\
\hline & 19 & 99 & 130 & 7.5 & 308 & 0.340 & \multicolumn{2}{|c|}{ OCT } & & 0.050 \\
\hline$\underset{D E C}{28 \ldots}$ & 28 & 139 & 220 & 9.9 & 429 & 0.240 & \multicolumn{2}{|c|}{$\mathrm{DEC}$} & & 0.570 \\
\hline \multicolumn{3}{|l|}{ MAR 1992} & 190 & 12 & 464 & 0.320 & & 0.040 & & 0.020 \\
\hline $25 \ldots$ & 25 & 123 & 160 & 13 & 354 & 0.200 & -. & 0.050 & $\cdots$ & $<0.010$ \\
\hline \multicolumn{11}{|l|}{ APR } \\
\hline$\underset{M A Y}{28 \ldots}$ & 25 & 127 & 150 & 8.2 & \multicolumn{5}{|c|}{ MAY } & $<0.010$ \\
\hline${ }_{\text {JUN }}^{18 \ldots}$ & 26 & 128 & 150 & 9.2 & 356 & 0.160 & $\cdots$ & 0.110 & $\cdots$ & 0.020 \\
\hline \multicolumn{11}{|l|}{ JUL } \\
\hline \multicolumn{9}{|l|}{ AUG } & $\cdots$ & $<0.010$ \\
\hline \multicolumn{9}{|l|}{ OCT } & $\cdots$ & 0.020 \\
\hline $\begin{array}{c}20 \ldots \\
\text { JAN } 1993\end{array}$ & 21 & 124 & 130 & 7.9 & 336 & 0.180 & $\cdots$ & 0.060 & $\cdot-$ & 0.050 \\
\hline $13 \ldots$ & 25 & 133 & 170 & 8.8 & 372 & $\cdots$ & 0.290 & $\cdots$ & 0.040 & 0.030 \\
\hline MAR & & & & & & & & & & \\
\hline $24 \ldots$ & 22 & 125 & 140 & 8.2 & 300 & $\cdots$ & 0.220 & $\cdots$ & 0.040 & 0.120 \\
\hline
\end{tabular}


Table 44. Onsite measurements and bacteriological and selected inorganic data for station 07099400 , Arkansas River above Pueblo--Continued

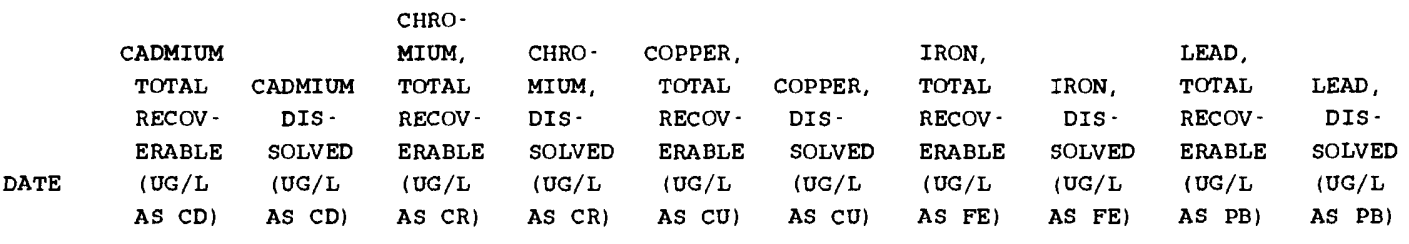

\begin{tabular}{|c|c|c|c|c|c|c|c|c|c|c|}
\hline \multicolumn{11}{|l|}{ APR 1990} \\
\hline $09 \ldots$ & $<1$ & 0.3 & 1 & 1 & 3 & 1 & 130 & 6 & 1 & $<0.5$ \\
\hline \multicolumn{11}{|l|}{ MAY } \\
\hline $14 \ldots$ & $<1$ & $<0.1$ & 1 & $<1$ & 2 & 1 & 30 & 4 & 1 & $<0.5$ \\
\hline \multicolumn{11}{|l|}{ JUN } \\
\hline $11 \ldots$ & 1 & 0.6 & $<1$ & $<1$ & 430 & 3 & 220 & 16 & 25 & 1.8 \\
\hline \multicolumn{11}{|l|}{ JUL } \\
\hline $20 \ldots$ & $<1$ & $<0.1$ & 1 & $<1$ & 3 & 2 & 700 & .10 & 6 & $<0.5$ \\
\hline $24 \ldots$ & $<1$ & $<0.1$ & 1 & $<1$ & 3 & 1 & 630 & 3 & 2 & $<0.5$ \\
\hline \multicolumn{11}{|l|}{ AUG } \\
\hline $20 \ldots$ & $<1$ & 0.5 & 2 & $<1$ & 3 & 2 & 4700 & 19 & 8 & $<0.5$ \\
\hline \multicolumn{11}{|l|}{ SEP } \\
\hline $28 \ldots$ & $<1$ & $<0.1$ & $<1$ & $<1$ & 2 & 1 & 280 & 3 & 2 & $<0.5$ \\
\hline \multicolumn{11}{|l|}{ OCT } \\
\hline $22 \ldots$ & $<1$ & $<0.1$ & 2 & $<1$ & 2 & 1 & 350 & 3 & 1 & $<0.5$ \\
\hline \multicolumn{11}{|l|}{$\mathrm{DEC}$} \\
\hline $17 \ldots$ & $<1$ & 0.3 & $<1$ & $<1$ & 3 & 1 & 110 & 9 & $<1$ & $<0.5$ \\
\hline \multicolumn{11}{|l|}{ MAR 1991} \\
\hline $27 \ldots$ & $<1$ & 0.4 & 2 & $<1$ & 2 & 2 & 100 & $<3$ & 1 & $<0.5$ \\
\hline \multicolumn{11}{|l|}{$\mathrm{APR}$} \\
\hline $15 \ldots$ & $<1$ & 0.9 & $<1$ & $<1$ & 2 & 1 & 60 & 5 & 2 & $<0.5$ \\
\hline \multicolumn{11}{|l|}{ MAY } \\
\hline $20 \ldots$ & $<1$ & $<0.1$ & $<1$ & $<1$ & 8 & 2 & 80 & 24 & 3 & $<0.5$ \\
\hline \multicolumn{11}{|l|}{ JUN } \\
\hline $10 \ldots$ & $<1$ & $<0.1$ & $<1$ & 2 & 10 & 4 & 110 & 6 & 6 & $<0.5$ \\
\hline \multicolumn{11}{|l|}{ JUL } \\
\hline $22 \ldots$ & $<1$ & $<0.1$ & 10 & $<1$ & 12 & 2 & $\cdots$ & $<10$ & 23 & $<0.5$ \\
\hline \multicolumn{11}{|l|}{ AUG } \\
\hline $15 \ldots$ & $<1$ & 2.9 & $<1$ & $<1$ & 12 & 6 & 500 & $<3$ & $\cdots$ & $<0.5$ \\
\hline \multicolumn{11}{|l|}{ Ост } \\
\hline $28 \ldots$ & $<1$ & $<0.1$ & 1 & $<1$ & 9 & $<1$ & 260 & $<3$ & 8 & 0.6 \\
\hline \multicolumn{11}{|l|}{ DEC } \\
\hline $16 \ldots$ & $<1$ & 0.8 & $<1$ & $<1$ & $\cdots$ & 1 & 130 & 5 & 5 & $<0.5$ \\
\hline \multicolumn{11}{|l|}{ MAR 1992} \\
\hline $25 \ldots$ & $<1$ & $<0.1$ & 1 & $<1$ & $<1$ & 1 & 40 & 28 & $<1$ & $<0.5$ \\
\hline \multicolumn{11}{|l|}{$A P R$} \\
\hline $28 \ldots$ & $<1$ & $<0.1$ & $<1$ & $<1$ & $<1$ & 1 & 60 & 15 & $<1$ & $<0.5$ \\
\hline MAY & & & & & & & & & & \\
\hline $18 \ldots$ & $<1$ & 0.1 & $<1$ & $<1$ & 1 & 1 & 70 & 8 & $<1$ & 1.5 \\
\hline JUN & & & & & & & & & & \\
\hline $16 \ldots$ & $<1$ & $<0.1$ & $<1$ & $<1$ & 4 & 3 & 110 & 5 & 1 & $<0.5$ \\
\hline JUL & & & & & & & & & & \\
\hline $16 \ldots$ & $<1$ & $<0.1$ & $<1$ & $<1$ & 4 & 1 & 150 & 4 & 1 & $<0.5$ \\
\hline AUG & & & & & & & & & & \\
\hline $17 \ldots$ & $<1$ & $<0.1$ & $<1$ & $<1$ & 2 & $<1$ & 180 & $<3$ & 1 & $<0.5$ \\
\hline OCT & & & & & & & & & & \\
\hline $20 \ldots$ & $<1$ & 0.3 & 1 & $<1$ & 3 & 2 & 340 & 4 & 3 & $<0.5$ \\
\hline JAN 1993 & & & & & & & & & & \\
\hline $13 \ldots$ & $<1$ & $<0.1$ & $<1$ & $<1$ & 1 & 1 & 30 & 6 & $<1$ & $<0.5$ \\
\hline MAR & & & & & & & & & & \\
\hline $24 \ldots$ & $<1$ & $<0.1$ & $<1$ & $<1$ & $<1$ & $<1$ & 20 & 5 & $<1$ & $<0.5$ \\
\hline
\end{tabular}


Table 44. Onsite measurements and bacteriological and selected inorganic data for station 07099400 , Arkansas River above Pueblo--Continued

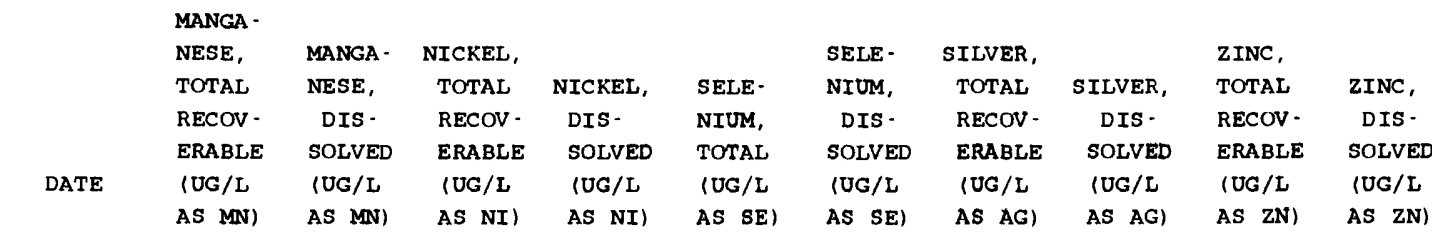

APR 1990

$09 .$.

MAY

$14 \ldots$

JUN

$11 \ldots$

JUL

$20 \ldots$

$24 \ldots$

AUG

$20 \ldots$

SEP

$28 \ldots$

OCT

$22 \ldots$

DEC

$17 \ldots$

MAR 1991

$27 \ldots$

APR

$15 \ldots$

MAY

$20 \ldots$

JUN

$10 \ldots$

$22 \ldots$

AS MN

AS MN)

AS NI) AS NI)

AS SE)

AS SE)

AS AG)

AS AG)

SN)

AS ZN)

AUG

$15 \ldots$

OCT

28 ...

DEC

$16 \ldots$

MAR 1992

$25 \ldots$

APR

$28 \ldots$

20

30

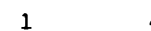

40

1

$$
1
$$

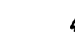

MAY

$18 \ldots$

JUN

$16 \ldots$

JUL

16 ...

AUG

$17 \ldots$

OCT

$20 \ldots$

JAN 1993

$13 \ldots$

MAR

$24 .$.

13

10

10

50

80

10

$<10$

$<10$

20

70

270

45

$50<1$

60

22

30

10

10

60

60

30

70

60

20

10

5

$<1$

$<1.0$

10

$<1.0<10$

$<1.0 \quad 30$

10

$4<1$

$<1<1.0 \quad 10$

$<10$

$<3$

$<1$

$<1.0$

$<10$

$<3$

18

$$
2
$$

$$
1
$$$$
<1
$$$$
<1.0
$$$$
20
$$$$
7
$$$$
<1<1.0 \quad 20<3
$$$$
<1<1.0<10
$$$$
<1<1.0 \quad 10
$$$$
<1<1.0<10
$$$$
<1<1.0<10
$$$$
<1<1.0<10
$$$$
<1
$$$$
<1.0
$$$$
50
$$$$
<1
$$$$
<1.0
$$$$
10
$$$$
<1<1.0<10
$$$$
<1<1.0
$$$$
<1
$$$$
<1.0
$$$$
40
$$$$
10
$$$$
<1
$$$$
<1.0
$$$$
<10
$$$$
<1<1.0
$$$$
<1<1.0
$$$$
<1
$$$$
<1,0
$$$$
<1<1.0 \quad 10<3
$$$$
37
$$$$
17
$$

43

$$
3
$$

3

3
3

3

2
$<1$ 
Table 45. Onsite measurements and bacteriological and selected inorganic data for station 07099970 ,

Arkansas River at Moffat Street, at Pueblo

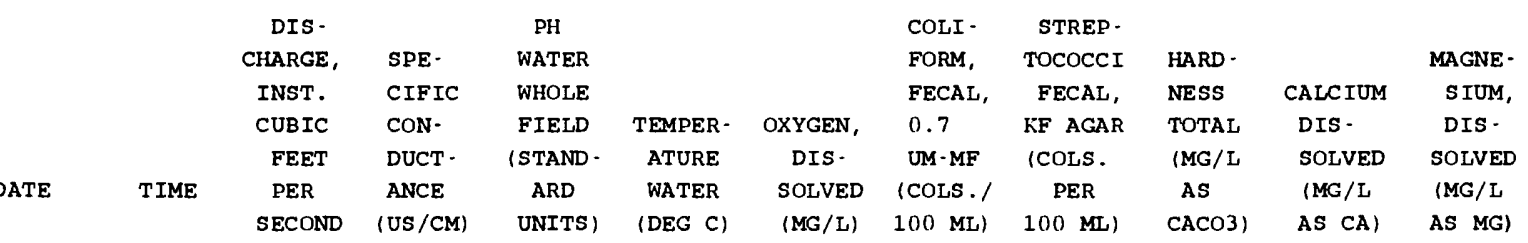

\begin{tabular}{|c|c|c|c|c|c|c|c|c|c|c|c|}
\hline APR 1990 & & & & & & & & & & & \\
\hline $10 \ldots$ & 0910 & 142 & 682 & 8.5 & 6.0 & 9.4 & $\cdots$ & $\cdots$ & 290 & 77 & 24 \\
\hline MAY & & & & & & & & & & & \\
\hline $14 \ldots$ & 1045 & 283 & 692 & 8.4 & 12.0 & 9.7 & K28 & K35 & 300 & 78 & 25 \\
\hline JON & & & & & & & & & & & \\
\hline $11 \ldots$ & 1130 & 3800 & 602 & 8.4 & 17.0 & 7.9 & 93 & 310 & 250 & 64 & 21 \\
\hline JUL & & & & & & & & & & & \\
\hline $20 \ldots$ & 1645 & 1460 & 353 & 8.1 & 22.5 & $\cdots$ & $\cdots$ & $\cdots$ & 140 & 41 & 10 \\
\hline $24 \ldots$ & 1135 & 629 & 427 & 8.4 & 21.5 & 7.8 & 98 & K560 & 180 & 51 & 13 \\
\hline AUG & & & & & & & & & & & \\
\hline $20 \ldots$ & 1215 & 863 & 445 & 8.3 & 22.5 & 7.9 & 52 & 110 & 180 & 52 & 13 \\
\hline SEP & & & & & & & & & & & \\
\hline $28 \ldots$ & 1100 & 270 & 558 & 8.1 & 18.0 & $\cdots$ & $>190$ & $>200$ & 220 & 60 & 18 \\
\hline ОСт & & & & & & & & & & & \\
\hline $22 \ldots$ & 1030 & 316 & 614 & 8.5 & 12.5 & 9.7 & E17 & 52 & 250 & 67 & 19 \\
\hline DEC & & & & & & & & & & & \\
\hline $17 \ldots$ & 1030 & 33 & 841 & 8.3 & 4.0 & 11.2 & E17 & E71 & 360 & 98 & 28 \\
\hline MAR 1991 & & & & & & & & & & & \\
\hline $27 \ldots$ & 1320 & 321 & 605 & $\cdots$ & 10.5 & $\cdots$ & E3 & E30 & 250 & 69 & 20 \\
\hline APR & & & & & & & & & & & \\
\hline $15 \ldots$ & 1005 & 232 & 639 & 8.1 & 8.0 & 9.9 & E1 & 22 & 260 & 70 & 20 \\
\hline MAY & & & & & & & & & & & \\
\hline $20 \ldots$ & 1115 & 502 & 607 & 8.2 & 12.5 & 9.4 & 46 & 97 & 250 & 68 & 20 \\
\hline JUN & & & & & & & & & & & \\
\hline $10 \ldots$ & 1145 & 1590 & 520 & 8.6 & 17.5 & 8.9 & E9 & 110 & 210 & 58 & 16 \\
\hline JUL & & & & & & & & & & & \\
\hline $22 \ldots$ & 1040 & 1630 & 402 & 8.4 & 21.5 & 7.6 & 51 & 96 & 160 & 45 & 12 \\
\hline AUG & & & & & & & & & & & \\
\hline $15 \ldots$ & 0955 & 1530 & 492 & 8.2 & 22.0 & 7.4 & 120 & 130 & 210 & 59 & 14 \\
\hline OCT & & & & & & & & & & & \\
\hline $28 \ldots$ & 1140 & 94 & 758 & 8.4 & 10.5 & 9.9 & E530 & 110 & 300 & 84 & 23 \\
\hline DEC & & & & & & & & & & & \\
\hline $16 \ldots$ & 1115 & 54 & 892 & 8.3 & 4.5 & 12.2 & E4 & E10 & 360 & 98 & 29 \\
\hline MAR 1992 & & & & & & & & & & & \\
\hline $25 \ldots$ & 1340 & 353 & 587 & 8.5 & 10.5 & 10.1 & $\cdots$ & -- & 240 & 65 & 19 \\
\hline APR & & & & & & & & & & & \\
\hline $28 \ldots$ & 0955 & 496 & 599 & 8.4 & 9.0 & 10.2 & E11 & 22 & 240 & 64 & 19 \\
\hline MAY & & & & & & & & & & & \\
\hline $18 \ldots$ & 1100 & 567 & 584 & 8.6 & 13.0 & 9.9 & E6 & 40 & 250 & 67 & 19 \\
\hline JUN & & & & & & & & & & & \\
\hline${ }_{\pi L}^{16}$ & 0930 & 1560 & 502 & 8.4 & 16.5 & 8.4 & E13 & 41 & 210 & 56 & 16 \\
\hline $16 \ldots$ & 1015 & 1020 & 423 & 8.4 & 19.5 & 8.1 & 26 & 33 & 170 & 48 & 13 \\
\hline AUG & & & & & & & & & & & \\
\hline${ }_{\text {OCT }}^{17 \ldots}$ & 0955 & 515 & 461 & 8.3 & 20.5 & 8.7 & E75 & 64 & 200 & 56 & 14 \\
\hline $20 \ldots$ & 1015 & 216 & 587 & 8.4 & 12.0 & 9.7 & E110 & 71 & 250 & 69 & 18 \\
\hline JAN 1993 & & & & & & & & & & & \\
\hline $14 \ldots$ & 0835 & 181 & 702 & 8.2 & 0.5 & 12.4 & E12 & E7 & 280 & 74 & 22 \\
\hline MAR & & & & & & & & & & & \\
\hline $24 \ldots$ & 1145 & 204 & 603 & 8.5 & 8.5 & 10.4 & E2 & $\cdots$ & 240 & 66 & 19 \\
\hline
\end{tabular}


Table 45. Onsite measurements and bacteriological and selected inorganic data for station 07099970 , Arkansas River at Moffat Street, at Pueblo--Continued

\begin{tabular}{|c|c|c|c|c|c|c|c|c|c|c|}
\hline & $\begin{array}{l}\text { SODIUM, } \\
\text { DIS - } \\
\text { SOLVED } \\
\text { (MG /L }\end{array}$ & $\begin{array}{l}\text { ALKA - } \\
\text { LINITY } \\
\text { LAB } \\
\text { (MG / L } \\
\text { AS }\end{array}$ & $\begin{array}{l}\text { SULFATE } \\
\text { DIS - } \\
\text { SOLVED } \\
\text { (MG /L }\end{array}$ & $\begin{array}{l}\text { CHLO- } \\
\text { RIDE, } \\
\text { DIS - } \\
\text { SOLVED } \\
\text { (MG/L }\end{array}$ & $\begin{array}{c}\text { SOLIDS, } \\
\text { RESIDUE } \\
\text { AT } 180 \\
\text { DEG C } \\
\text { DIS- } \\
\text { SOLVED }\end{array}$ & $\begin{array}{r}\text { NITRO- } \\
\text { GEN, } \\
\text { NO2+NO3 } \\
\text { TOTAL } \\
\text { (MG / L }\end{array}$ & $\begin{array}{l}\text { NITRO- } \\
\text { GEN, } \\
\text { NO2+NO3 } \\
\text { DIS - } \\
\text { SOLVED } \\
\text { (MG /L }\end{array}$ & $\begin{array}{l}\text { NITRO- } \\
\text { GEN, } \\
\text { AMMONIA } \\
\text { TOTAL } \\
\text { (MG /L }\end{array}$ & $\begin{array}{l}\text { NITRO- } \\
\text { GEN, } \\
\text { AMMONIA } \\
\text { DIS - } \\
\text { SOLVED } \\
\text { (MG /L }\end{array}$ & $\begin{array}{l}\text { PHOS - } \\
\text { PHORUS } \\
\text { TOTAL } \\
\text { (MG/L }\end{array}$ \\
\hline DATE & AS NA) & CACO3) & AS SO4) & AS CL) & (MG/L) & AS N) & AS N) & AS N) & AS N) & AS P) \\
\hline \multicolumn{11}{|l|}{ APR 1990} \\
\hline${ }_{M A Y}^{10} \ldots$ & \multicolumn{9}{|c|}{ MAY } & 0.020 \\
\hline$\underset{\pi U N}{14 \ldots}$ & 38 & 134 & 240 & 12 & 474 & 0.400 & $\cdots$ & 0.020 & $\cdots$ & 0.020 \\
\hline${ }_{\pi U L}^{11 \ldots}$ & 29 & $\cdots$ & 170 & 11 & 395 & 0.200 & $\cdots$ & 0.060 & $\cdots$ & 0.030 \\
\hline $20 \ldots$ & 15 & 87 & 86 & 7.3 & 210 & 0.400 & $\cdots$ & 0.070 & $\cdots$ & 0.280 \\
\hline $24 \ldots$ & 19 & 93 & 110 & 6.2 & 252 & 0.400 & $\cdots$ & 0.020 & $\cdots$ & 0.060 \\
\hline \multicolumn{11}{|l|}{ AUG } \\
\hline$\underset{\text { SEP }}{20}$ & \multicolumn{9}{|c|}{ SEP } & 0.030 \\
\hline \multicolumn{10}{|l|}{ OCT } & 0.030 \\
\hline $22 \ldots$ & 28 & 126 & 180 & 9.8 & 392 & 0.500 & $\cdots$ & 0.020 & $\cdots$ & 0.010 \\
\hline \multicolumn{11}{|l|}{ DEC } \\
\hline \multicolumn{11}{|l|}{ MAR 1991} \\
\hline $\begin{array}{l}27 \ldots \\
\text { APR }\end{array}$ & 28 & \multicolumn{7}{|c|}{ APR } & & 0.020 \\
\hline $15 \ldots$ & 31 & 134 & 180 & 10 & 423 & 0.500 & $\cdots$ & $<0.010$ & $\cdots$ & 0.010 \\
\hline \multicolumn{11}{|l|}{ MAY } \\
\hline \multicolumn{11}{|l|}{ JUN } \\
\hline $\int_{\pi L}^{10 \ldots}$ & 24 & \multicolumn{8}{|c|}{$\pi L$} & 0.030 \\
\hline$\underset{A U G}{22} \cdots$ & 17 & 89 & 110 & 6.6 & 237 & 0.210 & $\cdot-$ & $<0.010$ & $\cdots$ & 0.060 \\
\hline $\begin{array}{c}15 \ldots \\
\text { OCT }\end{array}$ & \multicolumn{9}{|c|}{ OCT } & 0.060 \\
\hline$\underset{D E C}{28 \ldots}$ & \multicolumn{9}{|c|}{ DEC } & $<0.010$ \\
\hline $16 \ldots$ & 45 & 151 & 290 & 18 & 663 & 1.10 & $\cdots$ & 0.010 & $\cdots$ & 0.010 \\
\hline \multicolumn{11}{|l|}{ MAR 1992} \\
\hline $25 \ldots$ & 28 & 123 & 180 & 14 & 390 & 0.110 & $\cdots$ & 0.020 & $\cdots$ & $<0.010$ \\
\hline \multicolumn{11}{|l|}{ APR } \\
\hline$\underset{M A Y}{28 \ldots}$ & 27 & 126 & 170 & \multicolumn{5}{|c|}{ MAY } & $\cdots$ & $<0.010$ \\
\hline $18 \ldots$ & 27 & 129 & 170 & 10 & 364 & 0.260 & $\cdots$ & 0.030 & $\cdots$ & 0.020 \\
\hline \multicolumn{11}{|l|}{ JUN } \\
\hline $16 \ldots$ & 22 & 108 & 130 & 8.6 & 294 & 0.170 & $\cdots$ & 0.020 & $\cdots$ & 0.020 \\
\hline $16 \ldots$ & 18 & 101 & 100 & 7.7 & 258 & 0.200 & $\cdots$ & 0.030 & $\cdots$ & $<0.010$ \\
\hline$A \cup G$ & & & & & & & & & & \\
\hline $\begin{array}{l}17 \ldots \\
\text { OCT }\end{array}$ & 19 & 108 & 120 & 6.7 & 276 & 0.290 & $\cdots$ & 0.030 & $\cdots$ & 0.030 \\
\hline $20 \ldots$ & 25 & 129 & 160 & 10 & 393 & 0.440 & $\cdots$ & 0.030 & $\cdots$ & 0.030 \\
\hline JAN 1993 & & & & & & & & & & \\
\hline $14 \ldots$ & 33 & 142 & 210 & 12 & 465 & $\cdots$ & 0.670 & $\cdots$ & 0.030 & 0.020 \\
\hline MAR & & & & & & & & & & \\
\hline $24 \ldots$ & 28 & 130 & 170 & 11 & 384 & $\cdots$ & 0.520 & $\cdots$ & 0.030 & 0.070 \\
\hline
\end{tabular}


Table 45. Onsite measurements and bacteriological and selected inorganic data for station 07099970 , Arkansas River at Moffat Street, at Pueblo--Continued

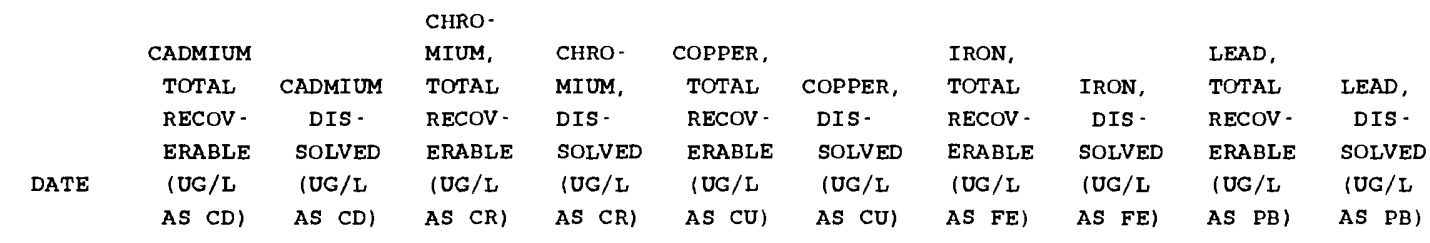

\begin{tabular}{|c|c|c|c|c|c|c|c|c|c|c|}
\hline \multicolumn{11}{|l|}{ APR 1990} \\
\hline $10 \ldots$ & $<1$ & $<0.1$ & 1 & 1 & 3 & 1 & 140 & 6 & 1 & $<0.5$ \\
\hline \multicolumn{11}{|l|}{ MAY } \\
\hline $14 \ldots$ & $<1$ & $<0.1$ & $<1$ & $<1$ & 2 & 1 & 80 & 4 & 1 & $<0.5$ \\
\hline \multicolumn{11}{|l|}{$\pi N$} \\
\hline $11 \ldots$ & $<1$ & 0.3 & $<1$ & $<1$ & 22 & 4 & 700 & 6 & 16 & 1.2 \\
\hline \multicolumn{11}{|l|}{ JOL } \\
\hline $20 \ldots$ & 2 & $\cdots$ & 6 & $<1$ & 15 & 5 & 5400 & 14 & 33 & $<0.5$ \\
\hline $24 \ldots$ & $<1$ & $<0.1$ & 1 & $<1$ & 3 & 1 & 1000 & 4 & 3 & $<0.5$ \\
\hline \multicolumn{11}{|l|}{ AUG } \\
\hline $20 \ldots$ & 1 & $<0.1$ & 4 & $<1$ & 3 & 1 & 490 & 4 & 2 & $<0.5$ \\
\hline \multicolumn{11}{|l|}{ SEP } \\
\hline $28 \ldots$ & $<1$ & 0.2 & $<1$ & $<1$ & 3 & 2 & 1000 & 7 & 4 & $<0.5$ \\
\hline \multicolumn{11}{|l|}{ OCT } \\
\hline $22 \ldots$ & $<1$ & $<0.1$ & $<1$ & $<1$ & 2 & 1 & 360 & 4 & $<1$ & $<0.5$ \\
\hline \multicolumn{11}{|l|}{ DEC } \\
\hline $17 \ldots$ & $<1$ & 0.2 & $<1$ & $<1$ & 3 & 1 & $\cdots$ & - & 1 & $<0.5$ \\
\hline \multicolumn{11}{|l|}{ MAR 1991} \\
\hline $27 \ldots$ & $<1$ & 0.2 & $<1$ & $<1$ & 3 & 1 & 120 & 7 & 1 & $<0.5$ \\
\hline \multicolumn{11}{|l|}{ APR } \\
\hline $15 \ldots$ & $<1$ & 0.4 & $<1$ & $<1$ & 2 & 1 & 80 & 7 & 1 & 0.7 \\
\hline \multicolumn{11}{|l|}{ MAY } \\
\hline $20 \ldots$ & $<1$ & $<0.1$ & $<1$ & $<1$ & 2 & 1 & 110 & 13 & 3 & $<0.5$ \\
\hline \multicolumn{11}{|l|}{ JUN } \\
\hline $10 \ldots$ & $<1$ & $<0.1$ & $<1$ & $<1$ & 4 & 1 & 200 & 15 & 4 & $<0.5$ \\
\hline \multicolumn{11}{|l|}{$\pi \mathrm{L}$} \\
\hline $22 \ldots$ & $<1$ & $<0.1$ & 6 & $<1$ & 6 & 3 & $\cdots$ & $<3$ & 9 & $<0.5$ \\
\hline \multicolumn{11}{|l|}{ AUG } \\
\hline $15 \ldots$ & $<1$ & 0.3 & 1 & $<1$ & 21 & 4 & 2300 & $<3$ & $\cdots$ & $<0.5$ \\
\hline \multicolumn{11}{|l|}{ OCT } \\
\hline $28 \ldots$ & $<1$ & $<0.1$ & 1 & $<1$ & 8 & $<1$ & 260 & 6 & 3 & 0.6 \\
\hline \multicolumn{11}{|l|}{ DEC } \\
\hline $16 \ldots$ & $<1$ & 0.8 & $<1$ & $<1$ & $\cdots$ & 1 & 80 & 5 & 1 & $<0.5$ \\
\hline \multicolumn{11}{|l|}{ MAR 1992} \\
\hline $25 \ldots$ & $<1$ & $<0.1$ & $<1$ & $<1$ & $<1$ & $<1$ & 140 & 7 & $<1$ & $<0.5$ \\
\hline \multicolumn{11}{|l|}{ APR } \\
\hline $28 \ldots$ & $\cdots$ & $<0.1$ & $\cdots$ & $<1$ & $\cdots$ & $<1$ & $\cdots$ & 7 & $\cdots$ & $<0.5$ \\
\hline MAY & & & & & & & & & & \\
\hline $18 \ldots$ & $<1$ & 0.3 & $<1$ & $<1$ & 1 & 1 & 100 & 9 & $<1$ & $<0.5$ \\
\hline JUN & & & & & & & & & & \\
\hline $16 \ldots$ & $<1$ & $<0.1$ & $<1$ & $<1$ & 10 & 7 & 140 & 9 & 2 & $<0.5$ \\
\hline JUL & & & & & & & & & & \\
\hline $16 \ldots$ & $<1$ & $<0.1$ & $<1$ & $<1$ & 8 & 5 & 690 & 5 & 3 & $<0.5$ \\
\hline AUG & & & & & & & & & & \\
\hline $17 \ldots$ & $<1$ & $<0.1$ & $<1$ & $<1$ & 2 & $<1$ & 290 & 8 & 1 & $<0.5$ \\
\hline ОСт & & & & & & & & & & \\
\hline $20 \ldots$ & $<1$ & 0.5 & $<1$ & $<1$ & 2 & 1 & 310 & 5 & 1 & $<0.5$ \\
\hline JAN 1993 & & & & & & & & & & \\
\hline $14 \ldots$ & $<1$ & $<0.1$ & $<1$ & $<1$ & 1 & 1 & 140 & 8 & $<1$ & $<0.5$ \\
\hline MAR & & & & & & & & & & \\
\hline $24 \ldots$ & $<1$ & $<0.1$ & $<1$ & 1 & $<1$ & 1 & 20 & 5 & $<1$ & $<0.5$ \\
\hline
\end{tabular}


Table 45. Onsite measurements and bacteriological and selected inorganic data for station 07099970 , Arkansas River at Moffat Street, at Pueblo--Continued

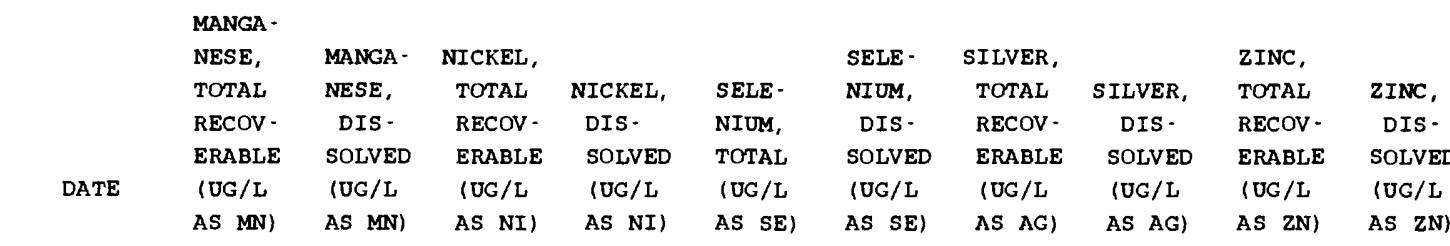

APR 1990

$10 \ldots$

MAY

$14 \ldots$

JUN

$11 \ldots$

JUL

$20 \ldots$

$24 \ldots$

AUG

$20 \ldots$

SEP

$28 \ldots$

OCT

$22 \ldots$

DEC

$17 \ldots$

MAR 1991

$27 \ldots$

$\mathrm{APR}$

15 ...

MAY

$20 \ldots$

JUN

$10 .$.

JUL

$22 \ldots$

AUG

$15 \ldots$

OCT

$28 \ldots$

DEC

$16 \ldots$

MAR 1992

$25 \ldots$

$\mathrm{APR}$

28 ...

MAY

$18 \ldots$

JUN

$16 \ldots$

NUL

$16 \ldots$

AUG

$17 \ldots$

OCT

$20 \ldots$

JAN 1993

MAR $14 .$.

$24 \ldots$

30

30

70

240

50

10

80

60

30

20

30

40

20

130

60

30

17

13

$$
9
$$$$
\begin{array}{r}
23 \\
7
\end{array}
$$$$
6
$$$$
26
$$$$
12
$$$$
16
$$

8

12

$$
9
$$

19

$$
3
$$

26

22

17

$$
9
$$$$
6
$$

50

50

50

50

60

50

20

9

$<1<1.0<10$

3

1

8

8

$<10<3$


Table 46. Onsite measurements and bacteriological and selected inorganic data for station 07106500 , Fountain Creek at Pueblo

\begin{tabular}{|c|c|c|c|c|c|c|c|c|c|c|c|}
\hline DATE & TIME & $\begin{array}{c}\text { DIS- } \\
\text { CHARGE, } \\
\text { INST. } \\
\text { CUBIC } \\
\text { FEET } \\
\text { PER } \\
\text { SECOND }\end{array}$ & $\begin{array}{l}\text { SPE- } \\
\text { CIFIC } \\
\text { CON- } \\
\text { DUCT- } \\
\text { ANCE } \\
\text { (US /CM) }\end{array}$ & $\begin{array}{c}\text { PH } \\
\text { WATER } \\
\text { WHOLE } \\
\text { FIELD } \\
\text { (STAND - } \\
\text { ARD } \\
\text { UNITS) }\end{array}$ & $\begin{array}{l}\text { TEMPER - } \\
\text { ATURE } \\
\text { WATER } \\
\text { (DEG C) }\end{array}$ & $\begin{array}{c}\text { OXYGEN, } \\
\text { DIS - } \\
\text { SOLVED } \\
\text { (MG/L) }\end{array}$ & $\begin{array}{l}\text { COLI- } \\
\text { FORM, } \\
\text { FECAL, } \\
0.7 \\
\text { UM-MF } \\
\text { (COLS.' } \\
100 \mathrm{ML} \text { ) }\end{array}$ & $\begin{array}{c}\text { STREP- } \\
\text { TOCOCCI } \\
\text { FECAL, } \\
\text { KF AGAR } \\
\text { (COLS. } \\
\text { PER } \\
100 \mathrm{ML} \text { ) }\end{array}$ & $\begin{array}{l}\text { HARD - } \\
\text { NESS } \\
\text { TOTAL } \\
\text { (MG/L } \\
\text { AS } \\
\text { CACO3) }\end{array}$ & $\begin{array}{l}\text { CALC IUM } \\
\text { DIS- } \\
\text { SOLVED } \\
\text { (MG/L } \\
\text { AS CA) }\end{array}$ & $\begin{array}{l}\text { MAGI } \\
\text { SI } \\
\text { DIS } \\
\text { SOLI } \\
\text { (MG } \\
\text { AS I }\end{array}$ \\
\hline $\begin{array}{c}\text { APR } 1990 \\
10 \ldots\end{array}$ & 1140 & 90 & 1160 & 8.3 & 13.0 & 7.0 & $\cdots$ & $\cdots$ & 360 & 91 & 32 \\
\hline $\begin{array}{l}\text { MAY } \\
14 \ldots\end{array}$ & 1300 & & $2 \times 0$ & & 20.0 & 1.0 & & & 200 & . & S \\
\hline JUN & 1300 & 88 & 1130 & 8.3 & 19.5 & 7.7 & K66 & K140 & 360 & 92 & 32 \\
\hline $\begin{array}{l}11 \ldots \\
\text { JUL }\end{array}$ & 1345 & 77 & 1250 & 8.2 & 25.0 & 6.5 & 420 & K2 800 & 380 & 98 & 33 \\
\hline$\underset{\text { AUG }}{24} \cdots$ & 1300 & 97 & 1230 & 8.3 & 25.5 & 6.3 & 700 & $\mathrm{~K} 2000$ & 390 & 98 & 35 \\
\hline$\underset{\text { SEP }}{20} \ldots$ & 1400 & 97 & 1070 & 8.1 & 26.0 & 6.1 & 1000 & 2800 & 340 & 88 & 29 \\
\hline${ }_{\mathrm{OCT}}^{05}$ & 1825 & 9.0 & 1390 & 8.5 & $\cdots$ & $\cdots$ & $\cdots$ & $\cdots$ & 440 & 86 & 54 \\
\hline${ }_{\mathrm{DEC}}^{22} \cdots$ & 1205 & 90 & 1230 & 8.5 & 10.5 & 9.4 & 190 & 560 & 370 & 94 & 33 \\
\hline MAR $17 \ddot{9} \dot{1}$ & 1220 & 119 & 1310 & 8.1 & 4.0 & 10.4 & E15 & 840 & 400 & 100 & 36 \\
\hline$\underset{A P R}{28} \cdots$ & 0825 & 68 & 1320 & 7.8 & 4.0 & 10.6 & 15 & 330 & 430 & 110 & 37 \\
\hline$\underset{M A Y}{15} \ldots$ & 1140 & 64 & 1370 & 8.3 & 15.0 & 8.8 & E23 & E110 & 420 & 110 & 36 \\
\hline $\operatorname{JUN}^{20} \cdots$ & 1305 & 50 & 1420 & 8.4 & 21.0 & 7.4 & 42 & 250 & 440 & 110 & 40 \\
\hline JUL $10 \ldots$ & 1320 & 190 & 941 & 8.3 & 24.5 & 6.7 & E200 & 1400 & 280 & 74 & 23 \\
\hline$\underset{A U G}{22} \cdots$ & 1300 & 253 & 909 & 8.3 & 21.0 & 7.2 & E110 & E2600 & 270 & 73 & 22 \\
\hline${ }_{\mathrm{OCT}}^{15} \ldots$ & 1145 & 119 & 1140 & 8.3 & 24.5 & 6.7 & 1100 & 1700 & 350 & 89 & 31 \\
\hline${ }_{\mathrm{DEC}}^{28 \cdots}$ & 1250 & 76 & 1440 & 8.3 & 7.0 & 10.0 & 240 & 870 & 440 & 110 & 39 \\
\hline MAR $16 \ddot{g}_{2}$ & 1315 & 114 & 1280 & 8.2 & 4.5 & 10.0 & E33 & 150 & 380 & 96 & 33 \\
\hline $\begin{array}{c}26 \ldots \\
\text { APR } \\
28 \ldots\end{array}$ & 0745 & 160 & 1090 & 8.4 & 6.0 & 9.8 & $\cdots$ & $\cdots$ & 330 & 84 & 28 \\
\hline MAY & 1110 & 96 & 1120 & 8.4 & 17.5 & 7.8 & E9 & E77 & 320 & 82 & 29 \\
\hline $\begin{array}{l}18 \ldots \\
\text { JUN }\end{array}$ & 1220 & 32 & 1480 & 8.4 & 24.5 & 6.8 & 68 & 79 & 460 & 110 & 44 \\
\hline JUL $16 \ldots$ & 1100 & 96 & 1160 & 8.4 & 18.0 & 7.7 & 180 & 230 & 350 & 90 & 30 \\
\hline${ }_{\text {AUG }}^{16 \cdots}$ & 1115 & 21 & 1530 & 8.4 & 20.5 & 6.9 & 340 & 330 & 480 & 110 & 50 \\
\hline$\underset{\mathrm{OCT}}{17}$ & 1110 & 34 & 1500 & 8.4 & 23.5 & 6.9 & 140 & 450 & 510 & 120 & 50 \\
\hline JAN $199 \dot{9}_{3}$ & 1145 & 80 & 1410 & 8.4 & 11.0 & 9.4 & 200 & 310 & 440 & 110 & 41 \\
\hline $\begin{array}{l}\operatorname{MAR} \ldots \\
25 \ldots\end{array}$ & 1000 & 100 & 1380 & 8.2 & 0.0 & $\cdots$ & E24 & 64 & 400 & 100 & 37 \\
\hline & 0745 & 96 & 1230 & 8.3 & 5.0 & 10.3 & E26 & 55 & 360 & 91 & \\
\hline
\end{tabular}

\begin{tabular}{|c|c|c|c|c|c|c|c|c|c|c|}
\hline DATE & $\begin{array}{l}\text { SODIOM, } \\
\text { DIS - } \\
\text { SOLVED } \\
\text { (MG/L } \\
\text { AS NA) }\end{array}$ & $\begin{array}{c}\text { ALKA- } \\
\text { LINITY } \\
\text { LAB } \\
\text { (MG/L } \\
\text { AS } \\
\text { CACO3) }\end{array}$ & $\begin{array}{l}\text { SULFATE } \\
\text { DIS- } \\
\text { SOLVED } \\
\text { (MG/L } \\
\text { AS SO4) }\end{array}$ & $\begin{array}{l}\text { CHLO- } \\
\text { RIDE, } \\
\text { DIS- } \\
\text { SOLVED } \\
\text { (MG/L } \\
\text { AS CL) }\end{array}$ & $\begin{array}{l}\text { SOLIDS, } \\
\text { RESIDUE } \\
\text { AT } 180 \\
\text { DEG C } \\
\text { DIS. } \\
\text { SOLVED } \\
\text { (MG/L) }\end{array}$ & $\begin{array}{l}\text { NITRO- } \\
\text { GEN, } \\
\text { NO2+NO3 } \\
\text { TOTAL } \\
\text { (MG /L } \\
\text { AS N) }\end{array}$ & $\begin{array}{c}\text { NITRO- } \\
\text { GEN, } \\
\text { NO2+NO3 } \\
\text { DIS - } \\
\text { SOLVED } \\
\text { (MG / } \\
\text { AS N) }\end{array}$ & $\begin{array}{l}\text { NITRO- } \\
\text { GEN, } \\
\text { AMMONIA } \\
\text { TOTAL } \\
\text { (MG /L } \\
\text { AS N) }\end{array}$ & $\begin{array}{l}\text { NITRO- } \\
\text { GEN, } \\
\text { AMMONIA } \\
\text { DIS - } \\
\text { SOLVED } \\
\text { (MG / } \\
\text { AS N) }\end{array}$ & $\begin{array}{l}\text { PHOS - } \\
\text { PHORUS } \\
\text { TOTAL } \\
\text { (MG/L } \\
\text { AS P) }\end{array}$ \\
\hline $\begin{array}{l}1990 \\
\ldots\end{array}$ & 120 & 169 & 310 & 53 & 828 & 6.40 & $\cdots$ & 0.030 & $\cdots$ & 1.60 \\
\hline$\ldots$ & 120 & 165 & 370 & 51 & 796 & 5.10 & $\cdots$ & 0.020 & . & 1.10 \\
\hline$\cdots$ & 130 & 181 & 390 & 51 & 854 & 4.40 & $\cdots$ & 0.010 & . & 0.820 \\
\hline$\ldots$ & 120 & 194 & 400 & 47 & 856 & 4.00 & $\cdots$ & 0.020 & $\cdots$ & 0.510 \\
\hline$\ldots$ & 110 & 165 & 310 & 40 & 724 & 3.60 & -. & 0.060 & $\cdots$ & 0.530 \\
\hline 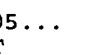 & 150 & 151 & 550 & 42 & 1010 & 6.90 & . & 0.200 & $\cdots$ & 0.330 \\
\hline$\ldots$ & 120 & 192 & 330 & 53 & 820 & 4.50 & $\cdots$ & 0.020 & $\cdots$ & 0.830 \\
\hline$\ldots$ & 130 & 200 & 400 & 61 & 904 & 6.50 & - & 0.090 & $\cdots$ & 1.60 \\
\hline
\end{tabular}


Table 46. Onsite measurements and bacteriological and selected inorganic data for station 07106500 , Fountain Creek at Pueblo--Continued

\begin{tabular}{|c|c|c|c|c|c|c|c|c|c|c|}
\hline DATE & $\begin{array}{c}\text { SODIUM, } \\
\text { DIS - } \\
\text { SOLVED } \\
\text { (MG /L } \\
\text { AS NA) }\end{array}$ & $\begin{array}{c}\text { ALKA - } \\
\text { LINITY } \\
\text { LAB } \\
\text { (MG/L } \\
\text { AS } \\
\text { CACO3) }\end{array}$ & $\begin{array}{c}\text { SULFATE } \\
\text { DIS - } \\
\text { SOLVED } \\
\text { (MG/L } \\
\text { AS SO4) }\end{array}$ & $\begin{array}{l}\text { CHLO- } \\
\text { RIDE, } \\
\text { DIS - } \\
\text { SOLVED } \\
\text { (MG/L } \\
\text { AS CL) }\end{array}$ & $\begin{array}{l}\text { SOLIDS, } \\
\text { RESIDUE } \\
\text { AT } 180 \\
\text { DEG C } \\
\text { DIS- } \\
\text { SOLVED } \\
\text { (MG/L) }\end{array}$ & $\begin{array}{c}\text { NITRO- } \\
\text { GEN, } \\
\text { NO2+NO3 } \\
\text { TOTAL } \\
\text { (MG/L } \\
\text { AS N) }\end{array}$ & $\begin{array}{c}\text { NITRO- } \\
\text { GEN, } \\
\text { NO2+NO3 } \\
\text { DIS - } \\
\text { SOLVED } \\
\text { (MG / } \\
\text { AS N) }\end{array}$ & $\begin{array}{c}\text { NITRO- } \\
\text { GEN, } \\
\text { AMMONIA } \\
\text { TOTAL } \\
\text { (MG / L } \\
\text { AS N) }\end{array}$ & $\begin{array}{l}\text { NITRO- } \\
\text { GEN, } \\
\text { AMMONIA } \\
\text { DIS - } \\
\text { SOLVED } \\
\text { (MG/L } \\
\text { AS N) }\end{array}$ & $\begin{array}{l}\text { PHOS - } \\
\text { PHORUS } \\
\text { TOTAL } \\
\text { (MG /L } \\
\text { AS P) }\end{array}$ \\
\hline MAR 1991 & & & & & & & & & & \\
\hline$\underset{A P R}{28} \cdots$ & 140 & 202 & 400 & 62 & 936 & 5.30 & $\cdots$ & 0.010 & $\cdots$ & 1.30 \\
\hline $15 \ldots$ & 130 & 206 & 380 & 56 & 942 & 4.90 & $\cdots$ & $<0.010$ & $\cdots$ & 0.960 \\
\hline${ }_{\text {JUN }}^{20} \ldots$ & 140 & 210 & 470 & 55 & 998 & 4.00 & $\cdots$ & $<0.010$ & $\cdots$ & 0.860 \\
\hline${ }_{\pi}^{10} \ldots$ & 95 & 147 & 280 & 35 & 624 & 3.40 & $\cdots$ & 0.070 & $\cdots$ & 0.830 \\
\hline$\underset{A U G}{22} \cdots$ & 87 & 146 & 270 & 34 & 586 & 2.80 & $\cdots$ & $<0.010$ & $\cdots$ & 3.00 \\
\hline${ }_{\text {OCT }}^{15 \ldots}$ & 110 & 179 & 330 & 41 & 755 & 3.60 & $\cdots$ & 0.030 & $\cdots$ & 0.700 \\
\hline${ }_{D E C}^{28} \cdots$ & 130 & 223 & 460 & 52 & 988 & 4.70 & $\cdots$ & 0.010 & $\cdots$ & 0.640 \\
\hline $\operatorname{MAR} 1692$ & 120 & 195 & 350 & 59 & 910 & 5.20 & $\cdots$ & 0.400 & $\cdots$ & 2.00 \\
\hline${ }_{A P R}^{26} \cdots$ & 110 & 159 & 310 & 52 & 728 & 5.30 & $\cdots$ & 0.030 & $\cdots$ & 1.40 \\
\hline$\underset{\operatorname{MAY}}{28 \ldots}$ & 100 & 162 & 320 & 46 & 750 & 4.30 & $\cdots$ & 0.010 & $\cdots$ & 1.00 \\
\hline${ }_{\pi N N}^{18} \ldots$ & 150 & 206 & 430 & 59 & 1040 & 4.50 & $\cdots$ & 0.020 & $\cdots$ & 0.640 \\
\hline${ }_{\text {JUL }}^{16} \ldots$ & 110 & 176 & 310 & 48 & 764 & 4.80 & $\cdots$ & 0.020 & $\cdots$ & 1.10 \\
\hline $16 \ldots$ & 160 & 220 & 490 & 57 & 1070 & 4.70 & $\cdots$ & 0.030 & $\cdots$ & 0.640 \\
\hline${ }_{\mathrm{OCT}} 17 \ldots$ & 150 & 215 & 500 & 57 & 1060 & 4.70 & $\cdots$ & 0.030 & $\cdots$ & 0.660 \\
\hline JAN 1993 & 140 & 220 & 440 & 56 & 1020 & 5.20 & $\cdots$ & 0.030 & $\cdots$ & 1.20 \\
\hline $14 \cdots$ & 140 & 207 & 370 & 63 & 934 & -. & 6.60 & . & $\cdots$ & 1.40 \\
\hline $25 \ldots$ & 120 & 185 & 330 & 55 & 818 & $\cdots$ & 6.80 & $\cdots$ & 0.020 & 1.40 \\
\hline DATE & $\begin{array}{l}\text { CADMIUM } \\
\text { TOTAL } \\
\text { RECOV } \\
\text { ERABLE } \\
\text { (UG/L } \\
\text { AS CD) }\end{array}$ & $\begin{array}{c}\text { CADMIUM } \\
\text { DIS - } \\
\text { SOLVED } \\
\text { (UG/L } \\
\text { AS CD) }\end{array}$ & $\begin{array}{l}\text { CHRO- } \\
\text { MIUM, } \\
\text { TOTAL } \\
\text { RECOV- } \\
\text { ERABLE } \\
\text { (UG/L } \\
\text { AS CR) }\end{array}$ & $\begin{array}{l}\text { CHRO- } \\
\text { MIUM, } \\
\text { DIS- } \\
\text { SOLVED } \\
\text { (UG/L } \\
\text { AS CR) }\end{array}$ & $\begin{array}{l}\text { COPPER, } \\
\text { TOTAL } \\
\text { RECOV- } \\
\text { ERABLE } \\
\text { (UG/L } \\
\text { AS CU) }\end{array}$ & $\begin{array}{l}\text { COPPER, } \\
\text { DIS - } \\
\text { SOLVED } \\
\text { (UG/L } \\
\text { AS CU) }\end{array}$ & $\begin{array}{l}\text { IRON, } \\
\text { TOTAL } \\
\text { RECOV. } \\
\text { ERABLE } \\
\text { (UG/L } \\
\text { AS FE) }\end{array}$ & $\begin{array}{l}\text { IRON, } \\
\text { DIS- } \\
\text { SOLVED } \\
\text { (UG/L } \\
\text { AS FE) }\end{array}$ & $\begin{array}{l}\text { LEAD, } \\
\text { TOTAL } \\
\text { RECOV. } \\
\text { ERABLE } \\
\text { (UG/L } \\
\text { AS PB) }\end{array}$ & $\begin{array}{l}\text { LEAD: } \\
\text { DIS: } \\
\text { SOLVED } \\
\text { (UG/L } \\
\text { AS PB) }\end{array}$ \\
\hline $\begin{array}{c}\text { APR } 1990 \\
10 \ldots \\
\text { MAY }\end{array}$ & $<1$ & 0.2 & 10 & $<1$ & 10 & 3 & 14000 & 7 & 15 & $<0.5$ \\
\hline$\underset{\pi N N}{14}$ & $<1$ & 0.1 & 6 & $<1$ & 8 & 2 & 8900 & 4 & 10 & $<0.5$ \\
\hline${ }_{\text {JUL }}^{11} \ldots$ & $<1$ & 0.2 & 18 & $<1$ & 25 & 4 & 20000 & 9 & 19 & $<0.5$ \\
\hline$\underset{A U G}{24} \cdots$ & $<1$ & $<0.1$ & 11 & $<1$ & 15 & 2 & 18000 & 4 & 16 & $<0.5$ \\
\hline$\underset{\text { SEP }}{20} \cdots$ & 1 & $<0.1$ & 16 & 1 & 24 & 2 & 20000 & 6 & 17 & $<0.5$ \\
\hline${ }_{\text {OCT }}^{05 \ldots}$ & 1 & $<0.1$ & 9 & $<1$ & 14 & 5 & 9000 & 16 & 64 & $<0.5$ \\
\hline$\underset{\mathrm{DEC}}{22} \cdots$ & $<1$ & $<0.1$ & 8 & $<1$ & 14 & 2 & 12000 & 7 & 16 & $<0.5$ \\
\hline MAR $17 \ddot{1}_{1}$ & 1 & 0.2 & 6 & $<1$ & 14 & 2 & 10000 & 37 & 13 & $<0.5$ \\
\hline$\underset{A P R}{28} \cdots$ & $<1$ & 0.3 & 4 & $<1$ & 11 & 2 & 4500 & 8 & 4 & $<0.5$ \\
\hline $\operatorname{MAY} 15$ & $<1$ & 0.5 & 3 & $<1$ & 8 & 2 & 6400 & 17 & 8 & $<0.5$ \\
\hline$\underset{\text { JUN }}{20 \ldots}$ & $<1$ & 0.1 & 4 & $<1$ & 6 & 2 & 2900 & 39 & 7 & $<0.5$ \\
\hline${ }_{\text {JUL }}^{10} \cdots$ & $<1$ & $<0.1$ & 14 & $<1$ & 5 & 2 & 18000 & 19 & 2 & $<0.5$ \\
\hline$\underset{A U G}{22} \cdots$ & 3 & $<0.1$ & 18 & $<1$ & 100 & 2 & $\cdots$ & 9 & 140 & $<0.5$ \\
\hline $\operatorname{OCT}^{15}$ & $<1$ & $<0.1$ & 6 & $<1$ & 15 & 2 & 11000 & $<3$ & $\cdots$ & $<0.5$ \\
\hline$\underset{D E C}{28} \cdots$ & $<1$ & $<0.1$ & 7 & $<1$ & 19 & 1 & 10000 & 6 & 19 & $<0.5$ \\
\hline $16 \ldots$ & $<1$ & 0.5 & 5 & $<1$ & -- & 2 & 1100 & 10 & 19 & 0.8 \\
\hline
\end{tabular}


Table 46. Onsite measurements and bacteriological and selected inorganic data for station 07106500 , Fountain Creek at Pueblo--Continued

\begin{tabular}{|c|c|c|c|c|c|c|c|c|}
\hline & $\begin{array}{l}\text { CADMIUM } \\
\text { TOTAL } \\
\text { RECOV- } \\
\text { ERABLE } \\
\text { (UG/L }\end{array}$ & $\begin{array}{c}\text { CADMIUM } \\
\text { DIS - } \\
\text { SOLVED } \\
\text { IUG/L }\end{array}$ & $\begin{array}{l}\text { CHRO- } \\
\text { MIUM, } \\
\text { TOTAL } \\
\text { RECOV- } \\
\text { ERABLE } \\
\text { (UG / I }\end{array}$ & $\begin{array}{l}\text { CHRO- } \\
\text { MIUM, } \\
\text { DIS - } \\
\text { SOLVED } \\
\text { (UG/L }\end{array}$ & $\begin{array}{l}\text { COPPER, } \\
\text { TOTAL } \\
\text { RECOV - } \\
\text { ERABLE } \\
\text { (UG/L }\end{array}$ & $\begin{array}{l}\text { COPPER, } \\
\text { DIS - } \\
\text { SOLVED } \\
\text { (UG/L }\end{array}$ & $\begin{array}{l}\text { IRON, } \\
\text { TOTAL } \\
\text { RECOV - } \\
\text { ERABLE } \\
\text { (UG/I }\end{array}$ & $\begin{array}{l}\text { IRON, } \\
\text { DIS - } \\
\text { SOLVED } \\
\text { (UG/I }\end{array}$ \\
\hline & & AS CD) & & & & & & \\
\hline
\end{tabular}

\begin{tabular}{|c|c|c|c|c|c|c|c|c|c|c|}
\hline $\begin{array}{l}26 \cdots \\
\text { APR }\end{array}$ & $<1$ & 0.1 & 5 & $<1$ & 8 & 2 & 8500 & 12 & 15 & $<0.5$ \\
\hline${ }_{\mathrm{MAY}}^{28} \ldots$ & $\cdots$ & $<0.1$ & $\cdots$ & $<1$ & -- & 2 & $\cdots$ & 4 & $\cdots$ & 0.7 \\
\hline $\int_{J N}^{18} \ldots$ & $<1$ & $<0.1$ & $<1$ & $<1$ & 4 & 4 & 940 & $<3$ & 2 & 0.5 \\
\hline${ }_{\text {JUL }}^{16 \ldots}$ & $<1$ & $<0.1$ & 1 & $<1$ & 6 & 3 & 3400 & 5 & 8 & $<0.5$ \\
\hline${ }_{A \cup G}^{16} \ldots$ & $<1$ & $<0.1$ & $<1$ & $<1$ & 3 & 2 & 690 & $<3$ & 2 & 0.6 \\
\hline${ }_{\mathrm{OCT}}^{17 \ldots}$ & $<1$ & $<0.1$ & $<1$ & 2 & 3 & 1 & 230 & 4 & 4 & 0.7 \\
\hline${ }_{\text {JAN }}^{20} 1993$ & $<1$ & $<0.1$ & 2 & $<1$ & 9 & 2 & 5800 & 7 & 20 & 0.8 \\
\hline $\operatorname{MAR}^{14} \cdots$ & $<1$ & $<0.1$ & 14 & $<1$ & 8 & 2 & 3000 & 13 & 8 & $<0.5$ \\
\hline $25 \ldots$ & $<1$ & 0.1 & 3 & 2 & 8 & 3 & 4700 & 14 & 6 & $<0.5$ \\
\hline
\end{tabular}

DATE

\begin{tabular}{|c|c|c|c|c|c|c|c|c|c|}
\hline $\begin{array}{l}\text { MANGA - } \\
\text { NESE, } \\
\text { TOTAL } \\
\text { RECOV - } \\
\text { ERABLE } \\
\text { IUG/L }\end{array}$ & $\begin{array}{l}\text { MANGA - } \\
\text { NESE, } \\
\text { DIS - } \\
\text { SOLVED } \\
\text { (UG / L }\end{array}$ & $\begin{array}{c}\text { NICKEL, } \\
\text { TOTAL } \\
\text { RECOV- } \\
\text { ERABLE } \\
\text { /UG / L }\end{array}$ & $\begin{array}{l}\text { NICKEL, } \\
\text { DIS- } \\
\text { SOLVED } \\
\text { (UG/L }\end{array}$ & $\begin{array}{l}\text { SELE- } \\
\text { NIUM, } \\
\text { TOTAL } \\
\text { (UG/L }\end{array}$ & $\begin{array}{l}\text { SELE - } \\
\text { NIUM, } \\
\text { DIS - } \\
\text { SOLVED } \\
\text { (UG / I }\end{array}$ & $\begin{array}{c}\text { SILVER, } \\
\text { TOTAL } \\
\text { RECOV - } \\
\text { ERABLE } \\
\text { (UG/L }\end{array}$ & $\begin{array}{c}\text { SILVER, } \\
\text { DIS - } \\
\text { SOLVED } \\
\text { (UG/L }\end{array}$ & $\begin{array}{l}\text { ZINC, } \\
\text { TOTAL } \\
\text { RECOV- } \\
\text { ERABLE } \\
\text { (UG / L }\end{array}$ & $\begin{array}{l}\text { ZINC, } \\
\text { DIS - } \\
\text { SOLVED } \\
\text { (UG/L }\end{array}$ \\
\hline AS MN) & AS MN) & AS NI) & AS NI) & AS SE) & AS SE) & AS AG) & AS AG) & AS ZN) & AS $\mathrm{ZN}$ ) \\
\hline
\end{tabular}

\begin{tabular}{|c|c|c|c|c|c|c|c|c|c|c|}
\hline $\begin{array}{c}\text { APR } 1990 \\
10 \ldots \\
\text { MAY }\end{array}$ & 350 & 2 & -- & 4 & 20 & 19 & $<1$ & 1.0 & 80 & 9 \\
\hline $\operatorname{JUN}^{14 \ldots}$ & 250 & 3 & -- & 3 & 11 & 10 & $<1$ & $<1.0$ & 50 & 4 \\
\hline${ }_{\text {JUL }} 11 \ldots$ & 370 & 5 & -- & 4 & 21 & 20 & $<1$ & $<1.0$ & 100 & 5 \\
\hline$\underset{\text { AUG }}{24 \ldots}$ & 480 & 4 & 14 & 3 & $\cdots$ & -- & $<1$ & $<1,0$ & 100 & 4 \\
\hline $\operatorname{seP}^{20 \ldots}$ & 460 & 3 & 21 & 3 & $\cdots$ & - & $<1$ & $<1.0$ & 120 & 9 \\
\hline$\stackrel{05 \ldots}{\text { OCT }}$ & 310 & 48 & 16 & 5 & - - & $\cdots$ & $<1$ & - & 110 & 8 \\
\hline${ }_{D E C}^{22 \cdots}$ & 410 & 3 & 14 & 4 & $\cdots$ & - & $<1$ & $<1.0$ & 80 & 8 \\
\hline$\stackrel{17}{17} \ddot{M A R}_{1}$ & 390 & 6 & 11 & 3 & $\cdots$ & 11 & 1 & $<1.0$ & 70 & 14 \\
\hline $\begin{array}{l}28 \ldots \\
\text { APR }\end{array}$ & 150 & 5 & 7 & 3 & - & 19 & $<1$ & $<1.0$ & 40 & 14 \\
\hline${ }_{\mathrm{MAY}}^{15} \cdots$ & 180 & 3 & 9 & 3 & -- & 6 & $<1$ & $<1.0$ & 40 & 10 \\
\hline$\underset{J U N}{20 \ldots}$ & 90 & 4 & 6 & 4 & 24 & 23 & $<1$ & $<1.0$ & 30 & 22 \\
\hline $10 \ldots$ & 460 & 4 & 19 & 4 & $\cdots$ & 12 & $<1$ & $<1.0$ & 100 & 7 \\
\hline$\underset{A U G}{22 \ldots}$ & 2000 & 2 & 78 & 2 & $\cdots$ & 12 & 1 & $<1.0$ & 420 & 8 \\
\hline${ }_{\text {OCT }}^{15 \ldots}$ & 300 & $<1$ & 14 & 4 & 16 & 5 & $<1$ & $<1.0$ & 50 & 4 \\
\hline DEC $28 \ldots$ & 390 & 9 & 12 & 2 & 29 & 33 & $<1$ & $<1.0$ & 80 & 8 \\
\hline MAR 16992 & 360 & 3 & 12 & 3 & 16 & 24 & $<1$ & $<1.0$ & 100 & 11 \\
\hline$\stackrel{26}{26} \cdots$ & 270 & 3 & 9 & 4 & 17 & 17 & $<1$ & $<1.0$ & 50 & 12 \\
\hline${ }_{\mathrm{MAY}}^{28} \ldots$ & $\cdots$ & 2 & $\cdots$ & 2 & 22 & 17 & $<1$ & $<1.0$ & $\cdots$ & 3 \\
\hline${ }_{\text {JUN }}^{18} \cdots$ & 50 & 3 & 4 & 3 & 42 & 33 & $<1$ & $<1.0$ & 20 & 4 \\
\hline $16 \ldots$ & 180 & 2 & 5 & 3 & 25 & 14 & $<1$ & $<1.0$ & 30 & 3 \\
\hline $16 \ldots$ & 40 & 6 & 3 & 3 & 32 & 31 & $<1$ & $<1.0$ & $<10$ & 3 \\
\hline${ }_{\text {OCT }}^{17 \ldots}$ & 50 & 11 & 4 & 2 & 56 & 53 & $<1$ & $<1.0$ & 40 & 3 \\
\hline JAN 20093 & 240 & 5 & 7 & 2 & 31 & 31 & $<1$ & $<1.0$ & 40 & 6 \\
\hline $\operatorname{MAR}^{14 \ldots}$ & 160 & 7 & 5 & 3 & 21 & 17 & $<1$ & $<1.0$ & 10 & 9 \\
\hline $25 \ldots$ & 200 & 3 & 7 & 3 & 19 & 17 & $<1$ & $<1,0$ & 40 & 11 \\
\hline
\end{tabular}


Table 47. Onsite measurements and bacteriological and selected inorganic data for station 381510104350601 , Arkansas River below Highway 227, at Pueblo

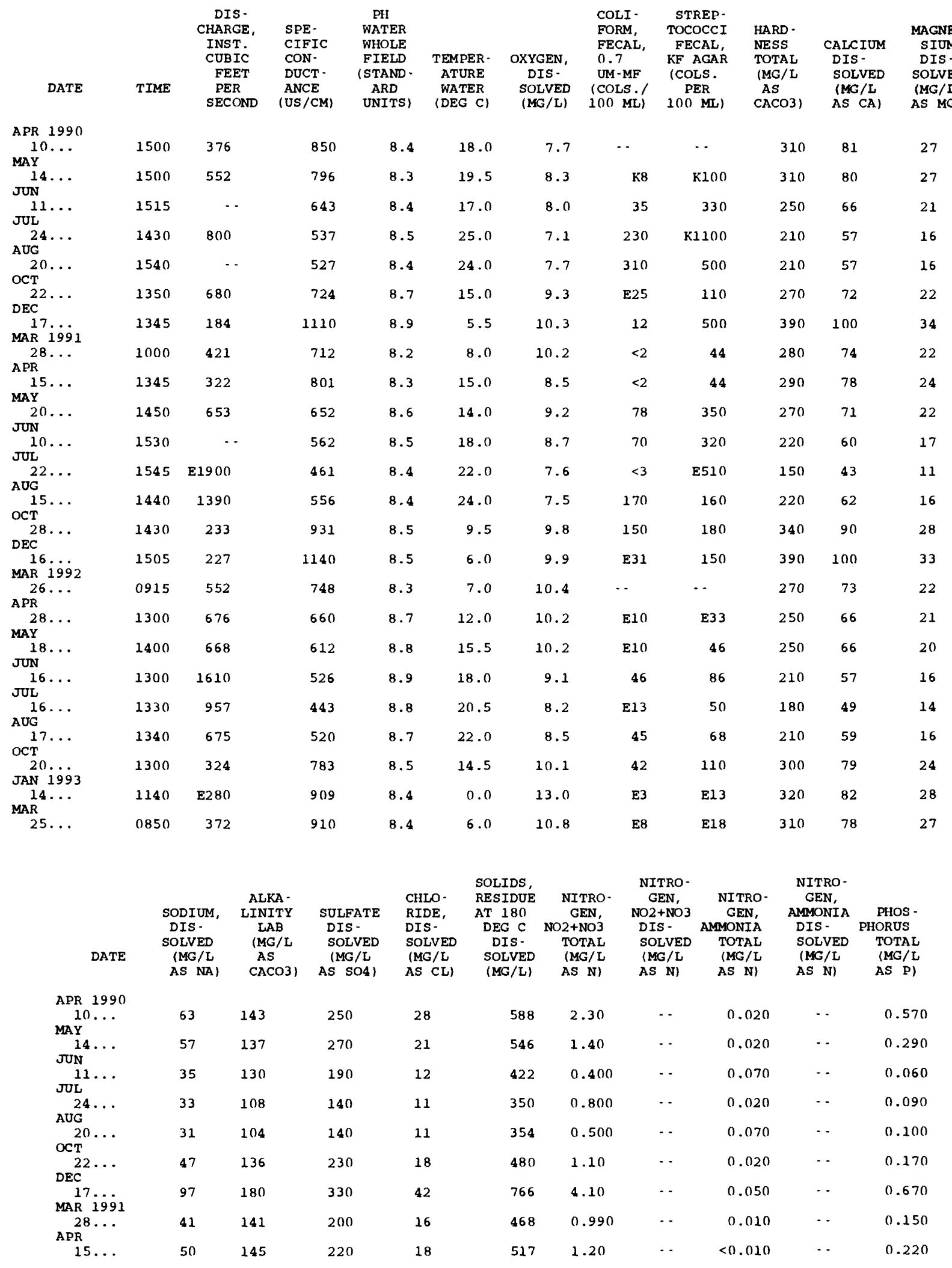


Table 47. Onsite measurements and bacteriological and selected inorganic data for station 381510104350601 , Arkansas River below Highway 227, at Pueblo--Continued

\begin{tabular}{|c|c|c|c|c|c|c|c|c|c|c|}
\hline DATE & $\begin{array}{l}\text { SODIOM, } \\
\text { DIS- } \\
\text { SOLVED } \\
\text { (MG/L } \\
\text { AS NA) }\end{array}$ & $\begin{array}{c}\text { ALKA- } \\
\text { LINITY } \\
\text { LAB } \\
\text { (MG/L } \\
\text { AS } \\
\text { CACO3) }\end{array}$ & $\begin{array}{c}\text { SULFATE } \\
\text { DIS - } \\
\text { SOLVED } \\
\text { (MG/L } \\
\text { AS SO4) }\end{array}$ & $\begin{array}{l}\text { CHLO- } \\
\text { RIDE, } \\
\text { DIS- } \\
\text { SOLVED } \\
\text { (MG/L } \\
\text { AS CL) }\end{array}$ & $\begin{array}{l}\text { SOLIDS, } \\
\text { RESIDUE } \\
\text { AT } 180 \\
\text { DEG C } \\
\text { DIS - } \\
\text { SOLVED } \\
\text { (MG/L) }\end{array}$ & $\begin{array}{c}\text { NITRO- } \\
\text { GEN, } \\
\text { NO2+NO3 } \\
\text { TOTAL } \\
\text { (MG/L } \\
\text { AS N) }\end{array}$ & $\begin{array}{c}\text { NITRO- } \\
\text { GEN, } \\
\text { NO2+NO3 } \\
\text { DIS- } \\
\text { SOLVED } \\
\text { (MG/L } \\
\text { AS N) }\end{array}$ & $\begin{array}{c}\text { NITRO- } \\
\text { GEN, } \\
\text { AMMNIA } \\
\text { TOTAL } \\
\text { (MG/L } \\
\text { AS N) }\end{array}$ & $\begin{array}{l}\text { NITRO- } \\
\text { GEN, } \\
\text { AMMONIA } \\
\text { DIS- } \\
\text { SOLVED } \\
\text { (MG/L } \\
\text { AS N) }\end{array}$ & $\begin{array}{c}\text { PHOS - } \\
\text { PHORUS } \\
\text { TOTAL } \\
\text { (MG/I } \\
\text { AS P) }\end{array}$ \\
\hline MAY 1991 & & & & & & & & & & \\
\hline $20 \ldots$ & 35 & 132 & 190 & 13 & 425 & 0.460 & $\cdots$ & 0.020 & $\cdots$ & 0.060 \\
\hline $10 \ldots$ & 30 & 118 & 160 & 9.2 & 351 & 0.450 & $\cdots$ & 0.040 & $\cdots$ & 0.110 \\
\hline$\underset{A U G}{22} \cdots$ & 16 & 98 & 99 & 6.0 & 213 & 0.190 & $\cdots$ & $<0.010$ & $\cdots$ & 0.050 \\
\hline${ }_{\text {ocT }}^{15 \ldots}$ & 27 & 107 & 150 & 11 & 348 & 0.640 & $\cdots$ & 0.040 & $\cdots$ & 0.110 \\
\hline$\underset{D E C}{28} \cdots$ & 55 & 163 & 310 & 22 & 627 & 1.50 & $\cdots$ & 0.020 & $\cdots$ & 0.030 \\
\hline${ }_{\operatorname{MAR}}^{16} 19992$ & 94 & 176 & 350 & 46 & 760 & 3.90 & $\cdots$ & 0.210 & $\cdots$ & 1.10 \\
\hline $\begin{array}{l}26 \cdots \\
\text { APR }\end{array}$ & 51 & 136 & 220 & 22 & 500 & 1.90 & $\cdots$ & 0.030 & $\cdots$ & 0.410 \\
\hline $\operatorname{MAY}^{28} \ldots$ & 37 & 129 & 200 & 17 & 384 & 0.800 & $\cdots$ & 0.020 & $\cdots$ & 0.160 \\
\hline${ }_{\mathrm{JUN}}^{18 \ldots}$ & 32 & 125 & 180 & 13 & 400 & 0.360 & $\cdots$ & 0.020 & $\cdots$ & 0.040 \\
\hline $16 \ldots$ & 26 & 111 & 140 & 11 & 328 & 0.390 & $\cdots$ & 0.020 & $\cdots$ & 0.060 \\
\hline $\begin{array}{l}16 \ldots \\
\text { AUG }\end{array}$ & 21 & 103 & 110 & 9.0 & 274 & 0.250 & $\cdots$ & 0.030 & $\cdots$ & 0.030 \\
\hline${ }_{\text {OCT }}^{17 \cdots}$ & 26 & 112 & 140 & 9.0 & 356 & 0.410 & $\cdots$ & 0.020 & $\cdots$ & 0.060 \\
\hline JAN 1993 & 50 & 148 & 230 & 19 & 536 & 1.40 & $-\cdot$ & 0.030 & $\cdots$ & 0.280 \\
\hline $\operatorname{MAR}^{14} \cdots$ & 69 & 161 & 270 & 28 & 630 & - & 2.70 & -- & 0.240 & 0.430 \\
\hline $25 \ldots$ & 74 & 158 & 270 & 32 & 598 & $\cdots$ & 3.60 & $\cdots$ & 0.020 & 0.650 \\
\hline DATE & $\begin{array}{l}\text { CADMIUM } \\
\text { TOTAL } \\
\text { RECOV- } \\
\text { ERABLE } \\
\text { (UG/L } \\
\text { AS CD) }\end{array}$ & $\begin{array}{c}\text { CADMI UM } \\
\text { DIS - } \\
\text { SOLVED } \\
\text { (UG/L } \\
\text { AS CD) }\end{array}$ & $\begin{array}{l}\text { CHRO- } \\
\text { MIUM, } \\
\text { TOTAL } \\
\text { RECOV- } \\
\text { ERABLE } \\
\text { (UG/L } \\
\text { AS CR) }\end{array}$ & $\begin{array}{l}\text { CHRO- } \\
\text { MIUM, } \\
\text { DIS- } \\
\text { SOLVED } \\
\text { (UG/L } \\
\text { AS CR) }\end{array}$ & $\begin{array}{l}\text { COPPER, } \\
\text { TOTALL } \\
\text { RECOV- } \\
\text { ERABLE } \\
\text { (UG/L } \\
\text { AS CU) }\end{array}$ & $\begin{array}{l}\text { COPPER, } \\
\text { DIS- } \\
\text { SOLVED } \\
\text { (UG/L } \\
\text { AS CU) }\end{array}$ & $\begin{array}{l}\text { IRON, } \\
\text { TOTAL } \\
\text { RECOV- } \\
\text { ERABLE } \\
\text { (UG/L } \\
\text { AS FE) }\end{array}$ & $\begin{array}{l}\text { IRON, } \\
\text { DIS- } \\
\text { SOLVED } \\
\text { (UG/L } \\
\text { AS FE) }\end{array}$ & $\begin{array}{l}\text { LEAD, } \\
\text { TOTAL } \\
\text { RECOV. } \\
\text { ERABLE } \\
\text { (UG/L } \\
\text { AS PB) }\end{array}$ & $\begin{array}{l}\text { LEAD, } \\
\text { DIS. } \\
\text { SOLVED } \\
\text { (UG/L } \\
\text { AS PB) }\end{array}$ \\
\hline $\begin{array}{c}\text { APR } 1990 \\
10 \ldots \\
\text { MAY }\end{array}$ & $<1$ & $<0.1$ & 4 & 2 & 5 & 2 & 3400 & 4 & 5 & $<0.5$ \\
\hline $14 \ldots$ & $<1$ & $<0.1$ & 1 & $<1$ & 1 & 2 & $<10$ & $<3$ & $<1$ & $<0.5$ \\
\hline${ }_{\text {JUL }}^{11} \ldots$ & $<1$ & $<0.1$ & 2 & $<1$ & 5 & 1 & 2600 & 7 & 9 & $<0.5$ \\
\hline$\underset{A U G}{24} \cdots$ & $<1$ & $<0.1$ & 2 & $<1$ & 6 & 2 & 3000 & 11 & 5 & $<0.5$ \\
\hline$\underset{\infty}{20} \cdots$ & $<1$ & $<0.1$ & 5 & $<1$ & 8 & 2 & 20000 & 9 & 17 & 0.9 \\
\hline${ }_{\text {DEC }}^{22} \cdots$ & $<1$ & $<0.1$ & 3 & $<1$ & 5 & 2 & 3100 & 33 & 5 & $<0.5$ \\
\hline$\stackrel{17}{\operatorname{MAR}} 1991$ & $<1$ & 0.1 & 4 & $<1$ & 10 & 2 & 6200 & 14 & 13 & $<0.5$ \\
\hline$\underset{A P R}{28} \cdots$ & $<1$ & 0.1 & 1 & $<1$ & 4 & 1 & 1900 & 9 & 1 & $<0.5$ \\
\hline $\operatorname{MAY}^{15 \ldots}$ & $<1$ & 0.3 & $<1$ & $<1$ & 3 & 1 & 940 & 5 & 2 & $<0.5$ \\
\hline$\underset{J U N}{20} \ldots$ & $<1$ & $<0.1$ & $<1$ & $<1$ & 3 & 1 & 380 & 11 & 2 & $<0.5$ \\
\hline${ }_{\text {JUL }}^{10} \cdots$ & $<1$ & $<0.1$ & 2 & 2 & 8 & 3 & 2600 & 360 & 1 & $<0.5$ \\
\hline$\underset{A \cup G}{22} \cdots$ & $<1$ & 0.2 & 4 & $<1$ & 39 & 5 & $\cdots$ & 34 & 7 & $<0.5$ \\
\hline${ }_{\infty}^{15} \cdots$ & $<1$ & 0.2 & $<1$ & $<1$ & 7 & 3 & 940 & $<3$ & $\cdots$ & $<0.5$ \\
\hline${ }_{\mathrm{DEC}}^{28} \ldots$ & $<1$ & $<0.1$ & 2 & $<1$ & 10 & 1 & 2500 & 6 & 6 & $<0.5$ \\
\hline $\operatorname{MAR} 1992$ & $<1$ & $<0.1$ & 2 & $<1$ & $\cdots$ & 2 & 5700 & 12 & 10 & $<0.5$ \\
\hline$\underset{A P R}{26 \ldots}$ & $<1$ & $<0.1$ & 2 & $<1$ & 4 & 1 & 2700 & 6 & 3 & $<0.5$ \\
\hline$\underset{\operatorname{MAY}}{28} \ldots$ & $<1$ & $<0.1$ & $<1$ & $<1$ & 2 & 1 & 500 & 5 & 2 & $<0.5$ \\
\hline $18 \ldots$ & $<1$ & $<0.1$ & $<1$ & $<1$ & 2 & 1 & 200 & 15 & 2 & $<0.5$ \\
\hline
\end{tabular}


Table 47. Onsite measurements and bacteriological and selected inorganic data for station 381510104350601 , Arkansas River below Highway 227, at Pueblo--Continued

\begin{tabular}{|c|c|c|c|c|c|c|c|c|c|c|}
\hline DATE & $\begin{array}{l}\text { CADMIUM } \\
\text { TOTAL } \\
\text { RECOV - } \\
\text { ERABLE } \\
\text { (UG/L } \\
\text { AS CD) }\end{array}$ & $\begin{array}{c}\text { CADMIUM } \\
\text { DIS - } \\
\text { SOLVED } \\
\text { (UG/L } \\
\text { AS CD) }\end{array}$ & $\begin{array}{l}\text { CHRO- } \\
\text { MIUM, } \\
\text { TOTAL } \\
\text { RECOV- } \\
\text { ERABLE } \\
\text { (UG /L } \\
\text { AS CR) }\end{array}$ & $\begin{array}{l}\text { CHRO- } \\
\text { MIUM, } \\
\text { DIS- } \\
\text { SOLVED } \\
\text { (UG/L } \\
\text { AS CR) }\end{array}$ & $\begin{array}{l}\text { COPPER, } \\
\text { TOTAL } \\
\text { RECOV- } \\
\text { ERABLE } \\
\text { (UG /L } \\
\text { AS CU) }\end{array}$ & $\begin{array}{l}\text { COPPER, } \\
\text { DIS- } \\
\text { SOLVED } \\
\text { (UG/L } \\
\text { AS CU) }\end{array}$ & $\begin{array}{l}\text { IRON, } \\
\text { TOTAL } \\
\text { RECOV- } \\
\text { ERABLE } \\
\text { (UG / L } \\
\text { AS FE) }\end{array}$ & $\begin{array}{l}\text { IRON, } \\
\text { DIS- } \\
\text { SOLVED } \\
\text { (UG/L } \\
\text { AS FE) }\end{array}$ & $\begin{array}{l}\text { LEAD, } \\
\text { TOTAL } \\
\text { RECOV- } \\
\text { ERABLE } \\
\text { (UG/L } \\
\text { AS PB) }\end{array}$ & $\begin{array}{l}\text { LEAD, } \\
\text { DIS- } \\
\text { SOLVED } \\
\text { (UG/L } \\
\text { AS PB) }\end{array}$ \\
\hline V 1992 & & & & & & & & & & \\
\hline $16 \cdots$ & $<1$ & 0.1 & $<1$ & $<1$ & 19 & 7 & 160 & 10 & 3 & $<0.5$ \\
\hline${ }_{G}^{16} \ldots$ & $<1$ & 0.2 & $<1$ & $<1$ & 18 & 14 & 170 & 8 & 2 & 0.5 \\
\hline $17 \ldots$ & $<1$ & $<0.1$ & $<1$ & $<1$ & 10 & 7 & 290 & 7 & 10 & 0.8 \\
\hline $\begin{array}{l}20 \ldots \\
N\end{array} 1993$ & $<1$ & $<0.1$ & $<1$ & $<1$ & 3 & 1 & 1500 & 4 & 5 & $<0.5$ \\
\hline $14 \ldots$ & $<1$ & $<0.1$ & 11 & $<1$ & 2 & 1 & 60 & 6 & $<1$ & $<0.5$ \\
\hline $5 \ldots$ & $<1$ & $<0.1$ & $<1$ & 2 & 3 & 2 & 1600 & 7 & 2 & $<0.5$ \\
\hline
\end{tabular}

\begin{tabular}{|c|c|c|c|c|c|c|c|c|c|c|}
\hline DATE & $\begin{array}{l}\text { MANGA - } \\
\text { NESE, } \\
\text { TOTAL } \\
\text { RECOV- } \\
\text { ERABLE } \\
\text { (UG/L } \\
\text { AS MN) }\end{array}$ & $\begin{array}{l}\text { MANGA- } \\
\text { NESE, } \\
\text { DIS- } \\
\text { SOLVED } \\
\text { (UG/L } \\
\text { AS MN) }\end{array}$ & $\begin{array}{l}\text { NICKEL, } \\
\text { TOTAL } \\
\text { RECOV - } \\
\text { ERABLE } \\
\text { (UG/L } \\
\text { AS NI) }\end{array}$ & $\begin{array}{l}\text { NICKEL, } \\
\text { DIS - } \\
\text { SOLVED } \\
\text { (UG/L } \\
\text { AS NI) }\end{array}$ & $\begin{array}{l}\text { SELE- } \\
\text { NIUM, } \\
\text { TOTAL } \\
\text { (UG/L } \\
\text { AS SE) }\end{array}$ & $\begin{array}{l}\text { SELE - } \\
\text { NIUM, } \\
\text { DIS - } \\
\text { SOLVED } \\
\text { (UG/L } \\
\text { AS SE) }\end{array}$ & $\begin{array}{c}\text { SILVER, } \\
\text { TOTAL } \\
\text { RECOV- } \\
\text { ERABLE } \\
\text { (UG/L } \\
\text { AS AG) }\end{array}$ & $\begin{array}{c}\text { SILVER, } \\
\text { DIS - } \\
\text { SOLVED } \\
\text { (UG/L } \\
\text { AS AG) }\end{array}$ & $\begin{array}{l}\text { ZINC, } \\
\text { TOTAL } \\
\text { RECOV- } \\
\text { ERABLE } \\
\text { (UG/L } \\
\text { AS ZN) }\end{array}$ & $\begin{array}{l}\text { ZINC, } \\
\text { DIS - } \\
\text { SOLVED } \\
\text { (UG/L } \\
\text { AS ZN) }\end{array}$ \\
\hline APR 1990 & & & & & & & & & & \\
\hline $10 \ldots$ & 120 & 9 & $\cdots$ & 3 & 12 & 11 & $<1$ & $<1.0$ & 30 & 10 \\
\hline${ }_{\pi N}^{14} \ldots$ & $<10$ & 8 & $\cdots$ & 2 & 11 & 11 & $<1$ & $<1.0$ & $<10$ & $<3$ \\
\hline${ }_{\pi L}^{11} \ldots$ & 100 & 11 & $\cdots$ & 2 & 7 & 6 & $<1$ & $<1.0$ & 30 & $<3$ \\
\hline$\underset{A U G}{24}$ & 100 & 7 & 4 & 1 & $\cdots$ & $\cdots$ & $<1$ & $<1.0$ & 20 & $<3$ \\
\hline${ }_{\text {OCT }}^{20} \ldots$ & 90 & 5 & 5 & 1 & $\cdots$ & $\cdots$ & $<1$ & $<1.0$ & 30 & 8 \\
\hline$\underset{D E C}{22} \cdots$ & 140 & 6 & 5 & 2 & $\cdots$ & $\cdots$ & $<1$ & $<1.0$ & 30 & $<3$ \\
\hline${ }_{\operatorname{MAR}}^{17} 19 \dot{9}_{1}$ & 240 & 8 & 17 & 4 & $\cdots$ & $\cdots$ & 1 & $<1.0$ & 30 & 5 \\
\hline$\underset{A P R}{28 \ldots}$ & 70 & 9 & 4 & 2 & $\cdots$ & $\cdots$ & $<1$ & $<1.0$ & 20 & 6 \\
\hline $15 \ldots$ & 50 & 10 & 5 & 1 & $\cdots$ & $\cdots$ & $<1$ & $<1.0$ & $<10$ & 3 \\
\hline${ }_{\pi N}^{20} \ldots$ & 40 & 8 & 2 & 2 & $\cdots$ & $\cdots$ & $<1$ & $<1.0$ & $<10$ & 6 \\
\hline 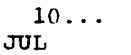 & 120 & 19 & 7 & 2 & $\cdots$ & $\cdots$ & $<1$ & $<1.0$ & 20 & 4 \\
\hline$\underset{A \cup G}{22} \cdots$ & 20 & 23 & 12 & 2 & $\cdots$ & $\cdots$ & $<1$ & $<1.0$ & 10 & 8 \\
\hline$\underset{\text { OCT }}{15 \ldots}$ & 70 & $<1$ & 5 & 3 & - & - & $<1$ & $<1.0$ & $<10$ & 4 \\
\hline${ }_{D E C}^{28} \cdots$ & 120 & 13 & 5 & 2 & $\cdots$ & $\cdots$ & $<1$ & $<1.0$ & 20 & 4 \\
\hline $\begin{array}{ll}16 \ldots & \ldots \\
\text { MAR } & 1992\end{array}$ & 220 & 7 & 8 & 3 & - & $\cdots$ & $<1$ & $<1.0$ & 70 & 5 \\
\hline $26 \ldots$ & 80 & 7 & 4 & 2 & $\cdots$ & $\cdots$ & $<1$ & $<1.0$ & 20 & 7 \\
\hline$\underset{\mathrm{MAY}}{28 \ldots}$ & 30 & 5 & 3 & 2 & $\cdots$ & - & $<1$ & $<1.0$ & 10 & $<3$ \\
\hline$\pi_{\pi N}^{18} \ldots$ & 40 & 14 & 2 & 2 & $\cdots$ & $\cdots$ & $<1$ & $<1.0$ & 10 & 3 \\
\hline$\pi_{\text {JL }}^{16 \ldots}$ & 50 & 13 & 2 & 2 & $\cdots$ & $\cdots$ & $<1$ & $<1.0$ & 10 & $<3$ \\
\hline${ }_{A \cup G}^{16} \ldots$ & 30 & 4 & 3 & $<1$ & $\cdots$ & $\cdots$ & $<1$ & $<1.0$ & 10 & 4 \\
\hline $\operatorname{OCT}^{17 \ldots}$ & 50 & 10 & 2 & $<1$ & $\cdots$ & $\cdots$ & $<1$ & $<1.0$ & 10 & $<3$ \\
\hline $\begin{array}{l}20 \ldots \\
\text { JAN } 1993\end{array}$ & 90 & 9 & 4 & 1 & $\cdots$ & $\cdots$ & $<1$ & $<1.0$ & 10 & $<3$ \\
\hline $\operatorname{MAR}^{14 \ldots}$ & 50 & 9 & 3 & 1 & $\cdots$ & $\cdots$ & $<1$ & $<1.0$ & $<10$ & $<3$ \\
\hline $25 \ldots$ & 90 & 7 & 4 & 2 & $\cdots$ & $\cdots$ & $<1$ & $<1.0$ & 20 & 7 \\
\hline
\end{tabular}


Table 48. Onsite measurements and bacteriological and selected inorganic data for station 07109500 , Arkansas River near Avondale

\begin{tabular}{|c|c|c|c|c|c|c|c|c|c|c|c|c|}
\hline DATE & T IME & $\begin{array}{c}\text { DIS- } \\
\text { CHARGE, } \\
\text { INST. } \\
\text { CUBIC } \\
\text { FEET } \\
\text { PER } \\
\text { SECOND }\end{array}$ & $\begin{array}{l}\text { SPE- } \\
\text { CIFIC } \\
\text { CON- } \\
\text { DUCT- } \\
\text { ANCE } \\
\text { (US /CM) }\end{array}$ & $\begin{array}{c}\text { PH } \\
\text { WATER } \\
\text { WHOLE } \\
\text { FIELD } \\
\text { (STAND - } \\
\text { ARD } \\
\text { UNITS) }\end{array}$ & $\begin{array}{c}\text { TEMPER - } \\
\text { ATURE } \\
\text { WATER } \\
\text { (DEG C) }\end{array}$ & $\begin{array}{c}\text { OXYGEN, } \\
\text { DIS - } \\
\text { SOLVED } \\
\text { (MG/L) }\end{array}$ & $\begin{array}{l}\text { COLI- } \\
\text { FORM, } \\
\text { FECAL, } \\
0.7 \\
\text { UM-MF } \\
\text { (COLS./ } \\
100 \mathrm{ML} \text { ) }\end{array}$ & $\begin{array}{l}\text { STREP- } \\
\text { TOCOCCI } \\
\text { FECAL, } \\
\text { KF AGAR } \\
\text { (COLS. } \\
\text { PER } \\
100 \mathrm{ML} \text { ) }\end{array}$ & $\begin{array}{l}\text { HARD- } \\
\text { NESS } \\
\text { TOTAL } \\
\text { (MG/L } \\
\text { AS } \\
\text { CACO3) }\end{array}$ & $\begin{array}{l}\text { CALCIUM } \\
\text { DIS- } \\
\text { SOLVED } \\
\text { (MG/L } \\
\text { AS CA) }\end{array}$ & $\begin{array}{l}\text { MAGNE- } \\
\text { SIUM, } \\
\text { DIS - } \\
\text { SOLVED } \\
\text { (MG/L } \\
\text { AS MG) }\end{array}$ & $\begin{array}{l}\text { SODIUM } \\
\text { DIS - } \\
\text { SOLVED } \\
\text { (MG /L } \\
\text { AS NA }\end{array}$ \\
\hline PR 1990 & & & & & & & & & & & & \\
\hline $10 \ldots$ & 1840 & 445 & 955 & 8.3 & 14.5 & 6.5 & $\cdots$ & $\cdots$ & 360 & 93 & 31 & 68 \\
\hline $\operatorname{suN}^{14 \ldots}$ & 1750 & 634 & 933 & 8.1 & 18.5 & 7.3 & K5 & 94 & 380 & 96 & 33 & 64 \\
\hline$J_{\pi L}^{12} \cdots$ & 0920 & 4000 & 648 & 8.1 & 16.0 & 7.9 & K28 & K550 & 270 & 70 & 22 & 35 \\
\hline $\begin{array}{l}24 \ldots \\
A U G\end{array}$ & 1700 & 960 & 649 & 8.2 & 23.0 & 6.3 & 230 & K670 & 250 & 69 & 20 & 39 \\
\hline $\mathrm{OCT}^{20 \ldots}$ & 1800 & 1170 & 610 & 8.2 & 24.5 & 6.7 & 460 & $\mathrm{~K} 1000$ & 230 & 63 & 18 & 34 \\
\hline $22 \ldots$ & 1615 & 628 & 871 & 8.5 & 14.5 & 8.8 & E31 & 160 & 320 & 85 & 27 & 55 \\
\hline${ }_{\text {MAR }}^{17} 1991$ & 1550 & 286 & 1120 & 8.4 & 5.5 & 11.0 & $<2$ & 420 & 420 & 110 & 36 & 77 \\
\hline$\underset{A P R}{28} \cdots$ & 1150 & 510 & 803 & 8.8 & 8.5 & 10.2 & $<3$ & E32 & 330 & 86 & 27 & 48 \\
\hline$\underset{\operatorname{MAY}}{15} \ldots$ & 1615 & 430 & 852 & 8.4 & 16.0 & $\cdots$ & $<2$ & E15 & 320 & 85 & 27 & 51 \\
\hline$\underset{\pi N}{20}$ & 1650 & 670 & 698 & 8.4 & 16.0 & 8.2 & E12 & 110 & 300 & 79 & 25 & 43 \\
\hline${ }_{\pi} 10 \ldots$ & 1800 & 2320 & 604 & 8.4 & 21.0 & 6.8 & 230 & 1000 & 230 & 62 & 18 & 33 \\
\hline $\begin{array}{l}22 \\
\text { AUG }\end{array}$ & 1830 & 1950 & 547 & 8.2 & 22.5 & 6.5 & 390 & E640 & 210 & 58 & 16 & 30 \\
\hline $\begin{array}{l}15 \ldots \\
\operatorname{SEP}\end{array}$ & 1715 & 1750 & 607 & 8.2 & 25.5 & 6.1 & 270 & 590 & 230 & 66 & 17 & 29 \\
\hline$\underset{\mathrm{OCT}}{04 \ldots}$ & 0935 & 740 & 617 & 8.0 & 20.0 & $\cdots$ & $\cdots$ & $\cdots$ & . & $\cdots$ & $\cdots$ & $\cdots$ \\
\hline$\underset{D E C}{28} \cdots$ & 1630 & 330 & 1050 & 8.8 & 9.0 & 10.3 & E52 & 110 & 410 & 110 & 34 & 59 \\
\hline${ }_{\text {MAR }}^{17} 19 \dot{9} 2$ & 0805 & 299 & 1230 & 8.2 & 3.5 & 10.2 & E28 & 140 & 470 & 120 & 41 & 87 \\
\hline$\underset{A P R}{26 \cdots}$ & 1115 & 654 & 813 & 8.4 & 9.0 & 9.8 & & $\cdots$ & 310 & 83 & 25 & 52 \\
\hline$\underset{\operatorname{MAY}}{28 \ldots}$ & 1500 & 810 & 765 & 8.3 & 16.0 & 8.7 & E7 & 20 & 290 & 75 & 24 & 42 \\
\hline${ }_{J U N}^{18 \ldots}$ & 1615 & 828 & 717 & 8.6 & 19.5 & 9.4 & E15 & 22 & 300 & 80 & 24 & 38 \\
\hline${ }_{J U L}^{16} \cdots$ & 1500 & 1720 & 593 & 8.4 & 19.5 & 8.0 & E16 & 75 & 230 & 64 & 18 & 30 \\
\hline${ }_{A \cup G}^{16} \cdots$ & 1520 & 1270 & 518 & 8.3 & 23.0 & 7.2 & 33 & 90 & 210 & 56 & 16 & 25 \\
\hline${ }_{\mathrm{OCT}}^{17} \cdots$ & 1530 & 733 & 617 & 8.3 & 24.0 & 6.8 & 58 & 69 & 260 & 70 & 20 & 30 \\
\hline JAN $20 \ldots 93$ & 1430 & 433 & 854 & 8.5 & 15.0 & 10.2 & E26 & 82 & 350 & 92 & 28 & 50 \\
\hline${ }_{\operatorname{MAR}}^{14} \cdots$ & 1330 & 444 & 973 & 8.2 & 0.0 & 11.5 & E1 & E8 & 360 & 93 & 30 & 64 \\
\hline & 1100 & 428 & 844 & 8.4 & 9.0 & 10.8 & E4 & E6 & 330 & 85 & 28 & 55 \\
\hline
\end{tabular}

\begin{tabular}{|c|c|c|c|c|c|c|c|c|c|c|}
\hline $\begin{array}{c}\text { ALKA- } \\
\text { LINITY } \\
\text { LAB } \\
\text { (MG / L } \\
\text { AS } \\
\text { CACO3) }\end{array}$ & $\begin{array}{l}\text { SULFATE } \\
\text { DIS- } \\
\text { SOLVED } \\
\text { (MG/L } \\
\text { AS SO4) }\end{array}$ & $\begin{array}{l}\text { CHIO- } \\
\text { RIDE, } \\
\text { DIS- } \\
\text { SOLVED } \\
\text { (MG/L } \\
\text { AS CL) }\end{array}$ & $\begin{array}{l}\text { SOLIDS, } \\
\text { RESIDUE } \\
\text { AT } 180 \\
\text { DEG C } \\
\text { DIS- } \\
\text { SOLVED } \\
\text { (MG/L) }\end{array}$ & $\begin{array}{c}\text { NITRO- } \\
\text { GEN, } \\
\text { NO2+NO3 } \\
\text { TOTAL } \\
\text { (MG /L } \\
\text { AS N) }\end{array}$ & $\begin{array}{c}\text { NITRO- } \\
\text { GEN, } \\
\text { NO2+NO3 } \\
\text { DIS - } \\
\text { SOLVED } \\
\text { (MG / L } \\
\text { AS N) }\end{array}$ & $\begin{array}{l}\text { NITRO- } \\
\text { GEN, } \\
\text { AMMONIA } \\
\text { TOTAL } \\
\text { (MG/L } \\
\text { AS N) }\end{array}$ & $\begin{array}{c}\text { NITRO- } \\
\text { GEN, } \\
\text { AMMONIA } \\
\text { DIS - } \\
\text { SOLVED } \\
\text { (MG / L } \\
\text { AS N) }\end{array}$ & $\begin{array}{c}\text { PHOS - } \\
\text { PHORUS } \\
\text { TOTAL } \\
\text { (MG / L } \\
\text { AS P) }\end{array}$ & $\begin{array}{c}\text { ARSENIC } \\
\text { TOTAL } \\
\text { (UG/L } \\
\text { AS AS) }\end{array}$ & $\begin{array}{l}\text { ARSENIC } \\
\text { DIS - } \\
\text { SOLVED } \\
\text { (UG/L } \\
\text { AS AS) }\end{array}$ \\
\hline
\end{tabular}

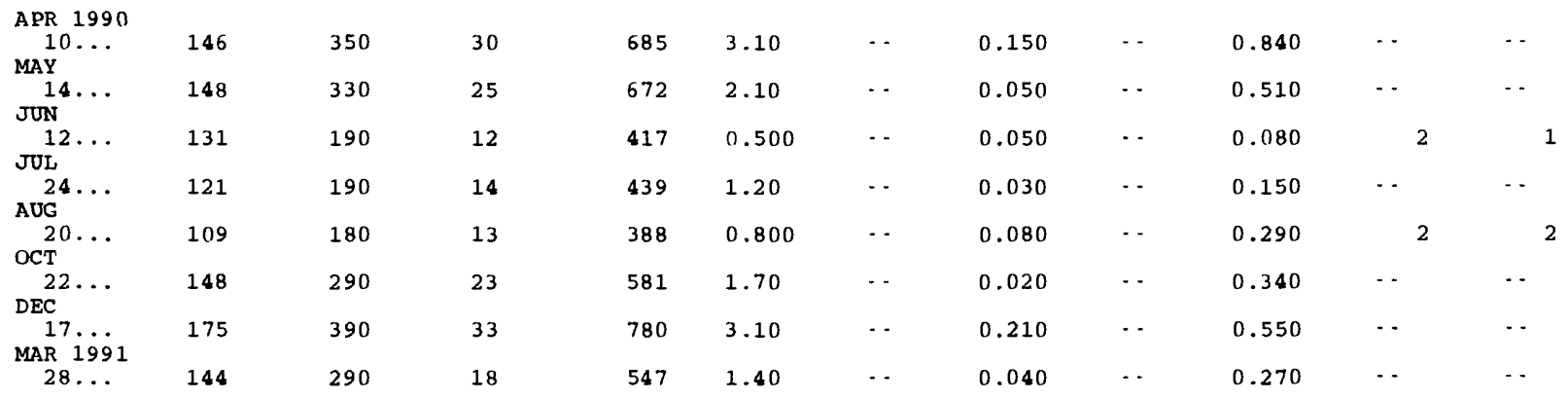


Table 48. Onsite measurements and bacteriological and selected inorganic data for station 07109500 , Arkansas River near Avondale--Continued

\begin{tabular}{|c|c|c|c|c|c|c|c|c|c|c|c|}
\hline DATE & $\begin{array}{c}\text { ALKA- } \\
\text { LINITY } \\
\text { LAB } \\
\text { (MG / } / \\
\text { AS } \\
\text { CACO3) }\end{array}$ & $\begin{array}{c}\text { SULFATE } \\
\text { DIS- } \\
\text { SOLVED } \\
\text { (MG/L } \\
\text { AS SO4) }\end{array}$ & $\begin{array}{l}\text { CHLO- } \\
\text { RIDE, } \\
\text { DIS- } \\
\text { SOLVED } \\
\text { (MG/L } \\
\text { AS CL) }\end{array}$ & $\begin{array}{l}\text { SOLIDS, } \\
\text { RESIDUE } \\
\text { AT } 180 \\
\text { DEG C } \\
\text { DIS- } \\
\text { SOLVED } \\
\text { (MG/L) }\end{array}$ & $\begin{array}{c}\text { NITRO- } \\
\text { GEN, } \\
\text { NO2+NO3 } \\
\text { TOTAL } \\
\text { (MG / L } \\
\text { AS N) }\end{array}$ & $\begin{array}{c}\text { NITRO- } \\
\text { GEN, } \\
\text { NO2+NO3 } \\
\text { DIS- } \\
\text { SOLVED } \\
\text { (MG/L } \\
\text { AS N) }\end{array}$ & $\begin{array}{c}\text { NITRO- } \\
\text { GEN, } \\
\text { AMMONIA } \\
\text { TOTAL } \\
\text { (MG/L } \\
\text { AS N) }\end{array}$ & $\begin{array}{l}\text { NITRO- } \\
\text { GEN, } \\
\text { AMMONIA } \\
\text { DIS- } \\
\text { SOLVED } \\
\text { (MG/L } \\
\text { AS N) }\end{array}$ & $\begin{array}{c}\text { PHOS - } \\
\text { PHORUS } \\
\text { TOTAL } \\
\text { (MG/L } \\
\text { AS P) }\end{array}$ & $\begin{array}{c}\text { ARSENIC } \\
\text { TOTAL } \\
\text { (UG /L } \\
\text { AS AS) }\end{array}$ & $\begin{array}{l}\text { ARSENIC } \\
\text { DIS- } \\
\text { SOLVED } \\
\text { (UG/L } \\
\text { AS AS) }\end{array}$ \\
\hline $\begin{array}{c}\text { APR } 1991 \\
15 \ldots \\
\operatorname{MAY} \cdots\end{array}$ & 146 & 260 & 21 & 582 & 1.50 & $\cdots$ & 0.020 & $\cdots$ & 0.310 & $\cdots$ & -- \\
\hline $\begin{array}{l}20 \ldots \\
\text { JUN }\end{array}$ & 137 & 230 & 16 & 487 & 1.10 & $\cdots$ & 0.030 & $\cdots$ & 0.230 & $\cdots$ & $\cdots$ \\
\hline JUL $10 \ldots$ & 119 & 180 & 11 & 372 & 0.660 & $\cdots$ & 0.040 & $\cdots$ & 0.420 & 3 & 2 \\
\hline$\underset{A U G}{22 \ldots}$ & 102 & 160 & 10 & 335 & 0.680 & $\cdots$ & $<0.010$ & $\cdots$ & 0.170 & $\cdots$ & $\cdots$ \\
\hline $\operatorname{sEP}^{15 \ldots}$ & 112 & 170 & 13 & 397 & 0.910 & $\cdots$ & 0.060 & $\cdots$ & 0.210 & $\cdots$ & $\cdots$ \\
\hline${ }_{\text {ост }}^{04 \ldots}$ & $\cdots$ & - & $\cdots$ & $\cdots$ & $\cdots$ & $\cdots$ & - & $\cdots$ & - & 4 & 2 \\
\hline${ }_{\text {DEC }}^{28} \ldots$ & 165 & 380 & 24 & 714 & 2.10 & $\cdots$ & 0.040 & $\cdots$ & $<0.010$ & $\cdots$ & $\cdots$ \\
\hline${ }_{\operatorname{MAR}}^{17} \mathrm{i} 9 \dot{9} 2$ & 173 & 370 & 37 & 975 & 3.60 & $\cdots$ & 0.610 & $\cdots$ & 0.840 & $\cdots$ & $\cdots$ \\
\hline$\underset{A P R}{26} \cdots$ & 139 & 250 & 24 & 548 & 2.10 & $\cdots$ & 0.030 & $\cdots$ & 0.450 & $\cdots$ & - \\
\hline $\operatorname{MAY}^{28} \ldots$ & 136 & 230 & 19 & 512 & 1.30 & $\cdots$ & 0.030 & $\cdots$ & 0.230 & $\cdots$ & $\cdots$ \\
\hline $18 \ldots$ & 135 & 200 & 18 & 472 & 0.790 & $\cdots$ & 0.020 & $\cdots$ & 0.140 & $\cdots$ & $\cdots$ \\
\hline${ }_{\text {JUL }}^{16 \ldots}$ & 116 & 150 & 12 & 366 & 0.660 & $\cdots$ & 0.040 & $\cdots$ & 0.090 & 1 & 1 \\
\hline${ }_{A \cup G}^{16} \ldots$ & 108 & 140 & 11 & 322 & 0.560 & $\cdots$ & 0.050 & $\cdots$ & 0.120 & $\cdots$ & $\cdots$ \\
\hline${ }_{\text {OCT }}^{17 \ldots}$ & 119 & 180 & 11 & 398 & 0.830 & $\cdots$ & 0.020 & $\cdots$ & 0.160 & 2 & $<1$ \\
\hline JAN 1993 & 150 & 260 & 19 & 609 & 1.60 & $\cdots$ & 0.030 & $\cdots$ & 0.340 & $\cdots$ & $\cdots$ \\
\hline $\operatorname{MAR}^{14 \ldots}$ & 159 & 300 & 27 & 669 & $\cdots$ & 2.70 & $\cdots$ & 0.480 & 0.440 & $\cdots$ & $\cdots$ \\
\hline $25 \ldots$ & 148 & 270 & 23 & 584 & $\cdots$ & 2.40 & $\cdots$ & 0.040 & 0.420 & $\cdots$ & $\cdots$ \\
\hline DATE & $\begin{array}{l}\text { CADMIUM } \\
\text { TOTAL } \\
\text { RECOV- } \\
\text { ERABLE } \\
\text { (UG/L } \\
\text { AS CD) }\end{array}$ & $\begin{array}{l}\text { CADMIUM } \\
\text { DIS - } \\
\text { SOLVED } \\
\text { (UG/L } \\
\text { AS CD) }\end{array}$ & $\begin{array}{l}\text { CHRO- } \\
\text { MIUM, } \\
\text { TOTAL } \\
\text { RECOV- } \\
\text { ERABLE } \\
\text { (UG/L } \\
\text { AS CR) }\end{array}$ & $\begin{array}{l}\text { CHRO- } \\
\text { MIUM, } \\
\text { DIS - } \\
\text { SOLVED } \\
\text { (UG/L } \\
\text { AS CR) }\end{array}$ & $\begin{array}{l}\text { COPPER, } \\
\text { TOTAL } \\
\text { RECOV- } \\
\text { ERABLE } \\
\text { (UG/L } \\
\text { AS CU) }\end{array}$ & $\begin{array}{l}\text { COPPER, } \\
\text { DIS- } \\
\text { SOLVED } \\
\text { (UG /L } \\
\text { AS CU) }\end{array}$ & $\begin{array}{l}\text { IRON, } \\
\text { TOTAL } \\
\text { RECOV- } \\
\text { ERABLE } \\
\text { (UG/L } \\
\text { AS FE) }\end{array}$ & $\begin{array}{l}\text { IRON, } \\
\text { DIS- } \\
\text { SOLVED } \\
\text { (UG/L } \\
\text { AS FE) }\end{array}$ & $\begin{array}{l}\text { LEAD, } \\
\text { TOTAL } \\
\text { RECOV. } \\
\text { ERABLE } \\
\text { (UG/L } \\
\text { AS PB) }\end{array}$ & $\begin{array}{l}\text { LEAD, } \\
\text { DIS - } \\
\text { SOLVED } \\
\text { (UG/L } \\
\text { AS PB) }\end{array}$ & $\begin{array}{l}\text { MANGA- } \\
\text { NESE, } \\
\text { TOTAL } \\
\text { RECOV. } \\
\text { ERABLE } \\
\text { (UG/L } \\
\text { AS MN) }\end{array}$ \\
\hline $\begin{array}{c}\text { APR } 1990 \\
10 \ldots \\
\text { MAY }\end{array}$ & 1 & $<0.1$ & 3 & $<1$ & 6 & 2 & 3600 & 6 & 5 & $<0.5$ & 130 \\
\hline $\begin{array}{l}\text { MAY } \\
14 \ldots \\
\text { JUN }\end{array}$ & $<1$ & $<0.1$ & 2 & $<1$ & 6 & 3 & 2900 & 5 & 21 & $\cdots$ & 120 \\
\hline${ }_{\text {JUL }}^{12 \ldots}$ & 1 & 0.2 & 8 & $<1$ & 22 & 3 & 8800 & 8 & 30 & $<0.5$ & 250 \\
\hline$\underset{A U G}{24} \ldots$ & $<1$ & $<0.1$ & 5 & $<1$ & 8 & 1 & 6400 & 22 & 9 & $<0.5$ & 210 \\
\hline $\begin{array}{l}20 \\
\text { OCT }\end{array}$ & $<1$ & $<0.1$ & 8 & $<1$ & 14 & 2 & 10000 & 4 & 10 & $<0.5$ & 230 \\
\hline$\underset{D E C}{22} \cdots$ & $<1$ & $<0.1$ & 3 & $<1$ & 7 & 2 & 2700 & 4 & 4 & $<0.5$ & 150 \\
\hline MAR $179 \dot{9}_{1}$ & $<1$ & $<0.1$ & $<1$ & $<1$ & 5 & 2 & 1900 & 18 & 3 & $<0.5$ & 110 \\
\hline$\underset{A P R}{28 \ldots}$ & $<1$ & $<0.1$ & 2 & $<1$ & 4 & 2 & 1200 & 10 & 1 & $<0.5$ & 70 \\
\hline${ }_{\operatorname{MAY}}^{15} \ldots$ & $<1$ & 0.1 & $<1$ & $<1$ & 3 & 1 & 890 & 10 & 5 & $<0.5$ & 50 \\
\hline $20 \ldots$ & $<1$ & 0.5 & $<1$ & $<1$ & 4 & 1 & 910 & 11 & 3 & $<0.5$ & 60 \\
\hline${ }_{J U L}^{10} \cdots$ & $<1$ & $<0.1$ & 2 & 2 & 12 & 3 & 7700 & 10 & 35 & $<0.5$ & 210 \\
\hline$\underset{A U G}{22} \ldots$ & $<1$ & $<0.1$ & 13 & $<1$ & 25 & 3 & $\cdots$ & 12 & 45 & $<0.5$ & 380 \\
\hline $\operatorname{SEP}^{15} \cdots$ & $<1$ & 0.2 & 3 & $<1$ & 11 & 4 & 4000 & $<3$ & $\cdots$ & $<0.5$ & 130 \\
\hline $\begin{array}{c}04 \ldots \\
\text { OCT }\end{array}$ & & . & & $\cdots$ & & $\cdots$ & $\cdots$ & $\cdots$ & $\cdots$ & $\cdots$ & $\cdots$ \\
\hline $\begin{array}{l}28 \ldots \\
\mathrm{DEC}\end{array}$ & $<1$ & $<0.1$ & $<1$ & $<1$ & 9 & $<1$ & 1200 & 21 & 4 & $<0.5$ & 90 \\
\hline $17 \ldots$ & $<1$ & 0.4 & 3 & $<1$ & $\cdots$ & 2 & 3200 & 18 & 11 & $<0.5$ & 150 \\
\hline
\end{tabular}


Table 48. Onsite measurements and bacteriological and selected inorganic data for station 07109500 , Arkansas River near Avondale--Continued

\begin{tabular}{|c|c|c|c|c|c|c|c|c|c|c|}
\hline $\begin{array}{l}\text { ADMIUM } \\
\text { TOTAL } \\
\text { RECOV- } \\
\text { ERABLE } \\
\text { (UG/L }\end{array}$ & $\begin{array}{c}\text { CADMIOM } \\
\text { DIS - } \\
\text { SOLVED } \\
\text { (UG/L }\end{array}$ & $\begin{array}{l}\text { CHRO- } \\
\text { MIUM, } \\
\text { TOTAL } \\
\text { RECOV- } \\
\text { ERABLE } \\
\text { IUG/L }\end{array}$ & $\begin{array}{l}\text { CHRO- } \\
\text { MIUM, } \\
\text { DIS- } \\
\text { SOLVED } \\
\text { /UG / L }\end{array}$ & $\begin{array}{c}\text { COPPER, } \\
\text { TOTAL } \\
\text { RECOV- } \\
\text { ERABLE } \\
\text { (UG/L }\end{array}$ & $\begin{array}{l}\text { COPPER, } \\
\text { DIS - } \\
\text { SOLVED } \\
\text { (UG /L }\end{array}$ & $\begin{array}{l}\text { IRON, } \\
\text { TOTAL } \\
\text { RECOV- } \\
\text { ERABLE } \\
\text { (UG/L }\end{array}$ & $\begin{array}{l}\text { IRON, } \\
\text { DIS- } \\
\text { SOLVED } \\
\text { IUG/L }\end{array}$ & $\begin{array}{l}\text { LEAD, } \\
\text { TOTAL } \\
\text { RECOV - } \\
\text { ERABLE } \\
\text { /UG/L }\end{array}$ & $\begin{array}{l}\text { LEAD, } \\
\text { DIS - } \\
\text { SOLVED } \\
\text { IUG/L }\end{array}$ & $\begin{array}{l}\text { MANGA - } \\
\text { NESE, } \\
\text { TOTAL } \\
\text { RECOV- } \\
\text { ERABLE } \\
\text { (UG/L }\end{array}$ \\
\hline AS CD) & AS CD) & AS CR) & AS CR) & AS CU) & AS CU) & AS FE) & AS FE) & AS PB) & AS PB) & AS $\mathbf{M N}$ ) \\
\hline
\end{tabular}

\begin{tabular}{|c|c|c|c|c|c|c|c|c|c|c|c|}
\hline $\begin{array}{l}\text { MAR } 1992 \\
26 \ldots \\
\text { APR }\end{array}$ & $<1$ & $<0.1$ & 2 & $<1$ & 5 & 1 & 2900 & 9 & 4 & $<0.5$ & 130 \\
\hline$\underset{M A Y}{28}$ & - & $<0.1$ & $\cdots$ & $<1$ & $\cdots$ & 1 & $\cdots$ & 6 & $\cdots$ & $<0.5$ & $\cdots$ \\
\hline $\operatorname{JUN}_{18}^{18}$ & $<1$ & $<0.1$ & $<1$ & $<1$ & 3 & 1 & 960 & 12 & 2 & 0.5 & 60 \\
\hline$J_{U L}^{16 \ldots}$ & $<1$ & $<0.1$ & $<1$ & $<1$ & 46 & 16 & 1400 & 12 & 5 & $<0.5$ & 90 \\
\hline${ }_{A \cup G}^{16} \ldots$ & $<1$ & $<0.1$ & $<1$ & $<1$ & 15 & 10 & 1600 & 7 & 4 & $<0.5$ & 120 \\
\hline $\mathrm{OCT}^{17 \ldots}$ & $<1$ & $<0.1$ & 2 & $<1$ & 2 & $<1$ & 830 & 7 & 3 & $<0.5$ & 80 \\
\hline${ }_{\text {JAN }}^{20} 1993$ & $<1$ & $<0.1$ & $<1$ & $<1$ & 3 & 1 & 1300 & 5 & 5 & $<0.5$ & 100 \\
\hline${ }_{\text {MAR }}^{14 \ldots}$ & $<1$ & $<0.1$ & 13 & $<1$ & 5 & 2 & 1100 & 35 & 2 & $<0.5$ & 100 \\
\hline $25 \ldots$ & $<1$ & $<0.1$ & $<1$ & $<1$ & 2 & 1 & 610 & 12 & 1 & $<0.5$ & 50 \\
\hline
\end{tabular}

DATE

\begin{tabular}{cccccc} 
MANGA- & MERCURY & \multicolumn{3}{c}{ NICKEL, } \\
NESE, & TOTAL & MERCURY & TOTAL, & NICKEL, & SELE- \\
DIS - & RECOV- & DIS- & RECOV- & DIS- & NIUM, \\
SOLVED & ERABLE & SOLVED & ERABLE & SOLVED & TOTAL \\
(UG/L & (UG/L & (UG/L & (UG/L & (UG/L & (UG/L \\
AS MN) & AS HG) & AS HG) & AS NI) & AS NI) & AS SE)
\end{tabular}

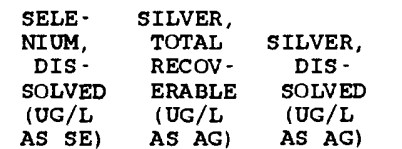

ZINC,

TOTAL ZINC

ERABLE SOLVED

(UG/L (UG/L

AS MN) AS HG) AS HG) AS NI) AS NI) AS SE)

AS SE) AS AG) AS AG)

AS $\mathrm{ZN}) \quad$ AS $\mathrm{ZN})$

APR 1990

$10 \ldots$

MAY $14 .$.

JUN

$12 \ldots$

24 ..

AUG

$20 \ldots$

22 ...

$17 \ldots$

MAR $19 \ddot{9} 1$

28 ...

APR $15 \ldots$

MAY

$20 \ldots$

10 ..

JUL

$22 \ldots$

15 ...

SEP

$04 .$.

OCT $28 \ldots$

DEC 17.

MAR 1992

$26 \ldots$

APR

MAY 28

18 .

JUN

16 ...

$16 \ldots$

AUG

${ }^{17 \ldots}{ }^{17}$

AN 1993

14 ...

MAR

25 ...

\begin{tabular}{|c|c|c|c|}
\hline$\cdots$ & $\cdots$ & 2 & 12 \\
\hline$\cdots$ & $\cdots$ & 3 & 13 \\
\hline$<0.1$ & $\cdots$ & 3 & 7 \\
\hline$\cdots$ & 7 & 1 & $\cdots$ \\
\hline$<0.1$ & 10 & 1 & $\cdots$ \\
\hline-- & 5 & 1 & - \\
\hline$\cdots$ & 6 & 3 & $\cdots$ \\
\hline-- & 3 & 3 & $\cdots$ \\
\hline$\cdots$ & 2 & 2 & $\cdots$ \\
\hline$\cdots$ & 3 & 2 & 8 \\
\hline$<0.1$ & 12 & 1 & $\cdots$ \\
\hline$\cdots$ & 27 & $<1$ & $\cdots$ \\
\hline - & 7 & 3 & 6 \\
\hline$<0.1$ & $\cdots$ & $\cdots$ & $\cdots$ \\
\hline$\cdots$ & 4 & 3 & 18 \\
\hline$\cdots$ & 8 & 3 & 25 \\
\hline$\cdots$ & 5 & 2 & 9 \\
\hline$\cdots$ & $\cdots$ & 3 & 9 \\
\hline - & 3 & 2 & 9 \\
\hline$<0.1$ & 3 & 2 & 5 \\
\hline - & 5 & 1 & 6 \\
\hline$<0.1$ & 2 & $<1$ & 8 \\
\hline - & 4 & 1 & 13 \\
\hline -. & 5 & 2 & 14 \\
\hline$\cdots$ & 4 & 2 & 12 \\
\hline
\end{tabular}

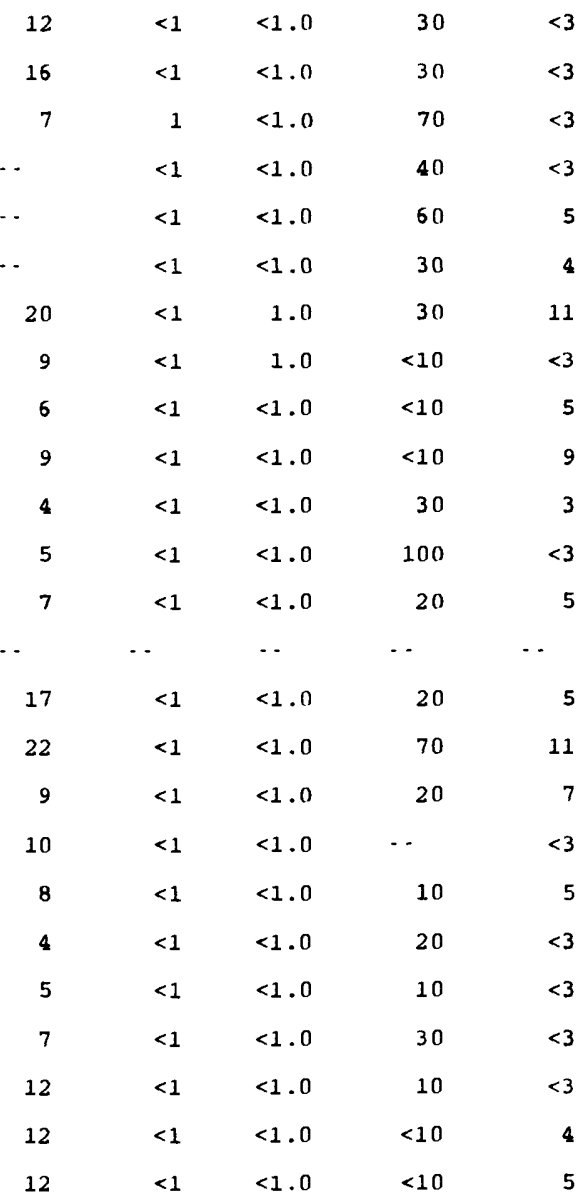


Table 49. Onsite measurements and selected inorganic data for station 07116500 , Huerfano River near Boone

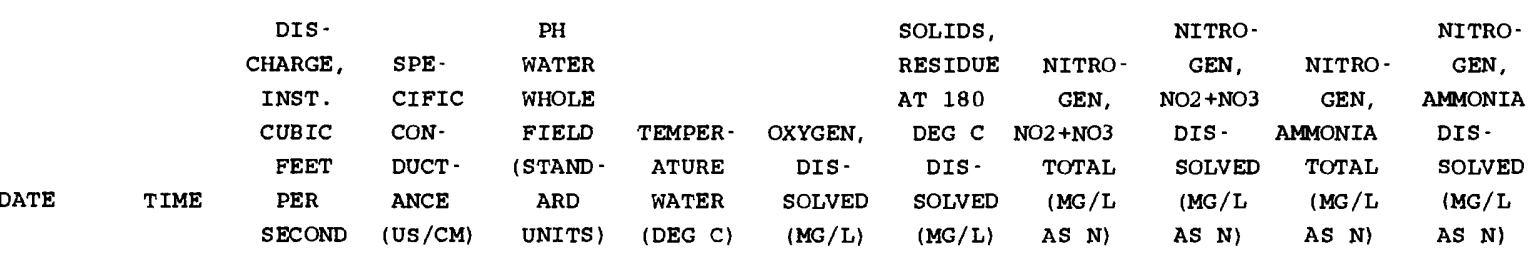

\begin{tabular}{|c|c|c|c|c|c|c|c|c|c|c|c|}
\hline \multicolumn{12}{|l|}{ APR 1990} \\
\hline $11 \ldots$ & 1135 & 0.35 & 5780 & 8.2 & 17.5 & 8.4 & 5640 & 0.900 & $\cdots$ & 0.010 & $-\cdot$ \\
\hline \multicolumn{12}{|l|}{ JUN } \\
\hline $12 \ldots$ & 1340 & 1.3 & 2540 & 8.2 & 35.5 & 5.2 & 2140 & $<0.100$ & $\cdots$ & 0.020 & $\cdots$ \\
\hline \multicolumn{12}{|l|}{ JUL } \\
\hline $25 \ldots$ & 0830 & 25 & 3040 & 8.2 & 17.0 & 7.8 & 2700 & 0.900 & $\cdots$ & 0.020 & $\cdots$ \\
\hline \multicolumn{12}{|l|}{ AUG } \\
\hline $21 \ldots$ & 1000 & 0.28 & 5000 & 8.2 & 24.0 & 7.7 & 4540 & $<0.100$ & $\cdots$ & 0.080 & $\cdots$ \\
\hline \multicolumn{12}{|l|}{ OCT } \\
\hline $23 \ldots$ & 0845 & 4.4 & 2440 & 8.5 & 6.5 & 10.3 & 1790 & 0.200 & $\cdots$ & 0.020 & $\cdots$ \\
\hline \multicolumn{12}{|l|}{ APR 1991} \\
\hline $16 \ldots$ & 0820 & 3.6 & 4050 & $\cdots$ & 6.0 & 10.3 & 3570 & 0.080 & $\cdots$ & $<0.010$ & $\cdots$ \\
\hline \multicolumn{12}{|l|}{ SEP } \\
\hline $04 \ldots$ & 1150 & 107 & 1080 & 8.0 & 20.0 & 7.0 & 774 & 2.10 & - & 0.240 & $\cdots$ \\
\hline \multicolumn{12}{|l|}{ DEC } \\
\hline $17 \ldots$ & 1000 & 27 & 3120 & 8.4 & 0.0 & 10.5 & 2560 & 0.860 & $\cdots$ & 0.030 & $\cdots$ \\
\hline \multicolumn{12}{|l|}{ APR 1992} \\
\hline $28 \ldots$ & 1610 & 2.1 & 5570 & 8.3 & 28.0 & 7.4 & 5480 & 0.290 & $\cdots$ & 0.030 & $\cdots$ \\
\hline \multicolumn{12}{|l|}{ JUN } \\
\hline $16 \ldots$ & 1610 & 7.7 & 3280 & 8.4 & 24.0 & 6.7 & 2840 & 0.270 & $\cdots$ & 0.030 & $\cdots$ \\
\hline \multicolumn{12}{|l|}{ AUG } \\
\hline $18 \ldots$ & 0730 & - & 5250 & 8.4 & 15.5 & 8.0 & 4530 & 0.091 & $\cdots$ & 0.040 & $\cdots$ \\
\hline \multicolumn{12}{|l|}{ MAR 1993} \\
\hline $25 \ldots$ & 1230 & 27 & 1700 & 8.4 & 17.0 & 8.3 & 1340 & $\cdots$ & 0.330 & $\cdots$ & 0.030 \\
\hline
\end{tabular}


Table 50. Onsite measurements and selected inorganic data for station 07117000 , Arkansas River near Nepesta

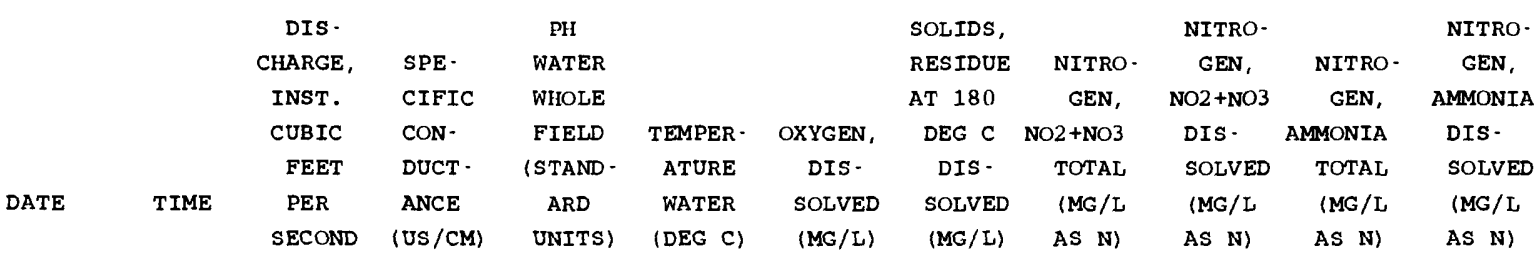

\begin{tabular}{|c|c|c|c|c|c|c|c|c|c|c|c|}
\hline \multicolumn{12}{|l|}{ APR 1990} \\
\hline $11 \ldots$ & 1300 & 360 & 1010 & 8.2 & 17.5 & 7.7 & 721 & 3.00 & $\cdots$ & $<0.010$ & $\cdots$ \\
\hline \multicolumn{12}{|l|}{ MAY } \\
\hline $15 \ldots$ & 0830 & 498 & 977 & 8.4 & 14.5 & 7.9 & 703 & 2.10 & $\cdots$ & $<0.010$ & $\cdots$ \\
\hline \multicolumn{12}{|l|}{ JUN } \\
\hline $12 \ldots$ & 1500 & 2720 & 674 & 8.3 & 20.0 & 7.4 & 431 & 0.500 & $\cdots$ & 0.020 & $\cdots$ \\
\hline \multicolumn{12}{|l|}{ JUL } \\
\hline $25 \ldots$ & 0940 & 216 & 768 & 8.2 & 19.5 & 7.2 & 539 & 1.20 & $\cdots$ & 0.010 & $\cdots$ \\
\hline \multicolumn{12}{|l|}{ AUG } \\
\hline $21 \ldots$ & 1130 & 675 & 653 & 8.1 & 23.0 & 6.8 & 447 & 0.900 & $\cdots$ & 0.060 & $\cdots$ \\
\hline \multicolumn{12}{|l|}{ OCT } \\
\hline $23 \ldots$ & 0950 & 675 & 883 & 8.6 & 11.0 & 8.8 & 590 & 1.60 & $\cdots$ & 0.010 & $\cdots$ \\
\hline \multicolumn{12}{|l|}{ DEC } \\
\hline $18 \ldots$ & 0825 & 93 & 1290 & 8.3 & 0.0 & 11.8 & 926 & 3.30 & $\cdots$ & 0.100 & $\cdots$ \\
\hline \multicolumn{12}{|l|}{ MAR 1991} \\
\hline $28 \ldots$ & 1445 & 345 & 897 & 8.5 & 13.5 & 8.8 & 606 & 5.60 & $\cdots$ & 0.010 & $\cdots$ \\
\hline \multicolumn{12}{|l|}{ APR } \\
\hline $16 \ldots$ & 0945 & 250 & 1000 & 8.3 & 10.0 & 9.4 & 660 & 1.90 & $-\cdot$ & $<0.010$ & $\cdots$ \\
\hline \multicolumn{12}{|l|}{ MAY } \\
\hline $21 \ldots$ & 0910 & 516 & 797 & 8.4 & 16.0 & 7.6 & 541 & 1.20 & $\cdots$ & 0.010 & $\cdots$ \\
\hline \multicolumn{12}{|l|}{ JUN } \\
\hline $11 \ldots$ & 1000 & 1820 & 629 & 8.3 & 17.5 & 7.8 & 383 & 0.750 & $\cdots$ & 0.020 & $\cdots$ \\
\hline \multicolumn{12}{|l|}{ JUL } \\
\hline $23 \ldots$ & 1050 & 1380 & 536 & 8.3 & 21.0 & 6.9 & 348 & 0.790 & $\cdots$ & $<0.010$ & $\cdots$ \\
\hline \multicolumn{12}{|l|}{ AUG } \\
\hline $16 \ldots$ & 0820 & 1170 & 645 & 8.2 & 22.0 & 6.8 & 415 & 1.10 & $\cdots$ & 0.030 & $\cdots$ \\
\hline \multicolumn{12}{|l|}{ OCT } \\
\hline $29 \ldots$ & 0915 & 156 & 1170 & 8.3 & 4.0 & 10.8 & 786 & 2.40 & - & 0.020 & $\cdots$ \\
\hline \multicolumn{12}{|l|}{ DEC } \\
\hline $17 \ldots$ & 1130 & 172 & 1410 & 8.4 & 2.5 & 11.2 & 1080 & 3.40 & $\cdots$ & 0.040 & $\cdots$ \\
\hline \multicolumn{12}{|l|}{ MAR 1992} \\
\hline $26 \ldots$ & 1305 & 505 & 902 & 8.4 & 13.5 & 8.8 & 621 & 2.30 & $\cdots$ & 0.010 & $\cdots$ \\
\hline \multicolumn{12}{|l|}{ APR } \\
\hline $29 \ldots$ & 0915 & 617 & 832 & 8.4 & 14.0 & 8.6 & 500 & 1.30 & $\cdots$ & 0.020 & $\cdots$ \\
\hline \multicolumn{12}{|l|}{ MAY } \\
\hline $18 \ldots$ & 1750 & 582 & 758 & 8.2 & 23.5 & 7.2 & 496 & 0.880 & $\cdots$ & 0.010 & $\cdots$ \\
\hline \multicolumn{12}{|l|}{ JUN } \\
\hline $17 \ldots$ & 0815 & 1530 & 650 & 8.5 & 16.0 & 8.2 & 384 & 0.730 & $\cdots$ & 0.020 & $\cdots$ \\
\hline JUL & & & & & & & & & & & \\
\hline $16 \ldots$ & 1700 & 971 & 554 & 8.3 & 23.0 & 6.8 & 638 & 0.670 & $\cdots$ & 0.060 & $\cdots$ \\
\hline AUG & & & & & & & & & & & \\
\hline $18 \ldots$ & 0845 & 624 & 691 & 8.4 & 20.0 & 7.2 & 466 & 1.10 & $\cdots$ & 0.020 & $\cdots$ \\
\hline OCT & & & & & & & & & & & \\
\hline $20 \ldots$ & 1555 & 280 & 984 & 8.5 & 17.0 & 8.8 & 717 & 2.10 & $\cdots$ & 0.030 & $\cdots$ \\
\hline JAN 1993 & & & & & & & & & & & \\
\hline $14 \ldots$ & 1510 & 479 & 1210 & 8.2 & 0.0 & 11.6 & 842 & $\cdots$ & 2.80 & $\cdots$ & 0.300 \\
\hline MAR & & & & & & & & & & & \\
\hline $25 \ldots$ & 1315 & 410 & 977 & 8.4 & 16.0 & 8.8 & 684 & $\cdots$ & 2.90 & - & 0.030 \\
\hline
\end{tabular}


Table 51. Onsite measurements and selected inorganic data for station 380715103564701 , Apishapa River at Highway 50, near Fowler



\begin{tabular}{|c|c|c|c|c|c|c|c|c|c|}
\hline \multicolumn{10}{|l|}{ APR 1990} \\
\hline $11 \ldots$ & 1630 & 7.0 & 2320 & 8.0 & 21.0 & 8.0 & 2110 & 2.70 & 0.030 \\
\hline \multicolumn{10}{|l|}{ JUN } \\
\hline $12 \ldots$ & 1715 & 26 & 1380 & 8.0 & 25.5 & 6.1 & 1040 & 1.10 & 0.080 \\
\hline \multicolumn{10}{|l|}{ JUL } \\
\hline $25 \ldots$ & 1130 & 55 & 1200 & 7.9 & 19.0 & 6.7 & 942 & 1.00 & 0.030 \\
\hline \multicolumn{10}{|l|}{ AUG } \\
\hline $21 \ldots$ & 1300 & 31 & 1640 & 7.8 & 24.5 & 6.5 & 1340 & 1.70 & 0.090 \\
\hline \multicolumn{10}{|l|}{ OCT } \\
\hline $23 \ldots$ & 1225 & 27 & 2100 & 8.4 & 12.5 & 9.7 & 1750 & 2.60 & 0.020 \\
\hline \multicolumn{10}{|l|}{ APR 1991} \\
\hline $16 \ldots$ & 1200 & 12 & 2430 & 8.2 & 13.0 & 12.2 & 1980 & 2.50 & 0.020 \\
\hline \multicolumn{10}{|l|}{ JUN } \\
\hline $11 \ldots$ & 1145 & 31 & 1150 & 7.8 & 23.5 & 5.9 & 789 & 1.50 & 0.190 \\
\hline \multicolumn{10}{|l|}{ JUL } \\
\hline $02 \ldots$ & 1645 & E800 & 1020 & $\cdots$ & $\cdots$ & $\cdots$ & $\cdots$ & $\cdots$ & $\cdots$ \\
\hline $23 \ldots$ & 1345 & 17 & 1590 & 8.2 & 23.0 & 8.4 & 1280 & 1.60 & $<0.010$ \\
\hline \multicolumn{10}{|l|}{ OCT } \\
\hline $29 \ldots$ & 1040 & 16 & 2230 & 8.1 & 6.0 & $\cdots$ & 1780 & 3.10 & 0.060 \\
\hline \multicolumn{10}{|l|}{ APR 1992} \\
\hline $29 \ldots$ & 1110 & 30 & 1490 & 8.2 & 16.5 & 9.2 & 1110 & 2.10 & 0.040 \\
\hline \multicolumn{10}{|l|}{ JUN } \\
\hline $17 \ldots$ & 1200 & 25 & 1450 & 8.1 & 20.0 & 7.8 & 1110 & 1.60 & 0.040 \\
\hline \multicolumn{10}{|l|}{ AUG } \\
\hline $18 \ldots$ & 1100 & E400 & 814 & 8.0 & 9.0 & 8.4 & 586 & $<0.050$ & 0.140 \\
\hline \multicolumn{10}{|l|}{ OCT } \\
\hline $21 \ldots$ & 0855 & 14 & 2460 & 8.0 & 9.5 & 9.2 & 2190 & 3.80 & 0.060 \\
\hline
\end{tabular}

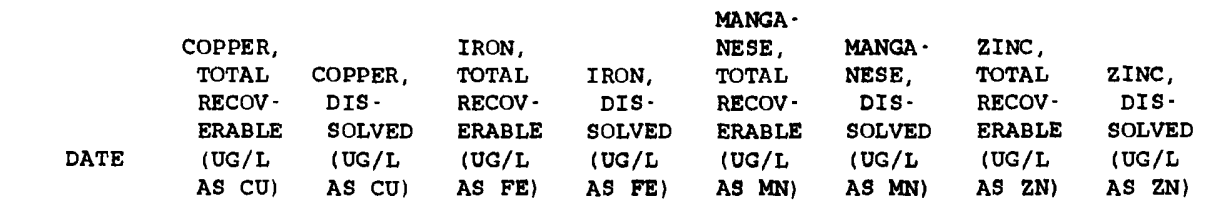

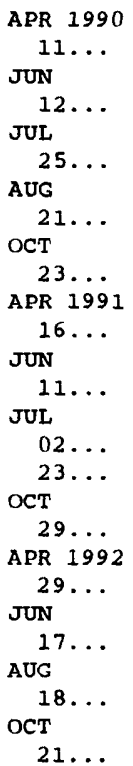


Table 52. Onsite measurements and bacteriological and selected inorganic data for station 07119700 , Arkansas River at Catlin Dam, near Fowler

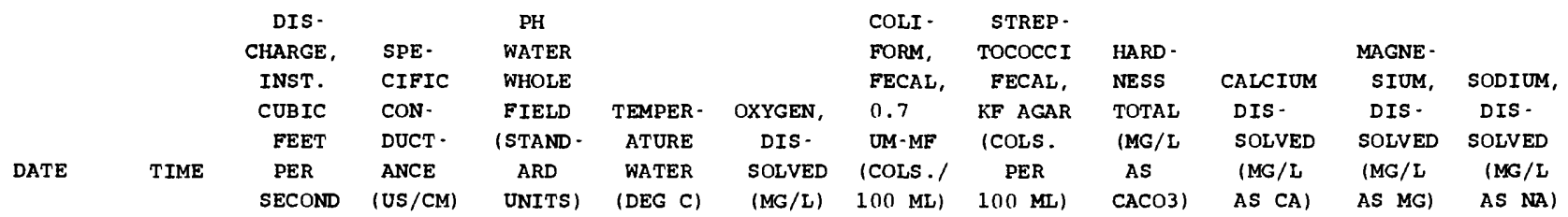

\begin{tabular}{|c|c|c|c|c|c|c|c|c|c|c|c|c|}
\hline $\begin{array}{c}\text { APR } 1990 \\
12 \ldots \\
\operatorname{MAY}\end{array}$ & 0845 & 112 & 1120 & 8.5 & 8.5 & 9.8 & 93 & 190 & 440 & 110 & 40 & 80 \\
\hline $15 \ldots$ & 1010 & 241 & 1050 & 8.3 & 15.5 & 8.0 & 94 & 210 & 400 & 100 & 37 & 73 \\
\hline JUN & & & & & & & & & & & & \\
\hline${ }_{\text {JUL }}^{13 \ldots}$ & 0920 & 2920 & 673 & 8.3 & 18,0 & 8.0 & 180 & K1100 & 280 & 73 & 24 & 39 \\
\hline$\underset{A U G}{25 \ldots}$ & 1320 & 83 & 927 & 7.8 & 23.0 & 6.4 & 1700 & 2000 & 370 & 100 & 28 & 52 \\
\hline$\underset{\text { OCT }}{21 \ldots}$ & 1600 & 445 & 721 & 8.1 & 26.0 & 6.7 & 690 & 1700 & 280 & 77 & 22 & 42 \\
\hline$\underset{D E C}{23}$ & 1315 & 308 & 1080 & 8.4 & 13.0 & 9.0 & 88 & 540 & 420 & 110 & 35 & 67 \\
\hline $\begin{array}{c}18 \ldots \\
M A R \quad 1991\end{array}$ & 0945 & 258 & 1510 & $\cdots$ & 0.0 & 11.8 & 30 & 200 & 660 & 170 & 57 & 100 \\
\hline$\underset{A P R}{28 \ldots}$ & 1630 & 112 & 1020 & 8.1 & 13.5 & 8.8 & E6 & 210 & 400 & 100 & 36 & 68 \\
\hline$\underset{\text { MAY }}{16 \ldots}$ & 1340 & 109 & 1210 & 8.4 & 13.0 & 9.7 & E8 & 110 & 470 & 120 & 41 & 73 \\
\hline $\operatorname{JUN}^{21 \ldots}$ & 1110 & 222 & 902 & 8.5 & 18.0 & 7.5 & 93 & 280 & 350 & 90 & 30 & 53 \\
\hline${ }_{J U L} 11 \ldots$ & 1430 & 1850 & 676 & 8.2 & 23.0 & 6.6 & 670 & 1600 & 250 & 67 & 19 & 37 \\
\hline $02 \ldots$ & 1835 & 1750 & 646 & - - & - & $\cdots$ & - & $\ldots$ & .. & - & -. & - \\
\hline $23 \ldots$ & 1600 & 948 & 594 & 8.3 & 23.0 & 8.0 & 680 & 1500 & 230 & 66 & 17 & 32 \\
\hline AUG & & & & & & & & & & & & \\
\hline$\underset{\text { SEP }}{16 \ldots}$ & 1130 & 1480 & 636 & 8.2 & 23.0 & 6.9 & $\cdots$ & $>3300$ & 250 & 68 & 19 & 32 \\
\hline $\begin{array}{c}04 \ldots \\
\text { Ост }\end{array}$ & 1445 & 1420 & 719 & 8.1 & 21.0 & 7.4 & $\cdots$ & $\cdots$ & . & $\cdots$ & .. & - \\
\hline$\underset{D E C}{29 \ldots}$ & 1200 & 77 & 1320 & 8.4 & 5.5 & 12.0 & 96 & 84 & 530 & 140 & 45 & 80 \\
\hline $\begin{array}{c}17 \ldots \\
\operatorname{MAR} 1992\end{array}$ & 1335 & 161 & 1500 & 8.4 & 3.0 & 11.4 & 57 & 160 & 600 & 150 & 55 & 100 \\
\hline $26 \ldots$ & 1505 & 291 & 963 & 8.5 & 14.5 & 8.6 & $\cdots$ & - & 370 & 96 & 32 & 63 \\
\hline APR & & & & & & & & & & & & \\
\hline $17 \ldots$ & 1515 & 690 & 868 & 8.3 & 19.0 & 7.9 & $\cdots$ & $\cdots$ & $\cdots$ & - & $\cdots$ & - \\
\hline $29 \ldots$ & 1220 & 300 & 914 & 8.4 & 18.5 & 7.9 & 54 & 130 & 350 & 89 & 30 & 54 \\
\hline MAY & & & & & & & & & & & & \\
\hline$\underset{\text { JUN }}{19 \ldots}$ & 0730 & 314 & 842 & 8.5 & 18.0 & 7.4 & 59 & 92 & 340 & 88 & 28 & 48 \\
\hline$\sigma_{J L} 17 \ldots$ & 1030 & 1150 & 648 & 8.4 & 17.0 & 8.2 & 96 & 190 & 250 & 66 & 20 & 32 \\
\hline${ }_{A U C}^{17} \cdots$ & 0740 & 581 & 634 & 8.2 & 19.5 & 7.5 & 490 & 870 & 250 & 68 & 20 & 31 \\
\hline $\begin{array}{l}18 \ldots \\
\text { OCT }\end{array}$ & 1230 & 613 & 781 & 8.1 & 14.5 & 8.2 & 5900 & 10000 & 320 & 94 & 21 & 38 \\
\hline $\begin{array}{c}21 \ldots \\
\text { JAN } 1993\end{array}$ & 1010 & 128 & 1140 & 8.4 & 11.0 & 9.2 & 47 & 180 & 460 & 120 & 39 & 75 \\
\hline${ }_{\mathrm{MAR}}^{14} \ldots$ & 1605 & 339 & 1360 & 8.2 & 0.5 & 11.4 & E8 & 39 & 530 & 130 & 49 & 93 \\
\hline $25 \ldots$ & 1430 & 269 & 1040 & 8.4 & 16.0 & 8.9 & E18 & 86 & 400 & 100 & 37 & 72 \\
\hline
\end{tabular}


Table 52. Onsite measurements and bacteriological and selected inorganic data for station 07119700 , Arkansas River at Catlin Dam, near Fowler--Continued

\begin{tabular}{|c|c|c|c|c|c|c|c|c|c|c|c|}
\hline DATE & $\begin{array}{c}\text { ALKA- } \\
\text { LINITY } \\
\text { LAB } \\
\text { (MG/L } \\
\text { AS }\end{array}$ & $\begin{array}{l}\text { SULFATE } \\
\text { DIS - } \\
\text { SOLVED } \\
\text { (MG/L }\end{array}$ & $\begin{array}{l}\text { CHLO- } \\
\text { RIDE, } \\
\text { DIS - } \\
\text { SOLVED } \\
\text { (MG / L }\end{array}$ & $\begin{array}{c}\text { SOLIDS, } \\
\text { RESIDUE } \\
\text { AT } 180 \\
\text { DEG C } \\
\text { DIS - } \\
\text { SOLVED }\end{array}$ & $\begin{array}{r}\text { NITRO- } \\
\text { GEN, } \\
\text { NO2+NO3 } \\
\text { TOTAL } \\
\text { (MG / L }\end{array}$ & $\begin{array}{l}\text { NITRO- } \\
\text { GEN, } \\
\text { NO2+NO3 } \\
\text { DIS - } \\
\text { SOLVED } \\
\text { (MG / L }\end{array}$ & $\begin{array}{l}\text { NITRO- } \\
\text { GEN, } \\
\text { AMMONIA } \\
\text { TOTAL } \\
\text { (MG/L }\end{array}$ & $\begin{array}{l}\text { NITRO- } \\
\text { GEN, } \\
\text { AMMONIA } \\
\text { DIS - } \\
\text { SOLVED } \\
\text { (MG/L }\end{array}$ & $\begin{array}{c}\text { PHOS - } \\
\text { PHORUS } \\
\text { TOTAL } \\
\text { (MG/L }\end{array}$ & $\begin{array}{r}\text { ARSENIC } \\
\text { TOTAL } \\
\text { (UG/L }\end{array}$ & $\begin{array}{l}\text { ARSENIC } \\
\text { DIS - } \\
\text { SOLVED } \\
\text { (UG/L }\end{array}$ \\
\hline & (АСС $)$ & AS SO4) & AS CL) & $(M G / L)$ & AS N) & AS N) & AS N) & AS N) & AS P) & AS AS) & AS AS) \\
\hline \multicolumn{12}{|l|}{ APR 1990} \\
\hline $12 \ldots$ & 166 & 360 & 29 & 833 & 2.70 & $\cdots$ & 0.040 & $\cdots$ & 0.480 & $\cdots$ & $\cdots$ \\
\hline \multicolumn{12}{|l|}{ MAY } \\
\hline $\operatorname{Jun}^{15 \ldots}$ & 159 & 400 & 26 & 768 & 2.00 & \multicolumn{4}{|c|}{ JUN } & & \\
\hline${ }_{\text {JUL }} 13 \ldots$ & 130 & 230 & 10 & 430 & 0.600 & \multicolumn{4}{|c|}{ JUL } & & \\
\hline${ }_{\text {AUG }}^{25 \ldots}$ & 120 & 320 & 14 & 693 & 1.30 & $\cdots$ & 0.090 & $\cdot$ & 0.050 & $\cdots$ & $\cdots$ \\
\hline$\underset{\mathrm{OCT}}{21 \ldots}$ & 119 & 230 & 13 & 494 & 0.800 & $\cdots$ & 0.080 & $\cdots$ & 0.140 & 4 & 2 \\
\hline${ }_{\mathrm{DEC}}^{23} 2$. & 164 & 380 & 23 & 732 & 1.70 & $\cdots$ & 0.020 & $\cdots$ & 0.210 & $\cdots$ & $\cdots$ \\
\hline $\begin{array}{c}18 \ldots \\
\text { MAR } 1991\end{array}$ & 217 & 560 & 34 & 1180 & 2.90 & - & 0.080 & $\cdots$ & 0.130 & $\cdots$ & $\cdot-$ \\
\hline $28 \ldots$ & 165 & 390 & 23 & 728 & 1.80 & $\cdots$ & 0.020 & $\cdots$ & 0.250 & $\cdots$ & $\cdots$ \\
\hline \multicolumn{12}{|l|}{ APR } \\
\hline $16 \ldots$ & 176 & 440 & 28 & 810 & 1.80 & $\cdots$ & 0.030 & $\cdots$ & 0.230 & $\cdots$ & $\cdots$ \\
\hline \multicolumn{12}{|l|}{ MAY } \\
\hline \multicolumn{12}{|l|}{ JUN } \\
\hline $11 \ldots$ & 122 & 210 & 11 & 426 & 0.780 & $\cdots$ & 0.050 & $\cdots$ & 0.170 & 3 & 1 \\
\hline \multicolumn{12}{|l|}{ JUL } \\
\hline $02 \ldots$ & $\cdots$ & $\cdots$ & $\cdots$ & $\cdots$ & $\cdots$ & $\cdots$ & $\cdots$ & $\cdots$ & $\cdots$ & $\cdots$ & $\cdots$ \\
\hline $23 \ldots$ & 106 & 190 & 10 & 371 & 0.710 & $\cdots$ & $<0.010$ & $\cdots$ & 0.450 & $\cdots$ & $\cdots$ \\
\hline \multicolumn{12}{|l|}{ AUG } \\
\hline $16 \ldots$ & 108 & 200 & 12 & 413 & 0.890 & $\cdots$ & 0.020 & $\cdots$ & 0.570 & $\cdots$ & $\cdots$ \\
\hline \multicolumn{12}{|l|}{ SEP } \\
\hline${ }_{\text {OCT }}^{04} \ldots$ & $\cdots$ & $\cdots$ & $\cdots$ & $\cdots$ & $\cdots$ & \multicolumn{3}{|c|}{ OCT } & $\cdots$ & 4 & 1 \\
\hline $29 \ldots$ & 192 & 440 & 28 & 874 & 2.10 & $\cdots$ & 0.030 & - & 0.210 & $\cdots$ & $\cdots$ \\
\hline \multicolumn{12}{|l|}{ DEC } \\
\hline $17 \ldots$ & 207 & 600 & 37 & 1160 & 3.00 & $\cdots$ & 0.060 & $\cdots$ & 0.350 & $\cdots$ & $\cdots$ \\
\hline \multicolumn{12}{|l|}{ MAR 1992} \\
\hline $26 \ldots$ & 151 & 320 & 26 & 690 & 2.20 & $\cdots$ & 0.020 & $\cdots$ & 0.490 & $\cdots$ & $\cdots$ \\
\hline \multicolumn{12}{|l|}{ APR } \\
\hline $17 \ldots$ & $\cdots$ & $\cdots$ & $\cdots$ & $\cdots$ & $\cdots$ & $\cdots$ & $\cdots$ & $\cdots$ & $\cdots$ & $\cdots$ & $\cdots$ \\
\hline $29 \ldots$ & 150 & 290 & 21 & 572 & 1.60 & $\cdots$ & 0.030 & $\cdots$ & 0.240 & $\cdots$ & $\cdots$ \\
\hline \multicolumn{12}{|l|}{ MAY } \\
\hline $19 \ldots$ & 144 & 250 & 19 & 550 & 0.920 & $\cdots$ & 0.020 & $\cdots$ & 0.150 & $\cdots$ & $\cdots$ \\
\hline \multicolumn{12}{|l|}{ JUN } \\
\hline $17 \ldots$ & 126 & 190 & 17 & 416 & 0.650 & $\cdots$ & 0.030 & $\cdots$ & 0.380 & 3 & 1 \\
\hline JUL & & & & & & & & & & & \\
\hline $17 \ldots$ & 118 & 180 & 13 & 400 & 0.760 & $\cdots$ & 0.020 & $\cdots$ & 0.660 & $\cdots$ & $\cdots$ \\
\hline AUG & & & & & & & & & & & \\
\hline $18 \ldots$ & 115 & 290 & 12 & 544 & 0.930 & $\cdots$ & 0.080 & $\cdots$ & 0.970 & 4 & 1 \\
\hline OCT & & & & & & & & & & & \\
\hline $21 \ldots$ & 171 & 410 & 25 & 858 & 1.90 & $\cdots$ & 0.020 & $\cdots$ & 0.180 & $\cdots$ & $\cdots$ \\
\hline JAN 1993 & & & & & & & & & & & \\
\hline $14 \ldots$ & 199 & 510 & 30 & 972 & $\cdots$ & 2.80 & $\cdots$ & 0.190 & 0.250 & $\cdots$ & $\cdots$ \\
\hline MAR & & & & & & & & & & & \\
\hline $25 \ldots$ & 165 & 340 & 27 & 754 & $\cdots$ & 2.70 & $\cdots$ & 0.030 & 0.370 & $\cdots$ & $\cdots$ \\
\hline
\end{tabular}


Table 52. Onsite measurements and bacteriological and selected inorganic data for station 07119700 , Arkansas River at Catlin Dam, near Fowler--Continued

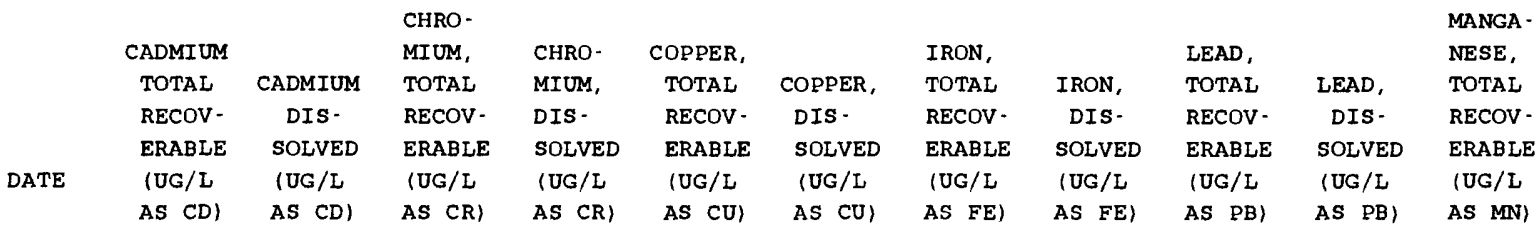

APR 1990

12.
MAY
15.

JUN 15.

$13 . .$.

JUL

$25 \ldots$

AUG

$21 \ldots$

OCT

$23 .$.

DEC

$18 \ldots$

MAR 1991

28 ...

APR

$16 \ldots$

MAY

21 ...

JUN

11 ...

AS CD

AS CU)

AS CU) AS FE)

AS FE)

$\begin{array}{llll}\cdots & 6 & 1 & 3900\end{array}$

6

$\begin{array}{lll}\cdots & \cdots & 30\end{array}$

JUL

$02 .$.

$23 .$.

AUG

$16 . .$.

SEP

$04 .$.

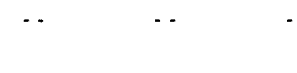

OCT

$29 .$.

DEC

$17 \ldots$

MAR 1992

$26 \ldots$
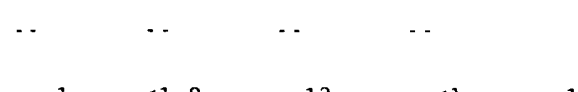

$6 \quad 1 \quad 7800$

3

300

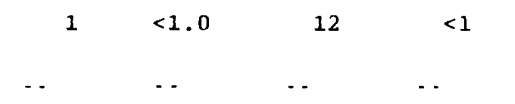

19

215000

42

140000

$4 \quad 26$

300

APR

$17 . .$.

$29 .$.

MAY

$19 .$.

JUN

$17 \ldots$

TUL

17 ..

$18 .$.

OCT

21...

JAN 1993

$14 \ldots$

MAR

$25 .$.

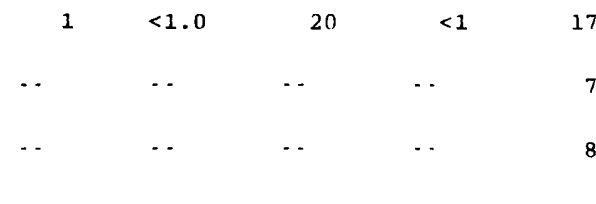

$2 \quad 23000$

47

1000

17

$4 \quad 21$

$<1 \quad 500$

\begin{tabular}{l}
$7 \quad 2 \quad 4300$ \\
\hline
\end{tabular}

4

200

$1 \quad 1000$

$<3$

60

$\begin{array}{lllll} & & & & \\ . & \ldots & \ldots & \ldots & 4\end{array}$

12100

8

80

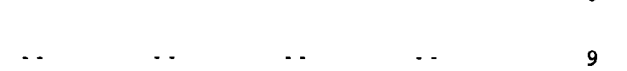

23100

$<3 \quad-$.

180

23500

6

150

$\begin{array}{llll}1<1.0 & 13 & <1 & 21\end{array}$

218000

$7 \quad 31$

$\begin{array}{rr}<1 & 490 \\ \cdots & 1900\end{array}$

$\begin{array}{llll}- & - & \ldots & - \\ . & - & \ldots & -\end{array}$

$88 \quad \ldots \quad 91000$

- $\quad$ -

34

$2 \quad \cdots$

7

40000

8

780

$5<1.0$

$<1 \quad$.

- $\quad$ -

.. 280

980

4

280

70

$\begin{array}{lll}- & 1 & 1900\end{array}$

7

90

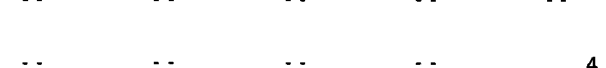

13900

$<3$

120

$\begin{array}{lllllll}\ldots & \ldots & \ldots & \ldots & \ldots & \ldots & \ldots\end{array}$

2400

-

$5 \quad 1 \quad 2300$

$<3$

.

130

5

6

110

$\begin{array}{lll}8 & 1 & 3400\end{array}$

- 14

29800

10

220

$5 \quad<1.0 \quad 58$

$<1 \quad 130$

$1 \quad 84000$

$9 \quad 120$

270

$\begin{array}{lll}3 & 2 & 480\end{array}$

$<3$

50

2

$<1 \quad 1200$

$<10$

80

2

1

1300

6

60 
Table 52. Onsite measurements and bacteriological and selected inorganic data for station 07119700 , Arkansas River at Catlin Dam, near Fowler--Continued

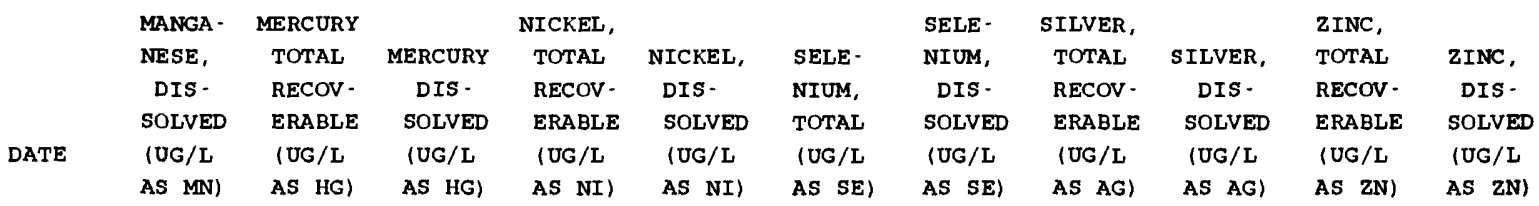

\begin{tabular}{|c|c|c|c|c|c|c|c|c|c|c|c|}
\hline \multicolumn{12}{|l|}{ APR 1990} \\
\hline $12 \ldots$ & 8 & $\cdots$ & $\cdots$ & $\cdots$ & $\cdots$ & 12 & 12 & $\cdots$ & . & $\cdots$ & 5 \\
\hline \multicolumn{12}{|l|}{ MAY } \\
\hline $15 \ldots$ & 5 & $\cdots$ & $\cdots$ & $\cdots$ & $\cdots$ & 12 & 14 & $\cdots$ & .. & 60 & 6 \\
\hline \multicolumn{12}{|l|}{ JUN } \\
\hline $13 \ldots$ & 7 & $<0.10$ & $<0.1$ & 16 & 1 & 9 & 6 & $<1$ & 1.0 & 110 & $<3$ \\
\hline \multicolumn{12}{|l|}{ JUL } \\
\hline $25 \ldots$ & 45 & $\cdots$ & - & $\cdots$ & $\cdots$ & 12 & 10 & $\cdots$ & - & 230 & 5 \\
\hline \multicolumn{12}{|l|}{ AUG } \\
\hline $21 \ldots$ & 5 & $<0.10$ & $<0.1$ & 21 & 2 & 21 & 8 & $<1$ & $<1.0$ & 130 & $<3$ \\
\hline \multicolumn{12}{|l|}{ OCT } \\
\hline $23 \ldots$ & 6 & $\cdots$ & $\cdots$ & $\cdots$ & - & 10 & 11 & - & .. & 40 & 4 \\
\hline \multicolumn{12}{|l|}{ DEC } \\
\hline $18 \ldots$ & 25 & $\cdots$ & - & $\cdots$ & - & 20 & 15 & - & -. & 20 & 6 \\
\hline \multicolumn{12}{|l|}{ MAR 1991} \\
\hline $28 \ldots$ & 9 & $\cdots$ & -. & $\cdots$ & - & 11 & 11 & - & -. & 30 & 6 \\
\hline \multicolumn{12}{|l|}{ APR } \\
\hline $16 \ldots$ & 23 & $\cdots$ & - & $\cdots$ & - & 15 & 13 & - & -. & 30 & $<3$ \\
\hline \multicolumn{12}{|l|}{ MAY } \\
\hline $21 \ldots$ & 9 & $\cdots$ & $\cdots$ & $\cdots$ & $\cdots$ & 10 & 10 & - & -. & 30 & 6 \\
\hline \multicolumn{12}{|l|}{ JUN } \\
\hline $11 \ldots$ & 7 & $<0.10$ & $<0.1$ & 25 & 7 & 4 & 7 & $<1$ & $<1.0$ & 110 & $<3$ \\
\hline \multicolumn{12}{|l|}{ JUL } \\
\hline $02 \ldots$ & $\cdots$ & $\cdots$ & -. & $\cdots$ & - & . & -. & $\cdots$ & . & 390 & $\cdots$ \\
\hline $23 \ldots$ & 2 & . & . & - & $\cdots$ & 6 & 5 & $\cdots$ & $\cdots$ & 150 & 4 \\
\hline \multicolumn{12}{|l|}{ AUG } \\
\hline $16 \ldots$ & $<1$ & $\cdots$ & $\cdots$ & $\cdots$ & $\cdots$ & 9 & 6 & -- & $\cdots$ & 200 & $<3$ \\
\hline \multicolumn{12}{|l|}{ SEP } \\
\hline $04 \ldots$ & $\cdots$ & 0.50 & $<0.1$ & 260 & 2 & $\cdots$ & $\cdots$ & 2 & $<1.0$ & $\cdots$ & $\cdots$ \\
\hline \multicolumn{12}{|l|}{ OCT } \\
\hline $29 \ldots$ & 16 & - & - & $\cdots$ & $\cdots$ & 19 & 15 & - & $\cdots$ & 10 & 3 \\
\hline \multicolumn{12}{|l|}{ DEC } \\
\hline $17 \ldots$ & 11 & - & . & $\cdots$ & $\cdots$ & 25 & 23 & $\cdots$ & - & 40 & $<3$ \\
\hline \multicolumn{12}{|l|}{ MAR 1992} \\
\hline $26 \ldots$ & 5 & -. & . & $\cdots$ & $\cdots$ & 11 & 11 & $\cdots$ & - & 20 & 7 \\
\hline APR & & & & & & & & & & & \\
\hline $17 \ldots$ & .. & $\cdots$ & . & $\cdots$ & - & - & - & - & . & -. & - \\
\hline $29 \ldots$ & 4 & . & . & $\cdots$ & . & 9 & 11 & $\cdots$ & - & 20 & $<3$ \\
\hline MAY & & & & & & & & & & & \\
\hline $19 \ldots$ & 3 & $\cdots$ & - & - & . & 10 & 8 & $\cdots$ & -. & 20 & $<3$ \\
\hline JUN & & & & & & & & & & & \\
\hline $17 \ldots$ & 3 & $<0.10$ & $<0.1$ & 7 & 1 & 6 & 6 & $<1$ & $<1.0$ & 50 & $<3$ \\
\hline JUL & & & & & & & & & & & \\
\hline $17 \ldots$ & $<1$ & $\cdots$ & . & $\cdots$ & - & 7 & 4 & $\cdots$ & . & 60 & $<3$ \\
\hline AUG & & & & & & & & & & & \\
\hline $18 \ldots$ & 27 & $<0.10$ & $<0.1$ & 160 & 1 & 20 & 8 & $<1$ & $<1.0$ & 430 & $<3$ \\
\hline OCT & & & & & & & & & & & \\
\hline $21 \ldots$ & 9 & - & - & $\cdots$ & - & 16 & 14 & $\cdots$ & $\cdots$ & 10 & 4 \\
\hline JAN 1993 & & & & & & & & & & & \\
\hline $14 \ldots$ & 20 & $\cdots$ & - & $\cdots$ & . & 15 & 16 & $\cdots$ & - & $<10$ & $<10$ \\
\hline MAR & & & & & & & & & & & \\
\hline $25 \ldots$ & 3 & . & -. & $\cdots$ & - & 13 & 11 & $\cdots$ & $\ldots$ & $<10$ & 4 \\
\hline
\end{tabular}


Table 53. Onsite measurements and selected inorganic data for station 380111103382101 , Timpas Creek at Highway 50, at Swink

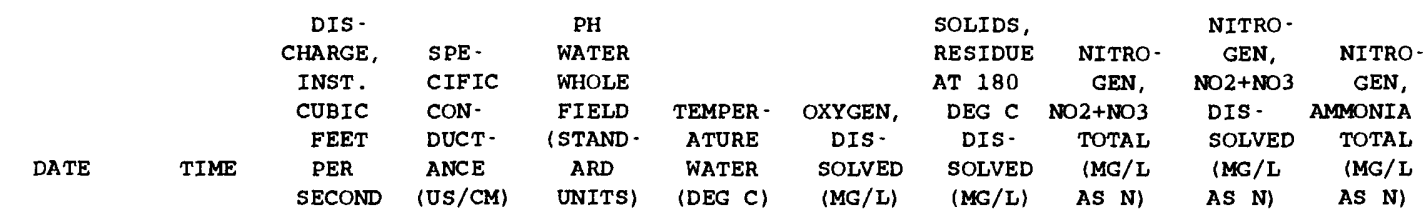

\begin{tabular}{|c|c|c|c|c|c|c|c|c|c|c|}
\hline $\begin{array}{c}\text { APR } 1990 \\
12 \ldots\end{array}$ & 1115 & 54 & 1950 & 8.1 & 10.0 & 8.3 & 1650 & 0.500 & $\cdots$ & 0.400 \\
\hline MAY & & & & 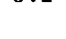 & & & & . & & 1.00 \\
\hline JUN $^{15 \ldots}$ & 1220 & 86 & 1710 & 7.8 & 19.0 & 7.0 & 1420 & 3.10 & $\cdots$ & 0.020 \\
\hline $\operatorname{sUL}^{13} \cdots$ & 1315 & $\cdots$ & 1620 & 8.0 & 21.0 & 7.1 & 1280 & 2.70 & $\cdots$ & 0.090 \\
\hline$\underset{A U G}{26 \ldots}$ & 0900 & 140 & 1460 & 7.9 & 20.0 & 6.9 & 1170 & 2.40 & $\cdots$ & 0.120 \\
\hline$\underset{\text { OCT }}{22} \cdots$ & 0830 & 102 & 1620 & 7.8 & 19.0 & 6.5 & 1300 & 2.60 & $\cdots$ & 0.080 \\
\hline $\begin{array}{l}23 \ldots \\
A P R \\
1991\end{array}$ & 1525 & 133 & 1560 & 7.9 & 12.5 & 8.7 & 964 & 2.80 & $\cdots$ & 0.060 \\
\hline $\begin{array}{l}16 \ldots \\
\text { JUN }\end{array}$ & 1540 & 50 & 2230 & 8.1 & 17.0 & 8.6 & 1800 & 3.90 & $\cdots$ & 0.020 \\
\hline${ }_{\text {JUL }}^{11} \cdots$ & 1745 & 65 & 1790 & 8.0 & 24.0 & 6.4 & 1380 & 3.20 & $\cdots$ & 0.050 \\
\hline${ }_{\mathrm{OCT}}^{23 \ldots}$ & 1905 & 70 & 1750 & 8.1 & 22.0 & 7.1 & 1430 & 2.90 & $\cdots$ & $<0.010$ \\
\hline${ }_{D E C}^{29} \cdots$ & 1330 & 97 & 1980 & 8.0 & 7.0 & 10.6 & 1540 & 3.40 & $\cdots$ & 0.030 \\
\hline $\begin{array}{c}17 \ldots \\
\text { APR } 1992\end{array}$ & 1530 & 19 & 3230 & 8.2 & 8.0 & 11.2 & 2790 & 6.30 & $\cdots$ & 0.030 \\
\hline${ }_{\text {JUN }}^{29} \cdots$ & 1435 & 65 & 1890 & 8.0 & 21.5 & 7.0 & 1480 & 3.00 & $\cdots$ & 0.350 \\
\hline${ }_{\text {JUL }}^{17 \ldots}$ & 1415 & 101 & 1410 & 8.0 & 21.5 & 7.1 & 1050 & 2.20 & $\cdots$ & 0.310 \\
\hline$\underset{A U G}{17} \cdots$ & 0900 & 181 & 1160 & 8.1 & 19.5 & 7.1 & 832 & 1.70 & $\cdots$ & 0.040 \\
\hline$\underset{\text { OCT }}{18 \ldots}$ & 1530 & E300 & 950 & 7.9 & 19.5 & 5.7 & 692 & 1.30 & $\cdots$ & 0.110 \\
\hline $\begin{array}{l}21 \ldots \\
\text { JAN } 1993\end{array}$ & 1200 & 66 & 2080 & 8.0 & 13.0 & 9.4 & 1800 & 3.70 & -- & 0.020 \\
\hline${ }_{\operatorname{MAR}}^{15} \ldots$ & 0820 & E30 & 3310 & 7.9 & 3.0 & 11.0 & 2890 & $\cdots$ & 6.60 & $\cdots$ \\
\hline $\begin{array}{r}M A R \\
26 \ldots\end{array}$ & 0710 & 124 & 1430 & 8.2 & 11.0 & 9.1 & 1040 & $\cdots$ & 2.80 & $\cdots$ \\
\hline
\end{tabular}

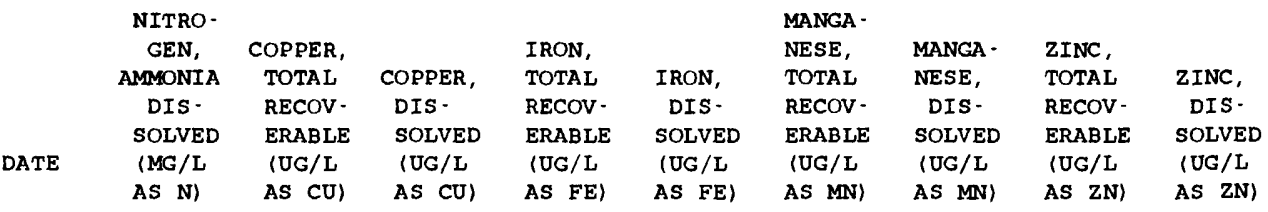

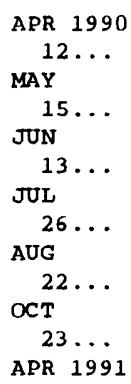

APR 1991

$16 \ldots$ 
Table 53. Onsite measurements and selected inorganic data for station 380111103382101 , Timpas Creek at Highway 50, at Swink--Continued

\begin{tabular}{|c|c|c|c|c|c|c|c|c|}
\hline NITRO- & & & & & MANGA - & & & \\
\hline GEN, & COPPER, & & IRON, & & NESE, & MANGA - & ZINC, & \\
\hline $\begin{array}{c}\text { AMIMUNIA } \\
\text { DIS - }\end{array}$ & $\begin{array}{l}\text { TOTAL } \\
\text { RECOV- }\end{array}$ & $\begin{array}{l}\text { COPPER, } \\
\text { DIS - }\end{array}$ & $\begin{array}{l}\text { TOTAL } \\
\text { RECOV - }\end{array}$ & $\begin{array}{l}\text { IRON, } \\
\text { DIS - }\end{array}$ & $\begin{array}{l}\text { TOTAL } \\
\text { RECOV- }\end{array}$ & $\begin{array}{r}\text { NESE, } \\
\text { DIS - }\end{array}$ & $\begin{array}{l}\text { TOTAL } \\
\text { RECOV - }\end{array}$ & $\begin{array}{l}\text { ZINC, } \\
\text { DIS - }\end{array}$ \\
\hline $\begin{array}{l}\text { SOLVED } \\
\text { (MG /L }\end{array}$ & $\begin{array}{c}\text { ERABLE } \\
\text { (UG/L }\end{array}$ & $\begin{array}{l}\text { SOLVED } \\
\text { (UG/L }\end{array}$ & $\begin{array}{l}\text { ERABLE } \\
\text { (UG/L }\end{array}$ & $\begin{array}{l}\text { SOLVED } \\
\text { (UG /L }\end{array}$ & $\begin{array}{l}\text { ERABLE } \\
\text { (UG/L }\end{array}$ & $\begin{array}{l}\text { SOLVED } \\
\text { (UG/L }\end{array}$ & $\begin{array}{l}\text { ERABLE } \\
\text { (UG/I }\end{array}$ & $\begin{array}{l}\text { SOLVED } \\
\text { (UG/L }\end{array}$ \\
\hline AS N) & AS CU) & AS CU) & AS FE) & AS FE) & AS $\mathrm{MN}\rangle$ & AS $\mathbf{M N}$ ) & AS $\mathrm{ZN}$ ) & AS ZN) \\
\hline
\end{tabular}

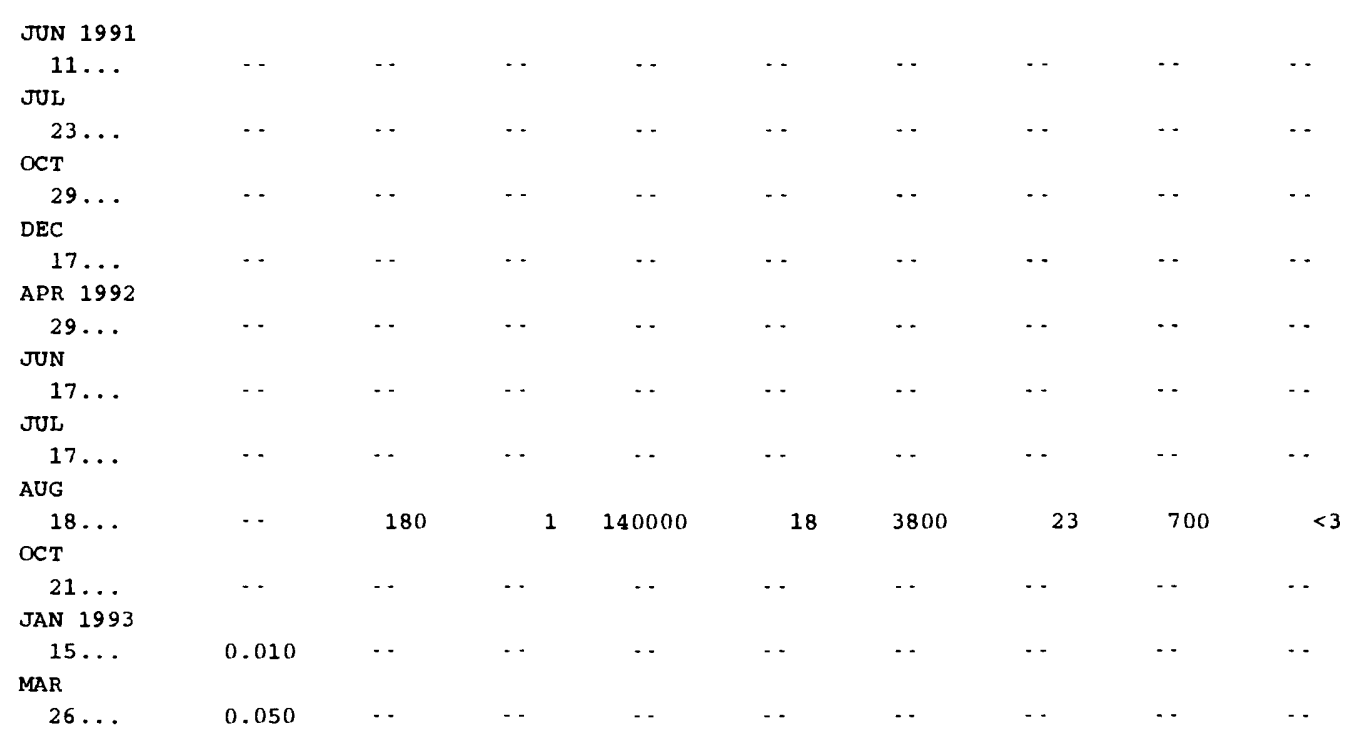


Table 54. Onsite measurements and selected inorganic data for station 07122500 , Crooked Arroyo near La Junta

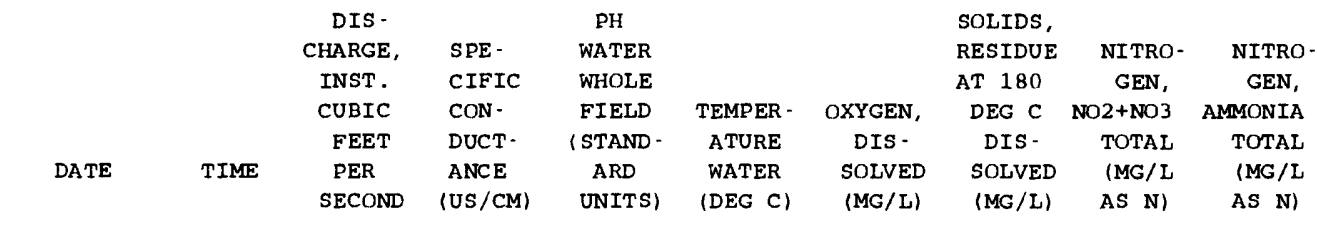

\begin{tabular}{|c|c|c|c|c|c|c|c|c|c|}
\hline \multicolumn{10}{|l|}{ APR 1990} \\
\hline $12 \ldots$ & 1340 & 12 & 1800 & 8.3 & 12.0 & 8.7 & 1500 & 2.40 & 0.040 \\
\hline \multicolumn{10}{|l|}{ JUN } \\
\hline $13 \ldots$ & 1425 & 13 & 1500 & 8.1 & 22.5 & 6.8 & 1140 & 2.50 & 0.100 \\
\hline \multicolumn{10}{|l|}{ JUL } \\
\hline $26 \ldots$ & 1045 & 19 & 1950 & 7.9 & 19.5 & 7.4 & 1660 & 2.40 & 0.050 \\
\hline \multicolumn{10}{|l|}{ AUG } \\
\hline $22 \ldots$ & 1030 & 15 & 2040 & 7.9 & 19.0 & 7.3 & 1730 & 2.60 & 0.100 \\
\hline \multicolumn{10}{|l|}{ OCT } \\
\hline $24 \ldots$ & 0820 & 11 & 2270 & 8.3 & 10.0 & 8.8 & 1770 & 3.80 & 0.040 \\
\hline \multicolumn{10}{|l|}{ APR 1991} \\
\hline $16 \ldots$ & 1715 & 7.5 & 2110 & 8.1 & 16.0 & 8.1 & 1650 & 2.50 & 0.070 \\
\hline \multicolumn{10}{|l|}{ JUN } \\
\hline $12 \ldots$ & 0820 & 14 & 1590 & 8.0 & 18.0 & 7.4 & 1170 & 1.90 & 0.060 \\
\hline \multicolumn{10}{|l|}{$\pi U L$} \\
\hline $24 \ldots$ & 0835 & 24 & 1530 & 8.0 & 17.5 & 7.6 & 1210 & 2.00 & 0.020 \\
\hline \multicolumn{10}{|l|}{ OCT } \\
\hline $29 \ldots$ & 1500 & 25 & 1940 & 8.2 & 8.5 & 10.8 & 1510 & 3.30 & 0.020 \\
\hline \multicolumn{10}{|l|}{ APR 1992} \\
\hline $29 \ldots$ & 1600 & 9.8 & 2240 & 8.1 & 22.0 & 6.7 & 1860 & 2.90 & 0.090 \\
\hline \multicolumn{10}{|l|}{ JUN } \\
\hline $17 \ldots$ & 1525 & 11 & 1920 & 8.0 & 22.0 & 6.6 & 1540 & 3.70 & 0.170 \\
\hline \multicolumn{10}{|l|}{ AUG } \\
\hline $18 \ldots$ & 1645 & 13 & 1730 & 8.0 & 21.5 & 7.1 & 1400 & 3.40 & 0.160 \\
\hline \multicolumn{10}{|l|}{ OCT } \\
\hline $21 \ldots$ & 1325 & 19 & 1730 & 8.2 & 14.0 & 8.8 & 1450 & 2.80 & 0.030 \\
\hline
\end{tabular}


Table 55. Onsite measurements and selected inorganic data for station 07123000, Arkansas River at La Junta

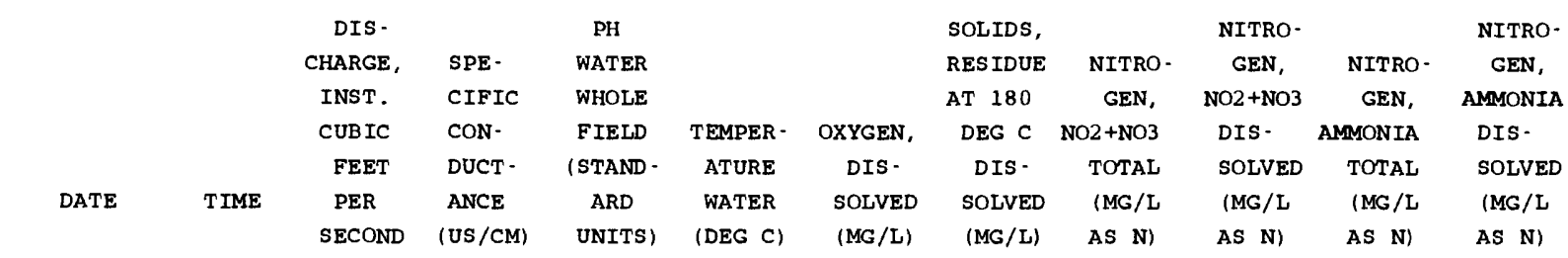

\begin{tabular}{|c|c|c|c|c|c|c|c|c|c|c|c|}
\hline \multicolumn{12}{|l|}{ APR 1990} \\
\hline $12 \ldots$ & 1510 & 38 & 2260 & 8.3 & 16.0 & 9.8 & 2000 & 3.70 & - & 0.030 & -. \\
\hline \multicolumn{12}{|l|}{ MAY } \\
\hline $15 \ldots$ & 1350 & 364 & 1530 & 8.0 & 21.0 & 7.2 & 1210 & 2.00 & $\cdots$ & 0.010 & $\cdots$ \\
\hline \multicolumn{12}{|l|}{ JUN } \\
\hline $13 \ldots$ & 1630 & 1310 & 848 & 8.3 & 22.5 & 6.9 & 590 & 0.900 & $\cdots$ & 0.020 & $\cdots$ \\
\hline \multicolumn{12}{|l|}{ JUL } \\
\hline $26 \ldots$ & 1215 & 244 & 1410 & 8.0 & 24.0 & 6.7 & 1080 & 2.00 & - & 0.040 & - \\
\hline \multicolumn{12}{|l|}{ AUG } \\
\hline $22 \ldots$ & 1200 & 331 & 1380 & 8.2 & 21.5 & 6.9 & 1050 & 1.90 & $\cdots$ & 0.100 & $\cdots$ \\
\hline \multicolumn{12}{|l|}{ OCT } \\
\hline $24 \ldots$ & 0930 & 474 & 1420 & 8.6 & 9.5 & 9.3 & 1030 & 2.30 & $\cdots$ & $<0.010$ & $\cdots$ \\
\hline \multicolumn{12}{|l|}{ DEC } \\
\hline $18 \ldots$ & 1110 & 113 & 2570 & 8.1 & 3.0 & 11.7 & 2140 & 4.50 & $\cdots$ & 0.070 & $\cdots$ \\
\hline \multicolumn{12}{|l|}{ MAR 1991} \\
\hline $29 \ldots$ & 0735 & 75 & 2150 & 8.1 & 5.0 & 10.9 & 1770 & 3.20 & $\cdots$ & 0.250 & $\cdots$ \\
\hline \multicolumn{12}{|l|}{ APR } \\
\hline $17 \ldots$ & 0810 & 40 & 2580 & 8.1 & 7.5 & 10.1 & 2110 & 4.60 & $\cdots$ & 0.050 & $-\cdot$ \\
\hline \multicolumn{12}{|l|}{ MAY } \\
\hline $21 \ldots$ & 1330 & 100 & 1970 & 8.3 & 23.5 & 7.2 & 1570 & 3.00 & $\cdots$ & 0.040 & $\cdots$ \\
\hline \multicolumn{12}{|l|}{ JUN } \\
\hline $12 \ldots$ & 0945 & 1020 & 978 & 8.1 & 21.5 & 6.8 & 672 & 1.30 & $\cdots$ & 0.040 & $\cdots$ \\
\hline \multicolumn{12}{|l|}{ JUL } \\
\hline $24 \ldots$ & 0940 & 511 & 999 & 8.2 & 19.5 & 7.2 & 712 & 1.40 & $\cdots$ & 0.160 & $\cdots$ \\
\hline \multicolumn{12}{|l|}{ AUG } \\
\hline $16 \ldots$ & 1420 & 410 & 944 & 8.2 & 26.0 & 6.5 & 645 & 1.40 & $\cdots$ & 0.030 & $\cdots$ \\
\hline \multicolumn{12}{|l|}{ OCT } \\
\hline $29 \ldots$ & 1610 & 123 & 1980 & 8.4 & 7.5 & 10.2 & 1530 & 3.30 & $\cdots$ & 0.020 & $\cdots$ \\
\hline \multicolumn{12}{|l|}{$\mathrm{DEC}$} \\
\hline $17 \ldots$ & 1705 & 84 & 2530 & 8.5 & 4.0 & 11.6 & 2010 & 4.40 & $\cdots$ & 0.120 & $\cdots$ \\
\hline \multicolumn{12}{|l|}{ MAR 1992} \\
\hline $26 \ldots$ & 1720 & 27 & 2360 & 8.4 & 14.5 & 8.7 & 2020 & 3.80 & $\cdots$ & 0.050 & $\cdots$ \\
\hline \multicolumn{12}{|l|}{ APR } \\
\hline $29 \ldots$ & 1730 & 348 & 1450 & 8.3 & 23.0 & 7.0 & 1080 & 2.00 & $\cdots$ & 0.070 & $\cdots$ \\
\hline \multicolumn{12}{|l|}{ MAY } \\
\hline $19 \ldots$ & 0930 & 260 & 1450 & 8.4 & 18.5 & 7.9 & 1050 & 2.20 & $\cdots$ & 0.020 & $\cdots$ \\
\hline \multicolumn{12}{|l|}{ JUN } \\
\hline $17 \ldots$ & 1630 & 492 & 925 & 8.3 & 22.5 & 7.2 & 622 & 1.30 & $\cdots$ & 0.060 & $\cdots$ \\
\hline JUL & & & & & & & & & & & \\
\hline $17 \ldots$ & 1050 & 324 & 1080 & 8.3 & 22.0 & 7.1 & 766 & 1.60 & $\cdots$ & 0.020 & $\cdots$ \\
\hline AUG & & & & & & & & & & & \\
\hline $18 \ldots$ & 1745 & 309 & 1510 & 8.3 & 24.0 & 6.7 & 1160 & 2.60 & - & 0.030 & $\cdots$ \\
\hline OCT & & & & & & & & & & & \\
\hline $21 \ldots$ & 1430 & 66 & 1890 & 8.3 & 17.5 & 8.7 & 1600 & 3.00 & - & 0.020 & $-\cdot$ \\
\hline JAN 1993 & & & & & & & & & & & \\
\hline $15 \ldots$ & 0905 & 145 & 2550 & 8.3 & 0.0 & 11.9 & 2120 & $\cdots$ & 4.80 & $\cdots$ & 0.050 \\
\hline MAR & & & & & & & & & & & \\
\hline $26 \ldots$ & 0825 & 25 & 2230 & 8.3 & 10.0 & 10.3 & 1810 & $\cdots$ & 3.90 & $\cdots$ & 0.040 \\
\hline
\end{tabular}


Table 56. Onsite measurements and selected inorganic data for station 380421103193101 , Horse Creek at mouth, near Las Animas

\begin{tabular}{|c|c|c|c|c|c|c|c|c|c|}
\hline DATE & TIME & $\begin{array}{c}\text { DIS - } \\
\text { CHARGE, } \\
\text { INST . } \\
\text { CUBIC } \\
\text { FEET } \\
\text { PER } \\
\text { SECOND }\end{array}$ & $\begin{array}{l}\text { SPE- } \\
\text { CIFIC } \\
\text { CON- } \\
\text { DUCT- } \\
\text { ANCE } \\
\text { (US/CM) }\end{array}$ & $\begin{array}{c}\text { PH } \\
\text { WATER } \\
\text { WHOLE } \\
\text { FIELD } \\
\text { (STAND- } \\
\text { ARD } \\
\text { UNITS) }\end{array}$ & $\begin{array}{l}\text { TEMPER - } \\
\text { ATURE } \\
\text { WATER } \\
\text { (DEG C) }\end{array}$ & $\begin{array}{c}\text { OXYGEN, } \\
\text { DIS - } \\
\text { SOLVED } \\
\text { (MG/L) }\end{array}$ & $\begin{array}{l}\text { SOLIDS, } \\
\text { RESIDUE } \\
\text { AT } 180 \\
\text { DEG C } \\
\text { DIS - } \\
\text { SOLVED } \\
\text { (MG/L) }\end{array}$ & $\begin{array}{c}\text { NITRO- } \\
\text { GEN, } \\
\text { NO2+NO3 } \\
\text { TOTAL } \\
\text { (MG/L } \\
\text { AS N) }\end{array}$ & $\begin{array}{l}\text { NITRO- } \\
\text { GEN, } \\
\text { AMMONIA } \\
\text { TOTAL } \\
\text { (MG/L } \\
\text { AS N) }\end{array}$ \\
\hline $\begin{array}{l}\mathrm{R} 1990 \\
12 \ldots \\
\mathrm{N}\end{array}$ & 1700 & 1.0 & 4360 & 8.1 & 14.5 & 8.9 & 4130 & 0.100 & 0.020 \\
\hline $13 \ldots$ & 1840 & 2.0 & 3920 & 8.1 & 25.0 & 7.7 & 3520 & $<0.100$ & 0.040 \\
\hline $26 \ldots$ & 1340 & 5.9 & 2540 & 8.0 & 25.5 & 7.9 & 2110 & 0.700 & 0.030 \\
\hline$\underset{\mathrm{T}}{22 \ldots}$ & 1330 & 0.88 & 2850 & 7.9 & 23.5 & 10.1 & 2530 & 0.300 & 0.080 \\
\hline $\begin{array}{c}24 \ldots \\
\text { PR } 1991\end{array}$ & 1110 & 0.48 & 4290 & 8.1 & 11.0 & 10.8 & 3610 & 0.400 & 0.020 \\
\hline $17 \ldots$ & 1000 & 0.83 & 4440 & 8.1 & 10.0 & 10.8 & 3790 & 0.150 & $<0.010$ \\
\hline $12 \ldots$ & 1125 & 0.16 & 4340 & 8.0 & 26.0 & 11.0 & 3780 & $<0.050$ & 0.040 \\
\hline $\begin{array}{c}05 \ldots \\
\text { CT }\end{array}$ & 1215 & 0.97 & 3400 & 8.0 & 20.0 & 8.3 & 2910 & 0.580 & 0.140 \\
\hline $\begin{array}{c}30 \ldots \\
\text { PR } 1992\end{array}$ & 0935 & 0.67 & 3880 & 8.1 & - - & $\cdots$ & 3390 & 0.640 & 0.040 \\
\hline$\underset{\text { UN }}{30 \ldots}$ & 0905 & 0.48 & 4040 & 7.7 & 14.5 & 5.7 & 3450 & 0.410 & 0.070 \\
\hline $17 \ldots$ & 1800 & 2.4 & 3270 & 8.2 & 23.0 & 8.1 & 2760 & 0.063 & 0.060 \\
\hline $19 \ldots$ & 0705 & 4.0 & 3620 & 7.9 & 18.0 & 5.2 & 3060 & 0.430 & 0.040 \\
\hline $21 \ldots$ & 1540 & 1.7 & 3620 & 8.2 & 13.0 & 9.9 & 3170 & 0.670 & 0.040 \\
\hline
\end{tabular}


Table 57. Onsite measurements and bacteriological and selected inorganic data for station 07124000 , Arkansas River at Las Animas

\begin{tabular}{|c|c|c|c|c|c|c|c|c|c|c|c|c|}
\hline DATE & TIME & $\begin{array}{c}\text { DIS- } \\
\text { CHARGE, } \\
\text { INST. } \\
\text { CUBIC } \\
\text { FEET } \\
\text { PER } \\
\text { SECOND }\end{array}$ & $\begin{array}{l}\text { SPE- } \\
\text { CIFIC } \\
\text { CON- } \\
\text { DUCT- } \\
\text { ANCE } \\
\text { (US/CM) }\end{array}$ & $\begin{array}{c}\text { PH } \\
\text { WATER } \\
\text { WHOLE } \\
\text { FIELD } \\
\text { (STAND - } \\
\text { ARD } \\
\text { UNITS) }\end{array}$ & $\begin{array}{c}\text { TEMPER- } \\
\text { ATURE } \\
\text { WATER } \\
\text { (DEG C) }\end{array}$ & $\begin{array}{c}\text { OXYGEN, } \\
\text { DIS - } \\
\text { SOLVED } \\
\text { (MG/L) }\end{array}$ & $\begin{array}{l}\text { COLI- } \\
\text { FORM, } \\
\text { FECAL, } \\
0.7 \\
\text { UM-MF } \\
\text { (COLS./ } \\
100 \mathrm{ML} \text { ) }\end{array}$ & $\begin{array}{c}\text { STREP- } \\
\text { TOCOCCI } \\
\text { FECAL, } \\
\text { KF AGAR } \\
\text { (COLS. } \\
\text { PER } \\
100 \mathrm{ML} \text { ) }\end{array}$ & $\begin{array}{l}\text { HARD - } \\
\text { NESS } \\
\text { TOTAL } \\
\text { (MG/L } \\
\text { AS } \\
\text { CACO3) }\end{array}$ & $\begin{array}{l}\text { CALCIUM } \\
\text { DIS - } \\
\text { SOLVED } \\
\text { (MG /L } \\
\text { AS CA) }\end{array}$ & $\begin{array}{l}\text { MAGNE- } \\
\text { S IUM, } \\
\text { DIS- } \\
\text { SOLVED } \\
\text { (MG/L } \\
\text { AS MG) }\end{array}$ & $\begin{array}{l}\text { SODIUM, } \\
\text { DIS- } \\
\text { SOLVED } \\
\text { (MG/L } \\
\text { AS NA) }\end{array}$ \\
\hline $\begin{array}{l}\text { APR } 1990 \\
13 \ldots \\
\text { MAY }\end{array}$ & 0810 & 24 & 3420 & 8.3 & 8.5 & 9.2 & 36 & E460 & 1400 & 330 & 130 & 370 \\
\hline $\begin{array}{l}16 \ldots \\
\text { JuN }\end{array}$ & 1110 & 230 & 1890 & 8.2 & 17.0 & 7.5 & K150 & 650 & 740 & 180 & 70 & 160 \\
\hline${ }_{\text {JUL }}^{14} \cdots$ & 0830 & 961 & 915 & 8.0 & 20.5 & 7.0 & 200 & K1700 & 360 & 93 & 31 & 58 \\
\hline$\underset{A U G}{26}$ & 1500 & 203 & 1710 & 8.0 & 28.0 & 6.4 & 1300 & 3200 & 630 & 170 & 50 & 140 \\
\hline $\mathrm{OCT}^{23} \cdots$ & 0800 & 287 & 1580 & 8.3 & 21.0 & 6.9 & 700 & 3100 & 610 & 160 & 52 & 130 \\
\hline $\mathrm{DEC}^{24 \cdots}$ & 1320 & 362 & 1620 & 8.4 & 12.0 & 9.3 & 180 & E1400 & 620 & 160 & 53 & 110 \\
\hline$\stackrel{18}{18} \ddot{M} 191$ & 1220 & 111 & 2710 & 8.3 & 3.0 & 11.9 & E2 & E110 & 1100 & 270 & 100 & 250 \\
\hline$\underset{A P R}{29} \ldots$ & 1005 & 23 & 3310 & 8.1 & 9.0 & 12.5 & E14 & 260 & 1300 & 300 & 130 & 350 \\
\hline $\operatorname{MAY} \cdots$ & 1215 & 19 & 3760 & 8.2 & 14.5 & 10.6 & E16 & $\cdots$ & 1300 & 300 & 140 & 400 \\
\hline$\underset{\pi N N}{21} \cdots$ & 1515 & 17 & 3730 & 8.2 & 26.5 & 8.2 & 120 & 240 & 1400 & 330 & 140 & 400 \\
\hline $\begin{array}{c}12 \ldots \\
\pi U L \\
24 \ldots\end{array}$ & 1300 & 538 & 1140 & 8.3 & 24.5 & 6.9 & 290 & 1200 & 420 & 110 & 36 & 82 \\
\hline AUG & 1230 & 375 & 1100 & 8.4 & 21.5 & 7.1 & 960 & 750 & 420 & 110 & 36 & 80 \\
\hline $\begin{array}{c}16 \ldots \\
\operatorname{SEP} \\
04 \ldots\end{array}$ & 1620 & 374 & 1110 & 8.3 & 27.0 & 6.8 & E2900 & E1800 & 420 & 110 & 35 & 77 \\
\hline $\begin{array}{c}\underset{04}{0 .} \ldots \\
\operatorname{OCT}_{30} \ldots\end{array}$ & 1815 & 207 & 1340 & 8.2 & 23.5 & 6.4 & $\cdots$ & $\cdots$ & $-\cdot$ & $\cdots$ & $\cdots$ & $\cdots$ \\
\hline $\begin{array}{c}30 \ldots \\
\text { DEC } \\
18 \ldots\end{array}$ & 0940 & 77 & 2430 & 8.2 & 1.0 & 11.9 & 100 & 340 & 1000 & 260 & 88 & 210 \\
\hline $\begin{array}{c}\text { MAR } 1992 \\
27 \ldots\end{array}$ & 0915 & 113 & 2730 & 8.3 & 0.0 & 11.5 & E5 & E55 & 1100 & 290 & 100 & 240 \\
\hline APR & 000 & 28 & 3480 & 8.2 & 9.0 & 10.7 & $\cdots$ & $\cdots$ & 1200 & 300 & 120 & 380 \\
\hline $\begin{array}{l}30 \cdots \\
\operatorname{MAY}\end{array}$ & 1015 & 264 & $\begin{array}{l}3610 \\
1660\end{array}$ & $\begin{array}{l}8.2 \\
8.3\end{array}$ & $\begin{array}{l}23.0 \\
18.5\end{array}$ & $\begin{array}{l}9.6 \\
7.8\end{array}$ & E180 & 570 & $\ddot{600}$ & 150 & 55 & 120 \\
\hline $\operatorname{sun}^{19} \ldots$ & 1125 & 174 & 1790 & 8.4 & 22.0 & 7.5 & 130 & 360 & 700 & 180 & 61 & 140 \\
\hline $\begin{array}{l}18 \ldots \\
\text { JUL }\end{array}$ & 0800 & 412 & 1130 & 8.3 & 19.0 & 7.6 & E1700 & 1100 & 440 & 110 & 39 & 77 \\
\hline AUG $\cdots$ & 1215 & 391 & 1290 & 8.2 & 23.0 & 6.7 & $>2000$ & $>3300$ & 500 & 130 & 42 & 98 \\
\hline $\mathrm{OCT}_{22} \ldots$ & 1000 & 264 & 1680 & 8.4 & 19.0 & 7.6 & E1200 & 1700 & 680 & 180 & 57 & 120 \\
\hline JAN 1993 & $0 / 43$ & 03 & 2620 & 8.2 & 12.0 & 8.3 & 48 & 86 & 1100 & 260 & 99 & 240 \\
\hline MAR & 1140 & 395 & 2760 & 8.3 & 0.0 & 11.6 & $<1$ & 40 & 1100 & 270 & 98 & 240 \\
\hline & 1030 & 24 & 3210 & 8.2 & 15.0 & 9.3 & E10 & E6 & 1200 & 300 & 120 & 340 \\
\hline
\end{tabular}

\begin{tabular}{|c|c|c|c|c|c|c|c|c|c|c|c|}
\hline DATE & $\begin{array}{c}\text { ALKA. } \\
\text { LINITY } \\
\text { LAB } \\
\text { (MG/L } \\
\text { AS } \\
\text { CACO3) }\end{array}$ & $\begin{array}{l}\text { SULFATE } \\
\text { DIS - } \\
\text { SOLVED } \\
\text { (MG/L } \\
\text { AS SO4) }\end{array}$ & $\begin{array}{l}\text { CHLO- } \\
\text { RIDE, } \\
\text { DIS- } \\
\text { SOLVED } \\
\text { (MG/L } \\
\text { AS CL) }\end{array}$ & $\begin{array}{l}\text { SOLIDS, } \\
\text { RESIDUE } \\
\text { AT } 180 \\
\text { DEG C } \\
\text { DIS- } \\
\text { SOLVED } \\
\text { (MG /L) }\end{array}$ & $\begin{array}{c}\text { NITRO- } \\
\text { GEN, } \\
\text { NO2+NO3 } \\
\text { TOTAL } \\
\text { (MG/L } \\
\text { AS N) }\end{array}$ & $\begin{array}{l}\text { NITRO- } \\
\text { GEN, } \\
\text { NO2+NO3 } \\
\text { DIS - } \\
\text { SOLVED } \\
\text { (MG /L } \\
\text { AS N) }\end{array}$ & $\begin{array}{c}\text { NITRO- } \\
\text { GEN, } \\
\text { AMMONIA } \\
\text { TOTAL } \\
\text { (MG/L } \\
\text { AS N) }\end{array}$ & $\begin{array}{l}\text { NITRO- } \\
\text { GEN, } \\
\text { AMMONIA } \\
\text { DIS - } \\
\text { SOLVED } \\
\text { (MG / } \\
\text { AS N) }\end{array}$ & $\begin{array}{c}\text { PHOS - } \\
\text { PHORUS } \\
\text { TOTAL } \\
\text { (MG / L } \\
\text { AS P) }\end{array}$ & $\begin{array}{c}\text { ARSENIC } \\
\text { TOTAL } \\
\text { (UG/L } \\
\text { AS AS) }\end{array}$ & $\begin{array}{l}\text { ARSENIC } \\
\text { DIS- } \\
\text { SOLVED } \\
\text { (UG/L } \\
\text { AS AS) }\end{array}$ \\
\hline 1990 & & & & & & & & & & & \\
\hline $13 \ldots$ & 252 & 1600 & 120 & 3120 & 1.30 & $\cdots$ & $<0.010$ & $\cdots$ & 0.020 & $\cdots$ & $\cdots$ \\
\hline $16 \ldots$ & 186 & 1000 & 48 & 1530 & 1.80 & $\cdots$ & 0.030 & $\cdots$ & 0.360 & $\cdots$ & $\cdots$ \\
\hline $14 \ldots$ & 145 & 350 & 17 & 633 & 1.50 & $\cdots$ & 0.010 & $\cdots$ & 0.520 & 3 & 1 \\
\hline $26 \ldots$ & 166 & 680 & 48 & 1330 & 1.40 & -- & 0.010 & $\cdots$ & 0.050 & $\cdots$ & $\cdots$ \\
\hline $23 \ldots$ & 169 & 570 & 42 & 1230 & 1.80 & $\cdots$ & 0.080 & $\cdots$ & 0.100 & 4 & 2 \\
\hline${ }_{i c}^{24} \ldots$ & 194 & 650 & 41 & 1190 & 2.20 & $\cdots$ & 0.020 & $\cdots$ & 0.100 & $\cdots$ & $\cdots$ \\
\hline${ }_{R}^{18} \ldots$ & 245 & 1200 & 81 & 2210 & 3.40 & $\cdots$ & 0.050 & $\cdots$ & 0.020 & $\cdots$ & $\cdots$ \\
\hline $29 \ldots$ & 250 & 2100 & 100 & 2850 & 1.50 & $\cdots$ & 0.010 & $\cdots$ & 0.020 & $\cdots$ & $\cdots$ \\
\hline
\end{tabular}


Table 57. Onsite measurements and bacteriological and selected inorganic data for station 07124000 , Arkansas River at Las Animas--Continued

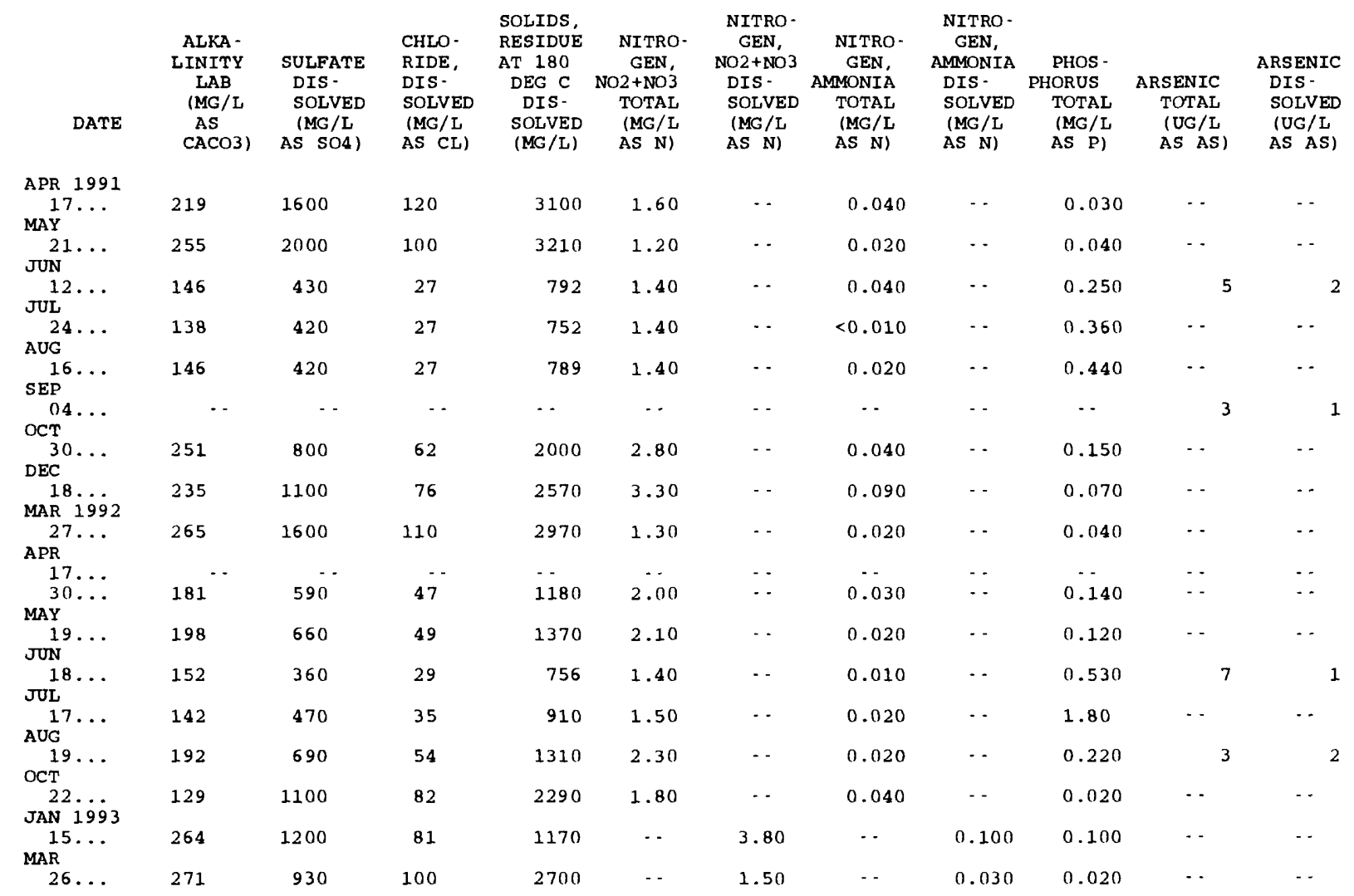

$\begin{array}{lc} & \\ \text { CADMIUM } & \\ \text { TOTAL } & \text { CADMIUM } \\ \text { RECOV- } & \text { DIS. } \\ \text { ERABLE } & \text { SOLVED } \\ \text { (UG } / L & \text { (UG } / L \\ \text { AS CD) } & \text { AS CD) }\end{array}$
CHRO CHRO- COPPER, DIS- RECOV. SOLVED ERABLE $\begin{array}{lll}\text { (UG/L } & \text { (UG/L } & \text { (UG/L } \\ \text { AS CR) } & A S C R \text { ) } & \text { AS CU) }\end{array}$
COPPER,

SOLVED (UG/L (UG/L
AS $\mathrm{CU})$
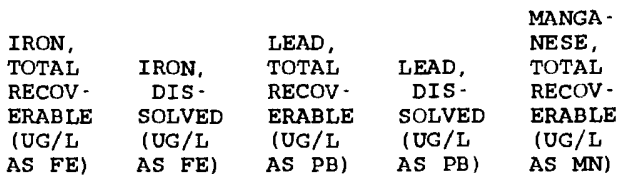

APR 1990

$13 \ldots$

MAY $16 .$.

JUN

$14 \ldots$

$26 \ldots$

AUG

ocr 33

$24 \ldots$

$18 \ldots$

MAR 1991

$29 \ldots$

APR

$17 \ldots$

MAY

JuN $21 \ldots$

$12 \ldots$

JUL

$24 \ldots$

AUG $16 .$.

SEP

$04 \ldots$

30.

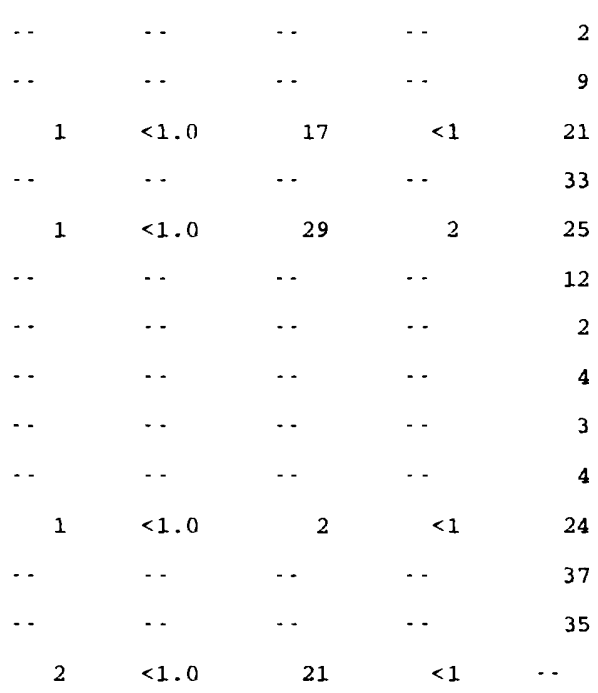

$\begin{array}{rr}<1 & 200 \\ 5 & 8100 \\ 1 & 18000 \\ 1 & 28000 \\ 2 & 34000 \\ 2 & 14000 \\ 1 & 1000 \\ 1 & 440 \\ 2 & 190 \\ 1 & 90 \\ 2 & 27000 \\ 1 & \cdots \\ 2 & 29000 \\ & \cdots \\ 1 & 1500 \\ <1 & 920\end{array}$

\begin{tabular}{|c|c|c|c|}
\hline 20 & $\cdots$ & $\cdots$ & 150 \\
\hline 5 & - & $\cdots$ & 350 \\
\hline 5 & 72 & $<1$ & 670 \\
\hline 14 & - & $\cdots$ & 740 \\
\hline 7 & 33 & $<1$ & 740 \\
\hline$<3$ & $\cdots$ & $\cdots$ & 620 \\
\hline 10 & $\cdots$ & $\cdots$ & 60 \\
\hline 10 & $\cdots$ & . & 160 \\
\hline 30 & - - & $\ldots$ & 220 \\
\hline 30 & 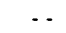 & $\cdots$ & 80 \\
\hline 11 & 19 & $<1$ & 870 \\
\hline 7 & - & $\cdots$ & 960 \\
\hline 6 & - & - & 940 \\
\hline . & 47 & $<1$ & $\cdots$ \\
\hline$<10$ & - & $\cdots$ & 130 \\
\hline 60 & - & . & 70 \\
\hline
\end{tabular}


Table 57. Onsite measurements and bacteriological and selected inorganic data for station 07124000 , Arkansas River at Las Animas--Continued

\begin{tabular}{|c|c|c|c|c|c|c|c|c|c|c|}
\hline $\begin{array}{l}\text { CADMIUM } \\
\text { TOTAL } \\
\text { RECOV - } \\
\text { ERABLE } \\
\text { (UG/L }\end{array}$ & $\begin{array}{c}\text { CADMIUM } \\
\text { DIS- } \\
\text { SOLVED } \\
\text { IUG/L }\end{array}$ & $\begin{array}{l}\text { CHRO - } \\
\text { MIUM, } \\
\text { TOTAL } \\
\text { RECOV- } \\
\text { ERABLE } \\
\text { IUG/L }\end{array}$ & $\begin{array}{l}\text { CHRO- } \\
\text { MIUM, } \\
\text { DIS - } \\
\text { SOLVED } \\
\text { /UG/L }\end{array}$ & $\begin{array}{l}\text { COPPER, } \\
\text { TOTAL } \\
\text { RECOV- } \\
\text { ERABLE } \\
\text { /UG/L }\end{array}$ & $\begin{array}{l}\text { COPPER, } \\
\text { DIS - } \\
\text { SOLVED } \\
\text { (UG/L }\end{array}$ & $\begin{array}{l}\text { IRON, } \\
\text { TOTAL } \\
\text { RECOV - } \\
\text { ERABLE } \\
\text { (UG/L }\end{array}$ & $\begin{array}{l}\text { IRON, } \\
\text { DIS - } \\
\text { SOLVED } \\
\text { /UG/L }\end{array}$ & $\begin{array}{l}\text { LEAD, } \\
\text { TOTAL } \\
\text { RECOV - } \\
\text { ERABLE } \\
\text { (UG/L }\end{array}$ & $\begin{array}{l}\text { LEAD, } \\
\text { DIS - } \\
\text { SOLVED } \\
\text { /UG /L }\end{array}$ & $\begin{array}{l}\text { MANGA - } \\
\text { NESE, } \\
\text { TOTAL } \\
\text { RECOV- } \\
\text { ERABLE } \\
\text { (UG/L }\end{array}$ \\
\hline AS CD) & AS CD) & AS CR & AS CR) & AS CU) & AS CU) & AS FE) & AS FE) & AS PB) & AS PB) & AS $\mathbf{M N}$ ) \\
\hline
\end{tabular}

\begin{tabular}{|c|c|c|c|c|c|c|c|c|c|c|c|}
\hline $\begin{array}{c}\text { MAR } 1992 \\
27 \ldots\end{array}$ & $\cdots$ & $\cdots$ & $\cdots$ & $\cdots$ & $<1$ & 2 & 90 & $<10$ & $\cdots$ & $\cdots$ & 100 \\
\hline APR & & & & & & & & & & & \\
\hline $17 \ldots$ & $\cdots$ & $\cdots$ & $\cdots$ & $\cdots$ & $\cdots$ & $\cdots$ & $\cdots$ & $\cdots$ & $\cdots$ & $\cdots$ & $\cdots$ \\
\hline $\operatorname{MAY}^{30} \cdots$ & $\cdots$ & $\cdots$ & $\cdots$ & $\cdots$ & 10 & 1 & 6600 & $<3$ & $\cdots$ & $\cdots$ & 370 \\
\hline${ }_{\text {JUN }}^{19} \ldots$ & $\cdots$ & $\cdots$ & $\cdots$ & -- & 7 & 1 & 4700 & 4 & $\cdots$ & $\cdots$ & 200 \\
\hline${ }_{J U L}^{18} \ldots$ & $<1$ & $<1.0$ & 7 & $<1$ & 19 & 3 & 15000 & $<10$ & 20 & 1 & 520 \\
\hline${ }_{A \cup G}^{17 \ldots}$ & $\cdots$ & $\cdots$ & $\cdots$ & $\cdots$ & 39 & 2 & 26000 & 8 & $\cdots$ & $\cdots$ & 890 \\
\hline $\mathrm{OCT}^{19 \ldots}$ & $<1$ & $<1.0$ & 6 & $<1$ & 9 & 2 & 9900 & 6 & 13 & $<1$ & 380 \\
\hline${ }_{\text {JAN }}^{22} 1993$ & $\cdots$ & -- & $\cdots$ & $-\cdot$ & 1 & 1 & 200 & $<10$ & $\cdots$ & $\cdots$ & 50 \\
\hline $\operatorname{MAR}^{15} \ldots$ & $\cdots$ & $\cdots$ & $\cdots$ & $\cdots$ & $<1$ & $<1$ & 710 & 4 & $\cdots$ & $\cdots$ & 90 \\
\hline $26 \ldots$ & $\cdots$ & $\cdots$ & $\cdots$ & $\cdots$ & $<1$ & $<1$ & 140 & $<10$ & $\cdots$ & $\cdots$ & 130 \\
\hline
\end{tabular}

$\begin{array}{llc}\text { MANGA- } & \text { MERCURY } & \\ \text { NESE, } & \text { TOTAL } & \text { MERCURY } \\ \text { DIS- } & \text { RECOV } & \text { DIS- } \\ \text { SOLVED } & \text { ERABLE } & \text { SOLVED } \\ \text { (UG/L } & \text { (UG /L } & \text { (UG/L } \\ \text { AS MN) } & \text { AS HG) } & \text { AS HG) }\end{array}$
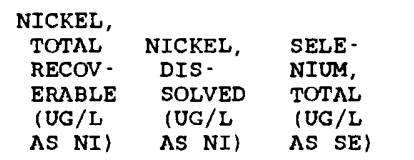

SELE -
NIUM,
DIS -
SOLVED
(UG/L
AS SE)
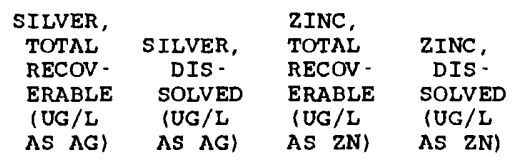

APR 199

$13 \ldots$
MAY
$16 \ldots$
JUN
$14 \ldots$
JUL
$26 \ldots$
AUG
$23 \ldots$
OCT
$24 \ldots$
DEC
$18 \ldots$

AS $A G$

ZN)

$29 \ldots$

APR
$17 \ldots$

MAY

21 ..

JUN $12 \ldots$

JUL

AUG

$16 \ldots$

SEP $04 \ldots$

OCT $30 .$.

DEC

MAR 1992

$27 . .$.

APR

$17 \ldots$

MAY

$19 \ldots$

JUN $18 \ldots$

JUL

$17 .$.

AUG $19 .$.

OCT

JAN 22.993

15 ...

MAR

$26 \ldots$

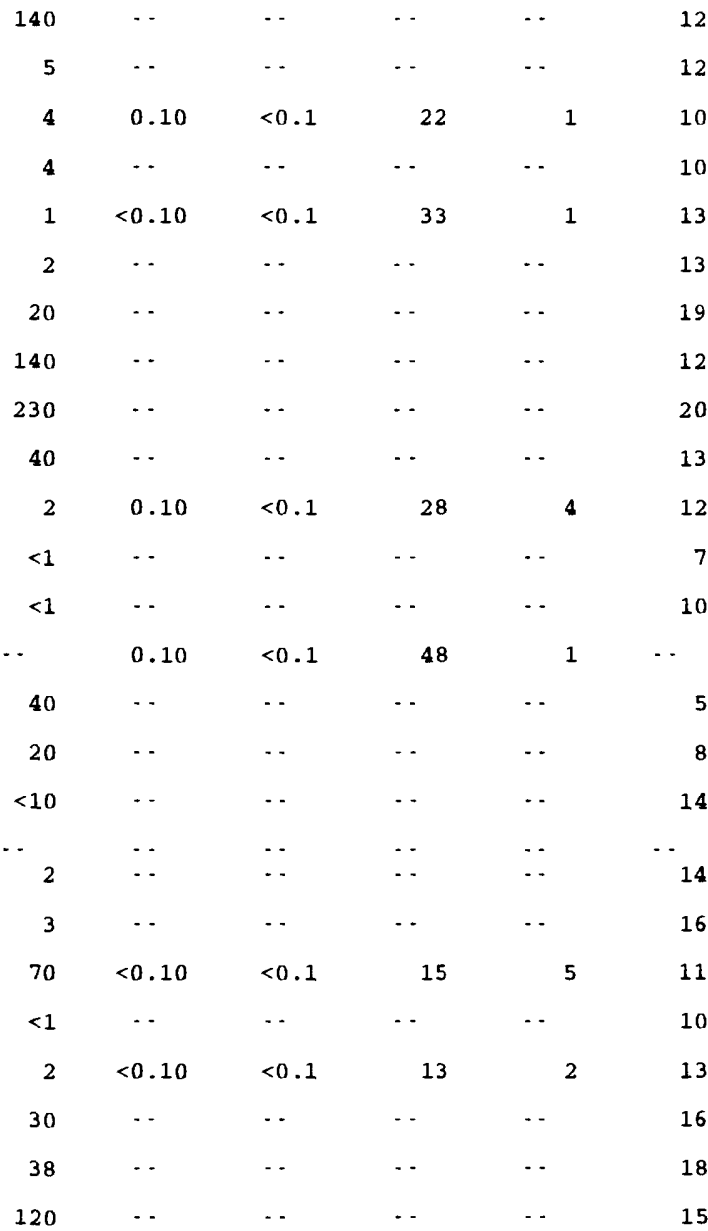

\begin{tabular}{|c|c|c|c|c|}
\hline 13 & $\cdots$ & $\cdots$ & 10 & $<10$ \\
\hline 14 & $\cdots$ & $\cdots$ & 60 & 6 \\
\hline 8 & $<1$ & $\cdots$ & 120 & $<3$ \\
\hline 11 & $\cdots$ & $\cdots$ & 150 & 11 \\
\hline 11 & $<1$ & $<1.0$ & 170 & $<3$ \\
\hline 12 & $\cdots$ & - & 100 & 5 \\
\hline 18 & $\cdots$ & $\cdots$ & 10 & $<10$ \\
\hline 12 & - & - & $<10$ & 10 \\
\hline 18 & - & - & $<10$ & $<10$ \\
\hline 13 & $\cdots$ & $\cdots$ & $<10$ & $<10$ \\
\hline 11 & $<1$ & $<1.0$ & 140 & 5 \\
\hline 9 & $\cdots$ & $\cdots$ & 180 & 4 \\
\hline 10 & $\cdots$ & $\cdots$ & 160 & $<3$ \\
\hline & $<1$ & $<1.0$ & $\cdots$ & $\cdots$ \\
\hline 4 & $\cdots$ & $\cdots$ & 20 & $<10$ \\
\hline 8 & $\cdots$ & $\cdots$ & 40 & $<10$ \\
\hline 14 & $\cdots$ & - & $<10$ & $<10$ \\
\hline & $\cdots$ & $\cdots$ & $\cdots$ & $\cdots$ \\
\hline 5 & $\cdots$ & $\cdots$ & 50 & $<3$ \\
\hline 14 & $\cdots$ & $\cdots$ & 40 & $<3$ \\
\hline 8 & $<1$ & $<1.0$ & 110 & $<10$ \\
\hline 7 & $\cdots$ & $\cdots$ & 170 & $<3$ \\
\hline 12 & $<1$ & $<1.0$ & 110 & $<3$ \\
\hline 16 & $\cdots$ & $\cdots$ & $<10$ & $<10$ \\
\hline 18 & $\cdots$ & - - & $<10$ & $<3$ \\
\hline 16 & $\cdots$ & $\cdots$ & $<10$ & $<10$ \\
\hline
\end{tabular}


Table 58. Onsite measurements and selected inorganic data for station 07128500 , Purgatoire River near Las Animas

\begin{tabular}{|c|c|c|c|c|c|c|c|c|}
\hline DATE & TIME & $\begin{array}{c}\text { DIS- } \\
\text { CHARGE, } \\
\text { INST. } \\
\text { CUBIC } \\
\text { FEET } \\
\text { PER } \\
\text { SECOND }\end{array}$ & $\begin{array}{l}\text { SPE- } \\
\text { CIFIC } \\
\text { CON- } \\
\text { DUCT- } \\
\text { ANCE } \\
\text { (US/CM) }\end{array}$ & $\begin{array}{c}\text { PH } \\
\text { WATER } \\
\text { WHOLE } \\
\text { FIELD } \\
\text { (STAND- } \\
\text { ARD } \\
\text { UNITS) }\end{array}$ & $\begin{array}{c}\text { TEMPER- } \\
\text { ATURE } \\
\text { WATER } \\
\text { (DEG C) }\end{array}$ & $\begin{array}{c}\text { OXYGEN, } \\
\text { DIS - } \\
\text { SOLVED } \\
\text { (MG/L) }\end{array}$ & $\begin{array}{l}\text { SOLIDS, } \\
\text { RESIDUE } \\
\text { AT } 180 \\
\text { DEG C } \\
\text { DIS - } \\
\text { SOLVED } \\
\text { (MG } / L)\end{array}$ & $\begin{array}{c}\text { NITRO- } \\
\text { GEN, } \\
\text { NO2+NO3 } \\
\text { TOTAL } \\
\text { (MG/L } \\
\text { AS N) }\end{array}$ \\
\hline $\begin{array}{c}\text { APR } 1990 \\
13 . \cdots \\
\operatorname{MAY}\end{array}$ & 0940 & 11 & 3780 & 8.1 & 11.0 & 8.8 & 3690 & 0.100 \\
\hline $\begin{array}{l}16 \ldots \\
\text { JUN }\end{array}$ & 0930 & 9.5 & 3350 & 7.9 & 15.0 & 8.9 & 3060 & 0.100 \\
\hline JUL $^{14} \cdots$ & 1100 & 26 & 2050 & 7.9 & 23.0 & 7.9 & 1710 & 0.300 \\
\hline${ }_{A \cup G}^{26} \cdots$ & 1620 & 331 & 1050 & 7.9 & 27.0 & 6.1 & 798 & 0.400 \\
\hline$\underset{\text { OCT }}{22} \cdots$ & 1500 & 5.8 & 3260 & 8.1 & 24.5 & 8.2 & 2960 & $<0.100$ \\
\hline${ }_{D E C}^{24 \cdots}$ & 1215 & 13 & 2960 & 8.3 & 11.5 & 12.3 & 2340 & 0.600 \\
\hline$\stackrel{18}{18} \ddot{1991}$ & 1330 & 45 & 3990 & 8.4 & 4.5 & 11.4 & 3730 & $<0.100$ \\
\hline$\underset{A P R}{29} \cdots$ & 0905 & 7.5 & 4260 & 7.9 & 6.5 & 9.8 & 4120 & 0.200 \\
\hline${ }_{\operatorname{MAY}}^{17} \cdots$ & 1105 & 5.8 & 4950 & 8.0 & 11.0 & 8.2 & 4610 & 0.240 \\
\hline$\underset{\text { JUN }}{21} \cdots$ & 1635 & 1.1 & 5140 & 8.1 & 22.5 & 7.1 & 5010 & 0.130 \\
\hline JuL $12 \ldots$ & 1435 & 5.4 & 4280 & 8.0 & 28.5 & 8.0 & 3750 & 0.058 \\
\hline$\underset{A \cup C}{24}$ & 1415 & 53 & 3640 & 8.1 & 20.0 & 7.0 & 3340 & 0.051 \\
\hline${ }_{\text {OCT }}^{16 \ldots}$ & 1745 & 40 & 1080 & 8.2 & 28.0 & 6.2 & 774 & 0.410 \\
\hline${ }_{D E C}^{30} \cdots$ & 0820 & 31 & 2460 & 8.2 & 2.5 & 11.6 & 1970 & 2.60 \\
\hline MAR $19 \dot{9} 2$ & 0730 & 12 & 3620 & 8.1 & 0.0 & 10.6 & 3350 & 0.100 \\
\hline $\begin{array}{c}27 \ldots \\
A P R \\
30 \ldots\end{array}$ & 0150 & 24 & 3200 & 8.2 & 9.5 & 9.3 & 2880 & 0.610 \\
\hline $\begin{array}{l}\text { MAY } \\
19 \ldots\end{array}$ & 1130 & 4.6 & $\mathbf{4 4 3 0}$ & 0.1 & 10.3 & 1.7 & 4060 & $<0.050$ \\
\hline JUN $18 \ldots$ & 0735 & 6.6 & 3200 & 8.0 & 18.0 & 7.2 & 2740 & 0.980 \\
\hline $\begin{array}{l}\text { JUL } \\
17 \ldots \\
\text { AUG }\end{array}$ & 1235 & 48 & 2760 & 8.2 & 23.5 & 7.3 & 2400 & $<0.050$ \\
\hline $\mathrm{OCT}^{19} \cdots$ & 0840 & 53 & 2370 & 8.1 & 16.5 & 7.6 & 2000 & 0.670 \\
\hline${ }_{\text {JAN }}^{21} 1993$ & 1700 & 33 & 2660 & 8.3 & 16.0 & 10.6 & 2350 & 1.20 \\
\hline$\stackrel{15 \cdots}{M A R}$ & 1015 & 48 & 4010 & 8.1 & 0.0 & 11.9 & 3900 & $\cdots$ \\
\hline $26 \ldots$ & 0940 & 76 & 2680 & 8.3 & 13.0 & 9.3 & 2250 & $\cdots$ \\
\hline DATE & $\begin{array}{c}\text { NITRO- } \\
\text { GEN, } \\
\text { NO2 +NO3 } \\
\text { DIS - } \\
\text { SOLVED } \\
\text { (MG/L } \\
\text { AS N) }\end{array}$ & $\begin{array}{c}\text { NITRO- } \\
\text { GEN, } \\
\text { AMMONIA } \\
\text { TOTAL } \\
\text { (MG /L } \\
\text { AS N) }\end{array}$ & $\begin{array}{l}\text { NITRO- } \\
\text { GEN, } \\
\text { AMMONIA } \\
\text { DIS- } \\
\text { SOLVED } \\
\text { (MG/L } \\
\text { AS N) }\end{array}$ & $\begin{array}{l}\text { COPPER, } \\
\text { TOTAL } \\
\text { RECOV- } \\
\text { ERABLE } \\
\text { (US/L } \\
\text { AS CU) }\end{array}$ & $\begin{array}{l}\text { IRON, } \\
\text { TOTAL } \\
\text { RECOV- } \\
\text { ERABLE } \\
\text { (UG/L } \\
\text { AS FE) }\end{array}$ & $\begin{array}{l}\text { MANGA- } \\
\text { NESE, } \\
\text { TOTAL } \\
\text { RECOV- } \\
\text { ERABLE } \\
\text { (UG/I } \\
\text { AS MN) }\end{array}$ & $\begin{array}{l}\text { SELE- } \\
\text { NIUM, } \\
\text { TOTAL } \\
\text { (UG/L } \\
\text { AS SE) }\end{array}$ & $\begin{array}{l}\text { ZINC, } \\
\text { TOTAL } \\
\text { RECOV- } \\
\text { ERABLE } \\
\text { (UG/L } \\
\text { AS ZN) }\end{array}$ \\
\hline $\begin{array}{c}\text { APR } 1990 \\
13 \ldots\end{array}$ & $\ldots$ & 0.020 & $\cdots$ & $\ldots$ & & & & \\
\hline MAY & & $0.0<0$ & - & $\cdots$ & $\cdots$ & $\cdots$ & $\cdots$ & $\cdots$ \\
\hline JUN & $\cdots$ & 0.020 & $\cdots$ & $\cdots$ & $\cdots$ & $\cdots$ & $\cdots$ & $\cdots$ \\
\hline${ }_{\text {JUL }}^{14} \cdot \cdots$ & $\cdots$ & 0.030 & $\cdots$ & $\cdots$ & $\cdots$ & $\cdots$ & $\cdots$ & $\cdots$ \\
\hline$\underset{A U G}{26}$ & $\cdots$ & 0.020 & $\cdots$ & 200 & 250000 & 5300 & 17 & 1000 \\
\hline$\underset{\mathrm{OCT}}{22} \cdots$ & $\cdots$ & 0.080 & $\cdots$ & $\cdots$ & $\cdots$ & $\cdots$ & $\cdots$ & $\cdots$ \\
\hline$\underset{D E C}{24 \cdots}$ & $\cdots$ & 0.020 & $\cdots$ & $\cdots$ & $\cdots$ & $\cdots$ & $\cdots$ & $\cdots$ \\
\hline$\stackrel{18}{18} 19{ }^{\prime} 1$ & $\cdots$ & 0.040 & $\cdots$ & $\cdots$ & $\cdots$ & $\cdots$ & $\cdots$ & $\cdots$ \\
\hline $\begin{array}{c}29 \ldots \\
\mathrm{APR} \\
17 \ldots\end{array}$ & $\cdots$ & 0.030 & $\cdots$ & $\cdots$ & $\cdots$ & $\cdots$ & $\cdots$ & $\cdots$ \\
\hline
\end{tabular}


Table 58. Onsite measurements and selected inorganic data for station 07128500 ,

Purgatoire River near Las Animas--Continued

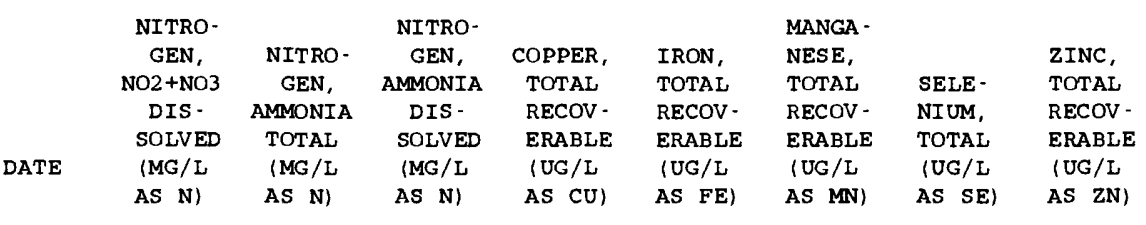

MAY 1991

21 ..

JUN

12 ...

JUL

$24 \ldots$

AUG

$16 \ldots$

OCT

$30 .$.

DEC

$18 \ldots$

MAR 1992

$27 \ldots$

$A P R$

$30 \ldots$

MAY

$19 \ldots$

JUN

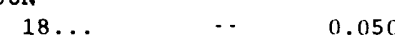

JUL

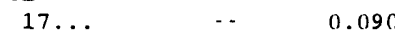

AUG

$19 \ldots$

OCT

$21 \ldots$

JAN 1993

$15 \ldots$

26 ...

- $\quad 0.140$

.. 0.030

$\cdots \quad<0.010$

- $\quad 0.050$

. $\quad 0.030$

. $\quad 0.030$

- 0.010

. $\quad 0.040$

. $\quad 0.040$

. 0.030

.
0.030

0.420

0.040

0.620

0.030 
Table 59. Onsite measurements and bacteriological and selected inorganic data for station 07130500 , Arkansas River below John Martin Reservoir

\begin{tabular}{|c|c|c|c|c|c|c|c|c|c|c|c|c|}
\hline DATE & TIME & $\begin{array}{c}\text { DIS - } \\
\text { CHARGE, } \\
\text { INST. } \\
\text { CUBIC } \\
\text { FEET } \\
\text { PER } \\
\text { SECOND }\end{array}$ & $\begin{array}{l}\text { SPE- } \\
\text { CIFIC } \\
\text { CON- } \\
\text { DUCT- } \\
\text { ANCE } \\
\text { (US/CM) }\end{array}$ & $\begin{array}{c}\text { PH } \\
\text { WATER } \\
\text { WHOLE } \\
\text { FIELD } \\
\text { (STAND- } \\
\text { ARD } \\
\text { UNITS) }\end{array}$ & $\begin{array}{c}\text { TEMPER- } \\
\text { ATURE } \\
\text { WATER } \\
\text { (DEG C) }\end{array}$ & $\begin{array}{c}\text { OXYGEN, } \\
\text { DIS - } \\
\text { SOLVED } \\
\text { (MG/L) }\end{array}$ & $\begin{array}{l}\text { COLI. } \\
\text { FORM, } \\
\text { FECAL, } \\
0.7 \\
\text { UM-MF } \\
\text { \{COLS./ } \\
100 \mathrm{ML} \text { ) }\end{array}$ & $\begin{array}{l}\text { STREP- } \\
\text { TOCOCCI } \\
\text { FECAL, } \\
\text { KF AGAR } \\
\text { (COLS. } \\
\text { PER } \\
100 \mathrm{ML} \text { ) }\end{array}$ & $\begin{array}{l}\text { HARD- } \\
\text { NESS } \\
\text { TOTAL } \\
\text { (MG/L } \\
\text { AS } \\
\text { CACO3) }\end{array}$ & $\begin{array}{l}\text { CALCIUM } \\
\text { DIS - } \\
\text { SOLVED } \\
\text { (MG/L } \\
\text { AS CA) }\end{array}$ & $\begin{array}{c}\text { MAGNE- } \\
\text { SIUM, } \\
\text { DIS- } \\
\text { SOLVED } \\
\text { (MG/L } \\
\text { AS MG) }\end{array}$ & $\begin{array}{l}\text { SODIUM } \\
\text { DIS - } \\
\text { SOLVED } \\
\text { (MG /L } \\
\text { AS NA }\end{array}$ \\
\hline $\begin{array}{c}\text { APR } 1990 \\
13 \ldots \\
\text { MAY }\end{array}$ & 1115 & 1.7 & 2860 & 7.9 & 13.0 & 8.6 & k1 & K17 & 1200 & 270 & 130 & 260 \\
\hline$\underset{J U N}{16} \cdots$ & 1330 & 403 & 2670 & 8.4 & 16.0 & 9.6 & K11 & 110 & 1100 & 230 & 120 & 270 \\
\hline$\pi^{14} \ldots$ & 1300 & 595 & 2430 & 8.2 & 22.0 & 8.7 & K26 & $\mathrm{K} 24$ & 910 & 200 & 100 & 260 \\
\hline$\underset{A \cup G}{27} \cdots$ & 0850 & 402 & 1530 & 8.1 & 23.0 & 8.4 & 84 & $\cdots$ & 530 & 120 & 56 & 130 \\
\hline$\underset{\text { OCT }}{23}$ & 1130 & 444 & 1490 & 8.2 & 23.0 & 8.0 & K85 & 89 & 560 & 130 & 58 & 130 \\
\hline$\underset{\mathrm{DEC}}{24} \cdots$ & 1515 & 268 & 1880 & 8.8 & 10.5 & 11.4 & E2 & E34 & 680 & 160 & 67 & 150 \\
\hline$\stackrel{18}{18} 1991$ & 1510 & 2.8 & 2590 & 7.9 & 3.5 & $\cdots$ & E18 & 52 & 1100 & 240 & 110 & 240 \\
\hline${ }_{\mathrm{APR}}^{29} \cdots$ & 1120 & 3.4 & 2460 & 8.3 & 10.0 & 14.7 & E2 & E3 & 1000 & 230 & 110 & 230 \\
\hline${ }_{\operatorname{MAY}}^{17} \cdots$ & 1400 & 500 & 2580 & 8.2 & 12.0 & 9.3 & E5 & E1 0 & 910 & 200 & 100 & 240 \\
\hline$\underset{\text { JUN }}{22} \cdots$ & 0810 & 53 & 2710 & 8.3 & 18.5 & 8.4 & 130 & 150 & 980 & 210 & 110 & 250 \\
\hline$\pi_{J}^{13} \cdots$ & 0840 & 575 & 2530 & 8.1 & 21.0 & 8.2 & E20 & E29 & 910 & 200 & 100 & 240 \\
\hline $\operatorname{sep}_{25}^{25}$ & 1305 & 539 & 1450 & 8.2 & 23.0 & 8.2 & 74 & 47 & 540 & 130 & 52 & 120 \\
\hline$\underset{\text { OCT }}{05 \ldots}$ & 0905 & 304 & 1530 & 8.0 & 23.0 & 7.9 & 110 & 43 & 550 & 130 & 55 & 120 \\
\hline${ }_{\mathrm{DEC}}^{30} \cdots$ & 1130 & 115 & 2330 & 8.3 & 7.0 & 11.7 & 100 & 110 & 900 & 210 & 90 & 220 \\
\hline MAR 18 & 1045 & 1.8 & 2600 & 7.9 & 3.5 & 12.1 & $<7$ & E6 & 1100 & 250 & 120 & 240 \\
\hline$\underset{A P R}{27} \cdots$ & 1005 & 1.6 & 2780 & 8.0 & 10.5 & 8.9 & $\cdots$ & $\cdots$ & 1100 & 240 & 120 & 260 \\
\hline${ }_{M A Y}^{30} \ldots$ & 1200 & 610 & 2680 & 8.3 & 14.0 & 9.9 & E13 & E2 & 1000 & 230 & 110 & 250 \\
\hline$\underset{\text { JUN }}{19} \cdots$ & 1400 & 490 & 2560 & 8.2 & 19.5 & 8.7 & E19 & E12 & 1100 & 240 & 120 & 240 \\
\hline${ }_{\pi U L}^{18} \ldots$ & 1100 & 411 & 2060 & 8.3 & 20.5 & 8.2 & 30 & 35 & 800 & 190 & 79 & 170 \\
\hline$\underset{A U G}{17} \cdots$ & 1445 & 845 & 1550 & 8.1 & 23.5 & 7.8 & 26 & 58 & 580 & 140 & 57 & 130 \\
\hline${ }_{\text {OCT }}^{19} \cdots$ & 1145 & 389 & 1700 & 8.1 & 22.5 & 8.4 & 110 & 77 & 620 & 140 & 65 & 140 \\
\hline JAN 1993 & 0930 & 120 & 2070 & 8.5 & 11.5 & 10.8 & 86 & 160 & 770 & 170 & 84 & 180 \\
\hline$\underset{M A R}{15} \cdots$ & 1315 & 89 & 2710 & 7.7 & 2.5 & 10.7 & $<3$ & E13 & 1100 & 260 & 120 & 240 \\
\hline $26 \ldots$ & 1130 & 3.1 & 2750 & 8.0 & 14.0 & 10.2 & E2 & $<1$ & 1100 & 250 & 120 & 250 \\
\hline
\end{tabular}

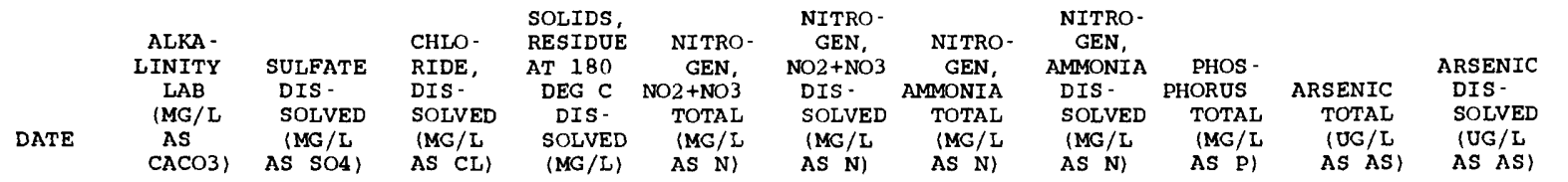

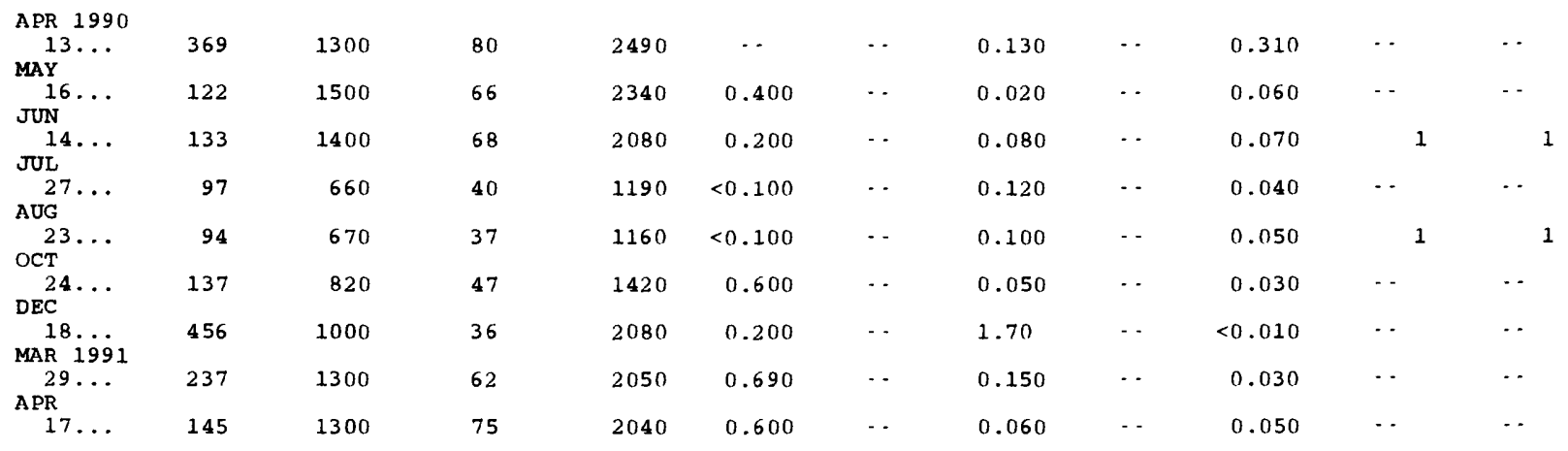


Table 59. Onsite measurements and bacteriological and selected inorganic data for station 07130500 , Arkansas River below John Martin Reservoir--Continued

\begin{tabular}{|c|c|c|c|c|c|c|c|c|c|c|c|}
\hline DATE & $\begin{array}{c}\text { ALKA- } \\
\text { LINITY } \\
\text { LAB } \\
\text { (MG / } \\
\text { AS } \\
\text { CACO3) }\end{array}$ & $\begin{array}{l}\text { SULFATE } \\
\text { DIS - } \\
\text { SOLVED } \\
\text { (MG /L } \\
\text { AS SO4) }\end{array}$ & $\begin{array}{l}\text { CHLO- } \\
\text { RIDE, } \\
\text { DIS- } \\
\text { SOLVED } \\
\text { (MG/L } \\
\text { AS CL) }\end{array}$ & $\begin{array}{l}\text { SOLIDS, } \\
\text { RESIDUE } \\
\text { AT } 180 \\
\text { DEG C } \\
\text { DIS - } \\
\text { SOLVED } \\
\text { (MG/L) }\end{array}$ & $\begin{array}{l}\text { NITRO- } \\
\text { GEN, } \\
\text { NO2+NO3 } \\
\text { TOTAL } \\
\text { (MG /L } \\
\text { AS N) }\end{array}$ & $\begin{array}{c}\text { NITRO- } \\
\text { GEN, } \\
\text { NO2+NO3 } \\
\text { DIS- } \\
\text { SOLVED } \\
\text { (MG/L } \\
\text { AS N) }\end{array}$ & $\begin{array}{l}\text { NITRO- } \\
\text { GEN, } \\
\text { AMMONIA } \\
\text { TOTAL } \\
\text { (MG / L } \\
\text { AS N) }\end{array}$ & $\begin{array}{l}\text { NITRO- } \\
\text { GEN, } \\
\text { AMMONIA } \\
\text { DIS- } \\
\text { SOLVED } \\
\text { (MG/L } \\
\text { AS N) }\end{array}$ & $\begin{array}{l}\text { PHOS - } \\
\text { PHORUS } \\
\text { TOTAL } \\
\text { (MG/L } \\
\text { AS P) }\end{array}$ & $\begin{array}{c}\text { ARSENIC } \\
\text { TOTAL } \\
\text { (UG /L } \\
\text { AS AS) }\end{array}$ & $\begin{array}{l}\text { ARSENIC } \\
\text { DIS- } \\
\text { SOLVED } \\
\text { (UG/L } \\
\text { AS AS) }\end{array}$ \\
\hline MAY 1991 & & & & & & & & & & & \\
\hline$\underset{\text { JUN }}{22 \cdots}$ & 146 & 1500 & 83 & 2260 & $<0.050$ & $\cdots$ & 0.040 & $\cdots$ & 0.070 & $\cdots$ & $\cdots$ \\
\hline $\begin{array}{l}13 \ldots \\
\text { JUL }\end{array}$ & 137 & 1300 & 69 & 2080 & 0.130 & $\cdots$ & 0.230 & $\cdots$ & 0.050 & 1 & 1 \\
\hline$\underset{\operatorname{SEP}}{25 \ldots}$ & 127 & 660 & 35 & 1090 & 0.200 & $\cdots$ & 0.410 & $\cdots$ & 0.070 & $\cdots$ & $\cdots$ \\
\hline${ }_{\text {OCT }}^{05 \ldots}$ & 72 & 750 & 38 & 1110 & 0.120 & $\cdots$ & 0.260 & $\cdots$ & 0.120 & 2 & 1 \\
\hline${ }_{D E C}^{30} \cdots$ & 151 & 970 & 74 & 1900 & 0.160 & $\cdots$ & 0.090 & $\cdots$ & 0.060 & $\cdots$ & $\cdots$ \\
\hline${ }_{\text {MAR }}^{18} \ddot{1992}$ & 426 & 910 & 81 & 2350 & 0.270 & $\cdots$ & 0.530 & $\cdots$ & 0.060 & $\cdots$ & $-\cdot$ \\
\hline$\underset{A P R}{27 \ldots}$ & 452 & 1100 & 80 & 2230 & 0.330 & $\cdots$ & 0.560 & $\cdots$ & 0.080 & $\cdots$ & $\cdots$ \\
\hline${ }_{\operatorname{MAY}}^{30} \ldots$ & 175 & 1100 & 89 & 2210 & 0.520 & $\cdots$ & 0.140 & $\cdots$ & $<0.010$ & $\cdots$ & $\cdots$ \\
\hline JUN $^{19} \cdots$ & 176 & 1100 & 77 & 2060 & 0.480 & $\cdots$ & 0.270 & $\cdots$ & 0.020 & $\cdots$ & $\cdots$ \\
\hline${ }_{J U L} 18 \ldots$ & 168 & 880 & 54 & 1490 & 0.520 & $\cdots$ & 0.250 & $\cdots$ & 0.070 & 2 & 1 \\
\hline${ }_{A U G}^{17} \ldots$ & 138 & 620 & 39 & 1160 & 0.430 & $\cdots$ & 0.200 & $\cdots$ & 0.070 & $\cdots$ & $\cdots$ \\
\hline${ }_{\text {OCT }}^{19}$... & 114 & 760 & 51 & 1300 & 0.650 & $\cdots$ & 0.120 & $\cdots$ & 1.80 & 2 & 1 \\
\hline JAN $22 \ddot{993}$ & 140 & 920 & 58 & 1730 & 0.140 & $\cdots$ & 0.090 & $\cdots$ & 0.070 & $\cdots$ & $\cdots$ \\
\hline${ }_{\text {MAR }}^{15} \ldots$ & 392 & 1200 & 74 & 2260 & $\cdots$ & 0.830 & $\cdots$ & 0.720 & 0.080 & $\cdots$ & $\cdots$ \\
\hline $26 \ldots$ & 380 & 1100 & 74 & 2240 & $\cdots$ & 0.490 & $\cdots$ & 0.550 & 0.030 & - & $\cdots$ \\
\hline DATE & $\begin{array}{l}\text { CADMIUM } \\
\text { TOTAL } \\
\text { RECOV. } \\
\text { ERABLE } \\
\text { (UG/L } \\
\text { AS CD) }\end{array}$ & $\begin{array}{c}\text { CADMIUM } \\
\text { DIS - } \\
\text { SOLVED } \\
\text { (UG/L } \\
\text { AS CD) }\end{array}$ & $\begin{array}{l}\text { CHRO- } \\
\text { MIUM, } \\
\text { TOTAL } \\
\text { RECOV- } \\
\text { ERABLE } \\
\text { (UG/L } \\
\text { AS CR) }\end{array}$ & $\begin{array}{l}\text { CHRO- } \\
\text { MIUM, } \\
\text { DIS. } \\
\text { SOLVED } \\
\text { (UG/L } \\
\text { AS CR) }\end{array}$ & $\begin{array}{l}\text { COPPER, } \\
\text { TOTAL } \\
\text { RECOV- } \\
\text { ERABLE } \\
\text { (UG/L } \\
\text { AS CU) }\end{array}$ & $\begin{array}{l}\text { COPPER, } \\
\text { DIS- } \\
\text { SOLVED } \\
\text { (UG /L } \\
\text { AS CU) }\end{array}$ & $\begin{array}{l}\text { IRON, } \\
\text { TOTAL } \\
\text { RECOV- } \\
\text { ERABLE } \\
\text { (UG/L } \\
\text { AS FE) }\end{array}$ & $\begin{array}{l}\text { IRON, } \\
\text { DIS- } \\
\text { SOLVED } \\
\text { (UG/L } \\
\text { AS FE) }\end{array}$ & $\begin{array}{l}\text { LEAD, } \\
\text { TOTALL } \\
\text { RECOV. } \\
\text { ERABLE } \\
\text { (UG/L } \\
\text { AS PB) }\end{array}$ & $\begin{array}{l}\text { LEAD, } \\
\text { DIS- } \\
\text { SOLVED } \\
\text { (UG/L } \\
\text { AS PB) }\end{array}$ & $\begin{array}{l}\text { MANGA- } \\
\text { NESE, } \\
\text { TOTAL } \\
\text { RECOV. } \\
\text { ERABLE } \\
\text { (UG/L } \\
\text { AS MN) }\end{array}$ \\
\hline APR 1990 & & & & & & & & & & & \\
\hline $\operatorname{MAY}^{13} \cdots$ & $\cdots$ & $\cdots$ & $\cdots$ & $\cdots$ & 2 & 1 & 1300 & 30 & $\cdots$ & $\cdots$ & 2500 \\
\hline${ }_{\text {JUN }}^{16} \ldots$ & $\cdots$ & $\cdots$ & $\cdots$ & $\cdots$ & 3 & 1 & 550 & 30 & $\cdots$ & $\cdots$ & 160 \\
\hline${ }_{\text {JUL }}^{14 \ldots}$ & $<1$ & $<1.0$ & 1 & $<1$ & 6 & 1 & 440 & 20 & 43 & 1 & 140 \\
\hline AUG & $\cdots$ & $\cdots$ & $\cdots$ & $\cdots$ & 3 & 1 & 330 & 6 & $\cdots$ & $\cdots$ & 270 \\
\hline $\mathrm{OCT}^{23} \ldots$ & $<1$ & $<1.0$ & 2 & $<1$ & 6 & 1 & 290 & 5 & 7 & $<1$ & 110 \\
\hline${ }_{\mathrm{DEC}}^{24 \cdots}$ & $\cdots$ & $\cdots$ & $\cdots$ & $\cdots$ & 3 & 1 & 250 & $<3$ & $\cdots$ & $\cdots$ & 110 \\
\hline MAR $18 . .991$ & $\cdots$ & $\cdots$ & $\cdots$ & - & 1 & 1 & 720 & 30 & $\cdots$ & $\cdots$ & 3000 \\
\hline$\underset{A P R}{29} \cdots$ & $\cdots$ & $\cdots$ & - & $\cdots$ & 3 & 1 & 470 & 10 & $\cdots$ & $\cdots$ & 710 \\
\hline${ }_{\operatorname{MAY}}^{17} \ldots$ & $\cdots$ & $\cdots$ & $\cdots$ & $\cdots$ & 3 & 1 & 380 & 20 & $\cdots$ & $\cdots$ & 110 \\
\hline$\underset{\text { JUN }}{22} \cdots$ & $\cdots$ & $\cdots$ & -. & $\cdots$ & 3 & 1 & 400 & $<10$ & $\cdots$ & $\cdots$ & 220 \\
\hline JUL $^{13} \ldots$ & $<1$ & $<1.0$ & 1 & $<1$ & 4 & 1 & 350 & $<10$ & 4 & $<1$ & 140 \\
\hline $\operatorname{SEP}_{\text {SEP }}^{25}$ & $\cdots$ & $\cdots$ & - & & 4 & $<1$ & $\cdots$ & 5 & $\cdots$ & $\cdots$ & 240 \\
\hline$\underset{\mathrm{OCT}}{05 \ldots}$ & $<1$ & $<1.0$ & $<1$ & $<1$ & 4 & 1 & 1400 & 9 & 3 & $<1$ & 230 \\
\hline${ }_{D E C}^{30} \cdots$ & $\cdots$ & $\cdots$ & $\cdots$ & $\cdots$ & 8 & 2 & 10 & $<10$ & $\cdots$ & $\cdots$ & 150 \\
\hline $\operatorname{MAR}^{18} 1992$ & $\cdots$ & $\cdots$ & $\cdots$ & $\cdots$ & $\cdots$ & $<1$ & 630 & 20 & $\cdots$ & $\cdots$ & 2200 \\
\hline$\underset{A P R}{27}$ & $\cdots$ & $\cdots$ & $\cdots$ & $\cdots$ & $<1$ & 1 & 1400 & 90 & $\cdots$ & $\cdots$ & 1900 \\
\hline $\operatorname{MAY}^{30} \ldots$ & $\cdots$ & $\cdots$ & $\cdots$ & $\cdots$ & 5 & 2 & 510 & $<10$ & $\cdots$ & $\cdots$ & 130 \\
\hline $19 \ldots$ & $\cdots$ & $\cdots$ & $\cdots$ & $\cdots$ & 1 & $<1$ & 280 & $<10$ & $\cdots$ & $\cdots$ & 120 \\
\hline
\end{tabular}


Table 59. Onsite measurements and bacteriological and selected inorganic data for station 07130500 , Arkansas River below John Martin Reservoir--Continued

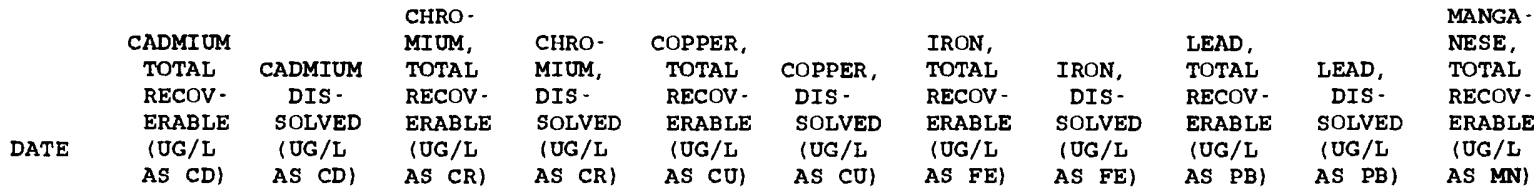

\begin{tabular}{|c|c|c|c|c|c|c|c|c|c|c|c|}
\hline $\begin{array}{c}\text { JUN } 1992 \\
18 \ldots \\
\text { JUL }\end{array}$ & $<1$ & $<1.0$ & $<1$ & $<1$ & 10 & 2 & 620 & $<3$ & 1 & $<1$ & 130 \\
\hline$\underset{A O G}{17} \cdots$ & -. & $\cdots$ & $\cdots$ & $\cdots$ & 5 & 2 & 710 & $<3$ & . & $\cdots$ & 130 \\
\hline $19 \ldots$ & $<1$ & $<1.0$ & $<1$ & $<1$ & 7 & $<1$ & 700 & 13 & 3 & $<1$ & 10 \\
\hline $\begin{array}{l}22 \ldots \\
\text { JAN } 1993\end{array}$ & $\cdots$ & $\cdots$ & $\cdots$ & $\cdots$ & 2 & $<1$ & 490 & $<10$ & $\cdots$ & $\cdots$ & 120 \\
\hline$\underset{\operatorname{MAR}}{15 \ldots}$ & $\cdots$ & $\cdots$ & $\cdots$ & $\cdots$ & 1 & $<1$ & 2400 & 30 & $\cdots$ & $\cdots$ & 2400 \\
\hline $26 \ldots$ & $\cdots$ & $\cdots$ & $\cdots$ & - & $<1$ & $<1$ & 770 & 10 & $\cdots$ & $\cdots$ & 2100 \\
\hline
\end{tabular}

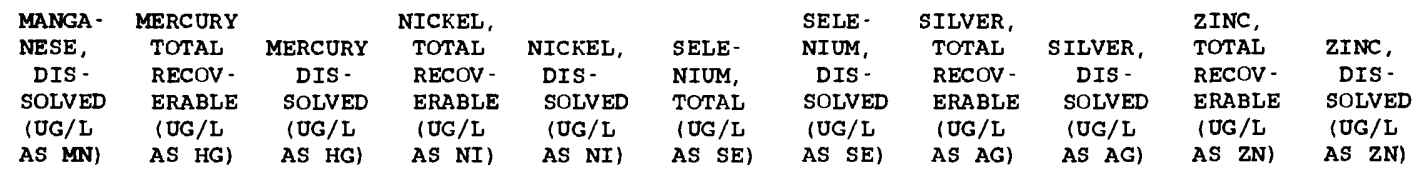

\begin{tabular}{|c|c|c|c|c|c|c|c|c|c|c|c|}
\hline $\begin{array}{c}\text { APR } 1990 \\
13 \ldots \\
\text { MAY }\end{array}$ & 2000 & $\cdots$ & $\cdots$ & $\cdots$ & - & 4 & 4 & $\cdots$ & - & 10 & $<10$ \\
\hline $16 \ldots$ & 30 & $\cdots$ & $\ldots$ & - & $\ldots$ & 8 & 9 & $\ldots$ & $\cdots$ & 10 & $<10$ \\
\hline JUN & & & & & & & & & & & \\
\hline$J_{J L}^{14 \ldots}$ & 40 & $<0.10$ & $<0.1$ & 4 & 2 & 9 & 9 & $<1$ & $<1.0$ & 20 & $<10$ \\
\hline$\underset{A U G}{27} \cdots$ & 76 & $\cdots$ & $\cdots$ & $\cdots$ & $\cdots$ & 6 & 7 & $\cdots$ & $\cdots$ & $<10$ & 4 \\
\hline$\underset{\text { OCT }}{23}$ & 20 & $<0.10$ & $<0.1$ & 3 & 2 & 5 & 6 & $<1$ & $<1.0$ & 10 & $<3$ \\
\hline$\underset{D E C}{24} \ldots$ & 32 & $\cdots$ & $\cdots$ & $\cdots$ & $\cdots$ & 9 & 9 & $\cdots$ & $\cdots$ & 10 & 6 \\
\hline $\begin{array}{l}18 \ldots \\
\text { MAR } 1991\end{array}$ & 3000 & $\cdots$ & $\cdots$ & $\cdots$ & $\cdots$ & $<1$ & 2 & $\cdots$ & $\cdots$ & 10 & $<10$ \\
\hline$\underset{A P R}{29 \ldots}$ & 640 & $\cdots$ & $\cdots$ & $\cdots$ & $\cdots$ & 8 & 8 & $\cdots$ & $\cdots$ & $<10$ & 10 \\
\hline${ }_{\mathrm{MAY}}^{17 \ldots}$ & 20 & $\cdots$ & $\cdots$ & $\cdots$ & $\cdots$ & 11 & 9 & $\cdots$ & $\cdots$ & $<10$ & $<10$ \\
\hline$\underset{\text { JUN }}{22} \cdots$ & 70 & $\cdots$ & $\cdots$ & $\cdots$ & $\cdots$ & 7 & 7 & $\cdots$ & $\cdots$ & $<10$ & $<10$ \\
\hline JUL $^{13 \ldots}$ & 90 & $<0.10$ & $<0.1$ & 12 & 7 & $\cdots$ & 6 & $<1$ & $<1.0$ & 30 & $<10$ \\
\hline$\underset{S E P}{25 \ldots}$ & 170 & - & $\cdots$ & $\cdots$ & $\cdots$ & 7 & 6 & $\cdots$ & $\cdots$ & $<10$ & 5 \\
\hline $\begin{array}{c}05 \ldots \\
\text { OCT }\end{array}$ & 64 & $<0.10$ & $<0.1$ & 4 & 1 & 8 & 8 & $<1$ & $<1.0$ & $<10$ & 7 \\
\hline${ }_{\mathrm{DEC}}^{30} \cdots$ & 60 & $\cdots$ & $\cdots$ & $\cdots$ & . & 8 & 8 & $\cdots$ & $\cdots$ & $<10$ & $<10$ \\
\hline $\begin{array}{l}18 \ldots \\
\text { MAR } 1992\end{array}$ & 2100 & $-\cdot$ & $\cdot-$ & $\cdots$ & $\cdots$ & 2 & 3 & -. & $\cdots$ & 30 & $<10$ \\
\hline$\underset{A P R}{27} \cdots$ & 1400 & $\cdots$ & $\cdots$ & $\cdots$ & $\cdots$ & 3 & 3 & $\cdots$ & $\cdots$ & $<10$ & $<10$ \\
\hline $\operatorname{MAY}^{30} \ldots$ & 60 & $\cdots$ & $\cdots$ & $\cdots$ & - - & 9 & 11 & $\cdots$ & $\cdots$ & 30 & $<10$ \\
\hline${ }_{\text {JUN }}^{19} \ldots$ & 80 & $\cdots$ & $\cdots$ & $\cdots$ & $\cdots$ & 10 & 9 & - & $\cdots$ & 10 & $<10$ \\
\hline${ }_{\text {JUL }}^{18 \ldots}$ & 76 & $<0.10$ & $<0.1$ & 4 & 4 & 8 & 8 & $<1$ & $<1.0$ & 10 & $<3$ \\
\hline$\underset{A U G}{17} \ldots$ & 49 & $\cdots$ & $\cdots$ & $\cdots$ & $\cdots$ & 8 & 8 & $\cdots$ & $\cdots$ & 20 & 5 \\
\hline$\underset{\text { OCT }}{19 . \ldots}$ & 49 & $<0.10$ & $<0.1$ & 2 & 2 & 6 & 7 & $<1$ & $<1.0$ & 40 & $<3$ \\
\hline JAN $22 \ldots 93$ & 20 & $\cdots$ & $\cdots$ & $\cdots$ & $\cdots$ & 8 & 8 & $\cdots$ & - & 10 & $<10$ \\
\hline$\underset{\operatorname{MAR}}{15 \ldots}$ & 2100 & $\cdots$ & $\cdots$ & $\cdots$ & $\cdots$ & 7 & 6 & $\cdots$ & $\cdots$ & $<10$ & $<10$ \\
\hline $26 \ldots$ & 1800 & $\cdots$ & $\cdots$ & $\cdots$ & $-\cdot$ & 5 & 5 & $\cdots$ & $\cdots$ & $<10$ & $<10$ \\
\hline
\end{tabular}


Table 60. Onsite measurements and selected inorganic data for station 07134100, Big Sandy Creek near Lamar

\begin{tabular}{|c|c|c|c|c|c|c|c|c|c|}
\hline DATE & TIME & $\begin{array}{c}\text { DIS - } \\
\text { CHARGE, } \\
\text { INST. } \\
\text { CUBIC } \\
\text { FEET } \\
\text { PER } \\
\text { SECOND }\end{array}$ & $\begin{array}{l}\text { SPE- } \\
\text { CIFIC } \\
\text { CON- } \\
\text { DUCT - } \\
\text { ANCE } \\
\text { (US/CM) }\end{array}$ & $\begin{array}{c}\text { PH } \\
\text { WATER } \\
\text { WHOLE } \\
\text { FIELD } \\
\text { (STAND- } \\
\text { ARD } \\
\text { UNITS) }\end{array}$ & $\begin{array}{l}\text { TEMPER - } \\
\text { ATURE } \\
\text { WATER } \\
\text { (DEG C) }\end{array}$ & $\begin{array}{c}\text { OXYGEN, } \\
\text { DIS - } \\
\text { SOLVED } \\
\text { (MG/L) }\end{array}$ & $\begin{array}{l}\text { SOLIDS, } \\
\text { RESIDUE } \\
\text { AT } 180 \\
\text { DEG C } \\
\text { DIS - } \\
\text { SOLVED } \\
\text { (MG/L) }\end{array}$ & $\begin{array}{l}\text { NITRO- } \\
\text { GEN, } \\
\text { NO2+NO3 } \\
\text { TOTAL } \\
\text { (MG/L } \\
\text { AS N) }\end{array}$ & $\begin{array}{l}\text { NITRO- } \\
\text { GEN, } \\
\text { AMMONIA } \\
\text { TOTAL } \\
\text { (MG/L } \\
\text { AS N) }\end{array}$ \\
\hline APR 1990 & & & & & & & & & \\
\hline $\mathrm{JUN}^{13} \ldots$ & 1130 & 6.8 & 4580 & 8.3 & 17.0 & 13.0 & 4170 & 1.30 & 0.010 \\
\hline $\begin{array}{l}14 \ldots \\
\text { JUL }\end{array}$ & 1530 & 5.0 & 4230 & 8.3 & 29.0 & 13.4 & 3750 & 1.00 & 0.060 \\
\hline${ }_{\text {AUG }}^{27 \ldots}$ & 1200 & 4.5 & 3980 & 8.4 & 24.5 & 15.9 & 3700 & 1.90 & 0.020 \\
\hline $\begin{array}{l}23 \ldots \\
\text { ост }\end{array}$ & 1430 & 8.7 & 3960 & 8.3 & 21.5 & 10.5 & 3560 & 1.50 & 0.080 \\
\hline $\begin{array}{c}24 \ldots \\
\text { APR } 1991\end{array}$ & 1630 & 5.9 & 4250 & 8.3 & 15.0 & 11.0 & 3580 & 2.10 & 0.020 \\
\hline $\mathrm{JUN}^{17 \ldots}$ & 1640 & 6.5 & 4620 & 8.2 & 21.0 & 9.4 & 4000 & 0.840 & 0.050 \\
\hline${ }_{\text {JUL }} 13 \ldots$ & 1030 & 6.1 & 3480 & 8.1 & 22.0 & 10.0 & 2800 & 1.00 & 0.020 \\
\hline$\underset{\text { OCT }}{25 \ldots}$ & 0905 & 5.9 & 4350 & 8.1 & 16.5 & 9.2 & 4070 & 2.10 & 0.150 \\
\hline $\begin{array}{c}30 \ldots \\
A P R \quad 1992\end{array}$ & 1315 & 4.7 & 4160 & 8.4 & 5.0 & 11.4 & 3720 & 3.00 & 0.040 \\
\hline$\underset{\text { JUN }}{30 \ldots}$ & 1415 & 7.8 & 4410 & 8.3 & 22.0 & 12.2 & 3960 & 1.50 & 0.040 \\
\hline${ }_{A U G}^{18 \ldots}$ & 1230 & 4.9 & 4610 & 8.3 & 20.0 & 16.0 & 4070 & 1.80 & 0.040 \\
\hline $\begin{array}{l}19 \cdots \\
\text { Ост }\end{array}$ & 1350 & 13 & 4070 & 8.4 & 20.0 & 13.0 & 3540 & 2.00 & 0.040 \\
\hline $22 \ldots$ & 1115 & 7.1 & 4050 & 8.2 & 14.0 & 9.6 & 3700 & 2.30 & 0.050 \\
\hline
\end{tabular}


Table 61. Pesticide data for selected surface-water stations on the Arkansas River, 1990-92

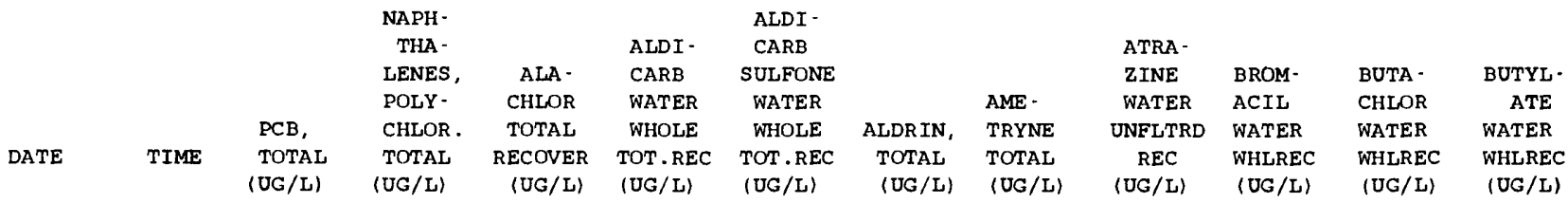

07097000 ARKANSAS RIVER AT PORTLAND

AUG 1990

30.

JUL 1991

18

1415

$<0.1<0.1<0.10$

$\begin{array}{lll}- & < & <0.010\end{array}$

$<0.10$

$<0.1$

1400

$<0.1<0.1<0.10$

$<0.5<0.5<0.010$

$<0.10$

$<0$.

$<0.20$

$<0.10<0.10$

SEP

1900

$<0.1<0.1<0.10$

$<0.5<0.5<0.010$

$<0.10$

$<0.1$

$<0.20$

$<0.10$

$<0.10$

APR 1992

0935

$<0.1<0.1<0.10$

$<0.5<0.5<0.010$

$<0.10$

$<0.1$

$<0.20$

$<0.10<0.10$

AUG

1330

$<0.1<0.1<0.10$

$<0.5$

$<0.5<0.010$

$<0.10$

$<0.1$

$<0.20$

$<0.10$

$<0.10$

07109500

AUG 1990

$20 \ldots$

JUL 1991

$22 \ldots$

SEP

$04 \ldots$

APR 1992

$17 \ldots$

AUG

17 ...

07119700

AUG 1990

21 ...

JUL 1991

23 ...

SEP

$04 . .$.

APR 1992

$17 \ldots$

AUG

$18 \ldots$

07124000

AUG 1990

23 ...

गUL 1991

$24 \ldots$

SEP

$04 \ldots$

APR 1992

17 ...

JUL

17 ...

\section{0}

1830

0935

1650

1530

$\begin{array}{lcc}<0.1 & <0.1 & <0.10 \\ <0.1 & <0.1 & <0.10 \\ <0.1 & <0.1 & <0.10 \\ \cdots & -. & <0.10 \\ <0.1 & <0.1 & <0.10\end{array}$

.

$<0.5$

$\ldots \quad<0.010$

$<0.5$

$<0.5$

$<0.5$

$<0.010$

$<0.10$

$<0.10$

$<0.5$

$<0.5$

$<0.10$

$<0.10$

$<0.5<0.5<0.010$

$<0.10$

ARKANSAS RIVER AT CATLIN DAM, NEAR FOWLER

$\begin{array}{llll}1600 & <0.1 & <0.1 & <0.10 \\ 1600 & <0.1 & <0.1 & <0.10 \\ 1445 & <0.1 & <0.1 & <0.10 \\ 1515 & <0.1 & <0.1 & <0.10 \\ 1230 & <0.1 & <0.1 & <0.10 \\ \text { ARKANSAS RIVER AT LAS ANIMAS }\end{array}$

0800

1230

$<0.1<0.1<0.10$

$<0.1<0.1<0.10$

$\cdots$

$\cdots \quad<0.010$

$<0.10$

$<0.5$

$<0.5$

$<0.010$

$<0.10$

1815

$<0.1<0.1<0.10$

$<0.5$

$<0.5<0.010$

$<0.10$

1230

$<0.1<0.1<0.10$

$<0.5$

$<0.5<0.010$

$<0.10$

$<0.1<0.1<0.10$

$<0.5$

$<0.5<0.010$

$<0.10$

ARKANSAS RIVER BELOW JOHN MARTIN RESERVOIR

$$
<0.1<0.1<0.10
$$$$
\text { .. }
$$

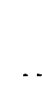

. \\ AUG 1990}

$+2$


Table 61. Pesticide data for selected surface-water stations on the Arkansas River, 1990-92--Continued

\begin{tabular}{|c|c|c|c|c|c|c|c|c|c|c|c|c|}
\hline & CARBO- & $\begin{array}{l}\text { CARBOX - } \\
\text { IN }\end{array}$ & & & & $\begin{array}{l}\text { CYCLO- } \\
\text { ATE }\end{array}$ & & & & & $\begin{array}{l}\text { DEETHYL } \\
\text { ATRA - }\end{array}$ & $\begin{array}{l}\text { DE-ISO } \\
\text { PROPYL }\end{array}$ \\
\hline & FURAN & WATER & CHLOR - & & & WATER & & & & & ZINE, & ATRAZIN \\
\hline & WATER & WHOLE & DYRIFOS & CHLOR - & CYAN - & WHOLE & & & & & WATER, & WATER， \\
\hline DATE & $\begin{array}{l}\text { WHOLE } \\
\text { TOT . REC } \\
\text { (UG/L) }\end{array}$ & $\begin{array}{l}\text { RECOV - } \\
\text { ERABLE } \\
\text { (UG } / L)\end{array}$ & $\begin{array}{l}\text { TOTAL } \\
\text { RECOVER } \\
\text { (UG/L) }\end{array}$ & $\begin{array}{l}\text { DANE, } \\
\text { TOTAL } \\
\text { (UG } / \mathrm{L} \text { ) }\end{array}$ & $\begin{array}{l}\text { AZINE } \\
\text { TOTAL } \\
\text { (UG/L) }\end{array}$ & $\begin{array}{l}\text { RECOV- } \\
\text { ERABLE } \\
\text { (UG/L) }\end{array}$ & $\begin{array}{l}\text { DDD, } \\
\text { TOTAL } \\
\text { (UG/L) }\end{array}$ & $\begin{array}{l}\text { DDE, } \\
\text { TOTAL } \\
\text { (UG/L) }\end{array}$ & $\begin{array}{l}\text { DDT, } \\
\text { TOTAL } \\
\text { (UG/L) }\end{array}$ & $\begin{array}{c}\text { DEF } \\
\text { TOTAL } \\
\text { (UG/L) }\end{array}$ & $\begin{array}{c}\text { WHOLE, } \\
\text { TOTAL } \\
\text { (UG/L) }\end{array}$ & $\begin{array}{l}\text { WHOLE, } \\
\text { TOTAL } \\
\text { (UG/L) }\end{array}$ \\
\hline
\end{tabular}

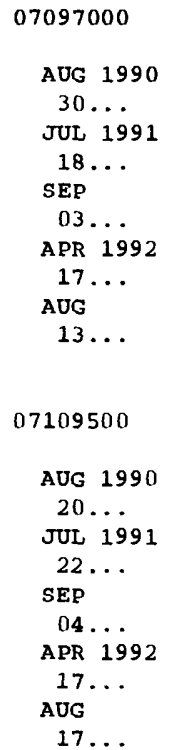

07119700

AUG 1990

$21 \ldots$

JUL 1991

$23 \ldots$

SEP

$04 . .$.

APR 1992

$17 \ldots$

AUG

18 ...

07124000

AUG 1990

$23 \ldots$

JUL 1991

24 ...

SEP

$04 . .$.

APR 1992

17 ...

JUL

17 ...

07130500

AUG 1990

$23 \ldots$
ARKANSAS RIVER AT PORTLAND

\begin{tabular}{|c|c|c|c|c|c|c|c|c|c|c|c|}
\hline$\cdots$ & $\cdots$ & $<0.01$ & $<0.1$ & $<0.10$ & $\cdots$ & $<0.010$ & $<0.010$ & $<0.010$ & $<0.01$ & $\cdots$ & $\cdots$ \\
\hline$<0.5$ & $<0.20$ & $<0.01$ & $<0.1$ & $<0.20$ & $<0.10$ & $<0,010$ & $<0,010$ & $<0.010$ & $<0.01$ & $<0.20$ & $<0.20$ \\
\hline$<0.5$ & $<0.20$ & $<0.01$ & $<0.1$ & $<0.20$ & $<0.20$ & $<0.010$ & $<0.010$ & $<0.010$ & $<0.01$ & $<0.20$ & $<0.20$ \\
\hline$<0.5$ & $<0.20$ & $<0.01$ & $<0.1$ & $<0.20$ & $<0.10$ & $<0.010$ & $<0.010$ & $<0.010$ & $<0.01$ & $<0.20$ & $<0.20$ \\
\hline$<0.5$ & $<0.20$ & $<0.01$ & $<0.1$ & $<0.20$ & $<0.10$ & $<0.010$ & $<0.010$ & $<0.010$ & $<0.01$ & $<0.20$ & $<0.20$ \\
\hline
\end{tabular}

ARKANSAS RIVER NEAR AVONDALE

\begin{tabular}{|c|c|c|c|c|c|c|c|c|c|c|c|}
\hline-- & $\cdots$ & $<0.01$ & $<0.1$ & $<0.10$ & $\cdots$ & $<0.010$ & $<0.010$ & $<0.010$ & $<0.01$ & $\cdots$ & $\cdots$ \\
\hline$<0.5$ & $<0.20$ & $<0.01$ & $<0.1$ & $<0.20$ & $<0.10$ & $<0.010$ & $<0.010$ & $<0.010$ & $<0.01$ & $<0.20$ & $<0.20$ \\
\hline$<0.5$ & $<0.20$ & $<0.01$ & $<0.1$ & $<0.20$ & $<0.10$ & $<0.010$ & $<0.010$ & $<0.010$ & $<0.01$ & $<0.20$ & $<0.20$ \\
\hline$<0.5$ & $<0.20$ & $<0.01$ & $\cdots$ & $<0.20$ & $<0.10$ & $\cdots$ & -. & $\cdots$ & $<0.01$ & $<0.20$ & $<0.20$ \\
\hline$<0.5$ & $<0.20$ & $<0.01$ & $<0.1$ & $<0.20$ & $<0.10$ & $<0.010$ & $<0.010$ & $<0.010$ & $<0.01$ & $<0.20$ & $<0.20$ \\
\hline
\end{tabular}

ARKANSAS RIVER AT CATLIN DAM, NEAR FOWLER

\begin{tabular}{|c|c|c|c|c|c|c|c|c|c|c|c|}
\hline$\cdots$ & -- & $<0.01$ & $<0.1$ & $<0.10$ & $\cdots$ & $<0.010$ & $<0.010$ & $<0.010$ & $<0.01$ & $\cdots$ & $\cdots$ \\
\hline$<0.5$ & $<0.20$ & $<0.01$ & $<0.1$ & $<0.20$ & $<0.10$ & $<0.010$ & $<0.010$ & $<0.010$ & $<0.01$ & $<0.20$ & $<0.20$ \\
\hline$<0.5$ & $<0.20$ & $<0.01$ & $<0.1$ & $<0.20$ & $<0.10$ & $<0.010$ & $<0.010$ & $<0.010$ & $<0.01$ & $<0.20$ & $<0.20$ \\
\hline$<0.5$ & $<0.20$ & $<0.01$ & $<0.1$ & $<0.20$ & $<0.10$ & $<0.010$ & $<0.010$ & $<0.010$ & $<0.01$ & $<0.20$ & $<0.20$ \\
\hline$<0.5$ & $<0.20$ & $<0.01$ & $<0.1$ & $<0.20$ & $<0.10$ & $<0.010$ & $<0.010$ & $<0.010$ & $<0,01$ & $<0.20$ & $<0.20$ \\
\hline
\end{tabular}

ARKANSAS RIVER AT LAS ANIMAS

\begin{tabular}{|c|c|c|c|c|c|c|c|c|c|c|c|}
\hline$\cdots$ & $\cdots$ & $<0.01$ & $<0.1$ & 0.10 & $\cdots$ & $<0.010$ & $<0.010$ & $<0.010$ & $<0.01$ & $\cdots$ & $\cdots$ \\
\hline$<0.5$ & $<0.20$ & $<0.01$ & $<0.1$ & $<0.20$ & $<0.10$ & $<0.010$ & $<0.010$ & $<0.010$ & $<0.01$ & $<0.20$ & $<0.20$ \\
\hline$<0.5$ & $<0.20$ & 0.01 & $<0.1$ & $<0.20$ & $<0.10$ & $<0.010$ & $<0.010$ & $<0.010$ & $<0.01$ & $<0.20$ & $<0.20$ \\
\hline$<0.5$ & $<0.20$ & $<0.01$ & $<0.1$ & $<0.20$ & $<0.10$ & $<0.010$ & $<0.010$ & $<0.010$ & $<0.01$ & $<0.20$ & $<0.20$ \\
\hline$<0.5$ & $<0.20$ & $<0.01$ & $<0.1$ & $<0.20$ & $<0.10$ & $<0.010$ & $<0.010$ & $<0.010$ & $<0.01$ & $<0.20$ & $<0.20$ \\
\hline
\end{tabular}

ARKANSAS RIVER BELOW JOHN MARTIN RESERVOIR

$<0.01<0.1<0.10<--<<0.010<0.010<0.010<0.01$ 
Table 61. Pesticide data for selected surface-water stations on the Arkansas River, 1990-92--Continued

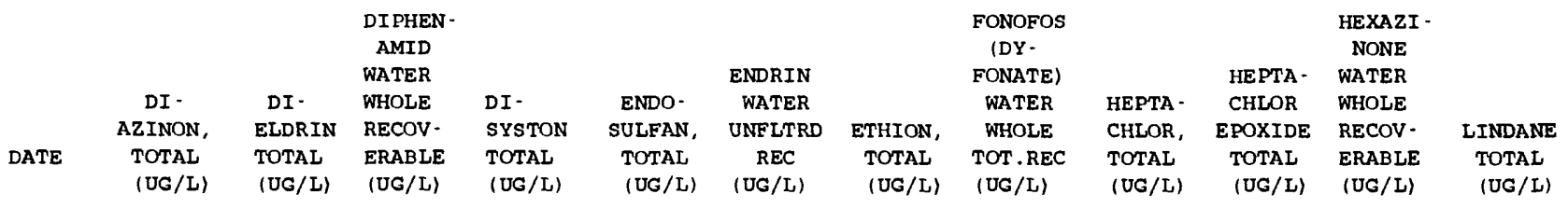

07097000 ARKANSAS RIVER AT PORTLAND

AUG 1990
$30 \ldots$
JUL 1991
$18 \ldots$
SEP
$03 \ldots$
APR 1992
$17 \ldots$
AUG
$13 \ldots$

$\begin{array}{rrrrrrrrrrrrr}<0.01 & <0.010 & \ldots & <0.01 & <0.010 & <0.010 & <0.01 & <0.01 & <0.010 & <0.010 & \cdots & <0.010 \\ <0.01 & <0.010 & <0.1 & <0.01 & <0.010 & <0.010 & <0.01 & <0.01 & <0.010 & <0.010 & <0.20 & <0.010 \\ <0.01 & <0.010 & <0.1 & <0.01 & <0.010 & <0.010 & <0.01 & <0.01 & <0.010 & <0.010 & <0.20 & <0.010 \\ <0.01 & <0.010 & <0.1 & <0.01 & <0.010 & <0.010 & <0.01 & <0.01 & <0.010 & <0.010 & <0.20 & <0.010 \\ <0.01 & <0.010 & <0.1 & <0.01 & <0.010 & <0.010 & <0.01 & <0.01 & <0.010 & <0.010 & <0.20 & <0.010\end{array}$

ARKANSAS RIVER NEAR AVONDALE

AUG 1990

$20 . .$.

JUL 1991

$22 \ldots$

SEP

$04 \ldots$

APR 1992

$17 \ldots$

AUG

$17 \ldots$

$\begin{array}{ccccccccccccc}0.01 & <0.010 & \ldots & <0.01 & <0.010 & <0.010 & <0.01 & <0.01 & <0.010 & <0.010 & \ldots & <0.010 \\ 0.01 & <0.010 & <0.1 & <0.01 & <0.010 & <0.010 & <0.01 & <0.01 & <0.010 & <0.010 & <0.20 & <0.010 \\ 0.16 & <0.010 & <0.1 & <0.01 & <0.010 & <0.010 & <0.01 & <0.01 & <0.010 & <0.010 & <0.20 & <0.010 \\ 0.01 & \ldots & <0.1 & <0.01 & \ldots & \ldots & <0.01 & <0.01 & \ldots & \ldots & <0.20 & \ldots & \\ 0.03 & <0.010 & <0.1 & <0.01 & <0.010 & <0.010 & <0.01 & <0.01 & <0.010 & <0.010 & <0.20 & <0.010\end{array}$

07119700

ARKANSAS RIVER AT CATLIN DAM, NEAR FOWLER

AUG 1990

$21 \ldots$

JUL 1991

$-\quad<0.01<0.010<0.010$

$<0.01$

$<0.01<0.010<0.010$

- $\quad<0.010$

. $<0.010$

$<0.1<0.01$

$<0.010<0.010$

$<0.01$

$<0.01$

$<0.010<0.010$

$<0.20$

$<0.010$

$04 .$.

APR 1992

$17 \ldots$

AUG

$0.01<0.010$

$<0.1<0.01$

$<0.010<0.010<0.01$

$<0.01$

$<0.010<0.010$

$<0.20$

$<0.010$

$0.01<0.010$

$<0.1<0.01$

$<0.010<0.010<0.01$

$<0.01$

$<0.010$

$<0.010$

$<0.20<0.010$

$<0.01<0.010$

$<0.1<0.01$

$<0.010<0.010<0.01$

$<0.01<0.010<0.010$

$<0.20<0.010$

07124000

ARKANSAS RIVER AT LAS ANIMAS

AUG 1990

$23 \ldots$

JUL 1991

$24 \ldots$

SEP

$04 \ldots$

APR 1992

$17 \ldots$

JUL

17 ...

$<0.01<0.010 \quad \cdots \quad<0.01$

.. $<0.010$

$<0.1<0.01$

$<0.010<0.010$

$<0.01$

$<0.01$

$<0.010$

$<0.010$

. $\quad<0.010$

$0.02<0.010$

$<0.01$

$<0.01$

$<0.010<0.010$

$<0.20<0.010$

$<0.01<0.010$

$<0.1<0.01<0.010<0.010$

$<0.01$

$<0.01$

$<0.010<0.010$

$<0.20<0.010$

$<0.01<0.010$

$<0.1<0.01<0.010<0.010$

$<0.01$

$<0.01$

$<0.010<0.010$

$<0.20<0.010$

ARKANSAS RIVER BELOW JOHN MARTIN RESERVOIR

07130500

AUC 1990

$23 \ldots$

$<0.01<0.010$

$<0.0$

$<0.010<0.010$

$<0.01$

$<0.01$

$<0.010<0.010$

$<0.010$ 
Table 61. Pesticide data for selected surface-water stations on the Arkansas River, 1990-92--Continued

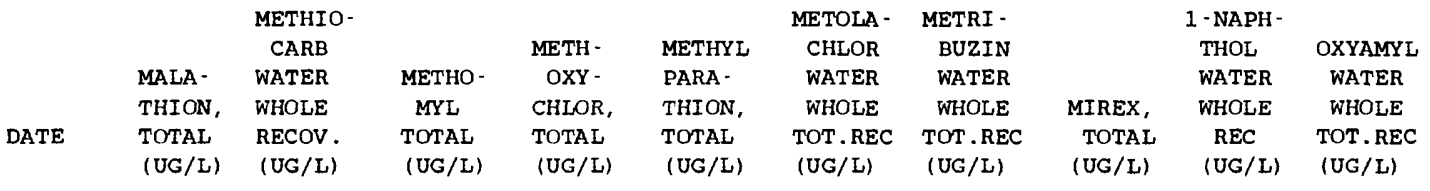

07097000

AUG 1990

$30 \ldots$

JUL 1991

$18 \ldots$

SEP

$03 \ldots$

APR 1992

$17 \ldots$

AUG

$13 \ldots$

ARKANSAS RIVER AT PORTLAND

$<0.01 \quad \cdots$

$<0.01$

$<0.01$

$<0.5$

$<0.5$

$<0.5$

$<0.5$

$<0.01$

$<0.5$

$<0.5$

$<0.01$

$<0.5$

$<0.5$

$<0.01$

$<0.01$

$<0.10$

$<0.01$

$<0.01$

$<0.10$

$<0.01$

$<0.01$

$<0.10$

$<0.01$

$<0.0$

$<0.20$

$<0.01$

ARKANSAS RIVER NEAR AVONDALE

07109500

AUG 1990

$20 .$.

JUL 1991

$22 \ldots$

SEP

$04 \ldots$

APR 1992

$17 . .$.

AUG

$17 \ldots$

$<0.01$

$<0.5$

$<0.5<0.01$

$<0.01$

$<0.20$

ARKANSAS RIVER AT CATLIN DAM, NEAR FOWLER

07119700

AUG 1990

$21 \ldots$

JUL 1991

$23 \ldots$

SEP

$04 \ldots$

APR 1992

$17 \ldots$

AUG

$18 \ldots$

07124000

AUG 1990

$23 \ldots$

JUL 1991

$24 \ldots$

SEP

$04 .$.

APR 1992

$17 \ldots$ JUL

$17 \ldots$

$<0.01$

-

$<0.5$

$<0.01$

$<0.0$

$<0.10$

$<0.01$

$<0.5$

$<0.5$

$<0.01$

$<0.01$

$<0.10$

$<0.10$

$<0.10$

$<0.01$

$<0.01$

$<0.5$

$<0$.

$<0.01$

$<0.01$

$<0.10$

$<0.10$

$<0.01$

$<0.01$

$<0.5$

$<0.5$

$<0.01$

$<0.0$

$<0.20$

$<0.10$

$<0.01$

$<0.5$

$<0$.

$<0.01$

$<0.01$

$<0.20$

$<0.10$

$<0.01$

$<0.01$

$<0.01$

ARKANSAS RIVER AT LAS ANIMAS

$<0.01$

-

$<0.5$

$<0.01$

$<0.01$

$<0.10$

$<0.01$

$<0.5$

$<0.5$

$<0.01$

$<0.01$

$<0.10$

$<0.10$

0.01

$<0.5$

$<0.5<0.01$

$<0.01$

$<0.20$

$<0.10$

$<0.01$

$<0.5$

$<0.5<0.01$

$<0.01$

$<0.20$

$<0.10$

$<0.10$

$<0.20<0.10$

$<0.5<0.5$

$<0.01$

$<0.01$

ARKANSAS RIVER BELOW JOHN MARTIN RESERVOIR

07130500

AUG 1990

$23 \ldots$

$<0.01$

$<0.5<0.01<0.01$
$<0.10$
$<0.01$

$<0.01$

$<0$.

$<0.01$

$<0.01$

$<0.5$

$<0.5$

$<0.5$ 
Table 61. Pesticide data for selected surface-water stations on the Arkansas River, 1990-92--Continued

07097000

AUG 1990

$30 \ldots$

JUL 1991

$18 \ldots$

SEP

$03 . .$.

APR 1992

$17 \ldots$

AUG

$13 \ldots$

$\begin{array}{lcc} & & \\ \text { PARA- } & \text { PER - } & \\ \text { THION, } & \text { THANE } & \text { PHORATE } \\ \text { TOTAL } & \text { TOTAL } & \text { TOTAL } \\ \text { (UG/L) } & \text { (UG/L) } & \text { (UG/L) }\end{array}$

PROPA CHLOR

WATER WHOLE

RECOV (UG/L) (UG/L)

\begin{tabular}{lclcc}
\multicolumn{5}{c}{ PROPO- } \\
XUR \\
PRO- & & WATER & \\
PAZINE & PROPHAM & WHOLE & SEVIN, & SILVEX, \\
TOTAL & TOTAL & RECOV. & TOTAL & TOTAL \\
(UG/L) & (UG/L) & (UG/L) & (UG/L) & (UG/L)
\end{tabular}

ARKANSAS RIVER AT PORTLAND

AUG 1990

20 ...

JUL 1991

$22 \ldots$

SEP

$04 \ldots$

APR 1992

$17 .$.

AUG

$17 \ldots$

07119700

AUG 1990

$21 \ldots$

JUL 1991

$23 \ldots$

SEP

$04 \ldots$

APR 1992

$17 \ldots$

AUG

$18 \ldots$
07109500

$<0.01$

$<0.01$

$<0.1<0.01<0.20$

$<0.01$

$<0.1<0.01<0.20$

$<0.01$

$<0$.

$<0.01$

$<0.20$

$<0.01$

$<0.1<0.01<0.20$

$<0.10$

-.

$<0.10$

$<0.5$

$-\cdot$

$<0.5<0.01$

$<0.10<0.10<0.10$

$<0.5$

$<0.5$

$<0.5<0.01$

$<0.10<0.10$

$<0.10$

$<0.5$

$<0.5$

$<0.5<0.01$

$<0.10<0.10$

$<0.10$

$<0.5$

$<0.5$

$<0.5<0.01$

$<0.10<0.10<0.10$

ARKANSAS RIVER NEAR AVONDALE

$<0.01$

$<0.01$

$<0.1<0.01$

0.10

$<0.10$

-

$<0.10$

$<0.5$

-

$<0.5<0.01$

$<0.01$

$<0.1<0.01$

$<0.20$

$<0.10<0.10$

$<0.10$

$<0.5$

$<0.5$

$<0.5<0.01$

$<0.1<0.01$

0.20

$<0.10$

$<0.10$

$<0.10$

$<0.5$

$<0.5$

$<0.5<0.01$

$<0.01$

$\begin{array}{ll}\text {. } & <0.01<0.20\end{array}$

$<0.10$

$<0.10$

$<0.10$

$<0.5$

$<0.5$

$<0.5<0.01$

$<0.01$

$<0.1<0.01$

$<0.20$

$<0.10$

$<0.10$

$<0.10$

$<0.5$

$<0.5$

$<0.5<0.01$

ARKANSAS RIVER AT CATLIN DAM, NEAR FOWLER

$<0.01$

$<0.1$

$<0.01$

0.10

$<0.10$

$+<\quad<0.10$

$<0.5$

$<0.10$

$<0.10$

$<0.5$

$<0$

$<0.01$

$<0.1<0.01$

$<0.20$

$<0.10$

$<0.10$

$<0.10$

$<0.5$

$<0.5$

$<0.5<0.01$

$<0.01$

$<0$.

$<0.20$

$<0.10$

$<0.10$

$<0.10$

$<0.5$

$<0.5$

$<0.5<0.01$

$<0.01$

$<0.1$

$<0.01$

$<0.20$

$<0.10$

$<0.10$

$<0.10$

ARKANSAS RIVER AT LAS ANIMAS

AUG 1990

$23 \ldots$

JUL 1991

24 ...

SEP

$04 \ldots$

APR 1992

$17 \ldots$

JUL

$17 .$.

$<0.01$

$<0.1<0.01$

0.10

$<0.10$

-

$<0.10$

$<0.5$

$-\cdot$

$<0.5<0.01$

$<0.01$

$<0.1<0.01$

$<0.20$

$<0.10$

$<0.10$

$<0.10$

$<0.5$

$<0.5$

$<0.5<0.01$

$<0.01$

$<0.1<0.01$

0.20

$<0.10$

$<0.10$

$<0.10$

$<0.5$

$<0.5$

$<0.5<0.01$

$<0.01$

$<0.1<0.01<0.20$

$<0.10$

$<0.10$

$<0.10$

$<0.5$

$<0.5$

$<0.5<0.01$

$<0.01$

$<0.1<0.01$

$<0.20$

$<0.10$

$<0.10<0.10$

$<0.5$

$<0.5$

$<0.5<0.01$

07130500

AUG 1990

$23 . .$.

ARKANSAS RIVER BELOW JOHN MARTIN RESERVOIR

$<0.1<0.01$

0.10

$<0.10$

-

$<0.10$

$<0.5$

$\cdots$

$<0.5<0.01$ 
Table 61. Pesticide data for selected surface-water stations on the Arkansas River, 1990-92--Continued

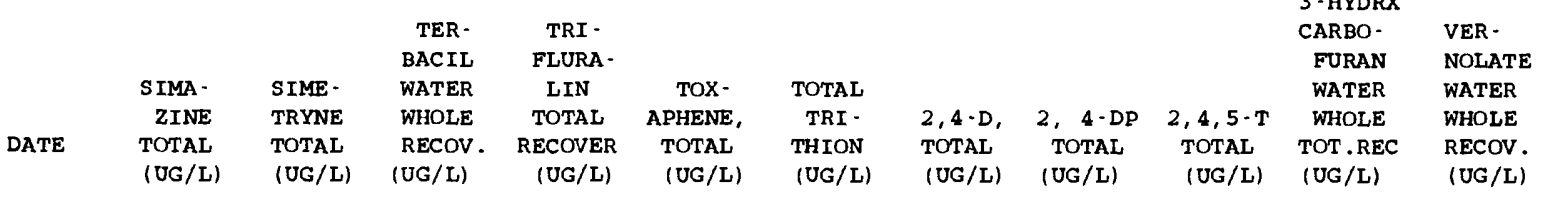

\begin{tabular}{|c|c|c|c|c|c|c|c|c|c|c|c|}
\hline 07097000 & ARKAN & RIVER & PORTLA & & & & & & & & \\
\hline AUG 1990 & & & & & & & & & & & \\
\hline $\begin{array}{l}30 \ldots \\
\text { JUL } 1991\end{array}$ & $<0.10$ & $<0.10$ & $\cdots$ & $<0.10$ & $<1$ & $<0.01$ & $<0.01$ & $<0.01$ & $<0.01$ & $\cdots$ & \\
\hline $\begin{array}{l}18 \ldots \\
\text { SEP }\end{array}$ & $<0.10$ & $<0.10$ & $<0.20$ & $<0.10$ & $<1$ & $<0.01$ & $<0.01$ & $<0.01$ & $<0.01$ & $<0.5$ & $<0.10$ \\
\hline $\begin{array}{l}03 \ldots \\
\text { APR } 1992\end{array}$ & $<0.10$ & $<0.10$ & $<0.20$ & $<0.10$ & $<1$ & $<0.01$ & 0.05 & $<0.01$ & $<0.01$ & $<0.5$ & $<0.10$ \\
\hline $\begin{array}{l}17 \ldots \\
\text { AUG }\end{array}$ & $<0.10$ & $<0.10$ & $<0.20$ & $<0.10$ & $<1$ & $<0.01$ & $<0.01$ & $<0.01$ & $<0.01$ & $<0.5$ & $<0.10$ \\
\hline $13 \ldots$ & $<0.10$ & $<0.10$ & $<0.20$ & $<0.10$ & $<1$ & $<0.01$ & 0.01 & $<0.01$ & $<0.01$ & $<0.5$ & $<0.10$ \\
\hline
\end{tabular}

$07109500 \quad$ ARKANSAS RIVER NEAR AVONDALE

\begin{tabular}{|c|c|c|c|c|c|c|c|c|c|c|c|}
\hline $\begin{array}{l}\text { AUG } 1990 \\
20 \ldots \\
\text { JUL } 1991\end{array}$ & $<0.10$ & $<0.10$ & $\cdots$ & $<0.10$ & $<1$ & $<0.01$ & 0.04 & $<0.01$ & $<0.01$ & - & - \\
\hline $\begin{array}{l}22 \ldots \\
\text { SEP }\end{array}$ & $<0.10$ & $<0.10$ & $<0.20$ & $<0.10$ & $<1$ & $<0.01$ & 0.04 & $<0.01$ & $<0.01$ & $<0.5$ & $<0.10$ \\
\hline $\begin{array}{l}04 \ldots \\
\text { APR } 1992\end{array}$ & $<0.10$ & $<0.10$ & $<0.20$ & $<0.10$ & $<1$ & $<0.01$ & 0.33 & 0.01 & $<0.01$ & $<0.5$ & $<0.10$ \\
\hline${ }_{A \cup G}^{17 \ldots}$ & $<0.10$ & $<0.10$ & $<0.20$ & $<0.10$ & $\cdots$ & $<0.01$ & 0.16 & 0.01 & $<0.01$ & $<0.5$ & $<0.10$ \\
\hline $17 \ldots$ & $<0.10$ & $<0.10$ & $<0.20$ & $<0.10$ & $<1$ & $<0.01$ & 0.01 & $<0.01$ & $<0.01$ & $<0.5$ & $<0.10$ \\
\hline
\end{tabular}

07119700 ARKANSAS RIVER AT CATLIN DAM, NEAR FOWLER

AUG 1990

$21 \ldots$

JUL 1991

$23 . .$.

SEP

$04 \ldots$

APR 1992

$17 \ldots$

AUG

$18 \ldots$

07124000

AUG 1990

$23 . .$.

JUL 1991

$24 \ldots$

SEP

$04 \ldots$

APR 1992

$17 \ldots$

JUL

$17 .$.

07130500

AUG 1990

$23 . .$.

$<0.10<0.10$

$<0.10<0.10$

$<0.10<0.10$

$<0.10<0.10$

$<0.10<0.10$

$<0.10$

$<0.20$

$<0.10$

$<0.20$

$<0.10$

$<0.20$

$<0.10$

$<0.20$

$<0.10$

$<1$
$<1$
$<1$
$<1$
$<1$

ARKANSAS RIVER AT LAS ANIMAS

$<0.10<0.10 \quad \ldots \quad<0.10<1$

$<0.10<0.10$

$<0.20$

$<0.10$

$<1$

$<0.20<0.10<1$

$<0.10<0.10$

$<0.10$

ARKANSAS RIVER BELOW JOHN MARTIN RESERVOIR

$<0.10<0.10$
-

$<0.01$

$<0.01$

$<0.01$

$<0.01$

$<0.01$

$<0.01$

0.04

0.02

$<0.01$

$<0.01$

0.01

$<0.01$

0.08

$<0.01$

0.30

$<0.01$

$<0.01$

0.26

$<0.01$

$<0.01$

$<0.01$

$<0.01$ 
Table 62. Radiochemical data for selected surface-water stations on the Arkansas River, 1990-92

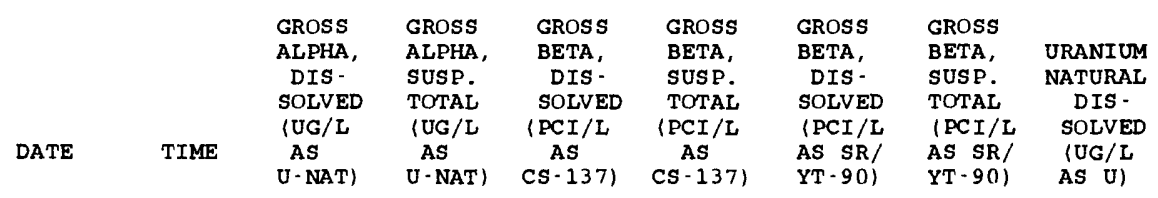

07087200 ARKANSAS RIVER AT BUENA VISTA

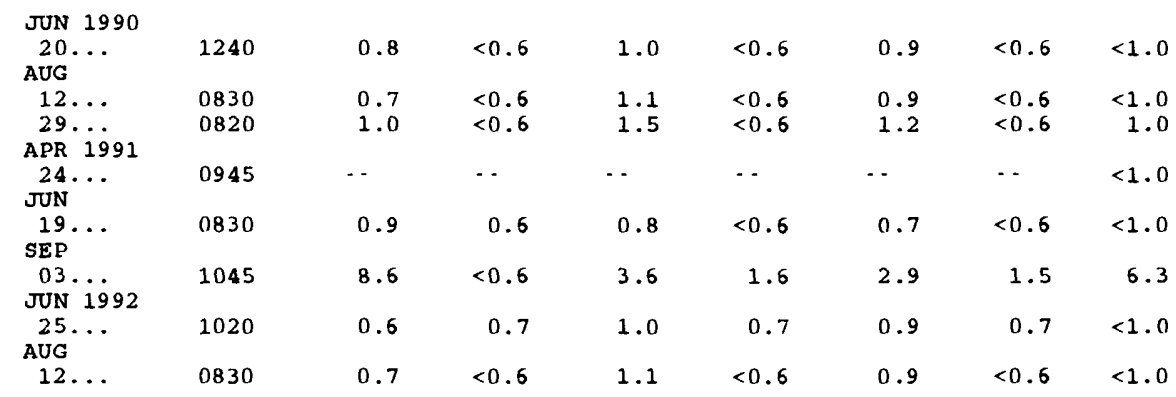

07093700 ARKANSAS RIVER NEAR WELLSVILLE

\begin{tabular}{|c|c|c|c|c|c|c|c|c|}
\hline $20 \ldots$ & 1730 & 1.7 & 1.2 & 1.5 & 1.5 & 1.3 & 1.2 & $<1.0$ \\
\hline $\begin{array}{l}12 \ldots \\
29 \ldots\end{array}$ & $\begin{array}{l}1450 \\
1400\end{array}$ & $\begin{array}{l}3.2 \\
7.0\end{array}$ & $\begin{array}{r}0.7 \\
<0.6\end{array}$ & $\begin{array}{l}2.9 \\
2.6\end{array}$ & $\begin{array}{l}0.8 \\
0.6\end{array}$ & $\begin{array}{l}2.4 \\
2.0\end{array}$ & $\begin{array}{r}0.8 \\
<0.6\end{array}$ & $\begin{array}{l}2.2 \\
3.7\end{array}$ \\
\hline APR 1991 & & & & & & & & \\
\hline JUN $25 \ldots$ & 0745 & $\cdots$ & $\cdots$ & -. & $\cdots$ & $\cdots$ & $\cdots$ & 2.3 \\
\hline$\underset{\text { SEP }}{19} \cdots$ & 1845 & 1.5 & 1.0 & 1.5 & 1.2 & 1.3 & 1.2 & 1.7 \\
\hline JUN 1992 & 1340 & 6.0 & 1.4 & 2.5 & 3.0 & 2.1 & 2.8 & 6.9 \\
\hline$\underset{\text { AUG }}{25} \ldots$ & 1430 & 2.1 & $<0.6$ & 1.6 & 1.0 & 1.4 & 0.9 & 3.4 \\
\hline $12 \ldots$ & 1450 & 3.2 & 0.7 & 2.9 & 0.8 & 2.4 & 0.8 & 2.2 \\
\hline
\end{tabular}

07094500 ARKANSAS RIVER AT PARKDALE

\begin{tabular}{|c|c|c|c|c|c|c|c|c|}
\hline $\begin{array}{c}\text { JUN } 1990 \\
21 . \ldots\end{array}$ & 0840 & 4.0 & 1.9 & 5.0 & 2.9 & 4.0 & 2.3 & $<1.0$ \\
\hline AUG & & & & & & & & \\
\hline $\begin{array}{l}30 \\
\text { APR }\end{array}$ & 1110 & 4.7 & $<0.6$ & 4.8 & 0.7 & 3.6 & $<0.6$ & 4.0 \\
\hline JUN & 1400 & $\cdots$ & - & $\cdots$ & $\cdots$ & $\cdots$ & $\cdots$ & 3.7 \\
\hline $20 \ldots$ & 0910 & 1.8 & 2.4 & 1.8 & 2.6 & 1.5 & 2.4 & 2.2 \\
\hline $\begin{array}{l}03 . . \\
\text { JUN } 1992\end{array}$ & 1625 & 12 & 0.7 & 5.2 & 2.3 & 4.0 & 2.2 & 7.5 \\
\hline$\stackrel{26}{26}$. & 0900 & 2.0 & 26 & 1.9 & 13 & 1.6 & 12 & 2.5 \\
\hline $13 \ldots$ & 1000 & 3.8 & 1.3 & 3.1 & 2.0 & 2.3 & 1.9 & 2.1 \\
\hline
\end{tabular}

07097000

ARKANSAS RIVER AT PORTLAND

\begin{tabular}{|c|c|c|c|c|c|c|c|c|}
\hline $\begin{array}{c}\pi U N 1990 \\
21 \ldots\end{array}$ & 1400 & 2.7 & 4.9 & 3.3 & 4.1 & 2.6 & 3.4 & 1.5 \\
\hline AUG & & & & & & & & \\
\hline $30 \ldots$ & 1415 & 10 & $<0.6$ & 5.3 & $<0.6$ & 4.0 & $<0.6$ & 5.4 \\
\hline $\begin{array}{l}\text { APR } 1991 \\
26 . \ldots \\
\text { JUN }\end{array}$ & 1015 & $\cdots$ & $\cdots$ & $\cdots$ & $\cdots$ & $\cdots$ & $\cdots$ & 5.0 \\
\hline$\underset{\text { SEP }}{20} \ldots$ & 1420 & 1.8 & 1.7 & 1.6 & 2.4 & 1.3 & 2.2 & 2.0 \\
\hline JUN 1992 & 1900 & 1.6 & $<0.6$ & 1.7 & 0.8 & 1.4 & 0.8 & 1.2 \\
\hline$\underset{\text { AUG }}{26} \cdots$ & 1400 & 3.7 & 12 & 2.9 & 8.6 & 2.3 & 8.0 & 4.3 \\
\hline $13 \ldots$ & 1330 & 4.9 & 2.0 & 3.6 & 3.2 & 2.6 & 3.0 & 3.8 \\
\hline
\end{tabular}


Table 62. Radiochemical data for selected surface-water stations on the Arkansas River, 1990-92-Continued

\begin{tabular}{|c|c|c|c|c|c|c|c|c|}
\hline & & $\begin{array}{l}\text { GROSS } \\
\text { ALPHA, } \\
\text { DIS - } \\
\text { SOLVED } \\
\text { (UG/L }\end{array}$ & $\begin{array}{l}\text { GROSS } \\
\text { ALPHA, } \\
\text { SUSP. } \\
\text { TOTAL } \\
\text { (UG /L }\end{array}$ & $\begin{array}{l}\text { GROSS } \\
\text { BETA, } \\
\text { DIS- } \\
\text { SOLVED } \\
\text { (PCI/L }\end{array}$ & $\begin{array}{c}\text { GROSS } \\
\text { BETA, } \\
\text { SUSP. } \\
\text { TOTAL } \\
\text { (PCI/L }\end{array}$ & $\begin{array}{l}\text { GROSS } \\
\text { BETA, } \\
\text { DIS- } \\
\text { SOLVED } \\
\text { (PCI/L }\end{array}$ & $\begin{array}{l}\text { GROSS } \\
\text { BETA, } \\
\text { SUSP. } \\
\text { TOTAL } \\
\text { (PCI /L }\end{array}$ & $\begin{array}{c}\text { URANIUM } \\
\text { NATURAL } \\
\text { DIS - } \\
\text { SOLVED }\end{array}$ \\
\hline TE & TIME & $\begin{array}{c}\text { AS } \\
\text { U-NAT) }\end{array}$ & $\begin{array}{c}\text { AS } \\
\text { U-NAT) }\end{array}$ & $\begin{array}{c}\text { As } \\
\text { Cs -137) }\end{array}$ & $\begin{array}{c}\text { AS } \\
\text { CS-137) }\end{array}$ & $\begin{array}{l}\text { AS SR/ } \\
\text { YT-90) }\end{array}$ & $\begin{array}{l}\text { AS SR/ } \\
\text { YT }-90)\end{array}$ & $\begin{array}{l}(U G / L \\
A S U)\end{array}$ \\
\hline
\end{tabular}

$07109500 \quad$ ARKANSAS RIVER NEAR AVONDALE

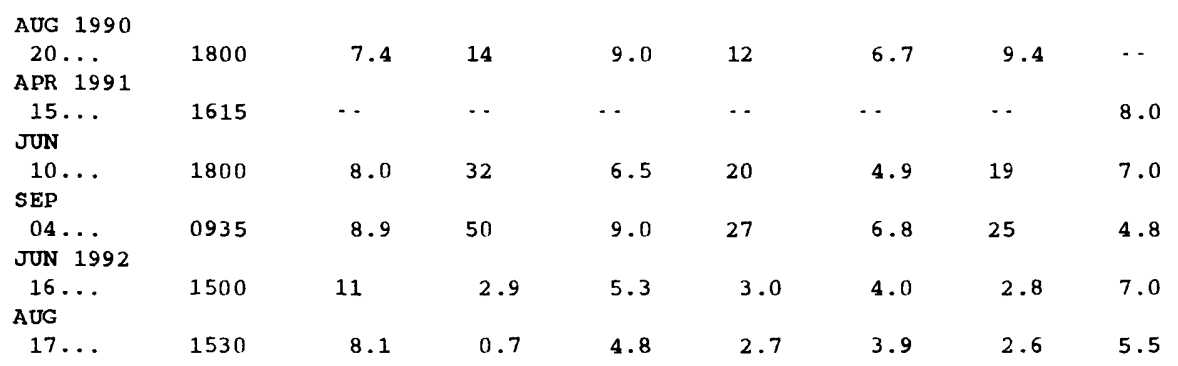

07119700

ARKANSAS RIVER AT CATLIN DAM, NEAR FOWLER

\begin{tabular}{|c|c|c|c|c|c|c|c|c|}
\hline \multicolumn{9}{|l|}{ AUG 1990} \\
\hline $21 \ldots$ & 1600 & 15 & 62 & 9.9 & 32 & 7.5 & 29 & $\cdots$ \\
\hline \multicolumn{9}{|l|}{ APR 1991} \\
\hline $16 \ldots$ & 1340 & $\cdots$ & $\cdots$ & $\cdots$ & $\cdots$ & $\cdots$ & $\cdots$ & 11 \\
\hline \multicolumn{9}{|l|}{ JUN } \\
\hline $11 \ldots$ & 1430 & 9.3 & 59 & 5.8 & 35 & 4.3 & 32 & 8.5 \\
\hline \multicolumn{9}{|l|}{ SEP } \\
\hline $04 \ldots$ & 1445 & 12 & 430 & 8.6 & 590 & 6.6 & 550 & 4.8 \\
\hline \multicolumn{9}{|l|}{ JUN 1992} \\
\hline $17 \ldots$ & 1030 & 10 & 9.5 & 5.8 & 8.4 & 4.3 & 7.6 & 7.4 \\
\hline \multicolumn{9}{|l|}{ AUG } \\
\hline $18 \ldots$ & 1230 & 8.9 & 550 & 7.3 & 330 & 6.0 & 310 & 5.2 \\
\hline
\end{tabular}

07124000

ARKANSAS RIVER AT LAS ANIMAS

\begin{tabular}{|c|c|c|c|c|c|c|c|c|}
\hline \multicolumn{9}{|l|}{ AUG 1990} \\
\hline $23 \ldots$ & 0800 & 28 & 460 & 18 & 410 & 14 & 390 & $\cdots$ \\
\hline \multicolumn{9}{|l|}{ APR 1991} \\
\hline $17 \ldots$ & 1215 & $\cdots$ & $\cdots$ & $\cdots$ & $\cdots$ & $\cdots$ & $\cdots$ & 36 \\
\hline \multicolumn{9}{|l|}{ JUN } \\
\hline $12 \ldots$ & 1300 & 16 & 51 & 5.6 & 33 & 4.3 & 30 & 12 \\
\hline \multicolumn{9}{|l|}{ SEP } \\
\hline $04 \ldots$ & 1815 & 20 & 320 & 12 & 160 & 9.1 & 150 & 13 \\
\hline \multicolumn{9}{|l|}{ JUN 1992} \\
\hline $18 \ldots$ & 0800 & 19 & 28 & 9.3 & 26 & 7.1 & 24 & 13 \\
\hline \multicolumn{9}{|l|}{ AUG } \\
\hline $19 \ldots$ & 1000 & 30 & 47 & 11 & 34 & 8.7 & 31 & 15 \\
\hline
\end{tabular}

ARKANSAS RIVER BELOW JOHN MARTIN RESERVOIR

\begin{tabular}{|c|c|c|c|c|c|c|c|c|}
\hline \multicolumn{9}{|l|}{ AUG 1990} \\
\hline $23 \ldots$ & 1130 & 14 & 0.7 & 13 & 1.7 & 9.6 & 1.4 & $\cdots$ \\
\hline \multicolumn{9}{|l|}{ JUN 1991} \\
\hline $13 \ldots$ & 0840 & 28 & 1.7 & 12 & 5.7 & 8.8 & 5.5 & 29 \\
\hline \multicolumn{9}{|l|}{ SEP } \\
\hline $05 \ldots$ & 0905 & 19 & 2.2 & 13 & 5.0 & 9.8 & 4.7 & 14 \\
\hline \multicolumn{9}{|l|}{ JUN 1992} \\
\hline $18 \ldots$ & 1100 & 21 & 0.8 & 18 & 4.1 & 13 & 3.9 & 29 \\
\hline \multicolumn{9}{|l|}{ AUG } \\
\hline $19 \ldots$ & 1145 & 20 & 1.4 & 12 & 7.1 & 9.2 & 6.8 & 11 \\
\hline
\end{tabular}


Table 63. Suspended-sediment data for selected surface-water stations on or near the Arkansas River, 1990-93

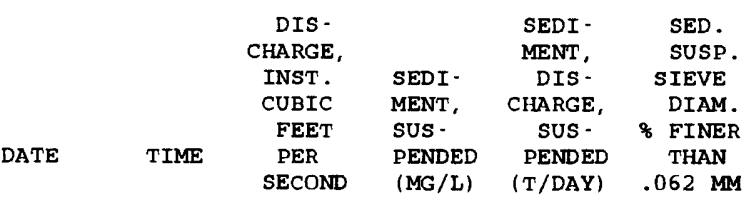

07081200

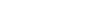

ARKANSAS RIVER NEAR LEADVILLE

\begin{tabular}{|c|c|c|}
\hline \multicolumn{3}{|l|}{ APR 1990} \\
\hline $17 \ldots$ & 1315 & - \\
\hline \multicolumn{3}{|l|}{ MAY } \\
\hline $22 \ldots$ & 1230 & 128 \\
\hline \multicolumn{3}{|l|}{ JUN } \\
\hline $05 \ldots$ & 1135 & 493 \\
\hline $19 \ldots$ & 1200 & 271 \\
\hline \multicolumn{3}{|l|}{ JUL } \\
\hline $17 \ldots$ & 1100 & 70 \\
\hline \multicolumn{3}{|l|}{ AUG } \\
\hline $28 \ldots$ & 1030 & 32 \\
\hline \multicolumn{3}{|l|}{ OCT } \\
\hline $29 \ldots$ & 1445 & 27 \\
\hline \multicolumn{3}{|l|}{$\mathrm{DEC}$} \\
\hline \multicolumn{2}{|l|}{ MAR 1991} & 18 \\
\hline $25 \ldots$ & 1350 & 17 \\
\hline \multicolumn{3}{|l|}{ APR } \\
\hline $23 \ldots$ & 0755 & 27 \\
\hline \multicolumn{3}{|l|}{ MAY } \\
\hline $14 \ldots$ & 0820 & 94 \\
\hline \multicolumn{3}{|l|}{ JUN } \\
\hline $18 \ldots$ & 0945 & 325 \\
\hline \multicolumn{3}{|l|}{ JUL } \\
\hline $16 \ldots$ & 0945 & 89 \\
\hline \multicolumn{3}{|l|}{ AUG } \\
\hline $13 \ldots$ & 0615 & 73 \\
\hline \multicolumn{3}{|l|}{ OCT } \\
\hline $22 \ldots$ & 0850 & 20 \\
\hline \multicolumn{3}{|l|}{ DEC } \\
\hline $16 \ldots$ & 1445 & 16 \\
\hline \multicolumn{3}{|l|}{ MAR 1992} \\
\hline $23 \ldots$ & 1340 & 32 \\
\hline \multicolumn{3}{|l|}{ APR } \\
\hline $20 \ldots$ & 1850 & 39 \\
\hline \multicolumn{3}{|l|}{ MAY } \\
\hline $20 \ldots$ & 1100 & 249 \\
\hline \multicolumn{3}{|l|}{ JUN } \\
\hline $24 \ldots$ & 1330 & 229 \\
\hline \multicolumn{3}{|l|}{ JUL } \\
\hline $13 \ldots$ & 1200 & 124 \\
\hline \multicolumn{3}{|l|}{ AUG } \\
\hline $11 \ldots$ & 1000 & 58 \\
\hline $26 \ldots$ & 0750 & 67 \\
\hline \multicolumn{3}{|l|}{ OCT } \\
\hline $26 \ldots$ & 1310 & 31 \\
\hline \multicolumn{3}{|l|}{ JAN 1993} \\
\hline $11 \ldots$ & 1200 & E26 \\
\hline MAR & & \\
\hline $22 \ldots$ & 1000 & E2 6 \\
\hline
\end{tabular}

\begin{tabular}{|c|c|c|}
\hline 6 & $\cdots$ & $\cdots$ \\
\hline 14 & 4.8 & 58 \\
\hline $\begin{array}{r}245 \\
28\end{array}$ & $\begin{array}{r}326 \\
20\end{array}$ & $\begin{array}{l}18 \\
52\end{array}$ \\
\hline 15 & 2.8 & 75 \\
\hline 2 & 0.17 & 82 \\
\hline 8 & 0.60 & - \\
\hline 1 & 0.04 & $\cdots$ \\
\hline 1 & 0.05 & - \\
\hline 181 & 13 & $\cdots$ \\
\hline 11 & 2.8 & 61 \\
\hline 37 & 32 & 24 \\
\hline 5 & 1.1 & $\cdots$ \\
\hline 13 & 2.5 & $\cdots$ \\
\hline 4 & 0.22 & 79 \\
\hline 6 & 0.24 & - \\
\hline 4 & 0.38 & - \\
\hline 4 & 0.42 & 59 \\
\hline 33 & 22 & 48 \\
\hline 19 & 12 & $\cdots$ \\
\hline 12 & 3.9 & $\cdots$ \\
\hline 3 & 0.49 & $\cdots$ \\
\hline 7 & 1.3 & 70 \\
\hline 2 & 0.17 & $\cdots$ \\
\hline 2 & - & 87 \\
\hline 2 & -. & 69 \\
\hline
\end{tabular}

ARKANSAS RIVER BELOW EMPIRE GULCH, NEAR MALTA

$\begin{array}{lrrrrr}\text { APR } 1990 & & & & \\ 17 \ldots & 1720 & 91 & 10 & 2.5 & 64 \\ \begin{array}{l}\text { MAY } \\ 22 \ldots\end{array} & 1615 & 140 & 16 & 6.0 & 52 \\ \text { JUN } & & & & & \\ 05 \ldots & 1430 & 512 & 73 & 101 & 59 \\ 19 \ldots & 1510 & 360 & 34 & 33 & 47 \\ \begin{array}{r}\text { JUL } \\ 17 \ldots\end{array} & 1420 & 139 & 24 & 9.0 & 7 \\ \begin{array}{l}\text { AUG } \\ 28 \ldots\end{array} & 1245 & 72 & 3 & 0.58 & 79 \\ \begin{array}{l}\text { OCT } \\ 30 \ldots\end{array} & 1300 & 99 & 1 & 0.37 & \ldots \\ \begin{array}{l}\text { DEC } \\ 19 \ldots\end{array} & 1415 & 48 & 5 & 0.65 & 54\end{array}$


Table 63. Suspended-sediment data for selected surface-water stations on or near the Arkansas River, 1990-93--Continued

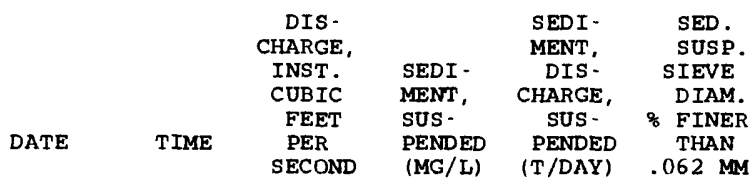

ARKANSAS RIVER BELOW EMPIRE GULCH, NEAR MALTA-Continued

\begin{tabular}{|c|c|c|}
\hline $25 \ldots$ & 1550 & 57 \\
\hline APR & & \\
\hline $23 \ldots$ & 1325 & 79 \\
\hline MAY & & \\
\hline $14 \ldots$ & 1400 & 156 \\
\hline JUN & & \\
\hline$\frac{18}{J U L} \cdots$ & 1345 & 427 \\
\hline $16 \ldots$ & 1600 & 137 \\
\hline AUG & & \\
\hline $13 \ldots$ & 1315 & 132 \\
\hline OCT & & \\
\hline $22 \ldots$ & 1430 & \\
\hline DEC & & \\
\hline $17 \ldots$ & 0900 & 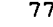 \\
\hline MAR 1992 & & \\
\hline APR & 1530 & \\
\hline $21 \ldots$ & 1030 & \\
\hline MAY & & \\
\hline $20 \ldots$ & 1520 & 297 \\
\hline JUN & & \\
\hline $24 \ldots$ & 1710 & 350 \\
\hline JUL & & \\
\hline $\begin{array}{l}13 \\
A U G\end{array}$ & 1615 & \\
\hline $11 \ldots$ & 1330 & 134 \\
\hline $26 \ldots$ & 1000 & 180 \\
\hline OCT & & \\
\hline${ }_{\text {JAN }}^{26} \ddot{1993}$ & 1515 & \\
\hline $11 \ldots$ & 1440 & 45 \\
\hline MAR & & \\
\hline & 1330 & \\
\hline
\end{tabular}

07086000

ARKANSAS RIVER AT GRANITE

JUN 1990

$19 . .$.

$1920 \quad 1220$

1240

72

07087200 ARKANSAS RIVER AT BUENA VISTA

\begin{tabular}{|c|c|c|c|c|c|}
\hline $\begin{array}{l}\text { APR } 1990 \\
\text { IB . . }\end{array}$ & 1530 & 171 & B & 3.7 & $\cdots$ \\
\hline$\underset{\text { JUN }}{23} \cdots$ & 0900 & 519 & 39 & 55 & 58 \\
\hline $\begin{array}{l}06 \ldots \\
20 \ldots\end{array}$ & $\begin{array}{l}1100 \\
1240\end{array}$ & $\begin{array}{l}2240 \\
1690\end{array}$ & $\begin{array}{r}187 \\
17\end{array}$ & $\begin{array}{r}1130 \\
77\end{array}$ & ${ }^{42}$ \\
\hline $\begin{array}{l}\text { JUL } \\
18 \ldots\end{array}$ & 1110 & 970 & 6 & 16 & 67 \\
\hline & 0820 & 227 & 2 & 1.2 & 82 \\
\hline $\begin{array}{l}\text { OCT } \\
31 \ldots \\
\text { DEC }\end{array}$ & 1000 & 167 & 2 & 0.77 & $\cdots$ \\
\hline JAN 1991 & 0900 & 219 & 2 & 0.95 & $\cdots$ \\
\hline${ }_{\text {MAR }}^{16}$ & 1310 & 517 & 11 & 15 & $\cdots$ \\
\hline$\underset{\mathrm{APR}}{26} \ldots$ & 0830 & 252 & 1 & 0.75 & $\cdots$ \\
\hline$\underset{\text { MAY }}{24} \cdots$ & 0945 & E378 & 5 & $\cdots$ & $\cdots$ \\
\hline$\frac{15}{\text { JUN }} \cdots$ & 1130 & 868 & 14 & 33 & 76 \\
\hline${ }_{\text {JUL }}^{19} \cdots$ & 0830 & 1780 & 27 & 130 & 25 \\
\hline $17 \ldots$ & 0730 & 648 & 4 & 6.5 & $\cdots$ \\
\hline $\begin{array}{l}\text { AUG } \\
14 \\
\text { OCT }\end{array}$ & 0800 & 423 & 3 & 3.1 & $-\cdot$ \\
\hline$\underset{D E C}{23} \cdots$ & 0825 & 114 & 2 & 0.52 & $\cdots$ \\
\hline $17 \ldots$ & 1320 & 254 & 5 & 3.6 & $\cdots$ \\
\hline
\end{tabular}


Table 63. Suspended-sediment data for selected surface-water stations on or near the Arkansas River, 1990-93--Continued

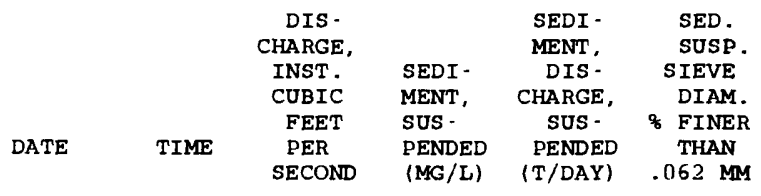

07087200 ARKANSAS RIVER AT BUENA VISTA--Continued

\begin{tabular}{|c|c|c|c|c|c|}
\hline \multicolumn{6}{|l|}{ MAR 1992} \\
\hline $\begin{array}{l}24 \ldots \\
\text { APR }\end{array}$ & 0830 & 219 & 2 & 1.4 & $\cdots$ \\
\hline $22 \ldots$ & 0815 & 192 & 2 & 0.93 & $\cdots$ \\
\hline MAY & & & & & \\
\hline$\frac{21}{\text { JUN }} \cdots$ & 0840 & 1210 & 42 & 137 & 50 \\
\hline $25 \ldots$ & 1020 & 1250 & 10 & 34 & 72 \\
\hline $\begin{array}{l}14 \\
\text { AUG }\end{array}$ & 0715 & 634 & 7 & 12 & $\cdots$ \\
\hline $\begin{array}{l}12 \ldots \\
25 \ldots\end{array}$ & $\begin{array}{l}0830 \\
1745\end{array}$ & $\begin{array}{l}595 \\
462\end{array}$ & $\begin{array}{r}3 \\
46\end{array}$ & $\begin{array}{l}5.1 \\
57\end{array}$ & 21 \\
\hline OCT & & & & & \\
\hline $\begin{array}{r}28 \\
\text { JAN } 1993\end{array}$ & 0950 & 154 & 3 & 1.0 & $\cdots$ \\
\hline$\underset{\text { MAR }}{12} \cdots$ & 1200 & 229 & 4 & 2.5 & 68 \\
\hline $23 \ldots$ & 0730 & 450 & 4 & 4.9 & 79 \\
\hline
\end{tabular}

07093700 ARKANSAS RIVER NEAR WELLSVILLE

\begin{tabular}{|c|c|c|}
\hline APR 1990 & & \\
\hline $19 \ldots$ & 1430 & 250 \\
\hline $23 \ldots$ & 1630 & 585 \\
\hline JUN & & \\
\hline $\begin{array}{l}06 \ldots \\
20 \ldots\end{array}$ & $\begin{array}{l}1700 \\
1730\end{array}$ & $\begin{array}{l}2620 \\
1690\end{array}$ \\
\hline JUL & & \\
\hline$\underset{\text { AUG }}{18} \cdots$ & 1550 & 1160 \\
\hline $\begin{array}{l}29 \ldots \\
\text { NOV }\end{array}$ & 1400 & 404 \\
\hline JAN & 0745 & 421 \\
\hline $17 \ldots$ & 0900 & 498 \\
\hline $\begin{array}{l}\text { MAR } \\
26 \ldots\end{array}$ & 1405 & 372 \\
\hline APR & & \\
\hline $\begin{array}{r}25 \ldots \\
\text { MAY }\end{array}$ & 0745 & 405 \\
\hline$\frac{15}{\text { JUN }} \cdots$ & 1620 & 1030 \\
\hline $19 \ldots$ & 1845 & 1930 \\
\hline $\begin{array}{l}\pi L \\
17 \ldots\end{array}$ & 2015 & 728 \\
\hline AUG & & \\
\hline $\begin{array}{l}15 \ldots \\
\text { OCT }\end{array}$ & 0615 & 547 \\
\hline $23 \ldots$ & 1420 & 270 \\
\hline DEC & & \\
\hline & 0900 & 500 \\
\hline $\begin{array}{l}24 \ldots \\
\text { APR }\end{array}$ & 1315 & 397 \\
\hline $22 \ldots$ & 1520 & 270 \\
\hline MAY & & \\
\hline $21 \ldots$ & 1200 & 1400 \\
\hline JUL & & \\
\hline $\begin{array}{l}14 . \cdots \\
\text { AUG }\end{array}$ & 2000 & 784 \\
\hline $\begin{array}{l}12 \ldots \\
25 \ldots\end{array}$ & $\begin{array}{l}1450 \\
1020\end{array}$ & $\begin{array}{r}742 \\
1180\end{array}$ \\
\hline OCT & & \\
\hline $\begin{array}{l}28 \\
\text { JAN }\end{array}$ & 1620 & 378 \\
\hline $12 \ldots$ & 1640 & 408 \\
\hline MAR & & \\
\hline $23 \ldots$ & 1200 & 638 \\
\hline
\end{tabular}

\begin{tabular}{|c|c|c|}
\hline 8 & 5.4 & $\cdots$ \\
\hline 120 & 190 & 58 \\
\hline $\begin{array}{r}498 \\
45\end{array}$ & $\begin{array}{r}3520 \\
205\end{array}$ & $\begin{array}{l}20 \\
27\end{array}$ \\
\hline 13 & 41 & 40 \\
\hline 4 & 4.4 & 77 \\
\hline 3 & 3.4 & $\cdots$ \\
\hline 3 & 3.9 & $\cdots$ \\
\hline 5 & 5.0 & 37 \\
\hline 5 & 5.9 & $\cdots$ \\
\hline 44 & 122 & 59 \\
\hline 50 & 261 & 22 \\
\hline 3 & 5.9 & $\cdots$ \\
\hline 7 & 10 & 51 \\
\hline 4 & 2.9 & 93 \\
\hline 4 & 5.3 & - \\
\hline 6 & 6.4 & 73 \\
\hline 5 & 3.6 & 83 \\
\hline 168 & 635 & 34 \\
\hline 5 & 9.5 & $\cdots$ \\
\hline $\begin{array}{l}12 \\
70\end{array}$ & $\begin{array}{r}23 \\
223\end{array}$ & 60 \\
\hline 3 & 3.0 & $\cdots$ \\
\hline 23 & 2.5 & 38 \\
\hline 5 & 8.6 & 73 \\
\hline
\end{tabular}


Table 63. Suspended-sediment data for selected surface-water stations on or near the Arkansas River, 1990-93--Continued

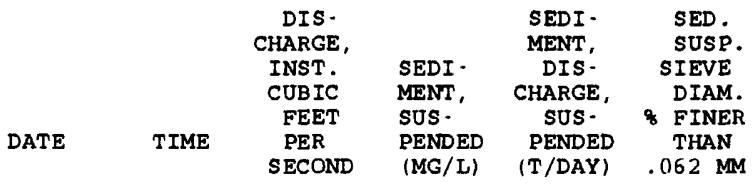

07094500

ARKANSAS RIVER AT PARKDALE
APR 1990

MAY..

$24 \ldots$

JUN

$07 \ldots$

$21 \ldots$

19.

AUG 19

30 ...

NOV

JAN 1919

JAN 1991
$17 \ldots$

APR

$25 \ldots$

$16 \ldots$

$20 \ldots$

JUL

$18 \ldots$

AUG

$15 \ldots$

OCT

DEC

$18 \ldots$

MAR 1992

24 ...

APR

$23 \ldots$

$22 \ldots$

JUN

$26 .$.

$15 \ldots$

AUG $13 .$.

$25 .$.

OCT

$29 \ldots$

Jan 1 (1)

$13 \ldots$

MAR $23 .$.

\section{4}

0900

0815

3110

$0840 \quad 1990$

$0940 \quad 1110$

$1110 \quad 395$

$1400 \quad 476$

1400

1400

1400

0830

0910

0945

194

1100

0820

1120

1630

0820

0820

0730

0900

0915

0915

1000

0700

0920

02

0815

1410

07097000

ARKANSAS RIVER AT PORTLAND

\begin{tabular}{|c|c|c|}
\hline APR 1990 & & \\
\hline MAY $\cdots$ & 1530 & 314 \\
\hline$\underset{\text { JUN }}{24} \cdots$ & 1345 & 509 \\
\hline $\begin{array}{l}07 \ldots \\
21 \ldots\end{array}$ & $\begin{array}{l}1530 \\
1400\end{array}$ & $\begin{array}{l}3210 \\
1820\end{array}$ \\
\hline JUL & & \\
\hline$\underset{A U G}{19} \cdots$ & 1345 & 1060 \\
\hline $\begin{array}{c}30 \\
\text { NOV }\end{array}$ & 1415 & 271 \\
\hline $\begin{array}{l}02 \\
\text { JAN } 1991\end{array}$ & 1015 & 514 \\
\hline$\underset{\text { MAR }}{17} \cdots$ & 1550 & 470 \\
\hline $\begin{array}{r}27 \\
\mathrm{APR}\end{array}$ & 1010 & 400 \\
\hline $\begin{array}{r}26 \\
\text { MAY }\end{array}$ & 1015 & 291 \\
\hline $16 \ldots$ & 1315 & 728 \\
\hline
\end{tabular}

$\begin{array}{rcr}26 & 22 & 76 \\ 524 & 720 & 68 \\ 897 & 7770 & 40 \\ 230 & 1130 & 24 \\ 51 & 146 & 44 \\ 5 & 3.7 & 81 \\ 39 & 54 & 43 \\ 52 & 66 & \ldots \\ 14 & 15 & 63 \\ 12 & 9.0 & \ldots \\ 130 & 256 & 40\end{array}$


Table 63. Suspended-sediment data for selected surface-water stations on or near the Arkansas River, 1990-93--Continued

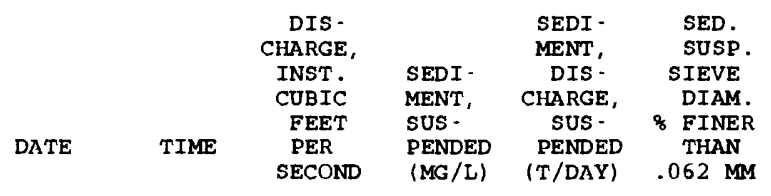

07097000 ARKANSAS RIVER AT PORTLAND--Continued

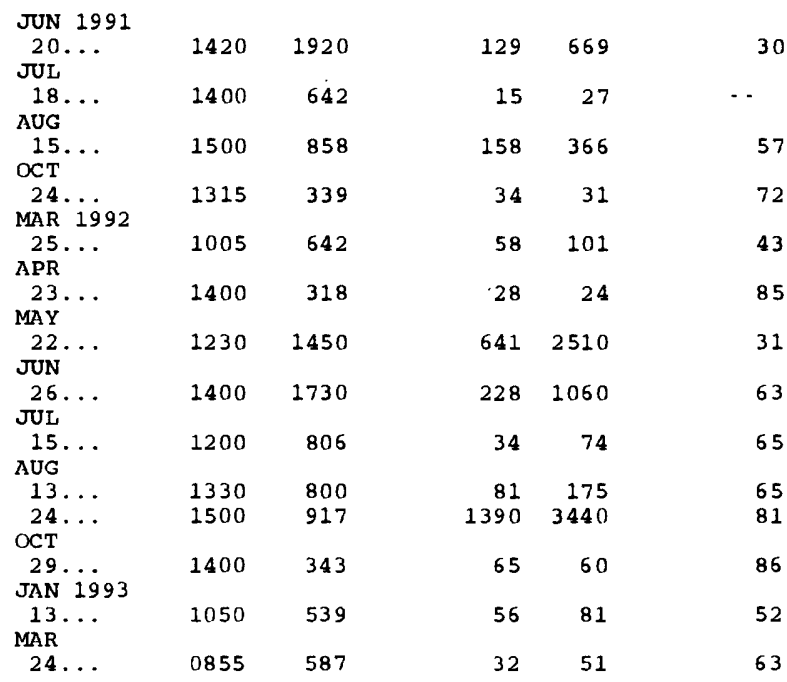

$07099400 \quad$ ARKANSAS RIVER ABOVE PUEBLO

$\begin{array}{llllll}\text { APR } 1990 & & & & & \\ 09 \ldots & 1440 & 228 & 16 & 9.8 & 54 \\ \text { JUL } & & & & & \\ 24 \ldots & 0950 & 695 & 33 & 62 & 98 \\ \text { AUG } & & & 18 & 47 & 99 \\ 20 \ldots & 1035 & 968 & & & \end{array}$

07099970 ARKANSAS RIVER AT MOFFAT STREET, AT PUEBLO

\begin{tabular}{|c|c|c|c|c|c|}
\hline $\begin{array}{c}\text { APR } 1990 \\
10 \ldots \\
\text { MAY }\end{array}$ & 0910 & 142 & 9 & 3.5 & 82 \\
\hline$\frac{14 \ldots}{\text { JUN }}$ & 1045 & 283 & 11 & 8.4 & 64 \\
\hline$\frac{11}{\pi} \cdots$ & 1130 & 3800 & 152 & 1560 & 21 \\
\hline$\underset{\text { AUG }}{24} \cdots$ & 1135 & 629 & 60 & 102 & 97 \\
\hline $28 \ldots$ & 1100 & 270 & 44 & 32 & 97 \\
\hline $\begin{array}{l}\mathrm{OCT} \\
22 \ldots\end{array}$ & 1030 & 316 & 19 & 16 & 97 \\
\hline $\begin{array}{l}\text { DEC } \\
17 \ldots \\
\text { MAR }\end{array}$ & 1030 & 33 & 18 & 1.6 & $\cdots$ \\
\hline $\begin{array}{l}27 \ldots \\
\text { APR }\end{array}$ & 1320 & 321 & 8 & 7.2 & $\cdots$ \\
\hline $\begin{array}{l}15 \ldots \\
\text { MAY }\end{array}$ & 1005 & 232 & 14 & 8.8 & 66 \\
\hline$\underset{\text { JUN }}{20} \ldots$ & 1115 & 502 & 14 & 19 & $\cdots$ \\
\hline$\frac{10 \ldots}{\pi U L}$ & 1145 & 1590 & 27 & 116 & 53 \\
\hline$\underset{A U G}{22} \cdots$ & 1040 & 1630 & 27 & 119 & 90 \\
\hline$\underset{\text { OCT }}{15}$ & 0955 & 1530 & 162 & 669 & 44 \\
\hline$\underset{D E C}{28} \cdots$ & 1140 & 94 & 17 & 4.3 & \\
\hline $16 \ldots$ & 1115 & 54 & 28 & 4.1 & 46 \\
\hline
\end{tabular}


Table 63. Suspended-sediment data for selected surface-water stations on or near the Arkansas River, 1990-93--Continued

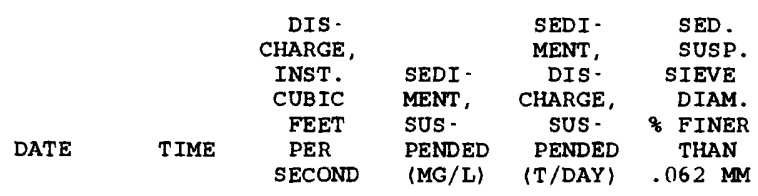

07099970 ARKANSAS RIVER AT MOFFAT STREET, AT PUEBLO--Continued

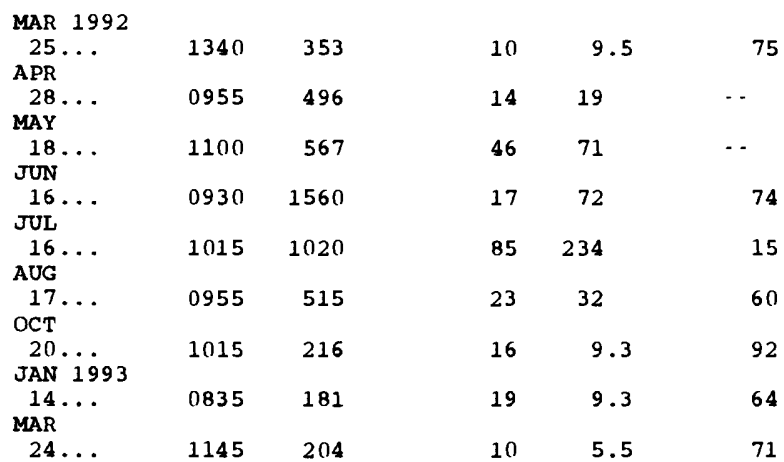

FOUNTAIN CREEK AT PUEBLO

\begin{tabular}{|c|c|c|c|c|c|}
\hline \multirow{2}{*}{\multicolumn{6}{|c|}{ APR 1990}} \\
\hline $10 \ldots$ & & & & & \\
\hline \multicolumn{6}{|l|}{ MAY } \\
\hline $14 \ldots$ & 1300 & 88 & 407 & 97 & 53 \\
\hline \multicolumn{6}{|l|}{ JUN } \\
\hline $11 \ldots$ & 1345 & 77 & 663 & 138 & 90 \\
\hline \multicolumn{6}{|l|}{ JUL } \\
\hline $24 \ldots$ & 1300 & 97 & 925 & 242 & 74 \\
\hline \multicolumn{6}{|l|}{ AUG } \\
\hline $20 \ldots$ & 1400 & 97 & 924 & 242 & 83 \\
\hline \multicolumn{6}{|l|}{ SEP } \\
\hline $05 \ldots$ & 1825 & 9.0 & 548 & 13 & 76 \\
\hline \multicolumn{6}{|l|}{ OCT } \\
\hline $22 \ldots$ & 1205 & 90 & 736 & 179 & 58 \\
\hline \multicolumn{6}{|l|}{ DEC } \\
\hline $17 \ldots$ & 1220 & 119 & 688 & 221 & 60 \\
\hline \multicolumn{6}{|l|}{ MAR 1991} \\
\hline $28 \ldots$ & 0825 & 68 & 289 & 53 & 48 \\
\hline \multicolumn{6}{|l|}{ APR } \\
\hline $15 \ldots$ & 1140 & 64 & 271 & 47 & 70 \\
\hline \multicolumn{6}{|l|}{ MAY } \\
\hline $20 \ldots$ & 1305 & 50 & 152 & 21 & 66 \\
\hline \multicolumn{6}{|l|}{ JUN } \\
\hline $10 \ldots$ & 1320 & 190 & 1020 & 523 & 63 \\
\hline \multicolumn{6}{|l|}{ JUL } \\
\hline $22 \ldots$ & 1300 & 253 & 3930 & 2680 & 85 \\
\hline \multicolumn{6}{|l|}{ AUG } \\
\hline $15 \ldots$ & 1145 & 119 & 541 & 174 & 74 \\
\hline \multicolumn{6}{|l|}{ OCT } \\
\hline $\begin{array}{r}28 \\
D E C\end{array}$ & \multicolumn{4}{|c|}{ DEC } & 28 \\
\hline $16 \ldots$ & 1315 & 114 & 663 & 204 & 50 \\
\hline \multicolumn{6}{|l|}{ MAR 1992} \\
\hline $26 \ldots$ & 0745 & 160 & 581 & 251 & 47 \\
\hline \multicolumn{6}{|l|}{ APR } \\
\hline $28 \ldots$ & 1110 & 96 & 239 & 62 & 54 \\
\hline \multicolumn{6}{|l|}{ MAY } \\
\hline $18 \ldots$ & 1220 & 32 & 45 & 3.9 & 83 \\
\hline \multicolumn{6}{|l|}{ JUN } \\
\hline $16 \ldots$ & 1100 & 96 & 223 & 58 & 62 \\
\hline \multicolumn{6}{|l|}{ JUL } \\
\hline $16 \ldots$ & 1115 & 21 & 69 & 3.9 & 58 \\
\hline \multicolumn{6}{|l|}{ AUG } \\
\hline $17 \ldots$ & 1110 & 34 & 72 & 6.6 & 87 \\
\hline OCT & & & & & \\
\hline $20 \ldots$ & 1145 & 80 & 365 & 79 & 63 \\
\hline JAN 1993 & & & & & \\
\hline $14 \ldots$ & 1000 & 100 & 436 & 118 & 15 \\
\hline MAR & & & & & \\
\hline $25 \ldots$ & 0745 & 96 & 254 & 66 & 54 \\
\hline
\end{tabular}


Table 63. Suspended-sediment data for selected surface-water stations on or near the Arkansas River, 1990-93--Continued

\begin{tabular}{|c|c|c|c|c|c|}
\hline & & $\begin{array}{c}\text { DIS - } \\
\text { CHARGE, } \\
\text { INST. } \\
\text { CUBIC }\end{array}$ & $\begin{array}{l}\text { SEDI - } \\
\text { MENT, }\end{array}$ & $\begin{array}{l}\text { SEDI - } \\
\text { MENT, } \\
\text { DIS - } \\
\text { CHARGE, }\end{array}$ & $\begin{array}{l}\text { SED. } \\
\text { SUSP. } \\
\text { SIEVE } \\
\text { DIAM. }\end{array}$ \\
\hline DATE & TIME & $\begin{array}{c}\text { FEET } \\
\text { PER } \\
\text { SECOND }\end{array}$ & $\begin{array}{l}\text { SUS - } \\
\text { PENDED } \\
\text { (MG/L) }\end{array}$ & $\begin{array}{c}\text { SUS - } \\
\text { PENDED } \\
\text { (T/DAY) }\end{array}$ & $\begin{array}{c}\text { \% FINER } \\
\text { THAN } \\
.062 \mathrm{MM}\end{array}$ \\
\hline
\end{tabular}

$07109500 \quad$ ARKANSAS RIVER NEAR AVONDALE

\begin{tabular}{|c|c|c|c|c|c|}
\hline $\begin{array}{c}\text { APR } 1990 \\
10 \ldots\end{array}$ & 1840 & 445 & 132 & 159 & 72 \\
\hline MAY & & & & & \\
\hline$\underset{\text { JUN }}{14} \cdots$ & 1750 & 634 & 154 & 264 & 58 \\
\hline$\underset{\pi U L}{12} \cdots$ & 0920 & 4000 & 501 & 5410 & 44 \\
\hline$\underset{\text { AUG }}{24} \ldots$ & 1700 & 960 & 345 & 894 & 82 \\
\hline$\underset{\text { OCT }}{20} \ldots$ & 1800 & 1170 & 408 & 1290 & 68 \\
\hline$\underset{D E C}{22} \cdots$ & 1615 & 628 & 1040 & 1760 & 10 \\
\hline MAR 17991 & 1550 & 286 & 76 & 59 & 63 \\
\hline $\begin{array}{l}28 \\
\mathrm{APR}\end{array}$ & 1150 & 510 & 102 & 140 & 45 \\
\hline $\begin{array}{l}15 \ldots \\
\text { MAY }\end{array}$ & 1615 & 430 & 59 & 68 & 43 \\
\hline$\underset{\text { JUN }}{20} \cdots$ & 1650 & E670 & 79 & $\cdots$ & 36 \\
\hline $\begin{array}{l}10 \\
\text { JUL }\end{array}$ & 1800 & 2320 & 849 & 5320 & 45 \\
\hline$\underset{A U G}{22} \cdots$ & 1830 & 1950 & 2160 & 11400 & 36 \\
\hline $\begin{array}{l}15 \cdots \\
\text { OCT }\end{array}$ & 1715 & 1750 & 249 & 1180 & 55 \\
\hline$\underset{D E C}{28} \cdots$ & 1630 & 330 & 304 & 271 & 96 \\
\hline${ }_{\text {MAR }}^{17} \ddot{1992}$ & 0805 & 299 & 819 & 661 & 14 \\
\hline$\underset{A P R}{26} \cdots$ & 1115 & 654 & 232 & 410 & 35 \\
\hline $\begin{array}{r}28 \ldots \\
\text { MAY }\end{array}$ & 1500 & 810 & 265 & 580 & 22 \\
\hline${ }_{\text {JUN }}^{18} \cdots$ & 1615 & 828 & 113 & 253 & 32 \\
\hline $16 \ldots$ & 1500 & 1720 & 163 & 757 & 38 \\
\hline$\underset{A U G}{16} \cdots$ & 1520 & 1270 & 2090 & 7170 & \\
\hline$\underset{\text { OCT }}{17 \ldots}$ & 1530 & 733 & 246 & 487 & 16 \\
\hline JAN 20.993 & 1430 & 433 & 765 & 894 & \\
\hline$\underset{M A R}{14} \cdots$ & 1330 & 444 & 848 & 1020 & \\
\hline $25 \ldots$ & 1100 & 428 & 74 & 85 & 50 \\
\hline
\end{tabular}

380715103564701 APISHAPA RIVER AT HIGHWAY 50, NEAR FOWLER

$\begin{array}{cccccc}\text { JUL } 1991 & & & & \\ 02 \ldots & 1645 & \text { E800 } & 20200 & \ldots & 96\end{array}$

07119700 ARKANSAS RIVER AT CATLIN DAM, NEAR FOWLER

\begin{tabular}{|c|c|c|c|c|c|}
\hline $\begin{array}{c}\text { APR } 1990 \\
12 \ldots\end{array}$ & 0845 & 112 & 120 & 36 & 82 \\
\hline $15 \ldots$ & 1010 & 241 & 1520 & 989 & 7 \\
\hline JUL & 0920 & 2920 & 789 & 6220 & 54 \\
\hline$\stackrel{25}{A U G}$ & 1320 & 83 & 3370 & 759 & 52 \\
\hline $21 \ldots$ & 1600 & 445 & 829 & 996 & 98 \\
\hline${ }_{D E C}^{23} \ldots$ & 1315 & 308 & 375 & 312 & 45 \\
\hline $\begin{array}{l}18 \ldots \\
\text { MAR } 1991\end{array}$ & 0945 & 258 & 147 & 102 & 44 \\
\hline $\begin{array}{l}28 \\
\text { APR }\end{array}$ & 1630 & 112 & 88 & 27 & 66 \\
\hline
\end{tabular}


Table 63. Suspended-sediment data for selected surface-water stations on or near the Arkansas River, 1990-93--Continued

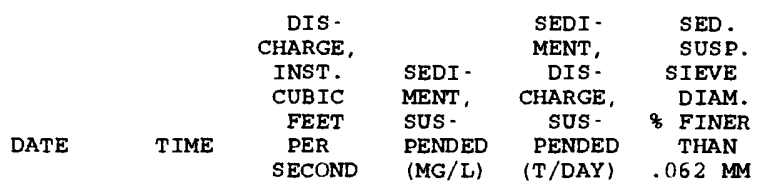

07119700

ARKANSAS RIVER AT CATLIN DAM, NEAR FOWLER--Continued

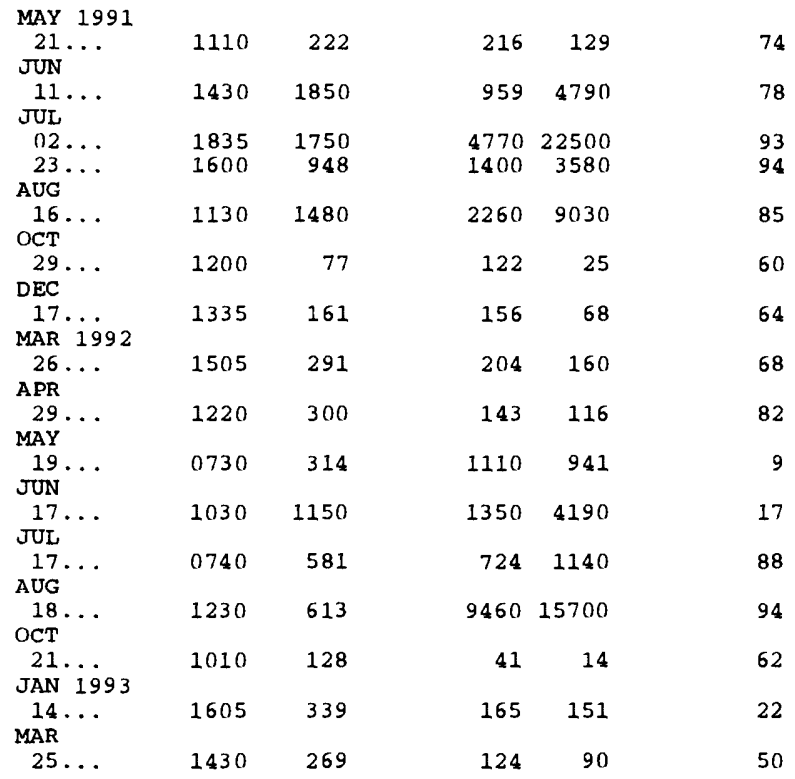

$07124000 \quad$ ARKANSAS RIVER AT LAS ANIMAS

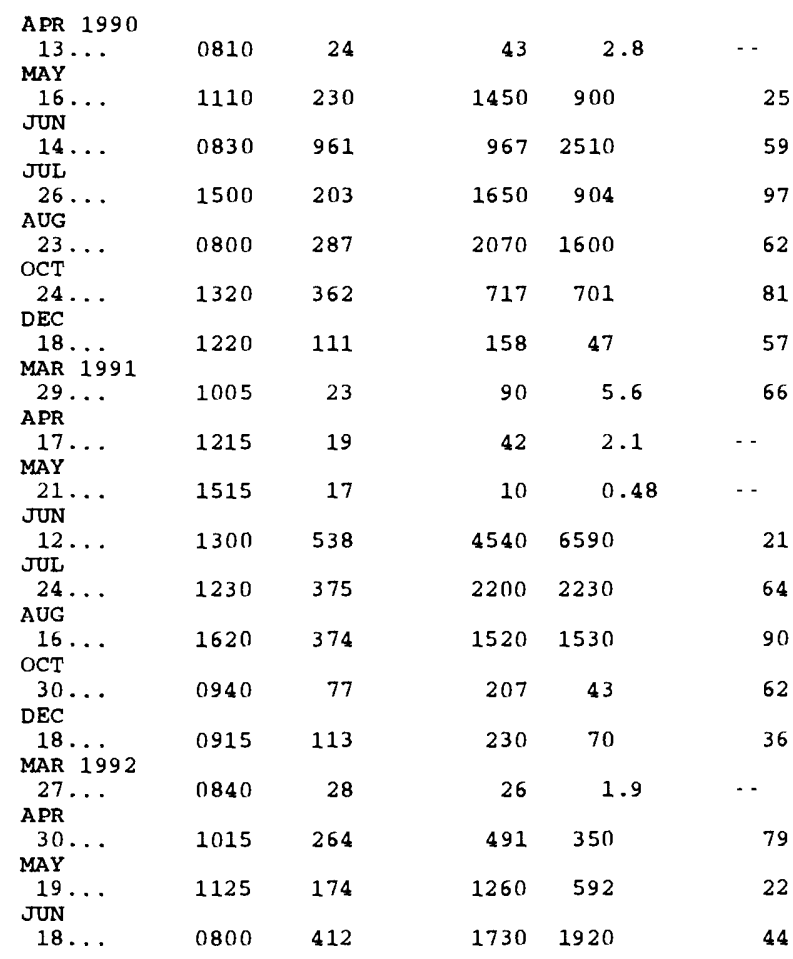


Table 63. Suspended-sediment data for selected surface-water stations on or near the Arkansas River, 1990-93--Continued

$\begin{array}{cccccc} & & \text { DIS - } & & \text { SEDI - } & \text { SED. } \\ & \text { CHARGE, } & & \text { MENT, } & \text { SUSP. } \\ & \text { INST. } & \text { SEDI - } & \text { DIS - } & \text { SIEVE } \\ & \text { CUBIC } & \text { MENT, } & \text { CHARGE, } & \text { DIAM. } \\ & \text { FEET } & \text { SUS - } & \text { SUS - } & \text { \% FINER } \\ \text { DATE } & \text { TIME } & \text { PER } & \text { PENDED } & \text { PENDED } & \text { THAN } \\ & & \text { SECOND } & (\text { MG/L) } & \text { (T/DAY) } & .062 \mathrm{MM}\end{array}$

$07124000 \quad$ ARKANSAS RIVER AT LAS ANIMAS - - Continued

$\begin{array}{llllll}\text { JUL } 1992 & & & & & \\ 17 \ldots & 1215 & 391 & 3930 & 4150 & 52 \\ \text { AUG } & & & & & \\ 19 \ldots & 1000 & 264 & 797 & 568 & 81 \\ \text { OCT } & & & & & \\ 22 \ldots & 0745 & 63 & 106 & 18 & 17 \\ \text { JAN } 1993 & & & & & \\ \begin{array}{l}15 \ldots \\ \text { MAR }\end{array} & 1140 & 395 & 126 & 134 & 44 \\ 26 \ldots & 1030 & 24 & 58 & 3.8 & 27\end{array}$

07130500 ARKANSAS RIVER BELOW JOHN MARTIN RESERVOIR

\begin{tabular}{|c|c|c|c|c|c|}
\hline \multicolumn{6}{|c|}{ APR 1990} \\
\hline $13 \ldots$ & 1115 & 1.7 & 231 & 1.1 & 38 \\
\hline \multicolumn{6}{|l|}{ MAY } \\
\hline $16 \ldots$ & 1330 & 403 & 51 & 55 & 31 \\
\hline \multicolumn{6}{|l|}{ JUN } \\
\hline $14 \ldots$ & 1300 & 595 & 34 & 55 & 62 \\
\hline \multicolumn{6}{|l|}{ JUL } \\
\hline $27 \ldots$ & 0850 & 402 & 30 & 33 & 90 \\
\hline \multicolumn{6}{|l|}{ AUG } \\
\hline $23 \ldots$ & 1130 & 444 & 9 & 11 & 79 \\
\hline \multicolumn{6}{|l|}{ OCT } \\
\hline $24 \ldots$ & 1515 & 268 & 14 & 10 & 51 \\
\hline \multicolumn{6}{|c|}{ MAR 1992} \\
\hline $27 \ldots$ & 1005 & 1.6 & 298 & 1.3 & 52 \\
\hline \multicolumn{6}{|l|}{ APR } \\
\hline $30 \ldots$ & 1200 & 610 & 242 & 399 & 8 \\
\hline \multicolumn{6}{|l|}{ MAY } \\
\hline $19 \ldots$ & 1400 & 490 & 56 & 74 & 75 \\
\hline \multicolumn{6}{|l|}{ JUN } \\
\hline $18 \ldots$ & 1100 & 411 & 276 & 306 & 62 \\
\hline \multicolumn{6}{|l|}{ JUL } \\
\hline $17 \ldots$ & 1445 & 845 & 204 & 465 & 30 \\
\hline \multicolumn{6}{|l|}{ AUG } \\
\hline $19 \ldots$ & 1145 & 389 & 44 & 46 & 94 \\
\hline \multicolumn{6}{|l|}{ OCT } \\
\hline $22 \ldots$ & 0930 & 120 & 32 & 10 & 94 \\
\hline \multicolumn{6}{|c|}{ JAN 1993} \\
\hline $15 \ldots$ & 1315 & 89 & 253 & 61 & 54 \\
\hline \multicolumn{6}{|l|}{ MAR } \\
\hline $26 \ldots$ & 1130 & 3.1 & 170 & 1.4 & 28 \\
\hline
\end{tabular}


Table 64. Quality-assurance data for source-solution blanks of deionized water for the Arkansas River Basin water-quality study, 1990-93

\begin{tabular}{|c|c|c|c|c|c|c|c|c|c|c|}
\hline DATE & TIME & $\begin{array}{c}\text { SPE - } \\
\text { CIFIC } \\
\text { CON- } \\
\text { DUCT - } \\
\text { ANCE } \\
\text { LAB } \\
\text { (US/CM) }\end{array}$ & $\begin{array}{c}\text { PH } \\
\text { WATER } \\
\text { WHOLE } \\
\text { LAB } \\
\text { (STAND - } \\
\text { ARD } \\
\text { UNITS) }\end{array}$ & $\begin{array}{l}\text { CALCIUM } \\
\text { DIS - } \\
\text { SOLVED } \\
\text { (MG/L } \\
\text { AS CA) }\end{array}$ & $\begin{array}{l}\text { MAGNE - } \\
\text { SIUM, } \\
\text { DIS - } \\
\text { SOLVED } \\
\text { (MG/L } \\
\text { AS MG) }\end{array}$ & $\begin{array}{l}\text { SODIUM, } \\
\text { DIS - } \\
\text { SOLVED } \\
\text { (MG/L } \\
\text { AS NA) }\end{array}$ & $\begin{array}{l}\text { SULFATE } \\
\text { DIS - } \\
\text { SOLVED } \\
\text { (MG/L } \\
\text { AS SO4) }\end{array}$ & $\begin{array}{l}\text { CHLO- } \\
\text { RIDE, } \\
\text { DIS- } \\
\text { SOLVED } \\
\text { (MG/I } \\
\text { AS CL) }\end{array}$ & $\begin{array}{l}\text { SOLIDS, } \\
\text { RESIDUE } \\
\text { AT } 180 \\
\text { DEG. C } \\
\text { DIS - } \\
\text { SOLVED } \\
\text { (MG/L) }\end{array}$ & $\begin{array}{c}\text { NITRO- } \\
\text { GEN, } \\
\text { NO2+NO3 } \\
\text { TOTAL } \\
\text { (MG/L } \\
\text { AS N) }\end{array}$ \\
\hline APR 1990 & & & & & & & & & & \\
\hline${ }_{\pi N}^{10} \cdots$ & 1752 & 7 & 6.3 & 0.03 & 0.02 & 1.1 & $<1.0$ & $<0.10$ & 11 & $<0.100$ \\
\hline${ }_{\pi L}^{21} \ldots$ & 1140 & 4 & 8.9 & 0.03 & 0.05 & $<0.20$ & $<1.0$ & $<0.10$ & $<1$ & $<0.100$ \\
\hline $18 \ldots$ & 1241 & 2 & 6.3 & 0.08 & $<0.02$ & $<0.20$ & $<1.0$ & 0.30 & $<1$ & 0.015 \\
\hline $\operatorname{Nov}^{28 \ldots}$ & 1121 & 2 & 6.4 & $<0.02$ & 0.06 & $<0.20$ & $<1.0$ & 0.20 & $<1$ & $<0.010$ \\
\hline $\begin{array}{l}02 \\
\text { JAN } 1991\end{array}$ & 1111 & 4 & 5.9 & 0.33 & $<0.01$ & $<0.20$ & $<1.0$ & 0.70 & 1 & $<0.010$ \\
\hline${ }_{\operatorname{MAR}}^{17} \cdots$ & 1243 & 24 & 4.4 & $<0.02$ & $<0.01$ & $<0.20$ & $<1.0$ & $<0.10$ & $<1$ & $\cdots$ \\
\hline$\underset{A P R}{26} \cdots$ & 1111 & 2 & 6.8 & 0.28 & $<0.01$ & $<0.20$ & $<1.0$ & $<0.10$ & 1 & $<0.005$ \\
\hline$\underset{\mathrm{MAY}}{23} \ldots$ & 0933 & 2 & 7.9 & $<0.02$ & 0.11 & $<0.20$ & $<0.10$ & 0.30 & 1 & $<0.005$ \\
\hline$\pi_{U N}^{14} \ldots$ & 1503 & 2 & 8.0 & 0.02 & $<0.01$ & $<0.20$ & $<0.10$ & $<0.10$ & $\cdots$ & $<0.005$ \\
\hline $\begin{array}{l}19 \ldots \\
19 \ldots\end{array}$ & $\begin{array}{l}1059 \\
1331\end{array}$ & $\begin{array}{l}1 \\
1\end{array}$ & $\begin{array}{l}7.1 \\
6.3\end{array}$ & $\begin{array}{l}0.03 \\
0.25\end{array}$ & $\begin{array}{r}0.02 \\
<0.01\end{array}$ & $\begin{array}{l}<0.20 \\
<0.20\end{array}$ & $\begin{array}{l}0.20 \\
0.30\end{array}$ & $\begin{array}{l}<0.10 \\
<0.10\end{array}$ & $\begin{array}{l}2 \\
2\end{array}$ & $\begin{array}{l}<0.005 \\
<0.005\end{array}$ \\
\hline $\begin{array}{l}\pi L \\
18 \ldots\end{array}$ & 1547 & 1 & 7.9 & 0.03 & $<0.01$ & $<0.20$ & 0.20 & $<0.10$ & 1 & $<0.005$ \\
\hline $\begin{array}{l}\text { AUG } \\
14 \ldots \\
\text { OCT }\end{array}$ & 1607 & 3 & 6.6 & 0.11 & 0.08 & 0.40 & 0.10 & $<0.10$ & 2 & $<0.005$ \\
\hline${ }_{\mathrm{DEC}}^{24 \ldots}$ & 0949 & 1 & 6.4 & $<0.02$ & $<0.01$ & $<0.20$ & 0.20 & 0.10 & $<1$ & $<0.005$ \\
\hline MAR 18 1992 & 1047 & 2 & 6.4 & $<0.02$ & $<0.01$ & $<0.20$ & $<0.10$ & $<0.10$ & 1 & $\cdots$ \\
\hline$\underset{A P R}{24 \cdots}$ & 1057 & 1 & 6.7 & $<0.02$ & $<0.01$ & $<0.20$ & $<0.10$ & $<0.10$ & 2 & 0.014 \\
\hline$\underset{\mathrm{MAY}}{23} \ldots$ & 1449 & 2 & 8.2 & $<0.02$ & $<0.01$ & $<0.20$ & $<0.10$ & 0.70 & $<1$ & $<0.005$ \\
\hline$\pi_{U N}^{22 \ldots}$ & 0951 & 1 & 8.1 & 0.16 & $<0.01$ & $<0.20$ & $<0.10$ & 0.30 & $<1$ & 0.015 \\
\hline$\pi^{26} \ldots$ & 1047 & 1 & 8.1 & $<0.02$ & $<0.01$ & $<0.20$ & 0.20 & 0.30 & $<1$ & $<0.005$ \\
\hline${ }_{\text {AUG }}^{13} \cdots$ & 1333 & 4 & 8.0 & $<0.02$ & $<0.01$ & $<0.20$ & $<0.10$ & 0.10 & 2 & $<0.005$ \\
\hline $\mathrm{OCT}^{11 \cdots}$ & 1423 & 2 & 7.5 & $<0.02$ & $<0.01$ & $<0.20$ & $<0.10$ & $<0.10$ & $<1$ & $<0.005$ \\
\hline JAN 1993 & 0952 & 2 & 7.9 & $<0.02$ & $<0.01$ & $<0.20$ & $<0.10$ & 0.10 & 1 & $<0.005$ \\
\hline $\operatorname{MAR}^{13 \ldots}$ & 1149 & 2 & 5.3 & $<0.02$ & $<0.01$ & $<0.20$ & 0.30 & 0.30 & $<1$ & $\cdots$ \\
\hline $23 \ldots$ & 1051 & 2 & 5.9 & 0.02 & $<0.01$ & $<0.20$ & $<0.10$ & $<0.10$ & $<1$ & $\cdots$ \\
\hline
\end{tabular}

\begin{tabular}{|c|c|c|c|c|c|c|c|c|c|}
\hline ATE & $\begin{array}{c}\text { NITRO - } \\
\text { GEN, } \\
\text { NO2+NO3 } \\
\text { DIS - } \\
\text { SOLVED } \\
\text { (MG / L }\end{array}$ & $\begin{array}{l}\text { NITRO- } \\
\text { GEN, } \\
\text { AMMONIA } \\
\text { TOTAL } \\
\text { (MG/L }\end{array}$ & $\begin{array}{l}\text { NITRO- } \\
\text { GEN, } \\
\text { AMMONIA } \\
\text { DIS- } \\
\text { SOLVED } \\
\text { (MG / L }\end{array}$ & $\begin{array}{l}\text { CADMIUM } \\
\text { DIS - } \\
\text { SOLVED } \\
\text { (UG/L }\end{array}$ & $\begin{array}{l}\text { COPPER, } \\
\text { DIS - } \\
\text { SOLVED } \\
\text { (UG/L }\end{array}$ & $\begin{array}{l}\text { IRON, } \\
\text { DIS - } \\
\text { SOLVED } \\
\text { (UG/L }\end{array}$ & $\begin{array}{l}\text { LEAD, } \\
\text { DIS- } \\
\text { SOLVED } \\
\text { (UG/L }\end{array}$ & $\begin{array}{l}\text { MANGA - } \\
\text { NESE, } \\
\text { DIS - } \\
\text { SOLVED } \\
\text { (UG/L }\end{array}$ & $\begin{array}{l}\text { ZINC, } \\
\text { DIS - } \\
\text { SOLVED } \\
\text { IUG/L }\end{array}$ \\
\hline & AS N) & AS N) & AS N) & & & AS FE) & AS PB) & & \\
\hline
\end{tabular}

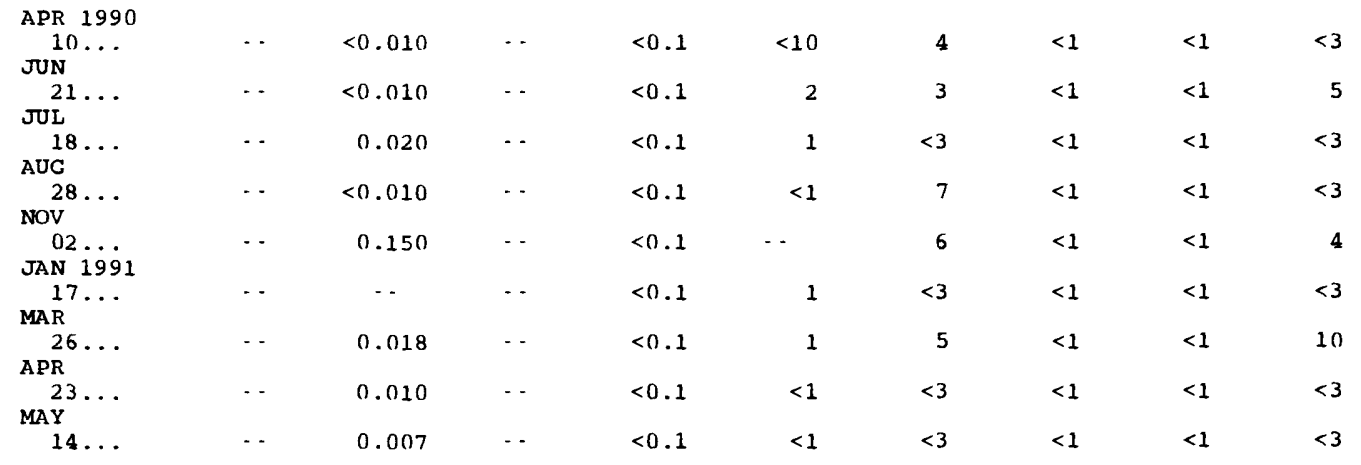


Table 64. Quality-assurance data for source-solution blanks of deionized water for the Arkansas River Basin water-quality study, 1990-93--Continued

\begin{tabular}{|c|c|c|c|c|c|c|c|c|c|}
\hline DATE & $\begin{array}{c}\text { NITRO- } \\
\text { GEN, } \\
\text { NO2+NO3 } \\
\text { DIS- } \\
\text { SOLVED } \\
\text { (MG/L } \\
\text { AS N) }\end{array}$ & $\begin{array}{l}\text { NITRO- } \\
\text { GEN, } \\
\text { AMMONIA } \\
\text { TOTAL } \\
\text { (MG/L } \\
\text { AS N) }\end{array}$ & $\begin{array}{c}\text { NITRO- } \\
\text { GEN, } \\
\text { AMMONIA } \\
\text { DIS - } \\
\text { SOLVED } \\
\text { (MG/L } \\
\text { AS N) }\end{array}$ & $\begin{array}{l}\text { CADMIUM } \\
\text { DIS - } \\
\text { SOLVED } \\
\text { (UG/L } \\
\text { AS CD) }\end{array}$ & $\begin{array}{l}\text { COPPER, } \\
\text { DIS - } \\
\text { SOLVED } \\
\text { (UG /L } \\
\text { AS CU) }\end{array}$ & $\begin{array}{l}\text { IRON, } \\
\text { DIS- } \\
\text { SOLVED } \\
\text { (UG/L } \\
\text { AS FE) }\end{array}$ & $\begin{array}{l}\text { LEAD, } \\
\text { DIS- } \\
\text { SOLVED } \\
\text { (UG/L } \\
\text { AS PB) }\end{array}$ & $\begin{array}{l}\text { MANGA - } \\
\text { NESE, } \\
\text { DIS - } \\
\text { SOLVED } \\
\text { (UG/L } \\
\text { AS MN) }\end{array}$ & $\begin{array}{l}\text { ZINC, } \\
\text { DIS- } \\
\text { SOLVED } \\
\text { (UG/L } \\
\text { AS ZN) }\end{array}$ \\
\hline JUN 1991 & & & & & & & & & \\
\hline $19 \ldots$ & $\cdots$ & 0.016 & $\cdots$ & $<0.1$ & 1 & $<3$ & $<1$ & $<1$ & 6 \\
\hline$\pi_{\pi L}^{19} \cdots$ & $\cdots$ & 0.014 & $\cdots$ & $<0.1$ & 2 & $<3$ & $<1$ & $<1$ & 5 \\
\hline$\underset{A U G}{18 \ldots}$ & $\cdots$ & 0.002 & $\cdots$ & $<0.1$ & 12 & 5 & 4 & $<1$ & 6 \\
\hline $\begin{array}{c}14 \ldots \\
\text { OCT }\end{array}$ & $\cdots$ & $<0.002$ & $\cdots$ & $<0.1$ & $<1$ & 5 & $<1$ & $<1$ & $<3$ \\
\hline $\begin{array}{c}24 \ldots \\
D E C\end{array}$ & $\cdots$ & 0.013 & $\cdots$ & $<0.1$ & $<1$ & $<3$ & $<1$ & $<1$ & $<3$ \\
\hline $\begin{array}{l}18 \ldots \\
\operatorname{MAR} 1992\end{array}$ & $\cdots$ & $-\cdot$ & $\cdots$ & $<0.1$ & $<1$ & $<3$ & $<1$ & $<1$ & $<3$ \\
\hline$\underset{A P R}{24 \ldots}$ & $\cdots$ & 0.010 & $\cdots$ & $<0.1$ & $<1$ & $<3$ & $<1$ & $<1$ & $<3$ \\
\hline $23 \ldots$ & $\cdots$ & 0.005 & $\cdots$ & $<0.1$ & $<1$ & $<3$ & $<1$ & $<1$ & $<3$ \\
\hline$\underset{\text { JUN }}{22}$ & $\cdots$ & 0.003 & - - & $<0.1$ & $<1$ & $<3$ & $<1$ & $<1$ & $<3$ \\
\hline${ }_{\pi L}^{26 \ldots}$ & $\cdots$ & $<0.002$ & $\cdots$ & $<0.1$ & $<1$ & $<3$ & $<1$ & $<1$ & $<3$ \\
\hline$\underset{A \cup G}{13} \ldots$ & $\cdots$ & $<0.002$ & $\cdots$ & $<0.1$ & $<1$ & $<3$ & $<1$ & $<1$ & $<3$ \\
\hline${ }_{\mathrm{OCT}}^{11 \ldots}$ & - & 0.003 & $\cdots$ & $<0.1$ & $<1$ & $<3$ & $<1$ & $<1$ & $<3$ \\
\hline $\begin{array}{l}28 \ldots \\
\text { JAN } 1993\end{array}$ & $\cdots$ & 0.004 & $\cdots$ & $<0.1$ & $<1$ & $<3$ & $<1$ & $<1$ & $<3$ \\
\hline $13 \ldots$ & $<0.005$ & $\cdots$ & 0.011 & $<0.1$ & $<1$ & $<3$ & $<1$ & $<1$ & $<3$ \\
\hline $23 \ldots$ & $<0.005$ & $\cdots$ & $<0.002$ & $<0.1$ & $<1$ & $<3$ & $<1$ & $<1$ & $<3$ \\
\hline
\end{tabular}


Table 65. Quality-assurance data for field-equipment blanks for the Arkansas River Basin waterquality study, 1990-93

\begin{tabular}{|c|c|c|c|c|c|c|c|c|c|c|}
\hline DATE & TIME & $\begin{array}{c}\text { SPE - } \\
\text { CIFIC } \\
\text { CON- } \\
\text { DUCT - } \\
\text { ANCE } \\
\text { LAB } \\
\text { (US/CM) }\end{array}$ & $\begin{array}{c}\text { PH } \\
\text { WATER } \\
\text { WHOLE } \\
\text { LAB } \\
\text { (STAND - } \\
\text { ARD } \\
\text { UNITS) }\end{array}$ & $\begin{array}{l}\text { CALCIUM } \\
\text { DIS - } \\
\text { SOLVED } \\
\text { (MG/L } \\
\text { AS CA) }\end{array}$ & $\begin{array}{l}\text { MAGNE- } \\
\text { SIUM, } \\
\text { DIS- } \\
\text { SOLVED } \\
\text { (MG/L } \\
\text { AS MG) }\end{array}$ & $\begin{array}{l}\text { SODIUM, } \\
\text { DIS - } \\
\text { SOLVED } \\
\text { (MG/L } \\
\text { AS NA) }\end{array}$ & $\begin{array}{c}\text { SULFATE } \\
\text { DIS - } \\
\text { SOLVED } \\
\text { (MG/L } \\
\text { AS SO4) }\end{array}$ & $\begin{array}{l}\text { CHLO- } \\
\text { RIDE, } \\
\text { DIS - } \\
\text { SOLVED } \\
\text { (MG/L } \\
\text { AS CL) }\end{array}$ & $\begin{array}{l}\text { SOLIDS, } \\
\text { RESIDUE } \\
\text { AT } 180 \\
\text { DEG. C } \\
\text { DIS - } \\
\text { SOLVED } \\
\text { (MG/L) }\end{array}$ & $\begin{array}{c}\text { NITRO- } \\
\text { GEN, } \\
\text { NO2+NO3 } \\
\text { TOTAL } \\
\text { (MG/L } \\
\text { AS N) }\end{array}$ \\
\hline \multicolumn{11}{|l|}{ APR 1990} \\
\hline $09 \ldots$ & 0600 & 6 & 7.4 & 0.42 & 0.05 & 0.80 & $<1.0$ & $<0.10$ & $<1$ & $<0.100$ \\
\hline $10 \ldots$ & 1803 & 7 & 6.8 & 0.09 & 0.05 & 1.1 & $<1.0$ & 0.80 & 5 & $<0.100$ \\
\hline \multicolumn{11}{|l|}{ MAY } \\
\hline $16 \ldots$ & 1703 & 12 & 7.9 & 0.57 & 0.25 & 0.60 & 2.1 & 0.50 & $<1$ & $<0.100$ \\
\hline $24 \ldots$ & 1018 & 42 & 4.5 & 0.10 & 0.08 & $<0.20$ & $<1.0$ & 5.3 & $<1$ & $<0.100$ \\
\hline \multicolumn{11}{|l|}{ JUN } \\
\hline $07 \ldots$ & 1717 & 7 & 7.2 & 0.10 & 0.03 & $<0.20$ & $<1.0$ & 1.4 & $<1$ & $<0.100$ \\
\hline $21 \ldots$ & 1120 & 18 & 4.9 & 0.32 & 0.04 & $<0.20$ & $<1.0$ & 3.0 & 1 & $<0.100$ \\
\hline \multicolumn{11}{|l|}{ JUL } \\
\hline $18 \ldots$ & 1213 & 8 & 6.7 & 0.17 & 0.02 & $<0.20$ & $<1.0$ & 1.4 & $<1$ & 0.018 \\
\hline \multicolumn{11}{|l|}{ AUG } \\
\hline $28 \ldots$ & 1117 & 13 & 6.1 & 0.11 & 0.08 & $<0.20$ & $<1.0$ & 2.9 & $<1$ & $<0.010$ \\
\hline \multicolumn{11}{|l|}{ NOV } \\
\hline \multicolumn{11}{|l|}{ JAN 1991} \\
\hline $17 \ldots$ & 1239 & 1 & 7.6 & 0.03 & $<0.01$ & $<0.20$ & $<1.0$ & 2.4 & $<1$ & $\cdots$ \\
\hline \multicolumn{11}{|l|}{ MAR } \\
\hline $26 \ldots$ & 1117 & 50 & 4.2 & 0.17 & 0.14 & $<0.20$ & 0.10 & 5.4 & 4 & $<0.005$ \\
\hline \multicolumn{11}{|l|}{$\mathrm{APR}$} \\
\hline $23 \ldots$ & 0937 & 0 & 3.7 & 0.09 & $<0.01$ & $<0.20$ & $<0.10$ & 9.1 & 1 & 0.006 \\
\hline \multicolumn{11}{|l|}{ MAY } \\
\hline $14 \ldots$ & 1507 & 5 & 6.7 & 0.03 & $<0.01$ & $<0.20$ & 1.0 & 0.60 & 1 & $<0.005$ \\
\hline \multicolumn{11}{|l|}{ JUN } \\
\hline $19 \ldots$ & 1057 & 3 & 5.8 & 0.03 & $<0.01$ & $<0.20$ & $<0.10$ & 0.20 & 6 & $<0.005$ \\
\hline $19 \ldots$ & 1344 & 56 & 4.0 & 0.31 & 0.05 & $<0.20$ & 0.10 & 6.4 & 6 & $<0.005$ \\
\hline \multicolumn{11}{|l|}{ JUL } \\
\hline $18 \ldots$ & 1401 & 4 & 8.0 & 0.05 & $<0.01$ & $<0.20$ & 0.60 & 0.20 & 1 & 0.011 \\
\hline \multicolumn{11}{|l|}{ AUG } \\
\hline $14 \ldots$ & 1611 & 8 & 5.6 & 0.25 & $<0.01$ & 0.40 & 0.20 & 1.2 & 3 & 0.011 \\
\hline \multicolumn{11}{|l|}{ OCT } \\
\hline $24 \ldots$ & 0947 & 4 & 6.6 & 0.06 & $<0.01$ & $<0.20$ & 0.20 & 0.50 & $<1$ & 0.005 \\
\hline \multicolumn{11}{|l|}{ DEC } \\
\hline $18 \ldots$ & 1051 & 15 & 5.6 & 0.05 & 0.04 & $<0.20$ & $<0.10$ & 1.2 & 3 & $\cdots$ \\
\hline \multicolumn{11}{|l|}{ MAR 1992} \\
\hline $24 \ldots$ & 0909 & 6 & 6.4 & 0.09 & 0.05 & $<0.20$ & 0.20 & 1.1 & 4 & 0.019 \\
\hline \multicolumn{11}{|l|}{ APR } \\
\hline $23 \ldots$ & 1447 & 3 & 7.5 & 0.02 & $<0.01$ & $<0.20$ & $<0.10$ & 0.60 & $<1$ & $<0.005$ \\
\hline \multicolumn{11}{|l|}{ MAY } \\
\hline $22 \ldots$ & 0949 & 5 & 6.3 & 0.04 & $<0.01$ & $<0.20$ & $<0.10$ & 2.0 & $<1$ & 0.009 \\
\hline JUN & & & & & & & & & & \\
\hline $26 \ldots$ & 1049 & 2 & 7.6 & 0.03 & $<0.01$ & $<0.20$ & 0.20 & 0.50 & $<1$ & $<0.005$ \\
\hline JUL & 1337 & $\cdots$ & $\cdots$ & 0.20 & & & & & & \\
\hline$\underset{A U G}{13 \ldots}$ & & & & 0.210 & $<0.10$ & $<0.10$ & $\cdots$ & $\cdots$ & $\cdots$ & $<0.005$ \\
\hline $11 \ldots$ & 1417 & 28 & 4.6 & 0.03 & $<0.01$ & $<0.20$ & $<0.10$ & 3.5 & $<1$ & $<0.005$ \\
\hline $26 \ldots$ & 1047 & 2 & 6.3 & $<0.02$ & $<0.01$ & $<0.20$ & 0.10 & 0.60 & $<1$ & $<0.005$ \\
\hline Ост & & & & & & & & & & \\
\hline $28 \ldots$ & 0957 & 4 & 8.1 & 0.03 & $<0.01$ & $<0.20$ & $<0.10$ & 0.50 & $<1$ & 0.005 \\
\hline JAN 1993 & & & & & & & & & & \\
\hline $13 \ldots$ & 1151 & 24 & 4.3 & 0.05 & $<0.01$ & $<0.20$ & $\cdots$ & $\cdots$ & $<1$ & 0.009 \\
\hline MAR & & & & & & & & & & \\
\hline $23 \ldots$ & 1113 & 34 & 4.1 & 0.14 & $<0.01$ & $<0.20$ & $<0.10$ & 3.2 & 6 & $\cdots$ \\
\hline
\end{tabular}


Table 65. Quality-assurance data for field-equipment blanks for the Arkansas River Basin waterquality study, 1990-93---Continued

\begin{tabular}{|c|c|c|c|c|c|c|c|c|c|}
\hline DATE & $\begin{array}{l}\text { NITRO- } \\
\text { GEN, } \\
\text { NO2+NO3 } \\
\text { DIS - } \\
\text { SOLVED } \\
\text { (MG / L }\end{array}$ & $\begin{array}{l}\text { NITRO- } \\
\text { GEN, } \\
\text { AMMONIA } \\
\text { TOTAL } \\
\text { (MG/L }\end{array}$ & $\begin{array}{l}\text { NITRO- } \\
\text { GEN, } \\
\text { AMMONIA } \\
\text { DIS- } \\
\text { SOLVED } \\
\text { (MG/L }\end{array}$ & $\begin{array}{l}\text { CADMIUM } \\
\text { DIS - } \\
\text { SOLVED } \\
\text { (UG/L }\end{array}$ & $\begin{array}{l}\text { COPPER, } \\
\text { DIS - } \\
\text { SOLVED } \\
\text { (UG / L }\end{array}$ & $\begin{array}{l}\text { IRON, } \\
\text { DIS - } \\
\text { SOLVED } \\
\text { IUG/L }\end{array}$ & $\begin{array}{l}\text { LEAD, } \\
\text { DIS - } \\
\text { SOLVED } \\
\text { (UG/L }\end{array}$ & $\begin{array}{l}\text { MANGA - } \\
\text { NESE , } \\
\text { DIS - } \\
\text { SOLVED } \\
\text { (UG/L }\end{array}$ & $\begin{array}{l}\text { ZINC, } \\
\text { DIS - } \\
\text { SOLVED } \\
\text { (UG /L }\end{array}$ \\
\hline & AS N) & AS NI & AS $\mathrm{N}$ ) & & & & & & AS $\mathrm{zN}$ ) \\
\hline \multicolumn{10}{|l|}{ APR 1990} \\
\hline $09 \ldots$ & $\cdots$ & $<0.010$ & $\cdots$ & 0.4 & $<10$ & 13 & $<1$ & 2 & 16 \\
\hline $10 \ldots$ & $\cdots$ & 0.030 & $\cdots$ & 0.6 & $<10$ & 50 & 1 & $<1$ & 18 \\
\hline \multicolumn{10}{|l|}{ MAY } \\
\hline $16 \ldots$ & $\cdots$ & 0.020 & $\cdots$ & $\cdots$ & 2 & 4 & 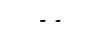 & $<1$ & 14 \\
\hline $24 \ldots$ & $\cdots$ & $<0.010$ & $\cdots$ & $<0.1$ & 2 & 13 & $<1$ & 2 & 14 \\
\hline \multicolumn{10}{|l|}{ JUN } \\
\hline $07 \ldots$ & $\cdots$ & 0.020 & $\cdots$ & 0.2 & 200 & 58 & 390 & 3 & 35 \\
\hline $21 \ldots$ & $\cdots$ & $<0.010$ & $\cdots$ & 1.1 & 260 & 24 & 160 & 2 & 100 \\
\hline \multicolumn{10}{|l|}{ JUL } \\
\hline $18 \ldots$ & $\cdots$ & 0.040 & $\cdots$ & 0.3 & 7 & 10 & 4 & $<1$ & 39 \\
\hline \multicolumn{10}{|l|}{ AUG } \\
\hline $28 \ldots$ & $\cdots$ & 0.020 & $\cdots$ & 0.4 & 2 & 19 & 2 & 1 & 30 \\
\hline \multicolumn{10}{|l|}{ NOV } \\
\hline $02 \ldots$ & $\cdots$ & 0.160 & $\cdots$ & 0.2 & $\cdots$ & 4 & $<1$ & $<1$ & 23 \\
\hline \multicolumn{10}{|l|}{ JAN 1991} \\
\hline $17 \ldots$ & $\cdots$ & $\cdots$ & $\cdots$ & $<0.1$ & $<1$ & $<3$ & $<1$ & $<1$ & 5 \\
\hline \multicolumn{10}{|l|}{ MAR } \\
\hline $26 \ldots$ & $\cdots$ & 0.036 & $\cdots$ & $<0.1$ & 1 & 10 & $<1$ & 2 & 13 \\
\hline \multicolumn{10}{|l|}{ APR } \\
\hline $23 \ldots$ & $\cdots$ & 0.011 & $\cdots$ & $<0.1$ & 1 & 10 & $<1$ & 3 & 39 \\
\hline \multicolumn{10}{|l|}{ MAY } \\
\hline $14 \ldots$ & $\cdots$ & 0.020 & $\cdots$ & $<0.1$ & 3 & 7 & $<1$ & 1 & 5 \\
\hline \multicolumn{10}{|l|}{ JUN } \\
\hline $19 \ldots$ & $\cdots$ & 0.007 & $\cdots$ & 0.1 & 100 & 4 & 12 & $<1$ & 10 \\
\hline $19 \ldots$ & $\cdots$ & 0.015 & $\cdots$ & $<0.1$ & 14 & 21 & 1 & $<1$ & 17 \\
\hline \multicolumn{10}{|l|}{ JOL } \\
\hline $18 \ldots$ & $\cdots$ & 0.009 & $\cdots$ & $<0.1$ & $<1$ & 4 & $<1$ & $<1$ & 7 \\
\hline \multicolumn{10}{|l|}{ AUG } \\
\hline $14 \ldots$ & $\cdots$ & $<0.002$ & $\cdots$ & $<0.1$ & $<1$ & $<3$ & $<1$ & $<1$ & 6 \\
\hline \multicolumn{10}{|l|}{ OCT } \\
\hline $24 \ldots$ & $\cdots$ & 0.016 & $\cdots$ & $<0.1$ & $<1$ & 3 & 1 & $<1$ & 5 \\
\hline \multicolumn{10}{|l|}{ DEC } \\
\hline $18 \ldots$ & $\cdots$ & $\cdots$ & $\cdots$ & 0.2 & $<1$ & $<3$ & 1 & $<1$ & $<3$ \\
\hline \multicolumn{10}{|l|}{ MAR 1992} \\
\hline $24 \ldots$ & $\cdots$ & 0.012 & $\cdots$ & 0.2 & $<1$ & $<3$ & $<1$ & $<1$ & 5 \\
\hline \multicolumn{10}{|l|}{ APR } \\
\hline $23 \ldots$ & $\cdots$ & 0.005 & $\cdots$ & $<0.1$ & $<1$ & $<3$ & $<1$ & $<1$ & $<3$ \\
\hline MAY & & & & & & & & & \\
\hline $22 \ldots$ & $\cdots$ & 0.008 & $\cdots$ & 0.3 & $<1$ & $<3$ & 3 & $<1$ & $<3$ \\
\hline JUN & & & & & & & & & \\
\hline $26 \ldots$ & $\cdots$ & $<0.002$ & $\cdots$ & $<0.1$ & 14 & $<3$ & $<1$ & $<1$ & 4 \\
\hline JUL & & & & & & & & & \\
\hline $13 \ldots$ & $\cdots$ & 0.010 & $\cdots$ & $<0.1$ & $<1$ & 10 & $<1$ & $<10$ & $<10$ \\
\hline AUG & & & & & & & & & \\
\hline $11 \ldots$ & $\cdots$ & 0.002 & $\cdots$ & $<0.1$ & $<1$ & $<3$ & 1 & $<1$ & 4 \\
\hline $26 \ldots$ & $\cdots$ & 0.006 & $\cdots$ & $<0.1$ & $<1$ & $<3$ & $<1$ & $<1$ & 3 \\
\hline OCT & & & & & & & & & \\
\hline $28 \ldots$ & $\cdots$ & 0.007 & - & $<0.1$ & $<1$ & $<3$ & 1 & $<1$ & $<3$ \\
\hline $13 \ldots$ & 0.009 & $\cdots$ & 0.011 & 0.3 & $<1$ & $<3$ & $<1$ & $<1$ & $<3$ \\
\hline MAR & & & & & & & & & \\
\hline $23 \ldots$ & $<0.005$ & $\cdots$ & $<0.002$ & $<0.1$ & $<1$ & $<3$ & $<1$ & $<1$ & 3 \\
\hline
\end{tabular}

